\title{
Nach dem Hochwasser ist vor dem Hochwasser: Eine Analyse zur Rationalität politischer Entscheidungsprozesse
}

\section{(Deutschland und Vietnam im späten 20. Jahrhundert)}

\author{
Dissertation \\ zur Erlangung des Doktorgrades \\ der Fakultät für Agrarwissenschaften \\ der Georg-August-Universität Göttingen \\ vorgelegt von
}

Nicole Kronenberg

geboren in Düren, NRW

Göttingen, November 2013 


\section{7}

1. Referentin/Referent: Prof. Dr. R. Marggraf

2. Korreferentin/Korreferent: Prof. Dr. K.-H. Pörtge

3. Korreferentin/Korreferent: Prof. Dr. B. Herrmann

Tag der mündlichen Prüfung: 25. November 2013 


\section{Erklärungen}

1. Hiermit erkläre ich, dass diese Arbeit weder in gleicher noch in ähnlicher Form bereits anderen Prüfungsbehörden vorgelegen hat.

Weiter erkläre ich, dass ich mich an keiner anderen Hochschule um einen Doktorgrad beworben habe.

Göttingen, den

(Unterschrift)

2. Hiermit erkläre ich eidesstattlich, dass diese Dissertation selbständig und ohne unerlaubte Hilfe angefertigt wurde.

Göttingen, den

(Unterschrift) 


\section{Zusammenfassung der Arbeit}

Im Zentrum dieser umwelthistorischen Untersuchung steht die Frage, ob die Reaktionen von politischen Entscheidungsträgern auf Überschwemmungsprobleme rational verliefen. Dazu werden zwei politische Organisationsansätze gegenübergestellt: Der zentralstaatlich organisierte Hochwasserschutz in An Giang, Vietnam wird mit föderalistischen Ansätze im Kölner Stadtgebiet kontrastiert.

Der Betrachtungsrahmen wird zwischen der multidimensionalen Struktur der Wohlfahrtsökonomie und der ethische Theorie des sozialen Subjektivismus aufgespannt. Die grundlegende Prämisse des sozialen Subjektivismus besteht im Transformationscharakter aller Wertungen in Aussagen über alle individuellen Präferenzen. Diese ethische Theorie dient als Grundlage, die Präferenzen aller Akteure gleichgestellt zu betrachten. Um die rezenten Präferenzen der politisch Verantwortlichen zu rekonstruieren, wurden die Entscheidungsprozesse aus Archivmaterial herausgearbeitet. Eine Hilfestellung, um das zentrale Instrument der Wohlfahrtsökonomie zur Bewertung der vernünftigsten Alternative, die Kosten-Nutzen-Analyse, in eine Diskursanalyse zu überführen, gibt Schnädelbachs Rationalitätsansatz, der die Verständlichmachung interpersoneller Kommunikation fokussiert. Die Analyse der politischen Auseinandersetzungen soll einen Beitrag leisten, zu erklären, wie Naturgefahren politische Entscheidungen beeinflussen, da diese vor dem Hintergrund zukünftiger Klimawandelszenarien häufiger werden.

Aus dem Diskurs zur Bewahrung der Stadtansicht von Köln mit einem Schwerpunkt auf Heimatdiskursen werden empirische Beispiele für die Wertvorstellungen politischer Entscheidungsträger gegeben. Unter Einbezug des Vulnerabilitätsansatzes wird gezeigt, wie negative Befürchtungen über zukünftige Hochwasserkosten in dem Konflikt zwischen ungeklärten Schuld- bzw. Verantwortungsfragen das Reaktionsvermögen der politisch Verantwortlichen schrumpfen bzw. wachsen ließ. In Vietnam stiftete die äußere Natur Identität für die lokale Bevölkerung. Die vietnamesische Regierung begriff die Umstrukturierung der äußeren Natur als einflussreiches Instrument, neben der Steigerung der landwirtschaftlichen Produktion auch die innere Natur der Menschen zu formen und ihnen eine neue Identität zu verleihen. Die Reaktionen der Entscheidungsträger des föderalistischen und des zentralistischen Systems verliefen verständlich. Paradoxerweise zeigte sich die Rationalität hinter den getroffenen Entscheidungen nachvollziehbar, obwohl durch die spezifischen Präferenzen der politischen Akteure monetär unbewertbare Kriterien ins Zentrum der Aushandlungsprozesse gestellt wurden. 
Die Erscheinung

ist vom Betrachter

nicht losgelöst, viel-

mehr in die Individualität

desselben verschlungen und

verwickelt!

Johann Wolfgang von Goethe

Diese Promotion wird als interdisziplinäres Projekt aus verschiedenen Fachperspektiven betrachtet. Die vielfältigen Betrachtungsweisen meiner Betreuer haben mich in den letzten drei Jahren angeleitet, meine persönliche Sicht weiter zu fassen. Hier ist zuallererst mein Doktorvater Rainer Marggraf zu nennen, der mich unterstützte, ökonomische Fragestellungen methodischkonzeptionell weiterzuentwickeln. Daneben stand mir Karl-Heinz Pörtge immer hilfsbereit als Experte in der historischen Aufarbeitung von Überschwemmungen zur Seite. Bernd Herrmann, als Ziehvater der Umweltgeschichte in Göttingen, sah in mir das Potential, mich von meinem bisherigen ingenieurswissenschaftlichen Arbeitsansatz $\mathrm{zu}$ lösen, und stattdessen Umweltgeschichte zu re-,,konstruieren“. Renate Bürger-Arndt begleitete mich bei der Fortentwicklung des Theoriekonzepts zur Anwendung in der Fallstudie zu Vietnam und Manfred Jakubowski-Tiessen unterstützte mich, die Quellenlage dieser Fallstudie kritisch einzuschätzen. Ihrer aller Zuversicht motivierte mich, mich in meinen Augen wissenschaftlich weit aus dem Fenster zu lehnen und eine umwelthistorische Betrachtungsweise einzunehmen.

Dieses komplexe Forschungsprojekt wäre allerdings ohne die großzügige finanzielle Unterstützung durch die Deutsche Forschungsgemeinschaft (DFG) nicht möglich gewesen. Auf meinen beiden Forschungsreisen nach Vietnam war es mir gegönnt, den lebhaften Austausch mit internationalen und lokalen Akteuren des Hochwassermanagements zu pflegen. Insbesondere ist da Dr. Bach Tan Sinh des Ministeriums für Forschung und Technik zu nennen, der für seine soziökonomische Untersuchung von Hochwassermaßnahmen bereits international bekannt ist, und sein starkes Interesse an meiner historischen Betrachtungsweise fruchtbare Ergebnisse mit sich brachte. Ferner waren mir Dr. Nguyen Van Be und Dr. Van Pham Dang Tri des Collage of Environment and Natural Resources der Can Tho University eine große Unterstützung. Sowohl wissenschaftlichen als auch persönlichen Beistand erfuhr ich aus dem Kreise meiner Kollegen im DFG-Graduiertenkolleg 1024 „Interdisziplinäre Umweltgeschichte“. Manuela Armenat, Jörg Cortekar und Patrick Masius schärften meine Sicht auf interessante Argumentationslinien der Protagonisten meiner Fallstudien. Jana Sprenger, Miriam Bader-Gassner, Linda Szücs, Andreas Kieseler, Peter Reinkemeier und Thore Lassen gestalteten eine konstruktive Arbeits- und Heimatatmosphäre nicht nur im Büro, sondern auch in Göttingen. Einen persönlichen Dank richte ich an meine Familie und Freunde, die in den letzten Monaten auf mich verzichtet haben, an dieser Stelle sind vor allem Kerstin Schmitz und Harry Bremschmit zu nennen. Außerdem gilt mein Dank insbesondere meinen Eltern, die mich in meinem Werdegang stets bestärkten.

Göttingen, im September 2013 


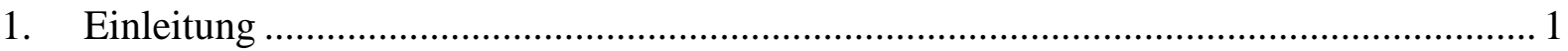

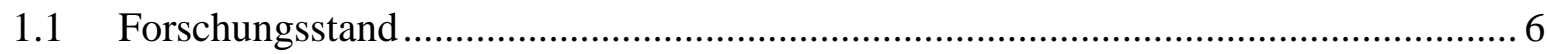

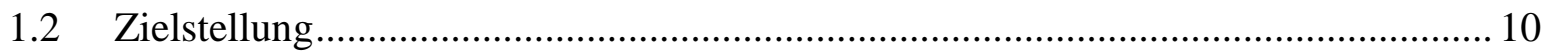

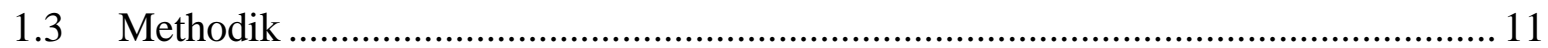

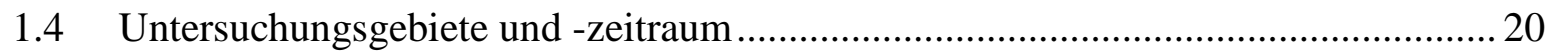

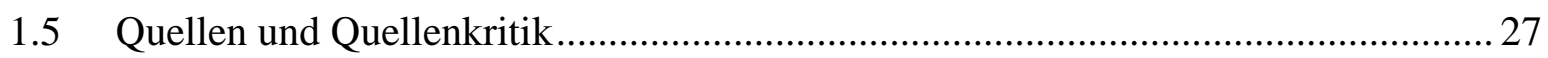

2. Wahrnehmung des Kölner Stadtbildes - Stadtgestaltung durch Hochwasserschutz ....... 30

2.1 Die Hochwasserschutzmauer 1960 - Der Not gehorchend ........................................ 31

2.2 Diskussionen zum Bau der Hochwasserschutzmauer ............................................. 32

2.2.1 Die treibenden Kräfte des Stadtrats .................................................................. 40

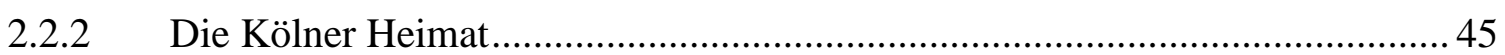

2.3 Bewertung des Diskurses aus der Kosten-Nutzen-Perspektive ...............................50

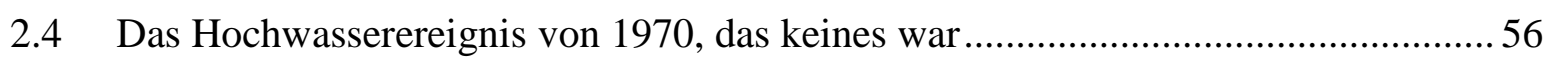

2.4.1 Reaktion: Die Diskussion nicht wiedereröffnen ............................................58

2.4.2 Die Bewährung der vorhandenen Schutzhöhen ............................................. 58

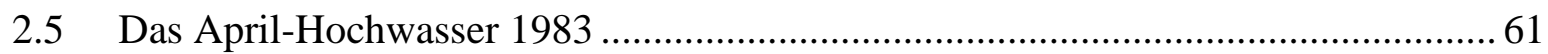

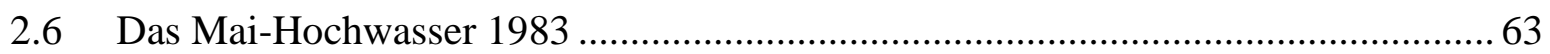

2.6.1 Reaktion: Anpassung der Hochwasserschutzmauer ......................................6 65

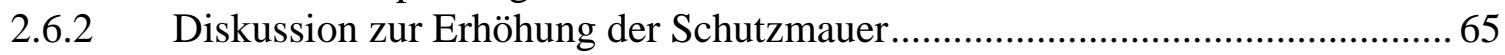

2.6.3 Bewertung des Diskurses aus der Kosten-Nutzen-Perspektive ........................ 67

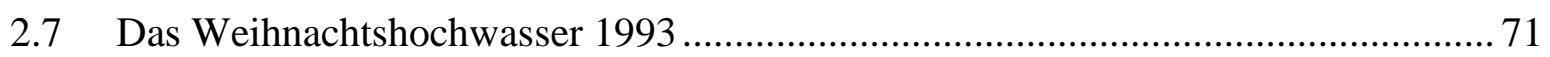

2.7.1 Reaktionen: Überregionale Ursachenbetrachtung ............................................. 74

2.7.2 Diskussion zur Strategie der tausend kleinen Aufgaben .................................. 75

2.7.3 Bewertung des Diskurses aus der Kosten-Nutzen-Perspektive ........................ 78

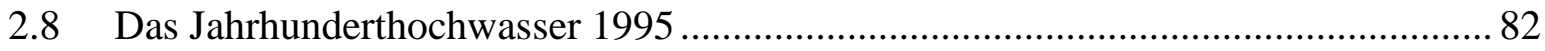

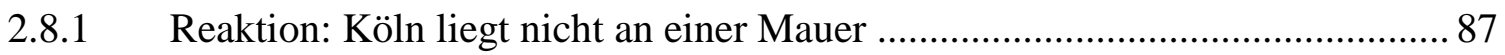

2.8.2 Diskussion zur Erhöhung der Schutzbauten ................................................... 93

2.8.3 Bewertung des Diskurses aus der Kosten-Nutzen-Perspektive ........................ 95

2.9 Abschlussdiskussion zur Wahrnehmung des Kölner Stadtbildes ........................... 102

3. Akteursrollen zwischen Hochwasserursachen und -verantwortung.............................. 111

3.1 Reaktionen von 1970: Streit um Entschädigungen ................................................. 112

3.1.1 Diskussionen über Schadensersatz ............................................................ 113

3.1.2 Bewertung des Diskurses aus der Kosten-Nutzen-Perspektive ...................... 120

3.2 Reaktionen von 1995: Ein ganz neues Konzept.................................................... 134

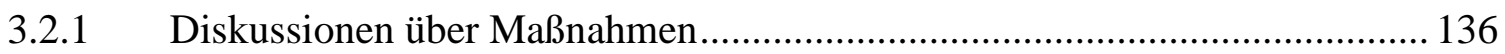

3.2.2 Bewertung des Diskurses aus der Kosten-Nutzen-Perspektive ...................... 140

3.3 Verschiebung der Rollen der politisch Verantwortlichen ....................................... 148

4. Politische Reaktionen auf Hochwasser im Mekong-Delta........................................... 151

4.1 Einführung: Traditionelles Leben im Wandel......................................................... 152

4.2 Reaktionen auf das Hochwasser im Mekong-Delta ............................................. 160

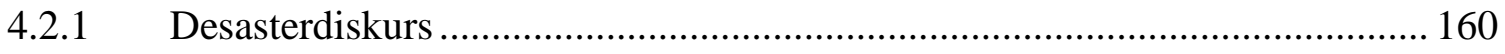

4.2.1.1 Interpretation des Desasterdiskurses ...........................................168 
4.2.2 Diskurs zur Intensivierung der Landwirtschaft und Flächennutzung............. 173

4.2.2.1 Interpretation des Landwirtschaftsdiskurses ................................177

4.2.3 Diskurs zum Hochwasserschutz im Mekong-Delta...................................... 182

4.2.3.1 Interpretation des Diskurses zum Hochwasserschutz .......................191

4.3 Bewertung der Diskurse aus der Kosten-Nutzen-Perspektive ................................ 202

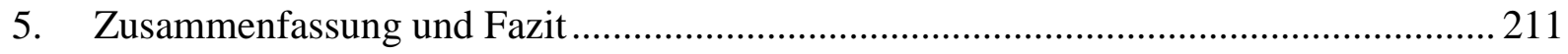

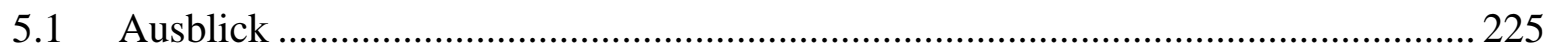

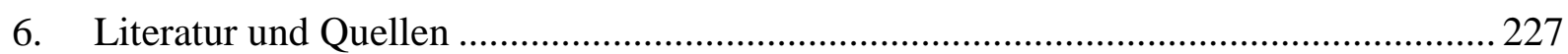

Abbildungsverzeichnis

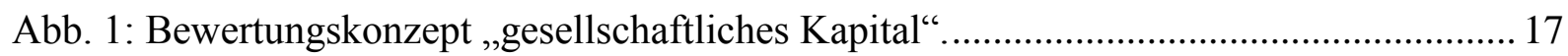

Abb. 2: Hilfsmethode zur Analyse der Entscheidungen. ..................................................... 18

Abb. 3: Institutionalisierung des Kölner Hochwasserschutzes seit 1985 ............................. 21

Abb. 4: Organisation des Hochwasserschutzes in Vietnam seit 1990.................................. 22

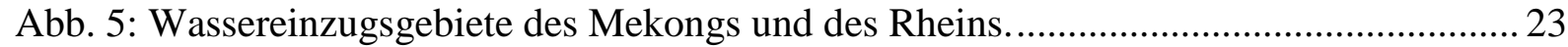

Abb. 6: Kölner Pegel an der Frankenwerft 2013 .............................................................. 24

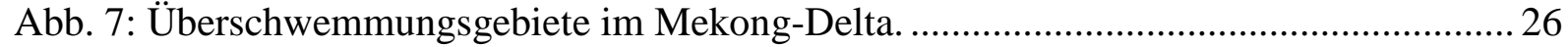

Abb. 8: Rheinuferstraße mit dem Hochwasserschutz -,Mäuerchen“ 1966........................... 32

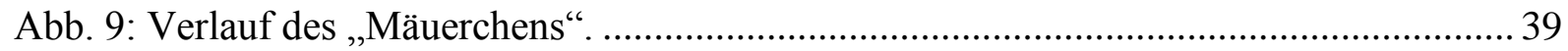

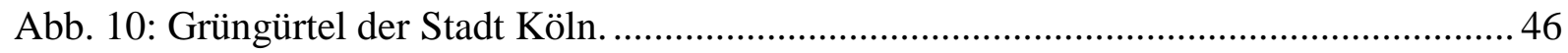

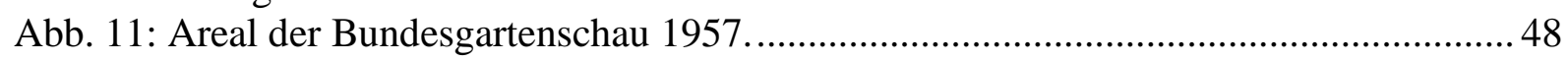

Abb. 12: Frankenwerft mit Kriegsschäden. ....................................................................... 49

Abb. 13: Erster Einsatz der mobilen Schutzmauer 1988. .................................................. 79

Abb. 14: Gruppierung der Kölner wasserbaulichen Maßnahmen........................................ 88

Abb. 15: 18 Planfeststellungsabschnitte im Hochwasserschutzkonzept Köln 1996............... 90

Abb. 16: Merkblatt für Bewohner hochwassergefährdeter Gebiete....................................... 92

Abb. 17: Mobile Hochwasserschutzwand am 01. Juni 2012 ............................................ 100

Abb. 18: Lagerhalle der mobilen Hochwasserschutzelemente. ............................................ 101

Abb. 19: Der Kasselberger Weg in der Hochwasserschutzkarte........................................ 122

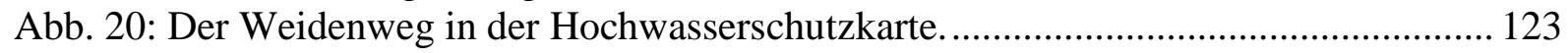

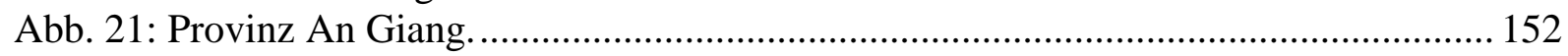

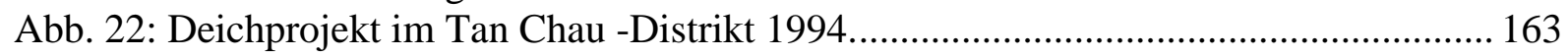

Abb. 23: Hochwassercluster und Umsiedlungsprojekt im An Phu -Distrikt. ........................ 190

Abb. 24: Typisches Haus auf Stelzen im Tan Chau-Distrikt. .............................................. 199

Abb. 25: Hochwasserschäden und Anzahl der Todesopfer 1991 - 2011 in An Giang.......... 204

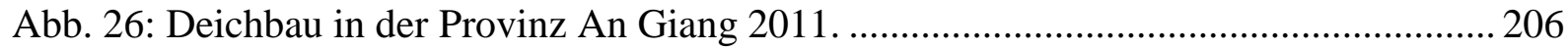

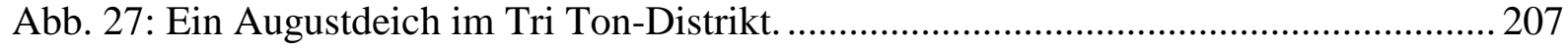


Tabelle 1: Verteilung von Investitionen und Schäden in Köln 1993/95 ................................ 89

Tabelle 2: Abgrenzung des Risikoansatzes gegenüber dem Sicherheitsansatz....................... 96

Tabelle 3: Kostenübersicht für bauliche Maßnahmen 1994 in Köln. .................................... 98

Tabelle 4: Hochwasserschäden in Köln 1970 .................................................................... 128

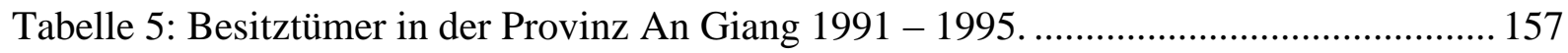

Tabelle 6: Steigerung der Pumpleistung in An Giang 1991 - 1995...................................... 158

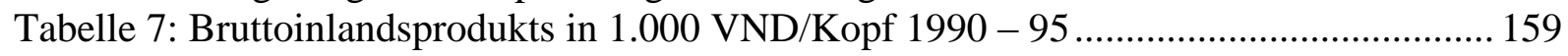

Tabelle 8: Hochwasserschäden im Can Tho-Distrikt in Mio. USD 1991 - 2001................... 193

Tabelle 9: Evakuierte Haushalte und staatliche Unterstützung 1991 - 2004........................ 194

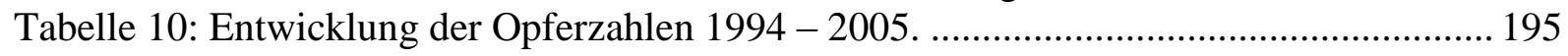

Tabelle 11: Bau fester Häuser in An Giang 1991 - 1995................................................. 199

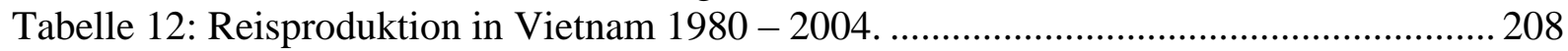

Abkürzungsverzeichnis

CCFSC Zentralkomitee für Flut und Sturm Kontrolle

DDMFCSP Department für Deich Management, Flut Kontrolle und Sturm Bereitschaft

DM Deutsche Mark

DRAGON Delta Research and Global Observation Network Institute

IKSR Internationale Kommission zum Schutz des Rheins

VNCIDNDR Viet Nam National Committee for the International Decade for Natural Disaster

Reduction

WISDOM Water-related Information System for the sustainable Development of the

Mekong-Delta in Vietnam

CDU Christliche Demokratische Union

FDP Freie Demokratische Partei

KNA Kosten-Nutzen-Analyse

K P Kölner Pegel

KPV Kommunistische Partei Vietnam

MARD Ministry of Agriculture and Rural Development

Mio. Million

MONRE Ministry of Natural Resources and Environment

MRC Mekong River Commission

NABU Naturschutzbund Deutschland

NRW Nordrhein-Westfalen

Steb Stadtentwässerungsbetriebe Köln AöR

SPD Sozialdemokratische Partei Deutschlands

THW Technischen Hilfswerks

USD United States Dollar

UN United Nations

VND Viet Nam Dong

WWF World Wide Fund of Nature 


\section{Einleitung}

Im Juni 2013 überschwemmte ein Jahrhunderthochwasser acht deutsche Bundesländer. Die zu beklagenden Schäden könnten das Hochwasser zum teuersten Naturereignis in der deutschen Geschichte werden lassen, konstatierte Peter Höppe, Leiter der Abteilung für Georisikoforschung der Munch $\mathrm{Re}^{1}$. Die Anwohner der überschwemmten Gebiete sowie die verantwortlichen Politiker waren von dem zweiten Jahrhundertereignis innerhalb von nur elf Jahren überrascht worden. Maßnahmen, welche bereits nach dem Schadensereignis von 2002 beschlossen worden waren, waren noch immer nicht umgesetzt worden ${ }^{2}$. Dieses Ereignis bot den Akteuren aller deutschen Verwaltungsebenen Gelegenheit, die Frage, ob die Organisation des Hochwasserschutzes aus der Verantwortung der Bundesländer herausgehoben und auf der Ebene der Bundesregierung zentralisiert werden soll ${ }^{3}$, erneut zu diskutieren.

Auch das südostasiatische Land Vietnam kämpft mit Hochwassern an Flüssen ${ }^{4}$. In dem Land wurde die Verantwortung für die Organisation des Hochwasserschutzes bereits 1992 auf nationaler Ebene institutionalisiert ${ }^{5}$. Ein Zusammenspiel von institutionellen, baulichen und vorsorgeorientierten Maßnahmen begründet seitdem den vietnamesischen Hochwasserschutz. Trotzdem entwickelten sich die volkswirtschaftlichen Kosten verursacht durch Flusshochwasser stetig nach oben.

Das Intergovernmental Panel on Climate Change (IPCC) prognostizierte 2007 immer stärker werdende und häufiger auftretende Hochwasser und damit zugleich die Forderung, den politischen Entscheidungsprozessen infolge von Überschwemmungen mehr Aufmerksamkeit zu schenken ${ }^{6}$. Im Zuge der administrativen Anpassung an den Klimawandel werden Hochwasserereignisse weltweit zum politischen Ereignis ${ }^{7}$ und zum „Thema multilateraler politischer Beratung “8. In der zweiten Hälfte des 20. Jahrhunderts wurde das Katastrophenmanagement allmählich zu einem Anliegen internationaler Organisationen ${ }^{9}$. Hinsichtlich des Klimawandels sind Naturgefahren Initialzündungen für internationale Abkommen. Nicht nur die westlichen Nationen vereinen sich, um die Folgen des Klimawandels abzuschwächen, auch die Entwicklungsländer beteiligen sich am Klimaschutz.

Die World Conference on Natural Disaster Reduction von 1994 bezeichnete das Wissen über

\footnotetext{
${ }^{1}$ Artikel: Deutschlands teuerste Naturkatastrophe, Süddeutsche Zeitung, 09.07.2013.

${ }^{2}$ Artikel: Warten auf die Schlammschlacht, Süddeutsche Zeitung, 05.06.2013.

${ }^{3}$ Artikel: Rösler fordert Hilfsfonds für Flutopfer, Süddeutsche Zeitung, 11.06.2013.

${ }^{4}$ Nguyen 2007.

${ }^{5}$ DR 0021992.

${ }^{6}$ Ebd.

${ }^{7}$ Artikel: Mehr Umweltpolitik wagen, Süddeutsche Zeitung, 16.07.2013.

${ }^{8}$ Jakubowski-Tiessen 2010.

${ }^{9}$ Masius/Sprenger/Mackowiak 2010.
} 
mögliche Einflüsse auf die Positionierung der administrativen Entscheidungsträger gegenüber alternativen Maßnahmen der potentiellen Reaktionsalternativen als essentiell ${ }^{10}$. „The role of the state in the creation and distribution of risk needs to be given much greater emphasis", forderte 2010 auch die Geografin Xiaomeng Shen nach ihren Untersuchungen des deutschen und chinesischen Hochwassermanagements ${ }^{11}$. Die verschiedenen Wahrnehmungen und Einstellungen der Entscheidungsträger werden durch das institutionelle Reaktionsvermögen in der Auswahl der Maßnahmen und in deren Effizienz beeinflusst ${ }^{12}$. Shen unterstreicht, es müsse verstanden werden, weshalb einige Strategien verfolgt werden, andere hingegen nicht. In diesem Zusammenhang stellen politische Handlungsträger ausdrucksstarke Akteure der Gesellschaft dar ${ }^{13}$. Generell scheint es ein ökonomischer Imperativ zu sein, die der Gesellschaft durch Hochwasser entstehenden Kosten zu reduzieren. Die Frage, ob es sinnvoll ist, diese Kosten zu reduzieren, entsteht vor dem Hintergrund nur begrenzt zur Verfügung stehender Ressourcen. In Notsituationen befindet sich die Reaktionsfähigkeit einer Gesellschaft zwischen den Grenzen des zu bilanzierenden Schadens und den zur Verfügung stehenden Ressourcen ${ }^{14}$. Entscheidungsträger müssen die Wahl treffen, welchen Herausforderungen begegnet werden kann und welchen nicht. So mag es beispielsweise von Vorteil sein, keinen Hochwasserschutz zu installieren, wenn die mit diesem verbundenen Opportunitätskosten, also die Kosten des entgangenen Nutzens, zu hoch sind. Die Effizienz der eingesetzten Mittel muss somit im Mittelpunkt der Betrachtung stehen. Folglich ergibt sich die Forschungsfrage für die vorliegende Arbeit: Verliefen die abgestimmten Handlungen als Reaktion auf ein Hochwasserereignis rational?

Der Terminus der Rationalität entstammt dem lateinischen „Denkvermögen“ und bezeichnet „das Wesen einer Sache, die menschliche Vernunft oder psychologisch, das auf Einsicht gründende Verhalten “15. Menschliches Handeln ist nach Aussage des Philosophen Karl Popper stets lösungsorientiert ${ }^{16}$, denn jede Gesellschaft versucht ihren Herausforderungen zu begegnen. In der Ökonomie wird dieses Handeln durch das Modell des „homo oeconomicus“ dargestellt. Es begreift den Menschen in einer Situation der Knappheit, in der er seine Bedürfnisse gegen verschiedenste Restriktionen, beispielsweise politischer oder ökonomischer Art, und unter unvollständigen Informationen erfüllt. ${ }^{17}$. Der Mensch wägt

\footnotetext{
${ }^{10}$ Ebd.

${ }^{11}$ Shen 2010, 4.

${ }^{12}$ Ebd.

${ }^{13}$ Ebd.

${ }^{14}$ Masius 2010.

${ }^{15}$ Duden 2013.

${ }^{16}$ Döring 1987, 49.

${ }^{17}$ Marggraf et al. 2005.
} 
gegen Alternativen ab, bevor er eine Entscheidung trifft. Seine Präferenzen lassen ihn die Alternative mit dem für ihn persönlich höchsten Nettonutzen wählen ${ }^{18}$. Nach Nicholas Rescher (Philosoph) ergibt sich eine evaluative Rationalität, die im Angesicht der limitierten Ressourcen Werturteile fällt ${ }^{19}$. „It is a mistake to view the perception of hazards and disasters in isolation from competing demands on people's attention from other events" ${ }^{20}$. Prozesse zwischen Mensch und Umwelt werden erkennbar, wenn die Beziehung zwischen Ressourcennutzung und Extremereignis wechselwirksam erforscht wird ${ }^{21}$. Ressourcen bedeuten in diesem Zusammenhang die Gesamtheit des gesellschaftlichen Kapitals, welches aufgrund eines Hochwasserereignisses wertmäßigen Veränderungen unterliegt. Handelt es sich um Vorhaben der öffentlichen Hand, dann wird auf Grundlage einer Kosten-NutzenAnalysen (KNA) über das zu bevorzugende Projekt entscheiden ${ }^{22}$. Die KNA geht ursprünglich auf wasserwirtschaftliche Planungsvorhaben des frühen 19. Jhs. zurück ${ }^{23}$. Sollen Entscheidungen über Allokationsverschiebungen gesellschaftlicher Ressourcen getroffen werden, wird das Instrument zur Unterstützung der Entscheidungsfindung herangezogen ${ }^{24}$, da eine Neuverteilung nur über die Optimierung der gesellschaftlichen Wohlfahrt zu legitimieren ist $^{25}$.

Es werden Kosten und Nutzen bestimmter Handlungen gegenübergestellt, um im Ergebnis abzulesen, ob die eingesetzten Mittel den gewünschten Resultaten gerecht werden ${ }^{26}$. Vorteile befördern dabei die Zielerreichung, dagegen verringern Kosten die Effektivität ${ }^{27}$. Ein Zweckbezug der einzusetzenden Mittel verdeutlicht im Vergleich mit alternativen Handlungsmöglichkeiten das effizienteste Verhältnis. Der Grad der erreichten Effizienz bedeutet einen Zuwachs von Wohlstand in der betroffenen Gesellschaft ${ }^{28}$. Das Ergebnis mit dem größten Wohlfahrtsgewinn stellt die effizienteste Lösungsoption dar. Somit ist die ökonomische Analyse ein Instrument, mit dem die vernünftigste Alternative identifiziert wird. Eine investierte Geldeinheit in eine bestimmte Schutzhöhe, die, statistisch betrachtet gegen ein 100jähriges Hochwasser schützt, kann schadenswirksamer und somit effizienter investiert sein, als eine Geldeinheit, die zum Aufbau einer Vorrichtung, die gegen ein 200jähriges

\footnotetext{
${ }^{18}$ Marggraf 2005, 63.

${ }^{19}$ Wüstenhube 1999, 80.

${ }^{20}$ Shen $2010,7$.

${ }^{21}$ Masius 2010.

${ }^{22} \S 7$, Abs. 2 betrifft die Bundeshaushaltsordnung, §6, Abs. 2 die Länder und $§ 10$, Abs. 2 die Gemeindehaushaltsverordnung.

${ }^{23}$ Hanley/Spash 2003; Marggraf/Streb 1997.

${ }^{24}$ Schofield 1989.

${ }^{25}$ Hanley/Spash 2003.

${ }^{26}$ FitzGerald 1978.

${ }^{27}$ Schofield 1989.

${ }^{28}$ Ebd.
} 
Hochwasser schützt, investiert wird. Eine Investition in die Schaffung eines Gefahrenbewusstseins in der Bevölkerung kann beispielsweise vorteilhafter sein, als der Bau von Deichen. Aber auch die Lösung anderer gesellschaftlicher Probleme kann für die jeweilige Gesellschaft vorteilhafter sein, als sich um gelegentlich auftretende Hochwasserschäden zu kümmern.

Die ökonomische Bewertung der Hochwasserschutzanlagen stellt die Entscheidungsträger vor einige Herausforderungen. Im Jahr 1968 erschien eine Überblicksveröffentlichung über die Anwendung der KNA in der Planungsphase von wasserwirtschaftlichen Projekten. Im Zusammenhang mit Hochwasserschutzanlagen fasste Walter Stolber die Unzulänglichkeiten der Methodik zusammen. Er war der Ansicht, dass es sich nicht bestimmen lasse, ob die direkten oder die indirekten Nutzen (und Kosten) überwiegen, weil sich dies ,infolge des Fehlens eines gemeinsamen Nenners nicht ohne subjektive Bewertung entscheiden [1ässt]، ${ }^{\text {29 }}$. Aus diesem Grund erhoffte er sich für den Projektplaner eine gute Portion „,common sense“630. Die Verbreitung der Anwendung solcher Wirtschaftlichkeitsberechnungen ist daher nicht flächendeckend. Dehnhardt et al. publizierten im Namen des Umweltbundesamts im August 2008 einen Statusbericht über KNA von Hochwasserschutzmaßnahmen ${ }^{31}$. In diesem wurde festgestellt, dass bis dato lediglich der technische Schutz - und dieser fast ausschließlich auf lokaler Ebene - durch KNA auf ihre jeweilige Vorteilhaftigkeit im Vergleich zu alternativen Reaktionen betrachtet wird ${ }^{32}$. Es wurde herausgefunden, dass weder die gewählten Systemgrenzen noch die methodischen Ansätze standardisierten Vorgehensweisen entsprechen und dabei intangible und indirekte Kosten und Nutzen ignoriert werden ${ }^{33}$. Des Weiteren fassten die Autoren des Berichts zusammen, dass hauptsächlich normative Zielvorgaben - z. B. die Beherrschung eines Hochwassers mit einer zweihundertjährigen Eintrittswahrscheinlichkeit $\left(\mathrm{Q}_{200}\right)$ - die Auswahl bestimmen ${ }^{34}$. Den Mangel begründen die Autoren mit der Schwierigkeit, die „Wirksamkeit der unterschiedlichen [...] Strategien auf

\footnotetext{
${ }^{29}$ Stolber 1968, 121.

${ }^{30}$ Ebd., 157. Kritik erfuhr die kalkulatorische Effizienzabwägung v. a. aus philosophischen Vernunftsdiskursen. Mitte der 1950er Jahre wurde die Kritik an der Rationalität durch Simon Herbst in Amerika angeführt. Er kritisierte die Rational Choice Theorie. Würde Rationalität ausschließlich auf Kosten-Nutzen-Abwägungen im ökonomischen Sinne beruhen, wäre nichts anderes vernünftig, als nach dem Erfolg einer Handlung zu bewerten. Viele seiner Kollegen, wie beispielsweise James March, Karl E. Weick, Cathrin E. Sadcliff, wiesen auf die weiche Seite der Vernunft hin und nahmen Abstand von dem Begriff der Ratio. Nach Auffassung Gerts sei die Kosten-Nutzen-Kalkulation das Ergebnis der Positivdefinition von Rationalität. Ihr entgehe ein signifikantes Problem, dass nämlich die Maximierung von üblen Wünschen (z. B. Selbstmord), ohne ein Übel zu vermeiden oder ein Gut zu gewinnen, auch als rational bezeichnet werden müsse. Er sah ein Problem, falls per Definition jede nicht maximierende Handlung eine irrationale Wahl wäre (vgl. Gert 1996; 1999; Wüstenhube 1996).

${ }^{31}$ Dehnhardt et al. 2008.

${ }^{32}$ Ebd., 7, 53.

${ }^{33}$ Ebd., 61. Intangible Kosten entstehen beispielsweise durch den Verlust an Lebensqualität oder durch Ängste und sind nicht unmittelbar monetär zu beziffern.

${ }^{34}$ Ebd.
} 
Grundlage naturwissenschaftlicher [...] Prognosen unter verschiedenen Rahmenbedingungen und auf unterschiedlichen Skalenebenen“ einzuschätzen ${ }^{35}$. Zweitens unterstreichen sie das Problem der „großen Unsicherheiten in der Einschätzung von UrsacheWirkungsbeziehungen“ für ökonomische Analysen ${ }^{36}$. Grenzen der Methode sehen die Autoren in der „Abhängigkeit von Bewertungskontexten, von der Art des Flussgebiets, dem Hochwasserereignis und der betrachteten Skalenebene“637. Letztlich bleibt „die Monetarisierung der Effekte ein zentrales Problem, ${ }^{38}$ welches sowohl die räumliche als auch die zeitliche Dimension umfasst ${ }^{\star 39}$. Die Autoren betonen, dass die Anwendung der KNA politökonomische Prozesse keinesfalls substituieren kann und in ihrer Instrumentalisierung von Bewertungskontexten abhängig ist ${ }^{40}$. Damit gewichten Autoren der UBA-Studie wie bereits Stolber 1968 neben harten ökonomischen Fakten das Bewusstsein und die Anschauung der zur Abstimmung aufgeforderten Akteure als einflussreiche Größen. Da die Wertvorstellungen der Entscheidungsträger durch die Veränderungen der Sachverhalte ersichtlich werden und sich unabhängig von der Anwendung einer KNA im Diskurs der einzelnen Reaktionen über die Zeit niederschlagen, stehen politische Diskurse im Mittelpunkt der vorliegenden Untersuchung. Auswirkungen der sich verändernden Mensch-UmweltBeziehung geben in der long durée Auskunft, über das Favorisieren der unterschiedlichen Alternativen potentieller Reaktionen auf Hochwasser ${ }^{41}$. In dieser Arbeit werden die beiden unterschiedlichen Organisationsansätze aus Deutschland und Vietnam beispielhaft gegenübergestellt und es wird neben der Rationalität der Entscheidungsprozesse untersucht, ob von der jeweiligen Organisationsform Vor- und Nachteile abzuleiten sind.

Die Arbeit ist wie folgt aufgebaut: Die Einleitung führt zu der Forschungsfrage, ob die abgestimmten Handlungen als Reaktion auf ein Hochwasserereignis rational verliefen. Die Aufarbeitung der bereits vorliegenden Untersuchungen der Hochwasser am Mekong und Rhein eröffnet die Forschungslücke, dich ich mit dieser Arbeit zu schließen versuche.

Im ersten Kapitel, das sich mit dem Kölner Hochwasser beschäftigt, wird der intangible Wert der Stadtansicht im Kontext des Baus von Hochwasserschutzmaßnahmen dargestellt. Die Stadtansicht wurde von den Kölner Akteuren in ihrem Wert so hoch eingeschätzt, dass auf normativ begründete Schutzbauten verzichtet wurde. Im zweiten Kapitel wird dargestellt, wie

\footnotetext{
${ }^{35}$ Ebd.,7.

${ }^{36}$ Ebd.

${ }^{37}$ Ebd.

${ }^{38}$ Ebd., 47.

${ }^{39}$ Ebd., 53.

${ }^{40}$ Ebd., 83.

${ }^{41}$ Shen 2010.
} 
der Wandel in der Wahrnehmung der Überflutungsursachen zum Auslöser für eine überregionale Auseinandersetzung zum Thema des Hochwasserschutzes wird. Außerdem wird herausgearbeitet, wie die intangiblen Kosten in negativen Erwartungen der politischen Akteure begründet lagen und die administrativen Reaktionen unmittelbar beeinflussten. Im dritten Kapitel werden drei Reaktionen auf Hochwasser im Mekong-Delta aus der NutzenKosten-Perspektive untersucht. Im Ergebnis der Analyse wird gezeigt, dass ein Verzicht auf die Reaktionen aufgrund der potentiell zu erwartenden Opportunitätskosten unlogisch gewesen wäre. Anzeichen werden sichtbar, die darauf hinweisen, dass hinter den Reaktionen auch Ziele standen, die die vietnamesische Regierung nicht kommunizierte. Abschließend wird eine Gesamtschau der Ergebnisse vorgenommen, die den Erkenntniswert für zukünftige Auswahlprozesse im Bezug zu Überschwemmungsproblemen begründet.

\subsection{Forschungsstand}

Der Auftakt zur historiographischen Betrachtung von Naturkatastrophen wird allgemein in der Darstellung des Erdbebens von 1348 durch Arno Borst aus dem Jahr 1981 gesehen $^{42}$, und liegt damit gerade einmal 30 Jahre zurück. Der allgemeine Fokus der Geschichtswissenschaft lag bis dahin auf politisch bedeutenden Figuren und deren Machtausübung. Eine weitere Begründung für die Ignoranz gegenüber Naturereignissen findet sich in der Einzigartigkeit der Naturereignisse und in deren Deutung ${ }^{43}$. Wieso sollte man etwas aufzeichnen, das man nie wieder erleben würde und durch das der eigene lasterhafte Lebensstil verdientermaßen zu Recht gewiesen wurde ${ }^{44}$ ? Die Klimaforschung war 1995 das erste Umweltthema, welches mit einem Nobelpreis ausgezeichnet wurde ${ }^{45}$. „Natur wurde zum Ereignis“, stellten Christof Mauch und Christian Pfister 2006 rückblickend fest ${ }^{46}$. Im Laufe der Jahre ergänzten gesellschaftliche Forschungsansätze die Klimaforschung, wobei sich die historischen Ansätze bis heute auf die Untersuchung von Fallbeispielen beschränken ${ }^{47}$.

Die historische Forschung zu Hochwassern konzentriert sich auf zwei Untersuchungsschwerpunkte. Nach den Ursachen aus meteorologischer, geologischer oder

\footnotetext{
${ }^{42}$ Borst 1981.

${ }^{43}$ Mauch 2009, 5.

${ }^{44}$ Ebd.

${ }^{45}$ Für ihre Untersuchung des Ozonabbaus erhielten F. Sherwood Rowland, Paul Crutzen, Mario Molina den Nobelpreis für Chemie.

${ }^{46}$ Mauch/Pfister 2006.

${ }^{47}$ Deutsch 2007.
} 
baulicher Sicht suchen die technisch-naturwissenschaftlichen Perspektiven. Den historischen Hochwasserschutz am Rhein beleuchtete Martin Schmidt (2000) ${ }^{48}$, während ein Beitrag über die baulichen Veränderungen am Mekong von dem amerikanischen Umwelthistoriker David Biggs (2004) geleistet wurde. Er promovierte zu der Entwicklung der Wasserstraßen und Bewässerungssysteme im vietnamesischen Teil des Deltas zwischen 1820 und $1975^{49}$. Dem gegenüber stehen die gesellschaftswissenschaftlichen Forschungsgebiete. Hochwasser werden aus politischer, wirtschaftlicher und verwaltungsbürokratischer Perspektive untersucht, von denen einige im Folgenden genannt werden.

Jürgen Weichselgartner nannte das Hochwasser im Jahr 2001 ein „soziales Ereignis“ und betrachtete die veränderten Wahrnehmungen unterschiedlicher Akteure anhand der Kölner Hochwasserereignisse der Jahre 1784 und 1882. 1784 waren durch das Hochwasser hauptsächlich Ackerflächen und Wiesen betroffen, während 1882 öffentliches und privates Eigentum zerstört wurde und diese Veränderung dem Staat als Akteur eine neue Rolle verlieh $^{50}$. Das Umweltbewusstsein knüpft Radkau 2001 in seinem Buch „Natur und Macht“ an Krisenerfahrungen und verbindet die Katastrophe mit der „Unvorhersehbarkeit gesellschaftlicher Prozesse“ sowie mit dem offenen Charakter einer Krise ${ }^{51}$. Wie Naturereignisse bewertet werden, hängt demnach von dem Verständnis von Risiko, Gefahr und den einzuschätzenden Wahrscheinlichkeiten ab. Fiona Miller promovierte im Jahre 2003 über die „Society-Water Relations in the Mekong-Delta: A Political Ecology of Risk” an der Universität Sydney. Sie stellte aufgrund der Entwicklung der Wasserressourcen eine räumliche, zeitliche und soziale Neuverteilung von Umweltrisiken fest ${ }^{52}$. Von Wolfgang Kron stammt der Ausdruck „Katastrophenpartnerschaft“; dieser Begriff beschreibt die Akteure der Versicherungsinstitute, die staatliche Fürsorge und die von der Naturgefahr betroffenen Opfer im Spannungsfeld der Schadensverminderung. Damit führte er die Diskussion um Rollen und Verantwortung fort ${ }^{53}$. Frühere Forschungsarbeiten $\mathrm{zu}$ Wasserthemen in der Mekongregion ergaben sich seit den 1980er Jahren in Zusammenarbeit mit der Can Tho University sowie mit dem International Rice Research Institute in O Mon, Can Tho Provinz. Diese verblieben jedoch im agrarökonomischen Bereich und behandelten die Entwicklung der Bewässerungssysteme, darunter Kanal-, Dränage- und Abschottungsanlagen, sowie die profitorientierte Verwendung von landwirtschaftlichen Inputprodukten ${ }^{54}$. Die Mekong River

\footnotetext{
${ }^{48}$ Schmidt 2000.

${ }^{49}$ Biggs 2004.

${ }^{50}$ Weichselgartner 2001.

${ }^{51}$ Radkau 2001.

${ }^{52}$ Miller 2003.

${ }^{53}$ Kron 2001.

${ }^{54}$ Interview mit Dr. Nguyen Van Be, Can Tho Univerity im März 2012.
} 
Commission (MRC) erarbeitet seit 1995 wissenschaftliche Strategien für eine effiziente und Ressourcen schonende Zusammenarbeit der Anrainerstaaten des Mekongflusses, bleibt aber als Forschungsinstitut ohne politisches Gewicht ${ }^{55}$. Die Kommission ging unter amerikanischem Einfluss aus dem bereits seit den 1950er Jahren tätigen Mekong Komitee hervor, welches sich zum Ziel setzte, die wirtschaftlichen Aktivitäten der Region unter stärkerer Berücksichtigung der Wasserressourcen $\mathrm{zu}$ steigern ${ }^{56}$. Pham Quang Minh veröffentlichte 2003 an der Humboldt-Universität zu Berlin eine Arbeit zur „Agrarpolitik in Vietnam seit 1945“. Er beleuchtet durch eine „top down“- und „bottom up“- Betrachtung der Agrarpolitik der Kommunistischen Partei Vietnams (KPV), inwieweit diese in den ländlichen Gebieten umgesetzt wurde und inwiefern die Bevölkerung Einfluss auf deren Formulierung nehmen konnte. Seit 2008 besteht das vietnamesisch-amerikanische Delta Research and Global Observation Network Institute (DRAGON) an der Can Tho Universität, das zur Stärkung der anthropogenen Anpassung an Naturgefahren vor allem technische Fragestellungen der Auswirkungen des Klimawandels untersucht ${ }^{57}$. Dabei steht das Jahrhunderthochwasser von 2000 als Auslöser der Untersuchungen im Vordergrund. Mit Blick auf das vietnamesische Mekong-Delta arbeitete das Water-related Information System for the sustainable Development of the Mekong-Delta in Vietnam (WISDOM) als deutschvietnamesischer Forschungszusammenschluss zwischen 2007 und 2011. Das WISDOM thematisierte auf den Wasserhaushalt bezogene Klimafragen im Mekong-Delta. Zwei Arbeiten sind dort zu dem Thema Flusshochwasser entstanden. Judith Ehlert legte 2011 ihre Promotion mit dem Titel „Beautiful Floods - Environmental Knowledge and Agrarian Change in the Mekong-Delta, Vietnam“ ab. Sie betrachtete die Überlieferung, Nutzung und den Zugang zu lokalem, umweltbezogenem Wissen, dessen Zugang abhängig von sozialen Standards ist ${ }^{58}$. Pham Cong Huu legte 2011 eine technische Abhandlung „Floods and Farmers, Planning and Implementation of the Dyke Systems in the Mekong-Delta, Vietnam“ vor. Er betrachtete administrative Herausforderungen beim Planungs- und Implementierungsprozess von Deichprojekten in der Stadt Can Tho, dem wirtschaftlichen Zentrum des vietnamesischen Mekong-Deltas. Daneben untersuchte er die Einflüsse der Bauwerke auf die natürliche Umwelt und auf die sozioökonomischen Entwicklungen sowie auf die Anpassungsfähigkeit der vom Hochwasser beeinflussten Landwirtschaft an den neuen Hochwasserschutz $^{59}$. David Biggs veröffentlichte 2010 sein zweites Werk zum Mekong. In

\footnotetext{
${ }^{55}$ Backer 2007.

${ }^{56}$ Browder, Ortolando, 2000.

${ }^{57}$ Internetbasierte Quelle 8.

${ }^{58}$ Ehlert 2011.

${ }^{59}$ Huu 2011.
} 
einem Rückblick in das 20. Jahrhundert erklärt er in seiner Studie „Quagmire“ die Zusammenhänge zwischen „Nation building and Nature“60.

Eine Veränderung im Umgang mit Naturgefahren unterliegt einem Lernprozess oder einer neuen Bewertung der Konsequenzen aus der Naturgefahr. Eine theoretische Untermauerung zur Untersuchung dieser Paradigmenverschiebungen liefern die folgenden Arbeiten. Eine naturwissenschaftliche Studie legte Cornelia Karger 1996 zur „Wahrnehmung und Bewertung von Umweltrisiken“ vor. Sie stellte das Vulnerabilitäts-, das Desaster- und das Chicagoer Konzept in dem Versuch zusammen, die Frage nach dem, was die betroffene Gesellschaft lernen kann, zu beantworten ${ }^{61}$. Als interkultureller Forschungsansatz gilt die 2010 erschienene Dissertation der Geographin Xiaomeng Shen über „Flood Risk Perception and Communication within Risk Management in Different Cultural Contexts“. Sie verglich dazu Hochwasserereignisse in Deutschland und China mit qualitativen sozialwissenschaftlichen Methoden an der United Nations University Bonn ${ }^{62}$. In dem 2009 ausgelaufenen DFGNachwuchsnetzwerk „Historische Erforschung von Katastrophen in Kultur vergleichender Perspektive“" unter Leitung von Gerrit Jasper Schenk und Franz Mauelshagen entstanden vier Forschungsarbeiten zur gesellschaftlichen Wahrnehmung von Desastern und zu aus diesen folgenden Lernprozessen in der Gesellschaft ${ }^{63}$. Weitere umwelthistorische Arbeiten untersuchen ebenfalls gesellschaftliche Lernprozesse und Handlungsmuster sowie historische Erklärungen für aktuelle Strategien. Eine historisch-wasserwirtschaftliche und geographische Arbeit zu Lernprozessen lieferte Matthias Deutsch im Jahr 2007, in der er explizit die Hochwasservorsorge an der Unstrut zwischen 1500 und 1900 betrachtete $^{64}$. Dabei sah er einen Dreischritt des Lernprozesses bei den untersuchten Akteuren. Gesellschaftliche Prägungen durch regelmäßige Katastrophenerfahrungen schlugen sich in den Begriffen „Katastrophenkultur“ nach Christian Rohr (2003) und „Cultures of Coping“ nach Greg Bankoff (2009) nieder ${ }^{65}$.

Festgehalten werden kann, dass zahlreiche Forschungsarbeiten Hochwasser aus Perspektiven unterschiedlicher Disziplinen bearbeiten; dabei bilden politische Betrachtungen den durchaus starken Veränderungscharakter von Wasserressourcen und Naturgefahren ab. Neben den politischen Untersuchungen wird ebenso versucht, die Frage, ob Gesellschaften aus

\footnotetext{
${ }^{60}$ Biggs 2010.

${ }^{61}$ Karger 1996.

${ }^{62}$ Shen 2010.

${ }^{63}$ Hierzu gehören die Arbeiten von Christian Pfister (2002), in welcher der Autor die Naturkatastrophen als Auslöser eines Lernprozesses ansieht, Dieter Groh, Michael Kempe und Franz Mauelshagen (2003), Greg Bankoff (2003), der die „Normalisierung der Gefahr“ aufgrund von kontinuierlich erlebten Naturgefahren beschreibt, und von Christian Pfister und Stephanie Summermatter (2004).

${ }^{64}$ Deutsch 2007.

${ }^{65}$ Rohr 2004; Bankoff 2003.
} 
Krisenerfahrungen lernen, wissenschaftlich zu beantworten. Komparative Aussagen scheinen aufgrund der „Flickenteppichgeographie in der historischen Forschungslandschaft ${ }^{\text {} 66}$ noch nicht aussagekräftig zu sein, so $\mathrm{M}$. Deutsch in Bezug auf die Vertiefung der umwelthistorischen Forschung sowie ferner auf die Weiterentwicklung methodischer Ansätze. Eine Forschungslücke scheint mit der Weiterentwicklung der Frage, ob Gesellschaften lernen, auch in der Fragestellung zu bestehen, ob Gesellschaften mit der Naturgefahr rational umgingen. Deswegen möchte ich diese Forschungslücke mit einer Untersuchung der politischen Reaktionen auf Naturgefahren unter den Gesichtspunkten der Kosten-NutzenAnalyse schließen.

\subsection{Zielstellung}

In der vorliegenden Forschungsarbeit soll spezifisch die Rationalität des politischen Umgangs mit Überschwemmungen herausgestellt werden. Die traditionelle Nutzung der KNA in Projekten der öffentlichen Hand ist im Kontext von Hochwasserschutzmaßnahmen aufgrund von Schwierigkeiten, Vor- und Nachteile monetär darzustellen, begrenzt. Deshalb wird eine prozessorientierte Untersuchung angewendet. Es wird die Hypothese aufgestellt, dass Handlungsalternativen von den Entscheidungsträgern betrachtet wurden, und Auswahlprozesse abgehalten wurden. Als zweite Hypothese soll bewiesen werden, dass die politisch Verantwortlichen in den von ihnen ausgewählten Handlungsoptionen eben auch negative Konsequenzen sahen und sich über Grenzen der favorisierten Auswahl bewusst waren. Es wird also geprüft, ob die entscheidungsbefugten Akteure Konsequenzen ihrer Handlungen reflektierten und bewerteten, bevor sie eine Entscheidung trafen. Um die zwei Hypothesen zu überprüfen, werden die Wertvorstellungen dieser Akteure auf Basis der politischen Auseinandersetzungen herausgearbeitet. Die Auseinandersetzungen werden auf der Basis von archivierten Protokollen, Gesprächsvorlagen und weiteren Dokumenten wie Postwechseln oder Veröffentlichungen rekonstruiert und untersucht, um die den diskursiven Prozessen inhärenten Interessen, Ziele, Abwägungen und Konsequenzen aufzuzeigen. Die hier angewendete Diskursanalyse dient dazu, neben den direkten Kosten und Nutzen die Gewichtung von nicht monetär bezifferten Vor- und Nachteilen sichtbar zu machen und deren Bewertung und Inwertsetzung durch die handelnden Akteure herauszuarbeiten. Des Weiteren

\footnotetext{
${ }^{66}$ Deutsch 2007, 35.
} 
soll der Wandel der Wertvorstellung der Akteure im Verlauf des Betrachtungszeitraums beschrieben werden, und die Frage nach den verwendeten und gegeneinander angeführten Argumentationsansätzen herausgearbeitet werden.

Zudem soll in Kapitel 3 die Frage beantwortet werden, in welchen Akteurskreisen die Entscheidungen getroffen wurden, welche Akteure Einfluss geltend machen konnten und warum sie dazu in der Lage waren? Konnte jedermann auf den Hochwasserschutz Einfluss nehmen oder spielten verschiedene administrative Einflussgrößen eine entscheidende Rolle? Aus den geäußerten Wertvorstellungen sollen Hinweisen auf eine veränderte MenschUmwelt-Beziehung abgeleitet werden.

Kapitel 4 widmet sich der Frage, wo die Stärken und Schwächen des Hochwasserschutzes als nationale und föderale Aufgabe lagen. Wie kam es zu der vietnamesischen Organisation des Hochwasserschutzes und wie ist diese über die Jahre gewachsen? Final soll die Frage beantwortet werden, wie sich im Vergleich dazu die Organisation dieser Aufgabe in Deutschland darstellte und wie diese im Betrachtungszeitraum verändert wurde? Welche Konfliktpunkte ergeben sich in diesen beiden Organisationsvarianten?

Die vorliegende Arbeit verfolgt neben inhaltlichen auch methodische Ziele. Die prozessorientierte Rationalitätsanalyse der jeweiligen politischen Handlung soll die rationale Vorgehensweise der entscheidungsbefugten Akteure herausstellen. Zur Analyse der Diskurse wurde ein Hilfsmodell erarbeitet, dass im Abschnitt 1.3 zur Methodik vorgestellt wird. Das Instrument transformiert die Grundstruktur der Kosten-Nutzen-Analyse aus dem finanzmathematischen Ansatz in eine prozessorientierte Untersuchungsstruktur, die in einer Diskursanalyse angewendet werden kann. Das primäre methodische Ziel dieser Arbeit ist die Erprobung des Modells in der praktischen Anwendung. Mit dieser Ausarbeitung sollen politische Entscheidungsprozesse im Bezug zum Umgang mit Naturgefahren erhellt werden und somit ein zukunftsgerichteter Beitrag zur globalen Diskussion möglicher Reaktionen auf den Klimawandel geleistet werden.

\subsection{Methodik}

In der vorliegenden Untersuchung werden ein zentralstaatlicher und ein föderalistischer Ansatz im Hochwasserschutz gegenübergestellt. Die in den jeweiligen Systemen durch die 
administrativen Akteure erarbeiten Reaktionen sollen auf ihre Rationalität hin überprüft werden.

Die oben vorgestellten Forschungsergebnisse des Umweltbundesamts zeigten bereits, dass finanzmathematische KNA in Hochwasserschutzprojekten keine relevante Anwendung fanden. Deshalb stehen in dieser Forschungsarbeit die politischen Diskurse der Entscheidungsträger im Vordergrund. Während in der Ökonomie die eingesetzten Mittel dem Nutzen kausal gegenübergestellt werden, gilt im Rahmen normativer Grundsätze verständliches Verhalten als vernünftig. Die Frage nach dem, was zu tun ist, kann in einen philosophischen Kontext gestellt werden. Julian Nida-Rümelin fokussiert die Konsequenzen, die Handlungen mit sich bringen. „Vernünftig“ ist, seiner Meinung nach, das zu tun, was die vertretbarsten Konsequenzen nach sich zieht $^{67}$. In kollektiven Entscheidungen müssen die Präferenzen über diese Konsequenzen als Entscheidungsgrundlage dienen ${ }^{68}$. Dazu schlug er als Handlungsempfehlung das Dominanzprinzip vor, bei dem die Alternative gewählt werden muss, deren Konsequenzen unter allen Umständen besser sind als die der anderen Handlungsmöglichkeiten ${ }^{69}$. Komplementär dazu steht in seinen Augen das Maximinkriterium. Es spielt in der politischen Philosophie, auch empfohlen durch John Rawls, eine Rolle ${ }^{70}$ : „Man setzt auf die Handlung, bei der im ungünstigsten Fall am wenigsten passiert ${ }^{\text {“71 }}$. Hartmut Esser unterstellt dem realen, unperfekten Akteur eine alltagstaugliche Rationalität, die nach Gewohnheiten und Codes (habits and frames) funktioniert ${ }^{72}$. „Aus der Sicht einer Theorie der Rationalen Wahl ist es also eine vernünftige Entscheidung, wenn man ohne viel nachzudenken Rezeptwissen verwendet ${ }^{\text {“73 }}$, erklärt Hartmut Esser „,das Zufriedensein mit der Erfüllung einer durchschnittlichen Erwartung und dem möglichen Verzicht auf die wahrscheinlich unbekannte beste Lösung. ${ }^{\text {“74 }}$ Nach Julian Nida-Rümelin spielt auch Einheit eine Rolle: „Eine Person verlangt nach Harmonie in ihrem Auftreten und charakterisiert sich durch ihre Aussagen, Handlungen, Gründe usw., die nach Einheit verlangen, Gründe nennen heißt, auf eine umfassende Struktur hinzuweisen, zu deren Konstitutiva die betreffende Handlung gehört“‘75. Da es aber nicht möglich ist, alle Individuen nach ihren Gründen zu befragen und deren Antworten $\mathrm{zu}$ vertrauen, können individuelle Präferenzen auf

\footnotetext{
${ }^{67}$ Nida-Rümelin 2009.

${ }^{68}$ Ebd.

${ }^{69}$ Nida-Rümelin 2009.

${ }^{70}$ Nida-Rümelin 2009, 35.

${ }^{71}$ Nida-Rümelin 2009, 34.

${ }^{72}$ Esser 1990.

${ }^{73}$ Abels 2004, 193.

${ }^{74}$ Abels 2004, 193.

${ }^{75}$ Nida-Rümelin 1996, 90.
} 
beobachtbares Verhalten zurückgeführt werden ${ }^{76}$. Bernhardt Gert fehlt die Definition der guten Gründe ${ }^{77}$. Die Funktion von Gründen liegt für ihn darin, Handlungsweisen rational zu machen, welche ansonsten irrational $\operatorname{sind}^{78}$. Auch Wüstenhube bleibt „unklar, was als guter Grund gelten darf ${ }^{\text {“79. }}$.

Für die hier vorliegende Ausarbeitung kann die ethische Theorie des sozialen Subjektivismus aushelfen. Diese Theorie stellt die Wertvorstellung der Menschen in den Mittelpunkt. Dabei werden diese nicht bewertet, sondern so wie sie sind präsentiert. Kern der Theorie ist die Annahme, dass die „Wertvorstellung der Menschen die Wertquelle“ ${ }^{* 80}$ menschlichen Wohlbefindens ist. Die Theorie erfreut sich unter Ökonomen großer Beliebtheit, denn sie ist neoklassisch, effizienz- und allokationsorientiert und strebt aufgrund der utilitaristischen Werttheorie das Wohlergehen der Menschen $a^{81}$. Die Begründungen der an den Reaktionen auf Hochwasser beteiligten politischen Akteure unterliegen in dieser Arbeit keiner Bewertung, Kategorisierung oder Stigmatisierung.

Julian Nida-Rümelin versucht, eine Kohärenz der Vernünftigen zu unterstellen ${ }^{82}$. Aufgrund des allgemeinen Menschenverstands oder einer ähnlichen Bildung etabliert sich eine kohärente Meinung, da eine homogene Personengruppe ähnliche Rationalitäten wahrnimmt ${ }^{83}$. Bernhardt Gert nimmt ebenfalls den Kohärenzgedanken auf, denn er schreibt, „dass dies [...] für ziemlich jeden mit vergleichbarem Wissen und Intelligenz offensichtlich wäre“ ${ }^{\text {84 }}$. Der kommunikative Gedanke wird hier bereits deutlich, da Konsensbemühungen dem Meinungsaustausch, dem Überzeugen von Menschen und Abstimmen zwischen Menschen unterliegen. Walter Stolber hat in Bezug auf die Planung von wasserbaulichen Maßnahmen zu Recht behauptet, dass Akteure in entscheidenden Positionen mit Gemeinsinn ausgestattet sein sollen. Eine ebenfalls offenere Definition des Rationalismus als die strikte ökonomische Auslegung fordert Herbert Schnädelbach. In seinem Aufsatz zu den verschiedenen „Rationalitätstypen“ bezieht er sich auf den nachvollziehbaren Charakter der Rationalität sowie auf das Verständliche in einer Aussage oder Handlung. Das Begründen durch das Beantworten von Warum-Fragen nennt er das Wesentliche der Rationalität. Begründungen im Zusammenhang von Redekontexten bilden eine Argumentation. Er konzentriert seine Definition vor dem Hintergrund der kommunikativen Aspekte, da „die Teilnehmer strittige

\footnotetext{
${ }^{76}$ Nida-Rümelin 2009.

${ }^{77}$ Gert 1996, 320.

${ }^{78} \mathrm{Ebd}$.

${ }^{79}$ Wüstenhube 1996.

${ }^{80}$ Marggraf 2005, 61.

${ }^{81}$ Marggraf 2005.

${ }^{82}$ Nida-Rümelin 1996; 2009.

${ }^{83}$ Nida-Rümelin 2009.

${ }^{84}$ Gert 1996, 320.
} 
Geltungsansprüche thematisieren und versuchen, diese mit Argumenten einzulösen und zu kritisieren. Ein Argument enthält Gründe, die in systematischer Weise mit dem Geltungsanspruch einer problematisierten Äußerung verknüpft sind. “ ${ }^{85}$ Er folgt hierbei Jürgen Habermas mit der Aussage, dass Kritik und Rechtfertigung Ausprägungen der Argumentation darstellen. Eine Argumentation muss Stringenz besitzen. Die Ansprüche „wahr“, „richtig“, „plausibel“ und „sinnvoll“ definieren die diskursive Ratio. Herbert Schnädelbach versteht die Urteilskraft als Werkzeug und Medium von Argumentation und Kritik als eine Beurteilung im Schein allgemeiner Kriterien. Er behauptet, dass Fehlbarkeit und Freiheit, Alternativen zu wählen, Elementarbedingungen der Rationalität ausmachen, wenn Rationalität vom Begriff der Regeln her verstanden wird. Das Befolgen der Regeln bildet eine grundlegende Voraussetzung für nachvollziehbares Verhalten. Die gleichen Regeln zu teilen, gilt für Schnädelbach als die Verbindung zwischen dem Verstandenwerden durch andere sowie dem Verstehen anderer. Da sich Begründungen und Argumentationen auf Regeln beziehen, begreift Schnädelbach die Regelrationalität als das Grundprinzip der Ratio. Als Anwendungsbereich der Regeln definiert er nicht nur Sprechakte, sondern alle Handlungen. Der Bereich des Verstehbaren dehnt er über das Sprachliche hinaus aus. Damit erklärt Schnädelbach die Ratio als das „Vermögen des Verständlichen - die Fähigkeit, etwas Verständliches zu tun, zu verstehen zu geben und zu verstehen “86. Schnädelbachs Ansatz sowie die Ethische Theorie des allgemeinen Subjektivismus bilden die Basis für die vorliegende Untersuchung.

Eine Übertragung der Kosten-Nutzen-Analyse (KNA) auf die Diskursanalyse bietet für die vorliegende Arbeit die Möglichkeit kommunikative Vorgänge zu untersuchen. Grundsätzlich lassen sich acht Schritte in der Erstellung einer KNA beschreiben ${ }^{87}$, die grundsätzlich zur Entscheidung über die Durchführung von Projekten angewandt werden. Zunächst muss das Projekt identifiziert werden, um die Neuverteilung der Ressourcen und die als Gewinner und Verlierer betroffene Bevölkerung abzustecken. Daraufhin sollen die Wirkungen des Vorhabens identifiziert werden; dazu muss beispielweise eine Liste der verwendeten Materialien sowie der resultierenden Effekte (Arbeitslosigkeit, Entwicklung der Grundstückspreise usw.) angefertigt werden. Diese Auswirkungen müssen in einem nächsten Schritt quantifiziert werden, um deutlich zu machen, welche Wirkungen einen ökonomischen Einfluss auf die Effektivität des Projektes ausüben. Die herausgestellten wirtschaftlichen Gewichte werden dann mit einer zeitlichen Perspektive versehen: Auf der Zeitachse früher

\footnotetext{
${ }^{85}$ Schnädelbach 2000, 262.

${ }^{86}$ Schnädelbach 2000, 276.

${ }^{87}$ Hanley/Spash 2003.
} 
auftretende Vorteile verbessern das Analyseergebnis, während hinausgezögerte Kosten eine spätere Generation belasten können. Alle monetären Angaben müssen in einem fünften Schritt vereinheitlicht werden, um in sechsten Schritt den heutigen Wert des erstrebten Projektes durch Abzinsung zum Bar- oder Kapitalwert zu errechnen. Die KNA muss ein positives Ergebnis ausweisen, damit das Projekt verwirklicht werden kann, das Projekt also effektiv das angestrebte Ziel erreicht. Stehen verschiedene Handlungsoptionen nebeneinander, muss diejenige mit dem vorteilhaftesten Ergebnis umgesetzt werden. In einem letzten Schritt wird die Veränderung der Effizienz unter der Bedingung, dass sensible Faktoren verschoben werden, betrachtet. Zur besseren Übersicht kann die KNA auch in drei Phasen zusammengefasst werden. Dabei sind die Identifizierung der Konsequenzen, die monetäre Bewertung der Nutzen und Kosten sowie die Begutachtung des Gesamtprojekts zu nennen ${ }^{88}$. Für die Bestimmung der Rationalität der gezeigten Reaktionen wäre eine vollständige Darstellung aller potentiellen Lösungsversuche aller im Moment zu lösenden Probleme einer Gesellschaft erforderlich. Dabei müsste die Auswertung in Form einer KNA der realisierten Hochwasserreaktion nicht nur positiv, im Sinne von effektiv, sondern auch noch größer als die der alternativen Projekte gewesen sein, um die effizienteste Handlung zu charakterisieren. Die folgende Zusammenfassung gibt einen Überblick über die theoretisch benötigten Informationen, wenn lediglich eine KNA für ein Hochwasserschutzprojekt berechnet werden soll: Angenommen, der Nutzen der realisierten Hochwasserschutzmaßnahme V läge darin, die potentiellen Schäden zu vermeiden, dann müsste gelten:

$$
\text { Nutzen }=(1+\mathrm{a}) \mathrm{V} \quad \text { mit der Annahme }(\mathrm{a}>0)
$$

Auf der Kostenseite der Analyse ständen die Bau- und Instandhaltungskosten (B) sowie die Kosten für Nutzeneinbußen oder Verluste (S), die durch die Realisierung der Hochwasserschutzmaßnahme auftreten:

Kosten $=\mathrm{bB}$ mit $(\mathrm{b}>1)$ und $\mathrm{cS}$ mit $(\mathrm{c}>1)$

Würde beispielsweise ein Schutz installiert, wäre dies nur zu verantworten, wenn gelten würde:
$(\mathrm{V}-\mathrm{B}-\mathrm{S})>(1+\mathrm{bB}+\mathrm{cS}-\mathrm{aV})$
bzw.
$(\mathrm{b}-1) \mathrm{B}+(\mathrm{c}-1) \mathrm{S}>\mathrm{aV}$

\footnotetext{
${ }^{88}$ Marggraf/Streb 1997.
} 
Im Laufe der Zeit akkumuliert der Mensch Besitz in den vor Hochwasser geschützten Gebieten. Damit steigt der potentiell gefährdete Wert. Da es in der Realität somit keine Angaben zu den tatsächlichen verhinderten Schäden geben kann, fällt auch die Bewertung des reellen Nutzens der Schutzanlage aus. Die Kosten bilden eine Mischkalkulation aus bekannten Baukosten. Die direkten Kosten sind den Rechnungen der Hersteller zu entnehmen. Hingegen sind die indirekten Kosten der tatsächlichen oder empfundenen Verschlechterung des Zustandes aufgrund von Einbußen in der bisherigen Nutzung nicht bestimmbar. Dabei kann die Verschlechterung des Zustandes für jeden Bürger etwas anderes bedeuten. Der eine mag sich an dem eingeengten Zugang zum Fluss stören, ein anderer allergisch auf die Pflanzen der neuen Blumenbeete reagieren. Zusätzlich zu der KNA der Hochwasserreaktion hätte ein Überblick über alle anderen alternativen Investitionsoptionen vorliegen müssen, um die Effizienz der Entscheidung zu beurteilen. Der Überblick hätte ein hohes Maß an Transparenz über alternative Handlungsoptionen sowie die Kenntnis interner und externer, sozialer und umweltbezogener Auswirkungen (zusätzlich zu dem Aspekt der Wirtschaftlichkeit) vorausgesetzt. Aufgrund der Quellenlage wäre eine vollständige Darstellung zu lückenhaft, um verlässliche Aussagen zu treffen.

Die Poppersche Dreiweltentheorie empfiehlt einen alternierenden Zugang. Die sachliche Strukturierung bezieht sich auf die Kosten, die ein Ereignis verursacht ${ }^{89}$. Diese können physisch oder psychisch sein. Demnach spiegeln die tatsächlich ergriffenen Reaktionen auf das Hochwasser und die dazugehörigen KNA die auf ihre Rationalität hin zu bewertende „1. Welt" wider. Popper bezeichnet mit diesem Begriff die physische Welt der Objekte. Da keine KNA vorhanden sind, wie die UBA-Studie zeigt, bleibt nur die Wahrnehmung der Entscheidungsträger aufgrund der in den Diskussionen und Veröffentlichungen genannten Präferenzen zu rekonstruieren. Popper beschreibt die Wahrnehmung und das Bewusstsein als die zwischen der 1. und der 3. Welt vermittelnde „2. Welt“. Der damit einhergegangene Diskurs über den Ressourceneinsatz muss folglich abgebildet werden, um die Beweggründe und Motive jener Zeit zu illustrieren. Durch eine Diskursanalyse werden die Logik und die Verständlichkeit, welche hinter den Hochwasserreaktionen standen, herausgearbeitet und verdeutlicht. Die „3. Welt“ bildet die Konsequenzen der Handlungen ab. Popper sieht in der 3. Welt den geistigen und kulturellen Gehalt. Ein Hochwasserereignis löst gesellschaftliche Reaktionen aus und diese wiederum beeinflussen durch den Ressourceneinsatz die sogenannte „3. Welt“. Diese Reaktionen lassen aufgrund des Ressourceneinsatzes gesellschaftliches Kapital entstehen oder verändern in einer weiterführenden Entwicklung das bereits

\footnotetext{
${ }^{89}$ Döring 1987, gilt für die Sätze zur Drei-Welten-Theorie.
} 
bestehende gesellschaftliche Vermögen. Abb. 1 zeigt schematisch die Unterscheidung zwischen der realisierten gesellschaftlichen Reaktion und dem Einfluss des Ereignisses auf das Kapital der Gesellschaft.

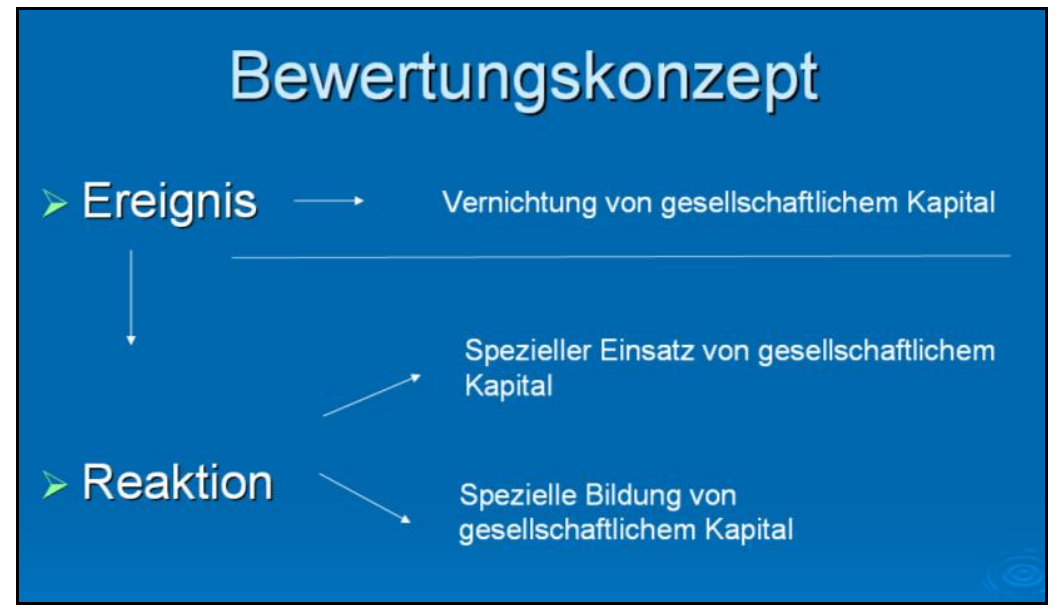

Abb. 1: Bewertungskonzept „,gesellschaftliches Kapital“.

Da in der „1. Welt“ keine finanzmathematische KNA aufzufinden ist, wird auf der zweiten Ebene - durch die Abstimmung der Ziele und die Vereinbarung der Mittel sowie der Abwägung der Investitions- und Opportunitätskosten - deutlich, dass der Prozess der Auswahl der spezifischen Hochwasserreaktionen vernünftig verlief. Zur Unterstützung und Strukturierung der Diskursanalyse wurde eine Hilfsmethode erarbeitet. Das Analyseinstrument wurde aufbauend auf philosophischen Diskursen zu den Begriffen der Rationalität und unter Einbezug der Arbeitsschritte einer KNA konzipiert. Es beinhaltet die folgenden Aspekte:

- Kosten und Nutzen gegenüber gestellt zu haben,

- Präferenzen der Entscheidungsträger zu erfüllen,

- Opportunitätskosten abgewogen zu haben,

- Risikoabschätzungen bzw. Grenzen der Alternativen zu erkennen,

- die Meinung der „Anderen“ mit einbezogen zu haben sowie

- Alternativen betrachtet zu haben.

Das Verfahren wird stufenweise angewandt, damit in der „2. Welt“ der Wahrnehmung und des Bewusstseins die Entscheidungsprozesse nachvollziehbar rekonstruiert werden können. Eine schematische Nachbildung des Abstimmungsverfahrens illustriert, dass eine Abwägung der Alternativen sowie der spezifischen Nutzen und Kosten stattfand. Abb. 2 zeigt das Schema der Hilfsmethode zur Analyse der Entscheidungen. 


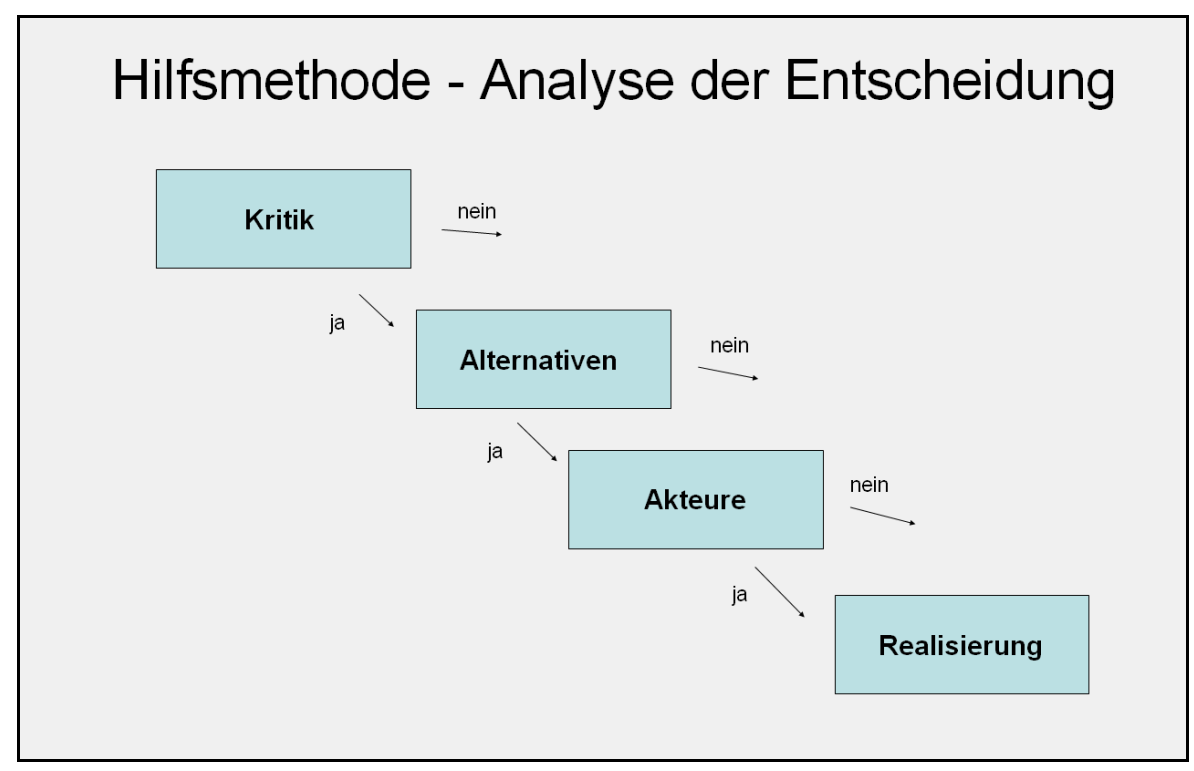

Abb. 2: Hilfsmethode zur Analyse der Entscheidungen.

Das Stufenmodell ist von unten rechts nach oben links zu lesen, obwohl es die Chronologie des Entscheidungsprozesses von der Diskussion zu der späteren Realisierung abbildet. Der Baustein der Realisierung spezifiziert, dass lediglich tatsächlich realisierte Reaktionen betrachtet werden. Nicht umgesetzte Reaktionen, etwa verworfene Alternativen, können nicht betrachtet werden, da diese erstens nur während der Untersuchung sichtbar werden und zweitens die Evaluierung der Rationalität im Nachhinein unbedeutend ist. Zum besseren Verständnis der einzelnen Maßnahmen erfolgt deren zeitliche Einordnung. Die Phasen des Katastrophenmanagementplans - Soforthilfe, Wiederaufbau, Prävention - lassen sich in operative und strategische Maßnahmen unterteilen. Dabei umfassen die operativen Maßnahmen alle Soforthilfeaktionen sowie den Wiederaufbau kurz nach dem Ereignis. Alle institutionellen Veränderungen sowie ursachenorientierte Präventionsmaßnahmen - besonders in Hinblick auf ein potentielles nächstes Hochwasser - fallen in den strategischen Bereich. Strategische Maßnahmen weisen auf grundlegende Veränderungen im Umgang mit dem Hochwasserereignis hin und geben Grund zu der Annahme, dass kontroverse Diskussionen geführt wurden. Dagegen weisen operative Maßnahmen nicht grundsätzlich auf Missstände hin.

Die Entscheidungen wurden in politischen Gremien gefasst. Diese Entscheidungsprozesse wurden von außen durch die allgemeine Wahrnehmung der Meinungsführer jener Zeit beeinflusst. Es soll gezeigt werden, wie die Entscheidungsfindung verlief. Welche Institutionen, Projekte, Regionen, Experten und Betroffene haben das Abstimmungsverfahren beeinflussen können? Gab es Gruppen, die sich durchsetzen konnten? Gab es ein Netzwerk von einflussreichen Persönlichkeiten? Besonderes Gewicht soll auf die Akteure und die 
Argumente, welche in dem Entscheidungsprozess angeführt wurden, gelegt werden. Ziel ist es herauszuarbeiten, welche wirtschaftlichen Interessen, Umweltaspekte, politischen Forderungen oder sozialen Faktoren den Hochwasserschutz in den betroffenen Gebieten beeinflussten? Waren alle Beteiligte in der Lage, Interessen zu formulieren, oder war dies einem ausgewählten Personenkreis vorbehalten? Wurde das Hochwasser von Entscheidungsträgern instrumentalisiert, um andere Interessen durchzusetzen?

Der begrenzte Zugang zu Ressourcen zwingt den Menschen, zu jeder Zeit zu entscheiden, welche Alternative jeweils seiner Präferenz entspricht. Anzunehmen ist deshalb, dass in Abstimmungsprozessen potentielle Alternativen und implementierte Maßnahmen gegeneinander abgewogen wurden. Der Vergleich gibt Aufschluss über die zeitgenössische Wahrnehmung, beweist bzw. begründet aber v. a. die Abwägung der Investition selbst, denn die Ressourcen hätten anderweitig eingesetzt werden können. Der Verzicht auf angeführte Interessen entspricht den unquantifizierbaren Opportunitätskosten in der „Berechnung“ von Nutzen und Kosten.

Die Kritik an den möglichen Handlungsalternativen kennzeichnet das Bewusstsein für die spezifischen Grenzen der jeweiligen Wahl. Besonders vor dem Hintergrund der kommunikativen Entscheidungsprozesse mit Konsenserwartung sind Einwände ein einfacher Bestandteil eines jeden Meinungsaustausches. Kritik an der Alternative kann einerseits Risiken erhellen oder andererseits genutzt werden, Akteure zu kompromittieren. Es ist kaum vorstellbar, dass die Akteure in einer von ihnen gewählten Handlungsweise keine Defizite sahen. Eine Alternative, an der keine Kritik geübt wurde, wäre eine unbedachte Wahl, da sie ohne die Betrachtung potentieller Konsequenzen realisiert worden wäre. Die Entscheidung wäre somit nicht ganz verständlich, denn eine fehlende Kritik wäre ein Hinweis auf mangelnde Rationalität.

Die Grenze der konstruierten Hilfsmethode liegt darin, dass ein Vorschlag, welcher zur Realisierung kommt, nicht per se die beste Wahl ausgibt. Außerdem kann nicht abschließend geklärt werden, ob die implementierte Reaktion die tatsächlich beste Lösung verkörperte. Das Absolvieren aller Phasen des Stufenplans wie die Betrachtung der beteiligten Akteurskreise, der Vergleich zwischen Alternativen sowie das Vorhandensein von Kritik beweist nicht automatisch die Rationalität der Reaktion. Zudem darf nicht angenommen werden, dass Vorschläge, welche aus dem Hilfskonzept herausfallen nicht ebenfalls vernünftig gewesen sein könnten. Die Annahme Poppers über den lösungsorientiert handelnden Menschen gilt als systemunabhängig und zeitlos. Es ist also anzunehmen, dass die gezeigten Reaktionen tatsächlich vernünftig waren, denn ein Hochwasserereignis und die getroffenen 
Entscheidungen erwachsen aus ihren spezifischen Kontexten und müssen auch in diesem betrachtet werden. Das erarbeitete Hilfsmodell dient als methodische Struktur für die folgenden Untersuchungen, während Schnädelbachs Ansatz der Verständlichkeit und die Theorie des sozialen Subjektivismus die theoretischen Grundlagen bilden.

\subsection{Untersuchungsgebiete und -zeitraum}

In der vorliegenden Untersuchung werden die politischen Reaktionen auf das Rheinhochwasser in der Stadt Köln und auf das Mekonghochwasser in der vietnamesischen Provinz An Giang gegenübergestellt. In Deutschland besorgen die Bundesländer den Hochwasserschutz, der in Nordrhein-Westfalen den kommunalen Verwaltungen obliegt ${ }^{90}$. In Vietnam hingegen wird der Hochwasserschutz als nationale Aufgabe verstanden und wird daher von einer zentralen politischen Abteilung gelenkt. Zwei unterschiedliche Organisationssysteme werden verglichen: Im ersten Fall werden seit zwei Dekaden Erfahrungen mit einer auf die nationale Politik zentrierte Hochwasserverantwortung zur Schadensreduzierung gesammelt, im zweiten Fall wurden im gleichen Zeitabschnitt wiederholt vorgeschlagen, diese Zentrierung auch in Deutschland einzurichten. Die Bundesrepublik Deutschland ist eine demokratische Marktwirtschaft während die Sozialistische Volksrepublik Vietnam als planwirtschaftlicher Einparteienstaat durch die Kommunistische Partei Vietnams (KPV) gelenkt wird. Die wirtschaftlichen und politischen Systemunterschiede der beiden Regionen lassen auf eine breite Varianz der Reaktionen auf Hochwasser hoffen ${ }^{91}$. Deswegen werden zunächst die beiden politischen Organisationsstrukturen der Hochwasserbekämpfung erläutert. Seit 1993 ist der Hochwasserschutz in Köln institutionalisiert worden. Nach dem verheerenden Hochwasser von 1993 sah die Stadt einen größeren Personalbedarf für die Betreuung des Hochwassers und gründete die Hochwasserschutzzentrale in dem Amt für Stadtentwässerung. Seit Dezember 1994 ist diese Zentrale ganzjährig im Einsatz. Vorher wurde lediglich nach einem Hochwasserereignis ein Bericht für den Stadtrat über die Vorkommnisse während der Soforthilfe aufgelistet. Das Amt für Stadtentwässerung wurde 2001 geschlossen und aus der

\footnotetext{
${ }^{90}$ Nach Art. 75 Abs. 2 Grundgesetz der BRD überlies der Bund den Hochwasserschutz der Landesebene. In Nordrhein-Westfalen (NRW) ist Hochwasserschutz, anders als in anderen Bundesländern, eine Aufgabe der Selbstverwaltung. Diese regelten in NRW zum Zeitpunkt des Jahrhunderthochwassers 199525 Verbände und 13 Kommunen (Landtag NRW, Ausschussprotokoll 12/651, 17. September 1997).

${ }^{91}$ Schweizer 1992.
} 
Verwaltung der Stadt Köln ausgegliedert und als Anstalt öffentlichen Rechts wiedereröffnet.

Die kommunalen Wasserthemen, wie beispielsweise die Behandlung von Abwasser, wurden zu Aufgaben der neu gegründeten Stadtentwässerungsbetriebe Köln AöR (Steb). Jedoch verblieb die Hochwasserschutzzentrale zunächst in der städtischen Behörde, bis diese 2003 ebenfalls in den Aufgabenbereich der Steb eingegliedert wurde. Zur Übersicht der Hochwasserzuständigkeiten in Köln zeigt Abb. 3 die Organisationsentwicklung seit 1985.

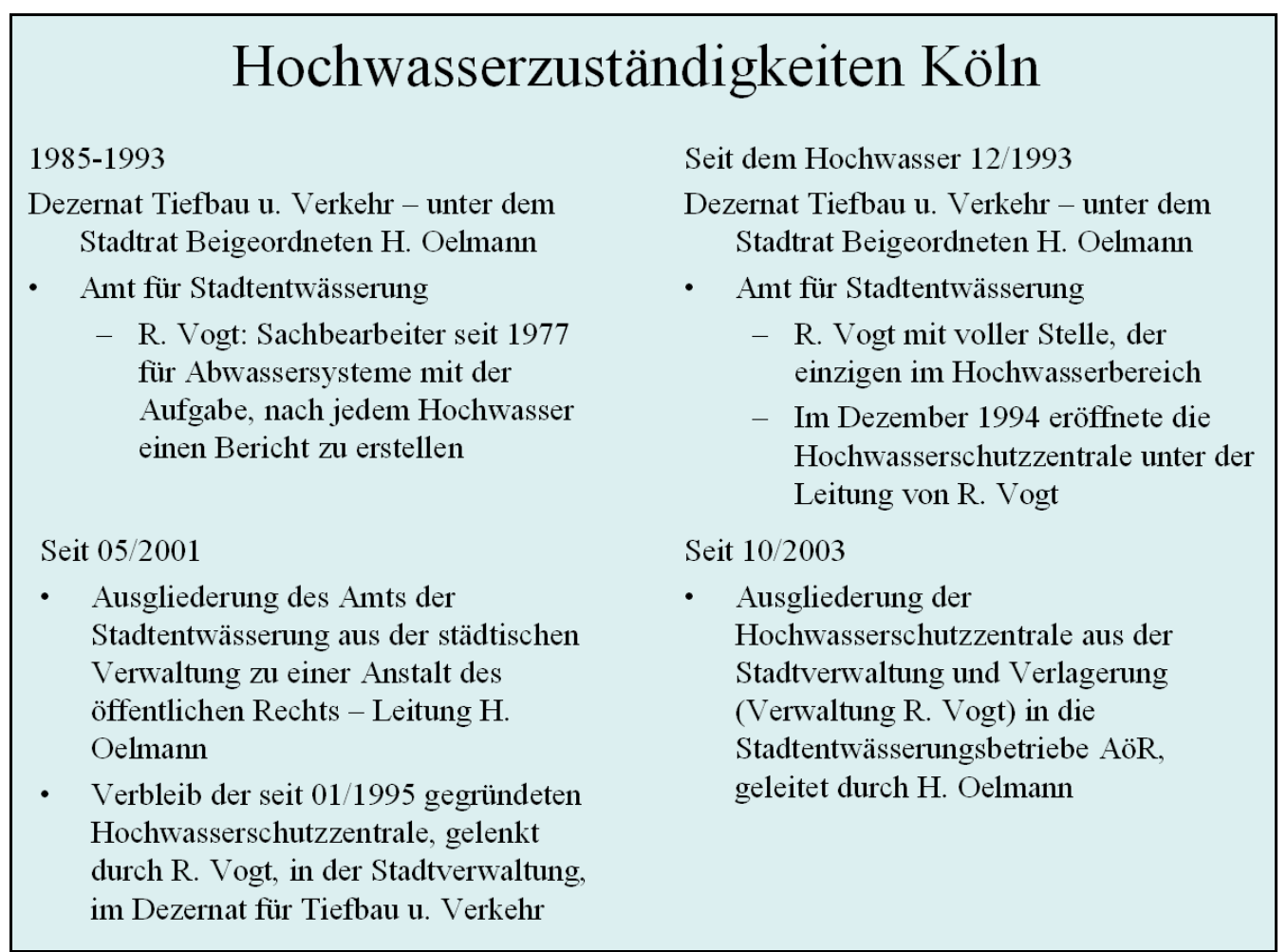

Abb. 3: Institutionalisierung des Kölner Hochwasserschutzes seit 1985.

In Vietnam ist der Hochwasserschutz keine kommunale Aufgabe. Dort entscheidet die staatliche Regierung über das $\mathrm{Maß}$ an Schutz vor Überschwemmungen. Nach der Wiedervereinigung von Nord- und Südvietnam 1976 wurde die Aufgabe, die Fluten des Mekongs zu kontrollieren, dem nordvietnamesischen Wasser Ressourcen Ministerium in Hanoi unterstellt. Das Ministerium führte zunächst eine Abteilung auf Provinzebene sowie Büros in den jeweiligen Distrikten. Die Verantwortlichen planten und führten alle technischen Maßnahmen, wie den Bau von Bewässerungssystemen, Kanälen und Deichen aus. Auf allen drei Ebenen waren dem Ministerium zunächst staatliche Handwerksbetriebe angeschlossen, bis diese nach der Wirtschaftsreform (Doi moi) von 1986 privatisiert wurden ${ }^{92}$. Der Name des Wasser Ressourcen Ministeriums wurde 1995 zum Ministerium für Landwirtschaft und

\footnotetext{
${ }^{92}$ Im Kapitel zu den politischen Reaktionen zu Hochwasser in Vietnam werden die Auswirkungen der Liberalisierungsreform auf die Ausführung der Deicharbeiten erläutert.
} 
Rurale Entwicklung weiterentwickelt. Der Hochwasserschutz wurde weiterhin durch das Department für Deich Management, Flut-Kontrolle und Sturm Bereitschaft - in Abbildung 4 aus Platzgründen als Department für Deich Management und Flut Kontrolle bezeichnet unter dem Schirm des Landwirtschaftsministeriums ausgeführt. 1990 wurde das Zentrale Komitee für Flut und Sturm Kontrolle gegründet, das direkt der Regierung unterstellt ist, und neben Deichreparaturen auch Hilfsmaßnahmen koordiniert. Abb. 4 zeigt schematisch die Hierarchie des Hochwasserschutzes in Vietnam. Das Zentralkomitee der Kommunistischen Partei Vietnam (KPV) sowie die Ministerien sind dem Premierminister unterstellt, die letztendliche Entscheidungsbefugnis liegt bei dieser Instanz. Beide Organe pflegen Repräsentanzen auf der Ebene der Provinzen sowie in den Bezirken und deren Kommunen.

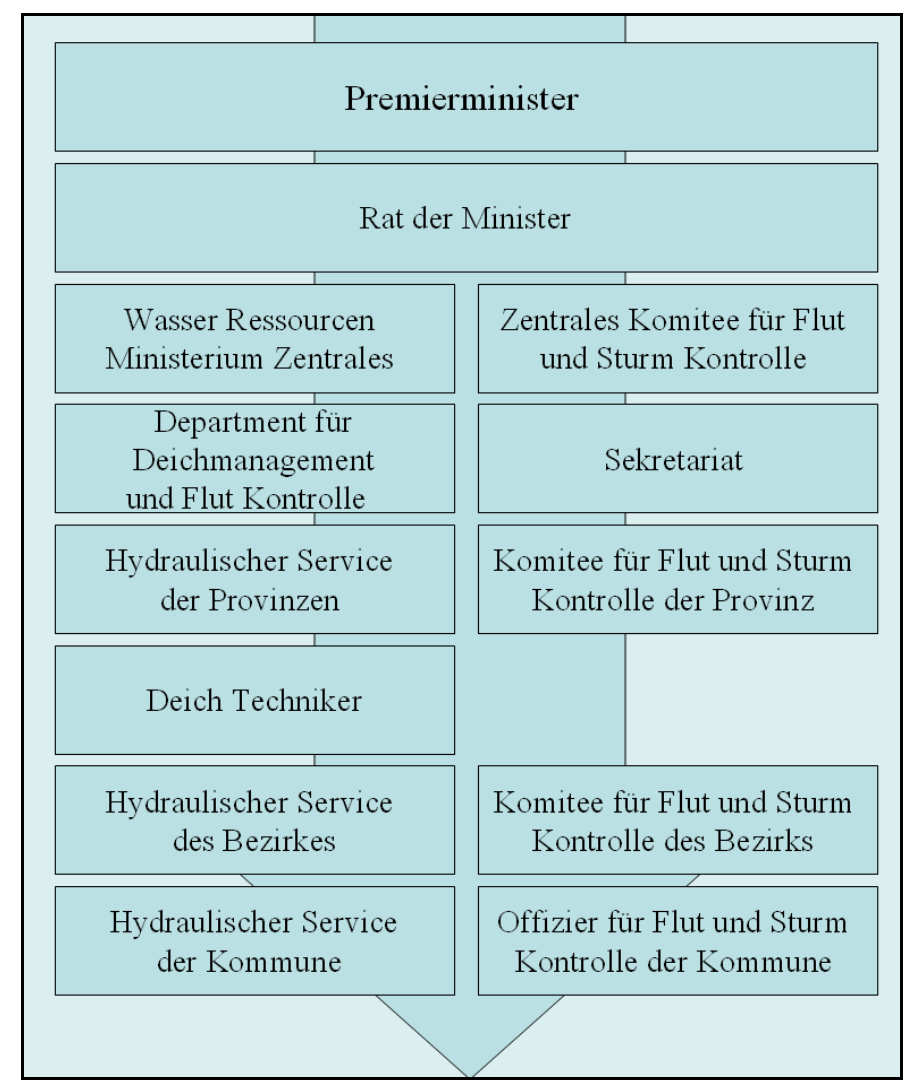

Abb. 4: Organisation des Hochwasserschutzes in Vietnam seit 1990.93

Beide Untersuchungsgebiete verbindet das geographische Problem, Unterlieger eines Flusslaufes zu sein, der von den Handlungen der Menschen am Oberlauf, den Oberliegern,

\footnotetext{
${ }^{93}$ Nach DR 004, 13.
} 
beeinflusst wird ${ }^{94}$. Abb. 5 zeigt die Wassereinzugsgebiete der beiden Flüsse. Der Mekong fließt von Norden nach Süden, während der Rhein von Süden nach Norden fließt.

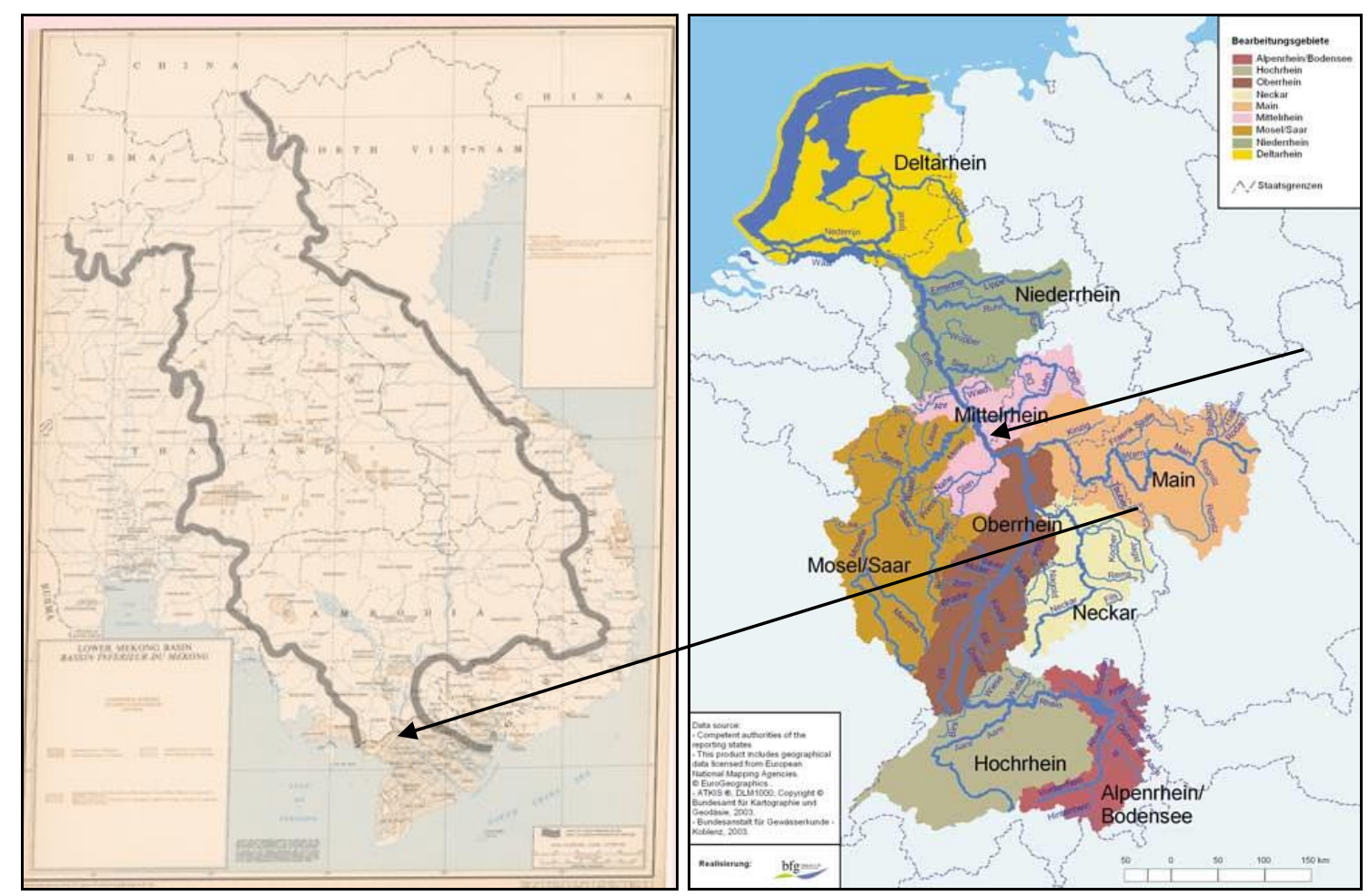

Abb. 5: Wassereinzugsgebiete des Mekongs und des Rheins. ${ }^{95}$

Die Stadt Köln hatte in dem Betrachtungszeitraum zwischen 600.000 bis 1 Mio. Einwohner und umfasst seit der Gebietsreform 1975 eine Fläche von 405,2 km². Die Provinz An Giang besitzt eine Fläche von $3.406 \mathrm{~km}^{2}$ auf der zischen 1990 - 20101.97 - 2,15. Mio. Einwohner lebten. In Köln liegt der Pegel des Mittelwassers bei einer Höhe von 3,21 m Kölner Pegel $(\mathrm{K} \mathrm{P})^{96}$. Hochwasser wird in Köln ab einem Wasserstand von 4,50 m K P ausgerufen. Der bauliche Hochwasserschutz variierte im Betrachtungszeitraum zwischen $7-11,70 \mathrm{~m} \mathrm{~K} \mathrm{P}$. Seit 1926 lag der bemessene Pegel eines Jahrhunderthochwassers (Q100) bei 10,96 m K P und einer Abflussmenge von $10.800 \mathrm{~m}^{3 /} \mathrm{s}$. Ein Bemessungshochwasser entspricht dem Wasserstand eines Hochwasserereignisses oder einer Extrapolation aus Wasserständen. Seit der Neuberechnung durch das Kölner Umweltamt wurde der Wasserstand für ein Hochwasserereignis mit einer statistischen Eintrittswahrscheinlichkeit von 100 Jahren bei

\footnotetext{
${ }^{94}$ Beide Flüsse haben beim Eintritt in das Untersuchungsgebiet bereits den Großteil ihrer Länge hinter sich gelassen. Das Wassereinzugsgebiet des $1.320 \mathrm{~km}$ langen Rheins ist $160.000 \mathrm{~km}^{2}$ groß (HWSK 1996, 3). Das Einzugsgebiet des $4.880 \mathrm{~km}$ langen Mekongs umfasst $795.000 \mathrm{~km}^{2}$ (Miller 2003, 80).

${ }^{95}$ Die Karte des Mekongeinzugsgebiets stammt aus dem Jahr 1968 und ist der Internetbasierten Quelle 11. entnommen worden. Die Quelle der Abbildung des Rheineinzugsgebiets ist die Internetbasierte Quelle 12.

${ }^{96}$ Internetquelle 6. Der Begriff Mittelwasser bezeichnet den über einen längeren Zeitraum und an einer bestimmten Stelle gemessenen Wasserspiegel.
} 
11,29 m K P, mit einem Durchfluss von $12.000 \mathrm{~m}^{3 /}$ s festgelegt $^{97}$. Dazu gab das Umweltamt in Köln die Referenzdaten für ein 200jähriges Ereignis (Q200) mit 11,89 m K P und ca. 13.000 $\mathrm{m}^{3 /} \mathrm{s}$ Wasservolumen $\mathrm{an}^{98}$. In Köln wird der Hochwasserstand an der Rheinuferstrasse durch den Pegel festgestellt. Abb. 6 zeigt das Pegelhäuschen auf der Promenade an der Frankenwerft.

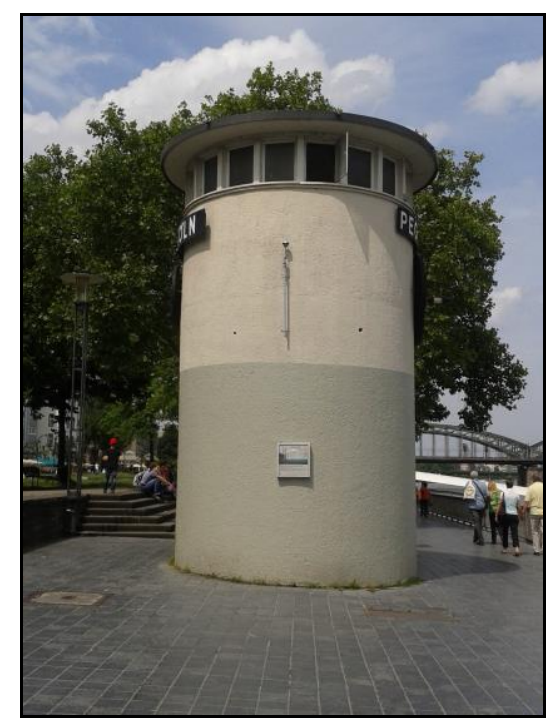

Abb. 6: Kölner Pegel an der Frankenwerft 2013. ${ }^{99}$

Der Pegel war bis zur Neukalibrierung am 1. November 1979 auf 35,972 m N. N. ausgerichtet. Dann wurde der Nullpunkt auf eine Fahrrinne von $150 \mathrm{~m}$ Breite ausgerichtet, bei dem noch ein Wasserstand von einem Meter garantiert werden kann. Die Umstellung hat zur Folge, dass alle Wasserstände und die Schutzvorrichtungen nun einem Meter höher als zuvor gemessen werden, obwohl diese faktisch unverändert blieben. Ein Hochwasser steigt mit ca. zwei Zentimetern pro Stunde ${ }^{100}$. Das Überschwemmungsgebiet betrifft die Anwohner entlang des Flusses und ist abhängig von der Höhe des Hochwassers. Eine Karte kann aufgrund dessen nicht allgemeingültig präsentiert werden.

\footnotetext{
${ }^{97}$ HWSK 1996. Auf Grundlage der Berechnungen der Bundesanstalt für Gewässerkunde ermittelte das Umweltamt Köln neue Grenzen. Das staatliche Umweltamt Köln ist der Behörde der Bezirksregierung nachgeordnet (Landtag NRW, Drucksache 12/248, 11.10.1995).

${ }^{98}$ Ebd.

${ }^{99}$ Eigene Aufnahme.

${ }^{100}$ Siehe Hochwasserbeschreibungen von 1970, 1983, 1993 und 1995.
} 
Im vietnamesischen Mekong-Delta ist Hochwasser eine saisonale Erscheinung. Jedes Jahr im August beginnt der Wasserspiegel im Mekong-Delta mit $5 \mathrm{~cm}$ pro Tag zu steigen ${ }^{101}$, bis er Ende September oder Anfang Oktober seinen Höchstpunkt erreicht und von da an bis Dezember wieder sinkt. In jedem Kubikmeter sind in etwa ein halbes Kilogramm Sediment, Lehm und organische Materialien enthalten, welche als natürliche Dünger wirken und den Boden des Mekong-Deltas fruchtbar machen ${ }^{102}$. In Zeiten des Hochwassers variiert die Höhe der Überschwemmung zwischen 1 und 5 m. Die Überschwemmung wird als hoch empfunden, wenn an der Messstation in Tan Chau mehr als 4,5 $\mathrm{m}$ gemessen werden, jedoch wird Hochwasser erst ab einem Wasserstand von 4 m ausgerufen ${ }^{103}$. Zwischen 1926 und 2006 war das 22 Mal der Fall ${ }^{104}$. Gemessen wird das Hochwasser zusätzlich an Stationen, die in der Trockenperiode nicht am Wasser liegen. Die Hochwasserereignisse aus den Jahren 2000, 1996, 1991, 1984, 1978, 1966, 1961 stachen durch besonders hohe Pegelstände hervor. Im Jahr 2000 wurden in Tan Chau 5,2 m gemessen ${ }^{105}$. Der Strom des Mekongflusses variiert stark im Zyklus eines Jahres. In den Trockenmonaten während des ersten Quartals fallen die Pegel auf statistische 2,55 $\mathrm{m}^{106}$. Das Wasservolumen verringert sich auf $33.309 \mathrm{~m}^{3} / \mathrm{s}^{107}$. Das Hochwasser bedeckt in der nassen Jahreshälfte $12.000-19.000 \mathrm{~km}^{2}$ des Mekong-Deltas ${ }^{108}$, was $48 \%$ der gesamten Fläche entspricht. Die Fluten basieren zu $60 \%$ auf dem saisonal angeschwollenen Wasservolumen des Mekong, zu $30 \%$ auf den Gezeiten, welche von den Küsten durch die Kanäle und Flussmündungen in das Delta eindringen und $\mathrm{zu} 10 \%$ auf Regenfällen vor Ort ${ }^{109}$. Diskussionen über unnatürliche Hochwasser aufgrund anthropogener Einflüsse wurden im Kontext zu Wasserkraftanlagen am Oberlauf des Flusses erst nach der Jahrtausendwende eröffnet. Zur Veranschaulichung der saisonalen Überschwemmung zeigt Abb. 7 zwei Hochwasserkarten.

\footnotetext{
${ }^{101}$ Artikel: Lu dong bang song Cuu Long len cao va co kha nang lan rong, Zeitung An Giang, 25.07.2000.

${ }^{102}$ Howie 2011; 2006.

${ }^{103}$ Tuan 2007, 2.

${ }^{104}$ Ebd.

${ }^{105}$ Interview Dr. Van Pham Dang Tri, Can Tho University, März 2011.

${ }^{106}$ Ebd.

${ }^{107} \mathrm{Ebd}$

${ }^{108}$ Tuan 2010.

${ }^{109}$ Ebd.
} 


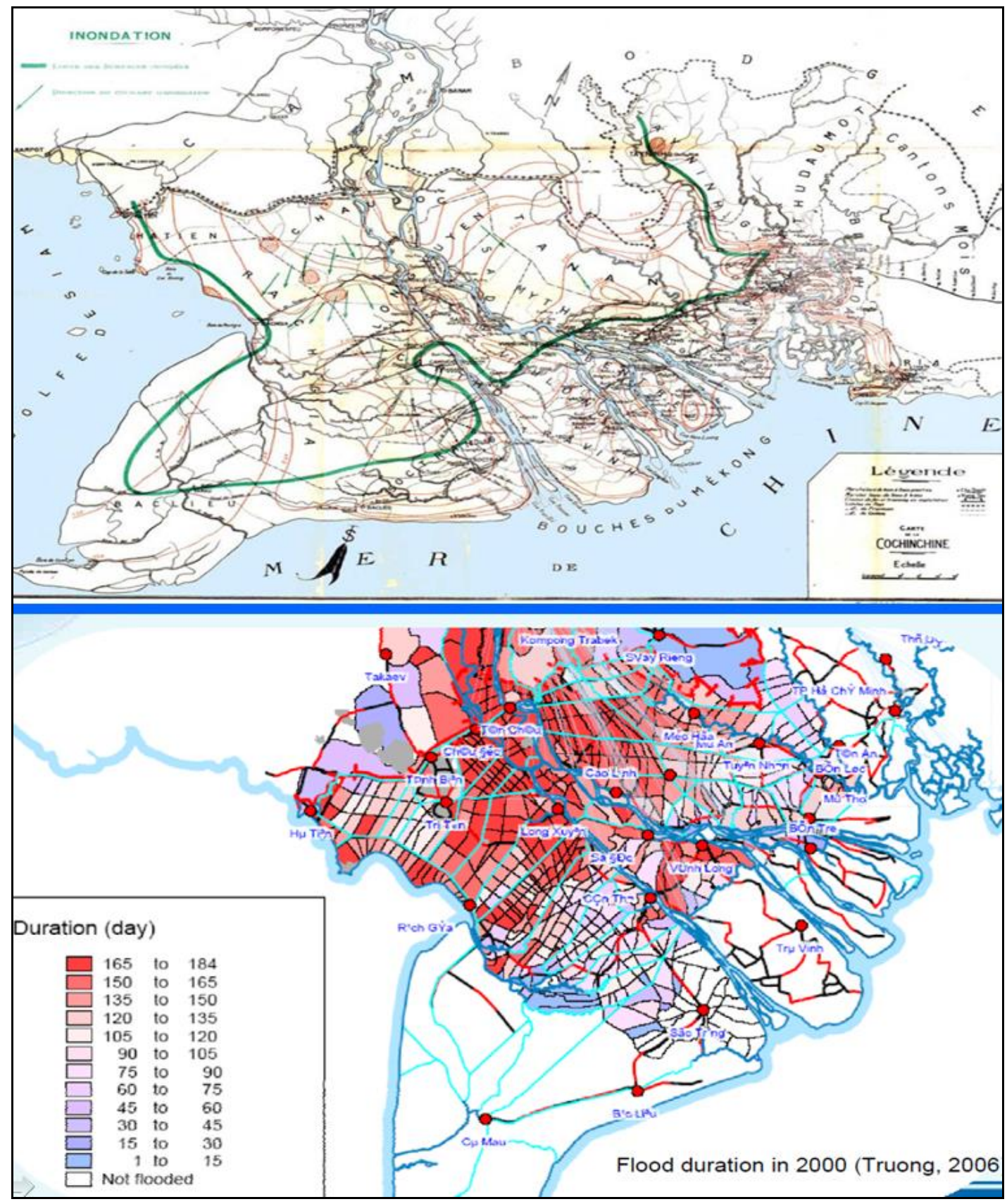

Abb. 7: Überschwemmungsgebiete im Mekong-Delta.

Eingezeichnet auf einer Französischen Karte von 1911, geben die grünen Pfeile Auskunft über die Wassertiefe. In der damaligen Provinz Chau Doc (An Giang) galt eine Wassertiefe von $4.0 \mathrm{~m}$ als durchschnittlich. Die roten Kreisflächen bedeuten gebirgiges Gelände. ${ }^{110}$ Die modellierte Gefahrenkarte aus 2006 kennzeichnet den Wasserstand von 2000. ${ }^{111}$

Hochwasser ist eine von vier Naturgefahren im vietnamesischen Mekong-Delta. Neben dem jährlichen Hochwasser besteht ein latenter Mangel an Süßwasser, eine Bedrohung durch die Versalzung der Böden durch das von den Seeseiten im Süden und im Westen eindringende Meereswasser, sowie das Versauern der Böden.

\footnotetext{
${ }^{110}$ Karte und Interpretation nach Biggs 2004, 126.

${ }^{111}$ Interview: Dr. Van Pham Dang Tri, Can Tho University, März 2011.
} 
Die konkreten Hochwasserereignisse für die vorliegende Untersuchung wurden nach zwei Kriterien ausgewählt. Da diese Arbeit einen Beitrag zum aktuellen und zukünftigen Klimaund Katastrophendiskurs politischer Entscheidungsträger leisten soll, wurden maximal die Zeiträume bis zur letzten kriegerischen Auseinandersetzung aufgegriffen. Deswegen werden die politischen Hochwasserdiskurse in Vietnam im Zeitraum 1976 bis 2000 betrachtet. Für die Betrachtungsregion am Rhein wurden die politischen Entscheidungen seit dem Ende des Zweiten Weltkriegs betrachtet. Das zweite Kriterium zur Auswahl der Naturereignisse liegt in der Unterstellung einer Veränderung der Mensch-Umwelt-Beziehung. In Vietnam begann mit der wirtschaftlichen Öffnung Vietnams 1986 die Industrialisierung der Landwirtschaft. Es vollzog sich ein Wandel von einer Nation in Hunger zum weltweit zweitgrößten Reisexporteur zu Beginn der 1990er Jahre ${ }^{112}$. In Deutschland begann in den 1960-70iger Jahren mit der Umweltbewegung die Diskussion zur umweltschonenden Industrie insbesondere in Nordrhein-Westfalen ${ }^{113}$.

\subsection{Quellen und Quellenkritik}

Maßnahmen und Institutionen erwachsen im Zeitverlauf und geben Auskunft über sich wandelnde gesellschaftliche Bedeutungen von Gefahr, Risiko und Natur. Die Basis der Analyse bilden Akteure aus der Politik, der Wirtschaft und der Wissenschaft. Für dieses Dissertationsprojekt wurden neben einigen Interviews mit vietnamesischen Wissenschaftlern und deutschen, derzeitig am Hochwassermanagement beteiligten Akteuren hauptsächlich schriftliche Quellen berücksichtigt.

Die betrachteten schriftlichen Quellen setzen sich aus lokalen und landesweiten Zeitungen, Akten und Kartenmaterial des Landesarchivs Nordrhein-Westfalens in Düsseldorf, des Stadtarchivs Can Tho, des Archiv der Entwicklungszusammenarbeit in Hanoi, der Provinzbibliothek Can Tho, der Stadtbibliotheken in Köln und Chau Doc in der Provinz An Giang, dem Archiv der Industrie- und Handelskammer zu Köln sowie ferner Dokumente der indirekt und direkt mit dem Hochwassermanagement beauftragten Institutionen zusammen ${ }^{114}$. Material des Stadtarchivs Köln konnte aufgrund des Einsturzes im Jahr 2008 nicht eingesehen werden. Die Unterlagen des Tiefbauausschusses, der in Köln die

\footnotetext{
${ }^{112}$ Miller 2003.

${ }^{113}$ Radkau 2011 und Willy Brandts Rede zum Blauen Himmel über der Ruhr, 1961.

${ }^{114}$ Ein Beispiel für eine direkt beteiligte Institution ist die Stadtreinigung Köln zu nennen und für indirekt beteiligte Institutionen können Hilfsorganisationen wie das Rote Kreuz angeführt werden.
} 
Hochwasserschutzmaßnahmen plante, wurden kurz vor dem Einsturz an das Archiv gegeben und bleiben bis auf weiteres verschollen ${ }^{115}$.

Wissenschaftliche Tätigkeiten im Kontext zum Hochwasser des Mekongs werden in Vietnam häufig von internationalen Forschungszusammenschlüssen behandelt (z. B. WISDOM, DRAGON, MRC). Die Ergebnisse spiegeln den jeweils aktuellen Stand wider. Im Kontext zueinander betrachtet wird der Verlauf der Diskussion erkennbar. Erst seit der verheerenden Flut von 2000 sind sozioökonomische Untersuchungen des Hochwassers in den akademischen Fokus gerückt ${ }^{116}$. Eine Systemkritik ergibt sich ausschließlich mündlich, während in schriftlichen Arbeiten neben der Konsenstendenz innovative Vorschläge und Kritik zwischen den Zeilen zu lesen sind. Der politische Einfluss dringt auch in die Akademien, da die Parteizugehörigkeit als Voraussetzung für eine Position an einer Universität gilt.

Die Analyse der Reaktionen auf das Rheinhochwasser bezog sich hauptsächlich auf die Diskussionen des Stadtrats und des Landtags bzw. des Umweltministeriums des Landes Nordrhein-Westfalens, dessen Bezeichnung über den Betrachtungszeitraum mehrfach verändert wurde. Zwischen 1946 - 1985 hieß es Ministerium für Ernährung, Landwirtschaft und Forsten, zwischen 1985 - 2000 wurde es als Ministerium für Umwelt, Raumordnung und Landwirtschaft bezeichnet und ab 2000 wurde es Ministerium für Umwelt und Naturschutz, Landwirtschaft und Verbraucherschutz genannt. Seit 2010 heißt die Behörde Ministerium für Klimaschutz, Umwelt, Landwirtschaft, Natur- und Verbraucherschutz des Landes NordrheinWestfalen.

In Vietnam wird Hochwassermanagement als zentralstaatliche Aufgabe betrachtet. Das Ministerium für Wasserressourcen war seit der Wiedervereinigung von Nord- und Südvietnam auch für die Mekongregion zuständig. Auch dieses Ministerium hatte im Laufe des Betrachtungszeitraums wechselnde Namen. Im Jahr 1995 wurde es zum Ministerium für Landwirtschaft und Rurale Entwicklung (MARD) umbenannt. Unter dem MARD operieren thematische Abteilungen wie das Department für Deich Management, Flut Kontrolle und Sturm Bereitschaft. Seit 2002 ist neben dem Landwirtschaftsministerium das Umweltministerium Ministerium für natürliche Ressourcen und Umwelt für Wasserressourcen zuständig, sodass Maßnahmen des Hochwasserschutzes von den Ministerien gemeinschaftlich entschieden werden. Dabei bleibt das Landwirtschaftsministerium technischen Aufgabenfeldern verhaftet, während das Umweltministerium in Qualitätsfragen tätig wird. Weitere Publikationen der Planungs- und Forschungsinstitute der vietnamesischen Regierung wie das Southern Institute

\footnotetext{
${ }^{115}$ Auskünfte der Stadtentwässerungsbetriebe Köln AöR, der Stadtverwaltung Köln und des Stadtarchivs Köln vom 03. Juli 2013.

${ }^{116}$ Interview, Dr. Nguyen Van Be, Can Tho University, März 2012.
} 
of Water Resource Research (SIWRR), das Southern Institute for Water Resource Planning (SIWRP), das National Institute for Sciences and Technology Policy and Strategy Studies und das Central Institute for Economic Management (CIEM) wurden für diese Untersuchung beachtet. Gesetzestexten können unproblematisch aus dem Internet heruntergeladen werden, während die interne Kommunikation wie beispielsweise Plenarprotokolle auf keinen Fall an Akteure außerhalb des Kreises der Entscheidungsträger ausgehändigt werden. Mit dem Ziel die Kosten-Nutzen-Vorstellungen der handelnden Akteure aus den politischen Institutionen herauszuarbeiten, wurde der Hochwasserdiskurs weitergefasst. Neben direkten Reaktionen auf das Hochwasser wurde der Diskurs zur Intensivierung der Landwirtschaft als auch der Desasterdiskurs betrachtet. Dieses Vorgehen begründet sich wie folgt. Die Landwirtschaft im Mekong Delta wir seit jeher aufgrund der saisonalen Überschwemmungen von dem Hochwasser eingeschränkt. Die monatelang überschwemmten Flächen sind zumindest in dieser Zeit nicht bestellbar. Das Interesse, die Landwirtschaft zu intensivieren entfachte eine Diskussion darüber, Überschwemmungsflächen trocken zu legen und der Agrarwirtschaft zu zuteilen. Es ist also zu erwarten, dass im Landwirtschaftsdiskurs auch Meinungen über das Mekong Hochwasser und wie man damit umzugehen hat, geäußert wurden. Die Erweiterung um den Desasterdiskurs soll erklären, ob die natürlichen Hochwasser im Mekong-Delta als Katastrophe empfunden wurden oder nicht. Außerdem soll die Beleuchtung des Diskurses erhellen, welche Ursachen als Begründung für die Fluten und die gestiegenen Schäden herangezogen wurden. Die erweiterte Perspektive verdichtet die Quellenlage, da Protokolle der politischen Auseinandersetzungen zum Hochwasserschutz kompensiert werden müssen. Lediglich ein Aushandlungsprozess kann beispielhaft herangezogen werden. Die Erläuterung basiert auf der Studie von Pham Cong Huu 2010 - die Daten konnte er in Interviews sammeln. Detaillierte Zeugnisse politischer Entscheidungsprozesse sind schlichtweg nicht zugänglich. Die hier analysierten Daten wurden aus sekundären Quellen herangezogen, dabei ist zu beachten, dass die Autoren möglicherweise mit zensierten Daten gearbeitet haben. Des Weiteren ist die im Ausland erschienene Literatur wahrscheinlich nicht zensiert worden, die jeweiligen Autoren aber evtl. an einer zukünftigen Zusammenarbeit mit vietnamesischen Kollegen interessiert und deshalb der Selbstzensur unterworfen. 


\section{Wahrnehmung des Kölner Stadtbildes - Stadtgestaltung durch Hochwasserschutz}

In der Nachkriegszeit wurde der Grundstein für den heutigen baulichen Hochwasserschutz in Köln gelegt. Nach einer zweijährigen Diskussionsphase wurde 1960 der Bau einer Hochwasserschutzmauer bis $\mathrm{zu}$ einem Schutzpegel von $8,40 \mathrm{~m}$ Kölner Pegel (K P) beschlossen. Erst 1970 wurde die umstrittene Vorrichtung zum ersten Mal auf die Probe gestellt. Obzwar die Mauer überspült wurde, ergaben sich keine neuen Debatten zur Vergrößerung des baulichen Schutzes. Daraus ergibt sich die Frage, warum die Höhe der Mauer nicht neu verhandelt wurde?

Erst zwanzig Jahre nach dem Bau der Mauer wurde sie dann zum zweiten und dritten Mal überströmt. Im April sowie im Mai 1983 mussten die Kölner Überschwemmungen mit fast 10 m K P hinnehmen. Die vorhandenen Schutzvorrichtungen wurden daraufhin ohne Disput der Entscheidungsträger auf eine Höhe von 10 m K P verstärkt. Eine Dekade lang wähnten sich die Kölner in Sicherheit, besonders da sich die Erhöhung der Hochwasserschutzmauer bereits 1988 für die Stadt bewähren sollte. Das Weihnachtshochwasser vom Dezember 1993 stellte die Wirksamkeit der Schutzvorrichtungen aufgrund der hohen Überschwemmungen und den daraus resultierenden Schäden allerdings erneut in Frage. Neben Überlegungen zur Verstärkung der Schutzbauten wurden alternative Hochwasserschutzmöglichkeiten, wie beispielsweise die Nutzung von Retentionsräumen gefordert. Das Jahrhunderthochwasser vom Januar 1995 bestärkte die Verantwortlichen in ihrer Auffassung, dass ein neues Konzept zur Schadensvermeidung - welches bis heute gilt - geschaffen werden musste. Das 1996 veröffentlichte Hochwasserschutzkonzept integrierte erstmals kommunikative Instrumente zur Steigerung des lokalen Gefahrenbewusstseins, überregionale Aktivitäten mit dem Ziel, die Pegelhöhen in Köln zu lindern, sowie den baulichen Hochwasserschutz in Köln. Unter anderem wurden die Schutzmaßnahmen in der Stadt durch Spundwände auf 11,30 m K P verstärkt und die für die Lagerung der mobilen Elemente benötigten Hallen als symbolische Repräsentation der Hochwassergefahr zur Steigerung des Gefahrenbewusstseins integriert.

Die Stadt lag nach dem Zweiten Weltkrieg in Schutt und Asche. Die politisch Verantwortlichen diskutierten nach dem Krieg intensiv, wie sie die bauliche Ästhetik der Stadt wieder aufleben lassen konnten ${ }^{117}$. Über die Jahre hinweg blieb die Verknüpfung von der Bewahrung des Stadtbildes und dem Ausbau des Hochwasserschutzes bestehen und galt als Argument, um keine Hochwasserschutzmauer zu bauen. Deshalb ist zu prüfen, ob die

\footnotetext{
${ }^{117}$ Stadt Köln, Rat der Stadt Köln, 4. Sitzung, 05.03.1959.
} 
Reaktionen auf die verschiedenen Hochwasserereignisse bis 1995 durch den Wiederaufbau der vom Krieg zerstörten Stadt beeinflusst wurden. Hierzu werden die Diskussionen nach den jeweiligen Hochwasserereignissen, die Bezug zu städtebaulichen Aspekten nehmen, auf Heimatdiskurse hin untersucht. Dabei soll nachgeprüft werden, ob die Präferenzen der Entscheidungsträger in der Nachkriegszeit bis zur Entwicklung des heutigen Hochwasserschutzkonzeptes in den jeweiligen spezifischen Argumentationslinien fortgeführt oder ob Brüche erkennbar wurden. Eine Betrachtung der Diskussionen aus der KostenNutzen-Perspektive soll abschließend Auskunft geben, ob die unterschiedlichen Bewertungen und Abwägungen verständlich nachvollzogen werden können, indem Alternativen gegeneinander abgewogen und spezifische Grenzen erkannt wurden. Einleitend werden zunächst die Hochwasserereignisse und die darauf abgestimmten Reaktionen referiert.

\subsection{Die Hochwasserschutzmauer 1960 - Der Not gehorchend}

Im Jahr 1960 wurde vom Stadtrat beschlossen, linksrheinisch „,im Zuge der Umprofilierung der Rheinuferstrasse“ ${ }^{118}$ den Hochwasserschutz zu verstärken, obwohl es in diesem Jahr kein Hochwasser gegeben hatte. Es wurde eine Mauer gebaut, die zwischen 0,6 und 1,0 m hoch war. Sie bot zusammen mit neu unterlegten Kragplatten (0,2 m Höhe) einen Hochwasserschutz bis zu einem Kölner Pegel von 8,40 m K P. Damit versuchten die Verantwortlichen das Überschwemmungsintervall von statistisch zu erwartenden drei auf sieben Jahre auszudehnen ${ }^{119}$. Die Mauer hatte darüber hinaus weitere Funktionen: Sie trennte die Spaziergänger von den Autofahrern ${ }^{120}$. Insbesondere dem ,„promenierenden Fußgänger“ bot sie Schutz vor dem motorisierten Straßenverkehr und diente als Sitzgelegenheit ${ }^{121}$. Die gesamte Baumaßnahme ermöglichte durch eine künstliche Verlängerung der vorhandenen Maße eine Vergrößerung der Promenadenfläche. Zwischen Lyskirchen und der Deutzer Brücke konnte die Fläche um 115 \% vergrößert werden, zwischen der Deutzer Brücke und der Hohenzollerbrücke wurden $40 \%$ dazu gewonnen, sowie zwischen der Hohenzollerbrücke und der Machabäerstrasse nochmals um $45 \%$. Die Kosten wurden zum Zeitpunkt des Beschlusses im Oktober 1960 mit 5,5 Mio. DM veranschlagt. Abb. 8 zeigt eine Aufnahme der Rheinuferstraße zwischen Hohenzollern- und Deutzer Brücke nach Fertigstellung der

\footnotetext{
${ }^{118}$ Bericht über das Hochwasser 1970, Hochwasserschutzzentrale der Stadt Köln, 03.04.1970.

${ }^{119}$ Stadt Köln, Rat der Stadt Köln, 12. Sitzung, 13.10.1959.

${ }^{120}$ Stadt Köln, Rat der Stadt Köln, 10. Sitzung, 20.10.1960.

${ }^{121}$ Ebd.
} 
Maßnahme aus dem Jahr 1966. Die damals neue Hochwasserschutzmauer ist zwischen den Blumenbeeten und den Gleisen bei näherem Hinsehen zuerkennen. Wie es zu der Entscheidung kam eine kleine Mauer zu verbauen ist nachfolgend erläutert.

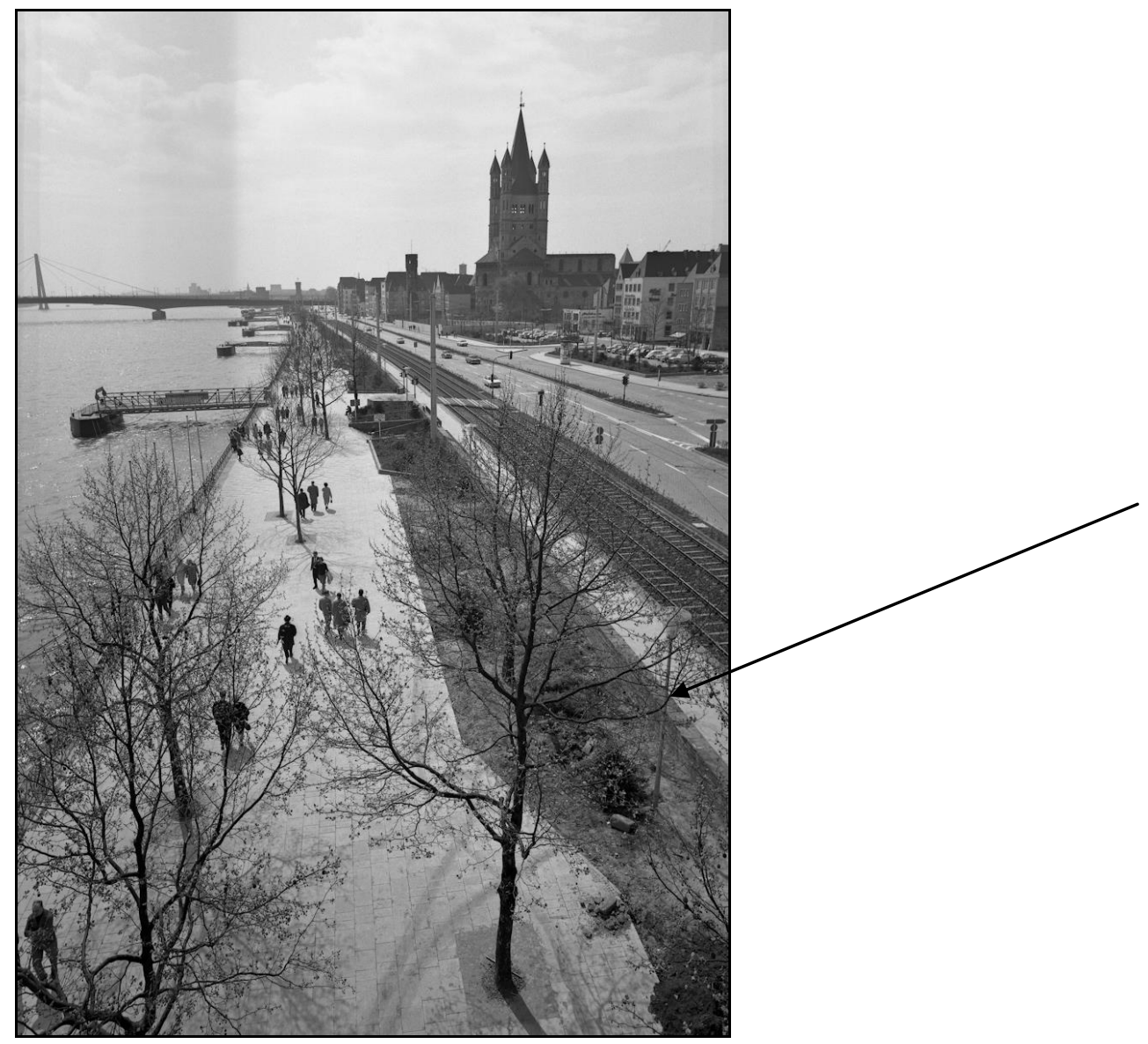

Abb. 8: Rheinuferstraße mit dem Hochwasserschutz-,Mäuerchen“ $1966 .{ }^{122}$

\subsection{Diskussionen zum Bau der Hochwasserschutzmauer}

Fast fünf Jahre nach dem letzten Hochwasserereignis von 1955 tagte der Stadtrat am 13. Oktober $1959 \mathrm{zu}$ dem Thema „Umprofilierung der Rheinuferstrasse“ in einer Sondersitzung auf dem Schiff „Stadt Köln“123. Bereits in einer vorhergegangenen Stadtratsdiskussion im März 1959 war eine Ortsbegehung aufgrund der fehlenden Visualisierungsmöglichkeiten beschlossen worden ${ }^{124}$. Köln litt zu jener Zeit an der Zunahme

\footnotetext{
${ }^{122}$ Internetbasierte Quelle 9.

${ }^{123}$ Stadt Köln, Rat der Stadt Köln, 12. Sitzung, 13.10.1959.

${ }^{124} \mathrm{Zu}$ der ersten Sitzung zur Umprofilierung der Rheinuferstraße und dem damit verbundenen Bau der Hochwasserschutzmauer vom März 1959 sowie zu der dritten Sitzung bezüglich dieses Themas zwischen Oktober
} 
des motorisierten Personenverkehrs. Oberstadtdirektor Maximilian Adenauer (CDU) gab an, sich bereits seit über einem Jahr mit dem Verkehrsproblem zu befassen ${ }^{125}$ :

„Die Entwicklung ist über uns hinweggegangen. [Man hoffte], mit der zunehmenden Zahl der Brücken, mit der Autobahn um die Stadt würde [sic] der Verkehr geringer, und es würde [sic] nicht mehr so schlimm werden. Die Verhältnisse sind aber schlimmer geworden, als man fürchtete [sic]. “126

Dr. Jacobs (FDP) vertrat die Meinung, in den Straßenverlauf muss eingegriffen werden, „,Sonst werden wir dem Verkehr nicht gerecht“" ${ }^{\text {127}}$. Sein Parteikollege Lercher nannte den Verkehr „entsetzlich“128. Auch Pflitsch (CDU) pflichtete bei, dass ,wir [...] bei dem Verkehr, den wir zu erwarten haben, zu einer Reglung am Rheinufer kommen [müssen]"129. Oberbaudirektor Schüssler (SPD) verwies auf die umfangreichen Beanstandungen der Verkehrsbehinderungen zwischen 1920 und 1958 aus der Bevölkerung, die gehäuft bei Hochwasser auftreten ${ }^{130}$. Außerdem präsentierte er die Ergebnisse einer neuen Erhebung: In diesem Bereich sind täglich mehr als 30.000 Fahrzeugen, davon $70 \%$ Pkw gezählt worden ${ }^{131}$. Oberbaudirektor Schüssler (SPD) erläuterte die Verkehrsprobleme anhand der Überschwemmungsgebiete von 1920 und 1926 und erklärte, als Lösung eine Mauer bauen zu wollen ${ }^{132}$. Diese Mauer solle den Verkehr vor Hochwasser bis zu einer Höhe von 8,60 m K P schützen, indem sie die Überschwemmungsintervalle auf statistische sieben Jahre ausdehnte $^{133}$. Auch die Opposition empfand es, wie Band (CDU) erklärte, als „oberstes Gebot, die Straße hochwasserfrei für den Verkehr zu halten, der nicht von der Rheinuferstraße wegzuziehen ist“\$134. Spontan wurden weitere Lösungsmöglichkeiten vorgeschlagen. Unter den Anwesenden entstand Uneinigkeit, denn der Hauptkonflikt galt bei allen Vorschlägen dem städtebaulichen Aspekt. „Ehe wir da Experimente machen, müssen wir uns alles dreimal überlegen“"135, mahnte Fröhlich von der SPD. Oberbürgermeister Burauen (SPD) warf die Frage auf, ob ,unsere Bürger noch an den Rhein kommen, oder ob dies [sic] dem Verkehr geopfert “136 wird? Hartmann (CDU) empfand die Mauer als wesentliche Einschränkung und

1959 und Oktober 1960 fehlen die Protokolle. Aus den zwei vorhandenen Mitschriften wird jedoch bereits ersichtlich, welche Akteure mit Einfluss ausgestattet waren und wie die Argumentationslinien verliefen.

${ }^{125}$ Stadt Köln, Rat der Stadt Köln, 12. Sitzung, 13.10.1959.

${ }^{126}$ Ebd., 461.

${ }^{127}$ Stadt Köln, Rat der Stadt Köln, 12. Sitzung, 13.10.1959, 456.

${ }^{128}$ Ebd., 457.

${ }^{129}$ Ebd., 455.

${ }^{130}$ Ebd.

${ }^{131}$ Ebd.

${ }^{132} \mathrm{Ebd}$

${ }^{133}$ Ebd.

${ }^{134}$ Ebd., 458.

${ }^{135}$ Ebd., 455.

${ }^{136}$ Ebd., 461. 
erklärte: Der Kölner ,,sieht nun mal gern das Leben des Rheins, das sich mit dem Leben der Stadt verbindet, gerade an dieser Stelle“ ${ }^{\text {137 }}$. Das Verkehrsproblem sei ihrer Meinung nach selbstgemacht:

„Man überließ den Verkehr einfach sich selber. Er wuchs und wuchs, und wir haben kaum etwas getan, um den Verkehr auf der Rheinuferstrasse zu regeln. Niemand kann sich davon freisprechen, daß wir nichts getan haben, um den diese Stadt durchflutenden Verkehr anderswie zu regeln. “" ${ }^{\text {(38 }}$

Das CDU-Mitglied Pflitsch bevorzugte eine Mauer mit einer Schutzhöhe von 8,20 m K P statt der vorgeschlagenen $8,60 \mathrm{mKP}$, da der steigende Verkehr zukünftig sowieso nach Neuregelungen verlangen würde ${ }^{139}$. Binot von der CDU machte den Vorschlag, stattdessen die Straße anzuheben. In seinen Augen behinderte eine Mauer die ,schiffahrende Bevölkerung und die am Rhein liegenden Gesellschaften““140. Er glaubte, „daß der Charakter der Rheinuferstraße völlig verändert wird, mit dem traditionellen Bild ist es aus ${ }^{\text {“141 }}$. Der Beigeordnete Dr. Kleppe (CDU) fokussierte das Wohl der Fußgänger. Eine Straßenerhöhung schätzte er als Nachteil für die Passanten ein ${ }^{142}$. Lercher (FDP) war „dafür, dass es schön wird“143, und favorisierte die Höherlegung der Straße. Fröhlich verwies auf zwei Sehenswürdigkeiten, „die Köln in der Welt berühmt gemacht haben: den Kölner Dom und die Stadtansicht“ ${ }^{144}$. Er betrachtete die Funktionen der Mauer einzeln und kam zu dem Schluss, dass man das Bauwerk weder für die Regelung des Verkehrs noch zum Hochwasserschutz braucht ${ }^{145}$. Denn seiner Meinung nach werde die Promenade dadurch ,belebt, daß der Spaziergänger auf der einen Seite den Strom und das, was sich auf ihm abspielt, und auf der anderen Seite das Stadtbild vor sich hatte $\left[\mathrm{sic}^{\text {“146 }}{ }^{146}\right.$. CDU-Parteikollege Stein sprach sich gegen unnötige Mauerhöhen aus und schlug vor $\mathrm{zu}$ prüfen, ob nicht eine Höhe von 8,40 m K P gebaut werden kann, wenn die Straße zusätzlich um 0,1 - 0,2 m angehoben wird. So wird eine kleine Mauer von nur 0,2 bis 0,3 m Höhe statt den vorgeschlagenen 0,6 bis 1,2 $\mathrm{m}$ - von Land her betrachtet - entstehen ${ }^{147}$.

\footnotetext{
${ }^{137}$ Ebd., 450.

${ }^{138}$ Ebd., 451.

${ }^{139}$ Stadt Köln, Rat der Stadt Köln, 12. Sitzung, 13.10.1959, 455.

${ }^{140}$ Ebd., 452.

${ }^{141}$ Ebd., 453.

${ }^{142}$ Ebd., 449.

${ }^{143}$ Ebd., 457.

${ }^{144}$ Ebd., 455.

${ }^{145}$ Ebd.

${ }^{146}$ Ebd., 456.

${ }^{147}$ Ebd.
} 
In Adenauers Augen lag der Kompromiss zwischen zwei Extremen; „dem heutigen und dem Zustand, den wir bei einem Pegelzustand von 9,69 m haben müssten“148. Der genannte Hochwasserstand von 9,69 m KP entsprach dem Jahrhundertpegel von 1926. Der Beigeordnete Kleppe wies darauf hin, dass das Hochwasser über einen Stand von 8,40 m K P hinweg gehen kann: „Geschieht das, müssen wir es hinnehmen. Der Rhein kann ja auch noch höher steigen. Das ist für uns nicht das Entscheidende. “149 Hartmann beklagte, dass die Mauer für immer bleibt, dagegen „starke Hochwasser sehr selten auftreten und nicht lange

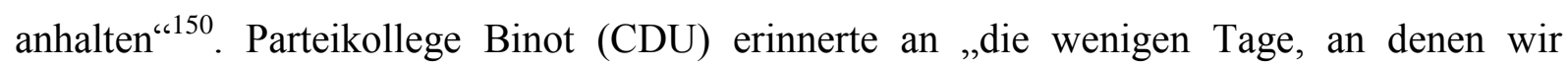
Hochwasser haben, und an die Jahresintervalle“151. Er war der Ansicht, „dann wird auch das, was dann [nach dem Eingriff] noch übrigbleibt, zu ertragen sein “152. Oberbürgermeister Burauen (SPD) bezweifelte, dass es bei der vorgeschlagenen Mauer in Zukunft bleiben wird: „Ich sage Ihnen an dieser Stelle beinahe das Jahr voraus, in dem die zweiten, dritten, vierten und fünften $60 \mathrm{~cm}$ draufkommen“153. Bamberger (SPD) sprach der Mauer gleich jede Hochwasserschutzfunktion ab: „Mit dem Mäuerchen halten Sie kein Wasser auf.“154 Band (CDU) glaubte gar nicht mehr an zukünftige Hochwasser:

„Der Rhein geht wirklich allmählich zurück, und zwar durch die Regulierung. Der Rhein fließt insgesamt viel besser ab. Bevor die Regulierung erfolgte, waren die Überschwemmungen viel stärker.“'155

Dr. Schwering äußerte sofort seine gegenteilige Ansicht ${ }^{156}$ und mutmaßte, dass der hier vorliegende Betrachtungszeitraum unglücklich gewählt und auf Grund dessen die tatsächliche Hochwassergefahr seltener ist: „Ich habe den Eindruck, daß sich der Rhein in den Jahren von 1920 bis 1958 besonders schlecht benommen hat.“157 Er schlug deshalb vor, „ernstlich darüber nachzudenken, ob man sich nicht mit einer [...] mittleren Linie begnügen könnte“،158. Lediglich Wacker (SPD) sprach sich für den Bau der Mauer nach der Vorlage der Verwaltung aus, denn er argumentierte, Köln liegt schon lange nicht mehr am Rhein ${ }^{159}$. Dabei nahm er Bezug zu früheren Veränderungen des Kölner Stadtbildes. Auch Oberbaudirektor Pecks

\footnotetext{
${ }^{148}$ Ebd., 464.

${ }^{149}$ Ebd., 463.

${ }^{150}$ Ebd., 450.

${ }^{151}$ Ebd., 453.

${ }^{152}$ Stadt Köln, Rat der Stadt Köln, 12. Sitzung, 13.10.1959, 453.

${ }^{153}$ Ebd., 461.

${ }^{154}$ Ebd., 457.

${ }^{155}$ Ebd., 458.

156 Ebd., 459.

${ }^{157}$ Ebd., 459.

${ }^{158}$ Ebd., 460.

${ }^{159}$ Ebd., 453.
} 
stimmte für die Mauer, wie sie vorgeschlagen wurde ${ }^{160}$. Eine ähnliche Meinung verdeutlichte Adenauer mit seiner Aussage, dass die Mauer „das Lebensgefühl der Besucher des Rheins so gut wie nicht tangiert" ${ }^{\star 161}$. In seinen Augen überwogen die Vorteile der Mauer ${ }^{162}$ insbesondere für die Lösung des Verkehrsproblems:

„Regeln wir den Verkehr so, wie er zu Hochwasserzeiten ist. Wir schaffen sozusagen ein künstliches Hochwasser. Wir sperren den Verkehr dort, wo sonst Hochwasser steht, und dann sehen wir uns mal den Verkehr in diesen Gegenden an. Sie werden mit mir zu der Überzeugung kommen, daß hier etwas geschehen muß.“163

Altbürgermeister Dr. Schwering (CDU) hoffte in einer späteren Sitzung einen Kompromiss, der für alle Beteiligten „erträglich“ ist, finden zu können, denn für ihn stand fest: „Das Mäuerchen ist eine böse Sache“6164. Nachdem seit März 1959 drei Stadtratssitzungen zu dem Thema stattgefunden hatten, kam es anlässlich der vierten Sitzung am 20. Oktober 1960 zur Abstimmung über den Bau der Hochwassermauer ${ }^{165}$. Der Beigeordnete des Dezernates Tiefbau Dr. Kleppe (CDU), der sein Dezernat 1950 übernommen hatte und bereits in der Hauptausschusssitzung zum Bau einer Hochwassermauer nach dem Schadensereignis von 1948 teilgenommen hatte ${ }^{166}$, eröffnete die Sitzung. Er führte einen neu ausgearbeiteten Höhenvorschlag von $8,40 \mathrm{~m} \mathrm{~K} \mathrm{P}$ aus und betonte dabei die flächenmäßigen Verbesserungen für alle Verkehrsteilnehmer. Die zusätzliche Fläche sollte durch die Verlegung von Kragplatten erreicht werden, die außerdem zusätzlich 0,2 $\mathrm{m}$ auf dem Boden aufliegen würden ${ }^{167}$. Der neue Vorschlag blieb um 0,2 m unter dem der Empfehlung der Verwaltung vom 30. Oktober 1959 und wurde ab nun nur noch als „das Mäuerchen““168 bezeichnet. Damit war die geplante Mauer nur noch zwischen 0,6 und 1,0 m hoch. „Man kann sich also noch draufsetzten.“169 Kleppe listete drei Punkte auf, die seiner Ausführung mehr Gewicht verleihen sollten. Erstens verglich er das rechte Rheinufer mit Verweis auf die 1957 durchgeführte Bundesgartenschau, welche „einen 6-7 km langen offenen Zugang zum Rhein

\footnotetext{
${ }^{160}$ Ebd., 449.

161 Ebd., 450.

162 Ebd.

${ }^{163}$ Ebd., S. 464.

${ }^{164}$ Stadt Köln, Rat der Stadt Köln, 12. Sitzung, 13.10.1959, 460.

${ }^{165}$ Es fanden insgesamt vier Sitzungen zur Erneuerung der Rheinuferstrasse statt, jedoch ist laut Auskunft des Mitarbeiters der Stadtverwaltung, Florian Schüler, das Protokoll zur ersten Sitzung nicht vorhanden. Im März 1959 wurde das Thema zum ersten Mal besprochen. Die zweite Sitzung fand am 13. Oktober 1959 auf dem Schiff „Stadt Köln“ statt. Danach fand eine weitere Sitzung auf dem Schiff statt, deren Protokoll laut Schüler auch nicht auffindbar ist. Der Stadtrat tagte in einer vierten und finalen Sitzung zu dem Thema am 20. Oktober 1960.

${ }^{166}$ Stadt Köln, Rat der Stadt Köln, 12. Sitzung, 13.10.1959.

${ }^{167}$ Stadt Köln, Rat der Stadt Köln, 10. Sitzung, 20.10.1960, 244.

${ }^{168}$ Ebd., 243.

${ }^{169}$ Ebd., 244.
} 
für Fußgänger" erlaubte ${ }^{170}$. Zweitens untermauerte Kleppe seine Ausführungen mit den neusten Erkenntnissen des Städtetages in Augsburg. Dort hat man in allen Foren festgestellt, dass „die Anpassung an den Verkehr unter besonderer Berücksichtigung des Fußgängers im

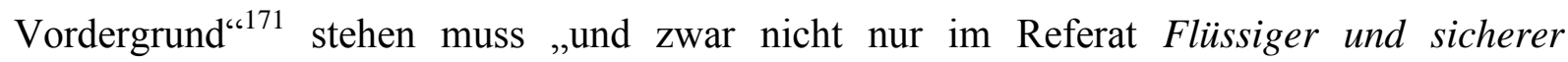
Stadtverkehr sondern in allen maßgeblichen Referaten und auch in der Schlußsitzung“ ${ }^{172}$ ist man zu dieser Schlussfolgerung gelangt. Darüber hinaus ergänzte er, dass zu diesem Konzept auch „die Entflechtung des Verkehrs durch Trennung von Schienenbahnen, Kraftfahrzeugen, Radfahrern und Fußgängerbezirken“173 gehört. Er vergaß dabei nicht zu betonen, dass „,der Ihnen heute zur Beschlussfassung vorliegende Plan ein gutes Beispiel für eine solche Maßnahme darstellt ${ }^{\text {‘174}}$. Drittens versuchte er die Qualität seines Vorschlages durch den Verweis auf den Besuch des Bundeskanzlers Konrad Adenauer (CDU) im November des Vorjahres zu stärken. Dieser hatte „Anregungen“"175 zur Umgestaltung der Rheinuferstraße gegeben. Der Kanzler favorisierte, den Fußgängern einen breiten Zugang zum Fluss zu ermöglichen ${ }^{176}$. Kleppe referierte, dass daraufhin bereits 1959/60 die Möglichkeit einer Tunnellösung für den Verkehr analysiert wurde, jedoch aufgrund der hohen Kosten und der langen Bauzeiten nicht in die Verhandlung gebracht wurde ${ }^{177}$. Während seiner Ausführung gab er lange Verhandlungen innerhalb der Verwaltung zu sowie dass es „strittig war, ob [...] der Stadtrat auch einen Hochwasserschutz wünsche““178. Weber (SPD) erinnerte noch einmal an die Eigenschaft einer Visitenkarte, die diese Straße am Rhein für Köln darstellt. Im Namen seiner Fraktion wertete er den Vorschlag als gut und zustimmungsreif ${ }^{179}$. Hartmann (CDU) schloss sich Weber an, schlug allerdings aufgrund der Beeinträchtigung des Allgemeinguts vor, die Bürger der Stadt über den Vorschlag abstimmen zu lassen und den Beschluss zu verschieben $^{180}$. Dr. Jacobs (FDP) pflichtete Hartmann bei, glaubte allerdings nicht, dass es noch $\mathrm{zu}$ neuen Verbesserungen kommen wird. Ihn interessierte vielmehr, wann der Bau beginnen kann und wie lange es bis zur Fertigstellung dauern wird. Auch van den Valentyn (CDU) glaubte an keine weitere Optimierung, obgleich er „das Mäuerchen technisch für fragwürdig“'181 hielt und bezweifelte, „ob der mit der Mauer verbundene Hochwasserschutz

\footnotetext{
${ }^{170}$ Ebd., 244.

${ }^{171}$ Ebd., 243.

172 Ebd.

173 Ebd.

${ }^{174}$ Stadt Köln, Rat der Stadt Köln, 10. Sitzung, 20.10.1960.

175 Ebd.

${ }^{176}$ Ebd.

${ }^{177}$ Ebd.

${ }^{178}$ Ebd., 245.

${ }^{179}$ Ebd.

${ }^{180} \mathrm{Ebd}$.

${ }^{181}$ Ebd., 248.
} 
uns diese Mauer wert“ ${ }^{182}$ ist. Dr. Schwering (CDU) äußerte, ,zufrieden sind wir noch immer nicht ${ }^{\star 183}$, fasste aber zusammen, dass alle ,ihr Bestes gegeben haben mit Einsatz aller Kraft“'184. Er glaubte, die ,wirklichen Schwächen und Vorzüge werden klar, wenn der Bau ausgeführt" ${ }^{\text {185 }}$ ist. Schwering sprach sich gegen ein Verschieben der Abstimmung aus und zitierte dazu aus dem Drama der Braut von Messina die Passage, „der Not gehorchend, nicht dem eigenen Trieb“"186.

Wacker (SPD) sprach sich ebenfalls gegen weiteres Warten aus und erinnerte noch einmal an den Hochwasserschutz aus dem die Idee des Mauerbaus entsprungen ist ${ }^{187}$. Stein (CDU) ging auf die Äußerung ein und erkundigte sich, ob der Hochwasserschutz denn nun $60 \mathrm{~cm}$ weniger beträgt als im ersten Vorschlag ${ }^{188}$. Kleppe beantwortete seine Frage mit den klaren Worten:

„Sie haben doch gemerkt, daß wir auf den Hochwasserschutz nicht mehr besonderen Wert gelegt haben; denn sonst würden wir Ihnen eine höhere Mauer vorgeschlagen haben. Wir sind heruntergegangen und haben gesagt, dieser Schutz reicht nach der Statistik aus, wenn wir etwa alle sieben Jahre Hochwasser in Kauf nehmen“" ${ }^{\text {"189. }}$.

Des Weiteren führte Kleppe zur Verdeutlichung den ersten Vorschlag für die Schutzhöhe an, dieser hatte bei $1,4-1,6$ m gelegen ${ }^{190}$. Stein vergewisserte sich daraufhin noch einmal gegen welchen Pegel die Mauer schützen soll ${ }^{191}$. Der Stadtbaudirektor Braun antwortete, die Mauer schützt bis zu einem Wasserstand von $8,40 \mathrm{~m} \mathrm{~K} \mathrm{P}$ und da teilweise der Boden mit einer Steigung verläuft, werden manche Strecken bis zu $9 \mathrm{~m} \mathrm{~K} \mathrm{P}$ abgesichert ${ }^{192}$. Mit seiner Aussage, dass er und auch van den Valentyn genau diesen über die Zielvereinbarung hinausgehenden Schutz für zu hoch halten, offenbarte Stein ein weiteres Missverständnis. Van den Valentyn widersprach allerdings umgehend. Parteikollege Pflitsch fasste noch einmal den finalen Plan für alle zusammen und klärte zumindest das Missverständnis bezüglich des Schutzbaus auf ${ }^{193}$. Zum Ende bat Oberbürgermeister Burauen (SPD) um das Wort, da er ,von der ersten Stunde leidenschaftlich in die Debatte eingegriffen“194 hat. Er nannte das

\footnotetext{
182 Ebd.

${ }^{183}$ Ebd., 248.

${ }^{184}$ Ebd., 249.

${ }^{185}$ Ebd.

${ }^{186}$ Ebd.

${ }^{187}$ Stadt Köln, Rat der Stadt Köln, 10. Sitzung, 20.10.1960.

${ }^{188}$ Ebd.

${ }^{189}$ Ebd., 250.

${ }^{190} \mathrm{Ebd}$.

191 Ebd.

${ }^{192}$ Ebd.

${ }^{193}$ Ebd.

${ }^{194}$ Ebd., 251.
} 
Hochwasser ein „Schreckgespenst“"195 welches zur Idee des Mauerbaus geführt hat und er kritisierte:

„Obwohl die in den ersten Sitzungen gezeigten Graphiken uns fast zu erschlagen drohten, hat man nun bei der Verwaltung nicht mehr entscheidenden Wert auf die größtmögliche Mauerhöhe gelegt. Vielmehr hat man dem Städtebaulichen Bild, der Diskussion und dem Widerstand aus dem Rat Rechnung tragend, die Mauer herabgezont [sic].“ 196

Da er immer noch die Meinung vertrat, diese Mauer sei nur der Beginn einer Bauära für eine wesentlich höhere Mauer, erklärte der Oberbürgermeister, gegen das Vorhaben zu stimmen:

„Ich habe nicht das Vertrauen, dass es bei dieser Mauerhöhe bleiben wird, [...] wir werden uns vor die Situation gestellt sehen, dass man sagt: [...] Ihr habt das letzte Hochwasser gesehen, Ihr müßt da noch etwas aufstocken. Im umgekehrten Sinne angewandt, hackt man der Katze stückweise den Schwanz ab, [...] baut man hier stückweise nacheinander die Mauer auf, und dann sind wir da, wo wir nicht hinwollten. Ich bedauere, die Zustimmung zu der Vorlage nicht geben zu können“ ${ }^{\star 197}$.

Burauen läutete daraufhin die Abstimmung ein, welche trotz vier Gegenstimmen und fünf Enthaltungen im Ergebnis für die Umsetzung der Vorlage ausfiel. Der Ausbau der Rheinuferstrasse wurde über 1,5 km zwischen der Großen Witschgasse und der Neuen Maximilianstraße beschlossen. Abb. 9 zeigt den Verlauf der Schutzmauer auf dem Stadtplan. Wieso Burauen die anderen Stadtratsmitglieder nicht überzeugen konnte, und welche Akteure mit mehr Einfluss ausgestattet waren, wird im nächsten Abschnitt betrachtet.

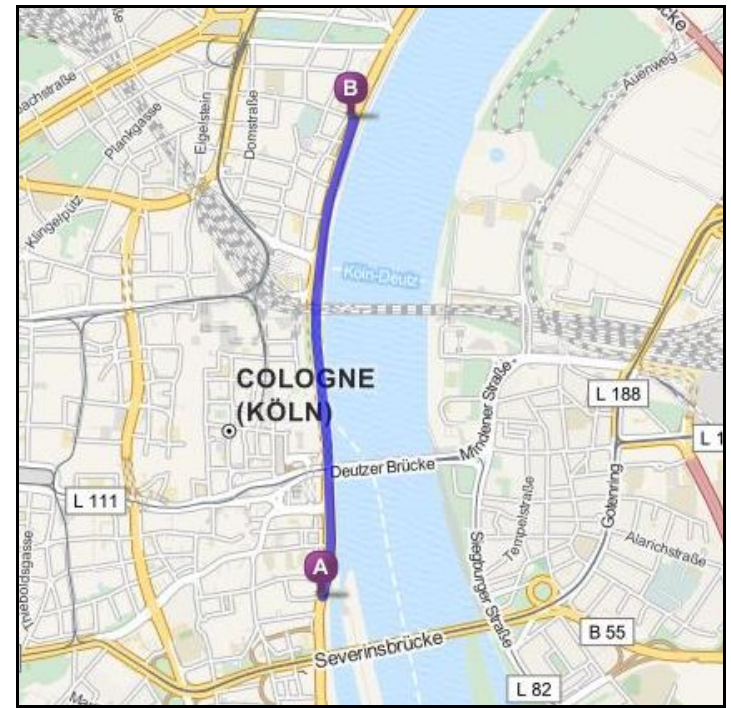

Abb. 9: Verlauf des „Mäuerchens“. ${ }^{198}$

\footnotetext{
${ }^{195} \mathrm{Ebd}$

196 Ebd.

${ }^{197}$ Stadt Köln, Rat der Stadt Köln, 10. Sitzung, 20.10.1960, 252.

${ }^{198}$ Die Karte erstellte der Autor mit Hilfe von OpenSourceMap (http://open.mapquest.de).
} 


\subsubsection{Die treibenden Kräfte des Stadtrats}

Im Folgenden wird der Fokus auf ein Quartett von überzeugenden Meinungsführern gelegt, um die Argumentationslinien genauer nachvollziehen zu können. Ein besonderes Augenmerk liegt auf der Doppelspitze der Kommunalpolitik, Oberbürgermeister Theodor Burauen (SPD) und Oberstadtdirektor Dr. Maximilian Adenauer (CDU), sowie auf dem Beigeordneten des Stadtrats und Leiter des Tiefbauamtes Dr. Kleppe (CDU) und dem ehemaligen Oberbürgermeister Dr. Ernst Schwering (CDU).

Der 1906 geborene Theodor Burauen (SPD) war im Jahr 1956 Dr. Ernst Schwering (CDU) im Amt des Oberbürgermeisters zu Köln gefolgt. Er behielt das Amt 17 Jahre lang, bis er 1973 zurück trat. Zwischen Anfang 1954 bis Mitte 1966 war er Mitglied des Landtages in NRW. Er gab in der Diskussion um den Bau der Hochwasserschutzmauer zu bedenken, dass der Bürger an den Rhein kommen müsse und diese Rheinnähe nicht dem Verkehr geopfert werden dürfe ${ }^{199}$; an dieser Stelle stimmte er mit Oberstadtdirektor Adenauer (CDU) überein. In der Sitzung vom 13. Oktober 1959 artikulierte Burauen (SPD) seine Bedenken, dass auf die debattierte Mauer zukünftig immer höhere Linien gebaut werden würden, um späteren Überschwemmungsereignissen zu trotzen. Der städtebauliche Aspekt stand für ihn klar im Vordergrund, darum sah er in den neuen Höhenvorschlägen die gleichen Probleme wie er sie bereits in vorherigen Treffen geäußert hatte und wandte sich demzufolge strikt gegen jeden Mauerbau. Damit bekundete Burauen öffentlich seinen Widerstand gegen eine willkürlich gesetzte Hochwasserschutzmauer. Bei der anschließenden Abstimmung lehnten allerdings lediglich drei weitere Regionalpolitiker den Bau der Mauer ab und die Mauer wurde gebaut. Offenkundig wirkte Burauen, obwohl Oberbürgermeister, nicht richtungweisend für seine Stadtratskollegen. Auch die ausführliche Darstellung seiner Bedenken bewog keine Umkehr oder ein Nachdenken unter den Anwesenden.

Der zweite Kommunalpolitiker, der im Rahmen der hier vorgestellten Diskussion von Interesse ist, ist Maximilian Adenauer. Er wurde 1910 als Sohn des zukünftigen Bürgermeisters von Köln, Konrad Adenauer (1917 - 1933), geboren, welcher zur Zeiten der Mauerdiskussion bereits Bundeskanzler (1949 - 1960) war. Nachdem Maximilian Adenauer das Amt des Oberstadtdirektors 1965 abgab, blieb er bis 1975 Mitglied des Stadtrats. Zu seinen Zielen gehörte die Wiederherstellung der historischen Kölner Altstadt ${ }^{200}$. Gerade die Hochwassermauer sollte den Fußgänger am Rhein von dem Verkehr trennen und ihm Platz

\footnotetext{
${ }^{199}$ Stadt Köln, Rat der Stadt Köln, 12. Sitzung, 13.10.1959.

${ }^{200}$ Brixius 2004.
} 
zum Flanieren bieten ${ }^{201}$. Bevor Adenauer 1953 Oberstadtdirektor wurde, war er im Stadtrat als Beigeordneter für Wirtschaft, Häfen und Verkehr tätig. Darum verwundert es nicht, dass er sich nach eigenen Angaben schon vor den ersten Stadtratssitzungen zu diesem Thema bereits intensiv mit dem Verkehrsproblem auf der Rheinuferstraße befasst hatte ${ }^{202}$. Des Weiteren versuchte er seine Empfehlung für den Mauerbau unter Heranziehung der Verkehrsprobleme bei Hochwasser zu bekräftigen und schlug vor, bei einem nächsten Treffen den Verkehr wie bei Hochwasser zu sperren, um das Problem für alle zu verdeutlichen. Mit dem Ziel, dem Mauerbau Gewicht zu verleihen, verwies Adenauer immer wieder auf die Bürgermeinung. Zunächst listete er die Beschwerden der Anwohner über die Verkehrsproblematik zu Hochwasserzeiten auf, dann erwähnte er Rundfragen der Presse über die diskutierte Mauerhöhe ${ }^{203}$. Er versuchte sich in die Betroffenen hineinzuversetzen und deren Bedenken aus verschiedenen Perspektiven nachzuvollziehen. Er stellte sich selbst als Verbündeten des Kölner Fußgängers dar und war der Auffassung, die Mauer brächte den Bürger stärker an den Rhein zurück, da er vor Unfällen, Lärm und Staub geschützt würde. Für ihn lag die nötige Mauerhöhe zwischen dem Status Quo und dem Jahrhunderthochwasser von 1926 mit 10,69 m K P, was als Hinweis darauf verstanden werden kann, dass er großen Wert auf einen Mauerbau, aber eben nicht auf eine bestimmte Schutzhöhe legte. Die Präsentation von Oberbaudirektor Schüssler (SPD) richtete sich nach statistischen Erfahrungswerten hinsichtlich der seit 1920 stattgefundenen Hochwasser und Adenauer unterstützte diese Einschätzung der Sachlage. Nichtsdestotrotz zählte für Adenauer offensichtlich weder der Hochwasserschutz noch die Ordnung des Verkehrs als zukünftiger Nutzen, sondern lediglich das Fußgängerwohl. Bereits bei dem Hochwasser im Jahr 1955 sprach Adenauer von einem harmlosen Ereignis, das die Kölner im Griff zu haben schienen, obwohl der Hochwasserpegel 1,80 m über die Kaimauer reichte. Für ihn stellte das Hochwasser keinerlei Gefahr dar, vielmehr schien er den Verkehr als Bedrohung für den Menschen zu empfinden. Gleichwohl wusste er, dass er eine Mauer, welche den Fußgänger vor dem Verkehr schützen würde, nur über den als unerträglich empfundenen PKW-Verkehr sowie über den Hochwasserschutz rechtfertigen konnte. An dieser Stelle scheint die Kritik von Dr. Schwering (CDU) durchaus angemessen, dass der Betrachtungszeitraum der vorgeführten Statistik für eine repräsentative Aussage sehr kurz gewählt worden $\operatorname{sei}^{204}$. Gerade dieser Zeitraum war durch das Auftreten einiger großer Hochwasser gekennzeichnet und ließ sich zum Zweck der Betonung der Notwendigkeit einer Hochwasserschutzmauer instrumentalisieren. Später ließ Adenauer nur

\footnotetext{
${ }^{201}$ Stadt Köln, Rat der Stadt Köln, 12. Sitzung, 13.10.1959.

${ }^{202}$ Ebd.

${ }^{203}$ Ebd.

${ }^{204}$ Stadt Köln, Rat der Stadt Köln, 12. Sitzung, 13.10.1959, 459.
} 
allzu offensichtlich durchblicken, dass ihm die Höhe gleich sei, da er ,jede Höhe als Kompromiss، ${ }^{205}$ begriff. Aus diesem Grund verteidigte er den Mauerbau mit eher schwachen oder weichen Argumenten. Zwar bediente er sich seiner Kenntnisse über den Verkehrssektor, doch blieben unausgewogene Statistiken, oberflächliche Befragungen und subjektive Gedanken ausdruckslos. Nichtsdestotrotz scheint Adenauer in der Lage gewesen zu sein, Stimmung zu machen. Er führte die Diskussion, ob eine Mauer gebaut werden solle, mit der Unterstützung seines Vaters zu der Frage, wie hoch die Mauer gebaut werden solle.

Der Beigeordnete des Stadtrats und Leiter des Tiefbauamtes Dr. Kleppe (CDU) hatte bereits nach dem Hochwasser von 1948 (10,41 m K P) Erfahrungen mit der Diskussion um eine Hochwassermauer gesammelt. Er kritisierte im Oktober 1959 eine weitere Herabsetzung der Höhe der geplanten Mauer, da „das Wasser über 9,40 hinweg gehen wird““206. Er versuchte sich zunächst an Erfahrungswerten zu orientieren. Die Sitzung im Oktober 1960 eröffnete er mit der Präsentation der wiederum neuen Mauerhöhen, und zwar genau die vorher von ihm kritisierten 9,40 m K P. Die Höhe war für ihn „nicht [mehr] das Entscheidende“6207, verkündete er zu dieser Gelegenheit. Er wechselte seine Meinung und ließ sich von den Meinungsführern beeinflussen. Als technischer Experte diente er dem Tiefbauamt als überzeugendes Sprachrohr für deren neue Vorschläge. Bei seiner Argumentation bediente er sich einiger rhetorischer Hilfen. Zunächst verharmloste er das zum Hochwasserschutz geplante „Mäuerchen“ zum „drauf sitzen“, dann sprach er von bereits wieder verworfenen Alternativen sowie von den Herausforderungen während der Ausarbeitung. Zu guter Letzt legte er seinen Zuhörern die überregional anerkannte Innovationskraft des Vorschlages, die Zustimmung des Bundeskanzlers sowie den direkten Zugang zum Rhein als Folge der Bundesgartenschau 1957 ans Herz. Als geübtem Redner traute Adenauer ihm Überzeugungskraft zu und setzte ihn deswegen bei der vierten Verhandlung ein. Kleppe gab offen zu, dass der neue Vorschlag nicht von Erfahrungen mit dem Hochwasser, sondern lediglich durch das Motiv der Bewahrung der Kölner Stadtansicht geleitet wurde. Dabei verschwieg er die statistisch verkürzten Überschwemmungsintervalle nicht, welche zukünftig als „Preis“ zu zahlen seien. Obwohl das Bauprojekt in seinen Augen nicht den kalkulierten Verhältnissen genügte, argumentierte er nicht gegen dessen Umsetzung. Er ließ zu, dass aufgrund emotionaler Einwände große Abstriche in der normativen Sicherheitsbemessung vorgenommen wurden. Er hatte keine emotionalen Einwände gegen die erste Mauerhöhe, da für ihn die berechnete technische Lösung als logischer Schritt für die Verbesserung der

\footnotetext{
${ }^{205}$ Ebd.

${ }^{206}$ Ebd., S. 462.

${ }^{207}$ Stadt Köln, Rat der Stadt Köln, 12 Sitzung, 13.10.1959, 463.
} 
Hochwassersicherheit galt. Außerdem war für das Tiefbaudezernat, welches er leitete, ein größtmögliches Bauprojekt die vorteilhafteste Lösung. Allerdings war ein kleineres Bauprojekt wiederum besser, als gar keine Aktivitäten vorzunehmen. Anzumerken ist, dass Kleppe (CDU) bei dem Bau der Severinsbrücke (1956 - 1959) beteiligt war, denn sie wurde von dem Tiefbauamt initiiert. Somit hatte er als Leiter des Tiefbauamtes den Besuch des Kanzlers zur Eröffnung der Brücke mitgetragen und war wohl auch in den Feierlichkeiten involviert. So ist nicht auszuschließen, dass der Kanzler auf ihn als Hauptverantwortlichen in Bauprojekten besonders Eindruck gemacht hatte.

Schwering (CDU) wurde 1886 geboren und war in den Jahren 1948, 1950 sowie von 1952 bis 1956 Oberbürgermeister der Stadt Köln. Während den Jahren 1954/55 sowie 1957/58 bekleidete Schwering das Amt des Präsidenten des Deutschen Städtetages. Ebenfalls engagierte er sich zwischen 1953 und 1956 sowie 1961/1962 als Vorsitzender der Landschaftsversammlung Rheinland. Bereits seit Mitte 1950 bis zu seinem Tod 1962 war er für seinen Wahlkreis Köln-Stadt Mitglied des Landtags NRW. In der Diskussion um den Mauerbau bezog er Position für die Bewahrung der Stadtansicht. Er kritisierte die statistische Auswertung der Hochwasserereignisse der Verwaltung, die lediglich Hochwasser seit 1920 betrachtete und meinte, der Zeitraum müsse bereits 1880 beginnen, da sich der Rhein „Zwischen 1920 und 1958 besonders schlecht benommen hat ${ }^{\text {‘208 }}$. Schwering personifizierte den Rhein und verlieh ihm menschliche Züge. Er betrachtete bei der Sitzung im Oktober 1959 die letzten Hochwasser unter der Voraussetzung, den vorgeschlagenen Schutz von 8,60 m K P bereits verbaut zu haben. Daraufhin bilanzierte er, dass diese Linie seit 1920 bereits dreimal überschritten worden wäre, während 30 Hochwasser unter einer Linie von 8 m K P geblieben waren $^{209}$. Damit schlug er vor, „60 cm für die Aussicht ${ }^{‘ 210}$ von dem Vorschlag der Verwaltung herab zu gehen. Er wünschte sich über „die böse Sache“211 einen „Kompromiß, der für alle Beteiligten erträglich ist“ ${ }^{\text {212 }}$. In der Sitzung vom 20. Oktober 1960 wollte Schwering offenkundig endlich zum Ende kommen, denn er zitierte das Drama von Friedrich Schiller, und verglich den Aushandlungsprozess mit einem Trauerspiel ${ }^{213}$. Schwering stellte heraus, dass er mit dem Vorschlag noch nicht zufrieden war, wohl aber anerkannte, dass viel

\footnotetext{
${ }^{208}$ Stadt Köln, Rat der Stadt Köln, 12. Sitzung, 13.10.1959, 459.

${ }^{209}$ Ebd.

${ }^{210}$ Ebd. Der erste Lattenpegel wurde in Köln 1810 errichtet. Zwischen 1901 bis zu seiner Zerstörung durch einen Bombenangriff 1943 stand das erste Pegelgebäude linksrheinisch in der Nähe der Deutzer Brücke. Das jetzige Pegelhäuschen wurde 1951 erbaut, und liefert den aktuellen Wasserstand unter anderem an ein Tonband. Dieses kann unter der Rufnummer (02 21) 19429 jederzeit abgehört werden (HWSK 1996, 11). Individueller ist die

Tonbandansage der Mitarbeiter der Kölner Hochwasserschutzzentrale, die unter (02 21) 22126161 abgehört werden kann.

${ }^{211}$ Ebd.

${ }^{212}$ Ebd.

${ }^{213}$ Stadt Köln, Rat der Stadt Köln, 10. Sitzung, 20.10.1960, 249.
} 
Arbeit, Kraft und Mühe in dieser Ausarbeitung steckte ${ }^{214}$. Obwohl er andeutete, dass jeder Kompromiss Schwächen aufweise und dass wirkliche Vor- und Nachteile erst klar würden, wenn der Bau ausgeführt sei, rief er nicht dazu auf, den Bau zu überdenken. Insgesamt vermittelte er den Eindruck einer kritischen als auch rationalen Persönlichkeit. Zweifelsfrei wünschte er den Bau einer Mauer, wenn sie nicht zu hoch wäre. Nachdem die Höhe der Mauer bis zur Hälfte des ersten Vorschlages abgesenkt worden war, drängte er die Kollegen zur Abstimmung. Aufgrund seines Alters sowie der zahlreichen schweren Erfahrungen, die Menschen aus dieser Zeit verbinden, kann in Erwägung gezogen werden, dass eine Verbundenheit mit Konrad Adenauer bestand. Beide Herren waren einmal im Amt des Oberbürgermeisters der Stadt Köln, so dass eine emotionale Verbundenheit zu Köln und dem Rhein abgeleitet werden kann. Beide trafen sich anlässlich des Deutschen Städtetags am 13. Juni 1958 und sprachen über eines der größten Probleme Kölns, den Verkehr ${ }^{215}$. Damit liegt nahe, dass der Bundeskanzler und Schwering bereits damals über das Vorhaben gesprochen haben, besonders weil man davon ausgehen kann, dass Konrad Adenauer durch seinen Sohn, dem Oberstadtdirektor Kölns, bereits vorher von dem Vorschlag, eine Hochwasserschutzmauer zu bauen, gehört hatte.

Zusammenfassend ist festzuhalten, dass Oberbürgermeister Burauen (SPD) neben den drei anderen Protagonisten den geringsten Einfluss ausübte. Er wirkte in dem Kölner Stadtrat mit seiner scharfen Kritik an dem Bau der Hochwassermauer kaum auf das abgestimmte Endergebnis ein. Stattdessen wirkte die zweite Kölner Spitze, Oberstadtdirektor Maximilian Adenauer (CDU) trotz fraglichen Argumenten sehr viel einflussreicher und wurde zudem von dem Bundeskanzler bei dessen Besuch im November 1959 öffentlich unterstützt. Diese Wirkung schien so nachhaltig, dass sie fast elf Monate später vom Beigeordneten des Tiefbaus Dr. Kleppe (CDU) in einer Sitzung aufgegriffen wurde ${ }^{216}$. Mit Schwering verband Konrad Adenauer nicht nur die Parteizugehörigkeit, sondern auch die Lebensabschnitte während der Kaiserzeit, der zwei Weltkriege und des Dritten Reichs sowie ihre berufliche Tätigkeit als Oberbürgermeister der Stadt Köln - und damit vielleicht eine emotionale Partnerschaft, die Schwering zum Fürsprecher der Mauer werden ließ. Im philosophischen Rationalitätsdiskurs fällt dieses Phänomen unter die „Kohärenz der Vernünftigen““217, Menschen mit ähnlichen Hintergründen entscheiden dementsprechend. Soziologen sehen das

\footnotetext{
${ }^{214}$ Ebd.

${ }^{215}$ Brixius 2004.

${ }^{216}$ Stadt Köln, Rat der Stadt Köln, 10. Sitzung, 20.10.1960.

${ }^{217}$ Nida-Rümelin 1996; 2009.
} 
ähnlich, denn sie halten den Konsens von „,ziemlich jedem mit vergleichbarem Wissen und Intelligenz ${ }^{\text {‘218 }}$ für offensichtlich.

Insbesondere macht es den Eindruck, dass die Vorkriegs-Eliten den Bau der Mauer hauptsächlich beeinflussten. Als Zwischenfazit lässt sich an dieser Stelle festhalten, dass die Reaktionen auf Hochwasser, speziell die Höhe der Schutzmauer, durch den Wiederaufbau der vom Krieg zerstörten Stadt beeinflusst waren. Infolgedessen werden im nächsten Abschnitt der Wiederaufbau der vom Krieg zerstörten Stadt und die Heimatproblematik zentriert.

\subsubsection{Die Kölner Heimat}

Während der kontroversen Diskussionen um den Bau der Hochwasserschutzmauer griff Bundeskanzler Konrad Adenauer im November 1959 in die Streitigkeiten ein. Dies ist verwunderlich, da der Hochwasserschutz in Nordrhein-Westfalen in den administrativen Gewalten der Gemeinden verankert war. Doch kommentierte Konrad Adenauer nicht die Qualität des Hochwasserschutzes, sondern lediglich den Nebeneffekt dessen, die Spaziergänger am Rhein vor dem Straßenverkehr zu schützen. Erst in den 1950er Jahren entwickelte sich das Verkehrsaufkommen zu einer beklagenswerten Belästigung auf der Rheinuferstrasse. Er erkannte in dem Verkehr eine Bedrohung für den Fußgänger am Ufer und forderte, dass der Aufenthalt am Rheinufer für den Fußgänger wieder ein positives Erlebnis werden müsse. Konrad Adenauer griff in den Kölner Diskurs ein, mit dem Ziel, die Erinnerungen an seine Heimat wieder Realität werden zu lassen. Heimat entstehe an Orten mit „ungewöhnlich vielen emotionalen Bindungen““219 und Erlebnissen. „Durch die Bürger oder Bewohner von Heimat wird eine Vorstellung über das typische Landschaftsbild im Sinne einer Welt in den Köpfen erstellt. Diese Welt besteht aus den für die Heimat typischen Bauformen, Land- und Raumnutzungen, welche aus der realen Welt ein Gemenge objektiver

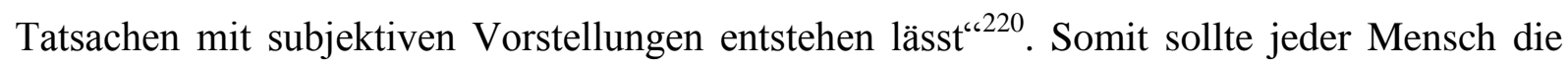
Möglichkeit haben, die Heimat des Konrad Adenauers zu erleben. Die Bestrebungen des Bundeskanzlers in seiner früheren Funktion als Oberbürgermeister Kölns ${ }^{221}$, das Stadtbild durch Parkanlagen zu optimieren, sind bekannt. Das Stadtbild wird nach dem Historiker Ferdinand Opll als Ikonographie, Ausdruck von Mentalität oder als aus der Geschichte der jeweiligen Stadt abgeleitet definiert ${ }^{222}$. Der Ausdruck der Mentalität wird an der unterschiedlichen Bebauung sichtbar und kann bestimmte Werte in den Vordergrund rücken,

\footnotetext{
${ }^{218}$ Gert 1996, 320.

${ }^{219}$ Spiegel 1995.

${ }^{220}$ Downs/Stea 1982; Spiegel 1995.

${ }^{221}$ Konrad Adenauer war zwischen 1917 und 1933 sowie einige Monate im Jahr 1945 Oberbürgermeister zu Köln.

${ }^{222}$ Opll 2004.
} 
während die Mentalität der Bewohner ihre Vorstellungen von der optimalen Bauart prägt ${ }^{223}$. Aus der eigenen Stadtgeschichte abgeleitet soll das Stadtbild eine Botschaft vermitteln und Identität stiften, bzw. gilt das Stadtbild als Identität der Stadt und wird als gegeben aufgefasst $^{224}$. So initiierte Adenauer 1922 die Umbauarbeiten des Preußenrings zu einem Park und Grünanlage von $8 \mathrm{~km}^{2}$ Größe um die Innenstadt. K. Adenauer wollte damit die Bürger in die Natur zurück bringen, „um sie von der urbanen Steinwüste zu befreien““225. Er mahnte, an die nächsten Generationen zu denken. Adenauer zielte darauf ab, den Kindern von Morgen die Möglichkeit zu bieten, unberührte Natur zu erfahren ${ }^{226}$. Er nannte es „eine Lebensfrage Kölns“ ein Band mit Wald, Wiesen und Feld aber auch Sportanlagen, Spielplätzen und Schwimmbädern um die Innenstadt zu legen, um durch eine gesunde, wohnliche Stadt den Handel erblühen zu lassen ${ }^{227}$. Abb. 10 zeigt den heutigen Kölner Stadtplan mit dem Grüngürtel, der wie ein Halbkreis auf der linken Rheinseite aufgespannt wurde.

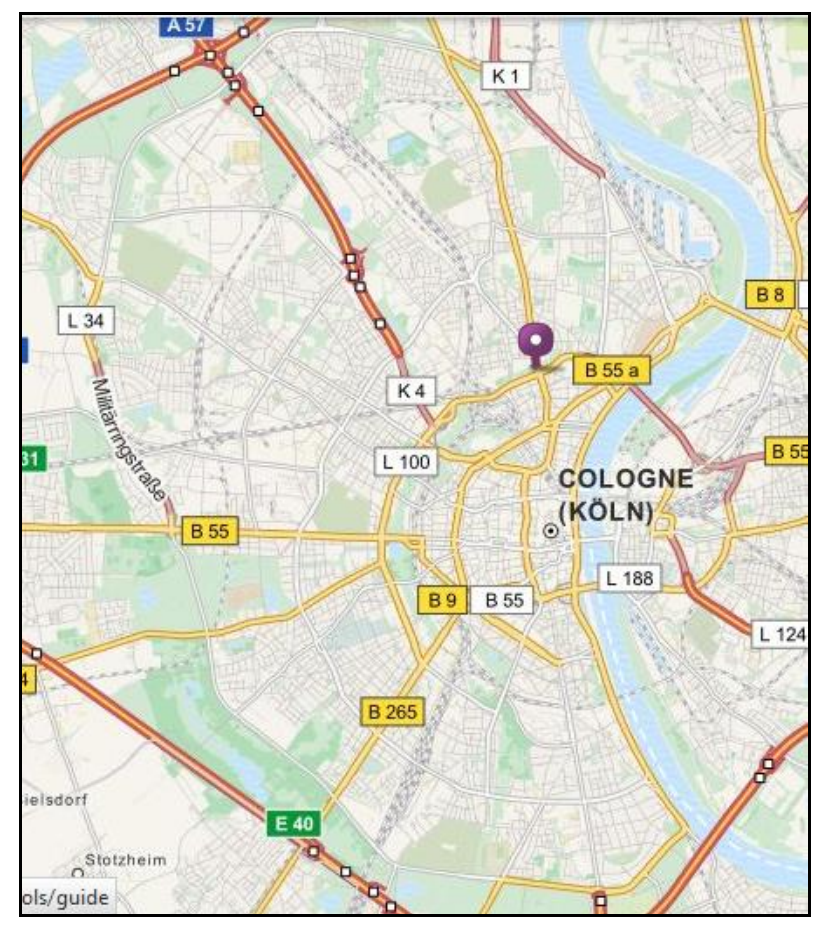

Abb. 10: Grüngürtel der Stadt Köln. ${ }^{228}$

Konrad Adenauer war offensichtlich bereits als Oberbürgermeister zu Köln damit beschäftigt, durch die Gestaltung des Stadtbildes Heimat zu kreieren und der Stadt ein ganz spezifisches Antlitz zu verleihen. Bis 1957 waren immer noch nicht alle Kriegsspuren beseitigt. Der

\footnotetext{
${ }^{223}$ Niedermaier 2004.

${ }^{224}$ Opll 2004.

${ }^{225}$ Frielingsdorf 2001.

${ }^{226}$ Ebd.

${ }^{227}$ Ebd.

${ }^{228}$ Die Karte erstellte der Autor mit Hilfe von OpenSourceMap (Internetbasierte Quelle 10).
} 
damalige Gartenbaudirektor Schönbohm skizzierte das rechtsrheinische Gelände gegenüber dem Dom als „nichts weiter als verwildertes und mit Trümmerschutt bedecktes Gebiet“‘229. Die erste Bundesgartenschau (BUGA) 1957 sollte folglich als Instrument dienen, die Verschönerungsarbeiten an dem Stadtbild voranzutreiben und einen Neubeginn nach den Kriegsjahren symbolisch einzuleiten ${ }^{230}$. Brixius hebt die duale Bedeutung der Leistungsschauen hervor. Sie schreibt ihnen die Lösungsfunktion für die vom Krieg zerstörten Städte zu, Schuttberge in „städtebaulich umfassende Konzeptionen“ zu verwandeln, während sie den Ausstellern eine Plattform bot. Die Schirmherrschaft der BUGA teilten sich der Bundespräsident Dr. Theodor Heuss sowie Konrad Adenauer in seiner Funktion als Bundeskanzler $^{231}$. Der ehemalige Bürgermeister Kölns blieb an der Wiederherstellung seiner Heimat interessiert, was sich auch darin widerspiegelt, dass die Organisatoren Wert darauf legten, die Gebäude, das Stadtbild und den Landschaftscharakter zu harmonisieren ${ }^{232}$. Damit wurde das Gelände von 0,424 km² Größe am Rheinufer ,zu einer der schönsten Schöpfungen der Gartenarchitektur in der Nachkriegszeit ${ }^{‘ 233}$. Die Homepage der Bundesgartenschau fasst das Vorhaben heute noch wie folgt zusammen:

„Mit der ersten BUGA in Köln nahm man sich vor, das durch große Trümmerschuttmassen verunstaltete Gelände zwischen Messe und Mülheimer Hafen neu zu gestalten. In Köln machte sich schon die positive Aufbruchstimmung der deutschen Wirtschaftswunderjahre bemerkbar: Auf dem Geländes des Rheinparks wurden üppige, farbenfrohe Blumenbeete angelegt und Brunnen mit hochschießenden Fontänen errichtet. Rosafarbene Flamingos bevölkerten weitgeschwungene Wasserbecken. “234

Brixius beschreibt die Sehnsucht der in den zerstörten Städten lebenden Menschen nach einem Stück heiler Welt, welches mit dem Erhalt der 200 Jahre alten Bäume auf dem Gelände durch die BUGA und dem daraus entwachsenen Garten geboten werden konnte ${ }^{235}$. Auch wurde wieder großes Gewicht auf die Stadtansicht gelegt. Brixius verdeutlicht Schönbohms Anliegen, dem Gelände den freien Blick auf das linke Rheinufer, „[das] Altstadtpanorama, [den] Dom und [die] romanische Kirche St. Kunibert ${ }^{\star 236}$, zu bewahren. Teilweise wurde die Stadtansicht in die Kunst integriert:

\footnotetext{
${ }^{229}$ Brixius 2004, 78.

${ }^{230}$ Brixius 2004 .

${ }^{231}$ Brixius 2004.

${ }^{232}$ Ebd.

${ }^{233}$ Der Rheinpark 2007, 4.

${ }^{234}$ Internetbasierte Quelle 13.

${ }^{235}$ Brixius 2004.

${ }^{236}$ Brixius 2004, 86.
} 
„[Eine Plastik,] die so platziert war, dass der Blick des Betrachters im Tanzbrunnengelände über den Rhein zum Dom hin über sie hinwegschweifen musste. Sie bildete einen markanten Punkt im Vordergrund - mit ihren weichen Formen im krassen Gegensatz zu den bizzaren

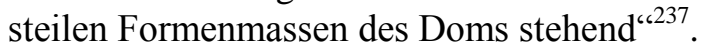

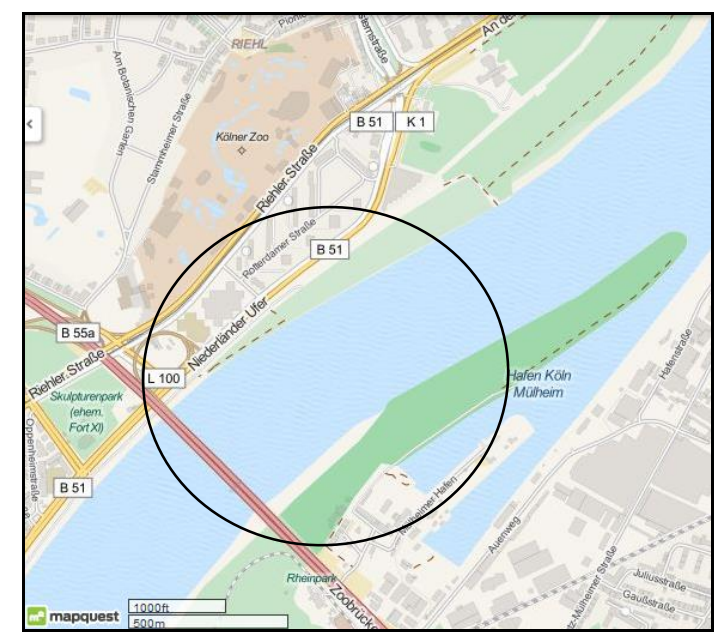

Abb. 11: Areal der Bundesgartenschau 1957. ${ }^{238}$

Abb. 11 zeigt die Lage der Bundesgartenschau zwischen Hohenzollern- und Zoobrücke auf der rechten Rheinseite sowie das Poster der Schau von 1957. Im Kontext der Mitte der 1950er Jahren noch zu beseitigenden Kriegsschäden wird die augenscheinliche Verharmlosung des Hochwasserereignisses von 1955 verständlich. Hier wird klar, weshalb die Überschwemmungen nach dem Krieg keine besondere Aufmerksamkeit von den Kölner Bürgern bekamen und weshalb diese in der Diskussion über den Mauerbau von 1960 ebenfalls keine Erwähnung fanden. Obwohl es 1955 Berichte über den „entfesselten Strom“‘239 sowie „über so etwas Elementar-Grausiges, dass es den Zuschauern fast die Sprache verschlägt“ ${ }^{\star 240}$ gab, empfanden die Menschen nicht, ein „KatastrophenHochwasser “241 zu sehen. Ein Autor eines Hochwasserberichtes bezeugte das noch vorhandene Gemeinschaftsgefühl der Kriegskameraden, welche im Angesicht der Überschwemmung zusammenhielten ${ }^{242}$. Die Zusammenarbeit der Rettungskräfte, wie auch der Ämter entlang des Rheins zum Erarbeiten und Kommunizieren der Prognosen wurde schon vor dem Erreichen des Höchststandes gelobt ${ }^{243}$. Die Notdienst leistenden Einsatzkräfte bemühten sich, einen normalen Ablauf des alltäglichen Lebens zu gewährleisten ${ }^{244}$. Dafür

\footnotetext{
${ }^{237}$ Brixius 2004, 124.

${ }^{238}$ Internetbasierte Quelle 13; Die Karte erstellte der Autor mit Hilfe von OpenSourceMap (Internetbasierte Quelle 10).

${ }^{239}$ Artikel: 8.80 m über Null um 14 Uhr, Kölnische Rundschau, 20.01.1955.

${ }^{240}$ Artikel: Nä, nä - wo kütt bloß all dat vile Wasser her! Kölnische Rundschau, 19.01.1955.

${ }^{241}$ Artikel: 8.80 m über Null um 14 Uhr, Kölnische Rundschau, 20.01.1955.

${ }^{242}$ Artikel: Deutz diesmal ohne Laufstege, Kölnische Rundschau, 21.01.1955.

${ }^{243}$ Artikel: Alarm beim Hochwasser-Schutz, Kölnische Rundschau, 18.01.1955.

${ }^{244}$ Artikel: Rheinuferstrasse wieder hochwasserfrei, Kölnische Rundschau, 22.01.1955.
} 
ernteten sie Lob aus dem Stadtrat, von der Presse sowie von Anwohnern ${ }^{245}$. Die Bewältigung der Überschwemmung wurde als gelungen empfunden, sodass offenkundig weder Diskussionen noch Aktionismus hervorgerufen wurden. In Anbetracht der Nachkriegssituation erscheint diese Haltung nachvollziehbar. Die Menschen erlebten die Stadt in Schutt und Asche zerlegt. Neben den Erlebnissen der zerstörten Heimat relativierten sich die Zerstörungen und Beschädigungen durch ein Hochwasser in der Wahrnehmung der politischen Akteure. Was sollte eine sich um den Rhein konzentrierende Überschwemmung schon anrichten können? Welchem Hochwasser sollte man nicht gewachsen sein, nachdem man selbst den Krieg überlebt und Köln wieder aufgebaut hatte? In Abb. 12 ist die Frankenwerft mit Kriegsschäden zu sehen. Das Bild zeigt beispielhaft das Ausmaß der Zerstörungen nach dem Zweiten Welt Krieg in Köln.

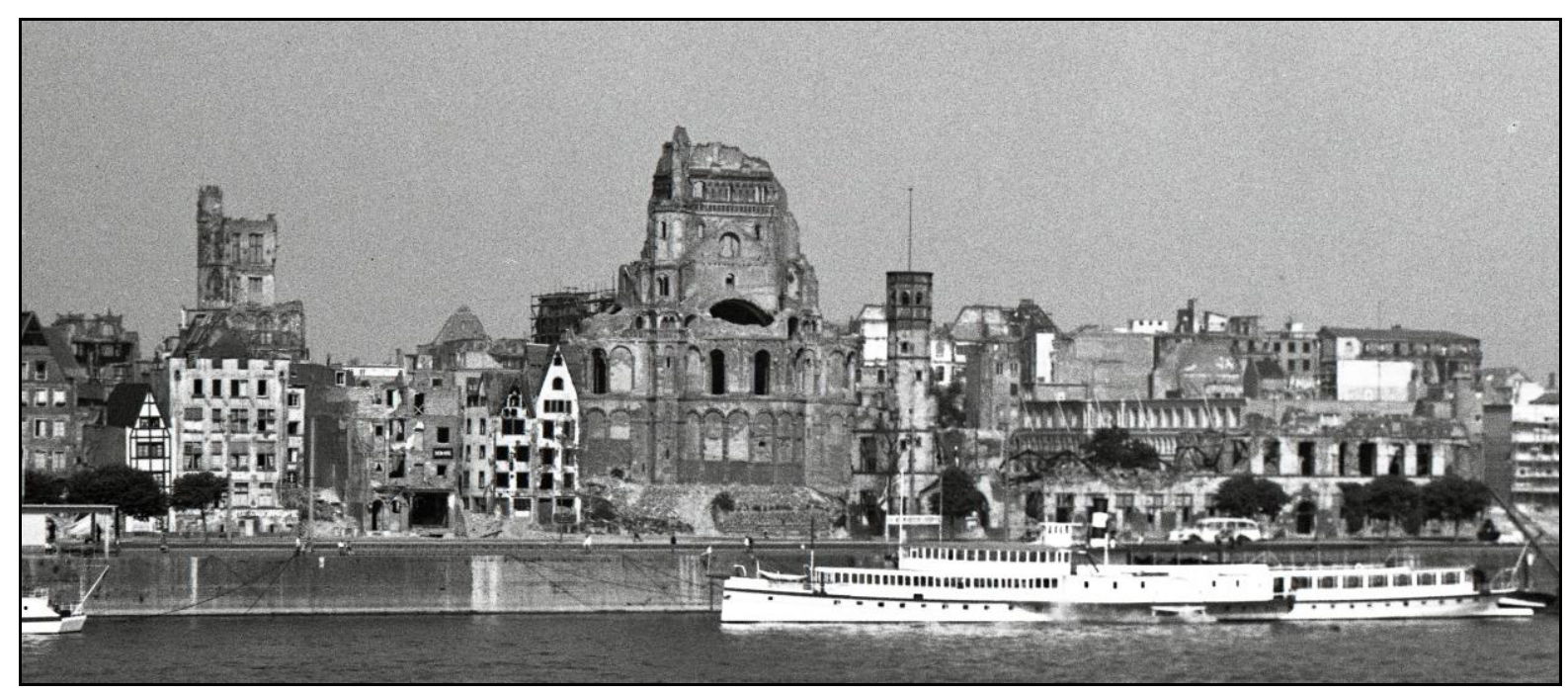

Abb. 12: Frankenwerft mit Kriegsschäden. ${ }^{246}$

Bereits im November 1959 setzte sich Bundeskanzler Konrad Adenauer wieder für die Gestaltung des Stadtbildes am Rhein ein. In seiner Rede zur Eröffnung der Severinsbrücke forderte er, dass die ehemaligen Voraussetzungen für den Besuch am Rhein wiederhergestellt würden. Die Schrägseilbrücke war die erste, welche nach dem Krieg in Köln neu errichtet wurde. Kanzler Adenauer hielt in seiner Ansprache mit dem Titel „Gebt den Kölnern den Rhein zurück ${ }^{، 247}$ neben der Würdigung des modernen Brückenkonstrukts eine Mahnrede über die Gestaltung des linksrheinischen Ufers ${ }^{248}$. Er redete den Verantwortlichen ins Gewissen, der Bürger müsse fußläufigen Zugang zum Fluss erhalten, während das Stadtbild gegen die

\footnotetext{
${ }^{245} \mathrm{Ebd}$.

${ }^{246}$ Internetbasierte Quelle 9.

${ }^{247}$ Becker 1998, 153.

${ }^{248}$ Stadt Köln, Rat der Stadt Köln, 10. Sitzung, 20.10.1960.
} 
Widrigkeiten der Moderne, also den starken PKW-Verkehr, geschützt werden müsse. Er verlangte die Wiederherstellung der Wasserkante, wie er sie aus Kindheit und Jugend kannte. Auch bis in die 1970er Jahre sollte der Fluss das markanteste Aushängeschild der Identität der Stadt Köln bleiben. Der Ratsbeschluss für eine weitere Veranstaltung im Rahmen der Bundesgartenschauen fiel am 26. Oktober $1961^{249}$. Die Kölner Verantwortlichen entschieden sich, das Gelände der ersten BUGA wieder zu benutzen und es um die Riehler Aue auf der gegenüberliegenden linken Rheinseite zu erweitern. Die Aue erstreckt sich 1,8 km entlang des Rheins und bot eine Ausstellungsfläche von $0,274 \mathrm{~km}^{2}$ Größe. Im Zusammenhang mit der Schaffung einer großen linksrheinischen Uferpromenade sollte das linksrheinische Gebiet am Grünzug Süd „gärtnerisch erschlossen werden“250. Gartenbaudirektor Schönbohm sprach im Rat der Stadt Köln von den Vorteilen der Gartenschau. Neben einer „städtebaulichen Sanierung mit starkem sozialen Charakter“'251 sollte der Rhein als „visuelle Verknüpfung“ zwischen den beiden Ausstellungsorten ${ }^{252}$ im Vordergrund stehen.

Vor diesem Hintergrund wird verständlich, welches Gewicht auf die Stadtansicht und dessen Bewahrung gelegt und weshalb eine Beeinträchtigung dessen 1960 mit einem Drama gleichgesetzt wurde. Das Drama hätte in den drei Akten der Zerstörung der Stadt durch den Krieg, des Wiederaufbaus der Stadt durch die Bevölkerung und der finalen Zerstörung der Stadt durch die Bevölkerung aufgebaut werden können. Die starke Betonung der Angst vor der Zerstörung des Antlitzes der Stadt wird als Reaktion auf eine befürchtete Gefährdung von Heimat und Stadtidentität nachvollziehbar und in einem abschließenden Abschnitt aus der Perspektive der Kosten-Nutzen-Analyse bewertet.

\subsection{Bewertung des Diskurses aus der Kosten-Nutzen-Perspektive}

In diesem Abschnitt wird der Entscheidungsfindungsprozess aus der Perspektive der KostenNutzen-Analyse beleuchtet, um die Rationalitäten der Entscheidungsträger aufzudecken. Das erarbeitete Hilfsanalysekonzept unterstützt die Bewertung, welche in die Kriterien Diskussion, Alternativen und Konsequenzen gegliedert ist.

Die Diskussionen verliefen über einen besonders langen Zeitraum von nahezu zwei Jahren. Die missliche Verkehrslage besaß einen starken Einfluss auf alle Kölner Politiker, wie die

\footnotetext{
${ }^{249}$ Brixius 2004.

${ }^{250}$ Brixius 2004, 144.

${ }^{251}$ Brixius 2004, 146.

${ }^{252}$ Ebd., 151.
} 
Einstimmigkeit der Mitglieder des Stadtrates zu dem Thema des Umbaus der Straßenführung verdeutlicht. Aus diesem Grund konnte das Thema von dem Verkehrsexperten Maximilian Adenauer (CDU) instrumentalisiert werden, den Bau einer Mauer am Rheinufer argumentativ zu befördern. Der Prozess der Entscheidungsfindung begann im März 1959 mit einem ersten Vorschlag über die Schutzhöhe seitens des Tiefbaudezernats. Zu Beginn der Diskussion über den Bau einer Hochwasserschutzmauer 1959 sprach sich Oberbaudirektor Schüssler (SPD) für eine Mauer mit einer Höhe von 1,4 - 1,6 $\mathrm{m}^{253}$ aus. Diese Höhe wies er aufgrund von Hochwasserstatistiken aus. Aufgrund der Kritik durch die Stadtratsmitglieder wurde in der zweiten Sitzung eine neue Ausarbeitung vorgestellt. An jenem Dienstag wurde eine Mauer, die nur noch 0,6 - 1,20 m hoch sein sollte, vorgeschlagen. In der vierten Sitzung des Stadtrates wurde eine Mauer von 0,6 - 1 m Höhe, mit der zusätzlichen Unterstützung durch 0,2 $\mathrm{m}$ hohe Kargplatten abgestimmt. Alleine die ersten beiden Höhenvorschläge der Verwaltung, vorgebracht durch Oberbaudirektor Schüssler (SPD) und ausgearbeitet unter Stadtdirektor Adenauer (CDU), orientierte sich an Hochwasserstatistiken und damit an normativen Erfahrungswerten. Anfänglich war also ein kalkulierender Ansatz erkennbar, als Oberbaudirektor Schüssler (SPD) in der Rolle des Experten bei der zweiten Sitzung am 13. Oktober 1959 Hochwasserstatistiken präsentierte und daraufhin eine Empfehlung für die Schutzhöhe aussprach. Alle späteren Höhenvorschläge blieben losgelöst von Hochwasserereignissen und damit in gewisser Weise willkürlich und zugleich emotional aufgeladen. Die scheinbar wahllos vorgetragenen Höhenvorschläge lassen erkennen, dass der Hochwasserschutzaspekt keine primäre Rolle spielte. Das Jahrhunderthochwasser von 9,69 m K P im Jahre 1926 diente bereits als Bemessungsgrenze für ein Ereignis mit hundertjähriger statistischer Wiederkehr, trotzdem ignorierten die Kölner Verantwortlichen diese normative Orientierungsgröße, denn die Pegelhöhe von 1926 wurde gar nicht in die Diskussion eingeführt. Ebenso wurde auf die letzten beiden großen Überschwemmungen von 1948 und 1955 kein Bezug genommen ${ }^{254}$. Nur am Rande wurde durch den Tiefbaudezernenten Kleppe (CDU) darauf verwiesen, dass der Vorschlag eine Mauer zu bauen bereits als Reaktion auf die hohen Schäden des Hochwassers von 1948 im Raum stand, aber damals als zu kostspielig verworfen wurde ${ }^{255}$. Als Maßstab für die alternativ diskutierten Schutzhöhen diente lediglich

\footnotetext{
${ }^{253}$ Stadt Köln, Rat der Stadt Köln, 12. Sitzung, 13.10.1959.

${ }^{254}$ Bis ins Jahr 1960 schützte eine Mauer die Kölner Innenstadt bis zu einem Wasserstand von 7 m K P. Die Bewohner waren Überschwemmungen in einem Dreijahresrhythmus gewohnt.

255 Der ungewöhnlich hohe Pegel des Hochwassers von 1948 wurde auf die im Rhein herumliegenden Kriegstrümmer zurückgeführt. Einige Brücken waren unter anderem in den Fluss gestürzt. Die verhältnismäßig hohen Schäden entstanden aufgrund der Überschwemmung von Lagerhäusern im Hafen in denen Nahrungsmittel gelagert wurden, die daraufhin verdarben. Der Zusammenhang wurde als die sogenannte Milchpulveraffäre bekannt. Als der Rheinpegel im Januar 1955 zu steigen begann, wurde ein Katastrophenhochwasser erwartet (Landesarchiv NRW, NW 112, Nr. 165, 1955). Die Landesregierung NRW bereitete den Bezirkspräsidenten darauf vor, dass die in Köln stationierten
} 
die Stadtansicht. Die Bewahrung dessen erforderte einen Verzicht auf einen erweiterten Hochwasserschutz. Aber ohnehin setzte man nur geringe Erwartungen an die Schutzwirkung der geplanten Mauer. Die Abgeordneten waren sich einig, das Hochwasser würde die Mauer eines Tages übersteigen, sie fanden aber, damit müsse man leben ${ }^{256}$. Die Sprecher trauten dem Fluss größere Kräfte zu und strebten offenbar gar nicht an, eine Dauerlösung auf den Weg zu bringen. In einem weiteren Redebeitrag gab Kleppe (CDU) offen zu, dass der Hochwasserschutz hinter der Bewahrung der Stadtansicht zurückgetreten sei. Die Kölner Verantwortlichen seien gewillt, das angestrebte Überschwemmungsintervall auszudehnen, wenn die Mauer damit der Stadtansicht zuträglicher wurde. Normativität fehlt insbesondere dem letzten Vorschlag. Mittlerweile fand nur noch ein einziges Argument Geltung, dass die Stadtansicht bewahrt werden müsse. Einige Mitglieder der CDU und der FDP haderten mit dem Verständnis für den Vorschlag und waren deswegen nicht in der Lage ihn vollends zu vertreten. Hartmann (CDU) wollte den Bürger entscheiden lassen, ihrer Meinung schlossen sich Parteikollege van den Valentyn (CDU) und Jacobs (FDP) an. Sie behandelten das Gelände am Rhein als öffentliches Gut, dessen Nutzung jedem Bürger kostenlos zusteht. Van den Valentyn versuchte in letzter Minute durch seine Provokation - ob der Schutz uns diese Mauer wert sei, fragte er in die Runde - Vernunft zu bewahren.

Die Mitglieder des Stadtrates brachten ihre persönlichen Anschauungen, Vorschläge und Kritik an der Hochwassermauer ein. Der Aushandlungsprozess ließ genügend Raum für Reflexion und demokratische Meinungsäußerungen, was auch daran erkennbar ist, dass innerhalb der Parteien und Ausschüsse bis zuletzt keine Einigkeit bestand. Für den Bau überhaupt irgendeiner Mauer sprachen sich vier CDU-Abgeordnete (Pflitsch, Stein, Adenauer und Schwering) und drei SPD-Abgeordnete (Pecks, Wacker und Schüssler) aus, dabei variierten die für nötig gehaltenen Höhenlinien fast von Sprecher zu Sprecher. Technische Experten waren in der Runde bis auf die Repräsentanten des Tiefbauamtes nicht vertreten. In

internationalen Pionierbataillone helfen sollten, die Stadt gegen die Fluten abzusichern (Ebd.). Die Ursachen der Flut wurden ausschließlich mit der nassen Witterung begründet (Artikel: Heute wird Höchststand erwartet, Kölnische Rundschau, 19.01.1955). Die zentrale Herausforderung verkörperte der aus dem Hochwasser resultierende Stau des Pkw-Verkehrs (Artikel: Wird in Köln die 9-Meter-Grenze erreicht? Kölnische Rundschau, 18.01.1955). Es ereignete sich ein „beängstigendes Verkehrswirrwarr“ denn ,,auch die zusätzlich eingesetzten Verkehrsposten wissen kaum, wie sie Ordnung in das Tohuwabohu bringen sollen“ (Ebd.). Dementsprechend folgten zum Ende der Überschwemmung einige Artikel, in denen die Verkehrsteilnehmer ,aufatmen“ (Artikel: Rheinuferstrasse wieder hochwasserfrei, Kölnische Rundschau, 22.01.1955), nachdem die sechs Tage andauernden „Verkehrskalamitäten“ (Ebd.) samstags vorbei gingen. Bereits am nächsten Tag fand eine reguläre Sitzung des Kölner Stadtrats statt. Oberstadtdirektor Dr. Maximilian Adenauer (CDU) berichtete über das Hochwasser und bewertete die Situation als: ,allgemein erfreulich und zufriedenstellend“ (Stadt Köln, Rat der Stadt Köln, 1. Sitzung, 20.01.1955, 4). Er fuhr fort, das „Notstandsbekämpfungsprogramm sei reibungslos [...] und die Evakuierungen ohne Schwierigkeiten“ verlaufen. Er hob hervor, „daß nichts, aber auch nichts irgendwie durch Wasserschäden verdorben oder sonst umgekommen“ sei und endete seine Ansprache damit, den Einsatz als ,gelungen“ zu bezeichnen. Da niemand der übrigen Anwesenden das Wort ergriff, kann angenommen werden, dass die Kölner Verantwortlichen gar kein Problem aufgrund der Überschwemmungen feststellten. Das Ereignis provozierte offenkundig weder Diskussionen noch Aktionismus.

${ }^{256}$ Stadt Köln, Rat der Stadt Köln, 10. Sitzung, 20.10.1960. 
der Analyse der Vor- und Nachteile des Bauwerks wurde ebenfalls über den Kreis der Betroffenen wie Anwohner, Kraftwagenfahrer und Touristen gesprochen und erörtert, ob eine dieser Gruppen an der Mauer Anstoß nehmen könnte. Kurz nach einem unbefriedigenden Treffen im Oktober 1959, als wieder neue Vorschläge über die Schutzhöhe von verschiedenen Meinungsträgern in die Diskussion des Stadtrats hineingeworfen wurden, griff auch der Bundeskanzler in die Debatten ein. Er nutzte im November 1959 als gebürtiger Kölner eine öffentliche Veranstaltung, um mit Autorität zu den lokalen Verantwortlichen und Bürgern zu sprechen und nahm dabei für sich in Anspruch die Kölner Sehnsüchte zu repräsentieren. Als ehemaliger Oberbürgermeister von Köln, der sich in dem Antlitz der Stadt durch die Gestaltung der Grünanlagen verewigt hatte, besaß Konrad Adenauer großen Einfluss auf die Entscheidungsträger und spielte das wissentlich aus. Nach seinem Besuch war der Mauerbau positiv durch die Heimatkonnotation besetzt. Die Verantwortlichen einigten sich also erst recht spät, ob die Mauer für die Fußgänger einen Gewinn oder einen Verlust darstellen würde. In der Abstimmungssitzung sprach man über den deutlichen Platzgewinn und den Schutz vor Unfällen mit Automobilen. Dass die Mauer als Sitzgelegenheiten dienen und bepflanzt werden könne, wurde als weiterer Nutzeneffekt wahrgenommen und schien den Grad der Vorteilhaftigkeit des Bauwerks ausschlaggebend zu erhöhen. Es wird deutlich, der Bau der Hochwasserschutzmauer nur auf die Agenda des Stadtrats gesetzt werden konnte, da das Verkehrsproblem eine ausreichende Diskussionsvorlage lieferte. Abschließend ist festzuhalten, dass die Entscheidung im Grunde von einem einzelnen Akteur beeinflusst wurde, dessen Meinung deutlich mehr Gewicht beigemessen wurde, als den Äußerungen der übrigen Verantwortlichen.

Zunächst wurde eine Lösung für das Verkehrsproblem gesucht. Deshalb wurde unter anderem überlegt, ob das Kernproblem, nämlich der Verkehr, nicht alternativ mit neuen Regelungen zu lösen wäre. Eine Option neben dem Status Quo, einer Straßenanhebung oder den Verkehr neuartig zu regeln schien der Bau einer Hochwassermauer. Die verschiedenen technischen Möglichkeiten blieben bis zuletzt jedoch nebeneinander bestehen, denn alle Alternativen boten verschiedene Nutzen und Einschränkungen, welche lediglich subjektiv bewertet wurden. Da keine monetäre Bewertung stattfand, blieb die Favorisierung einer Alternative Geschmackssache. Aufgrund der mangelnden Definition der Zielfunktion des Bauwerks wird verständlich, weshalb die Verantwortlichen sich bei der Auswahl der Schutzhöhe nicht von normativen Vorgaben, sei es eine Hochwasserstatistik oder die nach dem Jahrhunderthochwasser von 1926 festgeschriebene Bemessungsgrenze, inspirieren ließen. An 
dieser Stelle hätte eine traditionelle Kosten-Nutzen-Analyse sicher helfen können, eine Entscheidung zu treffen. Eine tatsächliche Durchführung der Analyse hätte den Akteuren verdeutlicht, dass sie sich zunächst über eine Zielfunktion im Klaren sein müssten. Was sollte mit der Mauer erreicht werden? Ohne definiertes Ziel blieb eine Bewertung des Zielerreichungsgrades und damit der Vergleich zwischen der Vorteilhaftigkeit alternativer Handlungsmöglichkeiten unmöglich ${ }^{257}$. Aufgrund der Uneinigkeit zwischen den kommunalen Politikern, ob diese Mauer gegen Hochwasser schützen oder den Verkehr regeln oder den Fußgänger vor dem Verkehr sichern sollte, blieben die Ansprüche an die Mauer ungeklärt. Das fehlen der Zieldefinition führte dazu, dass die Diskussionen fast zwei Jahre lang geführt werden musste. Da die Wirkungen der Mauer als intangibel begriffen wurden, konnte kein monetäres Kriterium an die Alternativen angelegt werden.

Der Bau der Mauer blieb umstritten, denn die Akteure waren sich wiederum einig, dass das Stadtbild besonders schützenswert ist und nicht entstellt werden darf. Die Handlung stand also in Konflikt $\mathrm{zu}$ dem $\mathrm{zu}$ bewahrenden Stadtbild. Auch wurden Bezüge $\mathrm{zu}$ den Wiederaufbaumaßnahmen nach dem Krieg hergestellt. So wurde darauf verwiesen, dass die alten Häuser an der Frankenwerft originalgetreu wie vor dem Krieg wiederaufgebaut wurden $^{258}$. Es ging also darum, Heimat zu schaffen, bzw. den Preis der Heimat festzulegen. Die Frage, was der Hochwasserschutz kosten darf, stand im Grunde im Zentrum der Argumentationen von 1959/60. Die attraktivste und somit auch Image bildende Stelle Kölns, die Front zum Rhein hin, durfte unter keinen Umständen beeinträchtigt werden. Die Befürchtung, die Hochwasserschutzmauer könne Köln ihr Image kosten, beschäftigte die Mitglieder des Stadtrats über fast zwei Jahre. Es wurde eine heftige Diskussion darüber geführt, ob die Mauer der Beginn der Verschandelung der Stadt sei, da Mitglieder des Stadtrats Sorge hatten, dass die Hochwasserschutzmauer der Anfang von noch immer höheren Mauerbauten nach zukünftigen Überschwemmungen sein könnte. Die Akteure der Diskussion mutmaßten über verschiedene Auswirkungen des Projektes. Es wurden wirtschaftliche Aspekte wie die möglichen Einbußen aufgrund der Mauer in der Touristenschifffahrt, deren Arbeitsplätze und deren Logistik besprochen ${ }^{259}$ und gegen die intangiblen Vorteile der Mauer, wie z. B. den Kölner Bürger wieder stärker an den Rhein zu holen, abgewogen. Ob die Mauer in der Lage wäre, den Verkehr zu regeln, wurde stark hinterfragt. Die Überlegung, ob sie das erhöhte Grundwasser bei Hochwasser verhindern könnte, wurde von den Verantwortlichen im

\footnotetext{
${ }^{257}$ Koester/Tangermann 1976.

${ }^{258}$ Stadt Köln, Rat der Stadt, 12. Sitzung, 20.10.1960.

${ }^{259}$ Ebd., 13.10.1959, 451.
} 
Stadtrat negativ bewertet. Zusätzlich sollte die Mauer für die Fußgänger ein Lärm- und Geruchsschutz bieten, da die Rheinuferstraße eine vielbefahrene „Rennbahn“ sei, aber auch das wurde nach den Gesprächen verworfen. Interessanterweise wurden der potentiell zu verhindernde Hochwasserschaden sowie die Investitions- und Instandhaltungskosten nicht in der Diskussion angeführt. Finanzielle Ressourcen stellten keine Restriktionen dar, weswegen die künftig vermeidbaren Schäden nicht den Hauptnutzen der Mauer ausmachten, der die Höhe der Kosten für Investition und Operation legitimiert hätte. Die unmittelbaren Nutzen und Kosten gingen in die Diskussion folglich nicht ein. Wäre der Hauptzweck der Mauer der Hochwasserschutz gewesen, hätten die Verantwortlichen die direkten Nutzen den direkten Kosten des Baus und seiner Operation entgegen setzten müssen. Ob in diesem Fall die intangiblen Kosten durch die empfundenen Einbußen an der Stadtansicht auch so gravierend angenommen worden wären, bleibt offen. Obwohl eine Betrachtung von Alternativen stattfand, blieben die verschiedenen Konsequenzen aufgrund der emotional aufgeladenen Debatte unevaluiert. Das Markenzeichen der Stadt musste bewahrt werden, auch um den Preis, Hochwasser in einem bestimmten statistischen Intervall in Kauf nehmen zu müssen. Am Ende der Auseinandersetzungen waren sich die Entscheidungsträger bewusst, dass die Mauer die Stadt keineswegs gegen jedwede Überschwemmung schützen werde, sondern dass nun der Verkehr nur noch alle sieben statt wie bisher alle zwei bis drei Jahre durch Hochwasser beeinträchtigt werden würde ${ }^{260}$.

Von einer überstürzten oder gar leichtfertigen Handlung kann keinerlei Rede sein, denn der Plan wurde intensiv überdacht, viele Male kritisiert und neu überarbeitet. Ein Akteur war letztlich in der Lage, eine ganz andere Problemstellung für den Bau der Hochwasserschutzmauer zu instrumentalisieren. Aufgrund seiner sozialen Stellung konnte er als Fürsprecher für die Mauer auftreten. Die Auseinandersetzung brauchte - wie gezeigt werden konnte - eine ausreichende Vorlage, um eingeleitet zu werden. Die fehlende monetäre Betrachtung ist den nicht vorhandenen finanziellen Restriktionen geschuldet. Ein begrenztes Budget hätte eine Gegenüberstellung von Kosten und Nutzen erforderlich gemacht. Aufgrund der immensen Wichtigkeit der Erhaltung des nach dem Zeiten Weltkrieg rekonstruierten Stadtbildes an der Rheinuferstraße wird die zähe und emotional aufgeladene Diskussion verständlich und nachvollziehbar.

\footnotetext{
${ }^{260}$ Stadt Köln, Rat der Stadt Köln, 10. Sitzung, 20.10.1960, 251.
} 


\subsection{Das Hochwasserereignis von 1970, das keines war}

Zehn Jahre nach der Entscheidung eine Mauer gegen Hochwasser zu bauen, sollte diese zum ersten Mal überspült werden. Ein Anschwellen des Flusses von ,stündlich 6 bis $7 \mathrm{~cm}$ kündete das Hochwasser an“261, welches zwischen Montag dem 23. Februar 1970 bis Sonntag dem 01. März 1970 die Stadt Köln heimsuchte ${ }^{262}$. Der Pegelanstieg wurde dem Tauwetter der letzten Tage zugeordnet ${ }^{263}$. Die Hochwasserschutzzentrale ${ }^{264}$ war für die Zeit des Hochwassers in dem Amt 68 für Stadtentwässerung eingerichtet und unter die Aufsicht des leitenden Stadtbaudirektor (Ltd. StBD.) Pelzer $^{265}$ gestellt worden. Die Zentrale übernahm die Steuerung von Meldungen aus der Bevölkerung und Informationen der Behörden sowie die Weitergabe an die jeweils zuständigen Stellen. Die Einsatzleitung des Hochwasserschutzdienstes unterstand dem Tiefbauverwaltungsdezernenten und dem Stadtratbeigeordneten Braun ${ }^{266}$. Der Beigeordnete Braun forderte alle Dienststellen auf, täglich bis 15.00 Uhr einen Tagesbericht mit Angaben zu den durchgeführten Maßnahmen, der Vorbereitung weiterer Maßnahmen sowie besonderen Schwierigkeiten an die Hochwasserschutzzentrale $\mathrm{zu}$ senden $^{267}$. An dem Montagnachmittag berief die Hochwasserschutzzentrale eine Pressekonferenz unter Mitwirkung der Polizei, dem Nachrichtenamt, der Berufsfeuerwehr, der Stelle zur Beseitigung von Wohnungsnotständen sowie den drei Ämtern für Straßenbau, Stadtentwässerung und Brücken- und U-Bahnbau $e^{268}{ }^{268}$, um die potentiell betroffenen Anwohner zu informieren. In eigener Verantwortung führten die städtischen Dienststellen wie Polizei, Gas- Elektrizitäts- und Wasserwerke (GEW), Kölner Verkehrsverbund (KVB) und Köln-Bonner Eisenbahn (KBE) die Hochwasservorschriften der Stadt Köln aus ${ }^{269}$. An jenem Montag prognostizierte die Wasserund Schifffahrtsdirektion in Duisburg für den darauf folgenden Tag bereits einen Wasserstand von $8,40 \mathrm{~m} \mathrm{KP}$ und damit die Überflutung der Rheinuferstrasse hinter der neuen

\footnotetext{
${ }^{261}$ Bericht über das Hochwasser 1970.

${ }^{262}$ Ebd.

${ }^{263}$ Ebd.

${ }^{264}$ Die Zentrale war ab einem Wasserstand von $7 \mathrm{~m} \mathrm{~K}$ P Tag und Nacht besetzt.

${ }^{265}$ Ebd.

${ }^{266} \mathrm{Ebd}$

${ }^{267}$ Ebd.

${ }^{268}$ Ebd.

${ }^{269}$ Die Hochwasservorschriften verbinden Handlungsweisungen mit normativen Vorgaben welche aus der Pegelhöhe resultieren.
} 
Schutzmauer $^{270}$. Nach der Hochwasservorschrift wurde die Straße für den Verkehr in der Nacht zum 24. Februar 1970 gesperrt, da das Überströmen der Mauer für die Morgenstunden zwischen fünf und sechs Uhr prognostiziert wurde ${ }^{271}$. Der Ltd. StBD. Pelzer unterstrich in der Besprechung an jenem Montag „die ernste Lage“ und ordnete an, die Menschen an der Rheinuferstrasse durch Lautsprecherwagen der Polizei über die „bevorstehende Überflutung $\mathrm{zu}$ unterrichten und sie auffordern, Heizungsanlagen im Keller [...] zu sichern“"272. Am gleichen Montag berichtete das Amt für Stadtentwässerung, alle „erforderlichen Maßnahmen bis zu einem Rheinwasserstand von $+9 \mathrm{~m} \mathrm{~K} \mathrm{P}^{\text {‘ }} 273$ abgewickelt zu haben.

Seinen Höhepunkt erreichte der Rhein am Mittwoch den 25. Februar 1970 mit einem Pegelstand von $8,87 \mathrm{~m} \mathrm{KP}$ und einer Überschwemmungsfläche von $0,41 \mathrm{~km}^{2}$. Am Donnerstag, den 26. Februar 1970 gab die Stelle zur Beseitigung von Wohnungsnotständen an, dass in Poll die Häuser noch ungefähr 1,50 m hoch vom Wasser umspült seien und sich Bewohner aus Porz aufgrund der mangelnden Versorgung von Strom und Wasser über die Stadt beschwerten ${ }^{274}$. Das Amt für Zivilschutz koordinierte die Kräfte der Bundeswehr zum Hochwasserschutz ${ }^{275}$. Des Weiteren wurde ein Fernschreiben des Innenministers des Landes NRW von Donnerstag erwähnt, in welchem mitgeteilt wurde, dass mit Genehmigung des Bundesinnenministers das THW zur Bekämpfung von Ölschäden und Aufräumarbeiten eingesetzt werden könne ${ }^{276}$.

Erstmalig konnte bei einem Hochwasser der Verkehr auf der Rheinuferstrasse bis zu einer Höhe von 8,40 m K P aufrecht erhalten bleiben, da die bereits 1960 erbaute Mauer solange Schutz gewährte ${ }^{277}$. Sie Straße konnte nach Reinigung und Überprüfung sogleich ab dem 04. März 1970 wieder voll befahren werden ${ }^{278}$. In der Stadt liefen während der Flutwoche 320 Keller mit Wasser voll, welche durch 127 Pumpgeräte der freiwilligen Feuerwehr, dem Katastrophenschutz oder der Stadt entleert wurden ${ }^{279}$. Die beteiligten Dienststellen leisteten insgesamt 8.050 Arbeitsstunden mit ca. $805 \mathrm{Mann}^{280}$, um die Soforthilfemaßnahmen zu gewährleisten. Der offizielle Hochwasserbericht endete mit folgenden vier operativen Verbesserungsvorschlägen für zukünftige Hochwasserereignisse:

\footnotetext{
${ }^{270}$ Stadt Köln, Hochwasserschutzzentrale beim Amt für Stadtentwässerung, Vermerk über die Besprechung 23.02.1970.

271 Ebd.

272 Ebd.

${ }^{273}$ Tagesbericht des Amtes für Stadtentwässerung, 24.02.1970.

${ }^{274}$ Ebd.

${ }^{275}$ Bericht des Amts für Zivilschutz, 27.02.1970.

${ }^{276}$ Ebd.

${ }^{277}$ Bericht über das Hochwasser 1970.

${ }^{278}$ Ebd.

${ }^{279}$ Ebd.

${ }^{280} \mathrm{Ebd}$
} 
„a) Einrichtung von neuen Telefonanschlüssen

b) Ausstattung der Pumpwerke mit dem gesetzlich vorgeschriebenen Explosionsschutz

c) Neueinstellung von Ingenieuren und Facharbeitern zur ordnungsgemäßen Bedienung der Hochwasserschutzeinrichtung bei den rd. $20 \%$ unbesetzten Stellen bei -68-, da hierdurch das vorhandene Personal in kaum noch zu verantwortender Weise überlastet ist.

d) Auswechslung der vorhandenen Hochwassertore aus hölzernen Dammbalken gegen wesentlich schneller einzubauende Stahltore.“281

\subsubsection{Reaktion: Die Diskussion nicht wiedereröffnen}

An dieser Stelle werden die Ergebnisse der Diskussionen der administrativ Verantwortlichen vorgestellt. Fünfzehn Jahre hatte der Rhein den Kölnern kein Hochwasser beschert. Obwohl das Hochwasser im Jahr 1970 die höchste Marke seit 1955 erreichte, blieben die Reaktionen verhältnismäßig gehemmt. Für die Kölner schien es kein großes Ereignis und schon gar keine Katastrophe zu sein, denn sogar die viel diskutierte und kritisierte neue 1,5 km lange Mauer an der Rheinuferstraße aus dem Jahr 1960 konnte sich bewähren ${ }^{282}$. Die Mauer hielt bis zu ihrer Überströmung bei 8,40 m K P stand. Eine Diskussion um deren Erhöhung blieb jedoch im Anschluss an das Schadensereignis vollständig aus. Im Folgenden wird erläutert, welche Akteure Einfluss auf die abgestimmten Reaktionen geltend machen konnten und welche Argumentationslinien sie verfolgten.

\subsubsection{Die Bewährung der vorhandenen Schutzhöhen}

Hier wird verdeutlicht, wie die Verantwortlichen 1970 die Bewährung der vorhandenen Schutzvorrichtungen feststellten und die entstandenen Schäden verhandelten. Die Auseinandersetzungen nach dem Hochwasser bezogen sich 1970 lediglich auf die Streitigkeiten bezüglich der Schadensersatzzahlungen an die Hochwasseropfer. Die Stadt Köln und die Landesregierung Nordrhein-Westfalens nahmen den vorhandenen Hochwasserschutz als ausreichend wahr, der obwohl Hochwasserschäden entstanden, von keinem Lokalpolitiker kritisiert wurde. Der abschließende Hochwasserbericht vom 3. April 1970 aus dem Tiefbauamt von dem Beigeordneten Dipl.-Ing. Braun hielt zu den vorhandenen Schutzvorrichtungen fest:

\footnotetext{
${ }^{281}$ Bericht über das Hochwasser 1970 .

${ }^{282}$ Ebd.
} 
„Es hat sich erstmalig erwiesen, daß bei voll angeströmter Mauer die Rheinuferstraße bis zu einem Rheinwasserstand von $+8,40 \mathrm{~m} \mathrm{~K} \mathrm{P}$ wasserfrei gehalten werden konnte, $\mathrm{d}$. h. sämtliche Einrichtungen (...) haben ihre Bewährungsprobe gut überstanden. “283

Im Landtag NRW wurde ebenfalls festgestellt, dass die nordrhein-westfälischen Schutzeinrichtungen standhaft waren: „Für unser Land haben sich die vorhandenen Hochwasserschutzanlagen bewährt. Eine ausgesprochene Hochwasserkatastrophe konnte mit ihrer Hilfe verhindert werden““284, kommentierte der Innenminister Weyer (SPD). Im Folgenden sprach sich auch der Minister für Ernährung, Landwirtschaft und Forsten, Deneke (SPD) für den bestehenden Schutz aus,

„daß wir nämlich die Hochwasserschäden am Rhein dieses Jahr als Unterlieger erleiden mussten, während sich die jahrelangen Hochwasserschutzmaßnahmen in unserem Lande durchaus bewährt haben. [...] Wir sind mit einem blauen Auge davon gekommen“'285.

Da auf das Ereignis von 1970 keine Reflexion über die Schutzhöhen folgte, stellt sich die Frage, weshalb die Verantwortlichen in Köln mit dem Verhältnis von Hochwasserhöhe zu Schadensumfang einverstanden waren. Welche Abwägungen trafen die Kölner Verantwortlichen, um zu diesem Einverständnis zu gelangen, und warum wurde der bereits vorhandene Hochwasserschutz nicht kritisiert? Welche Hochwasserereignisse kannte die Bevölkerung 1970 bereits, bzw. woher stammte das Bewusstsein, dass Überschwemmungen nicht ungewöhnlich seien?

In den letzten 50 Jahren vor 1970 wurde die Hochwassermarke von 8,40 m K P fünf Mal erreicht $^{286}$. Von den fünf Überflutungen, welche alle über die Rheinuferstraße traten, können die vier Fluten von 1920 (2x), 1926 und 1948 als besonders starke Hochwasser deklariert werden. Bei allen Ereignissen wurde ein Wasserstand von mindestens 10,41 m K P erreicht. Das Hochwasser von 1926 erreichte einen Pegel von 10,69 m K P und ging somit als sogenanntes Jahrhunderthochwasser in die Geschichte ein. Fortan galt es als Bemessungshochwasser mit einem Turnus von statistischen 100 Jahren und wurde erst wieder im Jahr 1995 erreicht. Das Hochwasser von 1948 überraschte Köln, als die Kriegsschäden noch nicht vollends beseitigt waren. Die im Rhein herumliegenden Trümmer wurden für die Einstauhöhen verantwortlich gemacht ${ }^{287}$. Aufgrund der Vernichtung von Lebensmitteln in Lagerhallen der Kölner Häfen wurde der Bau einer Mauer vorgeschlagen, welcher aber nicht

\footnotetext{
${ }^{283}$ Bericht über das Hochwasser 1970.

${ }^{284}$ Landtag NRW, 74. Sitzung, 22. April 1970, 3174.

${ }^{285}$ Ebd., 3175 .

${ }^{286}$ Die 1960 beschlossene Hochwasserschutzmauer war 8,40 m K P hoch.

${ }^{287}$ Stadt Köln, Rat der Stadt Köln, 1. Sitzung, 09.01.1948.
} 
finanzierbar war $^{288}$. Am 19. Januar 1955 wurde der Wasserstand von 8,80 m K P gemessen. Da 1955 kein Problem mit dem Hochwasser wahrgenommen wurde, entbrannte keine Diskussion über den Hochwasserschutz. In der hier knapp dargestellten Zusammenfassung der Überschwemmungen der letzten 50 Jahre wird ersichtlich, dass ein Hochwasser mit einer Pegelhöhe von mindestens 8,40 mKP ca. alle zehn Jahre zwischen 1920 und 1970 aufgetreten war. Somit kann verallgemeinert werden, dass sich jeder damals Sechzigjährige an mindestens fünf Überschwemmungen dieses Ausmaßes erinnern konnte. Besonders hervorzuheben scheint die Tatsache, dass ein Jahrhunderthochwasser in den Erinnerungen dieser Menschen archiviert gewesen war, welches seit 1926 die Maßstäbe für alle späteren Hochwasser setzte. Dagegen schien ein Hochwasser von 8,40 m KP nichts Außergewöhnliches zu bedeuten. Außerdem fielen vier von fünf Ereignissen mit einer Höhe von mindestens 10,41 m K P wesentlich höher aus als das Hochwasser von 1970. Lediglich die Flut von 1955 blieb mit einem Pegel von 8,80 m in Köln unter dem Pegel von 1970, hätte aber ebenfalls die seit 1960 vorhandene Schutzmauer um 0,4 m überspült. Somit erschließt sich aus den Überlegungen im Kontext der Vorgeschichte, dass das Ereignis von 1970 für die politisch Verantwortlichen nicht ausreichend außergewöhnlich war, um die vorhandenen Schutzvorrichtungen in Frage zu stellen. Seit dem letzten vergleichbaren Hochwasser dieses Ausmaßes waren bereits 15 Jahre vergangen und deshalb wurde auch der Zeitintervall neben der Stärke des Hochwassers als natürlich empfunden.

Der Stadtrat hatte 1960 beschlossen, an der Rheinuferstrasse eine Hochwasserschutzmauer zu errichten. Diese war eingerichtet worden und sollte bis zu einem Rheinpegel von 8,40 m K P vor einer Überflutung schützen. Wie bereits gezeigt werden konnte, war der Bau der Mauer sehr umstritten, da er als Bedrohung für die Stadtansicht empfunden wurde, Hartmann (CDU) aus dem Stadtrat zu Köln fasste zusammen: „Hier wird etwas versaut““ ${ }^{289}$ Es konnte gezeigt werden, dass das Hochwasser 1960 nicht als Gefahr empfunden wurde und diese Wahrnehmung auch 1970 noch Bestand hatte. Das absolute Ziel des Mauerbaus stellte nicht die Bekämpfung der Hochwasserproblematik dar, denn der Sicherheitsdiskurs wurde hinter der Bewahrung der Stadtansicht zurückgestellt. Die Kölner Entscheidungsträger waren sich 1960 bewusst, die Hochwasserintervalle lediglich auf statistische sieben Jahre auszudehnen. Die Kommunalpolitiker wussten, dass die Stadt schon bald wieder überschwemmt werden würde, doch sie beschlossen, damit würde man eben leben müssen. Bis zu der Überschwemmung von 1970 hatte es immerhin den statistisch berechneten Zeitraum gedauert, den die Entscheidungsträger bei der Verhandlung 1960 annahmen. Die Mauer hatte somit eine

\footnotetext{
${ }^{288}$ Ebd., 12. Sitzung, 13.10.1959, 462.

${ }^{289}$ Stadt Köln, Rat der Stadt Köln, 12. Sitzung, 13.10.1959.
} 
Berechtigung. Da die langjährigen Debatten über den Eingriff in die Stadtansicht noch immer der Hochwassermauer anhafteten, wurde das Hochwasser von 1970 instrumentalisiert, die endgültige Bewährung der Hochwasserschutzmauer festzustellen. An einer bewährten Methode musste folglich nichts verbessert werden.

\subsection{Das April-Hochwasser 1983}

„Hochwasser im April hatten wir noch nicht ${ }^{‘ 290}$, berichtete ein betroffener Anwohner. „Für den Monat April ist Hochwasser als Folge von staken Regenfällen vollkommen

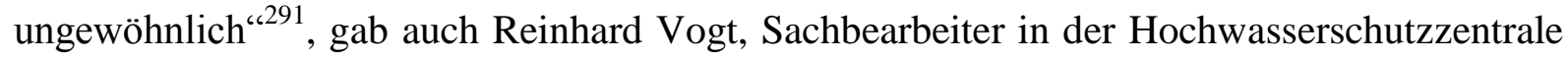
am 12. April 1983 zur Auskunft. „Im April steht der Rhein normalerweise bei nur vier Metern“ ${ }^{292}$, fügte er hinzu. Die Flüsse Saar, Mosel, Lahn, Main und Neckar führten allesamt Hochwasser, welches sie unweigerlich in den Rhein abgaben ${ }^{293}$. In den Kölner Bezirken Poll, Langel und Rodenkirchen standen bereits die ersten Straßen unter Wasser ${ }^{294}$. Die Hochwasserprognose lautete an jenem Dienstag, dass der Rhein $0.2 \mathrm{~m}$ unterhalb der Schutzmauer von 9,40 m K P Höhe bleiben würde. Vogt konstatierte die ungenaue Pegeluhr und wünschte sich eine digitale Anzeige sowie eine telefonische Standleitung zum Messgerät. Denn das Telefon an der Pegeluhr ist häufig überlastet und dann muss ein Mitarbeiter geschickt werden, um die Daten abzuholen. Die Schutzzentrale befand sich in der Hohe Straße, 700 m entfernt von der Pegeluhr.

Im Laufe des Dienstags wurde die Schutzmauer notdürftig um 0,1 m aufgemauert ${ }^{295}$. „Ich würde nie wieder am Rhein bauen““296, fluchte ein Anwohner. Am gleichen Tag brach aufgrund der Schließung des Rheinufertunnels ${ }^{297}$ ein Verkehrschaos in der Innenstadt sowie auf den Ringen ${ }^{298}$ aus $^{299}$. Trotz der Verkehrsprobleme wurde der Rheinufertunnel immer wieder als „perfektes Bauwerk ${ }^{\text {‘300 }}$ gelobt, denn zum ersten Mal seit seinem Bau wurde

\footnotetext{
${ }^{290}$ Artikel: Ruhe und Gelassenheit, Kölnische Rundschau, 13.04.1983.

${ }^{291}$ Artikel: Halten Wälle die Wellen? Kölnische Rundschau, 12.04.1983.

${ }^{292}$ Ebd.

${ }^{293}$ Artikel: Die Schifffahrt ab Köln ist gesperrt, Kölnische Rundschau, 12.04.1983.

${ }^{294}$ Ebd.

${ }^{295}$ Artikel: Aufgemauert, Kölnische Rundschau, 13.04.1983.

${ }^{296}$ Ebd.

${ }^{297}$ Der Rheinufertunnel wurde ein Jahr zuvor gebaut, um den Verkehr von der Rheinuferstraße unterirdisch verlaufen $\mathrm{zu}$ lassen und um am Rhein eine Promenade zu realisieren.

${ }^{298}$ Artikel: Die Kölner Altstadt überflutet, Kölnische Rundschau, 13.04.1983.

${ }^{299}$ Artikel: Am Dienstagabend: Statt „Dallas“ gab’s nasse Füße, Kölnische Rundschau, 13.04.1983.

${ }^{300}$ Artikel: Rhein bald hinter höherer Mauer? Kölnische Rundschau, 14.04.1983.
} 
erkennbar, dass der Tunnel wasserdicht war. Das Klärwerk in Stammheim wurde bereits ab einem Pegel von $7 \mathrm{~m} \mathrm{~K} \mathrm{P}$ überschwemmt, sodass es geschlossen werden musste ${ }^{301}$, und das Abwasser ungeklärt dem Rhein zulief. „In der Altstadt stand an jenem Mittwoch 13. April 1983 das Wasser bis zu einem Meter hoch““302. Dramatisch verschärfte sich die Situation, „auslaufende Öltanks, zusammenbrechende Telefonleitungen, reihenweise Kurzschlüsse und vorsorglich gesperrte Gasanschlüsse“ ${ }^{\text {(303 }}$ waren Begleiterscheinungen des Hochwassers, welches 650 Helfer ohne Nachtruhe beschäftigte ${ }^{304}$. Das Amt für Stadtentwässerung berichtete über ein in Summe $34,80 \mathrm{~km}^{2}$ großes Gebiet, das aufgrund seiner tiefen Lage von der Kanalisation abgeschottet und leer gepumpt werden musste ${ }^{305}$. Andernfalls wäre dieses Gebiet überschwemmt worden, obwohl es gar nicht am Rhein liegt. Die Flut erreichte am 14. April einen Pegel von 9,84 m und war damit drei Zentimeter geringer als das letzte Hochwasser im Jahr $1970^{306}$. Die Tageszeitung verglich das Ereignis mit früheren Fluten und kam zu dem Ergebnis, dass „,man wohl davon ausgehen kann, daß der Rhein zweimal im Jahrhundert die Zehn-Meter-Marke ${ }^{\text {‘307 }}$ überschreitet.

Bereits am Tage darauf titelte eine Tageszeitung „Rhein bald hinter höhere Mauer?“308 . „Rat und Bürger rennen mit dieser Anregung bei der Verwaltung offenen Türen ein“309, kommentierte Willi Leclaire von der Verwaltung. Die Ratsfraktion der CDU hatte einen Antrag für die folgende Hauptausschusssitzung gestellt: Dort soll die Verwaltung Maßnahmen vorstellen, die Hochwasserschäden in Zukunft verhindern oder begrenzen, „speziell fragt[e] die CDU nach Überlegungen, die Hochwasserschutzmauer zu erhöhen“310.

Nachdem die Flut zurückging, galten die Landwirte aufgrund der durch das Hochwasser angeschwemmten Umweltgifte als besonders betroffen, "in einigen Gebieten wurde damit gerechnet, daß die Felder bis zu zwei Jahren unbestellbar sind“311. Von der nordrheinwestfälischen Landesregierung wurde das Hochwasser als „Elementarereignis“ anerkannt. Dies ermöglichte den Bürgern, Soforthilfen bis zu 3.000 DM in Anspruch zu nehmen und den Unternehmern und freiberuflich Tätigen die Hochwasserschäden von der Steuer abzusetzen $^{312}$. Als offizielle Ursache des Hochwassers wurden „große Mengen

\footnotetext{
${ }^{301}$ Artikel: Hochwasser, Kölnische Rundschau, 14.04.1983.

${ }^{302}$ Artikel: Katastrophe weitet sich noch aus, Kölnische Rundschau, 14.04.1983.

${ }^{303}$ Artikel: Alle Befürchtungen übertroffen, Kölnische Rundschau, 14.04.1983.

${ }^{304}$ Ebd.

${ }^{305}$ Artikel: Rhein bald hinter höherer Mauer? Kölnische Rundschau, 14.04.1983.

${ }^{306}$ Artikel: Die Altstadt wird schon wieder naß, Kölnische Rundschau, 28.05.1983.

${ }^{307}$ Artikel: 1784 war der höchste Rheinwasserstand, Kölnische Rundschau, 13.04.1983.

${ }^{308}$ Artikel: Rhein bald hinter höhere Mauer?, Kölnische Rundschau, 14.04.1983.

${ }^{309}$ Ebd.

${ }^{310}$ Artikel: Nach dem Wasser fließt jetzt wieder das Kölsch, Kölnische Rundschau, 16.04.1983.

${ }^{311}$ Artikel: Nun läuft die braune Brühe ab, Kölnische Rundschau, 15.04.1983.

${ }^{312} \mathrm{Ebd}$
} 
Schmelzwasser und starke Niederschläge sowie gefrorener Boden, welcher das Regenwasser beim Versickern hindert" diskutiert ${ }^{313}$. In dem Hochwasserbericht der Stadt Köln hielten die Verantwortlichen aus dem Amt der Stadtentwässerung die „ergiebigen Niederschläge in den Einzugsgebieten der Mosel, des Mains und des Neckers““314 als Begründung fest, welche ,zu Überschwemmungen des Rheins und seiner Nebenflüsse“" geführt hatten.

\subsection{Das Mai-Hochwasser 1983}

„Nur 50 Tage nach dem ungewöhnlichen Rheinhochwasser wurde gestern in Köln und Bonn wieder Land unter gemeldet ${ }^{6315}$. Mit einer „Tendenz von plus 10 Zentimeter pro Stunde tritt der Rhein aller Voraussicht nach heute wieder über die 9,40 Meter hohe Hochwassermauer، ${ }^{\text {316. }}$. Die Autofahrer mussten wieder mit Sperrungen rechnen, da der Rheinufertunnel bei 9,30 m K P geschlossen wurde. ${ }^{317}$ „Die Eingänge unzähliger Häuser und Gaststätten wurden zum zweiten Mal zugemauert, das Straßenbauamt errichtete wieder Stege in der Altstadt und die Kanalisation wurde zum Schutz gegen Rückstau vollständig ,wasserdicht gemacht ${ }^{6318}$.

Gegen Mitternacht des 29. Mai 1983 erreichte die Flutwelle in Köln ihren Scheitelpunkt mit einem Pegel von $9.91 \mathrm{~m} \mathrm{~K} \mathrm{P.} \mathrm{„Die} \mathrm{Sicherheitsvorkehrungen} \mathrm{hatten} \mathrm{gegen} \mathrm{die} \mathrm{Fluten} \mathrm{wenig}$ genutzt. Da das Wasser nur sehr langsam sinkt, wird die Altstadt noch einige Tage unter Wasser stehen.“ 319 Aufgrund der Erfahrungen im April war zwar der Warndienst besser, „doch auch die findigsten Gegenmaßnahmen vermochten der Flut nicht standzuhalten“320. Dagegen ,verhielten sich die Menschen bemerkenswert diszipliniert und trugen durch ihre Besonnenheit wesentlich zum reibungslosen Ablauf der Katastrophen-Einsätze bei ${ }^{\text {‘321 }}$. Im südlichen Vorort Rodenkirchen breite sich das „Wasser 400 Meter weit aus und schloß ganze Häuserblocks ein “322 ${ }^{3}$. Die Bürgervereinigung Rodenkirchen brachte die Hochwasserprobleme dem Bürgermeister Dr. Heribert Blens vor. „Für Abhilfe könnte eigentlich nur der Bau einer

\footnotetext{
${ }^{313}$ Artikel: Hochwasser, Kölnische Rundschau, 15.04.1983.

${ }^{314}$ Bericht über das Hochwasser 1983.

${ }^{315}$ Artikel: Hochwasser bedroht wieder Kölner Altstadt, Kölnische Rundschau, 28.05.1983.

${ }^{316}$ Artikel: Die Altstadt wird schon wieder naß, Kölnische Rundschau, 28.05.1983.

${ }^{317}$ Ebd.

${ }^{318}$ Ebd.

${ }^{319}$ Artikel: Schlimmstes Hochwasser seit 36 Jahren, Kölnische Rundschau, 30.05.1983.

${ }^{320}$ Ebd.

${ }^{321}$ Artikel: Katastrophe am Rhein, Kölnische Rundschau, 01.06.1983.

${ }^{322}$ Artikel: Zukunft der Wirte droht zu ertrinken, Kölnische Rundschau, 30.05.1983.
} 
Hochwassermauer sorgen“‘323, konstatierten die Bürger. Die Gastronomen an der Wasserfront in der Altstadt wurden durch die wiederholt auflaufenden Kosten existenzbedrohlich geschädigt $^{324}$. Sie mussten nun - nach dem Hochwasser im April - ein zweites Mal renovieren. Dies bedeutete für die Besitzer hohe Kosten aber eine Versicherung gegen Hochwasserschäden bot lediglich Lloyds in London zu einem Preis von 34.000 DM pro Jahr $\mathrm{an}^{325}$, was so hoch war, dass die Gastronomen davon selten Gebrauch machten, kommentierte ein Mitglied der Zunft. Um ,,aus der Not eine Tugend zu machen“‘326, luden am Samstagabend die geschädigten Gastronomen für 20 DM Eintritt zur Hochwasserparty ein.

Der lokale Hotel- und Gaststättenverband wandte sich an den Oberbürgermeister. Sie forderten, „die Flutmauer ausreichend zu erhöhen, um das Eigentum der Bürger und Gewerbetreibenden vor der Überflutung zu schützen“6327. Eine „Erhöhung der Schutzmauer lehnte der Beigeordnete Braun jedoch erneut ab, mobile Aufstockungen seien dagegen überlegenswert, doch müssten dann neue Probleme wie Dammbruchgefahr oder dem verstärkten Auftrieb gelöst werden“328. Die Landesregierung stellte unterdessen klar, dass für die Stadt Köln der „Schutz vor Hochwasser weiterhin zu ihren originären Aufgaben gehöre, die Verwaltungskraft einer Stadt wie Köln reicht doch wohl aus, vorausschauende Schutzmaßnahmen für den Hochwasserfall zu treffen“3329, äußerte sich Innenminister Herbert Schnoor (SPD).

Die Ursache für das überraschende Hochwasser Ende Mai „liegt vor allem in den schweren Niederschlägen. Dieser Mai 1983 wird in den Wetterstatistiken enorm schlecht abschneiden. Am Monatsende werden wir wissen, ob er den ersten Platz seit 135 Jahren belegen wird“330, hieß es aus dem Wetteramt in Essen. Auch die Flurbereinigungen wurden als Ursache in Betracht gezogen. „Zwischen Umweltschützern und Landwirtschaftsministerium entbrannte ein Streit darüber, inwiefern durch die Flurbereinigungen vor allen in den Weinbergen an der Mosel, durch Flußregulierungen und Bodenversiegelungen wie Städte- und Straßenbau das Gleichgewicht der natürlichen Wasserwirtschaft nachhaltig gestört worden [sei] ${ }^{\text {‘331 }}$, glaubte Rüdiger Beiser von der Wasser- und Schifffahrtsdirektion in Mainz. Die Flurbereinigungen haben kaum eine Auswirkung، ${ }^{\star 332}$, meinte dagegen Herbert Voigt, leidender

\footnotetext{
${ }^{323}$ Artikel: Bei Hochwasser Probleme mit Heizung und Campern, Kölnische Rundschau, 07.05.1983.

${ }^{324}$ Ebd.

${ }^{325}$ Artikel: Zukunft der Wirte droht zu ertrinken, Kölnische Rundschau, 30.05.1983.

${ }^{326}$ Ebd.

${ }^{327}$ Ebd.

${ }^{328}$ Artikel: Rückkehr zur Normalität, Kölnische Rundschau, 31.05.1983.

${ }^{329}$ Artikel: Landeshilfen für Opfer des Hochwassers, Kölnische Rundschau, 07.05.1983.

${ }^{330}$ Artikel: Die Altstadt wird schon wieder naß, Kölnische Rundschau, 28.05.1983.

${ }^{331}$ Artikel: Aufatmen am Rhein, Kölnische Rundschau, 31.05.1983.

${ }^{332} \mathrm{Ebd}$
} 
Ministerialdirektor im Wasseramt des Mainzer Landwirtschaftsministeriums. Gerod Richter, Geographieprofessor bewertete als Experte im Sinne des Ministeriums. „Die Weinanbauflächen seien verschwindend gering, die Böden - bei den hiesigen Witterungsverhältnissen - gesättigt, der Ausbau der Moselstaustufen 1965/66 mache sich bei Hochwasser nicht bemerkbar، ${ }^{633}$, versicherte Richter.

\subsubsection{Reaktion: Anpassung der Hochwasserschutzmauer}

Am 14. April erreichte das Hochwasser seinen Scheitelpunkt von 9,84 m K P Wasserhöhe. Damit überspülte das Wasser die nach dem Beschluss aus 1960 gebaute Hochwasserschutzmauer, welche einen Wasserstand von 9,40 m halten konnte ein zweites Mal seit der Flut im Jahr 1970. Nur sechs Wochen später ereignete sich ein mit einem statistischen Turnus von 300 Jahren mögliches Ereignis ${ }^{334}$. Die Hochwassermauer wurde am 30. Mai um 0,56 m durch ein Hochwasser mit einer Höhe von 9,96 m K P überstiegen. Die Kölner Verantwortlichen entschieden, die Hochwassermauer an die Überschwemmungen anzupassen. Um das Kölner Stadtgebiet vor zukünftigen Schäden zu schützen, wurde die Mauer um 0,60 m auf eine Gesamtschutzhöhe von 10 m K P erhöht. Die verantwortlichen Behörden beabsichtigten, den oberirdischen Schutz auszubauen, damit dieser die Stadt bis zu dem statistischen Wert eines 40jährigen Hochwassers schützt. Teilweise wurde die bestehende Vorrichtung aufgemauert oder, wie in der Altstadt, durch die erste mobile Schutzwand just im Bedarfsfall verstärkt.

\subsubsection{Diskussion zur Erhöhung der Schutzmauer}

Der Tiefbauausschuss wurde in der Sitzung vom 9. Juni 1983 mit den beiden Hochwasserberichten der Ereignisse von April und Mai konfrontiert und kam zu dem Entschluss, die Hochwasserschutzmauer im Bedarfsfall zu erhöhen ${ }^{335}$. Daneben sollten am Weißen Bogen (Rodenkirchen) sowie in Porz-Langel, auf der rechten Rheinseite, Deichmaßnahmen geprüft werden. Bei einem Treffen der Bezirksvertretung Rodenkirchen am 5. September 1983 wurden jedoch Bedenken gegenüber den Vorschlägen geäußert. Die Bürger aus Rodenkirchen forderten, dass die Verwaltung mittels Holzbrettern verdeutliche, wie hoch die zusätzliche Mauer werden würde. Deswegen wurde in der darauf folgenden Woche ein Modell im Verhältnis von 1:1 gebaut und zur Veranschaulichung an der

${ }^{334}$ Neben der Pegelhöhe wird auch die Jahreszeit in die Berechnung der statistischen Wahrscheinlichkeit für ein Auftreten von einem bestimmten Hochwasserereignis einbezogen. In den Unterlagen der Kölner Behörden wird dieser Turnus häufig durch die Angabe der Zahl mit dem Anhang jährigen ausgedrückt.

${ }^{335}$ Rat der Stadt Köln, Sitzung des Ausschusses für Tiefbau und Verkehr, 09.06.1983.
} 
vorhandenen Mauer angebracht ${ }^{336}$. Zusätzlich wurde ein Gutachten von der Bundesanstalt für Gewässerkunde in Koblenz beauftragt, um festzustellen, ob sich die neue Schutzhöhe negativ auf das Grundwasser oder den Hochwasserspiegel auswirken würde. ${ }^{337}$

Der Tiefbauausschuss diskutierte nur einige Tage später in der Sitzung vom 8. September 1983 die Höhe der neuen Schutzmauer. Er fasste sogleich den Beschluss, dem Rat der Stadt Köln zu empfehlen, den ersten Bauabschnitt zwischen Kunibertkloster und Dreiköniginnenstraße in der Altstadt noch in diesem Jahr durchzuführen, sowie unter der Voraussetzung, dass das oben genannte hydrologische Gutachten keine wesentliche Beeinträchtigung nachweist, die unverzügliche Ausschreibung für die Hochwassermauer im gesamten Bereich von $6.000 \mathrm{~m}$ anzuordnen. Die Bezirksvertretung 1 (Köln-Altstadt) habe bereits zugestimmt, die Erhöhung vorzunehmen, während von der Vertretung 2 (Rodenkirchen) aufgrund der „Verärgerungen in der Sitzung vom 5. September 1983“338 noch keine Absprachen bestanden. Deswegen hielt der Ausschuss fest, „daß die Bedenken der Bezirksvertretung Rodenkirchen bis zur Ratsitzung ausgeräumt oder in Abstimmung mit der Bezirksvertretung 2 Alternativvorschläge für diesen Bereich erstellt sind“3339.

Der Stadtrat diskutierte die Erhöhung der Hochwassermauer am 22. September 1983. Kenter (CDU) stellte die Bedenken in Rodenkirchen vor, ein erzeugter Rückstau könnte in Zukunft eine zusätzliche Gefahr für den Ort herstellen. Der Beigeordnete Braun aus dem Tiefbaudezernat versuchte ihn $\mathrm{zu}$ beruhigen, und erklärte, dass der Beschluss aus dem Tiefbauausschuss mit einem Vermerk versehen worden sei, ein hydraulisches Gutachten müsse die Zweifel ausräumen ${ }^{340}$. Ohne dieses würde erstmal nicht abgestimmt oder gebaut ${ }^{341}$. Jansen (SPD) verdeutlichte die Dringlichkeit, im Bereich des Rheingartens die Arbeiten beginnen zu lassen, da dort „nur die Schiene hinter die Mauer gebaut werden soll, um einerseits den Ausbau des Rheingartens nicht aufzuhalten, und andererseits Zeit blieb, um die Bedenken (in Rodenkirchen) in Ruhe und sachlicher Sitzung zu erledigen“342. Der Rat beschloss einstimmig, mit dem ersten Bauabschnitt zu beginnen, dafür 470.000 DM zu bewilligen und in einer nächsten Ratssitzung über die restlichen Abschnitte zu entscheiden ${ }^{343}$. Der erste Abschnitt zog sich ca. 1.000 m zwischen der Hohenzoller- und der Deutzer Brücke entlang der Frankenwerft. Nachdem das hydraulische Gutachten der Bundesanstalt für

\footnotetext{
${ }^{336} \mathrm{Ebd}$

${ }^{337}$ Ebd.

${ }^{338}$ Ebd.

${ }^{339}$ Rat der Stadt Köln, Sitzung des Ausschusses für Tiefbau und Verkehr, 08.09.1983.

${ }^{340}$ Rat der Stadt Köln, Sitzung 22.09.1983.

${ }^{341}$ Ebd.

${ }^{342}$ Ebd., 352. Im Jahr 1982 wurde die Rheinpromenade gebaut, dies geschah durch eine Untertunnelung der Straße, die Fahrbahn verlief nun unterirdisch. Auf der Promenade entstanden Blumenbeete und Freiflächen.

${ }^{343}$ Ebd.
} 
Gewässerkunde in Koblenz die Bedenken widerlegte, stimmte der Rat der Stadt Köln in der Sitzung vom 27. Oktober des gleichen Jahres - mit einer Stimmenenthaltung - für den restlichen Bau der Erhöhung auf einer Gesamtlänge von $5.800 \mathrm{~m}^{344}$.

Die Umsetzung löste noch einmal eine Diskussion über die Art und Weise der Verstärkung zwischen dem Planungskreis im Ausschuss für Tiefbau und Verkehr und der Bezirksvertretung 2 Rodenkirchen aus. Es wurde durch die lokalen Entscheidungsträger erwogen, auch dort mobile Wände bei Bedarf einzusetzen. „Die Grundlange [...] war das Bestreben, das Ortsbild möglichst wenig zu verändern“6345. Auch in der Beratung zwischen dem Ausschuss für Tiefbau und Verkehr sowie der Stadt Köln, dem Regierungspräsidenten zu Köln wurde großer Wert darauf gelegt, auf eine „möglichst geringe Veränderung des Ortsbildes zu achten“346. Ferner, um „die Kosten und den Montageaufwand im Einsatzfall im wirtschaftl. u. technisch machbaren Bereich zu halten, musste der Einsatz mobiler Elemente auf ein Mindestmaß gebracht werden“3347.

Die Stadt Köln besprach das Hochwasserschutzprojekt mit dem Regierungspräsidenten zu Köln und kam zu dem Entschluss, dass die mobilen Wände sich als geeignet erwiesen, die Orte bis zu einer Höhe von $10 \mathrm{~m} \mathrm{~K} \mathrm{P} \mathrm{-} \mathrm{und} \mathrm{damit} \mathrm{bis} \mathrm{zu} \mathrm{einem} \mathrm{statistischen} \mathrm{Wert} \mathrm{des}$ 40jährigen Hochwassers - zu schützen ${ }^{348}$.

\subsubsection{Bewertung des Diskurses aus der Kosten-Nutzen-Perspektive}

Zunächst wird die Diskussion mit einem Fokus auf die Akteure und die vorgetragenen Argumentationslinien zusammengefasst. Die beiden Hochwasser von 1983 waren für die Kölner offenbar ein Schock. Gleich zweimal wurde die Stadt von extremen Pegelständen heimgesucht. Statistisch erreicht der Rhein alle 40 Jahre in Köln einen Pegel von 10 m, aber im April und Mai 1983 erreichte der Rhein 9,91 und 9,96 m K P. Beide Ereignisse fielen in die - statistisch betrachtet - hochwasserfreie Zeit nach den Wintermonaten, was die Kölner Behörden und Anwohner überraschte. Als Ursache wurden neben den widrigen Witterungsverhältnisse auch menschliche Eingriffe in das überregionale Einzugsgebiets des Flusses diskutiert, woraufhin die wissenschaftliche Experten, Vertreter der

\footnotetext{
${ }^{344}$ Rat der Stadt Köln, Sitzung 27.10.1983.

${ }^{345}$ Brief: Werth.

${ }^{346}$ Rat der Stadt Köln, Sitzung des Ausschusses für Tiefbau und Verkehr, Drucksache 19-214/83, 15.11.1983.

${ }^{347}$ Ebd.

${ }^{348}$ Ebd. Ein noch höherer Hochwasserschutz wurde in Erwägung gezogen, jedoch die hierfür bisher durchgeführten Planungen bezogen sich auf einen Wasserstand von 10,69 m, diesem sollte noch eine Schutzborte von 1,0 m zugefügt werden, sodass eine Höhe von 11,69 m K P diskutiert wurde. An diesem Wert scheiterten letztlich alle Konstruktionen aufgrund ,der optischen Beeinträchtigung des Stadtbildes, den hohen Bau-, Wartungs- und Montagekosten sowie der praktisch nicht durchführbaren Montage der erforderlichen überhohen mobilen Elemente im Einsatzfall“ (Rat der Stadt Köln, Sitzung des Ausschusses für Tiefbau und Verkehr mit der Bezirksvertretung 1, 01.12.1983).
} 
Verwaltungseinheiten und Umweltschützer keinen Konsens fanden. Die Kölner Politiker reagierten entschlossen. Die Ratsfraktion der CDU hatte bereites Mitte April einen Antrag gestellt, „die Hochwasserschutzmauer zu erhöhen“349. „Rat und Bürger rennen mit dieser Anregung bei der SPD-Verwaltung offenen Türen ein““350, kommentierte Willi Leclaire von der Verwaltung.

Aus der Mitte der Bevölkerung wurde nach dem zweiten Hochwasser der Wunsch nach einem ausgedehnten Schutz laut. Die Bürgervereinigung Rodenkirchen adressierte Bürgermeister Dr. Heribert Blens mit der Forderung „für Abhilfe könnte nur der Bau einer

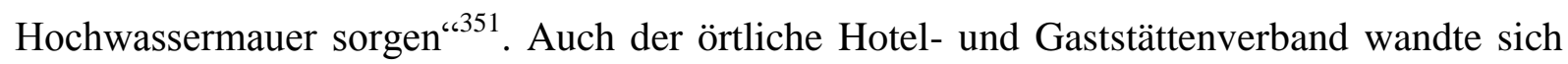
an die Kölner Behörde, sie trugen dem Oberbürgermeister den Wunsch zu, „die Flutmauer ausreichend $\mathrm{zu}$ erhöhen “352. NRW-Innenminister Schnoor stellte klar, dass Köln vorausschauende Schutzmaßnahmen für den Hochwasserfall treffen müsse ${ }^{353}$. Der Kölner Beigeordnete des Ausschusses für Tiefbau und Verkehr Braun lehnte eine Erhöhung der Schutzmauer jedoch erneut ab und plädierte stattdessen für mobile Aufstockungen ${ }^{354}$.

Mit der Sitzung vom 9. Juni 1983 wurde im Ausschuss für Tiefbau und Verkehr der Entschluss gefasst, die Hochwasserschutzmauer zu erhöhen ${ }^{355}$. Der Ausschuss diskutierte in der Sitzung vom 8. September 1983 die Stärke der Erhöhung sowie die Länge, über welche sich das Bauwerk erstrecken sollte ${ }^{356}$. Auch die Rodenkirchener Bürger nahmen durch ihre Vertretung in der Bürgervereinigung an der Diskussion teil. Sie forderten ein hydrologisches Gutachten. Offenbar wollten sie sicher gehen, keine negativen Folgewirkungen durch den Eingriff für ihren Ort südlich von Köln zu provozieren, denn sie äußerten Befürchtungen, der Grundwasserspiegel oder die Höhe der Hochwasserwellen könnten durch die Mauererhöhung schädlicher werden. Die Anwohner aus Rodenkirchen waren im Gegensatz zu anderen durch das Hochwasser betroffenen Bürgern in einer Bürgervereinigung organisiert, die die Interessen für den Ort, welcher erst am 1. Januar 1975 in den Kölner Stadtbezirk eingemeindet wurde, vertrat. Erstmalig in dem hier dargelegten Betrachtungszeitraum wurden, aufgrund des geforderten Gutachtens, auch hydrologische Experten in die Diskussion einbezogen und angehört. Bürger und Behördenmitglieder äußerten bei der letzten Erhöhung 1960 keine technischen Bedenken, die die Vorlage eines Gutachtens erfordert hätten.

\footnotetext{
${ }^{349}$ Artikel: Nach dem Wasser fließt jetzt wieder das Kölsch, Kölnische Rundschau, 16.04.1983.

${ }^{350}$ Ebd.

${ }^{351}$ Artikel: Bei Hochwasser Probleme mit Heizung und Campern, Kölnische Rundschau, 07.05.1983.

${ }^{352}$ Artikel: Zukunft der Wirte droht zu ertrinken, Kölnische Rundschau, 30.05.1983.

${ }^{353}$ Artikel: Landeshilfen für Opfer des Hochwassers, Kölnische Rundschau, 07.05.1983.

${ }^{354}$ Artikel: Rückkehr zur Normalität, Kölnische Rundschau, 31.05.1983.

${ }^{355}$ Rat der Stadt Köln, Sitzung des Ausschusses für Tiefbau und Verkehr, 09.06.1983.

${ }^{356}$ Rat der Stadt Köln, Sitzung des Ausschusses für Tiefbau und Verkehr, 08.09.1983.
} 
Verschiedene Gruppen der lokalen Gesellschaft waren sich einig, dass die Gefahr aufgrund des Hochwassers zu groß war, als auf eine Erhöhung der Schutzmauer zu verzichten. Unter den Fürsprechern für den Mauerbau befanden sich die Wirte in der Altstadt, die Bürger in Rodenkirchen, die verschiedenen politischen Parteien, die Stadtverwaltung und der Tiefbauausschuss, welcher für bauliche Planungen verantwortlich ist und bis dato den Wert der Kölner Heimat höher ansetzte, als die Hochwasserschäden. Es formierte sich eine Koalition von einflussreichen Akteuren, welche als Fürsprecher für die Erhöhung der Mauer auftrat. Die Schreckensereignisse beförderten eine Transformation der Haltung der Kölner Koalition. Niemand schien nach den zwei unverhofften Schadensereignissen vom April und Mai Einwände gegen die Pläne zu formulieren. Die Angehörigen der Leidensgemeinschaft teilten die Auffassung, als logische Reaktion auf die Hochwasserbedrohung den Schutz zu erhöhen. Sie klassifizierten diesen Schritt einstimmig als die vernünftigste Handlungsoption. Die Kohärenz der Vernünftigen ${ }^{357}$ ließ die Betroffenen zu einem übereinstimmen Entschluss kommen. Nida-Rümelin behauptet, eine homogene Personengruppe nehme ähnliche Rationalitäten wahr ${ }^{358}$. Die Hochwasseropfer verband der Schock über die beiden ungewöhnlichen Ereignisse miteinander, welcher die Perspektive auf mögliche Reaktionen und deren Bewertung vereinheitlichte.

Die Abwägung möglicher Handlungsalternativen soll nun Eingang in diese Betrachtung finden, denn die Erhöhung der Schutzwand wurde in Köln 1983 als einzige Lösung des Hochwasserproblems angesehen. Alternativen zu einer Anpassung an die zwei Ereignisse wurden weder von den Betroffenen noch von dem Tiefbauausschuss oder von den einzelnen Fraktionen im Stadtrat vorgebracht.

In einer Gesprächsrunde am 01.12.1983 wurde im Stadtrat darüber nachgedacht, welche Höhe die Aufstockung erreichen sollte. Der Vorschlag auf 10,69 m K P anzuheben wurde aber unmittelbar verworfen. Als Begründung wurden zu hohe Kosten, technische Probleme und Folgeprobleme durch Wasseraufstauungen in Gebieten, welche nördlich von Köln lagen aufgeführt. $^{359}$

In den Augen der Kölner Politiker schien klar zu sein, dass es nicht zu einer dauerhaften Veränderung des Stadtbildes kommen durfte. Von einer Aufmauerung in der Altstadt wurde bereits zu Beginn der Diskussionen nicht mehr gesprochen. Die Umsetzung der Erhöhung mit Hilfe der mobilen Schutzwände verhinderte eine auf die Bewahrung der Stadtansicht

\footnotetext{
${ }^{357}$ Nida-Rümelin 1996.

${ }^{358}$ Nida-Rümelin 1996; 2009.

${ }^{359}$ Artikel: Ufermauer hat schon Höchstmaß, Kölnische Rundschau, 30.03.1988.
} 
fokussierte Auseinandersetzung. Im kritischen Rückblick, scheinen die Kritikpunkte vorgeschobene Argumente zu sein, um den Bau eines höheren Schutzes, welcher ,aus gestalterischer Sicht nicht akzeptabel war ${ }^{\star 360}$, zu verhindern. Denn auch bei dieser strukturellen Maßnahme waren die Bedenken über eine mögliche „Beeinträchtigung des Stadtbildes“ präsent. Während man in Rodenkirchen schlicht die vorhandene Mauer erhöhte, konstruierte das Amt für Brücken- und U-Bahnbau für das Gebiet der Altstadt die erste mobile Schutzwand. Diese konnte von nun an im Bedarfsfall angebracht werden. Auf diese Weise entkamen die Verantwortlichen der Diskussion, ob auf Kosten der Stadtansicht die Hochwassermauer in der Altstadt erhöht werden könne. Kritisch betrachtet, hätte alternativ auch auf der gesamten Strecke die Mauer erhöht oder durch mobile Wände aufgestockt werden können. Doch auch die Menschen in Rodenkirchen setzten die Bewahrung ihres Stadtbildes über den Hochwasserschutz. Die Entscheidungsträger der kommunalen Behörde sowie die Vertreter des Vororts Rodenkirchen diskutierten, ob mobile Wände nicht auch in Rodenkirchen zum Einsatz kommen könnten. Besonders die von der Bezirksvertretung 2 geforderte Veranschaulichungen durch das Anbringen eines 1:1 Modells in der Woche vom 05. September 1983 beweist, dass die Menschen in Rodenkirchen sich mit baulichen Veränderungen an der Rheinkante schwer taten. Sie wollten sichergehen, dass das Erscheinungsbild des alten Ortskerns beibehalten wurde. Für Rodenkirchen wurde also über eine Alternative nachgedacht aber dann in der Diskussion verworfen. Abschließend ist daher die hier vorgetragene Kritik zu relativieren, denn vereinzelte Handlungsalternativen wurden aufgezeigt und von den Akteuren bewertet, bis ein Konsens gefunden wurde.

Die Kritik an der umgesetzten Reaktion, also die Überlegungen über mögliche Konsequenzen der abgestimmten Handlungsalternative, wird nunmehr spezifiziert. Dies dient der Analyse, ob die Akteure sich bewusst waren, dass auch ihre favorisierte Handlung negative Aspekte hätte mit sich bringen können. Die neue Schutzhöhe von $10 \mathrm{~m} \mathrm{~K} \mathrm{P}$ galt als die maximal mögliche Schutzhöhe, die durch eine mobile Konstruktion zu erreichen war. Diese wurde durch das Amt für Brücken- und U-Bahnbau in Eigenarbeit entworfen und konstruiert. Hier ist verständlich, dass die Verantwortlichen ohne Erfahrungswerte mit den technischen Einschätzungen über die Belastbarkeit der Vorrichtungen zurückhaltend agierten. Daher ist das Argument, die maximal mögliche Höhe wäre erreicht, nicht als Ausflucht zu werten, die Konstruktionen noch größer zu dimensionieren. Da die beiden Hochwasser aus den Monaten April und Mai eine Pegelhöhe von fast $10 \mathrm{~m}$ zeigten, bot der Vorschlag allerdings die

\footnotetext{
${ }^{360}$ Rat der Stadt Köln, Sitzung des Ausschusses für Tiefbau und Verkehr mit der Bezirksvertretung 1, 01.12.1983.
} 
geringste kausale Schlussfolgerung. Eine Erhöhung musste an den schockierenden Hochwassern aus April und Mai orientiert werden. Da beide Hochwasser innerhalb von 50 Tagen nur wenige Zentimeter unter der 10 Meter Marke blieben, die statistisch betrachtet alle 40 Jahre erreicht wird, setzten sie einen neuen Standard für ein Mindestmaß im Hochwasserschutz. Mit der Umsetzung wurde die vorhandene Hochwasserschutzmauer im Kölner Süden um 0,4 m erhöht, während in dem Altstadtbereich an der Frankenwerft die Mauer belassen wurde und nur im Bedarfsfall 0,4 m hohe mobile Elemente aufgebaut werden sollten. Die Mitglieder des Stadtrats äußerten keine Bedenken über eventuelle negative Folgen der technischen Veränderungen. Unsicherheiten über die Konsequenzen äußerte lediglich die Bezirksvertretung 2 aus Rodenkirchen. Die Vertretung forderte über die hydraulischen Zusammenhänge ein Expertengutachten von der Stadt Köln. Offenbar gab es kritische Überlegungen zu den möglichen Konsequenzen der ausgewählten Handlungsoption. Somit zeigten sich die vom Hochwasser besonders betroffenen Bürger aus Rodenkirchen sensibler als die Entscheidungsträger aus dem Stadtrat. Da Rodenkirchen aufgrund seiner tiefen Lage auch von kleinen und damit häufigeren Hochwassern in Mitleidenschaft gezogen wurde, wird offenbar, dass negative Konsequenzen für diese Bürger noch bedeutungsvoller und damit auch präsenter sind. Aus Sicht der Kosten-Nutzen-Perspektive ist diese Reaktion nachvollziehbar erarbeitet worden.

\subsection{Das Weihnachtshochwasser 1993}

Die Schifffahrt wurde bereits am Dienstagabend eingestellt ${ }^{361}$. Seit am Mittwoch um 17 Uhr die Sperrung des Rheinufertunnels erfolgte - ab 9,80 m K P muss dieser abgesperrt werden brach ein Verkehrschaos aus ${ }^{362}$. Am Donnerstag den 23. Dezember 1993 begann das Hochwasser auf breiter Front über die mobile Schutzwand in die Altstadt zu schwappen ${ }^{363}$. „In Minuten verwandelten sich diese Wellen in eine einzige gewaltige Kaskade, mit der die unteren Bereiche des Martinsviertels und die gesamte Uferzone des Stadtkerns unter Wasser gesetzt wurden. In kürzester Zeit dehnte sich der Strom über den gesamten Rheingarten und bis in die Gassen der Altstadt zu einer einzigen riesigen Wasserfläche aus “6364. Erstmals seit 1983 drang der Rhein so weit in die Altstadt vor. Durch das jetzige Hochwasser, das seinen

\footnotetext{
361 Artikel: Mit dem Wasserspiegel stieg die Angst, Kölnische Rundschau, 23.12.1993.

362 Artikel: Der Rhein schaffte die Mauer, Kölnische Rundschau, 23.12.1993.

363 Artikel: Jahrhundert - Hochwasser erreicht das Rheinland, Kölnische Rundschau, 23.12.1993.

${ }^{364}$ Artikel: Der Rhein schaffte die Mauer, Kölnische Rundschau, 23.12.1993.
} 
Höchststand bei einem Pegel von 10,61 m K P erreichte, wurden 25.000 Einwohner betroffen $^{365}$. Die Hochwasserschutzwand schützte Köln bis zu einer Pegelhöhe von $10 \mathrm{~m}$ seit $1984^{366}$. Karl-Heinz Schönbeck, Einsatzleiter des Technischen Hilfswerks (THW) schimpfte über die Schaulustigen. Er und seine Kollegen gingen regelmäßig an der Schutzwand entlang und zogen Schrauben nach und verhinderten Sabotageakte, wie sie 1988 vorgekommen waren $^{367}$.

Mehr als 1.000 Helfer von Feuerwehr, Polizei und THW waren im Einsatz ${ }^{368}$. Sie verlegten $2.500 \mathrm{~m}$ Stege und evakuierten 20 Menschen aus ihren Wohnungen ${ }^{369}$. Das Abwasser wurde bei diesem Ereignis aus der Kanalisation abgepumpt ${ }^{370}$. Diese wurde an 400 Eintrittsmöglichkeiten verschlossen, um das Hochwasser daran zu hindern, unterirdisch in die Stadt einzudringen, während das abgepumpte Abwasser $\mathrm{zu}$ den Klärwerken geleitete wurde $^{371}$. Der Stadt sparte diese Aktion täglich 300.000 DM ein, welche als Abwassergebühr fällig würden, würde das Abwasser ungeklärt dem Vorfluter zugeführt ${ }^{372}$.

Das Hochwasser breitete sich aufgrund von Regenfällen über ganz Südwestdeutschland aus, die Zeitungen berichteten, wie andere Bundesländer den betroffenen Menschen halfen, denn eine Versicherung für Hochwasserschäden gab es lediglich in Baden-Württemberg ${ }^{373}$. Während in Nordrhein-Westfalen erst seit wenigen Jahren eine Zusatzversicherung gegen Hochwasser angeboten wurde ${ }^{374}$, war diese obligatorisch für jedes Gebäude in BadenWürttemberg abzuschließen. Das Saarland stellte zehn Mio. DM Soforthilfe bereit, Rheinland-Pfalz offerierte Einzelhilfen von 3.000 DM und Hessen gar bis 20.000 DM, während Nordrhein-Westfalen seine Opfer an die Kommunen verwies ${ }^{375}$. Wie zu jedem

\footnotetext{
${ }^{365}$ Artikel: Das Hochwasser treibt Tausende in die Flucht, Kölnische Rundschau, 24.12.1993.

${ }^{366}$ Artikel: Mit dem Wasserspiegel stieg die Angst, Kölnische Rundschau, 23.12.1993.

${ }^{367}$ Ebd.

${ }^{368}$ Artikel: Mehr als tausend Helfer bekämpften Wassermassen, Kölnische Rundschau, 24.12.1993.

${ }^{369}$ Artikel: Das Hochwasser treibt Tausende in die Flucht, 24.12.1993.

${ }^{370}$ Artikel: Der Rhein schaffte die Mauer, Kölnische Rundschau, 23.12.1993.

${ }^{371}$ Ebd.

${ }^{372}$ Ebd. Seit 1988 plante die Stadt die Strafgebühren für das Einleiten der ungeklärten Abwässer aus der Kläranlage in Stammheim in Höhe von 180.000 DM pro Tag durch die Installation der ,modernsten Anlage der Welt mit Meß- und Regeltechniken“ einzusparen (Artikel: Köln macht Kanäle hochwassersicher, Kölnische Rundschau, 30.03.1988). Die Stadt plante 1,5 Milliarden Mark bis Mitte der 90er Jahre zu investieren und das System selbst zu bauen, deswegen war die Stadt motiviert, gegen die Strafgebühren zu klagen und argumentierte, dieses Hochwasser als ,höhere Gewalt“ anzusehen (Ebd.). Die Stadt befolge zudem nur das genehmigte Verfahren im Hochwasserfall und verstieß nicht gegen Regeln (Artikel: Meiner Meinung nach von M. Burkhard, Kölnische Rundschau, 30.03.1988). Ganz gegensätzlich sah das Kölns Regierungspräsident Dr. Franz-Josef Antwerpes, er beschuldigte die Stadt, ,stets bei der Bewältigung ihrer Abfallprobleme zu langsam gewesen zu sein“ (Artikel: Antwerpes: Stadt war zu langsam, Kölnische Rundschau, 02. April 1988). Höhere Gewalt wollte Antwerpes nicht anerkennen (Ebd.). Tatsächlich investierte die Stadt Köln in den folgenden Jahren über 100 Millionen DM und konnte die Klärwerke bei dem Hochwasser 1993 weiterhin funktionsfähig halten (Rat der Stadt Köln, Amt für Tiefbau und Verkehr, Amt für Stadtentwässerung, Pressekonferenz, Reinhard Vogt, 07.01.1994). Insgesamt musste die Stadt 1993 trotzdem 2,1 Millionen Mark als Strafe zahlen (Artikel: Flut kostet die Kölner über 100 Millionen Mark, Kölnische Rundschau, 08.01.1994).

${ }^{373}$ Artikel: Keine Entschädigung bei Überschwemmung, Kölnische Rundschau, 23.12.1993.

${ }^{374}$ Artikel: Hochwassergeschädigte können auf Steuererleichterungen hoffen, Kölnische Rundschau 24.12.1993.

${ }^{375}$ Ebd.
} 
Hochwasser wurden jedoch Steuererleichterungen und Leistungen über das Sozialamt für Hausrat und Bekleidung gewährt ${ }^{376}$. In Rodenkirchen gründete sich spontan ein Hilfskomitee, um für die Hochwasseropfer Geld und Gegenstände zu sammeln ${ }^{377}$, denn „halb Rodenkirchen sah Heiligabend aus, wie nach einem Bombenangriff ${ }^{\text {3378. }}$.

Lothar Ruschmeier (CDU), Oberstadtdirektor zu Köln, lobte die Helfer an Heiligabend für ihren Einsatz ${ }^{379}$. CDU-Ratsmitglied Heinrich Lohmer appellierte an die Arbeitgeber, die Helfer weiter freizustellen ${ }^{380}$. „Um alles wieder einsatzbereit $\mathrm{zu}$ machen, werden rund 14 Tage benötigt ${ }^{\star 381}$. Der Stadt kostete das Hochwasser alleine für die 200.000 Arbeitsstunden der Helfer zehn Mio. DM Personalkosten, rechnete Tiefbaudezernent Oelmann vor ${ }^{382}$. Der Leiter des Amtes für Straßenbau schätze die Kosten für die Reparaturen der Straßen in Höhe von vier bis fünf Mio. $\mathrm{DM}^{383}$. Am städtischen Eigentum insgesamt entstand ein Schaden von 13 Mio. DM ${ }^{384}$. Die Kosten für die 17.000 verteilten Sandsäcke würde die Stadt wohl auch übernehmen, da man „,nur bis zu einem gewissen Grad eine Selbstvorsorge der Bevölkerung erwarten kann, aber dieses Hochwasser den Rahmen sprengt $^{6385}$. Bei den Aufräumarbeiten waren 6.000 Menschen beteiligt ${ }^{386}$. Um die Kosten bezahlen zu können, richtete die Stadt ein Vorschusskonto für alle beteiligten Dienststellen $\operatorname{ein}^{387}$. Die unerwarteten Mehrkosten könnten nicht den für diesen Haushalt bereits gekappten und für das nächste Jahr weiter gekürzten Budgets der einzelnen Dezernate und Ämter angelastet werden, konstatierte Stadtdirektor Burkhard von der Mühlen. „Die Kosten würden fast den gesamten Ansatz für die Straßenunterhaltung verschlingen sowie bei der Feuerwehr dazu führen, dass der Feuer- und Katastrophenschutz nicht mehr sicher gestellt werden könnte، ${ }^{\text {‘38 }}$, verdeutlichte er Kölns prekäre Haushaltslage. Tiefbaudezernent Oelmann schätzte, die Kosten im privaten Bereich: „Ausgehend von einem durchschnittlichen Schaden von 20.000 DM an 5.000 Wohngebäuden, haben diese Betroffenen eine Belastung von mindestens 100 Millionen Mark zu verkraften“6389.

\footnotetext{
${ }^{376}$ Ebd.

377 Artikel: Komitee hilft Opfern der Katastrophe, Kölnische Rundschau, 30.12.1993.

${ }^{378}$ Artikel: Leben ohne Strom und ohne Heizung, Kölnische Rundschau, 30.12.1993.

${ }^{379}$ Artikel: Nach der Flut noch keine Pause, Kölnische Rundschau, 27.12.1993.

${ }^{380}$ Ebd.

${ }^{381}$ Artikel: Großeinsatz gegen Schlamm und eine Flut von Sperrgut, Kölnische Rundschau, 27.12.1993.

${ }^{382}$ Artikel: Flut kostet die Stadt Millionen, Kölnische Rundschau, 24.12.1993.

${ }^{383}$ Ebd.

${ }^{384}$ Dezernat für Tiefbau und Verkehr, Amt für Stadtentwässerung, Brief von Vogt an den Regierungspräsidenten Lampe, 23.02.1994.

${ }^{385}$ Artikel: Flut kostet die Stadt Millionen, Kölnische Rundschau, 24.12.1993.

${ }^{386}$ Artikel: Nach Hochwasser beginnt jetzt das große Aufräumen, Kölnische Rundschau, 27.12.1993.

${ }^{387}$ Artikel: Noch weiß keiner, wo die Millionen herzuholen sind, Kölnische Rundschau, 29.12.1993

${ }^{388}$ Artikel: Nach der Flut noch keine Pause, Kölnische Rundschau, 27.12.1993.

389 Artikel: Flut kostet die Kölner über 100 Millionen Mark, Kölnische Rundschau, 08.01.1994.
} 
Verursacht wurde das Hochwasser ,durch starke Niederschläge im Einzugsgebiet der Nebenflüsse Mosel, Neckar und Main. Im gesamten Mittelgebirgsraum gab es nur geringfügige Schneelagen und der Oberrhein südlich von Karlsruhe führte keine außergewöhnlichen Wassermengen und war für die Hochwasserentwicklung nur

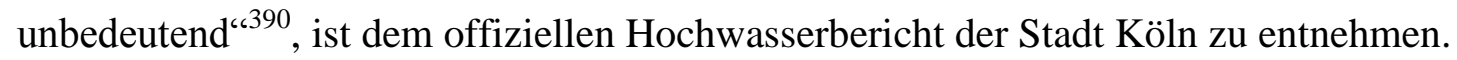

Die Katastrophe sei hausgemacht, urteilte dagegen Bundesumweltminister Klaus Töpfer (CDU): „Es könne nicht nur darum gehen, die Wasserfluten mit Talsperren und Dämmen zu beherrschen, die Selbstregulierung der Natur müsse wieder hergestellt werden und natürliche Überschwemmungsgebiete - Auen und Wiesen - erhalten werden“3391. Er drängte darauf, das Bodenschutzgesetz bis zur Bundestagswahl 1994 zu verabschieden ${ }^{392}$. Die Umweltministerin von Rheinland-Pfalz, Klaudia Martini (SPD) hielt die Flut nur zum Teil für durch den Menschen beeinflusst, „,bei solchen Regenfällen, wäre wohl auch dann „Land unter“ gewesen, wenn wir uns immer ordnungsgemäß ökologisch verhalten hätten“393. Eine rasche Handlung forderte dagegen der saarländische Umweltminister Josef Leinen (SPD), der für eine sofortige Kehrtwende in der Landschaftsgestaltung eintrat ${ }^{394}$ : Die Zubetonierung der Landschaft und Begradigung der Flüsse wurden als Ursachen verantwortlich gemacht“395. Ein Kölner Bürger erklärte in einem Leserbrief den Zusammenhang von natürlicher Überschwemmungsfläche und dem Hochwasserpegel und forderte ein Umdenken von den Behörden ${ }^{396}$. Rückblickend wurde ein Gesamtschaden von ca. 120 Mio. DM in Köln festgestellt ${ }^{397}$.

\subsubsection{Reaktionen: Überregionale Ursachenbetrachtung}

Das Hochwasser vom Dezember 1993 richtete mit ca. 120 Mio. DM den höchsten Schaden an. Die erste dauerhafte Hochwasserschutzzentrale wurde daraufhin im Dezember 1994 eingerichtet, um beim nächsten Ereignis die Koordination der Hilfskräfte sowie der Information für die betroffenen Anwohner reibungslos zu gestalten. Über die Schutzhöhen wurde jedoch nicht neu reflektiert. Auf breiter Ebene brachen Diskussionen zu den Ursachen des Hochwassers aus. Leserbriefe aus der Bevölkerung sowie die politischen Institutionen brachten die Flussbegradigungen, die Vernichtung von natürlichen Überschwemmungsgebieten und die zunehmende Bodenversiegelung mit der

\footnotetext{
${ }^{390}$ Pressekonferenz, Vogt, 07.01.1994.

${ }^{391}$ Artikel: Katastrophe hausgemacht, Kölnische Rundschau, 24.12.1993.

${ }^{392}$ Artikel: Rhein streckenweise für Schiffahrt wieder frei, Kölnische Rundschau, 28.12.1993.

${ }^{393}$ Artikel: Katastrophe hausgemacht, Kölnische Rundschau, 24.12.1993.

${ }^{394}$ Ebd.

395 Artikel: Rhein streckenweise für Schiffahrt wieder frei, Kölnische Rundschau, 28.12.1993.

${ }^{396}$ Artikel: Nadelöhr erweitern, Kölnische Rundschau, 29.12.1993.

${ }^{397}$ Artikel: Hochwasser, Köln entging nur knapp der Katastrophe, Kölnische Rundschau, 09.02.1995.
} 
Überschwemmung in Zusammenhang. „Zu Handeln“ wurde von allen verschiedenen Akteuren gefordert. Dieses Handeln sollte ein Umdenken in ökologischen Belangen zur Grundlage haben. Den Stadtratsmitgliedern war klar, dass es so wie bisher nicht weiter gehen konnte. Verschiedene Vorschläge wurden vorgebracht, die den Zusammenhang von menschlichen Eingriffen am Oberlauf und Geschehnissen am Unterlauf miteinander in Verbindung brachten. Die Mitglieder des Tiefbauamts begannen das spätere Hochwasserschutzkonzept unter dem Namen „Programm Hochwasserschutz 2020“ zu erarbeiten $^{398}$. In einem ersten Teil sollten die vorgeschlagenen organisatorischen Veränderungen innerhalb der Verwaltungsabläufe erläutert werden. Ein zweiter Teil sollte Aufschluss über ein ,übergreifendes Handlungskonzept der Rheinanlieger insgesamt ${ }^{\star 399}$ geben. Der Systemcharakter des Flusssystems sollte zukünftig durch eine verbesserte Kommunikation zwischen allen entlang des Rheins gelegenen Verwaltungsgebieten institutionalisiert werden. „Der Hochwasserschutz darf nicht an den Grenzen der Gemeinde enden“400, appellierte Reinhard Vogt, Sachbearbeiter des Amts für Stadtentwässerung im Januar 1994. Er konstatierte, dass ,eine Stadt initiierend wirken kann, aber eine Bewusstseinsänderung und ein Zusammenwirken aller mittelbaren und unmittelbar Beteiligten am Hochwasserschutz im gesamten Einzugsgebiet des Rheins notwendig sei“401.

\subsubsection{Diskussion zur Strategie der tausend kleinen Aufgaben}

Die Kölner Verantwortlichen stellten nach dem Ereignis erhebliche ökologische Probleme fest. Auftreibende Öltanks privater Haushalte hatten ein Wasser- und Ölgemisch von $2.000 \mathrm{t}$ freigesetzt, welches durch die Unternehmen Ford, Shell, ROW und EC-Bayer (Dormagen) unschädlich und kostenlos beseitigt wurde ${ }^{402}$. Von den 3.300 Einsätzen der Berufsfeuerwehr wurden 87 Einsätze mit dem Auslaufen von Heizungsöltanks begründeten ${ }^{403}$. Deswegen war das Umweltamt mit der Vermeidung von Boden- und Grundwasserverunreinigungen beschäftigt. Die im Rahmen des Öl- und Giftalarms entstanden Kosten beliefen sich auf 300.000 $\mathrm{DM}^{404}$. Das Umweltamt mahnte im Rahmen eines Zeitungsaufrufs, Öltanks in Zukunft vor einem Auftreiben abzusichern ${ }^{405}$. Lediglich aufgrund „des beispielhaften Verhaltens der Unternehmen sowie aufgrund der erheblich durch diese Naturkatastrophe

\footnotetext{
${ }^{398}$ Brief von Egelmann i. A. des Oberstadtdirektors Ruschmeier an Dezernat 1 Tiefbau, 22.12.1993.

${ }^{399}$ Ebd.

${ }^{400}$ Pressekonferenz, R. Vogt, 07.01.1994, 9.

401 Ebd.

${ }^{402}$ Rat der Stadt Köln, 52. Sitzung, 01.02.1994.

${ }^{403}$ Ebd.

${ }^{404}$ Ebd.

${ }^{405}$ Artikel: Bitte der Stadt: Öltanks gegen Auftrieb sichern, Kölnische Rundschau, 08.01.1994.
} 
geschädigten Bevölkerung“, verzichtete die Stadt auf die „Geltendmachung der Ansprüche der Berufsfeuerwehr sowie des Umweltamtes“406. Gegen Ende des Jahres 1994 wurde offiziell bekanntgegeben, dass alleine die Hilfsmaßnahmen der städtischen Einrichtungen 8.673.471,45 DM verschlungen hatten ${ }^{407}$.

Das Ereignis sollte Konsequenzen haben. Die Fraktion der Bündnis 90/DIE GRÜNEN forderten Maßnahmen im Stadtgebiet ${ }^{408}$. Sie meldeten Bedenken an, das Gebiet Weißer Bogen tatsächlich wie geplant am Sandpfad zu bebauen, da dort ein Meter hoch das Wasser gestanden habe. Sie entschieden sich gegen weitere Baumaßnahmen in Überschwemmungsgebieten. Für die Verantwortlichen ergab sich eine Zwickmühle, entweder eine hochwassersichere Bauweise für die geplanten Sozialwohnungen zu finanzieren, oder in Zeiten akuter Wohnungsnot auf sozialen Wohnungsbau zu verzichten ${ }^{409}$. Die Bürgervereinigung Rodenkirchen ließ durch ihren Vorsitzenden Dieter Maretz erklären, „daß die zunehmende Bodenversiegelung ufernaher Gebiete die Gefahr katastrophaler Hochwasser im Kölner Süden erhöhe. Die geplante Bauweise würde die Fluten nur noch weiter ansteigen lassen $^{\text {“410 }}$. Auch die Fraktion der FDP stellte sich hinter den Einspruch der Bündnis 90/DIE GRÜNEN. „Die schleichende Bebauung im Weißer Rheinbogen muß endlich gestoppt werden $^{\text {‘411 }}$, erklärte der FDP-Ortsverband Rodenkirchen. Auch die SPD teilte die Meinung, dass „über das Bebauungskonzept neu nachgedacht werden muss“. Johannes Waschek (SPD) erklärte, dass man „wohnungsbaupolitisch in der Zwickmühle stecke. Denn als städtisches Gelände biete sich das Grundstück für sozialen Wohnungsbau an“412. Die CDU verfolgte derweil „ein Konzept der sanften Bebauung“, wenn das Gebiet bebaut werden sollte, dann mit nur wenigen Einfamilienhäusern, aber die Bauherrn müßten mit einem gewissen Hochwasserrisiko leben““413.

Bereits am 13. Januar 1994 entstand eine rege Diskussion im Landtag von NRW durch die mündliche Anfrage der Abgeordneten Dr. Annemarie Schraps (CDU) ${ }^{414}$. Dr. Schraps

\footnotetext{
${ }^{406}$ Rat der Stadt Köln, 52. Sitzung, 01.02.1994.

${ }^{407}$ Rat der Stadt Köln, 3. Sitzung, 13.12.1994.

${ }^{408}$ Artikel: Grüne fordern: Weißer Bogen als Stauraum, Kölnische Rundschau, 08.01.1994.

${ }^{409}$ Artikel: Meiner Meinung von Volker Kutscher, Weißer Bogen, wer bezahlt den Schutz? Kölnische Rundschau, 10.01.1994.

${ }^{410}$ Artikel: Umdenken nach dem Hochwasser, Kölnische Rundschau, 11.01.1994.

${ }^{411}$ Ebd.

${ }^{412}$ Ebd.

${ }^{413}$ Ebd. Im Kontext zum sozialen Wohnungsbau stand der Deutsche Städtetag 1994, denn dort wurden die Trends im Wohnungsbau thematisiert: Der Deutsche Städtetag prognostizierte in jenem Jahr, dass der Wohnungsbau in den kommenden Jahren ein Dauerthema bleiben würde und sprach sich deutlich für die aktive Bekämpfung der Wohnungsnot und der direkten Förderung sozial benachteiligter Schichten aus (Artikel: Wohnungsbau bleibt Thema, Kölnische Rundschau, 15.01.1994).

${ }^{414}$ Landtag NRW, Drucksache 11/6588, 13.01.1994.
} 
interessierte sich für die Maßnahmen der Landesregierung, um Umweltschäden sowie Sekundärursachen zu beseitigen ${ }^{415}$. In seiner Antwort verwies der Minister für Umwelt, Raumordnung und Landwirtschaft Klaus Matthiesen (SPD) auf die Verunreinigungen durch auftreibende und berstende Öltanks ${ }^{416}$ und auf bereits laufende Maßnahmen. Außerdem verlangte er nach „einer Koordinierung all dieser Maßnahmen mit den übrigen Rheinanliegerländern. ${ }^{\text {“417 }}$ Er erklärte:

„Im Einzugsgebiet des Rheines gehört dazu die Rückhaltung entlang den Kanalnetzen, in Talsperren und Hochrückhaltebecken, die verstärkte lokale Versickerung des Wassers, die Entsiegelung der Flächen, die Schaffung und Erhaltung von Retentionsfläche in den Bachund Flusstälern, die weitre Umsetzung des Gewässerauenprogramms NRW und schließlich die Fortsetzung des Programms der Renaturierung der Gewässer zweiter Ordnung ${ }^{\text {‘4 }}{ }^{4}$.

Schraps erkundigte sich nach einer Verordnung zu dem Anbringen von Öltanks aufgrund der schweren Grundwassergefährdung ${ }^{419}$. Matthiesen antwortete, es für richtig zu halten, zu prüfen, ob eine gesetzliche Vorschrift generell möglich und dann vom Oberlauf bis zum Unterlauf des Rheins umsetzbar wäre ${ }^{420}$. Er nannte dies ein Problem, welches die Rheinanliegerländer mit dem Bund neben Förderprogrammen zu beraten hätten ${ }^{421}$. Kruse (CDU) erkundigte sich nach der Finanzierung des Auenschutzprogramms für Rückstauräume. Eine genaue Antwort konnte Matthiesen dazu nicht geben, gab aber zu verstehen, dass das Projekt „überhaupt nur in vielen, vielen Jahren verwirklicht werden kann und allein die Dimension von mehreren hundert Mio. DM erreicht ${ }^{\star 422}$. Daneben verwies er darauf, dass auch die Finanzierung eine Aufgabe für alle Rheinanlieger vom Ober- bis zum Unterlauf darstelle $e^{423}$

Bundesumweltminister Klaus Töpfer (CDU) äußerte sich zu der Überschwemmung mit „eine(r) Erinnerung daran, wie zerbrechlich das natürliche Gleichgewicht ist“"424 . „Jetzt blickt jeder nach Bonn, der Ruf nach schnellem Handeln wird laut, nach Programmen, Aktionen und nach rascher Abhilfe “425, fasste er zusammen. Er hoffte auf Einsicht bei den Stadt- und Gemeinderäten, bei der Landwirtschaft und am Kabinettstisch. Seiner Meinung nach haben

\footnotetext{
415 Ebd.

${ }^{416}$ Landtag NRW, Plenarprotokoll 11/118, 20.01.1994.

${ }^{417}$ Ebd., 14870.

${ }^{418}$ Ebd., 14869. Gewässer zweiter Ordnung werden von jedem Bundesland selbstständig definiert. Die Gewässer zweiter Ordnung sind im Wassergesetz NRW, Landeswassergesetz Anhang II, § 3 vom 25.06.1995 aufgeführt. 
die Städte und Gemeinden zu lange planerische Entscheidungen ohne Rücksicht auf die Umwelt getroffen. Auch die Bauernlobby habe die Verabschiedung eines wirksamen Bodenschutzes verhindert und die Politik habe es nicht geschafft, ein solides Naturschutzgesetz auf den Weg zu bringen, „denn massive wirtschaftliche Interessen bremsen die Umweltpolitik“426. Er empfahl eine „Strategie der tausend kleinen Aufgaben“427: Renaturierung müsse an großen Flüssen, wie auch an Bächen und Zuflüssen immer wieder geprüft werden, um wieder Wasser der Fläche zurück zu gegeben und Feuchtgebiete entstehen zu lassen. Dazu sah er als unverzichtbares Instrument den Dialog der Umweltminister der Länder an und begrüßte die Haltung des Umweltministers in NRW, nicht nur auf die Unterlieger zu verweisen, sondern auch in NRW Fehler einzuräumen ${ }^{428}$.

\subsubsection{Bewertung des Diskurses aus der Kosten-Nutzen-Perspektive}

Die erläuterte Diskussion der politischen Entscheidungsträger wird nun im Bezug zu den Akteuren, ihren Argumenten, den abgewogenen Alternativen und ihrer Kritik kommentiert.

Das Bewusstsein für die Zusammenhänge von natürlichen Überschwemmungsgebieten und Hochwasser wurde aufgrund des Ereignisses endgültig katalysiert. Die letzten Hochwasser vom April und Mai 1983, Winter $1988^{429}$ und dieses zu Weihnachten 1993 wurden als unnatürlich empfunden. Die Auffassung teilte die lokale Politik mit der Mitte der Kölner Gesellschaft. Für die Anlegung und Vergrößerung von Überschwemmungsgebieten sprachen sich auch Kölner Bürger als unmittelbar Betroffene aus. In Leserbriefen forderten sie eine Kehrtwende im Umgang mit der Natur. Abb. 13 zeigt den Ersteinsatz der Hochwasserschutzmauer 1988, die 1993 überströmt wurde.

\footnotetext{
${ }^{426} \mathrm{Ebd}$.

${ }^{427}$ Ebd.

${ }^{428}$ Ebd.

${ }^{429}$ Am 29. März 1988 stand das Hochwasser in Köln bei einem Pegel von 9,95 m. Die neue Aufstockung der Hochwassermauer bis zu einer Höhe von $10 \mathrm{~m} \mathrm{~K}$ P hielt das Wasser davon ab, in die Stadt zu schwappen. Nachdem das Ereignis keine bedeutenden Schäden hervorgerufen hatte, reagierten die Verantwortlichen der städtischen Verwaltung nicht auf das Hochwasserereignis. Vielmehr nahmen die Kölner eine Bewährung der neuen mobilen Hochwasserschutzmauer wahr, welche als Reaktion auf die zwei konsekutiven Überschwemmungen aus dem Jahr 1983 entworfen worden war. Der mobile Schutz kostete 2 Mio. DM und wurde von dem Amt für Brücken und UBahnbau konstruiert. Das Auf- und Abbauen, die Bewachung der mobilen Hochwasserschutzwand, die Sicherung des Rheinufertunnels und der Transport eingeschlossener Bürger verursachte ca. 1 Mio. Kosten für die Hilfseinsätze. Weitere 2,1 Mio. DM wurden als Strafzahlung an das Land NRW aufgrund der ungeklärten Einleitung von Abwässern fällig (Artikel: Rhein steht bedrohlich vor Häusern der Altstadt, 28.03.1988; Zentimeter trennte die Altstadt von Überflutung, 30.03.1988; Hochwasserschutzwand gestern wieder abgebaut, 07.04.1988; Flut kostet die Kölner über 100 Millionen Mark, 08.01.1994, Kölnische Rundschau).
} 


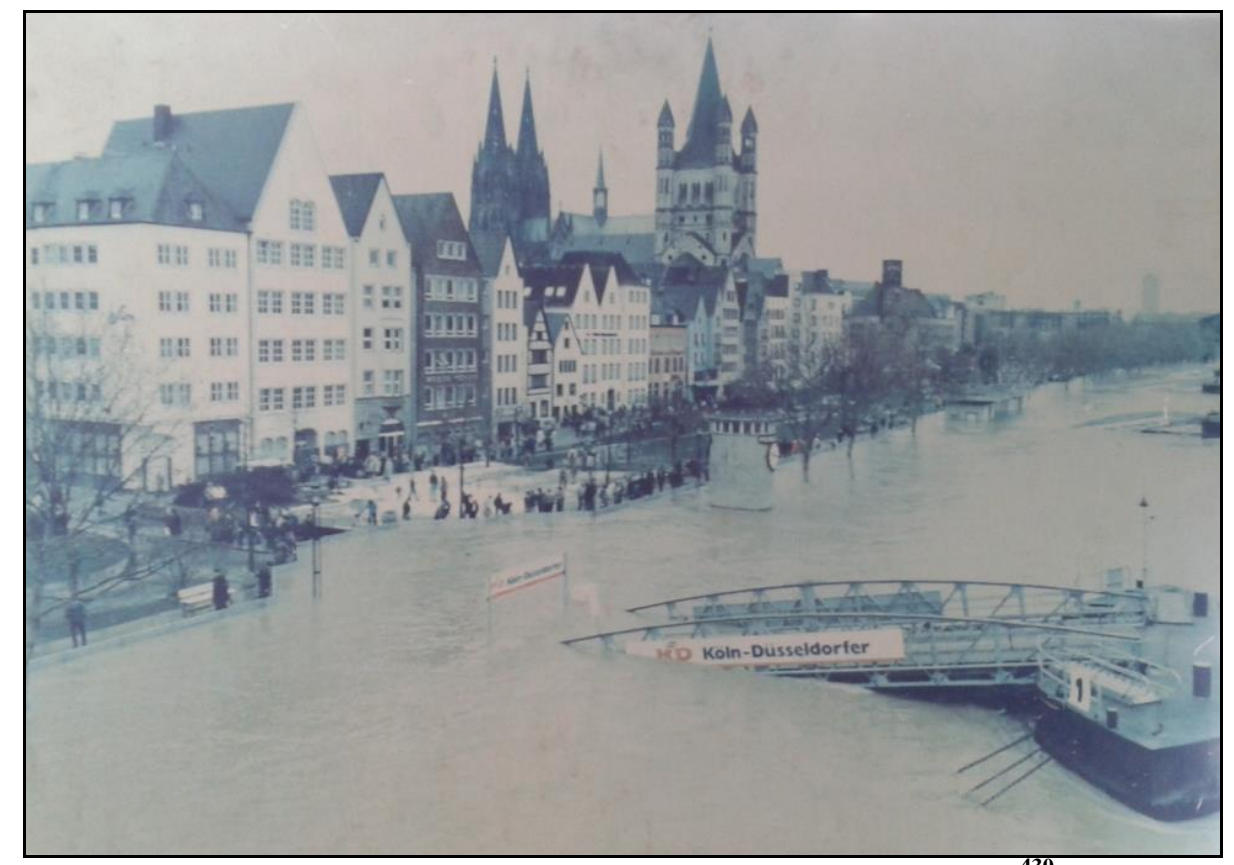

Abb. 13: Erster Einsatz der mobilen Schutzmauer 1988.

Die politisch Verantwortlichen zogen Konsequenzen aus dem Hochwasserschaden und begannen mit der Erarbeitung eines dauerhaften Konzepts zum Umgang mit dem Rheineinzugsgebiet. Das Programm „Hochwasser 2020“ wurde vom Ausschuss für Tiefbau und Verkehr in Zusammenarbeit mit dem Stadtrat, insbesondere durch den Auftrag des Oberstadtdirektors Ruschmeier (CDU), erarbeitet. An Ruschmeiers Verhalten ist zu erkennen, dass er als Kopf der Verwaltung die Ursachen für das Hochwasser ebenfalls in menschlichen Eingriffen begründet sah. Er versuchte durch das Konzept einen Weg zu finden, die verantwortlichen Rheinanlieger am Oberlauf zu beeindrucken und $\mathrm{zu}$ beeinflussen, damit diese sich ebenfalls beim überregionalen Hochwasserschutz engagieren und dieser nicht mehr an den Grenzen der Gemeinden endet ${ }^{431}$. Dieses Programm sollte eine Vielzahl von unterschiedlichen Maßnahmen enthalten und sich an mannigfache Akteure wenden. Es wurde auf den Weg gebracht, noch bevor das Hochwasser seinen Höchststand erreichte. Wann dieses Programm wirklich veröffentlicht werden sollte, ist in den Unterlagen jedoch nicht erkenntlich. Lediglich die faktische Publikation am 01. Februar 1996 lässt darauf schließen, dass das Konzept tatsächlich nach dem Weihnachtshochwasser von 1993 ausgearbeitet wurde. Der Umweltminister von Nordrhein-Westfalen Klaus Matthiesen (SPD) sah ebenfalls die Lösung in ganzheitlichen Ansätzen und teilte die Meinung der Stadt Köln, am Oberrhein müsse Retention betrieben werden. Der Minister machte in jeder seiner Antworten deutlich, dass eine Verbesserung der Zustände in der Hand aller Rheinanliegerländer läge und nicht nur

\footnotetext{
${ }^{430}$ Aufnahme der Hochwasserschutzzentrale Köln.

${ }^{431}$ Brief von Egelmann i. A. des Oberstadtdirektors Ruschmeier an Dezernat 1 Tiefbau, 22.12.1993.
} 
in NRW umgesetzt werden könne. Auch legte er Wert darauf zu erklären, dass diese Maßnahmen nur ,in vielen, vielen Jahren“ zu bewerkstelligen seien und dass finanzielle Ressourcen „,von mehreren hundert Millionen DM“ benötigt würden. Die Überregionalität des Flusses wurde auf der Ebene der Landesregierung ebenfalls priorisiert, jedoch schlug sich die Diskussion noch nicht in konkreten Handlungen nieder.

Außergewöhnlich war, dass sogar der Bundesumweltminister sich einschaltete. Klaus Töpfer (CDU) stärkte die Position der Stadt Köln. Er argumentierte ähnlich wie NRWUmweltminister Matthiesen, indem er zur Lösung eine „Strategie der tausend kleinen Aufgaben“ empfahl, denn auch ihm schien bewusst zu sein, dass das Flusssystem nur ganzheitlich durch viele verschiedene Maßnahmen zu beeinflussen sei. Töpfer schlug einen institutionalisierten Dialog über das Hochwasser zwischen den Umweltministern der Bundesländer vor, dieser setzte sich hingegen nicht durch. Sein persönliches Projekt war damals die gesetzliche Neuregelung des Bodenschutzgesetztes, welches nicht so recht zu

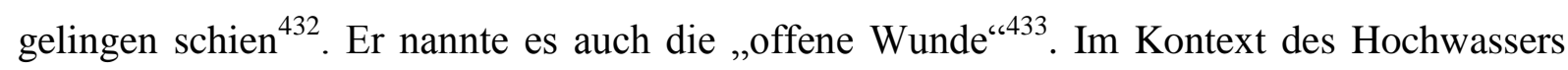
nannte er immer wieder die Umsetzung des Bodenschutzgesetzes als Lösung des Problems. Sein Handeln muss mit den bevorstehenden Bundestagswahlen in Verbindung gebracht werden. Da er sich für die Wahl im kommenden Herbst noch etwas profilieren musste, versuchte er sein Programm als Lösung der deutschlandweiten Probleme herauszustellen. Töpfer verteidigte trotzdem die passive Haltung der Bundesregierung gegen die Forderungen von ,,jedem, Abhilfe aus Bonn zu erwarten“434. Dazu machte er deutlich, dass es nicht ausschließlich an der Bundesregierung läge, die Bedingungen zum Besseren zu verändern. Er nannte zur Unterstützung seiner Aussage die allgemeinen wirtschaftlichen Interessen, welche bei den Städten und Gemeinden aber auch bei der Lobby der Landwirtschaft im Vordergrund ständen und ökologische Belange unbeachtete ließen.

Die durch die überregionalen Ansätze betroffenen Akteure empfanden die Hochwasserursachen durchaus unterschiedlich. Schon als das Hochwasser in den Straßen von Köln stand, entbrannte eine rege und überregionale Diskussion über die Ursachen des Hochwassers zwischen den Landesbehörden. Die Umweltminister der vom Hochwasser ebenfalls betroffenen Länder Baden-Württemberg und dem Saarland interpretierten die Ursachen der Überschwemmungen gegensätzlich. Aus dem Saarland wurde eine Kehrtwende in der Landschaftsgestaltung gefordert. Dort sah man offenbar die Begründungen für das verheerende Ereignis wie in NRW in menschlicher Hand. Der Aufruf nach ,raschen

\footnotetext{
${ }^{432}$ Bei der Wahl 1994 wurde Klaus Töpfer (SPD) durch die neue Umweltministerin Angela Merkel (CDU) abgelöst.

${ }^{433}$ Artikel: Natur ist keine Maschine, sie paßt sich der Technik nicht an, Kölnische Rundschau, 10.01.1994.

${ }^{434}$ Ebd.
} 
Handlungen“ verdeutlicht das Vorhandensein eines Bewusstseins für konstruierte Bedrohungen, denen man im Saarland künftig aus dem Weg gehen wollte. Von einer veränderten Landschaftsgestaltung versprach sich die saarländische SPD eine Verbesserung der Hochwasserproblematik an der Saar. Dagegen blieb die Umweltministerin des Nachbarlandes Rheinland-Pfalz zurückhaltender. Sie suchte die Gründe für die Fluten an Mosel und Rhein in den starken Regenfällen. Indes versuchten die Kölner Stadtratpolitiker dem Hochwasser mit überregionalen und lokalen Maßnahmen der Landschaftsgestaltung beizukommen. Ein Beispiel für sofortige Konsequenzen ist in den städtischen Auseinandersetzungen über den Erhalt natürlicher Überschwemmungsgebiete zu finden. Dort wurden die problematischen Folgekosten für die Stadt verdeutlicht, die zu einem Zielkonflikt führten. Aus dem Kölner Stadtrat, seinen Fraktionen sowie aus der Bürgerschicht wurde einstimmig danach verlangt, die Bodenversiegelung nicht weiter fortzutreiben und dem Wasser natürliche Abflussmöglichkeiten zurück zu geben. Konkrete Handlungen mündeten in der Diskussion, die Bebauung des Gebiets Weißer Bogen in Rodenkirchen zu stoppen. Die Bündnis 90/DIE GRÜNEN, die FDP sowie die SPD verweigerten die Fortführung und verwiesen auf eine gefährliche Bodenversiegelung. Ihnen zur Seite trat die lokale Bürgervereinigung Rodenkirchen, welche sich ebenfalls öffentlich gegen die Bebauung und gegen die weitere Bodenversiegelung aussprach. Dort hätten Sozialwohnungen entstehen sollen, die damals in Köln dringend gebraucht wurden. Der Bedarf an Sozialwohnungen, wie es 1994 bei dem Deutschen Städtetag thematisiert wurde, galt als eine allgemeine Herausforderung jener Zeit ${ }^{435}$. Die Kölner CDU wollte nicht von ihrem Vorhaben Abstand nehmen und suchte nach einem Mittelweg, die wirtschaftlichen Ziele nicht aufzugeben aber die künftige Bodenversiegelung gering zu halten. Sie schlugen vor, Einfamilienhäuser zu bauen und mit Bewohnern zu bevölkern, welche mit dem Risiko leben könnten. Der Aspekt „mit dem Hochwasser zu leben“ wurde im Zusammenhang mit dem Bewusstsein für ein generelles Hochwasserrisiko, welches niemals gänzlich vermeiden werden könne, gesetzt. Die CDU sah bereits damals in der Kommunikation mit dem betroffenen Bürger ein Potential zur Verbesserung des Hochwasserschutzes.

Im Folgenden werden die betrachteten Handlungsalternativen beleuchtet. Wie durch die Darstellung der Diskussion bereits demonstriert werden konnte, wurden verschiedenste Vorschläge zur Verbesserung des Hochwasserablaufs gemacht. Keine Diskussion lässt darauf schließen, dass Maßnahmen mit dem Ziel Ressourcen zu sparen, gegeneinander abgewogen

\footnotetext{
${ }^{435}$ Artikel: Wohnungsbau bleibt Thema, Kölnische Rundschau, 15.01.1994.
} 
wurden. Auf städtischer, nordrhein-westfälischer und Bundesebene war man sich sicher, dass eine Vielzahl mannigfacher Maßnahmen zu unternehmen sei, und dass es nicht die eine einfach Lösung geben würde. Allerdings konnten Maßnahmen nicht durchgeführt werden, nachdem die jeweiligen Vor- und Nachteile gegen alternative Handlungen abgewogen wurden. Nur eine Alternative wurde durch die Kölner strategisch ausgeblendet. Obwohl die Landesregierung bereits damals einen einheitlichen Hochwasserschutz in NRW verlangte, dachte die Stadt nicht darüber nach, ihre Schutzvorrichtungen zu erhöhen. In NRW sollte ein Schutz mit der Stärke von einem Ereignis mit einer statistischen Wiederkehr von 200 Jahren verbaut sein, aber in Köln war damals lediglich ein Schutz gegen statistisch alle 40 Jahre auftauchende Hochwasserereignisse vorhanden ${ }^{436}$. Erstaunlicherweise wurde die Frage, ob die Hochwasserschutzmauer zu erhöhen wäre, in Köln nicht besprochen. Die Kölner Verantwortlichen verfolgten also ein selektives Vorgehen.

Mit Kritik an den Konsequenzen der ausgearbeiteten Lösungen verfuhren die Verantwortlichen ähnlich wie mit der Abwägung von möglichen Handlungsalternativen. Der Status Quo des Hochwasserschutzes, die zu lokale Perspektive auf ein überregionales Phänomen, wurde vehement kritisiert und erneuert. Die absolute Unzufriedenheit mit den rezenten hydrologischen und politisch-organisatorischen Zusammenhängen lässt sich aus der kurzfristigen Veröffentlichung des Hochwasserschutzkonzepts ableiten. Der nun gewählte überregionale Ansatz zur Lösung des Hochwasserproblems wurde in Köln und in NRW nicht kritisiert. Eine spezifische Kritik der möglichen Grenzen und Konsequenzen blieb aus, nachdem die Kölner Politiker nun alles zu tun gewillt waren, was erfolgversprechend schien.

\subsection{Das Jahrhunderthochwasser 1995}

Eine Dichometrie aus den Ereignissen vor und nach dem Höchststand von 10,69 m K P bietet einen Überblick über die verschiedenen Phasen des Verlaufs des fast zweiwöchigen Hochwasserereignisses. Zunächst ließ sich von einer Routinesituation in Köln sprechen. Die ersten Tage waren von Vorbereitungen, kleinen Einschränkungen, Warnungen und Hoffnung gekennzeichnet. Mit Erfahrung wurden am 24. Januar 1995, einem Dienstag, die ersten Keller

\footnotetext{
${ }^{436}$ Siehe Bau der Hochwasserschutzmauer 1983.
} 
abgedichtet $^{437}$, während in Rodenkirchen, ein Vorort im linksrheinischen Süden der Stadt, Funkgeräte und Mobiltelefone für die Eröffnung eines Flutkoordinierungsbüros erwartet wurden $^{438}$. Die Mitarbeiter der Schutzzentrale hängten zur Warnung Plakate auf ${ }^{439}$ und veranlassten die ersten Spundwände zu positionieren sowie die Verteilung von 34.000 Sandsäcken vorzubereiten ${ }^{440}$. Der Wasserstand der Mosel galt als entscheidend für Köln ${ }^{441}$. Auf dem Flussabschnitt zwischen Koblenz und Dormagen, ca. zehn Kilometer flussabwärts der Domstadt gelegen, wurde während der Hochwasserwelle an jenem Mittwoch die Schifffahrt eingestellt ${ }^{442}$. Die erste Welle setzte „Straßen, Häuser und Keller unter Wasser“443. Die ca. 20 Häuser vom Stadtteil Kasselberg waren bereits abgeschnitten worden ${ }^{444}$ und sogar die Kölner Altstadt stand schon „,schenkelhoch“4445 unter Wasser. Nach einer weiteren Hochwasserwelle auf der Mosel warnte das Hochwassermeldezentrum Mainz vor der Möglichkeit einer „verheerenden Rheinflut“. Die bis Freitag eingetroffenen Soldaten halfen an der „Wasserfront“446 bei der Rettung von Möbeln und dem Anbringen von Sicherheitsabsperrungen, installierten Pumpen und transportierten Sandsäcke. Die Tiefbauten wurden am Freitag den 27. Januar geschlossen ${ }^{447}$, da bereits bekannt geworden war, dass an diesem „Nachmittag der Rhein über die mobile Schutzwand in den Rheingarten und in die Altstadt ${ }^{6448}$ schwappen werde.

Die zweite Phase der lokalen Hilfe und der überregionalen Hilferufe - begleitet von Kritik lief zum Wochenende hin an. In der Nacht zum Samstag verbreitete sich der Rhein um ca. 150 Meter $^{449}$. In einer Pressekonferenz verwies NRW-Umweltminister Klaus Matthiesen auf die Verantwortung der Bundesregierung für die Hochwassersituation in Köln ${ }^{450}$. Von einer Arbeitsgruppe der Kölner Stadtverwaltung wurde bereits an jenem Samstag erwogen, Überflutungsräume in Köln ${ }^{451}$ einzurichten und Joachim Pietrzenjuk, Abteilungsleiter

\footnotetext{
${ }^{437}$ Artikel: Zum Wochenende steigt das Hochwasser dramatisch, Kölnische Rundschau, 24.01.1995.

${ }^{438}$ Artikel: Die ersten Keller sind schon abgedichtet, Kölner Stadtanzeiger, 24.01.1995.

${ }^{439}$ Artikel: Zum Wochenende steigt das Hochwasser dramatisch, Kölnische Rundschau, 24.01.1995.

${ }^{440}$ Artikel: Das Bange Warten auf die Flut in Köln, Kölner Stadtanzeiger, 28.01.1995.

${ }^{441}$ Der Fluss mündet in Koblenz/Rheinland-Pfalz in den Rhein. Koblenz liegt 85 km südlich von Köln.

${ }^{442}$ Artikel: Angst vor bedrohlicher Rheinflut, Kölnische Rundschau, 26.01.1995.

${ }^{443}$ Artikel: Rodenkirchen: Einsatz der neuen Hochwasserboote, Kölnische Rundschau, 26.01.1995. Das Auenviertel ist statistisch alle zwei Jahre von Überschwemmungen betroffen (Stadt Köln, Drucksache 1141/095, Anlage 9, 18.01.1996).

${ }^{444}$ Kasselberg liegt in dem Bezirk Chorweiler, das ist im linksrheinischen Norden von Köln.

445 Artikel: Angst vor bedrohlicher Rheinflut, Kölnische Rundschau, 26.01.1995.

${ }^{446}$ Artikel: Köln Hilfe verweigert: Flut in Altstadt, Kölnische Rundschau, 27.01.1995.

${ }^{447}$ Artikel: Rheinufertunnel wurde am Abend geschlossen, Kölnische Rundschau, 28.01.1995; Artikel: Altstadttiefgaragen sind schon gesperrt, Kölnische Rundschau, 28.01.1995.

${ }^{448}$ Artikel: Flutwelle überschwemmt heute die Kölner Altstadt, Kölner Stadtanzeiger, 27.01.1995.

${ }^{449}$ Artikel: Wasser im Keller wird Dauerzustand, Kölnische Rundschau, 30.01.1995.

${ }^{450}$ Landtag NRW, Plenarprotokoll 11/157, 17.02.1995.

${ }^{451}$ Artikel: Polder für Köln, Kölner Stadtanzeiger, 28./29.01.1995. Tiefbaudezernent Oelmann nannte als potentielle Möglichkeiten für Rückhaltebecken Niederkassel, Langel und den Worringer Bruch. Bei letzterem äußerte er ökologische Bedenken. Er verwies auf das neue Hochwasserkonzept, welches Mitte des Jahres 1995 erscheinen solle.
} 
Wasserwirtschaft des NRW-Umweltministeriums, wies auf die seit Jahren fehlende Erhöhung der Kölner Schutzmauern hin ${ }^{452}$. Erste finanzielle Hilfen wurden an jenem Samstag in Form von Steuererleichterungen durch Bundesfinanzminister Theodor Waigel und NRWInnenminister Herbert Schnoor angekündigt. Wie schon bei dem Hochwasserereignis 13 Monate zuvor, wandte sich die Stadt Köln an die Landesregierung NordrheinWestfalens ${ }^{453}$ : Hätte Baden-Württemberg bereits am Dienstag seine Polder geöffnet, hätte man in Köln einen halben Meter weniger Hochwasser gehabt ${ }^{454}$. Pietrzenjuk teilte im Namen der NRW-Landesregierung mit, dass der Durchfluss des Rheins laut Umweltministerium Baden-Württemberg unterhalb der Öffnungskriterien läge ${ }^{455}$, die Polder somit geschlossen blieben. Das Rheinland-Pfälzische Umweltministerium verteidigte sich gegen die Vorwürfe der verpassten, seit 1982 vereinbarten, Einrichtungen von Poldern mit dem Versprechen, diese in den nächsten zehn Jahren einzubauen ${ }^{456}$. Der Umweltminister Nordrhein-Westfalens, Matthiesen, wurde „zur Zielscheibe der Umweltverbände“457, die einen „runden Tisch von Bund und Ländern“458 - wie der Hochwasserexperte Krug des Bundes für Umwelt und Naturschutz (BUND) - forderten. Parallel zu dem Streit uferte die Situation am Rhein aus. In dem Kölner Stadtteil Poll brach an dem Sonntag die Trinkwasserversorgung zusammen ${ }^{459}$. In den rechtsrheinischen Orten Mülheim und Deutz liefen an jenem Montag ebenfalls Keller voll $^{460}$. Bereits 30.000 Menschen waren direkt von dem Ereignis betroffen ${ }^{461}$. Das Grundwasser drückte auch in nicht unmittelbar am Fluss gelegene Gegenden in die Keller. Würde die Marke von 10,70 m K P überschritten, seien weitere 270.000 Menschen bedroht. Ein Katastrophenstab wurde vorsorglich unter der Leitung des Oberstadtdirektors Lothar Ruschmeier gebildet ${ }^{462}$. Arbeitgeber in der Region wurden von ihm aufgefordert, die freiwilligen Helfer von ihrer Arbeit freizustellen ${ }^{463}$. Er flehte auch an diesem Montag Baden-

\footnotetext{
${ }^{452}$ Ebd.

${ }^{453}$ Dieses Ausreichen nach der Hilfe der Oberlieger ist im Deutsch - Französischen Vertrag von 1982 (ein Name kann nicht gefunden werden) begründet. Dieser wurde zur Kompensierung des energetischen Ausbaus des Rheins und der damit verbundenen Erhöhung des Hochwasserrisikos abgeschlossen. Nach den Untersuchungen der Auswirkungen des Ausbaus durch die Hochwasserstudienkommission 1978 sollten in Baden-Württemberg, Hessen, Rheinland-Pfalz und in Frankreich Retentionsräume für 260 Mio. $\mathrm{m}^{3}$ Wasser gebaut werden. Während nur Frankreich den Anforderungen nachkam, begannen die deutschen Bundesländer teilweise oder gar nicht mit der Umsetzung. Da der energetische Rheinausbau nur in den südlichen Bundesländern stattfand, wurde NRW an dem Vertrag nicht beteiligt (Dr. Angela Merkel, 21.02.1996).

${ }_{454}$ Artikel: Kölns Altstadt erneut unter Wasser, Kölnische Rundschau, 28.01.1995.

${ }^{455}$ Artikel: Polder am Oberrhein bleiben dicht, Kölner Stadtanzeiger, 28./29.01.1995.

${ }^{456}$ Ebd.

${ }^{457} \mathrm{Ebd}$

458 Ebd.

${ }^{459}$ Artikel: Es kommt noch schlimmer, Kölnische Rundschau, 30.01.1995.

${ }^{460}$ Artikel: Neue Gefahr: „Flut von unten“ bedroht mehr als 300.000 Kölner, Kölnische Rundschau, 31.01.1995.

${ }^{461}$ Artikel: Es kommt noch schlimmer, Kölnische Rundschau, 30.01.1995.

${ }^{462}$ Artikel: Bei 10,70 Meter läuft die Schäl Sick voll, Kölnische Rundschau, 30.01.1995.

${ }^{463}$ Artikel: Ehrenamtliche Helfer von Arbeit freistellen, Kölnische Rundschau, 30.01.1995.
} 
Württemberg erneut um die „Gnade“464, die Polder zu öffnen. NRW-Umweltminister Matthiesen besuchte an jenem Montag Köln. Dort nahm er seine Kritik an Kölns fehlenden Schutzhöhen $^{465}$ als absurd zurück ${ }^{466}$. Außerdem „,beschimpfte [er] die Baden-Württemberger, die Polder noch nicht geöffnet zu haben“467. Nach seiner Abreise bezeichnete er am Tage darauf die Forderungen gegenüber den Baden-Württembergern als „dumm und sachlich falsch““468, denn sie hätten „,kaum Wirkung gezeigt“469. Zusätzlich verlangte er für die Zukunft ein „länderübergreifendes Hochwassermanagement unter Einbezug von Frankreich und Holland“4770. Aufgrund der Eskalation des Streits um die Hochwasserverantwortung zwischen den Ländern lud Bundeskanzler Helmut Kohl (CDU) die Staatssekretäre diverser Ministerien an jenem Montag ins Bonner Kanzleramt ein. Die Fraktionen der Bundesregierung schlossen sich seiner Meinung an, dass die Länder die Herausforderung untereinander „übergreifend““471 regeln sollten. Montagabends erreichte der Wasserstand in Köln die legendäre Jahrhundertmarke von $1926^{472}$.

In den auf den Höchststand folgenden Tagen eröffnete sich die letzte Phase des Hochwasserereignisses: Der Umgang mit dem Schaden und der Streit um Ursachen und Schuld. Der Beigeordnete Oelmann ${ }^{473}$ und Reinhard Vogt, Leiter der Hochwasserschutzzentrale wurden nur „,vorsichtig optimistisch“474, denn der fallende Rheinpegel hätte noch keine Auswirkungen auf den nach wie vor steigenden Grundwasserspiegel. Im Finanzausschuss der Stadt dachte Kämmerer Gleize am Dienstag den 31.01. über Möglichkeiten nach, im Haushalt für die Kosten des erneuten Hochwassers aufzukommen, denn das Hochwasser vor 13 Monaten belaste den Haushalt noch immer mit 9.2 Mio. $\mathrm{DM}^{475}$. Die Tageszeitung Kölnische Rundschau sowie die Stadtverwaltung riefen ab jenem Dienstag zu Spenden auf ${ }^{476}$. Gleich am ersten Tag kamen über 100.000 DM aus Wirtschaft, Industrie, Geldinstituten, Karnevalsvereinen sowie dem 1. FC Köln zusammen ${ }^{477}$,

\footnotetext{
${ }^{464}$ Ebd.

${ }^{465}$ Die Landesregierung mahnte bereits seit 1988 die Höhen der Schutzvorrichtungen in Köln an.

${ }^{466}$ Artikel: Wir brauchen ein länderübergreifendes Management, auch mit Frankreich und Holland, Kölner Stadtanzeiger, 31.01.1995.

${ }_{467}$ Landtag NRW, Plenarprotokoll 11/157, 17.02.1995.

${ }^{468}$ Artikel: Wir brauchen ein länderübergreifendes Management, auch mit Frankreich und Holland, Kölner Stadtanzeiger, 31.01.1995.

${ }_{469}$ Landtag NRW, Plenarprotokoll 11/157, 17.02.1995.

${ }^{470}$ Artikel: Wir brauchen ein länderübergreifendes Management, auch mit Frankreich und Holland, Kölner Stadtanzeiger, 31.01.1995.

${ }^{471}$ Ebd.

${ }^{472}$ Artikel: Jahrhundertflut: Höher als 1926, Kölnische Rundschau, 31.01.1995.

${ }^{473}$ Artikel: Arbeitslose zum Flut-Einsatz, Kölnische Rundschau, 01.02.1995.

${ }^{474}$ Artikel: Rekord-Flut geht langsam zurück, Kölnische Rundschau, 01.02.1995.

${ }^{475}$ Artikel: Solidarabgabe an die Flutopfer? Kölnische Rundschau, 31.01.1995.

${ }^{476}$ Artikel: Nach der Flut stehen viele vor dem Nichts, Kölnische Rundschau, 31.01.1995.

${ }^{477}$ Artikel: Am ersten Tag Spenden über 100.000 Mark, Kölnische Rundschau, 01.02.1995.
} 
hinzu kamen Spenden durch Privatleute ${ }^{478}$. Bundesfinanzminister Waigel bot 30 Mio. DM als zinsgünstige Darlehn für geschädigte Gewerbetreibende $\mathrm{an}^{479}$. Das Land NRW eröffnete, 15 Mio. DM neben Steuervergünstigungen und Barmittel ${ }^{480}$. Oberbürgermeister Norbert Burger rief an jenem 31.01. auf, gemeinsam alles „Menschenmögliche $\mathrm{zu}$ tun, um der Hochwasserkatastrophe Herr $\mathrm{zu}$ werden “481. Dagegen versprach Oberstadtdirektor Ruschmeier am gleichen Tag, das neue Hochwasserschutzkonzept bis zum Sommer vorzulegen, lehnte jedoch den Bau höherer Schutzwände ab. Denn Köln läge am Rhein und nicht an einer Mauer ${ }^{482}$. An jenem Dienstag meldete sich der Umweltminister von BadenWürttemberg Harald Schäfer zu Wort: Er argumentierte mit den technischen Bedingungen, festgeschrieben in dem Abkommen mit Frankreich, welche das Öffnen der Polder seit 1982 regelten. Dazu führte er die damaligen Einrichtungsziele aus, Städte in Baden-Württemberg zu schützen ${ }^{483}$. Außerdem hätte seiner Ansicht nach die Entfernung zwischen den Orten die Wirkung in Köln marginalisiert ${ }^{484}$. Damit widersprach er der Kölner Behauptung, sein Handeln hätte die Hochwasserwelle in Köln um 0,4 m abmildern können. In einem Leserbrief forderte ein wütender Bürger die „Verantwortlichen der Bausünden am Oberrhein zum harten Einsatz an der Hochwasserfront “485 auf. Am Mittwoch kündigten die Kölner Kreditinstitute ${ }^{486}$ und die Kreditanstalt für Wiederaufbau ${ }^{487}$ Sonderkonditionen für Hochwassergeschädigte an. Einen Tag später forderte die Kölner Ratsfraktion von Bündnis 90/DIE GRÜNEN, Vorreiter einer ökologischen Wende zu werden und endlich anzuerkennen, dass die Schuld nicht alleine in Baden-Württemberg zu suchen sei, die Flut vielmehr die Folge einer verfehlten Umweltpolitik verkörpere ${ }^{488}$. Der Biologe Klatt vom Naturschutzbund Deutschland (NABU) beschuldigte Stadt und Land mit dem von ihnen vorgebrachten Verweis auf die unterlassene Einrichtung von Rückhaltebecken am Oberrhein nur „,von eigenen Versäumnissen ablenken zu wollen“489. Das Öffnen der Polder ,hätte Köln nichts genützt ${ }^{\star 490}$, meinten auch Experten des World Wide Fund of Nature (WWF) sowie des NABU. Öffentlich Anregungen zur Verbesserung des Hochwasserschutzes gab der BUND durch seine Kritik an der Stadt Köln

\footnotetext{
${ }^{478}$ Artikel: Hilfswelle nach Aufruf zu Spenden in der Rundschau, Kölnische Rundschau, 01.02.1995.

${ }^{479}$ Artikel: Beratung für Flutopfer auch am Wochenende, Kölner Stadtanzeiger, 03.02.1995.

${ }^{480}$ Artikel: Hilfspakete werden geschnürt, Kölner Stadtanzeiger, 01.02.1995.

${ }^{481}$ Artikel: Gemeinsam die Not der Opfer lindern, Kölnische Rundschau, 01.02.1995.

${ }^{482}$ Artikel: Im Sommer neues Konzept, Kölner Stadtanzeiger, 01.02.1995.

${ }^{483}$ Artikel: Wir müssen an unsere eigenen Städte denken, Kölnische Rundschau, 31.01.1995.

${ }^{484}$ Ebd.

${ }^{485}$ Leserbrief von Walter Heilmann, Bergisch-Gladbach, Kölnische Rundschau 01.02.1995.

${ }^{486}$ Artikel: Hochwasserkredite werden gewährt, Kölnische Rundschau, 02.02.1995.

${ }^{487}$ Artikel: Hochwasser-Opfer können jetzt Mittel beantragen, Kölner Stadtanzeiger, 18./19.02.1995; Artikel: Keine niedrigen Zinsen für Privatleute, Kölner Stadtanzeiger, 03.02.1995.

${ }^{488}$ Artikel: Aktuelle Stunde zum Thema Überflutung, Kölnische Rundschau, 02.02.1995.

${ }^{489}$ Artikel: Viele Polder sind bis heute nicht gebaut, Kölner Stadtanzeiger, 02.02.1995.

${ }^{490}$ Ebd.
} 
bezüglich der Regenwasserversickerung ${ }^{491}$. Einige Tage später reagierte NRWUmweltminister Matthiesen und warf der Kölner Stadtverwaltung ebenfalls Verantwortungslosigkeit vor, wenn diese in Überschwemmungsgebieten weiterhin Baugebiete ausweisen wolle ${ }^{492}$. Am Donnerstag, den 02.02. kam der Rat der Stadt Köln zusammen und forderte die Regierungen von Bund und Ländern auf, eine einheitliche Koordination der Schutzmaßnahmen zu besorgen ${ }^{493}$. Dazu gehöre NRW in den DeutschFranzösischen Vertrag aufzunehmen ${ }^{494}$. Aufgrund der im Vergleich zum Hochwasser von 1993 geringeren ausgefallenen Schäden ${ }^{495}$, beurteilten die Verantwortlichen der Stadt Köln das Flutereignis von 1995 im Nachhinein als weniger dramatisch - man sei „der Katastrophe entgangen“496. Die Ursache für die geringeren Schäden führte die Stadtverwaltung auf den verbesserten Selbstschutz der Bürger zurück $^{497}$. In einem Interview mit dem Tiefbaudezernenten Oelmann an jenem Donnerstag nach dem Hochwasser klang bereits das spätere Hochwasserschutzkonzept an, indem er die Einrichtung längst beschlossener Retentionsräume am Oberrhein sowie eine Koordinierungsstelle für alle Rheinanlieger forderte ${ }^{498}$. Gleichzeitig müsse Köln Verantwortung zeigen, und vor Ort Überflutungsgebiete einrichten ${ }^{499}$.

\subsubsection{Reaktion: Köln liegt nicht an einer Mauer}

Das 216 Seiten umfassende Hochwasserschutzkonzept (HWSK) bündelte alle Reaktionen auf die Ereignisse von 1995 und 1993. Es wird hier verkürzt vorgestellt. Gleich zwei Kapitel des Konzeptes hielten die Auffassung des Rates zu Schuld- und Verantwortungsfragen fest. Ausführliche Darstellungen der Hochwasserursachen ${ }^{500}$ und -gefährdungen ${ }^{501}$ erklärten, dass die Hochwasserschuld bei den drei oberrheinischen Bundesländern läge, diese somit die

\footnotetext{
${ }^{491}$ Artikel: Versiegelung von großen Flächen, Kölnische Rundschau, 02.02.1995.

${ }^{492}$ Artikel: Schutz vor Hochwasser: Rasche Lösung Utopie, Kölnische Rundschau, 07.02.1995.

${ }^{493}$ Artikel: Für den Rhein wird ein Konzept gesucht, Kölner Stadtanzeiger, 03.02.1995.

${ }^{494}$ Ebd. Der Deutsch-Französische Vertrag regelte die Kompensation des energetischen Ausbaus des Rheins. Die Auswirkungen des Baus wurden durch die Rheinstudienkommission 1978 auch auf Regionen bezogen, welche nicht unmittelbar mit dem Projekt befasst waren. So ging die Kommission davon aus, dass es in Köln bei einem 200jährigen Hochwasser zu einem Anstieg von zusätzlichen 0,40 m kommen würde. Die am Ausbau beteiligten Länder einigten sich auf verbindliche Ausgleichsmaßnahmen untereinander, bezogen jedoch andere Regionen nicht mit in die Verträge ein. So kam es dann auch, dass Köln als eine von den Auswirkungen betroffene Stadt keine Stimme in dem Abkommen besaß (Dr. Angela Merkel, 21.02.1996).

${ }^{495}$ Bericht „Hochwasser 1995“. Im Jahr 1993 beliefen sich die Schäden in Köln auf 120 Mio. DM im Vergleich zu 199565 Mio. DM (45 Mio. bei privaten Haushalten, 9 Mio. an städtischen Gebäuden, 6 Mio. für Personal und Material beim Hochwassereinsatz, 5 Mio. bei den Kölner Verkehrsbetrieben KVB, GEW und anderen Versorgern).

${ }^{496}$ Artikel: Köln entging nur knapp einer Katastrophe, Kölnische Rundschau, 09.02.1995.

${ }^{497}$ Artikel: Flut-Schäden diesmal „,nur“ 65 Millionen DM, Kölner Stadtanzeiger, 09.02.1995.

${ }^{498}$ Artikel: Hochwasser, Köln entging nur knapp der Katastrophe, Kölnische Rundschau, 09.02.1995.

${ }^{499}$ Ebd.

${ }^{500}$ HWSK 1996, 14.

${ }^{501}$ Ebd., 43.
} 
Verantwortung für bislang unterlassene sowie zukünftig durchzuführende Maßnahmen tragen müssten. Realisiert wurden in Köln zwei Arten von baulichen Maßnahmen mit praktischen Zielen. Auf der einen Seite sollte das Stadtgebiet vor dem Hochwasser abgedichtet werden. Dazu dienten Deiche, Spundwände und Hochwassermauern sowie die Abschottung der Kanalisation. Im Kapitel zu Hochwassergefährdungen wurden die technischen Schutzoptionen sowie die noch vorhandenen Eindringmöglichkeiten des Hochwassers beschrieben. Der technische Nachweis, an welcher Stelle das Wasser in die Stadt eintrat, begründete die baulichen Aktivitäten ${ }^{502}$. Die zweite Komponente der baulichen Maßnahmen stimmte mit der physikalischen Notwendigkeit überein, dass das Hochwasser trotzdem Platz in Anspruch nehmen würde. Retentionsflächen sollten neben weiteren Raum spendenden Maßnahmen eine Möglichkeit bieten, zusätzliche Wassermassen aufzunehmen. Abb. 14 verdeutlicht die Gruppierung der baulichen Maßnahmen nach ihren wasserbaulichen Zielen.

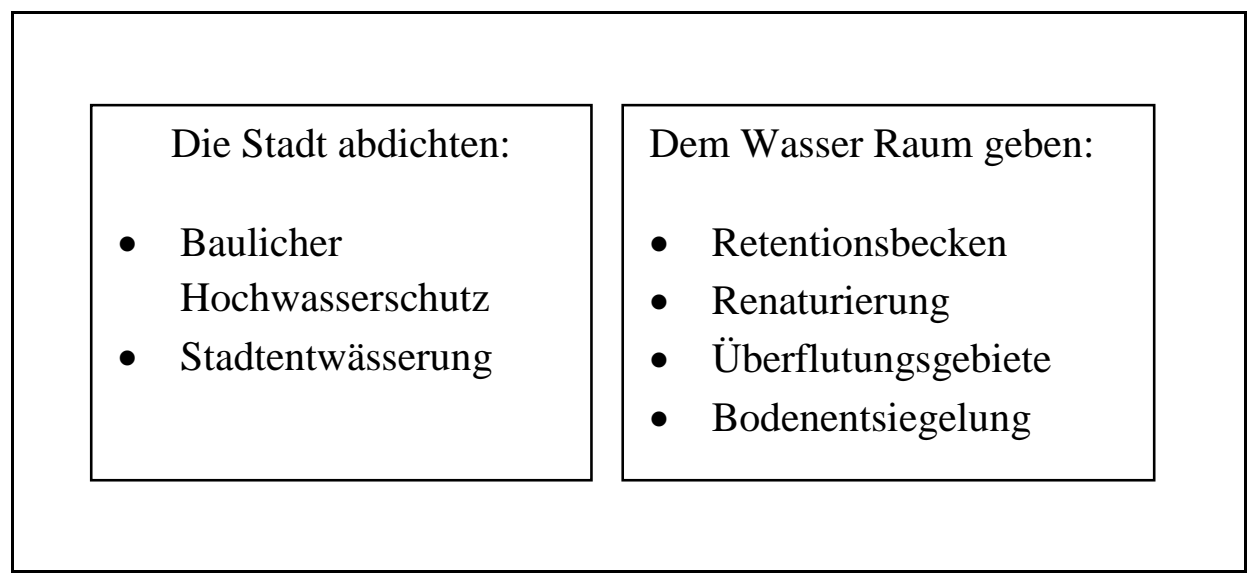

Abb. 14: Gruppierung der Kölner wasserbaulichen Maßnahmen.

Die 1996 beschlossenen Investitionen in den lokalen Hochwasserschutz werden in Tabelle 1 den Schäden der Flut von 1995 gegenüber gestellt. Zu der Belastung des städtischen Haushalts durch Hochwasser müssen die Schäden von 1993 dazugezählt werden. Diese umfassten 120 Mio. DM.

\footnotetext{
${ }^{502}$ An dieser Stelle erklärte die Kölner Verwaltung durch das Amt der Stadtentwässerung, dass sie zukünftig alles täte, was möglich sei.
} 


\begin{tabular}{|c|c|c|c|c|}
\hline & $\begin{array}{l}\text { Investition } \\
\text { nach HWSK }\end{array}$ & Finanzierung & Schäden 1995 & Schäden 1993 \\
\hline Total $^{\mathrm{A})}$ & 477,5 Mio. DM & & 65,0 Mio. $\mathrm{DM}^{\mathrm{B})}$ & 120 Mio. DM $^{503}$ \\
\hline $\begin{array}{l}\text { Oberirdischer } \\
\text { Schutz (Deiche) }\end{array}$ & 150,0 Mio. DM & $\begin{array}{l}\text { Eigenanteil der Stadt von } \\
67,5 \text { Mio. DM über } \\
\text { 10 Jahre, 6,75 Mio. DM/a zu } \\
\text { tragen }^{\text {C) }}\end{array}$ & 33.000 & k. A. \\
\hline $\begin{array}{l}\text { Unterirdischer } \\
\text { Schutz } \\
\text { (Kanalisation) }\end{array}$ & 322,5 Mio. DM & $\begin{array}{l}\text { Abwassergebühr über } 10 \text { Jahre, } \\
\text { 32,0 Mio. DM/a }\end{array}$ & 25.000 & k. A. \\
\hline & \multirow{2}{*}{\multicolumn{4}{|c|}{$\begin{array}{l}\text { A) Exklusive der Kosten für Retentionsräume. Diese standen } 1996 \text { noch nicht fest. } \\
\text { B) Betroffene sind hier Privathaushalte, auf sie entfielen } 45,0 \text { Mio. DM Schäden. Ob die } \\
\text { Schäden der direkten Rheinanlieger aufgrund der Nähe nicht tatsächlich relativ höher waren, } \\
\text { als die der durch Grundwasser geschädigten Haushalte, bleibt hier offen. Die restlichen } \\
\text { Schäden von 20,0 Mio. DM verteilten sich auf Infrastruktur, Personal und Material sowie die } \\
\text { Kölner Verkehrsbetriebe (KVB) }{ }^{504} \text {. }\end{array}$}} \\
\hline & & & & \\
\hline & \multicolumn{4}{|c|}{ C) Der Stadtrat ging von einer Unterstützung durch das Land NRW von 55 Prozent aus. } \\
\hline
\end{tabular}

Tabelle 1: Verteilung von Investitionen und Schäden in Köln 1993/95.

Die Rheinkilometer im Kölner Stadtgebiet wurden in 18 Planfeststellungsabschnitte unterteilt. Nach Fertigstellung sollte jeder einzelne Abschnitt zu dem übergeordneten Ziel, Köln für ein Hochwasser von 11,30 m bzw. 11,90 m K P zu sichern, beitragen. Abb. 15 erleichtert die geographische Einordnung:

\footnotetext{
${ }^{503}$ Das Hochwasser im Dezember 1993 richtete in Köln 120 Mio. DM Schaden an, die den Haushalt noch bis mindestens 1995 mit fast 10 Mio. DM pro Jahr belasteten (Artikel: Hochwasser, Köln entging nur knapp der Katastrophe, Kölnische Rundschau, 09.02.1995).

${ }^{504}$ Bericht „Hochwasser 1995“.

${ }^{505}$ HWSK 1996, 158; Bericht „Hochwasser 1995“.
} 


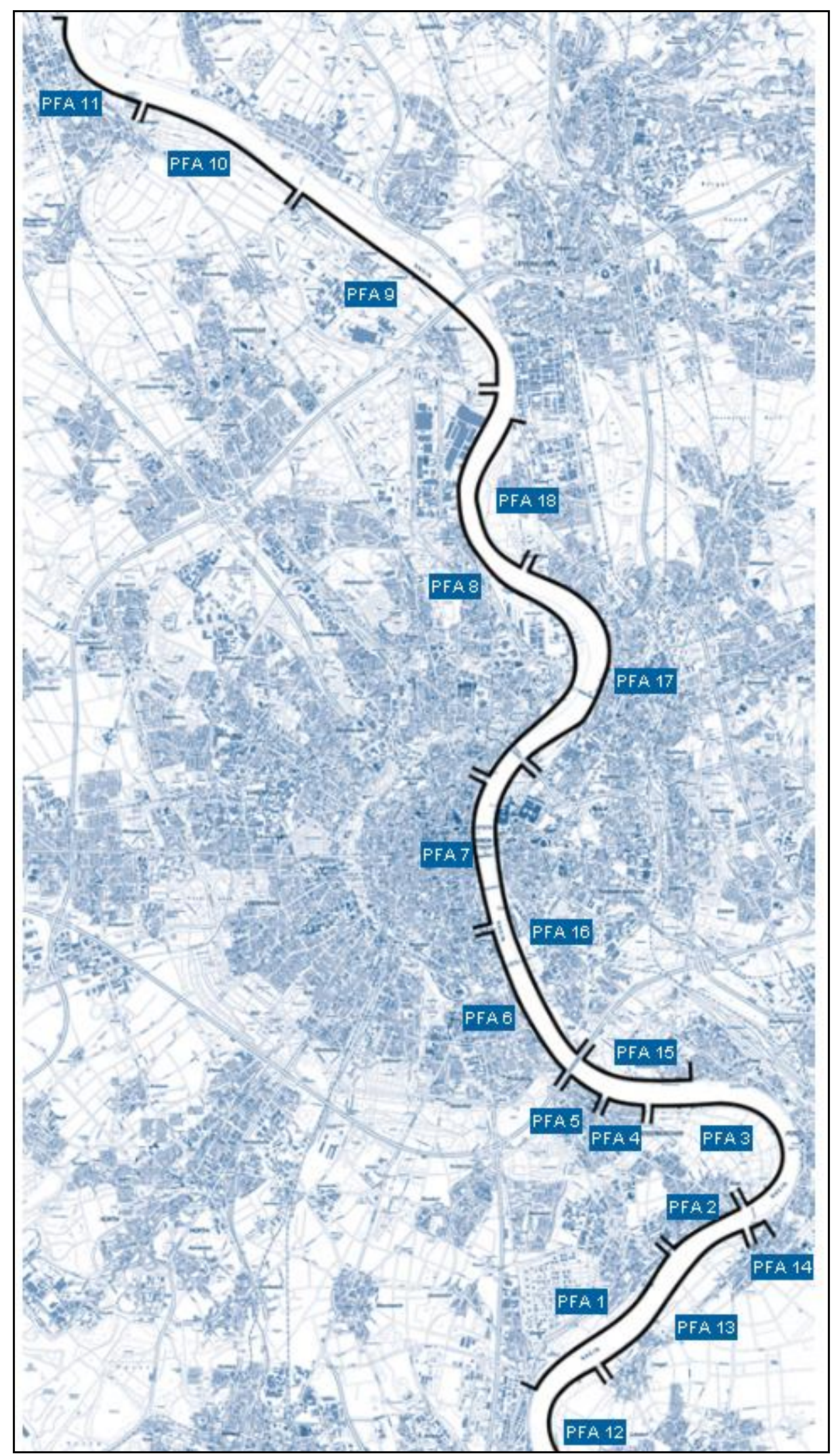

Abb. 15: 18 Planfeststellungsabschnitte im Hochwasserschutzkonzept Köln $1996 .{ }^{506}$

${ }^{506}$ Bild der Stadtentwässerungsbetriebe Köln AöR, Internetbasierte Quelle 2. 
Nachdem die Retentionsmaßnahmen durch komplementäre Handlungsoptionen im Bereich der Raumentwicklung verstärkt und systematisch $\mathrm{zu}$ einem ganzheitlichen Konzept ausgearbeitet wurden, folgten in der Niederschrift zahlreiche Forderungen an die nationalen

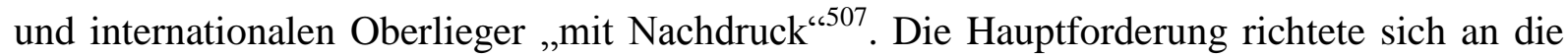
Vertragspartner des „Deutsch-Französischen Vertrags“ vom 06.12.1982 ${ }^{508}$. Nach den Untersuchungen der Auswirkungen des Ausbaus der Staustufen (z. B. in Iffezheim) durch die Hochwasserstudienkommission 1978 sollten in Hessen, Rheinland-Pfalz und BadenWürttemberg sowie in Frankreich Retentionsräume für 260 Mio. $\mathrm{m}^{3}$ Wasser gebaut werden. Während Frankreich den vertraglichen Anforderungen nachkam, hatten die deutschen Bundesländer bis 1995 nur teilweise oder gar nicht mit der Umsetzung begonnen. Um eine Beteiligung an dem Vertrag und die Einrichtung der Polder zu erreichen, forderte die Stadt Köln das Land NRW auf, eine Sprecherfunktion für Köln einzunehmen. Im Kontext der Koordinierung des Hochwasserschutzes forderte der Rat verschiedene Institutionen auf Vereins- und Bundesebene dazu auf, am Wissensaustausch teilzunehmen. Um diesem Anspruch mehr Kraft zu verleihen, wurden Allianzen mit anderen ,hochwassergeschädigten Städten und Gemeinden“6509 als nötig erachtet und etabliert.

Weitere präventive Maßnahmen im Hochwasserschutzkonzept der Stadt Köln bezogen sich auf die Möglichkeiten der privaten Vorsorge ${ }^{510}$, um einerseits Schäden zu minimieren und andererseits einen entstandenen Schaden besser verkraften zu können. Unter anderem wurde den im Gefahrengebiet lebenden Einwohnern eine viergliedrige Vorsorge aufgezeigt ${ }^{511}$, die auf der Verhaltens-, der Bau- und Flächenvorsorge, der Risikovorsorge durch den Abschluss einer „erweiterten Elementarschadenversicherung“ sowie auf bewusstseinsschaffenden Informationen zu dem Thema Grundhochwasser basierte ${ }^{512}$. Abb. 16 zeigt beispielhaft ein Merkblatt für Bewohner hochwassergefährdeter Gebiete.

\footnotetext{
${ }^{507}$ HWSK 1996, 80.

${ }^{508}$ Dr. Angela Merkel, 21.02.1996.

${ }^{509}$ HWSK 1996, 82.

${ }^{510}$ Eine Ressourcendiskussion kam nicht auf, denn Handlungsoptionen wurden gesammelt und an die entsprechenden Bauherren weitergegeben.

${ }^{511}$ Da die Maßnahmen in den privaten Bereich der betroffenen Bevölkerung fallen, entstand dazu keine Diskussion auf Entscheidungsebene.

${ }^{512}$ Grundhochwasser verbreitet sich wie eine unterirdische Welle mit Verzögerung nach Abklingen des

Flusshochwassers. Es gefährdet Flächen, die nicht direkt am Fluss liegen. Dadurch schädigt Grundhochwasser weitere Gebiete als das Wasser, welches über die Ufer tritt.
} 


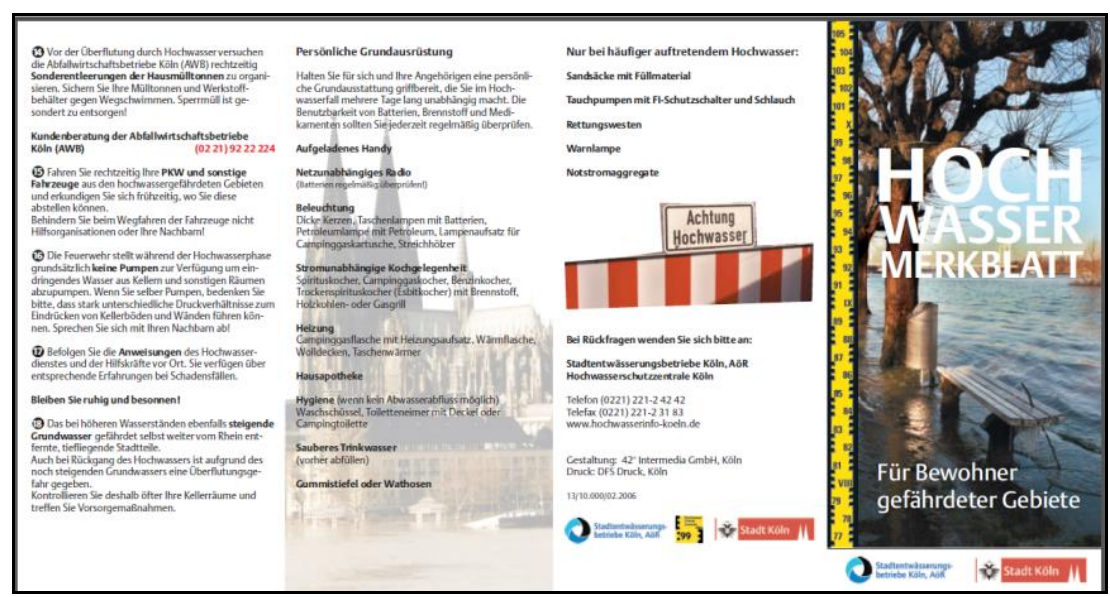

Abb. 16: Merkblatt für Bewohner hochwassergefährdeter Gebiete. ${ }^{513}$

Generelle Aufklärung nahm im HWSK eine weitere zentrale Rolle ein. Wie die vorliegende Ausarbeitung zeigen wird, wurden die Kommunikation mit der Bevölkerung sowie Appelle zur Erinnerung an die potentielle Gefährdung indirekt zur Grundlage des Hochwasserschutzkonzepts.

Bereits seit Dezember 1993 war das Schutzkonzept in Bearbeitung ${ }^{514}$ und konkretisierte sich zunehmend im Mai $1994^{515}$, es war also grundsätzlich von dem Weihnachtshochwasser 1993 inspiriert worden. Zur Revision in den einzelnen Bezirken Kölns konnte es bereits im Juni 1995, nur vier Monate nach dem Jahrhunderthochwasser von 1995, gegeben werden ${ }^{516}$. Der Rat der Stadt Köln stimmte am 1. Februar 1996 einstimmig für das Hochwasserschutzkonzept ${ }^{517}$. „Innerhalb der nächsten zehn Jahre sollen die Maßnahmen geplant, finanziert und umgesetzt werden“"518. Dies entsprach hauptsächlich den geplanten Retentionsräumen in Köln auf Höhe der Stromkilometer 669 bis 672 sowie 706 bis 708 zusammen $^{519}$. Eine neue Auseinandersetzung zu den Kölner Hochwasserschutzhöhen entstand zwischen der Stadt und dem Land NRW.

\footnotetext{
${ }^{513}$ Bild der Stadtentwässerungsbetriebe Köln AöR, Internetbasierte Quelle 2.

${ }_{515}^{514}$ Brief von Egelmann.

${ }^{515}$ Artikel: Im Sommer neues Konzept, Kölner Stadtanzeiger, 01.02.1995.

${ }^{516}$ Artikel: Millionen für den Schutz vor Fluten, Kölner Stadtanzeiger, 20./21.01.1996.

${ }^{517}$ Stadt Köln, Drucksachen - Nr. 1141/095, 29.01.1996.

${ }^{518}$ HWSK 1996.

${ }^{519}$ Unter einem Retentionsraum ist ein Wasserrückhaltebecken zu verstehen. Dies kann durch natürliche oder geplante Überflutung genutzt werden. Bei der natürlichen Überflutung übersteigt der Wasserstand eine bestimmte Hürde und füllt dann das Becken. Bei der geplanten Flutung wird ab einem bestimmten Wasserstand ein Einlass für das Wasser geöffnet. Nach dem Hochwasser zieht sich das Wasser auf natürlichem Wege zurück oder der Retentionsraum wird durch Schieber mechanisch entleert. Auf diese Weise ist die Dauer des Wassereinstaus beeinflussbar.
} 


\subsubsection{Diskussion zur Erhöhung der Schutzbauten}

Seit Beginn der 1980er Jahre wurde die Stadt durch die Landespolitik aufgefordert, die Höhe ihrer Schutzvorrichtungen den nordrhein-westfälischen Standards anzugleichen. In NRW galten uniforme Kriterien zum Hochwasserschutz, die durch das „Gesamtkonzept Rhein in NRW - Hochwasserschutz, Ökologie, Schifffahrt ${ }^{\star 520}$ - vorgegeben wurden ${ }^{521}$. Das Land empfahl - aufgrund der ausgemachten Priorität, durch Hochwassersicherheit Menschen, Tiere und Sachgüter nicht zu gefährden - einen 200jährigen Hochwasserschutz ${ }^{522}$. Die 1995 bereits vorhandenen Anlagen orientierten sich an den Erfahrungen aus den Jahren 1926 bzw. $1993^{523}$. Sie waren ausgelegt, einem Ereignis mit 40jähriger Wiederkehr von $10 \mathrm{~m} \mathrm{~K} \mathrm{P}$ standzuhalten $^{524}$. Das Weihnachtshochwasser von 1993 - mit einer Höhe von 10,63 m K P entfachte eine erneute Diskussion über die Schutzhöhen. Im Dezember 1994 reagierte das Umweltamt Köln mit der Berechnung neuer Hochwasserbemessungsgrenzen ${ }^{525}$. Ein zu erwartendes hundertjähriges Hochwasser wurde fortan mit einer Abflussmenge von $12.000 \mathrm{~m}^{3} / \mathrm{s}$ und einer Höhe von $11,30 \mathrm{~m} \mathrm{KP}$ charakterisiert, während ein zweihundertjähriges Hochwasser nun bei einem Durchfluss von $13.000 \mathrm{~m}^{3} / \mathrm{s}$ und 11,90 m K P ausgerufen werden sollte ${ }^{526}$. Gegenüber der Stadt Köln mahnte die Landesvertretung den standardisierten Minimalschutz in mindestens zwei Besprechungen über das neue Konzept $\mathrm{an}^{527}$. Dagegen hielt der Regierungspräsident ${ }^{528}$ von Köln, Franz Josef Antwerpes, auch den 100jährigen Schutz für „überzogen und in diesem Umfang sehr problematisch“, da „etwas niedriger besser finanzierbar sei““529 . Bereits im Februar 1995, während das Hochwasser noch in den Straßen stand, lehnte Oberstadtdirektor Ruschmeier den Bau höherer Schutzwände ab. Denn Köln läge am Rhein und nicht an einer Mauer ${ }^{530}$. Der Ausschuss für Umweltschutz und Abfallwirtschaft schlug im Stadtrat ebenfalls die Verfolgung des Minimalprinzips vor, ausschließlich einen Hochwasserschutz von 11,30 m K P zu installieren ${ }^{531}$. Der Ausschuss für Tiefbau und Verkehr betrat das Spannungsfeld zwischen anspruchsvollem Schutz und

\footnotetext{
${ }^{520}$ Dieses wurde zwischen 1990 und 1992 erarbeitet. Bewertet und ökologisch optimiert wurden Deichsanierungssowie Anpassungsmaßnahmen zur Verbesserung der Wasserstraße Rhein (Landtag NRW, Ausschussprotokoll 11/1505, 08.02.1995).

${ }^{521}$ Stadt Köln, Drucksache 1141/095, Anlage 8, 18.01.1996.

${ }^{522}$ Landtag NRW, Drucksache 12/248, 11.10.1995.

${ }^{523}$ HWSK 1996, 85.

${ }^{524} \mathrm{Ebd}$.

${ }^{525}$ Ebd., 86.

${ }^{526}$ HWSK 1996. Zuvor wurde ein Hochwasser von 10,69 m K P, begleitet von Ablaufmengen über $10.800 \mathrm{~m}^{3} / \mathrm{s}$, mit einer Wiederkehr von 100 Jahren erwartet.

${ }^{527}$ Ebd.

${ }^{528}$ Die Bezirksregierung ist mit der Beantragung von Fördermitteln bei der Landesregierung für die Hochwasserschutzmaßnahmen der Stadt Köln betraut.

${ }^{529}$ HWSK 1996

${ }^{530}$ Artikel: Im Sommer neues Konzept, Kölner Stadtanzeiger, 01.02.1995.

${ }^{531}$ Stadt Köln, Drucksache 1141/095, Anlage 5, 16.11.1995.
} 
entstehenden Kosten mit einem Kompromiss. Denn nur mit dem MURL und der Bezirksregierung abgestimmte Verwaltungsvorschläge waren genehmigungs- und zuwendungsfähig, doch ,letztlich hat die Stadt Köln in eigener Selbstverantwortung zu entscheiden, gegen welches Hochwasserereignis die Hochwasserschutzanlagen bemessen werden sollen“ ${ }^{532}$. Nach lokaler Einigung gelang es der Stadt, die beiden höheren Instanzen davon zu überzeugen, dass „das historische Stadtbild durch den Bau von drei Meter hohen

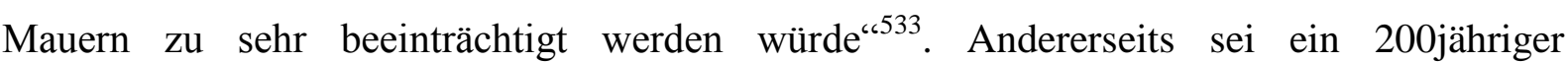
Hochwasserschutz aus technischen und finanziellen ${ }^{534}$ Gründen mit Spundwänden nicht durchgehend umsetzbar ${ }^{535}$. Für größere Einstauhöhen „,wären umfangreiche geologische Voruntersuchungen erforderlich“, welche eine ,erhebliche Kostensteigerung und Zeitverlust zur Folge hätten“‘536. Aufstockungen des vorhandenen Schutzes wären häufig technisch unmöglich und forderten den Neubau der kompletten Anlage, was deutlich zu teuer wäre ${ }^{537}$. Die Stadt setzte darauf, dass neue Retentionsräume am Oberrhein zukünftige Hochwasserwellen mildern würden. Der Ausschuss für Tiefbau und Verkehr beantwortete die Frage der Fraktion der SPD $^{538}$ im Rat der Stadt Köln im direkten Bezug zu dem dringenden und angemahnten Nachholbedarf im Hochwasserschutz in Köln mit einem Verweis auf die Überregionalität desselben ${ }^{539}$. Dezernent Oelmann erläuterte die Rückhaltebecken im Gefüge der lokalen Maßnahmen im August $1997^{540}$. „Ein absoluter Hochwasserschutz sei weder finanzierbar noch möglich ${ }^{541 ،,}$, bestätigte der Landtag bereits im Oktober 1995. Zwei Jahre später gab sich der Landtag aufgrund der Ergebnisse der Internationalen Kommission zum Schutz des Rheins (IKSR) überzeugt, dass nach Einrichtung der Retentionsräume Hochwasser mit seltenem Auftreten in Köln zukünftig kaum mehr durch den Oberrheinausbau beeinträchtigt sein würden ${ }^{542}$. Theoretisch seien also keine höheren Schutzmauern in Köln nötig. Deshalb wurde beschlossen, lediglich Anlagen mit „gravierendem Umweltgefahren“

\footnotetext{
${ }^{532}$ Landtag NRW, Drucksache 12/248, 11.10.1995.

${ }^{533}$ HWSK 1996, 86.

${ }^{534}$ Finanzielle Gründe wurden im Stadtrat nur vom Ausschuss für Tiefbau und Verkehr an dieser Stelle genannt (Stadt Köln, Drucksache 1141/095, Anlage 7, 30.11.1995).

${ }^{535}$ Aus den Kostenerwartungen für die einzelnen Bauabschnitte geht hervor, dass $1.000 \mathrm{~m}$ Spundwand etwa 4,25 Mio. DM kosteten, während der Aufwand für eine Verbesserung oder den Neubau von Deichen, Wänden und Mauern zwischen 2,27 und 2,5 Mio. DM pro 1.000 m Strecke liegen sollte (HWSK 1996, 98-146). In ganz NRW war damals bereits ein 200jähriger Hochwasserschutz implementiert (Stadt Köln, Drucksache 1141/095, Anlage 7, 30.11.1995).

${ }^{536}$ Oberstadtdirektor Ruschmeier erklärte in seinem Antwortschreiben an die Bürgervereinigung Rodenkirchen, weshalb die Schutzhöhen nur auf 11,30 m K P festgelegt wurden (Stadt Köln, Drucksache 1141/095, Anlage 7, 30.11.1995, Anlage II).

${ }_{537}^{537}$ Ebd.

${ }^{538}$ Stadt Köln, Ducksacke 1141/095, Anlage 6, 30.11.1995.

${ }^{539}$ Stadt Köln, Ducksacke 1141/095, Anlage 8, 18.01.1996.

${ }^{540}$ Landtag NRW, Drucksache 12/2452, 07.10.1997.

${ }^{541}$ Landtag NRW, Drucksache 12/248, 11.10.1995, 3.

${ }^{542}$ Es wird eine Tabelle aufgeführt, die zeigt, dass 50- und 100jährige Hochwasser dann immer noch einen erhöhten Wasserstand von 0,1-0,15 m in Köln zur Folge hätten, während 100- und 200jährige Hochwasser nur noch mit einer Pegelerhöhung von 0-0,05 m in Köln auflaufen würden (Landtag NRW, Drucksache 12/2452, 07.10.1997).
} 
sowie die Deiche, die ohnehin modernisiert werden müssten, und ferner Anlagen, welche keine technische Unsicherheit herstellen, mit einem 200jährigen Schutz zu versehen ${ }^{543}$. Die restliche Strecke wurde ebenfalls erhöht. Der ehemalige Schutz gegen Ereignisse mit einer statistischen Retour von allen 40 Jahren mit 10 m Pegelhöhe wurde auf alle 100 Jahre mit 11,30 m K P erweitert. Wie es zu dieser Wende, die Ruschmeier antizipierte, kam, soll im Folgenden dargelegt werden.

\subsubsection{Bewertung des Diskurses aus der Kosten-Nutzen-Perspektive}

Die Diskussion wird in diesem Abschnitt bezüglich der beteiligten Akteure, der betrachteten Alternativen sowie der Bewertung spezifischer Grenzen und Konsequenzen erhellt. Dazu werden die Argumentationslinien und die Konfliktpunkte aufgegriffen. Die Diskussion zur Erhöhung der Schutzlinien wurde zwischen dem Stadtrat und der Landesregierung geführt. Die Landesregierung NRW richtete sich nach normativen Vorgaben. Sie fokussierte keine Kosten-Nutzen-Analysen im Einzelfall, sondern sah einen Vorteil darin, im ganzen Land einheitlich zu verfahren. Sie wollte in dem gesamten Bundesland einen einheitlichen Hochwasserschutz durchsetzen, mit der Erwartung, Ereignisse mit einem statistischen Zyklus von 200 Jahren abzuwehren. Der Regierungspräsident zu Köln, Antwerpes (CDU), hatte die Funktion, die Vorschläge Kölns und die Forderungen der Landesregierung miteinander zu vermitteln. Er favorisierte die Kölner Vorschläge, keinen Hochwasserschutz für Ereignisse mit einer statistischen Wiederkehr von 200 Jahren $\mathrm{zu}$ installieren und verwies dabei auf finanzielle Hemmnisse. Eine Kostenvergleichsrechnung bot die Grundlage seiner Position. In dem Ausschuss für Tiefbau und Verkehr wurde ein Kompromiss aus einer Erhöhung der Schutzmauer und einer Kampagne für die Einrichtung von Retentionsräumen zur Abmilderung von Hochwasserwellen ausgearbeitet. Das Spannungsfeld aus Kosten, Schutzbedürfnissen und Ursachen wird in der Schutzhöhendiskussion der Stadt deutlich. Die möglichen Schutzstrategien können in einen Sicherheits- und einen Risikoansatz unterschieden werden ${ }^{544}$. Das Kernkonzept des Risikoansatzes bedeutet, der Bevölkerung keinen kompletten Schutz zu versprechen, sondern auf verbleibende Risiken aufmerksam zu machen. Das Konzept basiert auf der Grundfrage, welches Maß an Sicherheit zu welchem Preis geleistet werden kann. Eine Risikoanalyse der Eintrittswahrscheinlichkeiten und des potentiellen Schadensausmaßes bildet die Grundlage der Risikobewertung. Dazu werden Eintrittswahrscheinlichkeiten über niedrige, mittlere und hohe Wiederkehrzeiten berechnet.

\footnotetext{
${ }^{543}$ Stadt Köln, Drucksache 1141/095, Anlage 7, 30.11.1995.

${ }^{544}$ Wagner 2008, 775. Dieser, sowie die folgenden Sätze.
} 
Der dieser Methode gegenüberstehende Sicherheitsansatz bezieht nur mittlere und hohe Wiederkehrzeiten ein und ist folglich auf eine Betrachtung der kleineren Hochwässer beschränkt. In einem dritten Schritt werden die Möglichkeiten der Risikoreduktion festgelegt. Ausgerichtet ist der Risikoansatz an der Vorsorge, um damit mögliche Schäden präventiv zu umgehen. Dazu muss interdisziplinär gearbeitet werden. Die Betrachtung nur einer Fachdisziplin kann nicht hinreichen, wenn mehr als nur hohe Deiche gebaut werden sollen. Dazu richtet sich der Mitteleinsatz an einer Prioritätenauflistung aufgrund der Effizienz und Effektivität der Teilprojekte aus. Teilprojekte werden bewertet und nicht sektorbezogen abgearbeitet. Daraus folgen unterschiedliche Sicherheitsniveaus. Da unterschiedliche Standards aus dem Risikomanagement resultieren, ist die Beteiligung der Bevölkerung zentraler Bestanteil des Hochwasserschutzes. Tabelle 2 zeigt eine Abgrenzung des Risikoansatzes gegen den Sicherheitsansatz:

\begin{tabular}{|c|c|c|}
\hline & Risikoansatz & Sicherheitsansatz \\
\hline Basisorientierung & $\begin{array}{l}\text { „Welche Sicherheit zu welchem } \\
\text { Preis?“ }\end{array}$ & $\begin{array}{l}\text { „Auf welche Art und Weise können wir uns } \\
\text { schützen?“ }\end{array}$ \\
\hline Ausrichtung & präventiv & nachsorgend \\
\hline Aktivitätenplanung & interdisziplinär & spezifisch \\
\hline Mitteleinsatz & Prioritätenliste & sektorbezogen \\
\hline $\begin{array}{l}\text { Eintrittswahrscheinlichkeiten } \\
\text { der einbezogenen Ereignisse }\end{array}$ & seltene bis häufige Ereignisse & $\begin{array}{l}\text { seltene Ereignisse werden nicht mit } \\
\text { einbezogen. }\end{array}$ \\
\hline
\end{tabular}

Tabelle 2: Abgrenzung des Risikoansatzes gegenüber dem Sicherheitsansatz. ${ }^{545}$

Der bevorzugte Risikoansatz geht aus dem Antwortschreiben des Oberstadtdirektors

Ruschmeier an den Bezirk Rodenkirchen hervor:

„Ich verstehe voll und ganz die Sorgen der betroffenen Bürgerinnen und Bürger in den Überschwemmungsgebieten, denn für sie bedeutet jedes Hochwasser eine persönliche Katastrophe, verbunden nicht nur mit erheblichen materiellen Auswirkungen, sondern vor allem auch mit dem Vertrauensverlust in die Sicherheit der eigenen Lebensumstände. Das Vertrauen in die Sicherheit ist ein sehr hohes Gut. Unabhängig von der tatsächlichen Gefährdung wird durch die Angst vor erneuter Bedrohung durch extreme Hochwasser die Lebensqualität beeinträchtigt, teilweise mehr noch als durch einen tatsächlich eingetretenen Schaden. [...]. Bisher beträgt der vom Rat der Stadt Köln festgelegte Hochwasserschutz für den angesprochenen Bereich 10,69 m Kölner Pegel. [...]. Die in ihrem Anschreiben angesprochenen Gefährdungen beruhen auf einem möglichen höheren Wasserstand. [...] das Rheinwasser kann hier aber mittels Sandsackdamm ohne weiteres bis zu einem Wasserstand von knapp über 11,00 m Kölner Pegel gehalten werden. [...]. Es darf nicht übersehen werden, daß es einen absoluten Schutz vor Hochwasser

\footnotetext{
${ }^{545}$ Ebd.
} 
nicht geben kann. [...] alle staatliche Vorsorge kann dem Einzelnen nicht die Verantwortung für sein persönliches Umfeld abnehmen.“ ${ }^{546}$

Das natürliche Gefahrenbewusstsein wach zu halten, wurde zur Grundlage des Hochwasserschutzkonzeptes. Der Bürger wurde als einflussreicher Akteur erkannt, denn seither wurden Bewohner bedrohter Gebiete adressiert und aufgefordert, sich aktiv zu beteiligen. Damit hat die Stadt Köln die gesellschaftliche Konnotation des Katastrophenbegriffs erkannt. Das Konzept der Naturkatastrophe besteht generell aus zwei Teilen. Die Kraft der Natur erzeugt durch die Zerstörung gesellschaftlicher Werte einen Schaden. Somit kann die Schadenshöhe durch den bewussten Umgang der Gesellschaft mit ihren Besitztümern reduziert werden. Der „passive Hochwasserschutz“ in Form von Broschüren der Architekten- und Ingenieurkammer, adressiert an Eigentümer in Überschwemmungsbieten, welche nicht wirtschaftlich sinnvoll durch bauliche Maßnahmen geschützte werden können ${ }^{547}$, unterstützt den privaten Selbstschutz. Neben den Instrumenten der Risikoanalyse, wie beispielsweise der Erstellung von Hochwassergefahrenkarten ${ }^{548}$, fokussierte die Hochwasserschutzzentrale die Öffentlichkeitsarbeit. So entstanden Mitte der 1990er Jahre ein Film ${ }^{549}$, ein Modell zur Flussbegradigung ${ }^{550}$, ein Beitrag in einem Kindermagazin $^{551}$, eine Ausstellung sowie ein Schilderpfad für Fahrrad- und Wandertouren. Vorträge dienten als Appelle an das Gefahrenbewusstsein ${ }^{552}$. Auf Hochwassermessen konnten sich Betroffene erkundigen oder zum privaten Schutz ausstatten ${ }^{553}$. Der hohe Anspruch an die Öffentlichkeitsarbeit wurde durch die Ausstellung des Kölner Hochwasserschutzes auf der Expo 2000 in Hannover ausgezeichnet. Hochwasserschutz stand fortan in Interaktion mit den Bürgern.

Eine weitere Möglichkeit, den Hochwasserschutz und die allgegenwärtige Hochwassergefahr mit den Bürgern in Verbindung zu bringen, barg auch der bauliche Hochwasserschutz ${ }^{554}$. Darunter fielen 1996 Wände, Mauern, Deiche sowie die tragbaren Elemente. In dem Hochwasserschutzkonzept summierten sich die Kosten für den verbauten rechts- ${ }^{555}$ und

\footnotetext{
${ }^{546}$ Stadt Köln, Drucksache 1141/095, Anlage 7, 30.11.1995, Anlage II.

${ }^{547}$ Ebd., Anlage I.

${ }^{548}$ Internetbasierte Quelle 3.

${ }^{549}$ Der Film erschien unter dem Namen „Hochwasser was tun?““.

${ }^{550}$ Das Modell wurde von der RWTH Aachen als Wanderausstellung für die Hochwassernotgemeinschaft Rhein erarbeitet.

551 „Sendung mit der Maus“.

552 „Sensibilisierung der Bevölkerung für den Hochwasserschutz“ (Reinhard Vogt, 13.-14.12.2000).

${ }^{553}$ Die 1. Hochwasserschutz-Messe in Köln am 16.11.1996 wurde durch die Interessengemeinschaft „Altstadt, Kölner Bürgervereinigung gegr. 1995“ organisiert, ebenso die 2. Kölner Hochwasserschutz-Messe am 01.11.1997 mit der Fachtagung „Mit dem Hochwasser leben“.

${ }^{554}$ Köln verfügt über 67,4 km Uferstrecke, von welcher 1995 bereits $27 \mathrm{~km}$ mit Schutzanlagen ausgestattet waren. Davon bildeten zu diesem Zeitpunkt 16 km Flussdeiche und 11,4 km Schutzwände (HWSK 1996).

${ }_{555}$ Auf der rechten Rheinseite wurden 30,6 Mio. DM in bauliche Schutzvorrichtungen investiert (Ebd.).
} 
linksrheinischen ${ }^{556}$ Schutz auf 124,3 Mio. DM ${ }^{557}$. Eine Gruppierung hinsichtlich der Bautypen und Kosten kann den potentiell zu erwartenden Diskussionsumfang erhellen. Die verschiedenen Schutzvorrichtungen können in die Kategorien „Sofortmaßnahmen“, „Mobiler Schutz“ sowie „Neubau und Verbesserung ${ }^{558}$ fester Anlagen“ gegliedert werden ${ }^{559}$. Die folgende Tabelle 3 bietet auf der nächsten Seite einen nach diesen Kriterien gebildeten Überblick.

\begin{tabular}{|l|l|l|l|l|}
\hline & Anzahl & Kosten & Strecke & Kosten/Strecke \\
\hline Sofortmaßnahmen & 2 & 4,5 Mio. DM & $4,3 \mathrm{~km}$ & $1,05 \mathrm{Mio} . \mathrm{DM} / \mathrm{km}$ \\
\hline Mobiler Schutz & 6 & 15,5 Mio. DM & $3,65 \mathrm{~km}$ & $4,25 \mathrm{Mio} . \mathrm{DM} / \mathrm{km}$ \\
\hline Neubau & 13 & 50,9 Mio. DM & $20,43 \mathrm{~km}$ & $2,48 \mathrm{Mio} . \mathrm{DM} / \mathrm{km}$ \\
\hline Verbesserung & 23 & 59,2 Mio. DM & ca. $26 \mathrm{~km}$ & $2,28 \mathrm{Mio.} \mathrm{DM} / \mathrm{km}$ \\
\hline
\end{tabular}

Tabelle 3: Kostenübersicht für bauliche Maßnahmen 1994 in Köln. ${ }^{560}$

Sofortmaßnahmen erschienen nötig, daher wird keine kontroverse Diskussion erwartet. Neubau- und Verbesserungsmaßnahem beziehen sich auf die Errichtung von festen Mauern oder Deichen. Hier summierten sich durch lange Strecken hohe Investitionen, die allerdings aufgrund des relativ moderaten Preises, der Zuschüsse durch Land und Bund sowie der bedrohlichen Lage der Stadt keine scharfe Diskussion vermuten lassen. Im Vergleich waren die tragbaren Wände fast doppelt so teuer in der Anschaffung und müssen kontinuierlich gelagert, gepflegt und aufgebaut werden. Das System bestand aus nur zwei Komponenten: Senkrechte Stäbe hielten horizontale Elemente, welche der Hochwasserprognose angepasst werden konnten.

Der Hochwasserbeauftragte der Stadt Köln, R. Vogt ${ }^{561}$ durfte bereits zu Beginn der 1980er Jahre an einer Jahre an einer Übung zum Aufbau einer tragbaren Wand in Kirn teilnehmen ${ }^{562}$. Dort lernte er die die Vorzüge der mobilen Elemente kennen. Neben dem Erhalt des direkten Zugangs und Blickkontakts zu

\footnotetext{
${ }^{556}$ Auf der linksrheinischen Seite sah das Konzept eine Investition von 93,7 Mio. DM in feste Hochwasservorrichtungen vor (Ebd.).

${ }^{557}$ Ebd., 126; 146.

${ }^{558}$ Dazu zählen Sanierung, Verstärkung und Erhöhung vorhandener Anlagen.

${ }^{559}$ Eigene Klassifizierung.

${ }^{560}$ Zusammenfassung des HWSK 1996, 98-146.

${ }^{561}$ Reinhard Vogt (parteilos) betreute das Hochwasser seit 1978. Damals war er als Sachbearbeiter im Dezernat Tiefbau und Verkehr für die Kanalisation und Kläranlagen der Stadt Köln zuständig. In dieser Position initiierte er eine Hochwasserertüchtigung der Klärwerke. Erst nach dem Hochwasser von 1993 wurde eine ganze Stelle zur Betreuung des Hochwassers in der Stadtverwaltung geschaffen. Diese Stelle bekleidete Vogt. Im Januar 1995 wurde die erste Hochwasserschutzzentrale in Köln eingerichtet. Diese leitete Vogt. Wenige Tage später forderte das Jahrhundertereignis die Kompetenz der neuen Einrichtung heraus.

${ }^{562}$ Interview Reinhard Vogt, Leiter der Hochwasserschutzzentrale Köln, 09.08.2012 Köln. Kirn liegt an der Nahe in Rheinland-Pfalz. Das Konzept in Kirn beruhte auf dem Aufbau der Wand durch die Anwohner selbst. Die Intention, Hab und Gut zu schützen, personalisierte an der Nahe die Schutzmauerverantwortung. Ausreichend Helfer zeigten Bereitschaft, die Kenntnisse zum Aufbau zu erlernen, und die Anwohner mussten keine Sabotage fürchten.
} 
Blickkontakts zu dem Fluss steigerte die Übung das Gefahrenbewusstsein der Anwohner. Bereits seit 1984 Bereits seit 1984 war die erste tragbare Wand in Köln einsatzbereit und kam $1988 \mathrm{zum} \mathrm{Einsatz}^{563}$. Nach Einsatz $^{563}$. Nach dem Ereignis von 1995 wurden zu den bereits vorhandenen mobilen Schutzwänden von Schutzwänden von 1,4 km Länge zusätzliche $3,65 \mathrm{~km}$ angeschafft ${ }^{564}$. Das entspricht einem Zuwachs von Zuwachs von 260 Prozent. Die tragbaren Schutzvorrichtungen fanden offenbar - trotz zwei weiterer weiterer Einschränkungen - großen Anklang und überzeugende Fürsprecher. Die technische Grenze von Grenze von 2 m Höhe führte 1996 zu dem Entschluss $^{565}$, „wann immer möglich, feste [...] Anlagen Anlagen vorzuziehen, und diese gegebenenfalls mit mobilen Anlagen zu ergänzen“6566. Das Argument bot Argument bot die Chance, auf einer langen Strecke mit geringer Höhe Spundwände anzubringen und anzubringen und parallel bestehende Anlagen beizubehalten, statt neu investieren zu müssen.

Kostenmäßig kam diese Lösung auch der Bezirksregierung entgegen. Dazu galten mobile Elemente in der Elemente in der Aufbauphase $^{567}$ als personen- und zeitintensive Herausforderung ${ }^{568}$. Die Bildung einer Bildung einer Personengruppe, welche sich bereit erklärte, statt im Notfall ihr eigenes Hab und Gut zu und Gut zu sichern, bei dem Aufbau zu helfen sowie regelmäßig Übungen zu besuchen, bildete den bildete den Grundbedingung des Systems. Genau diese Schwachpunkte konnten als Instrumente der Instrumente der Aufklärung über die Hochwassergefahr genutzt werden.

Abb. 17 zeigt eine solche Spundwand nach Einsatz der mobilen Elemente.

\footnotetext{
${ }^{563}$ Reinhard Vogt, Leiter der Hochwasserschutzzentrale Köln, 09.08.2012 Köln. Die neue mobile Hochwasserschutzwand ist aus festem Zinkblech (Artikel: Metallwand trennt die Altstadt von der Flut, Kölnische Rundschau, 28.03.1988). „Die Investition nach der Flut vor fünf Jahren hat sich bisher gelohnt“ (Ebd.). Der mobile Schutz hat 2 Millionen DM gekostet, welche Oberbaurat Kurt Loose konstruiert hatte (Artikel: Hochwasserschutzwand gestern wieder abgebaut, Kölnische Rundschau, 07. April 1988). „Am Dienstagnachmittag begann die Polizei Teile der Altstadt zu sperren, um die neugierigen Hochwassertouristen von den Schutzvorrichtungen fern zu halten, denn Unbekannte hatten versucht, Schrauben aus den Stahlwänden herauszudrehen" (Artikel: Hochwasser ließ die Altstadt bis in die Nacht bangen, Kölnische Rundschau, 29.03.1988). Insgesamt waren 160 Polizeibeamte im Einsatz, die Hälfte von ihnen schützte den Rheingarten und die Hochwasserwand (Artikel: Polizeischutz für die Schutzwand, Kölnische Rundschau, 30.03.1988). Die Stadtverwaltung rechnete aufgrund des Aufwands der städtischen Verwaltung mit einer Million Kosten (Artikel: Zentimeter trennte die Altstadt von Überflutung, Kölnische Rundschau, 30.03.1988). Von der Summe ,entfällt der größte Teil auf Firmen, die im Auftrag der Stadt die Schutzwände auf- und abbauen, den Rheinufer-Tunnel sichern oder Bürger in Booten befördern“ (Artikel: Auf- und Abbau der Schutzmauer ist am teuersten, Kölnische Rundschau, 30.03.1988). Wobei „,der Auf- und Abbau genauso teuer ist, wie die tagelange Bewachung“ (Ebd.). Die Hochwasserschutzmauer blieb über das Osterwochenende für die Touristen stehen: „Wir sind ein wenig stolz darauf, Und die Touristen sollen sie ruhig einmal anschauen“, erklärte Oelmann (Artikel: Vater Rhein zieht sich weiter in sein Bett zurück, Kölnische Rundschau, 30.03.1988).

${ }^{564}$ HWSK 1996, 96.

${ }^{565}$ Aufgrund des Wasserdrucks, der in Abhängigkeit von der Höhe zunimmt, wurde die technische Grenze auf maximal $2 \mathrm{~m}$ Höhe festgelegt.

${ }^{566}$ HWSK 1996, 87.

${ }^{567}$ Internetbasierte Quelle 2: Zwei Personen benötigen eine Stunde, um über eine Länge von 100 Metern eine Schutzhöhe von 1,5 $\mathrm{m}$ zu errichten.

${ }^{568}$ Ebd.
} 


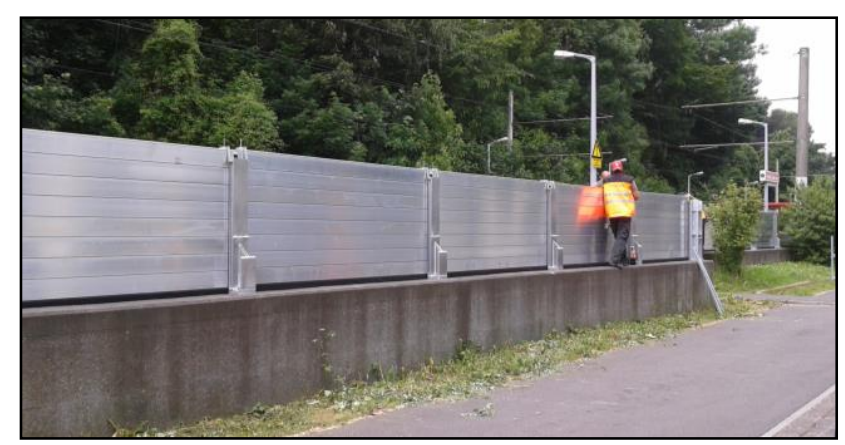

Abb. 17: Mobile Hochwasserschutzwand am 01. Juni 2012.

In der Folgezeit entwickelten sich die Spundwände zu einem öffentlichkeitswirksamen Kommunikationsinstrument: Die regelmäßigen Testaufbauten zum Trainieren der Helfer wurden in der Tat nicht nur auf möglichst viele Termine verteilt ${ }^{569} . \mathrm{Zu}$ den Übungen wurde die immerwährende Gefahr des Hochwassers sogar in einen Festtag transformiert. Neben den städtischen Dienststellen, DLRG, THW und der Feuerwehr, organisierte das Hochwasserteam Pressekonferenzen, Fachtagungen, Infozelte und Fototermine.

Um die Logistik der tragbaren Einzelteile im Hochwasserfall zu gewährleisten, wurde eine praktische Verteilung der Baukörper nötig. So wurden acht Lagerhallen für die mahnenden ${ }^{570}$ Schutzvorrichtungen entlang des Rheins von Süden nach Norden eingerichtet. Auch wenn die Magazine aufgrund der vorhandenen Bebauung nicht direkt an der Straßenfront installiert wurden, waren sie durch ihre Erscheinung im Gespräch der lokalen Bevölkerung. Im Zusammenhang mit den tragbaren Elementen wurden ebenso die Lagerhallen instrumentalisiert, das Gefahrenbewusstsein der Bevölkerung aufrecht zu erhalten. Auch diese Gebäude dienten offensichtlich als Kommunikationsinstrument, unter anderem indem sie im Laufe der Zeit mit Architektenpreisen ausgezeichnet wurden ${ }^{571}$. Bereits die Beschreibung ${ }^{572}$

\footnotetext{
${ }^{569}$ Internetbasierte Quelle 3. Testaufbauten wurden durchgeführt am 21.09.2007, 14.05.2009, 03.09.2009, 24.08.2010, 23.09.2011, 01.06.2012.

${ }^{570}$ Die mobilen Wände bezeichnet die Autorin als mahnend, da sie die Kölner Bevölkerung der Hochwassergefahr mahnen.

${ }^{571}$ Die Lagerhalle „hw rod“ wurde 2009 am Heinrich-Lübke-Ufer erbaut, später wurde die Halle als Lagerstätte für Hochwasserschutzelemente ausgezeichnet: ,wieweiterarbeiten - Arbeits-Orte der Zukunft“, Auszeichnung der Bundesstiftung Baukultur 2012 (Internetbasierte Quelle 1), Architekturpreis NRW, 2011. Kölner Architekturpreis, 2010 (Internetbasierte Quelle 5).

572 „Da das Grundstück für Halle plus Lkw-Wendeplatz eigentlich etwas zu klein war, wurde die bestimmende Entwurfsidee umgemünzt. Statt vor der Halle zu wenden, ließ man die Lkws die Halle umrunden und mit ihrer Schleppkurve die amorphe Form bestimmen. Das Ziel, alle größeren Bäume unversehrt zu erhalten, setzten die Architekten um, indem drei der Bäume in Höfen ins Gebäude integriert wurden. Nun überragen ihre Baumkronen die Halle deutlich und erzeugen die Illusion eines Waldes hinter der Hallenfassade - die Halle selbst wirkt wie eine
} 
der Halle „hw rod“ widmete sich beispielsweise einem himmlischen Schutzbau in Einklang mit allem Natürlichen und Gottgegebenen.

Abb. 18 zeigt links den Grundriss der prämierten Lagerhalle „hw rod“ und rechts eine Innenaufnahme derselben.

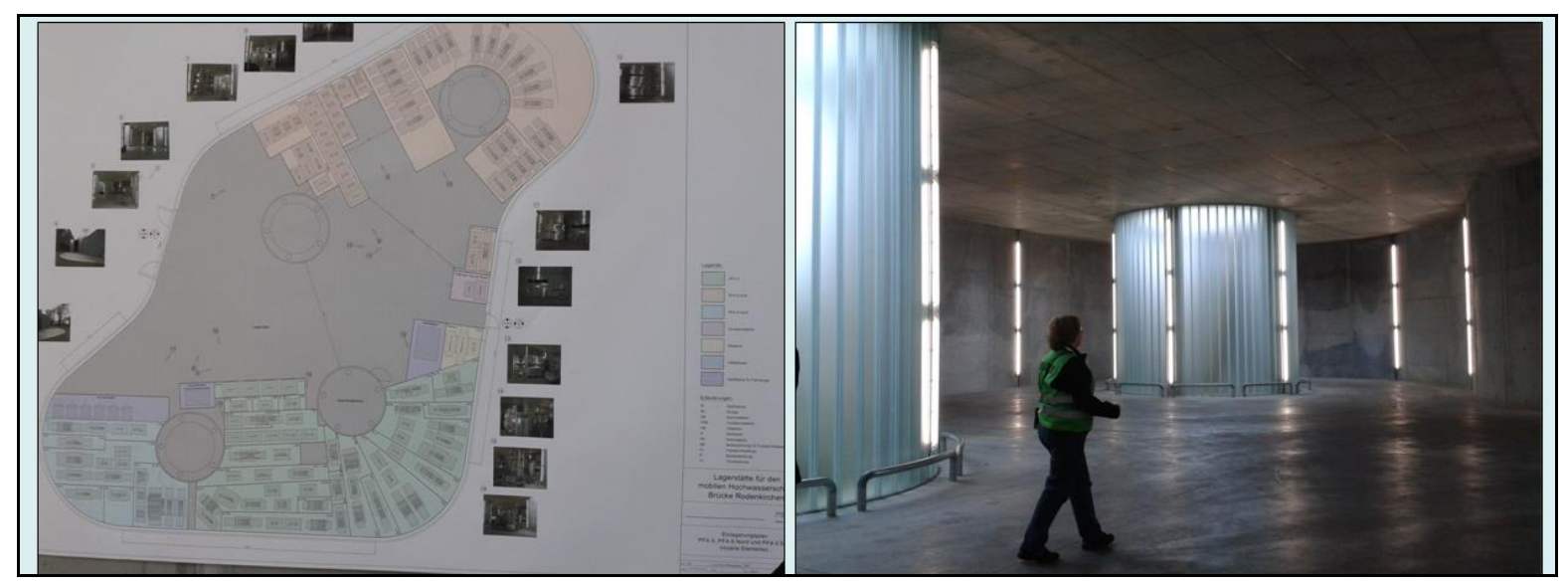

Abb. 18: Lagerhalle der mobilen Hochwasserschutzelemente.

Tragbare Wände und ihre Lagerhallen wurden also auch während der hochwasserfreien Zeit geschickt genutzt, indem durch diese regelmäßig an die kontinuierliche Gefahr erinnert wurde. Obwohl der mobile Hochwasserschutz mit Abstand die teuerste Variante abbildete, wurde die Kölner Wand fortan zur weltweit ${ }^{573}$ längsten ${ }^{574}$ mobilen Schutzwand weiterentwickelt. Der Schutz durch die mobilen Wände gehörte den Maßnahmen der Stadtentwässerung an. Eine Diskussion um eine Investition musste nicht in der Verwaltung der Stadt Köln geführt werden; stattdessen wurde die Anschaffung und Unterhaltung der Schutzvorrichtungen teilweise über die Abwassergebühren der Kölner Bevölkerung ${ }^{575}$ sowie durch die neuen Finanzierungsinstrumente nach der Gründung der Stadtentwässerungsbetriebe Köln AöR finanziert ${ }^{576}$.

In dem folgenden Absatz wird dargelegt, ob in der Abstimmungsphase Handlungsalternativen betrachtet wurden. Über die Erhöhung der Schutzhöhen wurde intensiv debattiert. Diesmal

\footnotetext{
Palisade. Der skulpturale Baukörper ist als fensterloser ca. 6 Meter hoher Monolith aus Beton ausgeführt, der ausreichend Schutz für die lagernden Elemente bietet. Da der Monolith im dichten Baumbestand liegt, ist auch seine Oberfläche von einem bestimmenden Motiv des Waldes geprägt, dem ständigen Wechsel von Licht und Schatten, der durch eine Stahlschalung mit Sinuswelle erzeugt wird. Da diese Welle auch für die Verkleidung der Tore verwendet wurde, läuft sie ohne Unterbrechung rund um die Halle herum. Im Inneren der Halle ist keine stützende Statik sichtbar. Diese ist in den vier zylindrischen Höfen versteckt, die mit ihrer Gussglashaut die Halle wie riesige Laternen in ein mildes Licht tauchen und eine annähernd sakrale Stimmung erzeugen. Die senkrechten Neonstreifen an Wänden und Höfen vervollständigen diese Wirkung“ (Internetbasierte Quelle 5).

${ }^{573}$ Der Hersteller IBS aus Thierhaupten in Bayern erhielt 2007 den Ingenieurspreis für Wasser-Bau-Werke der Bayerischen Ingenieurkammer (Internetbasierte Quelle 2).

${ }_{575}^{574}$ Die weltlängste mobile Hochwasserschutzwand wurde mit 9,3 km Länge im Jahr 2007 in Köln erreicht.

${ }^{575}$ Reinhard Vogt, Leiter der Hochwasserschutzzentrale Köln, 17.12.2011, Köln.

${ }^{576}$ HWSK 1996, 158.
} 
konnte die Stadt nicht wie 1960 und 1983 das Maß der Erhöhung selbst bestimmen. Andernfalls wäre eine neue Schutzhöhe in der Nähe der Pegelgrenze des Hochwassers von $10,96 \mathrm{~m}$ bei $11 \mathrm{~m} \mathrm{~K} \mathrm{P}$ sicher vorstellbar gewesen. Dieses Mal diktierten normative Zielvorgaben der übergeordneten Behörde ein Mindestmaß. Doch Köln schaffte es, die Landesregierung $\mathrm{zu}$ überzeugen, statt sich für ein Hochwasser von 200jähriger Wahrscheinlichkeit nur für eine Flut von einer 100jährigen Erwartung zu wappnen. Neben dem Argument, „das historische Stadtbild vor drei Meter hohen Mauern zu bewahren“577, führte die Kölner Verwaltung auch finanzielle Bedenken sowie technische Herausforderungen in ihrer Argumentation an. So viele Gründe wie möglich, sollten die gefürchteten Mauern verhindern. Abschließend ist festzuhalten, dass Handlungsalternativen zu der Erhöhung erwogen und diskutiert worden sind.

Als dritter und letzter Schritt wird die spezifische Kritik an der gewählten Lösung betrachtet. Ein Bewusstsein für die Grenzen der ausgewählten Handlungsoption kennzeichnet das Wissen um eventuelle Konsequenzen. Mit der Instrumentalisierung der Kommunikation zum Bewohner der durch Hochwasser bedrohten Gebiete manifestierte die Stadt Köln einen neuen Umgang mit der Hochwassergefahr. Ganz deutlich stellte sie sich der Erkenntnis, Hochwasser niemals völlig vermeiden zu können. Sie verband damit auch die Vorstellung, dass der Zeitraum nach einem Hochwasser dem Zeitraum vor einem Hochwasser entspricht. Mit dem Ausspruch; „Nach dem Hochwasser ist vor dem Hochwasser“ unterstrich Reinhard Vogt die Notwendigkeit, in ständiger Alarmbereitschaft zu bleiben. Mit dem daraus gewachsenen Bündel von Kommunikationswegen, wie z. B. der Instrumentalisierung des mobilen Hochwasserschutzes zur kontinuierlichen Ermahnung des Bürgers, ist deutlich zu erkennen, dass die spezifischen Grenzen der neuen baulichen Maßnahmen sehr ernst genommen wurden. Die Kölner Planungsverantwortlichen wussten, dass zukünftige Überschwemmungen nicht gänzlich verhindert, wohl aber der Umfang der künftigen Schäden durch alternative und komplementäre Handlungen zu lindern wäre. Wie dargelegt werden konnte, waren sich die Verantwortlichen über die Grenzen des Hochwasserschutzes und über die möglichen Konsequenzen bewusst. Der Entscheidungsprozess verlief daher nachvollziehbar.

\footnotetext{
${ }^{577}$ Ebd., 86.
} 


\subsection{Abschlussdiskussion zur Wahrnehmung des Kölner Stadtbildes}

In der Entwicklung der Argumentationslinien sind zwei Phasen erkennbar. Zunächst wurde der Hochwasserschutz als eine lokale Aufgabe betrachtet. Die Kölner Verantwortlichen agierten eigenmächtig und bauten den Hochwasserschutz, den sie für angemessen hielten. Dabei waren die Bautätigkeiten indirekt durch den Wiederaufbau nach dem Zweiten Weltkrieg beeinflusst. Besonders nach der Flut von 1955 wurde die Höhe der Überschwemmung in Anbetracht der Zerstörungen durch den Krieg relativiert. Das berühmte Stadtbild Kölns verkörperte durch die am Aufenthaltsort neben dem Rhein durchgeführten Traditionen Heimat und Wiedererkennung, welche unter allen Umständen bewahrt werden mussten. Auch wurden die Überflutungsstärken und -intervalle als natürlich wahrgenommen, eine Suche nach Ursachen und Schuldigen fand nicht statt. Obwohl die Stadt Köln seit der Währungsreform von zwei Hochwasserereignissen heimgesucht wurde, nämlich in den Jahren 1955 und 1970, kam es lediglich 1960 zu einer Reaktion auf allgemeine Hochwasserprobleme. Obzwar es kein unmittelbares Überschwemmungsereignis als Anlass gab, wurde eine Hochwassermauer gebaut. Den Auslöser stellte der als verheerend empfundene Straßenverkehr dar, damit war der Mauerbau offenkundig eine Reaktion auf ein Verkehrsproblem. Aber auch diese Begründung scheint nach näherer Betrachtung zweifelhaft. Wie zuvor gezeigt werden konnte, sollten vielmehr die Fußgänger durch die Mauer vor dem Verkehr geschützt werden, damit die Bürger in alter Tradition Heimat und Stadtbild zwischen Dom und Rhein genießen konnten. Unabhängig von einem Nutzen im Bereich des Hochwasserschutzes konnte die Hochwasserschutzmauer gebaut werden. Die Diskussionen spielten sich ausschließlich zwischen den Mitgliedern des Stadtrates und den der Verwaltung unterstellten Ausschüssen ab. Unabhängige Experten wurden nicht hinzugezogen. Die spezifischen Grenzen der Hochwasserschutzmauer wurden - wie gezeigt werden konnte vehement kritisiert, denn bis zur Entscheidung wurden die verschiedenen als Nutzen definierten Auswirkungen der Konstruktion, wie z. B. der Hochwasserschutz, in Frage gestellt. Alternativen zur Mauer wurden für einige Einzelwirkungen betrachtet, z. B. den Verkehr anders zu regeln. Da aber der Hauptzweck der Schutzmauer sich nicht aus dem Schutz vor Hochwassern ableitete, sondern durch eine Vielzahl von Nebenfunktionen, z. B. dem Schutz des Fußgängers vor Autounfällen, legitimiert wurde, konnte auch keine Alternative mit gleichen Eigenschaften gefunden werden. Die Diskussionsphase verlief verständlich und nachvollziehbar obwohl traditionelle Kosten-Nutzen-Analysen fehlten. In den Auseinandersetzungen wurden die Abwägungsvorgänge potentieller alternativer 
Handlungsmöglichkeiten sowie die Betrachtung der spezifischen Grenzen der ausgewählten Handlung, dem Bau der Hochwasserschutzmauer, deutlich, somit erfüllt die Diskussion die für diese Arbeit aufgestellten Kriterien der Rationalität. Zum Zeitpunkt des Beschlusses 1960 war den Verantwortlichen bereits bewusst, dass es auch Hochwasser geben werde, welche die Mauer übersteigen. Daher war es für die politisch Verantwortlichen keine Überraschung, als die Mauer nach sieben Jahren zum ersten Mal überströmt wurde. Dass diese Tatsache nicht allen Betroffenen bewusst war, spiegelt sich in den Streitigkeiten um Schadensersatz zwischen dem Stadtrat zu Köln und der Landesregierung NRW. Die Bürger hatten Vertrauen in die Hochwasserschutzmauer gefasst und lagerten nun Wertgegenstände in ehemals durch das Eindringen von Rheinwasser bedrohten Räumen. Die kommunalen Entscheidungsträger sahen das Versagen nicht im Hochwasserschutz begründet, vielmehr nahmen sie die Bewährung der neugebauten Schutzmauer wahr, die Mauer hielt bis zu ihrer Überströmung den Wassermassen stand. Für sie war das Übertreten der Mauer von Beginn an möglich. Sie verurteilten die Hochwasseropfer für die Höhe der Schäden selbst verantwortlich zu sein. Kritik am unzureichenden Schutz durch die öffentliche Hand fanden nicht statt - im Gegenteil. Die Mauer wurde 1970 durch die Kölner Verantwortlichen aus dem Tiefbauamt gelobt, denn sie zeigte augenscheinlich Wirkung. Das Lob wird nachvollziehbar, da die seit 1959 umstrittene Mauer sozusagen nachträglich rehabilitiert werden musste. Der Eingriff in das Antlitz der Stadt am Rhein musste auch 1970 noch vor der Bevölkerung legitimiert werden. Eine Mauererhöhung konnte aufgrund der umstrittenen Eingriffe im sensiblen Altstadtbereich nicht angesprochen werden. Bauliche Maßnahmen an der Promenade wurden tabuisiert. Das Hochwasserereignis von 1970 wurde im übertragenen Sinne instrumentalisiert, den Bau des Mäuerchens gut zu heißen. Aus der Perspektive der administrativen Ebenen wurde dieses Hochwasserereignis somit positiv besetzt und verlor seinen zerstörerischen Charakter. Deshalb wurden gegen das „positive Hochwasser“ von 1970 keine strategischen Maßnahmen ersonnen. Die Hochwassersicherheit konkurrierte weiterhin mit dem Wert der Stadtansicht. Eine Problemfeststellung konnte schlichtweg nicht stattfinden, somit musste auch keine Lösung ausgedacht und das Thema nicht in die Agenda ${ }^{578}$ der lokalen Politik eingebracht werden. Die spezifischen Grenzen der Hochwasserschutzmauer wurden aufgrund der Überschwemmungen im Februar 1970 den Anwohnern sowie den politisch Verantwortlichen vor Augen geführt. Diese wurden neben den Konsequenzen, binnen kurzem wieder eine Überschwemmung hinnehmen zu müssen, akzeptiert. Eben nicht mit neuen

\footnotetext{
${ }^{578}$ Kingdon 1995.
} 
Schutzvorrichtungen auf das Hochwasser zu reagieren, lässt sich aufgrund des bereits analysierten Verhaltens der Entscheidungsträger seit 1957 nachvollziehen.

In einer späteren Phase veränderte sich die Wahrnehmung des Hochwassers gänzlich. Seit 1983 wurde durch die Kölner Verantwortlichen an der Natürlichkeit der beiden FrühlingsHochwasser Zweifel geäußert. In einer Genese von der Dauer einer Dekade ist zu beobachten, wie sich die handelnden Akteure in Köln durch vier aufeinander folgenden Fluten in ihren Befürchtungen, die jüngsten Hochwasser seien durch die Eingriffe am Oberrhein entstanden, immer bestätigter fühlten. Zu Beginn der 1990er Jahre entwickelte die nordrhein-westfälische Landesregierung außerdem eigene Vorstellungen von einem landesweiten Hochwasserschutz, welcher in den Kommunen umsetzten werden sollte. Daraufhin verlor die Stadt Köln ihre bisherige Eigenständigkeit, das Maß des Hochwasserschutzes selbst zu bestimmen, und musste sich fortan mit der administrativen Ebene des Landes auseinandersetzen. Im Jahr 1983 überflutete der Rhein die Stadt im April wie im Mai mit zwei für die Jahreszeit ungewöhnlichen Hochwassern ${ }^{579}$. Obgleich die Hochwasserereignisse die Menschen schockierten, fanden sich die Verantwortlichen in den städtischen Gremien sowie die Bürger damit ab, dass es eben viel geregnet habe. Trotzdem wurde hervorgehoben, dass „so etwas noch nie vorgekommen sei““580. Die Verantwortlichen ergingen sich in den lokalen Kreisen in Überlegungen, wie die Stadt bei einem nächsten solchen Fall besser reagieren konnte. In der Diskussion involviert waren diesmal zudem die betroffenen Anwohner und Gewerbetreibende aus dem Überschwemmungsgebiet, sie waren durch die Bürgervereinigung Rodenkirchen sowie den Hotel- und Gaststättenverband vertreten. Neben den Hochwasseropfern setzte sich die CDU für die Erhöhung des baulichen Schutzes ein. Bedenken gegenüber der Erhöhung konnten durch ein Expertengutachten ausgeräumt werden. Nachdem die Stadtratsmitglieder 1960 keine negativen hydrologischen Auswirkungen durch die neue Mauer befürchteten, wurden 1983 erstmalig solche Zweifel geäußert und nur durch Expertenrat zerstreut. Die Auseinandersetzungen zur Erhöhung der Schutzvorrichtungen wurden innerhalb des Stadtrates ausgetragen, wo sich niemand mit einer kritischen Gegenstimme zu dem Vorhaben äußerte. Zum ersten Mal wurden mobile Elemente gegen das Hochwasser eingesetzt. Die kommunalpolitischen Zuständigkeiten betrachteten das Problem sehr selbstbewusst, denn sie beauftragten keine Experten, die tragbaren Teile anzufertigen. Diese wurden in Eigenarbeit durch das Amt für Brücken- und U-Bahnbau entworfen. Köln stellte ein Problem fest und trat

\footnotetext{
${ }^{579}$ Nachdem am 01.11.1979 der Pegel neu kalibriert wurde, wurde die Nulllinie um $1 \mathrm{~m}$ nach unten versetzt. Dies führte dazu, dass die Hochwasser von nun an alle $1 \mathrm{~m}$ stärker ausfallen. Daneben galt dies auch für den Hochwasserschutz, dieser lag seit 1979 bei 9,40 m K P statt wie bisher bei 8,40 m K P. Faktisch änderte sich an dem bestehenden Schutz nichts.

${ }^{580}$ Artikel: Ruhe und Gelassenheit, Kölnische Rundschau, 13.04.1983.
} 
diesem entgegen. Besonders auffällig ist hier die Unabhängigkeit, mit der die Kölner Behörden arbeiteten. Selbstständig orientierten sie sich an den normativ gemessenen Pegelhöhen der letzten beiden Monate. Sie vertrauten darauf, durch ihre selbstgebauten Vorrichtungen ein nächstes $10 \mathrm{~m} \mathrm{~K} \mathrm{P-Hochwasser} \mathrm{bezwingen} \mathrm{zu} \mathrm{können.} \mathrm{Nichtsdestotrotz}$ wurde der Hochwasserschutz noch als lokale Aufgabe wahrgenommen, dem mit ausschließlich technokratischen Maßnahmen begegnet wurde. Die natürliche Erklärung für die beiden statistisch betrachtet außergewöhnlichen Ereignisse unterstützte die als Panikreaktion auszumachende Handlung, mobile Elemente einer Schutzhöhe der gerade abgeflossenen Pegel eigenhändig zu bauen, ohne Bezug zu statistischen Auswertungen oder zu Hochwasserschutzexperten zu nehmen. Vielmehr hielten sich die Akteure selbst für Experten und waren der Ansicht, sich vor statistisch seltenen Hochwassern schützen zu können ${ }^{581}$. Eine Aufmauerung der vorhandenen Barriere wurde aufgrund der hohen Bewertung des Stadtbildes immer noch abgelehnt. Die geführten Diskussionen genügten den Merkmalen zur Rationalität, welche für diese Untersuchung herausgestellt wurden.

Das Hochwasser von 1993 ließ die Behörden in Köln ein überregionales Konzept fassen, welches nach der Überschwemmung von 1995 sinngemäß auf EU-Ebene institutionalisiert wurde. Ab 1993 zeichnete sich das Hochwasser in den Augen der Verantwortlichen Kölner als ein überregionales Problem ab. Die Ursachen des Weihnachtshochwassers wurden unmittelbar in menschlichen Eingriffen in die Natur gesehen. Dies blieb keineswegs eine Kölner Anschauung, auch die Umweltminister der Bundesländer des Saarlands und Nordrhein-Westfalens sowie der Bundesumweltminister begründeten die Überschwemmungen durch anthropogene Eingriffe in das Flusssystem. Die Diskussion wurde erstmalig aus ihrem lokalen Rahmen auf eine überregionale Ebene gehoben. Mögliche Lösungsvorschläge wie eine Kehrtwende in der Landschaftsgestaltung, die Verankerung des Bodenschutzgesetzes, und die Institutionalisierung des Dialoges der Umweltminister vorgeschlagen, sowie eine noch unkonkrete Empfehlung für eine überregionale Kooperation im Hochwasserschutz wurden entlang des Rheins diskutiert. Der Kölner Oberstadtdirektor Ruschmeier (CDU) gab verwaltungsintern das „Hochwasserschutzprogramm 2020“ in Auftrag, indem der Schwerpunkt auf ein übergreifendes Handlungskonzept der Rheinanlieger insgesamt gelegt werden sollte. Die erste ständige Hochwasserschutzzentrale wurde im Amt

\footnotetext{
581 Das Hochwasser von 1988 bestätigte augenscheinlich diese Einschätzung. Mit einem Pegel von 9,95 m K P blieb es nur fünf Zentimeter unter der Kante der mobilen Wand, welche zum ersten Mal zum Einsatz kam. Die Kölner Politiker fühlten sich in der Terminologie des Vulnerabilitäts-Diskurses ausgedrückt, unverwundbar. Trotz der Parierung des Hochwassers von 1988 verstärkte die Flut dennoch die Vermutung, dass Köln übernatürlich häufig von großen Hochwassern heimgesucht wurde. Ein Pegel von $10 \mathrm{~m}$ sollte statistisch betrachtet nur alle 40 Jahre eintreten. In den fünf Jahren zuvor wurde dieser Pegel jedoch drei Mal knapp erreicht.
} 
für Stadtentwässerung im Dezernat Tiefbau und Verkehr eingerichtet. Derweil formierte sich in der Kölner Politiklandschaft ein Widerstand, zukünftig weiter wie bisher mit der Bodenversiegelung fortzufahren. Die künftige Bebauung eines bisherigen Überschwemmungsgebiets wurde kritisiert. Dabei formulierte die CDU wie bereits 1963 das Dogma „mit dem Hochwasser zu leben“. Die neuen Handlungsmöglichkeiten wurden nicht als Alternativen zueinander sondern als Bestandteil eines kompletten Hochwasserschutzes betrachtet, der in den Augen der Kölner Meinungsführern einer alternativen Mauererhöhung vorzuziehen war. Die Option einer lokalen Mauererhöhung führten die Kölner Entscheidungsträger nicht in die Debatte ein, denn sie waren bereits der Ansicht, nur überregionaler Hochwasserschutz könnte die Bedrohung für die Stadt mildern. Die Debatte verschob sich von der Bekämpfung des Hochwassers zur Eindämmung der Hochwasserursachen. Am Beispiel dieser unterschiedlichen Verhaltensweisen ist erkennbar, dass sich die Wahrnehmung der Hochwasserursachen und der Umgang mit dem Hochwasserschutz im Laufe der Zeit veränderten. Deshalb entstand 1995 kein Abwägungsprozess zwischen den Vorteilen eines höheren Hochwasserschutzes und den Nachteilen durch eine eventuelle Beeinträchtigung des Kölner Stadtbildes. Damit wird ersichtlich, dass die Kölner Entscheidungsträger keine Alternative höher bewerteten, als Hochwasser bereits am Oberrhein durch verschiedene Maßnahmen vorzubeugen. Außerdem maßen sie den Konsequenzen einer Erhöhung ihrer eigenen Schutzvorrichtungen vor Ort keinen tatsächlichen Schutz bei. Die Forderung nach einer überregionalen Hochwasserursachenbekämpfung wird vor dem Hintergrund der als unnatürlich auf anthropogene Eingriffe in das Flusssystem beruhenden Schadensereignisse verständlich. Nur 13 Monate später beförderte das Jahrhunderthochwasser vom Januar 1995 die bereits zuvor begonnen Auseinandersetzungen. Das Ereignis besaß eine Katalysatorfunktion, denn der wiederaufflammende Streit katapultierte die Forderung nach einem überregionalen Hochwasserschutz auf die Ebene der EU-Politik. Anhand der Liste der sich an der Diskussion beteiligenden Akteure ist die interdisziplinäre und interregionale Hochwasserursachendeutung erkennbar. Neben den Kölner Behörden, den Umweltministern der Bundesländer, der Umweltministerin und dem Bundeskanzler beteiligten sich auch Bürger und NGOs aus den Bereichen des Boden-, Umwelt- und Naturschutzes an den Auseinandersetzungen. Die interdisziplinären und interregionalen Ansätze wurden in dem Hochwasserschutzkonzept 1996 der Stadt Köln aufgegriffen. Das Konzept entspricht der Fertigstellung des bereits 1993 begonnen „Programm Hochwasserschutz 2020“. Die fehlende Fertigstellung der Retentionsmaßnahmen aus dem Deutsch-Französischen Vertrag von 1982 stand im 
Mittelpunkt der Kölner Erklärungen für die Hochwasserschäden und bildete die Grundlage für die Kölner Forderungen nach einem überregionalen Hochwasserschutz. Alle Erklärungen für die Überschwemmung bezogen sich auf menschliche Eingriffe in das Einzugsgebiet des Rheins, obwohl ein Expertengutachten die meteorologischen Verhältnisse für das Hochwasser verantwortlich präsentierte. In Köln selbst entstand eine Debatte, die vorhandenen Schutzvorrichtungen seien nicht ausreichend und müssten verstärkt werden, denn die Landesregierung gab nicht nach, Köln aufzufordern, einen Hochwasserschutz nach den landeseinheitlichen Vorgaben einzurichten. Zum zweiten Mal nach 1983 wurde die Höhe des Hochwasserschutzes nach normativen Maßstäben ausgerichtet. $\mathrm{Zu}$ beachten ist hier, dass die Vorgaben erstmalig von außen gefordert wurden, denn weder 1960 noch 1983 gab die Landesregierung Standards für den Hochwasserschutz vor. Ein Aushandlungsprozess zwischen den Vertretern der Stadt, dem Regierungspräsidenten zu Köln und der Landesregierung entstand. Die kommunale Stadtverwaltung vertrat aufgrund der gesammelten Erfahrungen die Ansicht, dass immer ein Hochwasser über die vorhanden Schutzanlagen strömen könnte, sodass sich die Kölner Entscheidungsträger schlicht weigerten, die Stadtansicht für normative Vorgaben der Landesregierung zu opfern. Mit der Erfüllung der Minimalanforderung, den vorhanden Hochwasserschutz im ganzen Stadtgebiet von statistischen 40- auf 100jährige Ereignisse und nur in ausgewählten Zonen auf einen 200jährigen Schutz zu erhöhen, ließ sich die Stadt auf eine Verstärkung nach dem Risikoansatz ein. Bei diesem Ansatz steht die Frage, welcher Schutz zu welchem Preis gewählt werden soll, im Vordergrund. Deshalb ergänzten die Entscheidungsträger den baulichen Schutz um die Komponente „Erweiterung des Gefahrenbewusstseins der lokalen Bevölkerung“. Um ein Höhenniveau festzulegen, wurden Alternativen verglichen und mögliche Konsequenzen bewertet. Durch die Aufklärung der Anwohner nahmen die Verantwortlichen die Kritik an den neu eingerichteten Schutzhöhen vorweg, denn sie stellten öffentlich fest, dass kein Hochwasserschutz die totale Sicherheit bieten könne. Die Diskussionsphase zur Erhöhung des lokalen Hochwasserschutzes ist nachvollziehbar und verständlich. Sie beinhaltet alle Merkmale des Rationalitätsmodells und genügt den Kriterien, die für diese Arbeit festgelegt wurden. Köln konnte den Bau der geforderten hohen Schutzvorrichtungen in weiten Teilen abwenden, und damit wieder eine Beeinträchtigung des Stadtbildes verhindern.

Die beiden Phasen gehen im Laufe der 1970er Jahre ineinander über. Die technischen Veränderungen der hydrologischen Verhältnisse aufgrund der energetischen Wassernutzung 
des Rheins wurden abgeschlossen. Die letzte Staustufe von insgesamt zwölf Stück am Oberrhein wurde 1977 in Betrieb genommen. Die Eingriffe gaben Grund zur Beunruhigung, die Rheinstudienkommission studierte in den darauf folgenden Jahren die Auswirkungen der Staustufen und deren Kompensationsmöglichkeiten. Aufgrund dessen wurde im Jahr 1982 der Deutsch-Französische Vertrag über die Einrichtung von Retentionsgebieten zum Ausgleich der Baumaßnahem geschlossen. Das vollständige Einrichten der darin vereinbarten Retentionsräume wurde besonders von den deutschen Bundesländern hinausgezögert. Zwischen 1970 und 1983 trat kein nennenswertes Hochwasser in Köln auf, deswegen war das Ereignis vom Februar 1970 die letzte Überschwemmung in der ersten Phase während die Flut vom April 1983 das erste Hochwasser in der zweiten Phase darstellte.

In der ersten Phase ist eine Adenauer-Ära bis 1975 auszumachen, welche bereits 1917 begann. Konrad Adenauer (CDU) prägte in seiner Zeit als Oberbürgermeister der Stadt Köln die Stadtansicht stark. Mit dem Ziel Heimat entstehen zu lassen, legte er Gärten, Wälder, Schwimmbäder sowie Spiel- und Sportplätze in dem historischen Kölner Festungsrings auf einer Fläche von $8 \mathrm{~km}^{2}$ an. Seine Verbundenheit mit der Stadt gab er auch als Bundeskanzler nicht auf. So engagierte er sich 1957 als Schirmherr der Bundesgartenschau in Köln zur Gestaltung des Rheingartens auf der noch von Kriegsschutt begrabenen Deutzer Rheinseite. Außerdem gab er sein Projekt der Stadtgestaltung an seinen Sohn Maximilian Adenauer (CDU) weiter, dieser versuchte aus dem Tiefbauamt heraus mit Hilfe des Hochwasserschutzes die Stadt zu gestalten. Maximilian Adenauer bekleidete zwischen 1953 und 1965 das Amt des Oberstadtdirektors und setzte sich für die Wiederherstellung des fußläufigen Rheinzugangs für Besucher ein. Er sah den zunehmenden Verkehr als Bedrohung für den Ort am Fluss, der die Kölner Heimat verkörperte. Er schreckte deshalb nicht davor zurück, das Stadtbild weiter zu entwickeln, wenn es den alten Traditionen zuträglich war. Als dieser in unendlich anmutenden Diskussionen für den Bau der Hochwasserschutzmauer kämpfte, trat ihm sein Vater bei einem Kölnbesuch zur Seite. Konrad Adenauer sprach 1959 in seiner Rede die Entscheidungsträger der Neugestaltung des Rheinufers öffentlich an, den Besuchern den Genuss eines Spaziergangs am Rhein wieder zu ermöglichen. Ein Jahr später setzte sich Maximilian Adenauer mit dem Bau der Hochwassermauer durch, nachdem der Leiter des Tiefbauamtes in seinen Ausführungen u. a. auf die Ermahnung durch den Bundeskanzler verwies. Im Jahr 1975 verließ Maximilian Adenauer den Kölner Stadtrat. Das Abebben der Adenauer-Ära fällt zusammen mit dem Beginn des Wandels der Wahrnehmung der Überflutungsereignisse und dem steigenden Grad der Überregionalität des Hochwasserschutzes. Jedoch ist zu beobachten, dass die Entscheidungsträger der zweiten 
Phase sehr ähnlich wie in der Nachkriegszeit argumentierten. Die festen Schutzvorrichtungen in der Altstadt wurden nach 1960 nicht mehr in ihrer Höhe verändert ${ }^{582}$. Dies unterstreicht die enorme Wichtigkeit der Promenade und den direkten Zugang zum Rhein für die Kölner. In der zweiten Phase zeigte sich, dass die Auseinandersetzungen über die Schutzhöhen weiterhin mit Hinweisen auf die Bewahrung der Stadtansicht aufgeladen waren und eine Heimatkonnotation aufwiesen. Die Reaktion auf die beiden Hochwasser 1983 bildete keine Ausnahme, denn die ersten mobilen Elemente wurden konstruiert, damit das Stadtbild nicht tangiert würde. 1983 war wiederum die CDU als Treiber für die Fortentwicklung auszumachen. Die Argumentation, der Hochwasserschutz für Köln müsse am Oberrhein beginnen, untermauerte 1993/1995 den Widerstand der kommunalverantwortlichen CDUMitglieder, in Köln höhere Schutzvorrichtungen aufzubauen. Die Bewahrung der Stadtansicht wurde im Hinblick auf den Hochwasserschutz weiterhin durch die Partei der CDU fortgeführt. Die Argumentation bezog sich immer wieder auf die Stadt am Rhein, bzw. der Tradition des Spaziergangs an Dom und Rhein. Damit können Hinweise auf einen Heimatdiskurs bestätigt werden. Die Präferenzen der an den Debatten beteiligten Kölner Akteure weisen während der gesamten Betrachtungsperiode eine Kontinuität auf. Brüche in der Kritik des baulichen Hochwasserschutzes lassen sich nicht auffinden. Alle Diskussionen zum Hochwasserschutz konnten auf die hier festgelegten Kriterien zur Rationalität geprüft werden und genügten diesen. In allen Diskussionen wurden Alternativen verglichen und Konsequenzen betrachtet.

\footnotetext{
${ }^{582}$ HWSK 1996, 111.
} 


\section{Akteursrollen zwischen Hochwasserursachen und -verantwortung}

In diesem Kapitel werden die Rollen der am Hochwasserschutz beteiligten politischen Akteure im Spannungsfeld zwischen staatlicher Verantwortung und privater Vorsorge unter Berücksichtigung des Vulnerabilitätsansatzes ${ }^{583}$ analysiert ${ }^{584}$. Das Konzept dient hier als Kontrastfolie, die über die politischen Auseinandersetzungen gelegt wird. Die neu gefärbte Perspektive erhellt die politische Einschätzung des eigenen Verantwortungsbewusstseins im Kontrast zum empfundene Maß der erforderlichen Verantwortung der vom Hochwasser betroffenen Bürger und deren Notwendigkeit zu Handeln. Leitend für die Untersuchung ist die Frage, wie die Hochwasserursachen und die Verantwortung zum Handeln durch die Kölner Politiker wahrgenommen wurden und wie diese Wahrnehmung wiederum die Reaktionen auf ein Hochwasserereignis beeinflusste.

Zur Herausarbeitung der Verschiedenen Positionen werden zwei Hochwasser beispielhaft herausgegriffen. Die größtmögliche Differenz in den Wahrnehmungen verspricht ein Vergleich der Kölner Hochwasser von 1970 und $1995^{585}$. Mit Hilfe des Vulnerabilitätsansatzes ${ }^{586}$ wird die Gruppe der Geschädigten nach Umfang ihrer spezifischen Widerstandsfähigkeit unterteilt und gezeigt, wie unterschiedlich die Möglichkeiten zur Erholung bzw. der Resilience ${ }^{587}$ verteilt waren. Diese Unterschiede resultierten aus der historisch gewachsenen Verteilung von Ressourcen und äußerten sich in der unterschiedlichen Bemessung von Partizipationsmöglichkeiten durch die betroffenen Gruppen. Anhand des ersten Beispiels wird gezeigt, wie diese nicht bezifferbaren Kosten teilweise in negativen Erwartungen über die Zukunft begründet lagen und sodann die Wiederaufbauphase nach dem

\footnotetext{
${ }^{583}$ Das Konzept der Vulnerabilität beschreibt die Verletzlichkeit durch plötzliche Schocks und fungiert als Gegenstück zu m Konzept der Resilience. Dieses beschreibt die Kapazität, Schocks zu überwinden und Erholung zu erfahren. Damit verbunden ist in der Vulnerabilitätsliteratur der Zugriff auf verschiedene Ressourcen wie Kapital, Bildung, soziale Netzwerke und erlernte Fähigkeiten. Eine hohe Vulnerabilität resultiert folglich aus der Abwesenheit von Resilience (Vgl.: Weichselgartner 2001; Blaikie et al. 1994; Cutter et al. 2003; Turner et al. 2003; Voss 2008).

${ }^{584}$ Das Konfliktfeld zwischen staatlichen Institutionen, privaten Geschädigten und Versicherern nannte Wolfgang Kron eine „Risikopartnerschaft“ (Kron 2001. Lediglich wenn alle drei Akteure in einem aufeinander abgestimmten Verhältnis kooperieren, ließe sich ein effektiver Katastrophenschutz aus Risiko- und Schadensminimierung erzielen). Versicherungen gegen Elementarschadensereignisse wurden im Betrachtungszeitraum jedoch noch nicht in Anspruch genommen (Interview Reinhard Vogt, Leiter der Hochwasserschutzzentrale Köln am 17.12.2011 Köln).

${ }_{585}$ Im zweiten Kapitel ist ein Überblick über die Vorkommnisse an den Hochwassertagen zu finden. Das vorliegende Kapitel nimmt Bezug auf die vorhergegangenen Ausführungen, welche auf Zeitungsartikeln, den offiziellen Hochwasserberichten des Amts für Stadtentwässerung sowie auf umfangreich vorliegenden Tagesberichten der in der Bekämpfung des Hochwassers mitwirkenden Akteure basierten.

${ }^{586}$ Eine Vulnerabilitäts-Betrachtung bezüglich der Hochwassergefahr in der Stadt Köln wurde durch Alexander Fekete durchgeführt. Er betrachtete in seiner Dissertationsschrift „Social Vulnerability Assessment for River-Floods in Germany“ die Mobilität verschiedener Gruppen und kam zu dem Schluss, dass 2009 insbesondere Senioren aufgrund ihrer eingeschränkten körperlichen Mobilität zu den verletzlichen Personengruppen in Köln gehören (Fekete 2009).

${ }^{587}$ Voss betont die ,,participative capacity“ als entscheidenden Faktor der Resilience. Diese definiert er als „,degree of influence the unit can exert on its relevant conditions for subsistence“. Dazu führt er aus, dass soziale bzw. politische Prozesse des diskursiven Ausschlusses vom Zugang zu Ressourcen Einfluss auf die spezifische Vulnerabilität haben (Voss 2008, 39).
} 
Hochwasser von 1970 beeinflussten. Am Beispiel des Hochwassers von 1995 wird verdeutlicht, wie die Angst vor zukünftiger Hilflosigkeit die Hochwasserbekämpfung zu einem internationalen Anliegen werden ließ. Dazu wird der Vulnerabilitätsansatz auf das Wassereinzugssystem des Rheins übertragen. Köln als Stadt wird in einen überregionalen Kontext der, zu Deutsch, Vulnerabilität und Resilienz gesetzt, um zu erklären, mit welchen Mitteln die Stadt versuchte, ein internationales Mitspracherecht bei der Gestaltung des Flusssystems des Rheins zu erlangen. Zum Einstieg folgt nun eine Beschreibung der tatsächlich abgestimmten Reaktionen, um daraufhin das Ergebnis des Entscheidungsprozesses zu präsentieren. Mit dem Ziel, zu erläutern, welche Akteure an dem Prozess beteiligt waren und welche Argumentationslinien sie verfolgten, werden die wichtigsten Punkte der Diskussion daraufhin zusammengefasst. Die geführten Verhandlungen werden abschließend aus der Kosten-Nutzen-Perspektive kommentiert und die Bewertung intangibler Kosten verdeutlicht.

\subsection{Reaktionen von 1970: Streit um Entschädigungen}

Das Hochwasser vom 25. Februar 1970 rief Schäden hervor ${ }^{588}$, die für Diskussionen um adäquate Hilfsreaktionen in Stadt- und Landespolitik sorgten. Auf beiden administrativen Ebenen wogen die Verantwortlichen das Verhalten Schritt für Schritt ab, bis Hilfsmaßnahmen definiert werden konnten. Der Landtag verabschiedete am 8. März 1970 einen Kabinettsbeschluss über die Art und Weise der Landeshilfsmaßnahmen an die Kommunen. Da die Landesregierung den Kabinettsbeschluss nicht veröffentlichte, blieb für den Kölner Stadtrat ungeklärt, wie zu verfahren sei, worauf er sich handlungsunfähig gab ${ }^{589}$. Bis zum 22. April 1970 wurden die Hochwasseropfer der Stadt Köln lediglich nach Grundlage des Bundessozialhilfegesetzes durch das Kölner Sozialamt sowie mit Spendengeldern aus der Bevölkerung unterstützt ${ }^{590}$. Außerdem gewährte die Stadt Köln Unternehmern und selbstständig Tätigen Renovierungskosten am Jahresende steuerlich abzugelten ${ }^{591}$.

Überlegungen der Mitglieder des Stadtrats oder des Landtags, den Hochwasserschutz zu verbessern, gab es nach der Überschwemmung von 1970 nicht, wie bereits im zweiten Kapitel

\footnotetext{
${ }^{588}$ Die absolute Summe der Schäden konnte nicht nachvollzogen werden. Hauptsächlich fehlen Angaben zu Schäden im privaten Bereich.

${ }^{589}$ Stadt Köln, Rat der Stadt Köln, 2. Sitzung vom 12.03.1970; Stadt Köln, Rat der Stadt Köln, 3. Sitzung vom 16.04.1970.

${ }^{590}$ Landtag NRW, 74. Sitzung, 22.04.1970.

${ }^{591}$ Ebd.
} 
ausführlich dargelegt wurde. Es folgt die Herleitung der Reaktionen aufgrund der Zusammenfassung der markantesten Punkte des Diskussionsverlaufs. Dabei werden die Hintergründe und die Konsequenzen der Entscheidungen verdeutlicht.

\subsubsection{Diskussionen über Schadensersatz}

Nachdem am 4. März 1970 der Verkehr wieder vollständig eröffnet werden konnte, ${ }^{592}$ waren die Spuren der Flut noch nicht gänzlich von allen öffentlichen „Plätzen“ verschwunden, denn am 12. März stellte die CDU-Fraktion in ihrer Oppositionsfunktion im Stadtrat eine Anfrage betreffend der Übernahme der privaten Hochwasserschäden. Die SPD geleitete Verwaltung sollte darlegen, welche Hilfen die Stadt Köln den Bürgern anbieten kann und welche Unterstützung das Land Nordrhein-Westfalen gewährt ${ }^{593}$. Der Beigeordnete Dr. Petzborn (SPD) erklärte in seiner Funktion als Vertretung des Sozialdezernenten, dass die Stadt auf die Einforderung der angefallenen Kosten zur Unterstützung der Betroffenen, etwa für das Leerpumpen der Keller oder beispielsweise für die Aufräumarbeiten durch die Stadtreinigung, verzichten und diese selbst tragen werde ${ }^{594}$. Er nannte in seinen Beispielen konkrete Geldbeträge, so waren bei der Stadtreinigung Kosten in Höhe von 48.000 DM und bei der Berufsfeuerwehr in Höhe von 51.500 DM angelaufen ${ }^{595}$. Der Bund übernimmt sogar die Kosten für das THW, führte er weiterhin aus, bevor er sich den Schäden der betroffenen Anlieger zuwandte. Seit dem 02. März 1970 bot die Stadt für die Betroffenen eine Beratungsstelle an; in welcher seitdem „90 fernmündliche sowie etwa 200 persönliche Vorsprachen geschädigter Bürger registriert worden“"596 waren. Er verdeutlichte das Problem, dass die Geschädigten „nicht mal selbst den Schaden konkret beziffern konnten“, bevor er noch einmal von geschädigtem öffentlichem Eigentum am Hafen im Wert von 420.800 DM sprach $^{597}$. Weiterhin präsentierte er eine einzige städtische Unterstützungsmöglichkeit der betroffenen Bürger „im Rahmen der bestehenden Gesetze [...] nach dem Bundessozialhilfegesetz،598. Er wies darauf hin, dass nur der Erwerb von Hausrat und Bekleidung unterstützt werde, da sich die Höhe der Beihilfe nicht nach dem Schaden, sondern nach der Bedürftigkeit der Antragsteller richte ${ }^{599}$. Die Bedürftigkeit ist neben dem Schadensumfang abhängig von der Einkommenshöhe. Abschließend betonte er, dass er nicht

\footnotetext{
${ }^{592}$ Bericht über das Hochwasser 1970.

${ }^{593}$ Stadt Köln, Rat der Stadt Köln, 2. Sitzung vom 12.04.1970.

${ }^{594}$ Ebd.

${ }^{595}$ Ebd.

${ }^{596}$ Ebd., 67.

${ }^{597}$ Ebd.

${ }^{598}$ Ebd

${ }^{599}$ Stadt Köln, Rat der Stadt Köln, 2. Sitzung vom 12.04.1970.
} 
in er Lage sei, weitere Hilfen zu gewähren: Das Land hat Richtlinien zur Kanalisation von Hilfen erarbeitet, welche „bisher nicht in Erfahrung gebracht werden konnten“ ${ }^{\text {600 }}$. Die Stadt bevorzugte „abzuwarten, wie Art und Umfang der Landeshilfen sein werden“601 . Er schloss mit dem Hinweis, dass alle im Stadtgebiet gemeldeten Schäden beim Rechtsamt Köln gesammelt werden, da das Land Nordrhein-Westfalen - ebenfalls zu der Zeit von der SPD regiert - diese Aufstellung als Grundlage möglicher Hilfen voraussetzt ${ }^{602}$. Gruner von der CDU kritisierte: „Hat die Verwaltung [...] keine eigenen Vorstellungen über gezielte finanzielle Hilfsmaßnahmen für die mehr als 200 hochwassergeschädigten Bürger unserer Stadt? ${ }^{6603}$

Die Strategieentscheidung zwischen „Soforthilfe und Abwarten“ wurde durch das SPDMitglieder Surges und durch den CDU-Anhänger Schröder verdeutlicht. Surges wies durch seine Parole „Wer schnell hilft, hilft doppelt“" noch einmal auf die Not der Opfer hin, während Schröder vorschlug, ,,das Land zu drängen, die Richtlinien bekannt zu geben“604. Ein weiterer SPD-Vertreter, Dr. Vetter, spezifizierte die verschiedenen Charaktere der Geschädigten zwischen Privateigentum und Vereinen, um seinen Vorschlag zu bekräftigen, sofort Hilfe zu leisten $^{605}$. Dr. Pohl (CDU) schlug vor, im Rahmen des Härteausgleichs Mittel zur Überbrückung an die Hochwassergeschädigten zu verteilen, die von der Landespolitik an die Stadt Köln zurückgezahlt werden können ${ }^{606}$. Der Oberbürgermeister Burauen (SPD) schloss die Verhandlung mit dem Hinweis, dass zumindest die Spendengelder bereits verteilt werden. SPD-Mitglied Gruner konstatierte, dies sind „keine städtischen Mittel““607, während der FDP jede Hilfe recht zu sein schien, denn „Geld ist Geld!،6008, stellte der Abgeordnete Baum (FDP) fest.

Etwa einen Monat später, am 16. April 1970, stellte die CDU-Fraktion des Stadtrats eine erneute Anfrage an die Verwaltung, um festzustellen, ob erstens einkommensunabhängige Überbrückungsgelder gezahlt werden können, zweitens durch Steuererleichterungen eine Unterstützung möglich ist und drittens Gelder der Stadt für vorbeugende Maßnahmen des Hochwasserschutzes fließen können ${ }^{609}$. Durch Überbrückungsgeld wird Hausrat und Kleidung finanziert, erklärte der Beigeordnete Dr. Schaefer (SPD), dies ist aber bereits durch die

\footnotetext{
${ }^{600}$ Ebd., 67.

${ }^{601}$ Ebd., 68 .

${ }^{602}$ Ebd.

${ }^{603}$ Ebd., 67.

${ }^{604}$ Ebd.

${ }^{605} \mathrm{Ebd}$

${ }^{606}$ Ebd.

${ }^{607}$ Ebd.

${ }^{608}$ Ebd.

${ }^{609}$ Stadt Köln, Rat der Stadt Köln, 3. Sitzung vom 16.04.1970.
} 
Spenden geleistet worden, ,weshalb bisher ein Einsatz von städtischen Mitteln [...] nicht erforderlich gewesen ist ${ }^{\star 610}$. Er betonte wie sein Parteikollege, Dr. Petzborn (SPD) in der vorhergegangen Sitzung die Arbeit der Verwaltung und klagte über die Problematik, Schäden genau anzugeben ${ }^{611}$. Neben den Problemen in der städtischen Verwaltung nannte er die Herausforderungen der Landespolitik, da sich ,immer noch nicht absehen läßt, wann die Landesrichtlinien ergehen werden “612. Über seinen persönlichen Besuch im Schadensgebiet in Köln-Poll referierte der CDU-Abgeordnete Dr. Pohl, dass er Gebäudeschäden festgestellt hatte $^{613}$. Gebäudeschäden fanden aber keine Erwähnung in der Stadtratsdebatte, kritisierte Pohl den dafür verantwortlichen Dr. Schäfer (SPD), der „ein Beweissicherungsverfahren an Ort und Stelle [...] in einem Schreiben abgelehnt" hatte ${ }^{614}$. Er plane die Schadenshöhen festzustellen, wenn die Landesrichtlinien bekannt seien ${ }^{615}$, wehrte sich Schaefer gegen den Angriff des CDU-Mitgliedes. Er habe sich bereits „,bei den Leuten erkundigt und eine grobe Umfrage veranstaltet. Das mussten wir binnen zwei Tagen dem Minister melden“616. Dann wies er noch einmal auf das Generalproblem hin, dass es ,in allen Fällen ziemlich schwierig sein wird, die Schadenshöhe festzustellen“617. Er begründete sein Abwarten mit dem limitierten Zugang zu finanziellen Ressourcen sowie mit einem Vergleich von Naturgefahren mit Unfällen:

\begin{abstract}
„Selbstverständlich muss auch die finanzielle Situation der Stadt Köln berücksichtigt werden. Es treten ja nicht nur bei Hochwasser Schäden für einzelne auf. Jemand brennt ab oder jemand verunglückt. Das ist für den einzelnen genau so schlimm, als wenn durch ein Hochwasser Hunderte geschädigt werden. Auch in diesen Fällen wird nicht ohne weiteres die öffentliche Hand einspringen. Ich meine, es ist richtig, auf die Landesrichtlinien zu warten. “618
\end{abstract}

Zur Beantwortung des zweiten Punktes der CDU-Anfrage referierte der Stadtkämmerer Meyer über drei Möglichkeiten der Steuererleichterung. Adressaten dieser Hilfsmaßnahme waren vordringlich Unternehmer. „Wegen der außergewöhnlichen Umstände sollte gegenüber Gewerbetreibenden [...] großzügig verfahren werden“"619, bekräftigte er sein Vorgehen. Meyer gab sich verbindlich durch seine Ausführung, er habe das Steueramt angewiesen, Anträge mit Hochwasserfolgeschäden ihm persönlich zur Entscheidung vorzulegen ${ }^{620}$. Die dritte Anfrage,

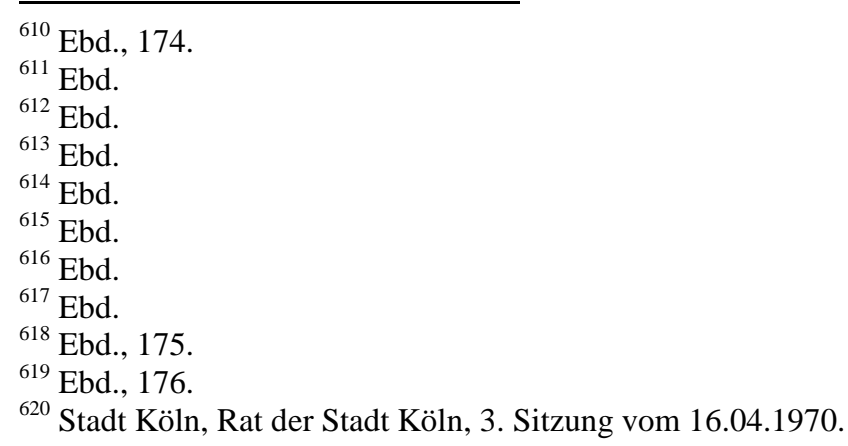


inwieweit die Stadt vorbeugende Maßnahmen unterstützen könne, wurde kurz abgehandelt ${ }^{621}$. Der Beigeordnete Tiefbaudezernent und Leiter des Hochwasserschutzdienstes Dipl.-Ing. Braun war der Auffassung, dass „von der Investition her, vorbeugen besser als entschädigen ist“, aber auch, „daß jeder Hauseigentümer nach der jetzigen Rechtslage verpflichtet ist, sich selbst zu schützen“622. Als vorbeugende Maßnahme schlug Braun den „mechanischen Verschluß von Einflußöffnungen“623 vor, stellte dann aber die Frage: „Inwieweit ist es zweckmäßig, daß die Stadt Köln sich bei dieser Hilfe für vorbeugende Maßnahmen mit engagiert?“624 Das Thema der Vorsorge wurde im Einverständnis aller verschoben ${ }^{625}$.

Auf der Landesebene entstanden ebenfalls Diskussionen über die Leitlinien für Entschädigungszahlungen. Am 22. April 1970 wurde im nordrhein-westfälischen Landtag über den „Schadensersatz bei Hochwasserschäden“626 diskutiert. Der Antrag der CDU wurde bereits am 17. März 1970 gestellt. Zunächst führte der Antragsteller Dr. Hüsch (CDU) sein Anliegen aus. Drei wesentliche Punkte lassen sich zusammenfassen. Zunächst beschrieb er die Überschwemmung als „ein Unglück ungewöhnlichen Ausmaßes“6627 und forderte,

„den Menschen die Sicherheit zu verschaffen, daß sie leben können, ohne Furcht und ohne Angst davor, über Nacht Leben und Gesundheit zu verlieren, oder alles das, was sie in mühsamer Arbeit in Jahren geschaffen haben, den Naturgewalten geopfert werden muß, nur, weil ausreichende Vorkehrungen zum Schutz nicht vorhanden waren." ${ }^{\circ 28}$

Jedoch galt sein eigentliches Anliegen der Transparenz der Landesrichtlinien über Entschädigungsleistungen:

„Die Geschädigten sollen und müssen wissen, woran sie sind, und sie müssen darüber vollständige Klarheit bekommen. [...] Aus der Sicht des Parlaments ist es erforderlich, daß alle Maßnahmen allgemein bekannt werden. [...] Es ist daher erforderlich, daß in neuen Richtlinien, [...], auch Klarheit über diese Maßnahmen getroffen wird, und daß diese Richtlinien die Mittel, den Weg und den Umfang des Ersatzes unkonventionell beschreiben.“629

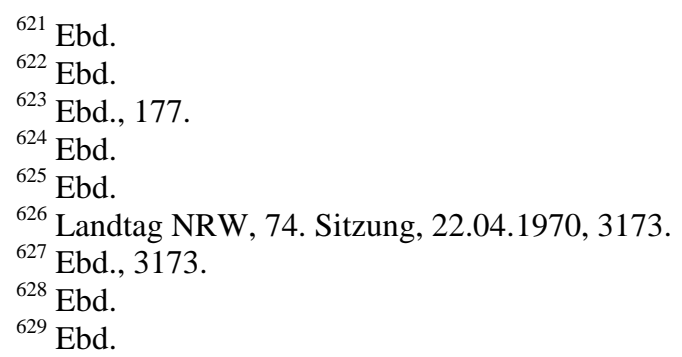


Drittens lässt sich anmerken, dass er mehrfach auch die Vorsorge für zukünftige Hochwasser thematisierte, aber nicht konkretisierte: „Vorbeugen ist und bleibt besser als Heilen“630. Er sprach davon, ,,auf Dauer“ den Hochwasserschutz sicherzustellen sowie ihn ,,als vordringliche Aufgabe" anzusehen ${ }^{631}$.

Als erster Gegenredner formulierte Innenminister Weyer (SPD) seine Meinung und zitierte den umstrittenen Kabinettsbeschluss vom 3. März 1970. Darin hieß es, dass unter Anwendung der Richtlinien, welche aus Anlass der Hochwasserkatastrophe Ostwestfalen-Lippe 1961 beschlossen wurden, Zuschüsse und Bürgschaften $\mathrm{zu}$ gewähren seien ${ }^{632}$. Soforthilfsmaßnahmen sollten gegebenenfalls unter Überarbeitung der Richtlinien durch die verschiedenen Ressorts eingeleitet werden. Er hielt fest, dass die „Exekutivmaßnahmen seit geraumer Zeit liefen“, bevor er die Aufgaben von fünf Ressorts als „Fächer vielfältiger Hilfsmaßnahmen“ 633 beschrieb. Er betonte die Hilfsbereitschaft der Landesregierung, während ,weder das Land noch eine Gemeinde oder ein Gemeindeverband eine Rechtspflicht haben, für Katastrophenschäden jeder Art Schadensersatz zu leisten “634. Auch malte er die Rollen der lokalen Behörden sowie die Aufgabe der Landesbehörde aus: „Die Gemeinden und Gemeindeverbände werden in der Regel die ersten sein, die im Rahmen ihrer Finanzkapazität und ihrer sonstigen Möglichkeiten ihren Bürgern zu helfen haben. Die Hilfeleistung des Landes kann nur subsidiär sein.“635 Die Richtlinien sollten seiner Ansicht nach nicht zugänglich sein, da er ihre Transparenz als „Gefährlichkeit" ${ }^{\text {6636 }}$ ansah:

„Die Hilfsmaßnahmen des Landes müssen [...] so flexibel wie eben möglich bleiben. Dementsprechend werden auch die Ressorts jeweils der Lage angepasst werden müssen. Vor allem aber wird der Umfang der Hilfsmaßnahmen entscheidend auch von der Finanzlage der Helfenden mit abhängen. Es dürfte daher bedenklich, wenn nicht gefährlich sein, solche Richtlinien ein für alle Mal zu fixieren und der Öffentlichkeit bekanntzugeben." ${ }^{\text {(637 }}$

Weyer schloss seine Ausführungen und betonte, dass ,sich die Hochwasserschutzanlagen für unser Land bewährt haben“ und, dass die „Landesregierung glaubt, alles getan zu haben, was aus Anlaß des Hochwassers im Frühjahr [...] getan werden muss “6638.

\footnotetext{
${ }^{630} \mathrm{Ebd}$

${ }^{631}$ Ebd.

${ }^{632}$ Ebd.

${ }^{633}$ Landtag NRW, 74. Sitzung, 22.04.1970, 3174.

${ }^{634}$ Ebd.

${ }^{635}$ Ebd.

${ }^{636}$ Ebd.

${ }^{637}$ Ebd.

${ }^{638}$ Ebd.
} 
Parteikollege Toetemeyer (SPD) äußerte sich ebenfalls zum Thema, da sein „eigener Wahlkreis von diesen Katastrophen betroffen wurde“639. Gewissermaßen gratulierte er der

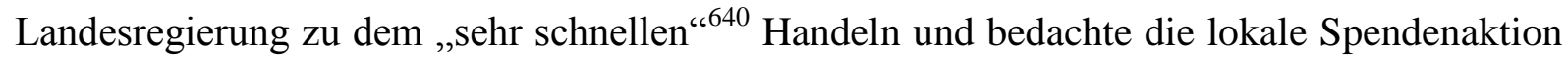
einer Kölner Tageszeitung durch ein ,,außerordentlich herzliches Wort des Dankes“6641. Für seinen Wahlkreis richtete er eine persönliche Bitte an Innenminister Weyer. Als Ressortminister für Kommunalaufsicht solle er „die Zusagen des Kämmerers, beispielsweise in der Stadt Köln, decken, damit es in den Städten nicht zu finanziellen Schwierigkeiten kommt ${ }^{\text {“642 }}$. Trotz des Bezugs zu seinem Wahlkreis merkte Toetemeyer an,

„[...] daß es eine ganze Reihe von Menschen gibt, die, obwohl sie rechtzeitig durch den Rundfunk darauf hingewiesen wurden, daß zu einem bestimmten Zeitpunkt ein bestimmter Pegel erreicht würde, nichts unternommen haben. [...] man kann nicht verlangen, daß Menschen, die gewarnt wurden und auf diese Warnung nicht hörten, nachher für ihre eigene Nachlässigkeit entschädigt werden.“"643

Zum überregionalen Kontext des Hochwasserproblems nahm er ebenfalls Stellung und bat den Innenminister,

„Sorge zu tragen, daß im Rahmen der [gerade durch Bundesinnenminister Hans-Dietrich Genscher (FDP) verhandelten] Bundeskompetenz die anderen Länder mit ihren großen Zuflüssen in den Rhein gezwungen werden, entsprechende Rückhaltebecken einzubauen, [...], damit wir nicht am Ende der Prügelknabe sind, der das ausbaden muß, was in andern Ländern der Bundesrepublik versäumt worden ist ${ }^{\text {“644 }}$.

Zu den Landesrichtlinien äußerte sich auch FDP-Mitglied Tornau. Er pflichtete Innenminister Weyer bei, da einerseits eine „Naturkatastrophe in ihrem Ablauf nicht zu berechnen“645 ist und andererseits „die Gefahr [bestände], daß dann Anlieger und Bürger eventuell die eigene Vorsorge etwas vernachlässigten in der Hoffnung, daß dann, wenn ihnen ein Schaden zugefügt wird, dieser sowieso großzügig ersetzt wird“646. Auch er besaß Zuversicht in das weiterhin „schnelles Handeln“6477 der Landesregierung, und verwies auf die überregionale Herausforderung der „engeren Zusammenarbeit mit allen übrigen Beteiligten. [Denn] es ist stets ein sehr zweifelhaftes Vergnügen, Unteranlieger eines Stroms oder Flusses zu sein“648.

\footnotetext{
${ }^{639}$ Ebd., 3175.

${ }^{640}$ Landtag NRW, 74. Sitzung, 22.04.1970.

${ }^{641}$ Ebd., 3176.

${ }^{642} \mathrm{Ebd}$

${ }^{643}$ Ebd., 3176.

${ }^{644}$ Ebd., 3176.

${ }^{645} \mathrm{Ebd}$

${ }^{646}$ Ebd.

${ }^{647}$ Ebd.

${ }^{648}$ Ebd., 3176.
} 
Antragsteller Hüsch begegnete den Wortmeldungen mit zwei Punkten. Zunächst verdeutlichte er die Sinnlosigkeit, bei dieser Gelegenheit über Spendenaktionen zu sprechen, da dies keine öffentlichen Gelder seien:

„Denn die Betroffenen wollen kein Almosen; sie wollen nicht die karikativen Leistungen, sondern sie verlangen öffentliche Leistungen in einem geordneten Verfahren, frei von jeder etwa diskriminierenden Beigebung irgendwelcher sozialbedingter oder sozialhilfebedingter Akzente.“649

Seine zweite Anmerkung zum „Mangel im Verfahren“650 richtete er an Innenminister Weyer und Finanzminister Wertz. Hüsch kritisierte scharf, dass Weyer „dem Parlament [...] diesen [Kabinetts-]Beschluss nicht bekanntgegeben“ ${ }^{\text {hatte }}{ }^{651}$. „Bei einer Frage, die das Land in einem solchen Maße berührte, hätte es, so meine ich, doch zur Informationspflicht der Landesregierung gehört. Sie hätten den vollen Wortlaut des Kabinettsbeschlusses den Mitgliedern dieses Hohen Hauses bekanntgeben sollen“652. Finanzminister Wertz tadelte er ebenfalls bezüglich des nicht einsehbaren Kabinettsbeschlusses: „Das, was Sie am 03. März beschlossen haben wollen und was als Ausarbeitung Ihrer Herrn dort geschehen sein soll, das ist bis in die vorderen Linien hinein, dort, wo die Schadensersatzfragen geregelt werden sollten, nicht vorgedrungen“653. Zur Bestätigung seiner Ausführungen zitierte er aus einem Schreiben von Oberstadtdirektor Kölns Heinz Mohnen vom 23. März 1970 sowie aus dem Stadtratprotokoll vom 16. April desselben Jahres.

Innenminister Weyer stellte sich dem Vorwurf mit einer Erklärung über die Aufgabenverteilung. Nicht er ,hat in jedem einzelnen Falle die Ressorts aufgefordert, sondern die Ressorts haben von sich aus die einzelnen Stellen aufgefordert, die Schadensfeststellungen zu treffen“654. Weyer bekräftigte seine Aussagen mit dem Verweis auf Landwirtschaftsminister Deneke. Dieser machte bereits vor dem Kabinettsbeschluss Gebrauch, denn er bot Ende Februar den geschädigten Land-, Forst- und Fischereibetrieben Unterstützung nach den Katastrophenrichtlinien $a^{655}$. Zur Unterstützung des Innenministers gab Toetemeyer (SPD) ein Gegenzitat an. Er führte ebenfalls das Ratsprotokoll der Stadt Köln von Mitte April 1970 an und schloss daraus den „Vollzug des Kabinettsabschlusses“656 . FDPMitglied Tornau unterstellte Antragsteller Hüsch, „Opposition mit Sicht auf die Wahl am 14.

\footnotetext{
${ }^{649}$ Landtag NRW, 74. Sitzung, 22.04.1970, 3177.

${ }^{650}$ Ebd.

${ }^{651}$ Ebd.

${ }^{652} \mathrm{Ebd}$

${ }^{653}$ Ebd.

${ }^{654}$ Ebd., 3178.

${ }^{655}$ Ebd.

${ }^{656}$ Ebd.
} 
Juni zu treiben“657 und wies auf das Versäumnis der Kölner Verantwortlichen hin, denn ,auch die Herrn Beigeordneten oder Beauftragten für Sicherheitsfragen in Köln hätten ja [einen] Anruf beim Innenminister tätigen können, dann wäre manches geklärt worden“658. In der Abstimmung wurde der Antrag der CDU, das Thema im Hauptausschuss zu besprechen, abgelehnt ${ }^{659}$.

Nachdem die Darstellung der Diskussionen die Argumentationslinien und Standpunkte der administrativen Akteure verdeutlichte, folgt nun die Bewertung der Handlungsstränge aus der Kosten-Nutzen-Perspektive.

\subsubsection{Bewertung des Diskurses aus der Kosten-Nutzen-Perspektive}

Im vorangegangenen Abschnitt wurden die wichtigsten Eckpunkte des politischen Diskurses vorgestellt, welche die Standpunkte sowie Argumente der Akteure widerspiegelten. Der Verhandlungsprozess besaß jedoch eine zweite Betrachtungsperspektive. Schadensersatzzahlungen konstituieren ein Verhältnis zwischen staatlichen Akteuren und betroffenen Bürgern. Die Situation der verantwortlichen Entscheidungsträger muss betrachtet werden, jedoch darf für eine umfassende Bewertung die Seite der Hochwasseropfer nicht außer Acht gelassen werden. Die Streitigkeiten über die Schadensersatzansprüche sprechen dafür, dass die Bürger Vertrauen in die nach 1960 erbaute Hochwasserschutzmauer gefasst hatten und zum Zeitpunkt des Hochwassers von 1970 ihre Wertgegenstände in ehemals durch regelmäßiges Eindringen von Rheinwasser bedrohten Räumen lagerten. Für die betroffenen Bürger war das Hochwasser also eine Überraschung, sie wurden verwundbar. Im $0,41 \mathrm{~km}^{2}$ großen Überschwemmungsgebiet lassen sich verschiedene Gruppen von Hochwasseropfern einteilen. Diese Gruppen können nach ihrer spezifischen Vulnerabilität klassifiziert werden. Unter den Betroffenen ist ein differenzierter Zugang zu Ressourcen erkennbar. Die Verteilung von Ressourcen, bzw. von Kapital ${ }^{660}$, stellt das entscheidende Kriterium für das Maß der Resilience und der Kapazität, nach dem Schadensereignis Erholung zu finden, dar. Die nachfolgend vorgestellten Beispiele illustrieren die ungleiche Verteilung von Resilience zum Zeitpunkt des Hochwassers von 1970.

\footnotetext{
${ }^{657}$ Ebd. 3179.

${ }^{658}$ Landtag NRW, 74. Sitzung, 22.04.1970, 3179.

${ }^{659}$ Ebd.

${ }^{660}$ Voss bezieht seine Beobachtung auf den Foucaultschen „microphysics of power“, denn das zur Verfügung stehende Kapital reguliert, welche Thematiken zu welchem Zeitpunkt verhandelt werden, welche Rahmenbedingungen für den Aushandlungsprozess gewählt werden und welche Akteure überhaupt teilhaben dürfen. Die Kapitalverteilung ist das grundlegende Kriterium, welches die generelle Problemwahrnehmung und die Lösungsansätze beeinflusst. Besonders unter Einbezug wirtschaftlicher Interessen bleiben die Betroffenen aufgrund ihres mangelhaften Ressourcenzugangs gegenüber anderen Akteuren ungehört. Voss bezieht den Begriff des Kapitals auf die Definition nach Bourdieu (Voss 2008, 39).
} 
Der tief an der Wasserkante gelegene Ort Rodenkirchen war stets einer der ersten Orte, welche vom Hochwasser überschwemmt wurden. Doch während der Rettungsaktionen sind von dort im Jahr 1970 weder Schwierigkeiten noch Beschwerden bekannt. In Rodenkirchen wohnen wirtschaftlich besser gestellte Familien, u. a. aus der lokalen Politik. Die Zeitungen berichteten nach dem Hochwasser über Besuche der Kölner Politiker, darunter Gemeindedirektor Dr. Kühn, der stellvertretende Landrat Müller sowie weitere Vertreter des Kreises und der Gemeinde. Sie kamen vor der Presse zu dem Schluss, „dass die Familien unmöglich die Auswirkungen alleine tragen können" ${ }^{661}$.

Auf der anderen Rheinseite wurde ebenfalls der Vorort Porz überschwemmt. Die Menschen des dicht besiedelten Örtchens beschwerten sich über die mangelnde Versorgung mit Strom und Wasser, wie bereits in der Hochwasserskizze im zweiten Kapitel zu lesen war. Anders als die Bewohner von Rodenkirchen hatten diese Hochwasseropfer keine Lobby.

In der linksrheinischen Altstadt waren die Straßen auch überschwemmt worden. Die Touristengegend war zu der Zeit bereits mit Cafés und Kneipen gesäumt und die Sicht auf den Kölner Dom und den Rhein gehörten zum Geschäftskonzept eines jeden Wirts. Deshalb war der Hochwasserschutz dort vergleichsweise niedrig ${ }^{662}$, jedoch verliefen die Soforthilfemaßnahmen reibungslos. Die Anwohner lobten die Hochwasserzentrale und die Einsatzkräfte.

Nördlich der Altstadt, in der Nähe der Fordwerke, waren angeworbene ausländische Arbeiter in Rheinkassel untergebracht. Die Presse berichtete an jenem Mittwoch, 25. Februar 1970 über die Feuerwehr, die ausländischen Arbeiter zu dem von der Flut abgetrennten Wohnheim transportierte ${ }^{663}$. Die Siedlung rund um den Kasselberger Weg war vor Hochwasser nicht geschützt und wurde deshalb zu einer Insel. „Am Abend zuvor war der Vorort noch über eine schnell gezimmerte Notbrücke zu erreichen gewesen“664. Doch diese wurde bei einer Pegelhöhe von 8,87 m K P fortgespült und am Morgen des 25. Februar 1970 lagen 100 Meter Wasserfläche zwischen den Siedlungen und dem Kölner Stadtteil Rheinkassel ${ }^{665}$. Abb. 19 zeigt die Lage der Siedlung am Kasselberger Weg bei einem simulierten Hochwasser von $9,50 \mathrm{~K} \mathrm{P}$.

\footnotetext{
${ }^{661}$ Artikel: Geschädigten muß geholfen werden, Kölner Stadtanzeiger, 28.02.1970.

${ }^{662}$ Siehe dazu das vorhergegangene Kapitel zur Stadtansicht, speziell die Diskussion zum Bau der

Hochwasserschutzmauer 1960.

${ }^{663}$ Bericht der Berufsfeuerwehr, 25.02.1970.

${ }^{664}$ Artikel: Hundert auf einer Insel, Kölner Stadtanzeiger, 25.02.1970.

${ }^{665}$ Ebd.
} 


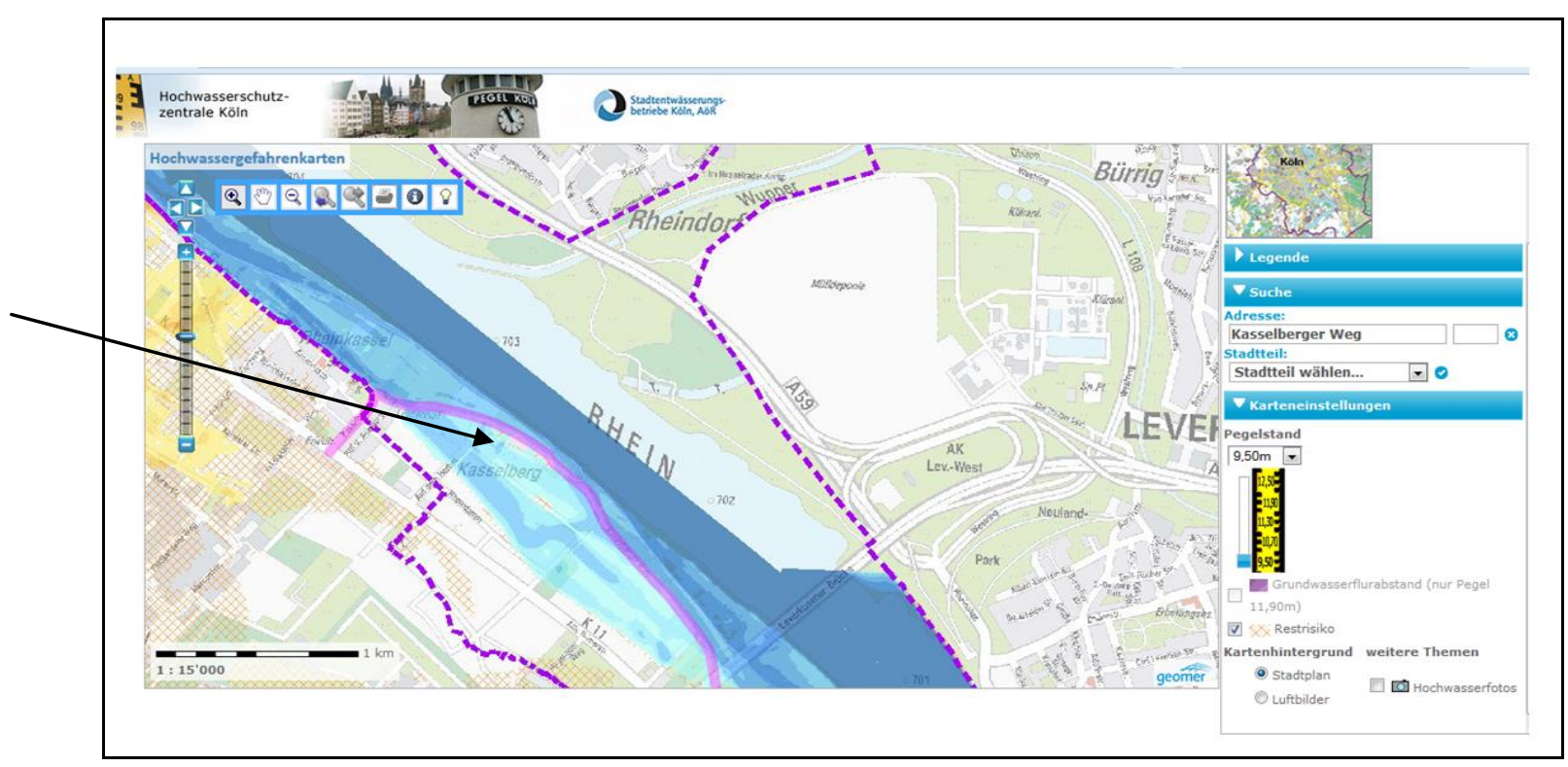

Abb. 19: Der Kasselberger Weg in der Hochwasserschutzkarte.

Der Pfeil verweist auf die Lage der Siedlung am Kasselberger Weg bei einer Hochwasserhöhe von 9,50 m K P und einer Schutzhöhe von 11,90 m K P im Jahr 2013. ${ }^{666}$

Auf der rechten Rheinseite wurde die ungleiche Vulnerabilität der vom Hochwasser betroffenen Menschen noch deutlicher. Die Stelle zur Beseitigung von Wohnungsnot alarmierte die Hochwasserschutzzentrale am 25. Februar 1970 über „besondere Schwierigkeiten“6677: Familie Schurtz aus dem Weidenweg in Poll „weigerte sich kategorisch, ihr Haus zu räumen“668. Da sie ihre ablehnende Haltung nicht ablegte, wurde das Bauaufsichtsamt herangezogen. Dieses deklarierte kurzerhand das Haus als einsturzgefährdet, worauf die Familie mitsamt der ,scharfen deutschen Dogge und gleichfalls zu beherbergendem Hausgetier “669 durch die Polizei und Berufsfeuerwehr zwangsgeräumt wurde $^{670}$. Neben Familie Schurtz gab es 16 weitere Fälle in dieser Nachbarschaft, die ebenfalls persönlich aufgefordert werden mussten, ihre Häuser zu räumen ${ }^{671}$. Der Weidenweg in Poll ist Bestandteil der Westhovener Aue, welche heute den Kölner Retentionsraum bildet, Abb. 20 zeigt die Lage des Weidenwegs im heutigen Retentionsraum. Die Gegend um die „Behelfsheimsiedlung،672 wurde in der Regel bei Hochwasser überschwemmt und war zumindest im Jahr 1970 von mittellosen Bürgern bewohnt.

\footnotetext{
${ }^{666}$ Internetbasierte Quelle 7. Die gestrichelte Linie zeigt den Verlauf des Hochwasserschutzes bis zu einem Pegel von 11,90 m K P an, die durchgezogene Linie beschreibt den Grundwasserflurabstand bei einem Hochwasserpegel von $11,90 \mathrm{~m} \mathrm{~K}$ P.

${ }^{667}$ Bericht der Stelle zur Beseitigung von Wohnungsnotständen, 25.02.1970.

${ }^{668}$ Ebd.

${ }^{669} \mathrm{Ebd}$

${ }^{670}$ Ebd.

${ }^{671}$ Ebd.

${ }^{672}$ Artikel: Sie verließen ihre Häuser doch, Kölner Stadtanzeiger, 25.02.1970.
} 


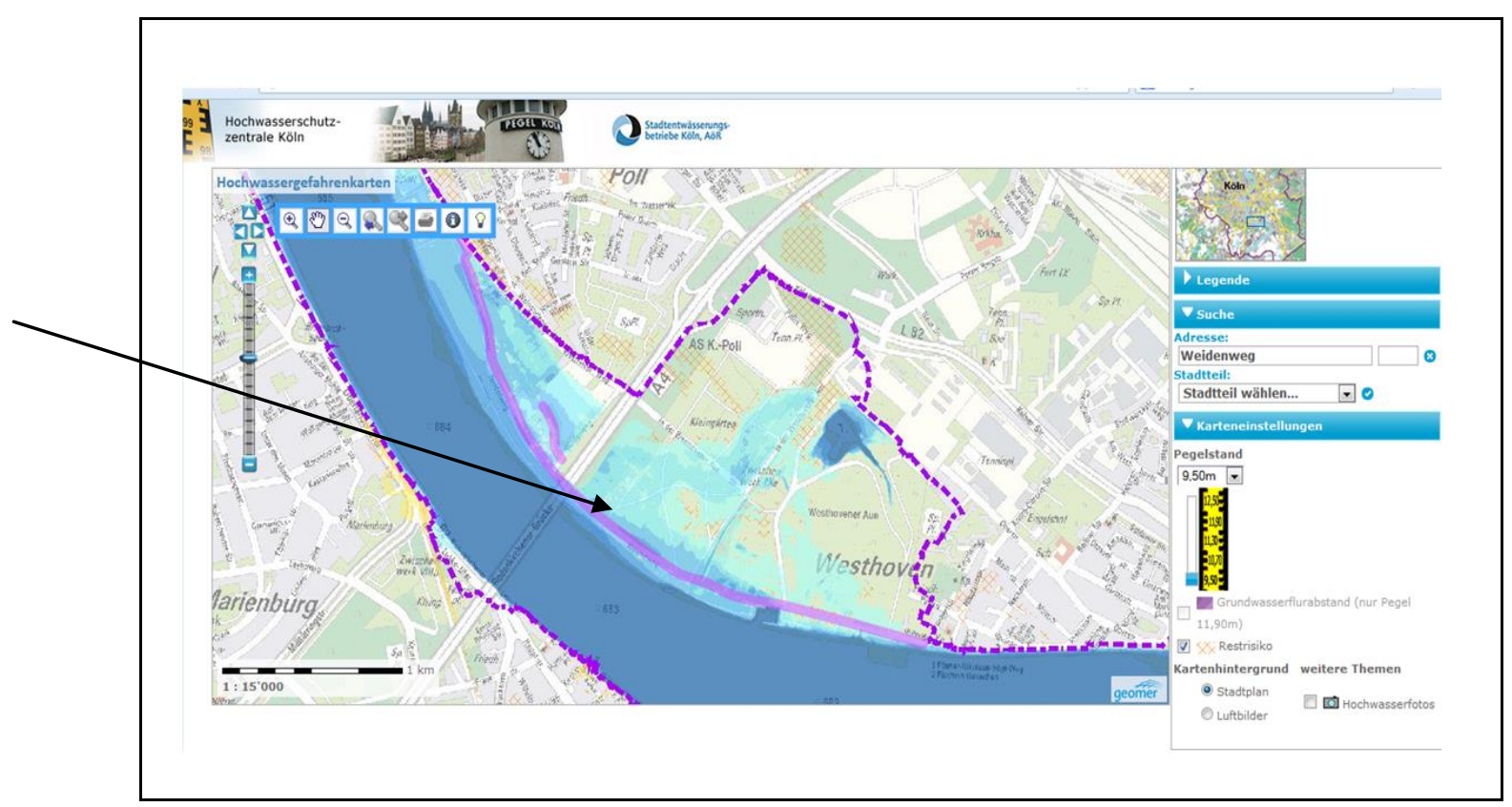

Abb. 20: Der Weidenweg in der Hochwasserschutzkarte.

Der Pfeil verweist auf die Lage der Behelfsheimsiedlung am Weidenweg. Die Karte zeigt die Westhovener Aue rechtsrheinisch bei einer Hochwasserhöhe von 9,50 m K P im Jahr 2013. ${ }^{673}$

Deutlich wird, dass alle diese Menschen zu den Opfern der Überschwemmung gehörten, dass sie aber alle unterschiedlich mit der Flut umgehen konnten. Die Bevölkerung von Rodenkirchen kann als Gruppe des Bürgertums zusammengefasst werden, die über die nötigen finanziellen Rücklagen verfügt, um sich eigenständig in einen ähnlichen Zustand wie vor dem Ereignis bringen zu können. Diese Nachbarschaft war und ist durch einen hohen Stand in der Gesellschaft und eine gute Vernetzung mit politischen Instanzen gekennzeichnet. Einige der Lokalpolitiker wohnten zudem selbst in Rodenkirchen ${ }^{674}$. Die Politiker versuchten, sich in einflussreichen Hochwassergebieten beliebt zu machen. Die Wirte in der Altstadt bildeten eine dominante Interessengruppe inmitten des zu erhaltenden historischen Zentrums. Bereits nach den zwei Überschwemmungen von 1983 vertrat der Hotel- und Gaststättenverband die Interessen der geschädigten Gastronomen gegenüber den Kölner Behörden ${ }^{675}$. Dies mag dem traditionsreichen Bild zu Grunde liegen, welches das Image der Stadt in der Welt verkörpert. Der Dom neben dem Rhein und Wirte, die gastfreundlich lokales Bier, ein Kölsch, ausschenken, zieht Touristen wie Einheimische in die Altstadt Kölns. Die Pflege des historischen Ortskerns lag den Verantwortlichen der Stadt sehr am Herzen, wie bereits im ersten Kapitel zur Bewahrung der Stadtansicht dargelegt wurde. Deswegen wurde dort ein sehr aktiver behördlicher Einsatz gegen das Hochwasser gezeigt und die

\footnotetext{
${ }^{673}$ Internetbasierte Quelle 7. Die gestrichelte Linie zeigt den Verlauf des Hochwasserschutzes bis zu einem Pegel von 11,90 m K P an, die durchgezogene Linie markiert den Grundwasserflurabstand bei einem Hochwasserpegel von 11,90 $\mathrm{m}$ K P.

${ }^{674}$ Reinhard Vogt, Leiter der Hochwasserschutzzentrale Köln, am 17.12.2011 Köln.

${ }^{675}$ Vgl. Das Mai-Hochwasser von 1983.
} 
Gastronomen wurden durch die lokalen Politiker zumindest theoretisch mit einer Entschädigung bedacht. Ihnen wurde zugestanden, ihre Renovierungskosten an der zu zahlenden Einkommenssteuer abzugelten ${ }^{676}$. Die Bewohner von Porz hingegen waren vergleichsweise ,abgeschnitten“. Obwohl sie nicht wie die ausländischen Arbeiter durch Sprache, fehlenden gesellschaftlichen Rückhalt und durch das Wasser selbst abgeschnitten waren, gab es in Porz weder Strom noch Wasser. Die Beschwerden dieser Anwohner deuten darauf hin, dass die städtischen Helfer in ihren Augen die Arbeit in anderen Teilen der Stadt vorrangig behandelten, während die Probleme im Vorort Porz außer Acht gelassen wurden. Diese Menschen fühlten sich, anders als die Rodenkirchener, durch die Verantwortlichen (Retter) im Stich gelassen. Die Kritik der Porzer Bevölkerung an den Verantwortlichen aus dem Notfallmanagement weist auf ihre Unzufriedenheit hin.

Es konnte hingegen kein Zeitungsbericht gefunden werden, welcher den immigrierten ausländischen Arbeitern eine Stimme verlieh; diese wurden offenbar nicht zu ihren Erlebnissen befragt. Lediglich aus der Berichterstattung über die „tatkräftige Feuerwehr“ ist nachzuvollziehen, dass ihnen Hilfe angeboten wurde und dass sie in einem Gebiet vor dem Deich lebten, welches von der Flut unmittelbar betroffen war. Im Jahr 1970 war das Konzept der Gastarbeiter mit einem begrenzten Zeitraum konnotiert. Es wurde aus der deutschen wie auch aus der Perspektive des Gastes angenommen, dass dieser nach getaner Arbeit wieder in sein Heimatland zurückkehren würde. Im Moment des Hochwassers schienen die Verantwortlichen nicht berücksichtigt zu haben, dass Gäste zu Besuch waren, und übersahen diese hilfsbedürftige Gruppe inmitten des Überschwemmungsgebietes. Aber nicht nur die ausländischen Mitbürger lebten in relativ ungeschützten Hochwassergebieten. Am Weidenweg in Poll, auf der anderen Rheinseite, lag eine Siedlung mit sozial benachteiligten Familien. Wohlmöglich wussten diese Anwohner sich nicht $\mathrm{zu}$ helfen und verharrten deswegen in der vom Hochwasser eingeschlossenen „Behelfsheimsiedlung“ in ihrer ausweglosen Situation. In dem Resilience-Diskurs liegt der Fokus auf den „,soft skills“ der Betrachtungsgruppe $^{677}$, wie z. B. der Anpassungsfähigkeit. Obwohl die Stadt Köln während der Hochwassertage Notunterkünfte bereitstellte, nutzte diese Gruppe das Angebot nicht. Diese Familien waren sich entweder nicht sicher, ob sie sich den städtischen Unterstützern anvertrauen konnten oder sie wussten nicht, wie gefährlich die Situation wirklich war und wie man die Notunterkünfte beziehen sollte. Die Anwohner zeigten äußerste Zurückhaltung, den Beistand der Stadt anzunehmen ${ }^{678}$, und verteidigten ihren Standpunkt, in ihrer Wohnung zu

\footnotetext{
${ }^{676}$ Stadt Köln, Rat der Stadt Köln, 3. Sitzung vom 16.04.1970.

${ }^{677}$ Voss 2008, 52.

${ }^{678}$ Artikel: Ungern umgezogen - Kaum jemand wollte in die Notunterkunft, Kölner Stadtanzeiger, 25.02.1970.
} 
bleiben, sogar aggressiv. Die Beamten sahen sich gezwungen eine Familie zu bevormunden, um sie aus der Gefahr heraus zu bringen. Die Stadt verfuhr mit dieser Gruppe, wie Eltern mit ihren uneinsichtigen Kindern, und entband sie ihrer Rechte. Durch einen administrativen Trick wurde der Widerstand der Familie gebrochen und diese gezwungen, den Anweisungen der Stadt zu folgen. Diese Gruppe wurde reglementiert und ihrer Selbstständigkeit entbunden. Das Verhalten besagter Familie wurde lediglich in Zeitungsartikeln über die Hilfeleistungen der Stadt, nämlich dem Spendensammeln durch Oberbürgermeister Burauen, erwähnt. Dabei wurde der Fall sogar zweifach von der Presse ausgenutzt. Erstens unterstützte die Darstellung der Bedürftigen die Aktivitäten Burauens noch sinnvoller herauszustellen und zweitens konnte die Presse ihren eigenen Artikel für die Leserschaft noch dramatischer ausgestalten. Die Presse erfuhr von den Vorfällen aus den Berichten des Amtes für Stadtentwässerung bei dem der Bericht des Amts zur Beseitigung von Wohnungsnotständen am 25. Februar 1970 eingegangen war. Diesen Berichten ist deutlich zu entnehmen, dass die Helfer keinen anderen Weg sahen als zum Schutz der Anwohner das Haus als baufällig zu deklarieren, da nur so einer Zwangsräumung stattgegeben werden konnte ${ }^{679}$. Die Tageszeitung Kölner Stadt Anzeiger berichtete dagegen: „In Poll wurde ein Haus so stark in Mitleidenschaft gezogen,

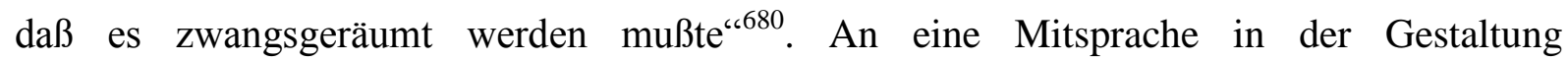
administrativer Reaktionen auf das Hochwasser war keineswegs zu denken. Diese vulnerablen Gruppen besaßen keine Stimme. Aufgrund ihrer fehlenden „soft skills“ übernahmen die Behörden die Verantwortung für die Gruppe und trafen für sie Entscheidungen. Die Berichterstattung der lokalen Zeitungen war vergleichbar mit derjenigen über die Arbeitsmigranten $^{681}$. Beiden Gruppen wurde keine Stimme verliehen oder Partizipation zugestanden. Weder die Gastarbeiter noch die Anwohner vom Weidenweg waren in der Lage, die vorhandenen Maßnahmen zu kritisieren und auf diesem Weg eine Mitbestimmung zur Verbesserung zu realisieren. Diese beiden Randgruppen besaßen keine Interessenvertreter und hatten somit ,no voice ${ }^{\text {“682 }}$.

Wie standen die Verantwortlichen aus dem Stadtrat zu den Opfern? Unter Anwendung des erarbeiteten Hilfsmodells wird im Folgenden der Aushandlungsprozess der Hilfeleistungen betrachtet. Die CDU-Fraktion in der Opposition der Stadt Köln forderte eine Sondersitzung,

\footnotetext{
${ }^{679}$ Bericht der Stelle zur Beseitigung von Wohnungsnotständen, 25.02.1970.

${ }^{680}$ Artikel: Mehr als 100000 DM gespendet, Kölner Stadtanzeiger, 11.03.1970.

${ }^{681}$ Der Begriff der Arbeitsmigranten geht auf den Soziologen Thomas Schiller zurück.

${ }^{682}$ (Voss, 2008).Voss unterstreicht, die Beobachtung, dass die Verletzlichen ,no voice“ besitzen, also kein Mitspracherecht oder keine Fürsprecher haben, welche ihnen eine Stimme verleihen. Stattdessen werden die Stimmen der Missachteten auf verschiedenen gesellschaftlichen Ebenen durch ein Bündel verschiedener Methoden ausgeschlossen, überhört oder zum Schweigen gebracht. Diese Missachtung vollzieht sich meist im Verborgenen.
} 
um sich über die Hilfsmaßnahmen, welche das Land Nordrhein-Westfalen angeregt hatte, zu informieren $^{683}$. Die Diskussionen um die Hilfsmaßnahmen und Schadensersatzleistungen wurden hauptsächlich durch zwei Akteure geführt: Dr. Petzborn und sein SPD-Parteikollege Dr. Schäfer verteidigten die zurückhaltenden Reaktionen der durch die SPD geführten Verwaltung gegenüber den Forderungen aus der Opposition (CDU), aber auch aus der eigenen Partei, mehr Unterstützung für die Hochwassergeschädigten zu leisten. In der ersten Stadtratssitzung ist eine Abfolge der Argumentation der Regierungspartei zu erkennen, die sich genauso in der zweiten Sitzung beobachten lässt. Zunächst wurde auf die Leistungen der Stadt hingewiesen. Dr. Petzborn schien es wichtig, die städtischen Leistungen an die Bürger, wie das Auspumpen der Keller und der Straßenreinigung, monetär zu beziffern und diesen Betrag als von der Stadt an die Opfer geleistet anzuerkennen. Demgegenüber stand die Betonung der Unmöglichkeit, Hochwasserschäden genau festzustellen. Da die Schadensaufstellung als Grundlage für Hilfsmaßnahmen diente, deutete er bereits hier an, dass nicht weiter verfahren werden könne. In einem dritten Schritt akzentuierte er die städtische Abhängigkeit von den noch nicht ergangenen Landesrichtlinien. Er beabsichtigte auszudrücken, dass nicht er Schuld habe, wenn die Unterstützung der Hochwasseropfer begrenzt ist. Mitte April übernahm Parteikollege Dr. Schäfer die Ausführung dieser drei Punkte. Er erwähnte zuerst die „Arbeit der Verwaltung“ und damit indirekt die Aufwendungen der Stadt. In einem zweiten Punkt hob er die Schwierigkeiten bei der Schadensbezifferung hervor, um zuletzt wieder darauf zu verweisen, dass niemand absehen könne, wann die Landesrichtlinien ergingen. Sein letzter Punkt lässt sich mit der Bedeutung übersetzen, dass niemand absehen könne, ob überhaupt Hilfe geleistet würde. Wie Dr. Petzborn einen Monat zuvor, versuchte Dr. Schäfer sich ebenfalls hilflos darzustellen und sich somit bei den ebenso hilflos wartenden Bürgern einzureihen und hierdurch an deren Verständnis für die Zurückhaltung der Administration zu appellieren. Die Abgabe der Verantwortung an höhere Instanzen zeigt, dass es keine Lösungs- oder Handlungsbereitschaft innerhalb des zuständigen Personenkreises in Köln gab. Der Oberbürgermeister Theodor Burauen (SPD) verhielt sich in den Verhandlungen zurückhaltend, schmückte sich aber als Vorsitzender des Kreisverbandes des Deutschen Roten Kreuzes mit der Verteilung der Spendengelder, welche in Zusammenarbeit mit dem Herausgeber des Kölner Stadt Anzeigers, Alfred Neven DuMont, gesammelt worden waren ${ }^{684}$. Es waren ca. 100.000 DM gesammelt worden, welche nun als ausreichend betitelt wurden, um die Schaden privater Betroffener auszugleichen.

\footnotetext{
${ }^{683}$ Artikel: In der guten Stube schwimmen die Möbel, Kölner Stadtanzeiger, 28.02.1970.

${ }^{684}$ Artikel: Mehr als 100000 DM gespendet, Kölner Stadtanzeiger, 11.03.1970.
} 
Die Maßnahmen, welche die Stadt im April in Form von Steuererleichterungen in die Wege leitete, richteten sich lediglich an Gewerbetreibende und Freiberufliche. Der Kämmerer Meyer rechnete offensichtlich nicht damit, viele Anträge aus dieser Gruppe zu bekommen, schließlich plante er alle Eingänge persönlich zu bearbeiten ${ }^{685}$. Hochwasserschäden an Hausrat und Bekleidung der privaten Haushalte sollten nur über das Bundessozialhilfegesetz abgegolten werden. Bei der Anerkennung der Schäden wurden die Einkommen der Antragsteller betrachtet, um die Bedürftigkeit eines Antragstellers und Hochwasseropfers festzustellen $^{686}$. Des Weiteren bestimmte ein nach sozialen Standards festgelegter „Warenkorb“ die möglichen Gegenstände, welche als Schaden anerkannt werden konnten. Der Wert des tatsächlich zerstörten Artikels wurde nicht ersetzt. Im Bundessozialhilfegesetz war ein Gegenwert für entsprechende Objekte gesetzlich festgelegt, der nun an Bedürftige erstattet wurde. Eine von den Kommunalpolitikern verkündete vollständige Hilfe ${ }^{67}$ bedeutete also keineswegs, dass die Menschen nach dem Hochwasser genauso gestellt waren wie vor dem Ereignis. Das Ergebnis des Aushandlungsprozesses zeigt eine Zweiteilung der Hochwasseropfer in sozial schwache Bürger und Unternehmer auf. Durch diese Einteilung wurden zwei Fakten geschaffen:

Erstens wurde damit die Gruppe der hilfebedürftigen Hochwasseropfer organisatorisch geschmälert. Die Verantwortlichen des Stadtrats definierten, wer als Opfer gelten durfte und wer nicht. Gruppen zu bilden bedeutete Kriterien zu entwerfen, welche diese Gruppen charakterisieren. Nicht alle durch Schaden betroffenen Menschen konnten sich aber der einen oder der anderen Gruppe zuordnen. Auch durften nicht alle politischen Akteure bei der Gestaltung der Gruppen teilhaben. Beispielsweise schlug Dr. Vetter (SPD) eine weitere Unterteilung zwischen „Schäden am Privateigentum und bei bestimmten Sportvereinen“688 vor, welche Dr. Petzborn abwehrte, um die Erweiterung erst „,von der Verwaltung prüfen“6689 zu lassen. Die mit Macht ausgestatteten Entscheidungsträger lenkten die Verhandlungen und wollten sichergehen, diese Macht weiterhin zu verteidigen. Wohlweislich wurden scharfe Grenzen gezogen, um nicht am Ende den gesamten Schaden in Köln ersetzen zu müssen. Auch schien die Gruppenbildung die Komplexität der Geschädigten zu vereinfachen. Gruppen zu bilden, welche bereits über eine eigene „Zuständigkeit“ verfügten, schmälerte die Verantwortung der Verwaltung. Das Sozialamt ist so eine Zuständigkeit, da es wie ein Netz die gesellschaftlich Schwachen auffängt: Die Bedürftigen nach dem Bundessozialhilfegesetz

\footnotetext{
${ }^{685}$ Stadt Köln, Rat der Stadt, 2. Sitzung, 12.03.1970.

${ }^{686}$ Ebd.

${ }^{687}$ Artikel: So wurde den Opfern geholfen, Kölner Stadtanzeiger, 29.04.1970.

${ }^{688}$ Stadt Köln, Rat der Stadt, 2. Sitzung, 12.03.1970, 68.

${ }^{689}$ Ebd.
} 
würden also nicht durch spezifische Hochwasserhilfen einer politischen Ebene unterstützt werden müssen.

Zweitens definierten die Verantwortlichen der Stadt nicht nur die Anspruchsgruppen, sondern auch die Schadenshöhen. Sie selbst bestimmten, in welchem Umfang finanzielle Verluste durch das Hochwasser hervorgerufen wurden. Als anerkannte Schäden lassen sich wiederum zwei Klassifikationen benennen. Auch hier wurden Gruppierungen vorgenommen, die Zugehörigkeit und Anerkennung einstuften. Die Schäden an öffentlichem Eigentum und die Schäden an Privateigentum stehen beispielhaft für solche Gruppen. In den beiden Stadtratssitzungen wurden lediglich die wie in Tabelle 4 aufgeführten Schadensaufzählungen aufgelistet:

\begin{tabular}{|c|c|c|c|c|}
\hline & \multicolumn{2}{|c|}{ Sitzung vom 12. März 1970 ${ }^{690}$} & \multicolumn{2}{|c|}{ Sitzung vom 16. April 1970 ${ }^{69 \Gamma}$} \\
\hline & Schadensart & Umfang & Schadensart & Umfang \\
\hline $\begin{array}{l}\text { Öffentliche } \\
\text { Schäden }\end{array}$ & $\begin{array}{l}\text { Stadtreinigung } \\
\text { Berufsfeuerwehr } \\
\text { Häfen: Einsatz von } \\
\text { Personal, Folge der } \\
\text { Schifffahrtssperre }\end{array}$ & $\begin{array}{l}48.000 \mathrm{DM} \\
51.500 \mathrm{DM} \\
420.800 \mathrm{DM}\end{array}$ & & \\
\hline \multirow{3}{*}{$\begin{array}{l}\text { Private } \\
\text { Schäden }\end{array}$} & & & $\begin{array}{l}\text { Schäden an } \\
\text { Sachanlagevermögen der } \\
\text { Wirtschaftsunternehmen }\end{array}$ & 4-6 Mio. DM \\
\hline & & & $\begin{array}{l}\text { ca. } 234 \text { Anträge zum Ersatz } \\
\text { von Hausrat und Bekleidung }\end{array}$ & $\begin{array}{l}70.000 \mathrm{DM} \\
\text { (Sozialhilfemittel) } \text { 100.000 DM } \\
\text { (Spendengelder) }^{692}\end{array}$ \\
\hline & Betroffene Häuser & 400 Stück & Gebäudeschäden & N.N. \\
\hline Summe & 6.690.300 DM & & & \\
\hline
\end{tabular}

Tabelle 4: Hochwasserschäden in Köln 1970.

Private Hochwasseropfer hatten per Definition nur einen finanziellen Schaden, wenn sie entweder selbstständig Gewerbetreibende waren und in dem Kalenderjahr einen Gewinn zu versteuern hatten, von dem sie die Neuinvestitionen aufgrund des Hochwassers abziehen konnten, oder wenn sie Sozialhilfeberechtigte waren ${ }^{693}$. Alternative Schäden, die hinter den Klassifikationen verblassten, wurden durch die Verantwortlichen nicht mehr wahrgenommen. Hochwasseropfer, welche in keine der beiden Kategorien fielen, wurden nicht durch öffentliche Hilfe bedacht, denn die Akteure im Stadtrat lehnten die Übernahme der Verantwortung für weitere finanzielle Schäden ab. Das Hochwasser wurde ausschließlich als Naturgewalt wahrgenommen und so schien der Schaden von einem unvorhersehbaren Zufall abhängig zu sein, und in seiner Eigenschaft nicht an Verwaltungen adressierbar zu sein. Da

\footnotetext{
${ }^{690}$ Stadt Köln, Rat der Stadt, 2. Sitzung, 12.03.1970.

${ }^{691}$ Ebd., 3. Sitzung, 16.04.1970.

${ }^{692}$ Ebd., 175. „Mit diesen Zahlungen des Roten Kreuzes sind die wesentlichen Schäden behoben.“

${ }^{693}$ Stadt Köln, Rat der Stadt, 2. Sitzung, 12.03.1970.
} 
ein vergleichbares Schadensereignis nach Auffassung der Stadtratsmitglieder jedoch jederzeit wieder auftreten könnte, schien es unmöglich, diese Last auf die öffentlichen Kassen zu übertragen. Die Wahrnehmung des Hochwassers schien bei der Verwaltung und der Opposition ähnlich zu sein; es bestehen keine Hinweise auf eine differenzierte Wahrnehmung. Die SPD-Vertreter Dr. Petzborn und Dr. Schäfer stellten die Schäden am öffentlichen Eigentum gleichberechtigt neben die privaten Schäden und die Stadt neben die privaten Hochwasseropfer. Sie hegten ein hohes Anliegen, die Stadt als eines der vielen Opfer darzustellen. Sie kommunizierten, die Stadt leide ebenfalls unter der unberechenbaren Naturgewalt des Rheins und demnach sei es nur angemessen, dass die Verwaltung nicht auch noch die privaten Schäden aufgebürdet bekommen sollte.

Alternativen wurden von verschiedenen Stadtratsmitgliedern eingefordert. Bemerkenswert ist dabei, dass ein alternatives Handeln nicht nur von der Opposition gefordert wurde. In der Diskussion mit der CDU zeigten sich auch immer wieder SPD-Kollegen hilfsbereit. Surges von der SPD skandierte in der ersten Sitzung Mitte März die Parole „Wer schnell hilft, hilft doppelt ${ }^{\text {“694}}$. Sein Kollege Vetter stand ihm in der gleichen Besprechung zur Seite und versuchte „ad hoc Hilfe zu leisten“695. Dem Lob des Bürgermeisters Burauen (SPD), dass immerhin die Spenden in Höhe von ca. 100.000 DM ausgezahlt würden, widersprach sein Parteikollege Gruner mit der Einforderung städtischer Gelder. An diesen innerparteilichen Auseinandersetzungen zeigt sich die Unsicherheit der Verantwortlichen im Umgang mit den Schäden und Hilfsmitteln. Aus der städtischen Diskussionsabfolge wird ersichtlich, dass die Reaktion auf die entstandenen Schäden keine alltägliche Aufgabe der Lokalpolitik war und deren Grundlage erst gefunden werden musste. Ein „Tauziehen“ um institutionelle Verantwortlichkeiten, Risiko- und Rollenverteilungen zwischen Betroffenen und staatlichen Behörden prägte monatelang die Argumentationslinien im Aushandlungsprozess der Hilfsleistungen. Zwischen moralisch-gebotenen Maßnahmen und strategisch-staatlichem Verhalten entstand ein Zwiespalt. Die Verantwortlichen der Stadt wurden von einer Verhaltensunsicherheit ergriffen und versuchten im Angesicht der Geschädigten Haltung zu bewahren. Die Frage, ob nun erst Schäden zu beziffern seien oder das Wissen über Hilfen vorhanden sein müsste, erzeugte eine Zeit des Stillstandes, die den Akteuren willkommen schien. Abwarten wurde seit der ersten Debatte - durchgesetzt durch die Vertretung des Sozialdezernenten Dr. Petzborn - zur lokal-politischen Strategie. Die unsichere Situation, in der die Verantwortlichkeiten erst noch definiert werden mussten, führte dazu, dass die Akteure sich die Strategie des Abwartens zu Nutzen machten, denn das Hinauszögern

\footnotetext{
${ }^{694}$ Stadt Köln, Rat der Stadt Köln, 2. Sitzung, 12.03.1970, 68.

${ }^{695}$ Ebd.
} 
versprach den größten Nutzen zu bergen. Jede Regung war nun zukunftsweisend und mit großen Unsicherheiten verbunden, denn niemand wollte in die Verlegenheit geraten, auch bei zukünftigen Hochwasserschäden wieder Entschädigungen zahlen zu müssen. Für keinen Politiker in einer Machtposition schien die Alternative der breiten Unterstützung der Hochwassergeschädigten von Vorteil zu sein. Die Strategie des Abwartens wird in der Rede der beiden SPD-Sprecher deutlich: Beide betonten, dass die Landesregierung auf die Schadensmeldungen warte, um daraufhin Richtlinien zu erarbeiten. Darum ist anzunehmen, dass die Parteikollegen genau Bescheid wussten und nach der Flut Zeit gewonnen werden sollte. Eine weitere Bestätigung gibt Schäfer, der aufgrund von „groben Umfragen“696 ungenaue Zahlen an den Minister meldete. Er beabsichtige offenbar, ungenau zu bleiben und sich in der allgemeinen Problematik, Schäden genau zu beziffern, zu verstecken. Es ist unwahrscheinlich, dass er die Gebäudeschäden zu einem späteren Zeitpunkt noch erheben wollte. Noch deutlicher wurde Surges (SPD), als er Mitte März wissen ließ, dass die „Richtlinien des Landes noch etwas auf sich warten lassen“697. Schäfer (SPD) offenbarte sogar den Grund, weshalb er bevorzugte, keine Hilfe zu leisten. Mitte April erklärte er sein Verhalten mit der finanziellen Situation der Stadt Kölns. Der CDU-Vertreter Pohl hatte in dem Streit um die Gebäudeschäden einen „wunden Punkt“ getroffen, als er von seiner Inaugenscheinnahme der Gebäude vor Ort berichtete. Schäfers Ausführungen zu den Opferbefragungen dienten dazu, die Schadensfeststellung aufzuschieben und damit zusätzlich $\mathrm{zu}$ erschweren. Auch sein Vergleich mit Brandopfern verdeutlichte seine Haltung, die öffentliche Hand sei nicht zu belangen. An dieser Stelle zeigt sich das Spannungsfeld, Risiko und Verantwortung auf andere zu verteilen, par excellence.

An der gewählten Strategie des Abwartens wurde von der Opposition mehrmals Kritik ausgedrückt. In der ersten Sitzung forderte Gruner „eigene Vorstellungen über gezielte finanzielle Hilfsmaßnahmen“698, während in der zweiten Konferenz seine Parteikollegin Müller verlangte, „selber Überlegungen an [zu] stellen“6699. In beiden Besprechungen verteidigte die SPD, keine eigenen Ideen zur Unterstützung der Bürger vorweisen zu können, wie die CDU das Verhalten der SPD umschrieb. Würde man jetzt großzügig in der Schadensersatzfrage verfahren, so würden die Bürger im Hochwasserschutz noch nachlässiger, erwartete die SPD. Der Schaden würde künftig größer und die Stadt müsste dann wieder dafür aufkommen. Dies schienen die einzigen negativen Konsequenzen zu sein,

\footnotetext{
${ }^{696}$ Stadt Köln, Rat der Stadt Köln, 3. Sitzung, 16.04.1970, 174.

${ }^{697}$ Ebd., 2. Sitzung, 12.03.1970, 68.

${ }^{698}$ Ebd., 68.

${ }^{699}$ Ebd., 3. Sitzung, 16.03.1970, 175.
} 
welche bedacht wurden. Dagegen blieben Gedanken über eventuelle Folgen dieser passiven Haltung aus, stattdessen verwiesen die Akteure wie zum Schutz auf die gesetzliche Lage. Laut rezenter Gesetzgebung war die Zahlung von Schadensersatz nach Naturereignissen der Freiwilligkeit der politischen Instanzen überlassen. Dr. Petzborn referierte in der ersten Sitzung, „im Rahmen der bestehenden Gesetze Hilfe [zu] leisten“700, während er in der zweiten Sitzung darauf verwies, „daß jeder Hauseigentümer nach der jetzigen Rechtslage verpflichtet ist, sich selbst zu schützen““701. Den Verantwortlichen war bewusst, dass die Bürger über die gesetzlichen Regelungen hinaus Unterstützung oder Anerkennung verlangten und versuchten ihr Verhalten daher mit den Hinweisen auf die rechtliche Lage zu entschuldigen. Die Landespolitik verhielt sich in diesem Punkt sehr ähnlich. Parallelen sind besonders in der Betonung der Rechtslage sowie in der Zurückhaltung der Hilfen zu finden. In seiner verteidigenden Haltung verwies Innenminister Weyer auf die rechtliche Obliegenheit und bekräftigte, bereits mehr Hilfe zu leisten als er verpflichtet sei. Er nutzte die Debatte, um an die Freiwilligkeit und Großzügigkeit der landespolitischen Hilfen zu erinnern. Kurz vor der Wahl am 14. Juni 1970 war ein solcher Hinweis nicht unzweckmäßig. Während er die Rollen der Behörden definierte - für ihn stand fest, dass erstrangig die Gemeinden die Betroffenen unterstützen müssten, während die Leistungen der Landesregierung lediglich subsidiär sind wurde erkennbar, dass die Schäden auch für ihn persönlich ein Spannungsfeld zwischen lokalen und landespolitischen Behörden und dem Betroffenen aufspannten. Auch er war angesichts der zu übernehmenden Verantwortung und deren Bedeutung für die Zukunft vorsichtig. Seine Vorsicht konnte er mit einem Hinweis auf die Abhängigkeit der Landesregierung von dem jeweiligen Zustand der Landeskassen verständlich darlegen. Innenminister Weyer gelang es, mit fadenscheinigen Zitaten aus einem noch immer unbekannten Kabinettsbeschluss Paroli zu bieten. Er konnte demonstrieren, dass seine Botschaft in Köln angekommen sein musste und zitierte dazu aus Stadtrat-Protokollen. SPDMitglied Toetemeyer präsentierte wie sein Parteikollege Weyer Stadtratzitate. Anhand von Kölner Dokumenten versuchte er zu beweisen, dass die Kommunalregierung von dem Kabinettsbeschluss Kenntnis besaß. Damit behauptete er indirekt wie Weyer, die Kölner SPDVerwaltung führe eine Strategie des Hinauszögerns, um keine Hilfen leisten zu müssen. Toetemeyer demonstrierte Einigkeit in der Regierungspartei und ließ den Wahlkampf nicht außen vor. Er stellte durch seine Anspielung auf die Presseaktion einen deutlichen lokalen Bezug her. Toetemeyer lokalisierte sich in der Gruppe hilfsbereiter (SPD-)Retter und

\footnotetext{
${ }^{700}$ Stadt Köln, Rat der Stadt Köln, 2. Sitzung, 16.04.1970, 175.

${ }^{701}$ Ebd., 3. Sitzung, 16.04.1970, 177.
} 
verheimlichte nicht, dass er sich ohnehin nur zu Wort meldete, da es in der Verhandlung um seinen Wahlkreis ging.

Als dritte Parallele wird augenscheinlich, dass auch die Landesregierung den als unberechenbar wahrgenommen Bürger als Gefahr betrachtete. Innenminister Weyer sprach von einer Gefahr, womit er nicht das Hochwasser meinte. Es erschien ihm geradezu leichtsinnig, die Bevölkerung über die Entschädigungsmaßnahmen aufzuklären. Zwar erklärte er in der Abhängigkeit der Landesregierung von dem jeweiligen Zustand der Landeskassen seine Vorsicht, doch fürchtete er den Bürger als die unberechenbare Komponente. Für ihn verkörperte das Hochwasser nicht Gefahr, sondern er verstand die Überschwemmung als eine gewöhnliche Laune der Natur. Würde dem Bürger nun Zahlungen zugesichert, würde der Betroffene diese bei einem zukünftigen Ereignis wieder einfordern. Er war überzeugt, der Bürger könne mit soviel Transparenz nicht umgehen. Weyer verstand den Menschen als Auslöser für den Schaden. In Anlehnung an das Katastrophenkonzept interpretierte Weyer den Schaden als Produkt natürlicher und sozialer Vorgänge. Ohne eine Ansammlung von Werten wäre ein Schaden unmöglich, somit verstand er in der Unvorhersehbarkeit menschlichen Handelns die größte Gefahr, welche im Folgenden mit dem Begriff des unpredictable civil belegt wird. FDP-Mitglied Tornau machte drei Gefahren für die Landesregierung aus: Erstens sah er die Bevölkerung als gierige Masse, welche nicht mehr versuchen würde den Schaden einzudämmen, wenn die Landesregierung nun großzügig verfahre. Er warnte davor, dass die private Vorsorge der Bürger durch Schadensersatzzahlungen der öffentlichen Hand nachlassen könnte. Zweitens sah er sich der „unberechenbaren Naturkatastrophe“ ausgeliefert und drittens sah er ein Problem in der seiner Meinung nach fehlenden Eigeninitiative der Kölner Verantwortlichen. Auch er war sich des Zielkonflikts um zukünftige Schadensminimierung im Spannungsfeld zwischen staatlichen Institutionen und Bürgern bewusst. Dabei gebar er sich dominanter als seine Vorsprecher und warf den Kölner Verwaltungsmitgliedern vor, selbst Schuld zu tragen, sollten sie den Beschluss nicht gekannt haben. Er unterstellte der Kölner Verwaltung, durch Passivität Zeit vertrödelt zu haben. Somit wird die Schwierigkeit, in einem plötzlichen Unglücksfall handlungsfähig zu bleiben, ganz allgemein verdeutlicht.

Abschließend wird erkennbar, dass das Vorgehen der städtischen SPD-Regierung dem Verhalten der SPD auf Länderebene ähnelte: Der Kabinettsbeschluss wurde angeblich bereits am 03. März 1970 gefasst, aber erstmalig am 22. April 1970 besprochen. Auch hier ist eine Phase der Passivität augenscheinlich. In diesem Zusammenhang kann einerseits die Strategie 
des Abwartens weiter verfolgt und das Spannungsfeld der Rollen auf nächst höherer Ebene aufgezogen werden. Andererseits sperrte Stillstand faktisch den Raum für die Opposition, sich im Wahlkampf als Helfer zu profilieren. Insbesondere die bevorstehende Wahl könnte also ein Grund für das zähe Vorgehen gewesen sein. Im Landtag waren alle Redner vom Wahlkampf ergriffen, auch wenn es keinem Sprecher überzeugend gelang, das Hochwasser im Wahlkampf zu nutzen. Hüsch versuchte nicht mal das Ereignis selbst zu instrumentalisieren, sondern er bemühte sich, das Thema der mangelhaften Transparenz der regierenden Partei als allgemeines Versagen in Notsituationen publikzumachen.

An beiden Schauplätzen bildete die CDU die Opposition. Unter anderem versuchten die CDU-Kollegen des Stadtrats Schröder und Pohl umgehende Hilfe zu ermöglichen. Sie thematisierten verschiedene konkrete Optionen, während sie ebenfalls versuchten, die Schäden auszudifferenzieren. Auch auf der Landesebene war die Fraktion der CDU aktiv mit der Transparenz um die Schadensersatzfrage beschäftigt, wie der Antrag vom 17. März 1970 zeigte. Bemerkenswert bleibt der Zwiespalt zwischen Stadtrat und Landtag, welcher nie direkt angesprochen wurde. Beide Ebenen beschäftigten sich nicht mit dem Ereignis als Naturgefahr, seinen Ursachen oder seiner Bekämpfung. Während der Stadtrat sich mit den Hochwasserschäden auseinandersetzte, wurde im Landtag Wahlkampf betrieben und nur über Transparenz gestritten. Dabei ging das Hochwasser völlig unter. Das Ereignis selbst stand für die Entscheidungsträger nicht im Vordergrund. Lediglich die Konsequenzen einer zu großzügigen Schadensübernahme wurden gefürchtet. Damit verkörperte der potentielle zukünftige Antragsteller nach Schadensersatz die eigentliche Bedrohung für die öffentlichen Kassen und nicht die Wahrscheinlichkeit der Überflutung selbst.

Das Abwarten und Hinauszögern kann als Taktik interpretiert werden, um die Unsicherheit bezüglich der Rollen um Verantwortlichkeiten zu überspielen. In der Zwischenzeit könnten andere Akteure die unerwünschten Tätigkeiten übernehmen, so die Hoffnung. Beispielsweise könnten die Anwohner selbst einen Weg finden, mit den Schäden umzugehen. Es wurde die Notwendigkeit der privaten Vorsorge betont, um die Höhe der Schäden gering zu halten. Resultierend daraus wurde davor gewarnt, die private Vorsorge als obsolet darzustellen, sollte die öffentliche Hand den Bürger entschädigen. Keine staatliche Institution wollte sich als „Auffangbecken für versäumte bürgerliche Vorsorge“ ausnutzen lassen. Die Landesregierung betrachtete ihre Unterstützungsleistung als subsidiäre Hilfe, wohingegen sie die kommunalen Behörden als Institutionen betrachteten, die als Erste den Bürgern aushelfen müssten. Diese Argumentation ist aus der akteursspezifischen Perspektive verständlich. 


\subsection{Reaktionen von 1995: Ein ganz neues Konzept}

Als Reaktion auf das Hochwasser von 1995 wurde das neue Hochwasserschutzkonzept Köln fertiggestellt. Im Kapitel zwei wurde das Konzept vorgestellt. Der Schwerpunkt der verschiedenen Maßnahmen lag von nun an auf der Raumentwicklung anstatt an der Wasserkante des Flusses. Außerdem wurde das Prinzip der Verantwortung gegenüber Unterliegern anerkannt. Nach dem Verursacherprinzip wurde der Hochwasserschutz neu organisiert, damit Unterlieger zukünftig nicht mehr durch Eingriffe der Oberlieger betroffen seien $^{702}$. Der Rat beschloss mit gutem Beispiel voran zu gehen und lokale Retentionsräume einzurichten. Er spekulierte, sich somit in die Position zu rücken, selbst Forderungen an die Kölner Oberlieger ${ }^{703}$ richten zu können. Er forderte von den politisch Verantwortlichen in Hessen, Rheinland-Pfalz und Baden-Württemberg endlich bereits 1982 vereinbarte Retentionsräume einzurichten ${ }^{704}$. Mit dem Ziel, die Umsetzung des Deutsch-FranzösischenVertrags von 1982 und eine Beteiligung an den Entscheidungen zur Bewirtschaftung des Rheineinzugsgebiets zu erreichen, forderten die Verantwortlichen der Stadt Köln das Land NRW auf, eine Sprecherfunktion für Köln in dem Vertrag einzunehmen.

In Köln wurde 2009 ein erster Retentionsraum am südlichsten Punkt des zu schützenden Bereichs mit dem Planfeststellungsabschnitt 12 zur Aufnahme von 4,5 Mio. m³ Hochwasser realisiert $^{705}$. Der zweite Rückzugsraum im Kölner Stadtgebiet soll zukünftig im Naturschutzgebiet Worringer Bruch eingerichtet werden und ca. 30 Mio. $\mathrm{m}^{3}$ Wasser fassen können ${ }^{706}$. Die Regenwasserversickerung, die Renaturierung von Bachläufen sowie die Vereinbarung gesetzlicher Überschwemmungsgebiete wurden von den Verantwortlichen in Köln ebenfalls als Möglichkeiten zur Reduzierung von Hochwasserpegeln in Betracht gezogen. Bei der Regenwasserversickerung teilten die Verantwortlichen der Stadt das Areal in bestehende Bauten und Neubaugebiete. Das erste Ergebnis zeigte sich im Februar 1996 in Form einer Forderung: Bereits seit dem 01. Juli 1995 wurde durch die Novellierung des $\S 51$ des Landeswassergesetzes NRW die sogenannte dezentrale Regenwasserbewirtschaftung für Neubaugebiete verpflichtend. Dabei wird das Regenwasser entweder gespeichert, abgeleitet oder versickert $^{707}$. Für Neubaugebiete forderte der Rat, das Regenwasserversickern in den

\footnotetext{
${ }^{702}$ HWSK 1996.

${ }^{703}$ Ebd., 79.

${ }^{704}$ Ein Name des Deutsch - Französischen Vertrags von 1982 kann nicht gefunden werden. Da der energetische Rheinausbau nur in den südlichen Bundesländern statt fand, wurde NRW an dem Vertrag nicht beteiligt (Dr. Angela Merkel, 21. Februar 1996).

${ }^{705}$ Internetbasierte Quelle 6.

${ }^{706}$ Planfeststellungsabschnitt 10, (Präsentation vom 18.07.2011).

${ }^{707}$ Bis dato war die Ableitung des Regenwassers über die Kanalisation gängig.
} 
Satzungen der Gemeinden zu verankern. Das zweite Ergebnis der Stadt Köln betraf die bereits vorhandene Bebauung im Stadtgebiet. Für diese wurde der zu erzielende Regenanteil, der natürlich abgeführt werden könnte, durch den Stadtrat auf deutlich geringer als zehn Prozent geschätzt $^{708}$. Vorschläge zur Regenwassernutzung wurden aufgelistet, um dem restlichen Wasservolumen einen alternativen ökologischen Sinn zu geben. Daneben wurde ein wirtschaftlicher Anreiz betont: Eine Verringerung der Regenwassergebühr entlohnt eine Entsiegelung sowie ein Ableiten anderweitig als über die Kanalisation. Der Rat forderte, dass diese Handlungen auch durch das Bundesnaturschutzgesetz (BNatSchG) finanziell gefördert werden sollten. In dem Hochwasserschutzkonzept der Landesebene bildete die Entsiegelung von Boden eines der Hauptziele. Das seit Dezember 1996 bestehende Programm der „Initiative zur ökologischen und nachhaltigen Wasserwirtschaft NRW“ sollte für diesen Punkt instrumentalisiert werden ${ }^{709}$, da es finanzielle Anreize schaffte ${ }^{710}$. Die 1996 in Köln bestehenden gesetzlichen Überschwemmungsgebiete ${ }^{711}$ stammten aus der Zeitspanne von 1906 bis 1912 und basierten auf dem Gesetz zur Verhütung von Hochwassergefahren vom 16.08.1905 in Anlehnung an eine Überschwemmungslinie von 8,30 m K P $\mathrm{P}^{712}$. Zwei Ergebnisse wurden zur Bebauung von gesetzlichen Überschwemmungsgebieten erarbeitet. Erstens erweiterte der Rat die Sicherheit der natürlichen Überflutungsflächen durch eine Vergrößerung der gesetzlich festgelegten Überschwemmungsgebiete bis zu einer Höhe von $11,30 \mathrm{~m} \mathrm{~K} \mathrm{P}^{713}$. Zweitens formulierte der Rat eine weitere Forderung und fasste den Fokus hier ebenfalls eine politische Ebene weiter: Zum seit 19. Juni 1994 bestehenden Verbot von Handlungen, „die zu einer Beeinträchtigung von regelmäßig überfluteten Bereichen führen“714, verankert im Landschaftsgesetz ${ }^{\text {(7RW }}{ }^{715}$, sollten diese Ziele zukünftig in den „Wassergesetzen““716 des Bundes wieder zu finden sein. Die Stadtratsmitglieder kamen am 01. Februar 1996 überein, von der Ausweisung neuer Baugebiete in potentiellen Überflutungsflächen abzusehen und bereits erstellte Flächennutzungspläne

\footnotetext{
${ }^{708}$ HWSK 1996.

${ }^{709}$ Landtag NRW, Plenarprotokoll 12/91, 18.06.1998, 7527.

${ }^{710} \mathrm{Ebd}$. Die finanziellen Anreize für die Bodenentsiegelung sind aus dem Aufkommen der Abwassergebühren gedeckt.

${ }^{711}$ Gesetzliche Überschwemmungsgebiete werden durch das Wasserhaushaltsgesetz auf eine hundertjährige Wiederkehr festgelegt. In Köln entspricht das einer Höhe von 11,30 m K P. In dem Bereich gelten durch die Länderebene gestellte Anforderungen und Verbote. Die gesetzlichen Überschwemmungsgebiete werden in NRW von den Bezirksregierungen festgestellt (hier das Umweltamt Köln) und von den Kommunen umgesetzt

(Wasserhaushaltsgesetz).

712 HWSK 1996, 67.

${ }^{713}$ Ebd.

${ }^{714}$ Ebd., 66.

${ }^{715}$ Landschaftsgesetz NRW, Gesetz zur Sicherung des Naturhaushalts und zur Entwicklung der Landschaft, § 62, Gesetzlich geschützte Biotope.

${ }^{716}$ Landeswassergesetz des Landes, Wasserhaushaltsgesetz des Bundes.
} 
zurückzunehmen $^{717}$. Als Beispiele nannte der Rat zwei Flächen ${ }^{718}$. Die Renaturierung von Bachläufen wurde durch die Kölner Verantwortlichen lediglich auf unbebauten Flächen für möglich gehalten.

Der folgende Abschnitt dient der Darstellung der verschiedenen Diskussionsphasen der städtischen Entscheidungsträger sowie der Diskussionen zwischen den Verantwortlichen des Landtags.

\subsubsection{Diskussionen über Maßnahmen}

Am 30. November 1995 diskutierten die Mitglieder des Kölner Stadtrats die Vor- und Nachteile der Retentionsmaßnahmen. Oelmann, der Vorsitzende des Ausschuss für Tiefbau und Verkehr erklärte, dass der zukünftige Retentionsraum im Worringer Bruch die Wasserhöhe in Köln nicht beeinflussen wird und deswegen lediglich „die Vorbildfunktion der Stadt Köln manifestiert" ${ }^{\text {7719 }}$. Von örtlichen Renaturierungen wurden lediglich „Wasserstandsabsenkungen im Zentimeter-Bereich“720 erwartet. Der Rat der Stadt Köln war sich 1996 einig, dass die Polder ausschließlich für die Unterlieger der Stadt von Bedeutung seien $^{721}$. Zum Zeitpunkt der Entscheidung über die Installation der Retentionsräume, waren die Kosten hierfür noch nicht bekannt ${ }^{722}$. Der Rat nahm einzig an, dass diese „,voraussichtlich höher bezuschusst werden könn[t]en“"723, als die Kosten der baulichen Schutzmaßnahmen. Die nordrhein-westfälische Landesregierung unterstützte die Umstrukturierung des Geländes zum Hochwasserretentionsraum ebenfalls, denn das Projekt wurde vom Land NRW vollständig bezuschusst $^{724}$. NRW-Umweltministerin Bärbel Höhn (Bündnis 90/DIE GRÜNEN) war von den Einrichtungen der Polder im Juli 1996 so angetan, dass gerade diese beiden Vorhaben sie dazu veranlassten, dem weiteren Ausbau der „technischen Hochwasserschutzanlagen“ zuzustimmen und ,in angemessenem Umfang mit Mitteln des Landes zu unterstützen““725. Im Fall der Westhovener Aue musste keine Ressourcendiskussion auf städtischer Ebene geführt werden, denn man war sich einig, dass die Umwidmung des Geländes eine erstrebenswerte Maßnahme sei, da sie Köln schütze und die Attraktivität der Aue sowie ihrer Umgebung

\footnotetext{
${ }^{717}$ HWSK 1996, 65; 79.

${ }^{718}$ Ebd., 68. Die beiden genannten Beispiele galten dem „Sandpfad“ in Köln-Weiß sowie der ehemaligen belgischen Kaserne in Westhoven.

${ }^{719}$ Stadt Köln, Drucksachen 1141/095, Anlage 7, 16.11.1995.

${ }^{720}$ Ebd.

${ }^{721}$ Ebd.

${ }^{722}$ HWSK 1996.

${ }^{723}$ Stadt Köln, Drucksachen 1141/095, Anlage 8, 18.01.96, 4. Der Rat hoffte bei den Maßnahmen einen Zuschuss zwischen 40 - 80 Prozent durch die Unterstützung verschiedener Behörden zu bekommen.

${ }^{724}$ Landtag NRW, Drucksache 12/1577, 02.12.1996. Dabei wurde darauf hingewiesen, dass „Verbände und Gemeinden einen Eigenanteil von mindestens 20 Prozent selbst finanzieren“ (Ebd., 3).

${ }^{725}$ NRW-Umweltministerin Höhn, 08.07.1996.
} 
steigere $^{726}$. Die Argumentationslinien im Fall des Worringer Bruchs verliefen dagegen kontrovers $^{727}$. In der Planung des Hochwasserschutzkonzeptes wurden von der Bezirksvertretung Chorweiler, in deren Gebiet Worringen liegt, Fragen an den Rat der Stadt Köln zur Umwidmung des Naturschutzgebietes gestellt. Diese Fragen betrafen den Einbezug privater Flächen, die Wasser führend werdenden Altlasten in der Umgebung, die Einflüsse des steigenden Grundwassers auf eine Tankstelle, das Eindringen des Grundwassers in Keller sowie die Auswirkungen des belasteten Rheinwassers auf Flora und Fauna des Naturschutzgebietes $^{728}$. Die Verantwortlichen der Stadt antworteten Ende 1995, dass ein geplantes Gutachten die Fragen der Chorweiler in Zukunft klären würde ${ }^{729}$. Im daraufhin verabschiedeten Hochwasserschutzkonzept hielt der Rat ein Budget von 80.000 DM für eine Machbarkeitsstudie fest. In dieser sollte die „besondere ökologische Problematik““730 untersucht werden. Schwerpunkt der Studie waren, unter anderem, die unterschiedlichen Einstautiefen und -zeiten sowie die Auswirkungen einer kontrollierten und einer natürlichen Retention $^{731}$. Um der Gefahr vor eventuellen negativen Einflüssen durch die „relativ hohe Schadstofffracht ${ }^{\text {6732 }}$ des Flusses auf das Grundwasser vorzubeugen, sollten die Wasserversorgungsunternehmen bei der Planung beteiligt werden. Bis heute wurde der Umbau des Worringer Bruchs zum Retentionsraum noch nicht realisiert.

In der Diskussion über die Renaturierung von Bachläufen wurde durch die Stadtratsmitglieder daran erinnert, dass vor allem rechtsrheinisch Bäche bereits vor Jahrzehnten verrohrt worden

\footnotetext{
${ }^{726}$ Einstimmiger Beschluss vom 01. Februar 1996 (Stadt Köln, Drucksachen 1141/095). Die Westhovener Aue wurde durch die Umwandlung wiederbelebt (Internetbasierte Quelle 14). Die Anlage, die bereits seit den 1930er Jahren als Kaserne diente, wurde seit dem Zweiten Weltkrieg durch belgische Besatzungstruppen als Übungsgelände genutzt. 1995 zogen die belgischen Truppen ab. Zunächst war die Anlage aufgrund von Kampfmittelresten nur auf ausgewiesenen Wegen für die Öffentlichkeit nutzbar. Im Jahr 2002 erwarb die Stadt Köln die Fläche, die dann ab 2009 als Retentionsraum für das Rheinhochwasser und als Naherholungsgebiet dient. Wiesen wurden angelegt und ca. 2000 auentypische Pflanzen angesiedelt. Die Einrichtung der neuen Überflutungsbereiche in der Westhovener Aue, südlich des Kölner Stadtgebiets gelegen, dient unter anderem dazu, das Wasser, welches sonst von der Stadt selbst aufgenommen worden wäre, zu speichern (Umweltbundesamt 2003; Landtag NRW, Plenarprotokoll 12/91, 18.06.1998).

${ }^{727}$ Gegen den zu erwartenden Nutzen des Retentionsraums in der Westhovener Aue wird die hydrologische Auswirkung des Retentionsraums im Worringer Bruch keinen Schutzeffekt für die Stadt ergeben (Stadt Köln, Drucksache 1141/095, Anlage 7, 30.11.1995, Anlage I): „Es wird jedoch darauf hingewiesen, dass der Bereich des Worringer Bruchs zu keiner unmittelbaren Reduzierung einer Hochwassergefährdung für die Stadt Köln führt. Dies ist nur für die Kölner Unterlieger der Fall. [...] jedoch würde hierdurch eine Vorbildfunktion der Stadt manifestiert"(Ebd.). Flussabwärts der Stadt gelegen, könnte die Anlage alsbald die Region nördlich von Köln beeinflussen. Der Worringer Bruch ist ein „verlandeter Altarm des Rheins“ (Internetbasierte Quelle 4). Das Gebiet ist aufgrund seiner Ausprägung über die Stadtgrenzen hinaus im „Korridor der Rheinschiene von erheblicher Bedeutung und steht seit 1989 unter Naturschutz“(Ebd.). Das Ziel der Stadt lag darin, durch die Übernahme einer Vorbildfunktion die Akteure entlang des Rheins zu überzeugen, die gleichen Maßnahmen durchzuführen.

${ }^{728}$ Rat der Stadt Köln, Drucksachen 1141/095, Anlage 3.

${ }^{729}$ Rat der Stadt Köln, Drucksachen 1141/095, Anlage 4. Das genaue Datum fehlt auf der Anlage 4 des Dokuments Rat der Stadt Köln, Drucksachen 1141/095.

${ }^{730}$ HWSK 1996, 62.

${ }^{731}$ Ebd., 61.

${ }^{732}$ Ebd., 64.
} 
waren, um Bauland als ganze Fläche ausweisen zu können ${ }^{733}$. Deswegen sollte sich die Renaturierung lediglich auf unbebaute Flächen konzentrieren ${ }^{734}$. Die Renaturierung von Bachläufen wurde ebenfalls von den Mitgliedern des Landtags besprochen. Allerdings wurde lediglich darauf verwiesen, dass bereits seit 1990 das Gewässerauenprogramm in NRW lief, welches die Renaturierung von Bachläufen beinhaltete. Dieses Programm wurde 1996 von den Mitgliedern des Landtags als Bestandteil des Hochwasserschutzkonzepts NRW ${ }^{735}$ betrachtet, um der Forderung nach Renaturierung eine abgestimmte Maßnahme entgegenzusetzen.

Außerdem wurde die Regenwasserversickerung in Köln kontrovers diskutiert. Im Hochwasserschutzkonzept wurde festgehalten, dass „lang anhaltende Niederschläge“6736 verantwortlich waren für eine Sättigung der Böden im Einzugsgebiet des Rheins und sich somit die Art der Bodenversiegelung als „unwesentlich““737 erwies. Trotzdem versprach sich der Stadtrat durch die Verankerung des $§ 51$ des Landeswassergesetzes NRW in den Satzungen der Gemeinden, auf großen Flächen „einen wirkungsvollen Beitrag““738 zum Hochwasserschutz leisten zu können. Obwohl dieser lediglich Neubaugebiete betraf, sollte Regenwasser dort künftig ökologisch versickern, statt sich in der Kanalisation zu konzentrieren. Die Landesregierung hingegen beurteilte das Konzept der Regenwasserversickerung gegenteilig. Sie traute dem Prinzip nicht zu, einen großen Anteil zur Lösung des Hochwasserproblems beitragen zu können. Johannes Remmel (Bündnis 90/DIE GRÜNEN) fasste den Effekt als marginal zusammen ${ }^{739}$. Da nur ein Prozent der Bebauung aus Neubaugebieten bestünde, müsse im Bestand vermehrt nach Möglichkeiten der Regenwasserabführung gesucht werden ${ }^{740}$. Genauso uneinig waren sich die zwei Instanzen bei dem Thema der Entsiegelung der Böden. Die Landesregierung kam 1998 zu dem Schluss, dass ,anthropogene Einflüsse keine Hochwasser verursachen“741 würden. Sie räumte allerdings ein, dass die Bodenversiegelung den Effekt der Überschwemmungen allgemein verstärke, was sich jedoch bei weiten Flusseinzugsgebieten wieder relativiere ${ }^{742}$. Bezogen auf

\footnotetext{
${ }^{733}$ Ebd., 70.

${ }^{734}$ Ebd.

${ }^{735}$ Landtag NRW, Plenarprotokoll 12/91, 18.06.1998, 7527.

${ }^{736}$ HWSK 1996, 70.

${ }^{737}$ HWSK 1996.

${ }^{738}$ Ebd. 73.

${ }^{739}$ Landtag NRW, Plenarprotokoll 12/91, 18.06.1998, 7525.

${ }^{740}$ Ebd.

${ }^{741}$ Landtag NRW, Drucksache 12/2870, 04.03.1998. In einem Überblicksschreiben listete die Landesregierung wissenschaftliche Quellen auf, welche allgemein zu dieser Ansicht führten. Es ist hervorzuheben, dass die Literatur zum größten Teil auf die Bundesanstalt für Gewässerkunde in Koblenz oder auf ihre Mitarbeiter in Verbindung mit weiteren Forschungseinrichtungen zurückzuführen ist.

${ }^{742}$ Ebd.
} 
das konkrete Ereignis von 1995, so erklärte die Landesregierung, bestand ein Großteil der Bodenversiegelung aus natürlich entstandener Sättigung, die auf lang anhaltenden Regen, Bodenfrost und Schnee zurückzuführen war $^{743}$. Eine Studie der Universität Kaiserslautern von 1993 wurde im Gespräch über die Abflussmöglichkeiten von Regenwasser angeführt ${ }^{744}$. Auf den Streit im Landtag besaßen die Ergebnisse aufgrund fehlender empirischer Messungen am Rhein jedoch keinen Einfluss ${ }^{745}$. Neben der Untersuchung der Universität Kaiserslautern wurde 1996 durch Remmel eine Studie der Universität Darmstadt ignoriert, indem er schimpfte: „Ich kann Ihnen [...] Gutachten und Ausführungen zitieren, die genau das Gegenteil besagen“"746. Die Ansichten der verschiedenen Fraktionen zur Wichtigkeit der Speicherfähigkeit der Böden lassen sich aus einer Diskussion ableiten, die aufgrund eines Antrags der Bündnis 90/DIE GRÜNEN am 17. Februar 1995 stattfand $^{747}$. In dieser betonte Kruse (CDU) die Wichtigkeit der Flächenentsiegelung sowie die Wichtigkeit aller Maßnahmen, welche die Fließgeschwindigkeit verlangsamten. Kuhl (FDP) schloss sich dem an, während Umweltminister Matthiesen ${ }^{748}$ (SPD) zum Thema der Bodenversiegelung ausschließlich die Wichtigkeit der Renaturierung von Bachläufen unterstrich. Matthiesen erklärte, dass der neue Landesentwicklungsplan Freiraum innerhalb der natürlichen Überschwemmungsgebiete vorsah und den Gemeinden somit fortan kein Bebauungsplan für diese Flächen mehr genehmigt werden würde ${ }^{749}$. Fünf Monate nach dem Hochwasserereignis fanden Landtagswahlen statt. Die daraus hervorgegangene neue Umweltministerin Bärbel Höhn $^{750}$ (Bündnis 90/DIE GRÜNEN) wurde nach einem Besuch in Köln von der Landesregierung kritisiert. Ihr Parteikollege Remmel beschwerte sich über die im Hochwassergebiet angesprochenen Themen ${ }^{751}$ : „Während Sie [Höhn] nur von Naturgewalten palaverten, hätten Sie die Flächenversiegelung, Fehler in der Landwirtschaft, [...] und die

\footnotetext{
${ }^{743}$ Ebd.

${ }^{744}$ Ebd. Die Studie wurde von Prof. Dr. -Ing. Köhler betrieben.

${ }^{745}$ Landtag NRW, Plenarprotokoll 12/91, 18.06.1998.

${ }^{746}$ Landtag NRW, Plenarprotokoll 12/38, 10.10.1996, 3094.

${ }^{747}$ Landtag NRW, Plenarprotokoll 11/157, 17.02.1995. Die Bündnis 90/DIE GRÜNEN forderten nach dem
}

Hochwasserereignis von 1995, die Erhöhung der Speicherfähigkeit der Böden zu besprechen. Dazu reichten sie bereits eine Liste mit Handlungsvorschlägen ein. Die Vorschläge betrafen die Umstellung auf eine ökologische Landwirtschaft, die Wiederherstellung der Humus- und Grünfutterlandwirtschaft, eine Einrichtung einer leistungsstarken Forstverwaltung, eine Bodenversiegelungsabgabe, die Maßnahme, Flächen sparendes Bauen zur Bedingung für öffentliche Fördermittel zu erheben, verpflichtende Ausgleichsmaßnahmen, die Idee, eine geringe Versiegelung aus kommunalen Abwassergebühren zu entlohnen, sowie den Vorschlag, die Regenwasserversickerung im Landeswassergesetz zu verankern. In der Abstimmung wandten sich SPD, CDU und FDP gegen die Stimmen der Bündnis 90/DIE GRÜNEN, denn die Parteien unterstellten den Antragstellern, das Hochwasser zur Beförderung ihre parteilichen Kernziele auszunutzen.

${ }_{748}^{7}$ Klaus Matthiesen (SPD) war zwischen 05.06.1985 - 17.06.1995 Minister für Umwelt, Raumordnung und Landwirtschaft in NRW.

${ }^{749}$ Landtag NRW, Plenarprotokoll 11/157, 17.02.1995.

${ }^{750}$ Nach den Landtagswahlen am 17.07.1995 übernahm Bärbel Höhn (Bündnis 90/DIE GRÜNEN) das Amt von Klaus Matthiesen.

${ }^{751}$ Landtag NRW, Plenarprotokoll 12/38, 10.10.1996. 
Baulandausweisung ansprechen müssen.“"752 Die FDP, die Bündnis 90/DIE GRÜNEN sowie die SPD waren sich einig, dass ein Zusammenhang zwischen Erosion, Bodenversiegelung, Waldsterben und der mangelhaften Aufnahmefähigkeit der Böden bestünde ${ }^{753}$.

Im Zuge der Diskussion über die gesetzlich festgelegten Überschwemmungsgebiete wurde vom Rat der Stadt Köln im Hochwasserschutzkonzept festgehalten, dass ,,unbekümmert“6754 angelegte Siedlungsflächen für die Behinderung des Hochwasserabflusses verantwortlich zu machen sind. Über die Höhe der neuen gesetzlich festgelegten Überschwemmungsgebiete stritten die Mitglieder des Kölner Stadtrats nicht, da diese vom Land NRW vorgeschrieben wurden.

Die Erhellung der Diskussionen auf Stadt- und Landesebene beschreibt die verschiedenen Gewichtungen der Maßnahmen aus den beiden Perspektiven. Außerdem wurde deutlich, dass die beiden politischen Organe unterschiedliche Wirkungen der Maßnahmen wahrnahmen. Nachfolgend werden die Diskussionen aus der Sicht der der Kosten-Nutzen-Perspektive kommentiert.

\subsubsection{Bewertung des Diskurses aus der Kosten-Nutzen-Perspektive}

Im Folgenden wird die Genese aus der Kosten-Nutzen-Perspektive berücksichtigt. Das Vergleichen von Alternativen und das Kritisieren der diskutierten Handlungsoptionen stehen dabei im Vordergrund. Es wird dem erarbeiteten Hilfsmodell in der folgenden Betrachtung gefolgt.

Die Berichterstattung der Presse ließ erkennen, dass ganz Köln sich verletzlich und ausgeliefert, also vulnerable, fühlte. Die Kölner Akteure schätzten ihren Einfluss auf den Rhein als gering ein, was sich aus dem Hochwasserschutzkonzept ableiten lässt. Die Veröffentlichung des Konzepts weist darauf hin, dass die politisch Verantwortlichen in Köln nun endgültig die Hochwasserhäufigkeiten und -intensitäten als unnatürlich empfanden und sich als ohnmächtig gegenüber den menschlichen Eingriffen am Oberrhein betrachteten. Die Kölner Entscheidungsträger empfanden die beteiligungslose Stellung der Stadt bei Entscheidungsprozessen am Oberlauf als zentrales Hindernis, ihre Hochwassersicherheit erhöhen zu können. Sie versuchten bereits während der Hochwassertage, bei höheren Instanzen wie zum Beispiel bei der Landesregierung in Nordrhein-Westfalen, um Fürsprecher $\mathrm{zu}$ werben, welche in ihrem Namen mit den Entscheidungsträgern am Oberrhein kommunizieren sollten. Die Verantwortlichen der Stadt Köln sahen sich abhängig vom

\footnotetext{
${ }^{752}$ Ebd.

${ }^{753}$ Landtag NRW, Drucksache 11/8495, 17.02.1995.

${ }^{754}$ HWSK 1996, 66.
} 
Wohlwollen der südlichen Bundesländer, Baden-Württemberg, Hessen und Rheinland-Pfalz. Dieses Gefühl drückte Oberstadtdirektor Lothar Ruschmeier beispielhaft aus, als er die Bundesländer „um Gnade flehte“6755. Die Stadtverwaltung erkannte angesichts der Überflutung, dass sie starke Verbündete brauchen würde, um sich aus dieser Situation befreien zu können. Die Hilferufe zeigen, dass die Verantwortlichen der Stadt Köln sich außer Stande sahen, die Hochwassergefahr selbst zu beherrschen. Die Reaktionen der Kölner Politiker belegen, dass das Problem der Überschwemmungen in einen überregionalen Kontext gerückt wurde. Die Kölner Verwaltungsmitglieder betrachteten die Hochwassergefahr nicht mehr als lokal überwindbar, sondern nur noch durch systembezogene Handlungen ${ }^{756}$. Um dies zu bewerkstelligen, brauchten sie einflussreiche Fürsprecher.

Besonders Umweltverbände unterstützten die Kölner Forderungen. Sie verlangten ebenso wie der Hochwasserexperte Krug des Bundes für Umwelt und Naturschutz (BUND), einen „runden Tisch von Bund und Ländern““757. Die unabhängigen Experten bestätigten, dass Köln sich nicht alleine vor der Hochwassergefahr retten konnte, und somit abhängig von überregionalen Veränderungen und Vertretern war. Um weitere Fürsprecher zu gewinnen, demonstrierte die Stadt, dass der Bau von Retentionsgebieten auch im urbanen Bereich möglich ist. Hiermit wollte die Kölner Kommunalverwaltung Verantwortung für ihre Unterlieger im Norden der Stadt übernehmen und hoffte mit dieser Handlung eine überregionale Wirkung einzuleiten.

Sowohl die Stadt Köln als auch das Land NRW betrachteten verschiedene Maßnahmen aus dem Bereich der Raumentwicklung als mögliche Lösungsansätze, um den Wasserabfluss durch die Bereitstellung von Flächen zur Ausdehnung des Wassers zu entschärfen. Zu den Möglichkeiten gehörten der Einbau von Retentionsbecken, die Renaturierung von Bachläufen sowie die Versickerung von Regenwasser. Trotzdem wurden die Handlungsmöglichkeiten unterschiedlich wahrgenommen und kritisiert. Wie in den Diskussionen zu den Raumentwicklungsmaßnahmen zu sehen ist, wurden die Retentionsräume mit Nachdruck von beiden Instanzen als besonders wichtig erachtet. Alle anderen Aktionen der möglichen Hochwassergefahrenbeseitigung wurden in Köln sowie in NRW aufgrund von entgegen gesetzten wirtschaftlichen Interessen nur marginal umgesetzt.

Bei der Bewertung der Bachläufe ist ein deutlicher Unterschied festzustellen. Die Bachläufe der Stadt Köln verlaufen größtenteils unterirdisch durch das urbane Gebiet. Der Konflikt

\footnotetext{
${ }^{755}$ Artikel: Ehrenamtliche Helfer von Arbeit freistellen, Kölnische Rundschau, 30.01.1995.

756 „Die Landesregierung teilt die Meinung der Stadt Köln zu Retentionsräumen am Mittel- u. Niederrhein sowie die Annahme, dass der Hochwasserspiegel in Köln im Durchschnitt um $40 \mathrm{~cm}$ aufgrund des Oberrheinausbaus zw. 1955 u. 1977 ansteigt" (Landtags NRW, Drucksache 12/2452, 07.10.1997).

${ }^{757}$ Artikel: Naturflächen am Rhein nicht weiter bebauen, Kölnische Rundschau, 23.02.1995.
} 
zwischen Grundstückswerten und Hochwassersicherheit beherrschte den Diskurs des Stadtrats im Februar 1996. Die Renaturierung von Bächen im urbanen Bereich wurde von den Politikern im Stadtrat im Vergleich zu den ökonomischen Faktoren als zweitrangig eingestuft. Eine Diskussion um die Nutzung von bereits ausgewiesenem Bauland versuchte der Stadtrat zu vermeiden. Demgegenüber stand die Einschätzung der Landespolitik, dass eine Mischung aus ländlicher und städtischer Fläche die Möglichkeit eröffne, Bäche zu renaturieren. Der Renaturierung kam im Hochwasserschutzkonzept NRW neben dem Schutz der Deiche, der Schaffung von Rückhalteraum und der Bodenentsiegelung die gleiche Wertschätzung $\mathrm{zu}^{758}$. Eine Weiterentwicklung der Maßnahmen, die auf Landesebene zur Verfügung standen, wurde allerdings durch die Nutzung bereits bestehender Konzepte behindert.

Das Potential der Regenwasserversickerung wurde im Stadtrat und im Landtag völlig unterschiedlich bewertet. Die kommunale Stadtverwaltung betrachtete das Regenwasserproblem auf zwei unterschiedliche Arten: Sie differenzierte zwischen Neubaugebieten und bereits bestehender Bebauung. Daraus resultierten unterschiedliche Handlungsempfehlungen. Während in Köln Informationsblätter zur Regenwasserversickerung an Hausbesitzer verteilt wurden, empfand die Landesregierung diese als nicht vielversprechend genug, um tätig zu werden. In der Anerkennung von Maßnahmen mit relativ kleinen Auswirkungen wurde der Handlungsdrang der Stadt sichtbar. Einzelne Aktionen wurden - solange keine alternativen wirtschaftlichen Interessen dagegensprachen strategisch ausgenutzt, um die Aktivitäten der Stadt als umfangreich darzustellen und besonders auf den überregionalen Dialog zu projizieren.

Des Weitern wurden in Köln zwei Lösungen für das Problem der Bebauung der Überschwemmungsgebiete erarbeitet. Dann zeigte sich jedoch, dass finanzielle Interessen die Stadt Köln an der Umsetzung hinderten: Der erste Lösungsansatz beinhaltete die Verlegung der Grenze für gesetzliche Überschwemmungsgebiete auf eine Höhe von 11,30 m K P und resultierte aus einer Forderung der Landespolitik, Überflutungsflächen für Hochwässer mit einer 100jährigen Wiederkehr freizugeben und diese in zukünftigen Bauplänen auszuweisen. Die gesetzlichen Überschwemmungsgebiete wurden durch die Bundespolitik, im Rahmen des Wasserhaushaltgesetzes, festgelegt und damit auch nicht auf der Landesebene diskutiert ${ }^{759}$. Die Stadt kam demgemäß nur landes- bzw. bundestypischen Forderungen nach. Mit dem Ziel, ihre Aktivitäten zu betonen, zeigte der Stadtrat zwei lokale Beispiele für die Räumung der gesetzlichen Überschwemmungsbiete auf: Zumindest eine der sofort zum Abriss

\footnotetext{
${ }^{758}$ Landtag NRW; Plenarprotokoll 12/91, 18.06.1998.

${ }^{759}$ Der $§ 76$ Abs. 2 WHG befähigt die Landesregierungen Gebiete, welche statistisch einmal in 100 Jahren überschwemmt werden als Überschwemmungsgebiet festzulegen.
} 
freigegebenen Bebauungen kann als Mogelpackung entlarvt werden: Die Kaserne des Retentionsraums Westhovener Aue sollte ohnehin entfernt werden. Nur die gestellte Forderung, das Verbot von Handlungen, die zu einer Beeinträchtigung von regelmäßig überfluteten Bereichen führen, aus dem Landschaftsgesetz NRW auch auf Bundesebene zu verankern, kann als neue Reaktion bewertet werden. Jedoch entsprach die genannte Forderung dem Landesprogramm zur „Initiative für den ökologischen und nachhaltigen Wasserhaushalt NRW“, folglich nahm die Stadt eine Botenrolle ein, anstatt einen eigenen Ansatz zu entwickeln. Allerdings sind in der Forderung, diese Maßnahme in der Bundesgesetzgebung zu verankern, drei Wünsche erkennbar: Erstens wäre ein viel größeres Rheineinzugsgebiet von den positiven Effekten betroffen, zweitens wäre der Stadt Köln somit die Unterstützung durch die Bundesregierung sicher und drittens würde sich der Belohnungsanreiz vergrößern. Da diese Effekte keine Kosten verursachten, gab es somit auch keine Akteure, die sich gegen diese Maßnahmen aussprachen.

In Anbetracht des Hochwasserschutzkonzepts wird deutlich, dass die Verantwortlichen der Stadt Köln motiviert waren, die bekannten Hochwasserursachen auf regionaler Ebene zu bekämpfen, jedoch gerieten sie aufgrund des urbanen Umfelds schnell an finanzielle Grenzen. Dennoch versuchten sie, über die lokalen Umsetzungsmöglichkeiten hinaus, die Handlungsalternativen in einen überregionalen Rahmen zu setzen und stellten eine Vielzahl von Forderungen an höhere politische Instanzen. Sie nutzten diese Forderungen, um die übergeordneten Instanzen auf sich aufmerksam zu machen und ihre Anliegen auf die Agenden höherer Entscheidungsebenen zu setzten ${ }^{760}$. Bei der Gegenüberstellung der städtischen und landespolitischen Einschätzungen wurde deutlich, dass die Landesregierung in NRW sich durch die bestehenden Programme als ausreichend gewappnet ansah, da über die existierenden Maßnahmen hinaus keine Neuerungen erarbeitet wurden. Die nähere Betrachtung der Raumentwicklungsmaßnahmen lässt ableiten, dass die Entscheidungsträger aufgrund der Fortentwicklung des lokalen Hochwasserschutzes mit vielfältigen Zielkonflikten konfrontiert wurden. Aufgrund von umfangreichen Abwägungsprozessen mit alternativen Interessen mussten einige Maßnahmen gegen das Hochwasser eingeschränkt werden. An diesen Beispielen wurde offenbar, dass die Möglichkeiten des Hochwasserschutzes in der Raumordnung intensiv hinterfragt und kritisiert wurden. Wie wirkten sich die Handlungen der Stadt Köln während der Hochwassertage und in der Folgezeit auf ihre potentiellen Fürsprecher aus?

\footnotetext{
${ }^{760}$ Kingdon 1995.
} 
Die Hilferufe der Stadt während der Hochwassertage überforderten zunächst die Landesregierung. Insbesondere der Umweltminister NRWs, Klaus Matthiesen, versuchte in Anbetracht der Schäden für Köln Partei zu ergreifen, verstrickte sich allerdings in wahllosen Vorschlägen und Anschuldigungen. Er beschuldigte abwechselnd die Verantwortlichen der südlichen Bundesländer ihren Pflichten nicht nachzukommen und warf der Stadt Köln vor, zu geringe Schutzhöhen aufgebaut zu haben und somit an den entstandenen Schäden zumindest teilweise selbst Schuld zu sein. Er änderte während der Hochwassertage mehrfach seine Meinung über die Schuld- und Ursachenfragen, was ihn unentschlossen und hilflos erscheinen ließ. Er konnte keinen Einfluss auf das Handeln der Oberlieger Kölns im Süden Deutschlands ausüben, da sich diese alleine an normativen Vorgaben orientierten. Seinen Forderungen, die vorhandenen Retentionsräume zu öffnen, kamen die Bundesländer nicht nach, da sie argumentierten, die Pegelstände vor Ort wären noch nicht ausreichend hoch genug, um eine Öffnung zu rechtfertigen. In seiner Ratlosigkeit wandte Matthiesen sich an die Bundesregierung, um sich Unterstützung durch eine höhere Ebene zu sichern. Er gebar sich, als fühlte er sich den Akteuren am Rhein ausgeliefert. Die Verantwortlichen in Köln mussten während dieser Tage einsehen, dass der Umweltminister ihres Landes kein geeigneter Fürsprecher sein würde, um das Hochwasserproblem zu lösen. Dass Bundesfinanzministers Theodor Waigel anbot, den Hochwasseropfern finanzielle Hilfe zukommen zu lassen, kann als Anerkennung der dramatischen Situation gewertet werden. Die Bundesregierung versuchte jedoch die Hochwasserproblematik nicht auf ihre nationale Agenda zu setzten. Bundeskanzler Helmut Kohl (CDU) organisierte ein Treffen der Staatssekretäre diverser deutschen Ministerien, welche entschieden, die [Bundes]Länder sollten die Herausforderung untereinander ,,übergreifend“761 ${ }^{\text {regeln. }}$

Obwohl bereits 1970 im Landtag über erforderliche Retentionsbecken am Oberrhein für die Hochwassersicherheit in NRW gesprochen wurde ${ }^{762}$, verlangte der Landtag von der Stadt Köln 1995, die Schutzvorrichtungen anzupassen. Um nicht nur Fürsprecher am Oberrhein, sondern auch in NRW von ihren neuen Vorstellungen zum Hochwasserschutz zu überzeugen, erarbeiteten die Verantwortlichen der Stadt Köln ein möglichst umfangreiches und ganzheitliches Konzept. Die spätere NRW-Umweltministerin Höhn bewertete dieses Konzept als sehr gut, denn sie akzeptierte den zukünftigen, weit unter den landespolitischen Anforderungen gelegenen, Hochwasserschutz aufgrund der Retentionsplanung und unterstützte diesen mit Landesmitteln ${ }^{763}$. Daraus lässt sich ableiten, dass die Stadt Köln durch

\footnotetext{
${ }^{761}$ Artikel: Kohl erklärt die Fluten zur Chefsache, Rhein-Lahn-Zeitung, 31.01.1995.

${ }^{762}$ Landtag NRW, 74. Sitzung, 22.04.1970.

${ }^{763}$ NRW-Umweltministerin Höhn, 08.07.1996.
} 
ihre Bemühungen eine Vorbildfunktion im Hochwasserschutz zu übernehmen, die Unterstützung durch die NRW-Umweltministerin spätestens seit Juni 1996 genoss. Da der Hochwasserschutz in Nordrhein-Westfalen den Gemeinden selbst überlassen ist, stellte Höhn eine wichtige Fürsprecherin dar, da sie die Ressourcenverteilungen für den Hochwasserschutz maßgeblich beeinflussen konnte. Außerdem fungierte sie als Sprachrohr bei den Auseinandersetzungen mit den südlichen Bundesländern Rheinland-Pfalz, Hessen und BadenWürttemberg.

Wie zuvor gezeigt werden konnte, wurde der Bau des südlichen Retentionsbeckens in der Westhovener Aue von lokaler Seite befürwortet und durch die Landesregierung finanziert. Lediglich die Diskussion um den Bau des nördlichen Retentionsraums verlief kontrovers. Die Mitglieder des Kölner Stadtrats wogen 1996 öffentlich die zu erwartende landespolitische Unterstützung $\mathrm{ab}$ und signalisierten somit investitionsbereit $\mathrm{zu}$ sein. Der durch die Betroffenen Bürger des Bezirks in Chorweiler erstellte Fragenkatalog beinhaltete Überlegungen $\mathrm{zu}$ Folgeerscheinungen und externen Kosten ${ }^{764}$. Der Rat der Stadt Köln befürchtete im Februar 1996 bereits, dass „Konflikte zwischen der landwirtschaftlichen Nutzung und dem Freizeit- und Erholungsverkehr im Naturschutzgebiet auftreten“765 könnten. Der Hauptverantwortliche Oelmann bestätigte eine Dekade später, dass gravierende Auseinandersetzungen eine konkrete Planung des zweiten Retentionsraums verhindert haben $^{766}$. In dieser Konfliktsituation wurde für die Kölner Stadtratmitglieder erkennbar, dass Hochwasserschutzprojekte schwer durchsetzbar sind, wenn lokal auftretende Einbußen nicht durch lokal auftretenden Nutzen kompensiert werden können. Der Retentionsraum in Worringen würde nicht Köln, sondern Gebiete weiter nördlich schützten und das Naturschutzgebiet des Worringer Bruchs im Bedarfsfall beeinträchtigen. Im Gegensatz zum ersten und bereits installierten Retentionsraum in der Westhovener Aue hatte der Versuch, im Worringer Bruch Wasser zu sammeln, ausschließlich Symbolcharakter. Die geplante Installation des Retentionsraums diente damals vor allem dazu, um an die Oberlieger Kölns zu appellieren ebenfalls Retention zu betreiben. Außerdem half das Vorhaben Forderungen an andere Verwaltungsgebiete zu bekräftigen.

Im Umweltausschuss des NRW-Landtags wurde die Hochwasserschutzverantwortung ebenfalls diskutiert. Man war sich einig, dass am Oberrhein Polder angelegt werden müssten. Zudem stand die Installation einer einheitlichen Koordinierungsstelle aller Rheinanlieger im Vordergrund der Gespräche. Die Koordinierung des Hochwasserschutzes wurde bereits vor

\footnotetext{
${ }^{764}$ Stadt Köln, Stadt Köln, Drucksachen 1141/095, Anlage 3, 12.09.1995.

${ }^{765}$ HWSK 1996, 64.

${ }^{766}$ Oelmann 2008.
} 
dem Jahrhunderthochwasser von 1995 auf der Ebene von Bund und Ländern diskutiert. „Doch in den vergangenen Jahren hätten die Bundesländer Wert darauf gelegt, dass der Hochwasserschutz eine klassische landespolitische Aufgabe bleibe“6767, kommentierte Matthiesen die Abwehrhaltung der Bundesländer, die ihre Eigenverantwortung schützten. In der Umweltministerkonferenz am 18./19.05.1994 wurde eine Bund-LänderArbeitsgemeinschaft unter der Schirmherrschaft des Bundes abgelehnt ${ }^{768}$, führte er weiterhin aus. Als nationales Forum der Zusammenarbeit konnte lediglich die Länderarbeitsgemeinschaft „Wasser“ (LAWA) gewertet werden. Wie der NRWUmweltminister feststellte, „mache es wenig Sinn, nur die vier Bundesländer im Rahmen der Umweltministerkonferenz oder der LAWA an einen Tisch zu bringen“6769. Der Vorschlag, international aktiv $\mathrm{zu}$ werden, soll nach dem Hochwasserereignis bei der damaligen Bundesumweltministerin Merkel (CDU) laut Matthiesen auf Zuspruch gestoßen $\operatorname{sein}^{770}$. Das Anliegen könne in die Internationale Kommission zum Schutz des Rheines (IKSR) getragen werden $^{771}$, um von den Umweltministern der Rheinanliegerstaaten diskutiert zu werden. Im Rahmen der durch die Kommission veranstalteten internationalen Treffen fand die Stadt Köln Gehört und erreichte somit erstmals ihr Ziel. Allerdings stellt sich die Frage, ob die Gespräche in der IKSR tatsächlich auf die Bemühungen der Stadt Köln zurückzuführen sind oder, ob diese durch andere Akteure angeregt wurden.

Der Kommission mit Sitz in Koblenz fehlte seit der Beendigung der 2. Phase des „Aktionsprogramms Rhein“ im Dezember 1994 das Mandat ${ }^{772}$ : „Die intensive Behandlung früherer Themen ist weitgehend abgeschlossen“"773, erklärte ein Sprecher der Kommission. Deswegen sollte zukünftig in einem neuen Übereinkommen das Abflussverhalten des Rheins eine Rolle spielen ${ }^{774}$. Im Vorfeld zu dem Treffen der europäischen Umweltminister am 04. Februar 1995 in Arles hatte der innerdeutsche Streit zwischen den Städten und Bundesländern, der zunehmend durch die Presse verstärkt wurde, die Diskussion des Hochwasserproblems auf die Bundesebene gehoben. Am 30. Januar zitierte Bundeskanzler Helmut Kohl (CDU) die Staatsekretäre der Ministerien in das Bonner Kanzleramt, um über das Hochwasserproblem zu sprechen ${ }^{775}$. Bereits am 02. Februar 1995 sprach die damalige

\footnotetext{
${ }^{767}$ Landtag NRW, Ausschussprotokoll 11/1505, 08.02.1995.

${ }^{768}$ Landtag NRW, Drucksache 11/8490, 16.02.1995.

${ }^{769}$ Landtag NRW, Ausschussprotokoll 11/1505, 08.02.1995. Matthiesen sprach von Baden-Württemberg, RheinlandPfalz, Hessen und Nordrhein-Westfalen am Rhein.

${ }^{770} \mathrm{Ebd}$.

${ }^{771}$ Ebd.

${ }^{772}$ IKSR, PLEN 3/95.

${ }^{773}$ IKSR, Presseerklärung, 06./07.07.1995.

${ }^{774}$ Anne Schulte-Wülwer-Leidig, Stellvertretende Geschäftsführerin der IKSR, 06.08.2012 Koblenz: „Von Hochwasser traute sich damals niemand zu sprechen."

${ }^{775}$ Artikel: Kohl erklärt die Fluten zur Chefsache, Rhein-Lahn-Zeitung, 31.01.1995.
} 
Bundesumweltministerin Angela Merkel (CDU) während der Eröffnung des europäischen Naturschutzjahres in Konstanz von überregionalen und interdisziplinären Mahnahmen, die gegen das Hochwasser helfen sollten ${ }^{776}$. Zwei Tage später wurde die „Erklärung von Arles“ von allen Staaten im Rheineinzugsgebiet unterzeichnet ${ }^{777}$. Diese Erklärung beurkundete die Geburtsstunde des von der IKSR 1998 erstellten „Aktionsplans Hochwasser“6778. Das Treffen der EU-Umweltminister in Arles im Februar 1995 fand zu dem Zeitpunkt statt an dem das Thema nicht aktueller hätte sein können. Das Wasser schwappte in Köln gerade über die Vorrichtungen und in den Niederlanden mussten 240.000 Menschen evakuiert werden ${ }^{779}$. „Die jüngsten Hochwässer hatten aufgezeigt, dass auf europäischer Ebene kurzfristig Maßnahmen vereinbart werden müssten“780. Ziel war es, eine interdisziplinäre Zusammenarbeit $^{781}$ aller Rheinanlieger auf internationaler Ebene aufzubauen ${ }^{782}$. Die Erklärung von Arles bezog die Raumordnung und Bodennutzung in die Hochwassermaßnahmen mit ein ${ }^{783}$. Ein Treffen der Raumordnungsminister am 30. März 1995 in Strasbourg brachte eine Erklärung zwischen Frankreich, Deutschland, den Niederlanden, Belgien und Luxemburg hervor, Raumordnungsmaßnahmen im Hochwasserkontext zu ergreifen und die erforderlichen Instrumente, einschließlich finanzieller Art, zu finden ${ }^{784}$. Erst bei dem IKSR-Treffen in Echternach im Dezember 1997 entstand der Beschluss, Nichtregierungsorganisationen ${ }^{785}$ den Konferenzen zukünftig mit einem Beobachterstatus beiwohnen zu lassen ${ }^{786}$. Bei dem Treffen in Rotterdam am 22.01.1998, zur Vorstellung des „Aktionsplans Hochwasser“, waren erstmalig Nichtregierungsorganisationen zugelassen ${ }^{787}$.

\footnotetext{
${ }^{776}$ Artikel: Merkel will Ökosysteme sichern, Kölner Stadtanzeiger, 03.02.1995.

${ }^{777}$ Die „Erklärung von Arles“ beinhaltete die Bereitschaft der Umweltminister, Maßnahmen der Wasserwirtschaft, der Raumordnung und Bodennutzung mit dem Ziel der Verbesserung des Schutzes von Menschen und Gütern vor Hochwasser unter Einbindung des Ziels der ökologischen Verbesserung des Rheins und seiner Aue zu aktivieren (IKSR, Aktionsplan Hochwasser).

${ }^{778}$ In der Rheinministerkonferenz am 22.01.1998 in Rotterdam wurden die erarbeiteten Handlungen u. a. für Retentionsmaßnahem zwischen der Quelle und der Mündung mit Kostentabellen versehen vorgestellt. In dem Aktionsplan wurde der Deutsch-Französische Vertrag von 1982 nicht aufgegriffen, allerdings wurden die vereinbarten Ausgleichsmaßnahmen der Staustufen in die Projektliste der Rückhaltebecken integriert. Neben der Verringerung der Hochwasserstände wurden drei Ziele formuliert: die Verstärkung des Hochwasserrisikobewusstseins, die Verbesserung der Meldesysteme sowie die Verringerung des Schadensrisikos (Aktionsplan Hochwasser, IKSR, 1998;

Rheinministertreffen, Rotterdam, 22.01.1998; Erklärung von Arles, 04.02.1995).

${ }^{779}$ Artikel: 240.000 Holländer fliehen vor der Flut, Kölner Stadtanzeiger, 01.02.1995.

${ }^{780}$ Artikel: Aktion gegen Hochwasser geplant, Rhein-Lahn-Zeitung, 06.02.1995.

${ }^{781}$ Landtag NRW, Drucksache 12/248, 11.10.1995.

782 Die Projektgruppe „Hochwasser“ der IKSR legte schon im März 1995 einen Plan vor. IKSR, PLEN 16/95; IKSR, PLEN 3/95.

${ }^{783}$ IKSR, Tätigkeitsbericht 1995/1996, 47.

${ }^{784}$ Ebd., 49.

${ }^{785} \mathrm{Zu}$ den Vorbereitungstreffen durften die ausgewählten NGOs bereits früher kommen. So beispielsweise R. Vogt, H. Oelmann und Dr. B. Manthe als Repräsentanten der Hochwassernotgemeinschaft Rhein am 02.07.1997 zur

63. Vollversammlung der IKSR (IKSR, Beschlussprotokoll der 63. Vollversammlung).

${ }^{786}$ IKSR, Luxemburg, PLEN 43/97.

${ }^{787}$ IKSR, Pressemitteilung, PLEN 42/97.
} 
Die Stadt Köln war im Rahmen der Hochwassernotgemeinschaft Rhein ${ }^{788}$ in Rotterdam vertreten, bis April 1998 allerdings ohne bestimmten Status. Hier zeigt sich, dass die Stadt Köln ihr Ziel erreichte, international Einfluss zu nehmen, nachdem der Aktionsplan bereits erarbeitet worden war. Es kann folglich nicht davon ausgegangen werden, dass überregionale und internationale Dialoge durch die Kölner Retentionsbemühungen motiviert wurden. Dagegen ist deutlich zu erkennen, dass das Ober-/Unterlieger-Problem einen starken politischen Druck sowohl auf den Kanzler als auch auf die EU-Umweltminister ausübte. Es lässt sich also somit festhalten, dass die Eröffnung der internationalen Dialoge in dem Handlungszwang begründet lag, und somit fand eine Entwicklung statt, die sich, parallel zu den Kölner Bemühungen, in dieselbe Richtung bewegte.

\subsection{Verschiebung der Rollen der politisch Verantwortlichen}

Die in den vorherigen Abschnitten beschriebenen Reaktionen zeigen, zusammenhängend betrachtet, eine Verschiebung der Rollen der Kölner Akteure zwischen 1970 und 1995 auf. Während eine Überschwemmung zunächst ein lokales Ereignis verkörperte, welches vor Ort diskutiert und bereinigt werden musste (1970), veränderte sich die Wahrnehmung der Lokalpolitiker des Charakters der Schadensereignisses im Laufe der Zeit. Die Betroffenen waren sich im ersten Beispiel selbst überlassen, im zweiten Beispiel jedoch wurde der Hochwasserschutz zur Gemeinschaftsaufgabe. Hochwasser wurde zunehmend als anthropogenes Ereignis begriffen (1995), die Ursache für Hochwasser und die damit verbundenen Schäden waren nicht mehr die zu niedrigen Schutzvorrichtungen vor Ort, sondern die durch menschliche Eingriffe erschwerten Abflussverhältnisse. Diese Eingriffe verstärkten die natürliche Hochwasserwelle, die an der dargestellten Schadensstelle, in der Stadt Köln, von den lokalen Akteuren als unbeherrschbar wahrgenommen wurde. Dementsprechend wandelten sich die Rollen der politischen Akteure. Die ehemals lokal agierenden Stadtratsmitglieder engagierten sich nach dem Hochwasser von 1995 über die nordrhein-westfälischen Grenzen hinaus und strebten einen überregionalen Hochwasserschutzdiskurs an. Dabei gerieten sie in die Position der vulnerablen

\footnotetext{
${ }^{788}$ Diese ist eine Solidargemeinschaft der Gemeinden am Mittel- und Niederrhein, (Stadt Klön,

Besprechrechungsniederschrift, 14.06.1996). Die Notgemeinschaft verfügt über eine strukturierte Verwaltung in Form einer Geschäftsstelle in dem Gemeinde- und Städtebund Rheinland-Pfalz (Brief des Oberbürgermeisters Norbert Burger an die IKSR, 08.04.1998). Die Hochwassernotgemeinschaft Rhein wurde am 16.11.1996 gegründet, (Einladung zur Mitgliederversammlung). Kölns Oberbürgermeister Burger wurde zum Vorstandsvorsitzenden der 62 Mitglieder gewählt (Stadt Köln, Besprechungsniederschrift, 16.12.1996).
} 
Hochwasseropfer, die sich nicht selbstständig zu den diskutierten Maßnahmen äußern durften. Politische Hierarchien unterbanden eine direkte Kommunikation der kommunalen Verwaltung der Stadt Köln mit den Entscheidungsbefugten anderer Bundesländer und somit ein Mitwirken in der Verbesserung des Kölner Hochwasserschutzes. Das Verhalten der administrativen Akteure hat sich ebenfalls grundlegend geändert. Während die Kölner Verantwortlichen 1970 sich nicht mit dem Hochwasser auseinandersetzen wollten, suchten sie 1995 mittels des Einbaus von Retentionsbecken nach Fürsprechern auf allen politischen Ebenen, die ihre verletzliche Lage verbessern könnten. Während die politisch Verantwortlichen in Köln 1970 die unberechenbare Gefahr im Bürger (unpredictable civil) sahen und versuchten diesen abzuwehren, suchten sie 1995 den Dialog mit den Betroffenen, um durch die Stärkung des Gefahrenbewusstseins die Anhäufung materieller Werte im Gefahrenbereich zu minimieren. Zunächst nahmen die Kölner Stadtratsmitglieder die Hochwasser als natürliche Ereignisse wahr, die durch Hochwasserschutzmauern zumindest in ihrer Häufigkeit zu schmälern waren. Ab 1995 jedoch nahmen sie die Hochwasser aufgrund ihrer Stärke und häufigen Intervalle als unnatürliche Schadensereignisse wahr, dem sie nichts entgegenzusetzen hatten. Ähnlich wie die Bürger der Stadt Köln 1970 suchten die Kölner Politiker 1995 einen Weg, an den Entscheidungen über das Einzugsgebiet des Rheins mitzubestimmen. Die Stadt verfügte über ausreichende Ressourcen, um den beispielhaften Einbau von Retentionsbecken zu planen, denn eine geeignete Fläche konnte für diesen Zweck gefunden werden und die finanziellen Mittel wurden mit der Vorstellung, eine Vorbildfunktion zu übernehmen, welche den Hochwasserschutz für Köln beflügelt, effizient investiert.

Beide Reaktionen der handelnden Akteure lassen sich aus Sicht der Kosten-NutzenPerspektive nachvollziehen. Während 1970 ausschließlich im Stadtrat Kölns und dem Landtag NRWs über die Hochwasserschäden verhandelt wurde, entbrannte nach den Überschwemmungen von 1995 ein deutschlandweiter Streit, der auch in die angrenzenden Nachbarländer getragen wurde. Das Ereignis wurde im Rahmen der EUUmweltministerkonferenz vom 04. Februar 1995 verhandelt. In beiden Fällen verglichen die politischen Akteure alternative Handlungsoptionen und versuchten gesellschaftliche Konsequenzen zu bewerten. Die beschlossenen Reaktionen wurden von den handelnden Akteuren nach beiden Hochwasserereignissen spezifisch kritisiert und es wurde der Versuch unternommen, die Grenzen der jeweiligen Handlungsoptionen aufzuzeigen. Die intangiblen Kosten wurden 1970 in der Diskussion, ob die Stadt Köln Schadensersatz leisten könnte, als zu hoch erachtet. Die Ängste der politisch Verantwortlichen stellten eine Hemmnis dar, aktiv 
zu werden. Die negativen Befürchtungen lassen sich jedoch nicht in Geldeinheiten ausweisen, obwohl sie den Abwägungsprozess aus Kosten-Nutzen-Perspektive unmittelbar beeinflussten. Die politisch Aufgrund der Angst vor der Hilflosigkeit gegenüber den Entscheidungsträgern am Oberrhein, setzten sich die Kölner Stadtratmitglieder für eine überregionale Auseinandersetzung zur Hochwasserproblematik am Rhein ein. 


\section{Politische Reaktionen auf Hochwasser im Mekong-Delta}

Im vorliegenden Kapitel werden drei zentralstaatliche Diskurse als politische Reaktionen auf die jährlichen Hochwasser im Mekong-Delta untersucht. Die Rationalität hinter den gewählten Strategien des Hochwasserschutzes soll durch die Kontextualisierung des Desaster, Landwirtschafts- und Hochwasserschutzdiskurs verdeutlicht werden. Dazu werden die von der Regierung genannten jeweiligen Ziele im Zusammenhang mit ihren Handlungen im Hochwasserschutz betrachtet. Die drei gewählten Diskurse verfolgen die hier aufgezählten politischen Ziele:

1. Diskurs zum Desaster- und Katastrophenmanagement ${ }^{789}$

- Verringerung der Opferzahlen

○ Verringerung der Schadenshöhe

2. Diskurs zur Intensivierung der Landwirtschaft ${ }^{790}$

- Steigerung des landwirtschaftlichen Outputs

○ Steigerung der Einkommen der lokalen Bevölkerung

3. Diskurs zum Hochwasserschutz

- Deichbau ${ }^{791}$

- Vergrößerung der landwirtschaftlich nutzbaren Fläche durch Landgewinnung in den Überschwemmungsgebieten

- Steigerung des landwirtschaftlichen Outputs

- Steigerung der Einkommen der lokalen Bevölkerung

○ Richtlinie Living with Floods ${ }^{792}$

- Verringerung der Opferzahlen

- Verringerung der Schadenshöhen

Eine Einordnung in den historischen Kontext erleichtert die Bewertung der Diskurse, darum wird zunächst ein Überblick über die Entwicklungen im Mekong-Delta in dem Zeitraum zwischen der Wiedervereinigung Vietnams 1976 bis zum Beginn der betrachteten Diskurse ca. 1990 vermittelt. Es folgt die Präsentation der drei Diskurse. Zunächst wird das jeweilige Ergebnis vorgestellt, sodann wird der Verlauf des politischen Entscheidungsprozesses aufgrund historischer Quellen, soweit möglich, rekonstruiert. Angeschlossen an die jeweiligen Diskurse findet eine Interpretation der Auseinandersetzungen unter Berücksichtigung des

\footnotetext{
${ }^{789}$ DR 0021992.

${ }^{790}$ AG 461993.

${ }^{791}$ White 2002.

${ }^{792}$ Howie 2006.
} 
Hilfsmodells aus der Kosten-Nutzen-Perspektive statt. Anschließend werden die Entwicklungen vor Ort mit den kommunizierten Zielen der Regierung abgeglichen, um Hinweise auf die Rationalität der Handlungen der Zentralregierung ableiten zu können.

\subsection{Einführung: Traditionelles Leben im Wandel}

Nach der Wiedervereinigung von Nord- und Südvietnam im Jahr 1976 wurden die administrativen Strukturen des Nordens auf den Süden übertragen ${ }^{793}$. Somit wurde Südvietnam von der Regierung des Einparteiensystems und der dort herrschenden Planwirtschaft einbezogen. Abb. 21 zeigt das Untersuchungsgebiet der Provinz An Giang.

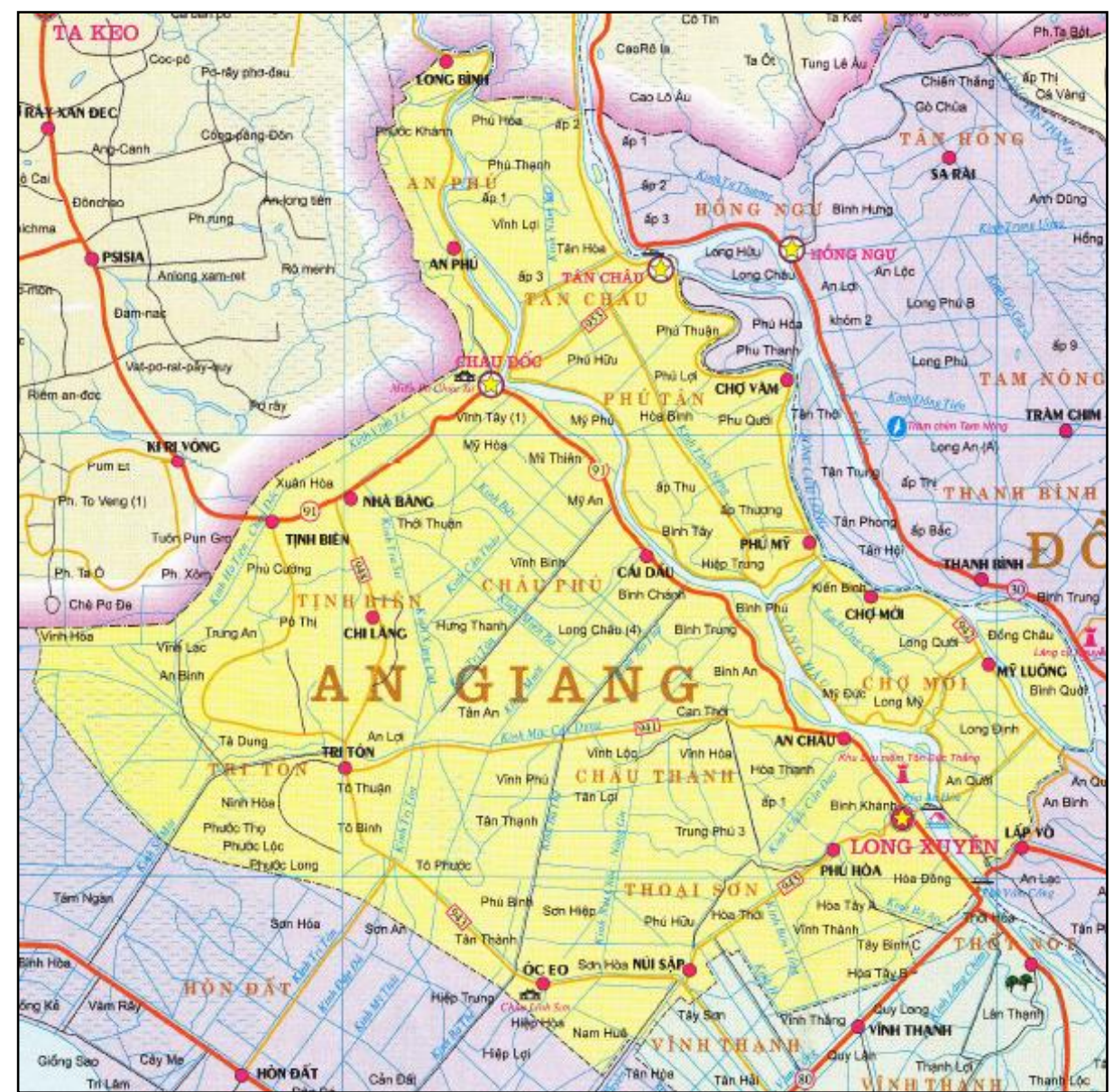

Abb. 21: Provinz An Giang. ${ }^{794}$

\footnotetext{
${ }^{793}$ Evers/Benedikter 2009.

${ }^{794}$ Ban do du lich mien tay nam bo, nha xuat ban tai nguyen moi truong va ban do viet nam, xong va nop luu chieu thang 10 nam 2011. Landkarte des Mekong-Deltas, Verlagshaus: ban tai nguyen moi truong va ban do viet nam, Oktober 2011.
} 
Die Festigung der neuen politischen Strukturen und die wirtschaftliche Entwicklung galten als Hauptziele der vietnamesischen Regierung ${ }^{795}$. Doch schon im Jahr 1976 gab es erste Probleme: Die geringe Ernte löste Hungersnöte aus ${ }^{796}$. Nachdem nur eine Menge von $300.000 \mathrm{t}$ Reis in der Provinz An Giang eingeholt worden war $^{797}$, reagierte der Staat mit einem Hilfsprogramm ${ }^{798}$. Zudem stellte eine verlängerte Trockenzeit im Jahr 1977 die Akteuren aus Landwirtschaft und Politik vor weitere Herausforderungen. Als Reaktion auf die Wasserknappheit wurden neue Bewässerungsprojekte umgesetzt ${ }^{799}$. Ein weiteres Projekt der vietnamesischen Regierung war die Einführung von Hochleistungssorten (Reis) und in Folge dessen der Anbau in einer Zwei-Phasen-Bewirtschaftung der Felder ${ }^{800}$.

Doch trotz der Bemühungen der Hanoier Politiker entwickelten die Menschen im Mekonggebiet kein intaktes Verhältnis zur neuen Regierung, denn die lokale Bevölkerung lehnte sich gegen die Überstülpung des sozialistischen Systems auf. Die Bewohner des Deltas wehrten sich bereits gegen die ersten Phasen auf dem Weg zu einer Kooperative nach sozialistischem Vorbild ${ }^{801}$. Mit dem Ziel, die Abgaben an die Zentralregierung zu vermeiden, bauten die Haushalte in den darauf folgenden Jahren Nahrungsmittel nur noch für den Eigenbedarf $a^{802}$. Mehr als $66 \%$ der regionalen Bevölkerung waren in der Landwirtschaft tätig, bearbeiteten aber nur $1.800 \mathrm{~km}^{2}$ Reisfelder der ca. $20.000 \mathrm{~km}^{2}$ großen landwirtschaftlichen Fläche des Deltas, welche sie zwischen den Hochwasserzeiten einmal ernteten $^{803}$. Die Wehrhaftigkeit nahm eine ,dialogische ${ }^{\text {(604 }}$ Form an, wie der Geograph David Howie das Beispiel der „everyday politics ${ }^{\text {(805 }}$ von 1978 kommentierte: Einige Landwirte hatten 1978 eigenständig einen Deich um ihre Aussaat von Hochleistungssorten gebaut. Sie wollten diese vor dem auflaufenden Hochwasser mit provisorischen Deichkonstruktionen schützen $^{806}$. Obgleich die Bauern von den lokalen Behörden aufgefordert wurden, die Ernte zum Schutz vor dem kommenden Hochwasser einzuholen, damit diese nicht gänzlich durch das Hochwasser zerstört werden würde, fuhren die Bauern mit dem Deichbau fort. Nach der erfolgreichen Ernte am Ende des natürlichen Reifungsprozess soll der stellvertretende Prime Ministers Do Muoi bei einem Besuch in der Provinz die Konstruktion gelobt haben und

\footnotetext{
795 Tong 1997.

${ }^{796}$ Ebd.

${ }^{797}$ Die Menge entsprach ca. 7,5 \% des Gesamterzeugnisses von 1976 und ca. 0,8 \% der heutigen Reisproduktion.

${ }^{798}$ Tong 1997.

${ }^{799}$ Ebd.

${ }^{800}$ Ebd.

${ }^{801}$ Howie 2011.

${ }^{802}$ Tong 1997.

${ }^{803}$ Ebd.

${ }^{804}$ Howie 2011, 7.

${ }^{805}$ Benedikter/Waibel 2013.

${ }^{806}$ Howie 2011.
} 
angegeben haben, er würde der Regierung empfehlen, den Deich zu behalten ${ }^{807}$. Außerdem beschreibt Howie die ,farmers resistance to collectivization in the Mekong-Delta in the early

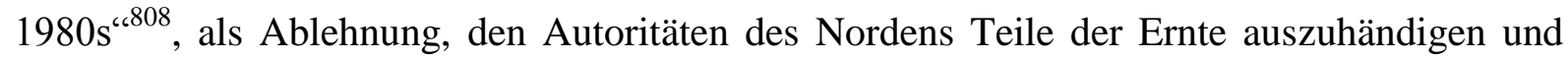
begründete diesen Widerstand mit den Landallokationen der Regierung ${ }^{809}$. „The reforms followed many years of peaseant resistance“ ${ }^{\text {810. }}$. Ein Widerstand der südlichen Landbevölkerung gegenüber der Ausdehnung der Kollektive war zu Beginn der 1980er Jahre verbreitet $^{811}$. Zwischen der Wiedervereinigung Vietnams 1976 und der wirtschaftlichen Erneuerung Doi moi $1986^{812}$ blieb die vietnamesische Landbevölkerung an den abgelegenen Armen des Mekongflusses wehrhaft gegenüber den Plänen der Zentralregierung. Diese Gruppe war in der Lage, auf politische Entscheidungen Einfluss zu nehmen, gerade weil der Staatsapparat sie nicht gänzlich kontrollieren konnte ${ }^{813}$. Da die lokalen Behörden den nationalen Ebenen verschwiegen hatten, mit welchen Konflikten sie in ihren Gebieten zu kämpfen hatten, konnte der die Städte nicht mehr mit dem Grundnahrungsmittel Reis versorgen ${ }^{814}$. Neben Reis konnten auch andere natürliche Ressourcen nicht mehr bis in die Städte geliefert werden ${ }^{815}$. Die bedrohliche Lage der Nahrungsmittelknappheit führte zu der Konsequenz, dass einige Mitglieder des Zentralkomitees der Kommunistischen Partei Vietnams (KPV) in Hanoi ersetzt wurden und die Reform der Doi moi-Erneuerung verabschiedet wurde ${ }^{816}$. Die ländlichen Widerstände wurden in der Wissenschaft als ein Treiber für die Reformen Doi moi in Jahr 1986 identifiziert ${ }^{817}$. Aus diesem Grund fürchtet die Zentralregierung die Landbevölkerung des Mekonggebietes. Der Widerstand der Landbevölkerung, der in der Reform 1986 gipfelte, verdeutlichte den Entscheidungsträgern die Fragilität ihrer Macht. Der Erlass des Bodengesetzes von 1988 diente als ein Instrument „to promote permanent agriculture and settlement ${ }^{4818}$. Nachdem das neue Gesetzt eingeführt worden war, lebten $32,67 \%$ der Einwohner von weniger als 0,5 ha Land $^{819}$. Im krassen Gegensatz dazu besaßen 98,99\% der Bevölkerung weniger als 5 ha Land ${ }^{820}$. Einigen wenigen blieben deutlich größere Flächen zur Verfügung, so hatten 0,07 \% der Bevölkerung Zugang

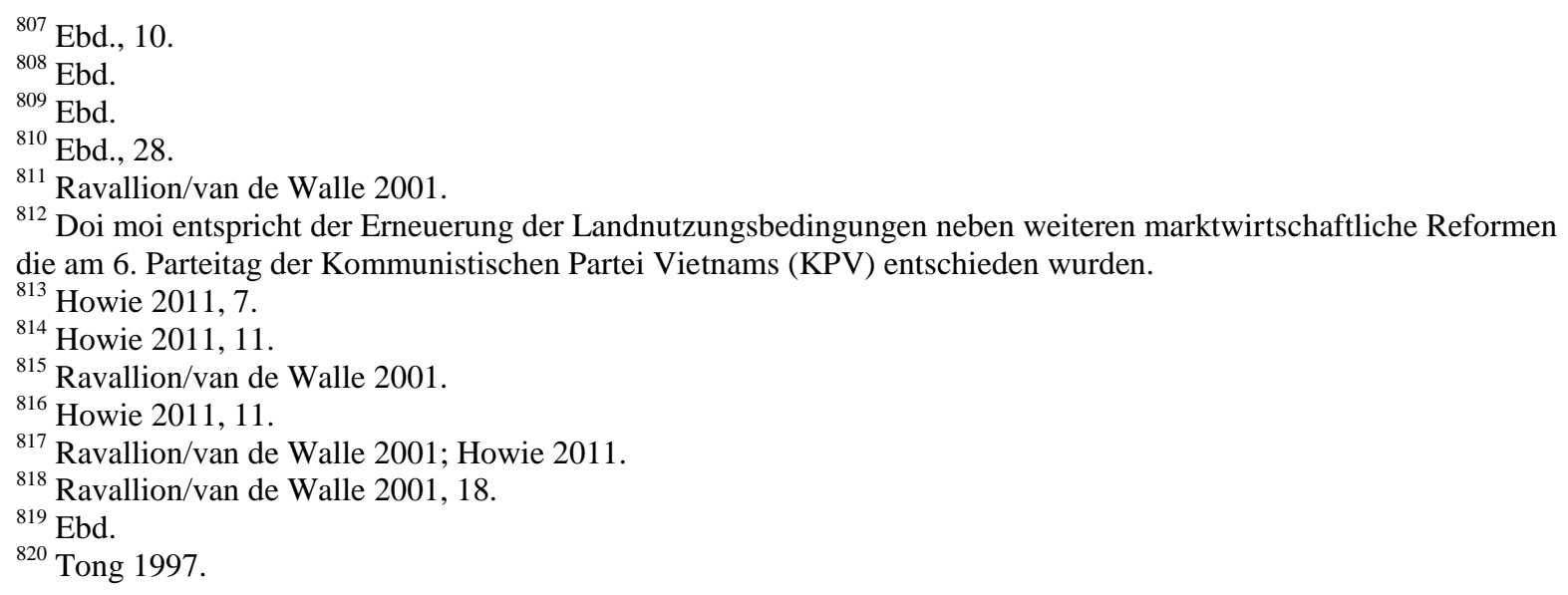


zu mehr als 10 ha $^{821}$. Die Größe der Flächen halbierte sich zwischen 1980, als ein Landwirt noch über durchschnittlich $0,0063 \mathrm{~km}^{2}$ verfügte auf $0,0035 \mathrm{~km}^{2}$ in 1990 und $0,0032 \mathrm{~km}^{2} \mathrm{im}$ Jahr $1994^{822}$. 1994 gehörten 2,19\% der Haushalte in An Giang (42.529 Menschen) der landlosen Bevölkerung an ${ }^{823}$.

Der Ökonom Ngo Van Tong betont in seiner Studie zur sozioökonomischen Entwicklung der Provinz zwischen 1990 und 1995 die Problematik des Bevölkerungswachstums als ein Limit für die wirtschaftliche Entwicklung. Die ca. 30.000 neuen Arbeitskräfte, die jährlich auf den Arbeitsmarkt strömten, versetzten die Regierung in Alarm, denn sie sahen sie als Hemmschwelle für die als nötig erachtete Einführung der Mechanisierung in der Landwirtschaft, da diese die menschliche Arbeitskraft zusätzlich rationalisieren würde ${ }^{824}$. Für das Jahr 2010 wurde eine Bevölkerungszahl von 2,6 Mio. Menschen in der Provinz An Giang erwartet, die der Sektor der Landwirtschaft nicht alle aufnehmen könnte ${ }^{825}$. Eine Lösung versprach die Ausbildung der Arbeitskräfte für die Sektoren der Verarbeitung und Dienstleistung ${ }^{826}$. Landbesitz sollte keine Voraussetzung für Einkommen mehr bedeuten. Die vorhandene Arbeitskraft stellte sich aufgrund der steigenden Bevölkerungszahlen und den kleiner werdenden Flächen noch überflüssiger dar, die Arbeitstage reduzierten sich aufgrund der geringer zu beackernden Fläche von ehemals 120 Tagen pro Jahr auf nur noch $60-70$ Tage. Die Menge der landwirtschaftlichen Arbeitskraft war bereits ausreichend, dies zeigte sich auch an den großen Leerlaufzeiten der Landwirte. Freie Zeit gab es in der Landwirtschaft reichlich $^{827}$. Die Entwicklung der Bevölkerungszahlen wurde von der Regierung mit Sorge betrachtet. Als soziale Herausforderungen wurden 1997 die begrenzt zur Verfügung stehende landwirtschaftlich Fläche, das schnelle Bevölkerungswachstum, die hohen Arbeitslosenzahlen ${ }^{828}$, die unmodernen Arbeitsmethoden, die fehlende Industrialisierung, sowie instabile Preise wahrgenommen ${ }^{829}$. Ebenso fehlte es an wissenschaftlich und technisch ausgebildeten Fachkräften. Die Quote der schulisch ausgebildeten Erwachsenen lag in der Provinz An Giang unter dem Durchschnitt des ganzen Landes ${ }^{830}$. Im Jahr 1992 besuchte, statistisch betrachtet, eins von 6,54 Kindern die Grundschule ${ }^{831}$.

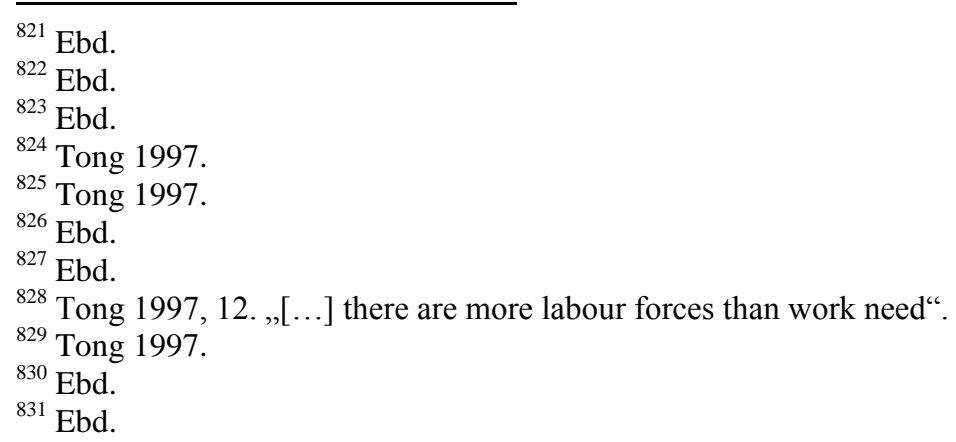


Die Einführung der Hochleistungssorten erforderte in den späten 1970er Jahren erste Maßnahmen, das Hochwasser aus landwirtschaftlichen Anbaugebieten fern zu halten ${ }^{832}$. Dazu bauten die Landwirte mit einfachen Mitteln Wälle auf, die das Hochwasser einige Tage zurück hielten und dann eingerissen wurden ${ }^{833}$. Diese Provisorien heißen Augustdeiche. Die Ausdehnung der hochwasserfreien Zeit verlängerte die landwirtschaftlich nutzbaren Phasen und so versuchten die Landwirte durch die Errichtung ihr Einkommen zu steigern und ihren Lebensstandard zu erhöhen. Während der Staat den Anbau der neuen Hochleistungssorten förderte, beförderte die neue Einsaat in der Trockenzeit das Verlangen der Landwirte, Augustdeiche aufzubauen. Das International Rice Research Institute führte erstmalig 1966 in Zusammenarbeit mit vietnamesischen Forschern des Rice Research Institutes in O Mon bei Can Tho innovative Reisvarianten ein, die einen Ertrag von 5-8 t/ha einbrachten ${ }^{834}$. Im Gegensatz dazu wirkte die Ernte der traditionell angebauten Sorten von 2 t/ha gering. Die neuen Pflanzen mussten im Gegensatz zum schwimmenden Reis in den trockenen Boden gepflanzt und dann bewässert werden ${ }^{835}$. Sie bedürfen für ihre Entwicklung von der Aussaat bis zur Ernte ca. 100 Tage. Wenn die Zeit zwischen zwei Hochwassern künstlich um einige Tage ausgedehnt wurde, waren folglich zwei Ernten möglich. Zum Ziel der Landwirte wurde es, den Boden in der trockenen Jahreszeit für zwei Ernten zu nutzen. Sie sehnten sich nach dem Wohlstand, den die deutlich umfangreicheren Ernten versprachen, weswegen sie die Nutzung der Böden intensivierten. Für den Landwirt bedeutete diese umfangreiche Ertragssteigerung eine erste Veränderung des traditionellen Lebenswandels. Nach dem Ablaufen des Hochwassers zwischen Januar bis Ende April war es den Landwirten möglich, das neue Saatgut auszubringen, um es vor dem nächsten Hochwasserzyklus im August zu ernten. Im Sommer begannen die Landwirte Hindernisse gegen das Wasser zu errichten. Nach Ablaufen des Hochwassers bzw. nach der letzten Ernte wurden diese Deiche wieder entfernt oder im August einfach dem Hochwasser überlassen, um den Feldern die Überschwemmung zu ermöglichen ${ }^{836}$. Um die Bewässerung der Reisanbaugebiete zu gewährleisten, organisierten die Bauern Pumping Clubs. Bis zum Beginn der 1990er Jahre nutzte annähernd die ganze Provinz provisorische Augustdeiche ${ }^{837}$, sodass Pumpen zur Bewältigung der Flächenbe- und Entwässerung angeschafft wurden.

\footnotetext{
${ }^{832}$ Howie 2011.

${ }^{833}$ Ebd.

${ }^{834}$ Howie 2011; 2006.

${ }^{835}$ Ebd.

${ }^{836}$ Howie 2011.

${ }^{837}$ Howie 2011.
} 


\begin{tabular}{|l|l|l|l|l|l|}
\hline In Stückzahlen & 1991 & 1992 & 1993 & 1994 & 1995 \\
\hline Mopeds & 14 & 15 & 16 & 18 & 21 \\
\hline Fernseher & 18 & 22 & 22 & 26 & 33 \\
\hline Uhren & 74 & 80 & 82 & 94 & 157 \\
\hline Wasserpumpen & 24.513 & 26.453 & 24.982 & 30.258 & 33.067 \\
\hline
\end{tabular}

Tabelle 5: Besitztümer in der Provinz An Giang 1991 - 1995. ${ }^{83}$

Tabelle 5 zeigt das Vorhandensein von Gütern, deren Besitz noch 1995 als Luxus aufgefasst wurde $^{839}$, im Vergleich zu einem landwirtschaftlichen Arbeitsgerät, der Wasserpumpe. Auffällig steigt die Anzahl der Uhren über den Betrachtungszeitraum zwischen 1991 und 1995 rapide an. Das Wirtschaftswachstum stellte der Bevölkerung eine höhere Kaufkraft zur Verfügung. Der Besitz eines Fernsehers in der Provinz verzeichnete einen Zuwachs von $83 \%$ in fünf Jahren, dagegen verhielten sich die Bewohner bei der Investition in ein Moped etwas zurückhaltender. Entweder musste für das Aufbringen der Kaufsumme länger gespart werden, oder die Menschen bevorzugten ein Fernseher, da der Verkehr auf dem Wasser keine Probleme bereitete und ein Fernseher die moderne Welt in die eigene Unterkunft transportiert. Bis Dezember 1993 verliefen 2.715 km Straßennetz durch die Provinz An Giang, davon waren jedoch lediglich $8,07 \%$ betoniert ${ }^{840}$. Das landwirtschaftliche Arbeitsinstrument der Wasserpumpen war in An Giang im Vergleich zu den Luxusgütern schon 1991 verbreitet. Die Kontrolle über die Bewirtschaftung des Ackerlands, sowie dessen Überflutung lag in der Verantwortung der lokalen Bauern ${ }^{841}$. Auf die 1.967.805 Einwohner der Provinz kamen 1991 24.513 Pumpgeräte. Einen Teil der steigenden Kaufkraft investierten die Bauern in ihre Arbeitsgeräte, der Besitz der Pumpen wurde bis ins Jahr 1995 um ca. 35 \% vergrößert. Besonders entwickelte sich die technische Leistung der Pumpen, wie Tabelle 6 zeigt. Die vorhandene Pumpkapazität wurde um $126 \%$ auf $2.155 .074 \mathrm{~m}^{3} /$ Tag gesteigert. Die Verantwortung über landwirtschaftliche Arbeiten trug der Landwirt für sein Anbaugebiet selbst. Die Eigenständigkeit der Landwirte manifestierte die Stellung des Bauers als Entscheidungsträger. Obwohl bereits große Stückzahlen von Pumpen vorhanden waren, sahen die Menschen einen Nutzen darin, noch mehr Wasser pumpen zu können. Je schneller Wasser bewegt werden konnte, desto eher konnten die Felder nach dem Hochwasser wieder eingesät werden. Die Bauern verzögerten mit Hilfe der Augustdeiche den Jahreszyklus und sie waren motiviert, den natürlichen Gegebenheiten durch ihre modifizierten Anbaumethoden noch größere Erträge abzutrotzen.

\footnotetext{
${ }^{838}$ Nach Tong 1997.

${ }^{839}$ Tong 1997.

${ }^{840}$ Ebd. Das Netz der Wasserstrassen umfasste eine Strecke von 2.461 km Länge, pro Quadratkilometer Land verliefen $0,719 \mathrm{~km}$ Wasserstraße. Mehr als $1.000 \mathrm{t}$ konnten nur auf den großen Wasserstraßen transportiert werden, welche $6,56 \%$ des Netzes ausmachten, auf 80,79\% war ein Transportgewicht bis zu 10 t bereits möglich.

${ }^{841}$ Howie 2011.
} 


\begin{tabular}{|l|l|l|l|l|l|}
\hline An Giang & 1991 & 1992 & 1993 & 1994 & 1995 \\
\hline Leistung der Pumpen $\left(\mathrm{m}^{3} / \mathrm{Tag}\right)$ & 953.443 & 1.066 .254 & 1.150 .430 & 2.052 .097 & 2.155 .074 \\
\hline
\end{tabular}

Tabelle 6: Steigerung der Pumpleistung in An Giang 1991 - 1995.

Bis 1995 gewann die Provinz An Giang $1.600 \mathrm{~km}^{2}$ Land aus einstigen Überschwemmungsgebieten, damit wuchs die landwirtschaftlich genutzte Fläche um $16,58 \%{ }^{843}$. Der Umsatz des Exportgeschäftes der Provinz stieg durch die gesteigerten Erntemengen seit 1991 von 30 Mio. USD auf 130 Mio. USD in 1995, davon machten landwirtschaftliche Erzeugnisse bis zu $76 \%$ aus. Davon waren $67 \%$ - 90 \% Reis, was im Jahr 1995367.000 t Reis entsprach ${ }^{844} .1991$ war der Anteil des Reises noch bei $65 \%$ der angebauten Erzeugnisse, was 140.000 t Reis gleichkam ${ }^{845}$. Die Intensität der Bodennutzung stieg 1995 auf einen Bodennutzungskoeffizient von 1,8 an ${ }^{846}$. Fast alle Bauern schienen eine doppelte Einsaat zu praktizieren. Der Reisanbau in der Regenzeit wurde, seitdem flächendeckend Hochleistungssorten abgebaut wurden, nur noch von wenigen Bauern gepflegt $^{847}$.Das Monatseinkommen für Bauern, die zwei Reisernten in der Trockenzeit pflegten, stieg zwischen 1991 - 1995 von 104.000 VND auf 237.000 VND, während es für Bauern mit nur einer Ernte von 97.000 VND auf 193.000 VND anstieg ${ }^{848}$. Tabelle 7 gibt einen Überblick über die wirtschaftliche Situation der Provinz An Giang im Vergleich zum Mekong-Delta und zum ganzen Land. Die Daten belegen den wirtschaftlichen Wandel, den sowohl die Provinz, das Delta, sowie das Land zu Beginn der 1990er Jahre vollzogen haben. In Relation zum landesweiten BIP pro Kopf wuchs der Anteil des Bruttoinlandsprodukts der Provinz fast genauso stark, während der Anteil in der Provinz stärker ausgeprägt war, als im Mekong-Delta insgesamt. Zwischen 1990 und 1995 war An Giang im Verhältnis zu den Nachbarprovinzen eine der wirtschaftlich stärkeren Administrationen. Der Umrechnungskurs zwischen Vietnam Dong (VND) und amerikanischen Dollar (USD) wurde 1992 mit 11.700 VND/USD angegeben ${ }^{849}$.

\footnotetext{
${ }^{842}$ Nach Tong 1997.

${ }^{843}$ Tong 1997.

${ }^{844}$ Ebd.

${ }^{845}$ Ebd.

${ }^{846} \mathrm{Ebd}$

${ }^{847}$ Howie 2011.

${ }^{848}$ Ebd.

${ }^{849}$ DR 0021992.
} 


\begin{tabular}{|l|l|l|l|l|l|l|}
\hline & 1990 & 1991 & 1992 & 1993 & 1994 & 1995 \\
\hline Vietnam & 576 & 1.132 & 1.593 & 1.923 & 2.348 & 3.013 \\
\hline Mekong-Delta & 602 & 1.199 & 1.406 & 1.632 & 2.023 & 2.450 \\
\hline An Giang & 648 & 1.149 & 1.538 & 1.830 & 2.208 & 2.772 \\
\hline
\end{tabular}

Tabelle 7: Bruttoinlandsprodukts in 1.000 VND/Kopf $1990-95^{850}$

Mitarbeiter der FAO 1993 empfahlen der Regierung, das Kreditwesen auszubauen. Das Ministerium für Landwirtschaft und rurale Entwicklung legte ein Kreditprogramm über die AgriBank Viet Nam auf, um Landwirten den Zugang zu Kapital zur Beschaffung der ertragreichen Reissorten und des nötigen Düngers zu ermöglichen ${ }^{851}$.

„For increasing agricultural productivity the use of modern inputs is facilitated by increasing the availability of agricultural credit. Credit is an important instrument in enabling farmers to acquire command over the use of working capital, fixed capital and consumption goods."

Als Zielgruppe machten sie „relatively lower-income farmers those who are close to poverty line ${ }^{\text {c853 }}$ aus. Bis dahin war das Kreditsystem in den Augen der FAO-Mitarbeiter „,not a rural financial markets system "854 und dies wollten sie ändern. Sie zielten auf, die „deposit mobilization, [as] it is important that such financial resources tie into a national financial market ${ }^{855} \mathrm{ab}$. Im Dezember 1994 wurden an 2.664 assoziierte Produktionseinheiten 115,11 Milliarden VND verliehen ${ }^{856}$. Durch die Produktionseinheiten kamen 71.133 Haushalte in den Genuss, staatlich finanziertes Saatgut zu kaufen ${ }^{857}$. Neben der Steigerung der Einkommen brachte die Einführung des modifizierten Saatguts eine Verschiebung des ökonomischen Gleichgewichts in die Region ${ }^{858}$. Traditionell wurde ein Teil der Ernte für den Zweck der nächsten Aussaat zurück gehalten, doch die modifizierten Reissorten eigneten sich dazu nicht. Das Saatgut musste nun eingekauft werden. Von der Regierung wurde der modifizierte Reis mit einem modernen Lebensstandard beworben. Aufgrund der Einführung des Kreditsystems proklamierten die Mitarbeiter der AgriBank Viet Nam die Vorteile des neuen Reis bis in entlegene Gebiete. Die saisonale Überschwemmung wurde zum Hindernis, dem modernen Lebensstil nachzugehen. Im Folgenden werden die politischen Reaktionen auf das Hochwasser analysiert. Dazu werden drei Diskurse betrachtet, die die Reaktionen auf Hochwasser betreffen können.

\footnotetext{
${ }^{850}$ Daten nach Tong 1997.

${ }^{851}$ Artikel: Toan tinh xuong duoc 116.194 ha lua dong xuan, dat $65 \%$ dien tich ke hoach. Zeitung An Giang, 29.12.1994.

${ }^{852}$ AG 46 1993, 63.

${ }^{853}$ Ebd., 62.

${ }^{854}$ Ebd.

${ }^{855}$ Ebd., 63.

${ }^{856}$ Artikel: Toan tinh xuong..., Zeitung An Giang, 29.12.1994.

${ }^{857}$ Ebd.

${ }^{858}$ Vietnam ist in acht administrative Regionen gegliedert.
} 


\subsection{Reaktionen auf das Hochwasser im Mekong-Delta}

Dieser Abschnitt fokussiert die Wende in der Handhabung der Überschwemmungen. Die Auslöser den Wandel werden im Folgenden analysiert, dazu werden drei staatliche Reaktionen auf Hochwasser vorgestellt. Im Jahr 1987 traten Vertreter der vietnamesischen Regierung und Mitarbeiter des United Nations Development Programme (UNDP) in einen Diskurs, durch den neue Institutionen zur Bekämpfung von „Wasserdesastern“ geschaffen wurden. Parallel verlief der Diskurs zur Intensivierung der Landwirtschaft zwischen dem vietnamesischen State Planning Committee und der UN. Zusätzlich verfolgte die Regierung bauliche Maßnahmen gegen die jährlichen Überschwemmungen. Die Ausarbeitung des Mekong-Delta-Master-Plans, in Kooperation mit der Weltbank, und der Erlass der Living with Floods-Richtlinie, beschreiben die infrastrukturellen Maßnahmen des Hochwasserschutzes.

\subsubsection{Desasterdiskurs}

Anders als in Deutschland wurden Hochwasserbekämpfungsmaßnahmen in Vietnam nicht auf lokaler, sondern auf nationaler Ebene koordiniert. Der Desasterdiskurs in Vietnam wurde durch die Verkündung der Internationalen Dekade der Katastrophenvorbeugung des UNDP am 11. Dezember 1987 gefestigt $^{859}$. Die Hochwasserbekämpfung wurde daraufhin in Vietnam politisch institutionalisiert, denn das Nationale Komitee für die Dekade der Katastrophenvorbeugung wurde unter der Leitung des Ministers für Wasserressourcen Nguyen Canh Dinh gegründet ${ }^{860}$. Im Juni 1992 eröffnete der Minister den Internationalen Workshop Flood Mitigation, Emergency Prepardness and Flood Disaster Mitigation der UNDP und der vietnamesischen Regierung, der die Etablierung der neuen Institutionen manifestierte. Während des Workshops vom 22. - 25. Juni in Hanoi wurden vier Schwerpunkte diskutiert. Es sollte eine Strategie entwickelt werden, Hochwasser in den beiden Deltas des Landes zu minimieren, Hochwasser in Küstengebieten und Gebirgen zu minimieren und das Hochwasserkatastrophenmanagement sowie die Notfallvorsorge zu verbessern $^{861}$. In seiner Eröffnungsrede sprach Nguyen Canh Dinh die Hochwasserschäden an, darunter den Verlust hunderter Menschenleben und finanzielle Einbußen in Höhe von Billionen amerikanischer Dollar. Gleichzeitig erinnerte er an anthropogene Einflüsse auf die natürliche Umwelt und sprach von ,man-made changes increasing the severity of the

\footnotetext{
${ }^{859}$ DR 0021992.

${ }^{860} \mathrm{Ebd}$.

${ }^{861}$ Ebd.
} 
calamities ${ }^{\text {‘862 }}$. Jedoch verdeutlichte er auch die Hoffnung, der Fluten zukünftig Herr zu werden und forderte von der internationalen Gemeinschaft: ,accumulated experience, advanced technology, more complete understanding of natural processes, and close

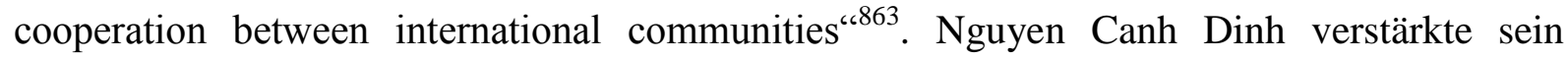
Anliegen, die internationale Gebergemeinschaft solle in Vietnam investieren, noch einmal mit der Betonung:

„[disaster mitigation is] most effective when supported by the interest of the international community $[\ldots]$ this interest and support includes technology transfer for improved disaster mitigation and preparedness. ${ }^{\prime 864}$

Die Gäste des Workshops waren 66 Mitglieder verschiedenster vietnamesischen Ministerien, 52 stellvertretende Vorsitzende der Kommunistischen Partei Vietnam (KPV) mit den jeweils zuständigen Direktoren der Wasserressourcenabteilungen auf Provinzebene sowie 54 internationale Gäste. Darunter waren hauptsächlich die in Hanoi eingesetzten Botschafter, aber auch Mitarbeiter der Asian Development Bank, United Nations Organisationen, World Food Programme, Federation of Red Cross, Save the Children, Mekong Secretariat, und zwei Ingenieure der Delft University of Technology. Die Veranstaltung wurde von der Swedish International Development Authority, der Regierungen des United Kingdom, der Niederlande, Norwegen und Italien mitfinanziert ${ }^{865}$.

Roy Morey, Vorsitzender des UNDP und Lokalkoordinator der Vereinten Nationen in Hanoi, hielt die Eröffnungsrede im Namen der internationalen Organisationen. Morey betonte die hohen Schäden, unter welchen Vietnam jedes Jahr litt, „Vietnam continues to suffer floods and disastrous magnitude which claim human lives and result in untold human suffering and massive economic loss" ${ }^{\$ 66}$. Morey adressierte die Vertreter aller durch Hochwasser bedrohten Provinzen, doch aus dem Mekong-Delta war niemand angereist. Der nächste Sprecher war K. A. Edwards, Chef der Wasser Ressourcenabteilung der Vereinten Nationen, er setzte den Workshop in den Kontext der gerade stattgefundenen Konferenz der Vereinten Nationen über Umwelt und Entwicklung in Rio de Janeiro vom 03. - 14. Juni 1992 und spannte den Bogen von „ensure sustainable development ${ }^{4867}$ bis $\mathrm{zu}$ „Vietnam has placed emphasis on improving the system of river [...] dykes during the next five year plan $(1991-1996)^{\text {، } 868}$. Morey

\footnotetext{
${ }^{862}$ DR 002 1992, 2.

${ }^{863} \mathrm{Ebd}$.

${ }^{864} \mathrm{Ebd}$

${ }^{865} \mathrm{Ebd}$

${ }^{866}$ Ebd., 3.

${ }^{867}$ DR 002 1992, 5.

${ }^{868}$ Ebd., 6.
} 
präsentierte eine vorläufige Kosten-Nutzen-Analyse von Hochwassermaßnahmen, ,which is convincing enough even without taking into account the unquantifiable social benefits accruing from such programmes“ ${ }^{\text {(869 }}$. Die Analyse bestand in einem Überblick der Auswirkungen von verschiedenen Ereignissen in verschiedenen Regionen des Landes. Die potentiellen Schäden wurden aufgrund von Interviews mit Anwohnern und der lokalen Industrie erhoben und mit statistischen Daten abgeglichen. Ingenieure des Departments für Deich Management, Flut Kontrolle und Sturm Bereitschaft wurden in die Studie einbezogen und schätzten die Wahrscheinlichkeiten für Deichbrüche in verschiedenen Szenarien ab. „When undertaking risk analyses of projects for the World Bank, one should develop appropriate probability distributions with the project appraiser ${ }^{6870}$, begründete Dr. Terry Dr. Lustig in seiner Präsentation. Der Nutzen der Maßnahmen wurde aufgrund der vermiedenen Schäden durch die Ingenieure der Behörde geschätzt. Lustig stellte verschiedenen Wassergefahren und deren Bekämpfung vor, sowie die Priorität aus lokalem Wissen zu schöpfen. Lustig verdeutlichte, dass eine KNA keine finalen und akkuraten ökonomischen Evaluationen liefert: „It is sufficient if one is able to make judgements whether certain strategies appear feasible, and which of them appear to have the highest priority."

Als Ergebnis des Workshops ist die Veröffentlichung des Hochwasserrahmenprogramms „Strategy and Action Plan for Mitigating Water Disasters in Viet Nam“ von 1994 zu nennen. Der Strategieplan fixierte den Diskurs, der im Jahr 1992 begonnen hatte ${ }^{872}$. Der Aktionsplan legte 18 Maßnahmen in den drei Bereichen „Frühwarnsysteme“, „Bereitschaft und Linderung“" sowie „Soforthilfe“ vor ${ }^{873}$. Der erste Bereich umfasste vier Bauprojekte und ein Training zur Steigerung des Gefahrenbewusstseins der breiten Öffentlichkeit. Der Punkt der „Bereitschaft und Linderung“ wurde mit Priorität behandelt, denn er enthielt 11 der 18 Maßnahmen, worunter das nachhaltige Management der Deiche an Küsten und Flüssen, sowie die Kontrolle von Flusssystemen fielen. Der Bereich der „Soforthilfe“ beinhaltete zwei Maßnahmen, die Instandsetzung von Schutzvorrichtungen, welche während des Hochwassers zu brechen drohten, sowie den Institutionsaufbau für die Soforthilfe. Von den insgesamt 18 Projekten wurden 15 von vietnamesischen Provinzen vorgeschlagen, wovon wiederum 12 Stück aus baulichen Teilprojekten im Bereich der „Bereitschaft und Linderungen“ bestanden ${ }^{874}$. Ein Deichprojekt sollte in der Provinz An Giang entstehen, da die Menschen

\footnotetext{
${ }^{869}$ Ebd., 4.

${ }^{870}$ Ebd., 57.

${ }^{871}$ Ebd.

${ }^{872}$ DR 0041994.

${ }^{873}$ Ebd., 98.

${ }^{874}$ Ebd.,102.
} 
stets saisonale Augustdeiche aufbauen ${ }^{875}$. Feste Deiche und Schleusen sollten die Reparatur der bereits existierenden Erddeiche erübrigen ${ }^{876}$. Die Höhe der Deiche richtete sich nach den empfohlenen Standards aus dem Mekong-Delta-Master-Plan von $1993^{877}$. Die Größe der Maßnahme wurde mit 500.000 USD veranschlagt, was nur $1.62 \%$ des für bauliche Maßnahmen vorgesehenen Budgets der Komponente „Sustainable Management of River Dykes“ entsprach $^{878}$. Abb. 22 zeigt die Karte der nördlichen Region An Phu in An Giang aus dem Projektkatalog von 1994. Hinter dem Deich werden seit seinem Bau Reisflächen und Fruchtbäume kultiviert. Das Projekt in An Phu besaß Pilotcharakter, da es der erste Deich war, der am Mekong in Vietnam gebaut wurde.

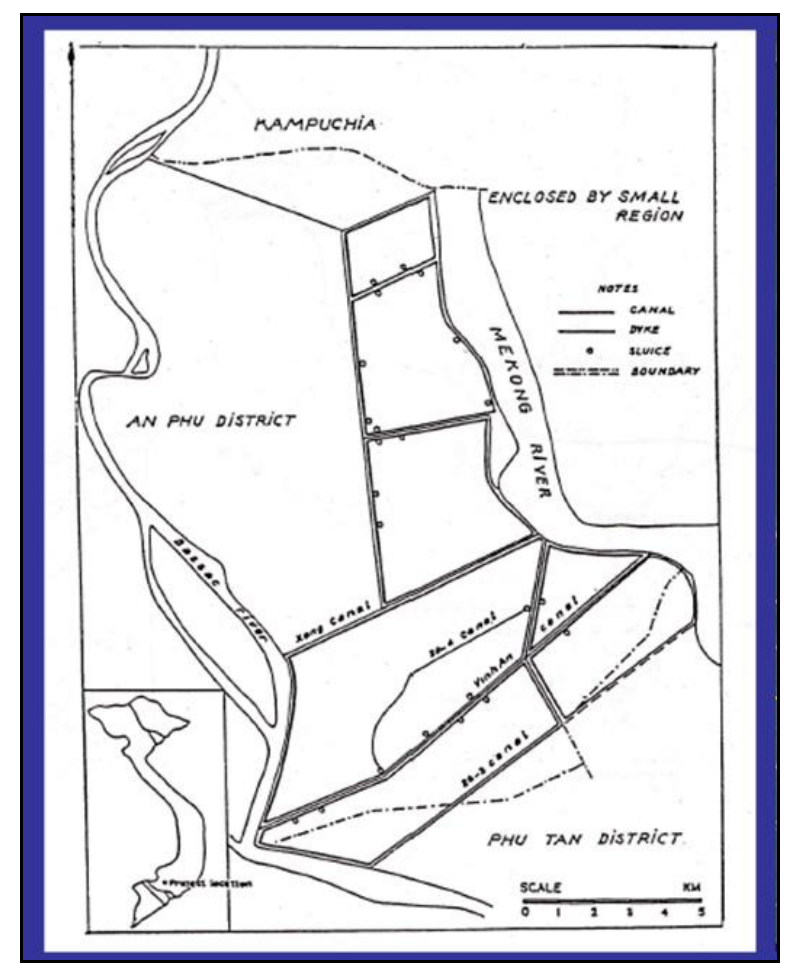

Abb. 22: Deichprojekt im Tan Chau -Distrikt 1994. ${ }^{879}$

Die zweite bauliche Komponente „Sustainable Management of the Sea and Estuary Dykes“ war mit 21 Mio. USD angelegt, wovon 4,75 \% im Mekong-Delta investiert werden sollten. In der östlich gelegenen Provinz Ben Tre sollte ein $11 \mathrm{~km}$ langer Deich entlang der Meeresküste gebaut werden, um das Eindringen von Salzwasser in die landwirtschaftlich genutzten Flächen zu vermeiden. In der Nachbarprovinz sollte eine Verkleidung gegen fortschreitende Erosionsprobleme an einem Mekong-Arm für 350.000 USD angebracht werden, was $28 \%$

\footnotetext{
${ }^{875}$ Ebd., 131.

${ }^{876} \mathrm{Ebd}$

${ }^{877}$ Ebd.

${ }^{878} \mathrm{Ebd}$

${ }^{879}$ DR 0041994.
} 
der dritten baulichen Komponente „Sustainable Control of the Rives“ entsprach. Die baulichen Komponenten vier und fünf (Emergency Communication System und Watershed Management and Deforestation) sahen keine Investitionen im Mekong-Delta vor.

In dem Aktionsplan wurden zwei weitere Ziele erhoben: Erstens wurden die politischen Autoritäten adressiert, der vorliegenden Wasser-Desaster-Bekämpfungs-Strategie in Zukunft mehr Gewicht zu verleihen und in ein nationales Programm zu verwandeln, das stärkere politische Priorität besaß. Zweitens nutzen die UN-Mitglieder den Plan, um an das bereits bestehende Department für Deich Management, Flut Kontrolle und Sturm Bereitschaft zu appellieren, sich für die Koordination der Projekte des Aktionsplans zu verpflichten ${ }^{880}$. Neben kommunikativen Aufgaben wurde das DDMFC mit der Kontrolle über die Finanzen internationaler Projekte, sowie mit der Verwaltung und dem Wissensmanagement der vietnamesischen Daten bevollmächtigt. Zur Unterstützung dieser Aufgaben sollte das DDMFC mit einer Water-Disaster-Database und einem Water-Disaster Geographic Information System (GIS) ausgestattet werden.

Im April 1995 folgte die erste Aktualisierung des Aktionsplans. Dabei einigten sich die Akteure auf eine stärkere Betonung der nicht-technischen Komponenten. Die Kooperationspartner bewerteten die nicht-physikalischen Aufgaben „more cost-effective“ ${ }^{\text {“881. }}$. Die Annahme „,communal preparedness is essential for sustainable disaster mitigation“ ${ }^{\text {“882, }}$ veränderte die Gewichtung der Aufgabenbereiche des Aktionsplans. Der ganze Aktionsplan wurde dezentralisiert, indem die Regionen durch stärkere Partizipation in die Organisationsabläufe eingebunden wurden ${ }^{883}$. Die 1992 abgehaltene Konferenz in Rio de Janeiro prägte nun noch stärker den Desasterdiskurs in Vietnam. So wurde die Versalzung durch Meerwasser mit einem zukünftigen Anstieg des Meeresspiegels in Verbindung gesetzt und fortan „Sea-water Intrusion and Greenhouse Effect“ genannt. In der Institutionslandschaft Vietnams ergab sich eine erste Entwicklung. Die Disaster Management Unit stand kurz vor der Gründung ${ }^{884}$. Sie sollte drei Funktionen besitzen: Erstens sollten alle Informationen zu bereits existierenden Mechanismen in der Soforthilfe gesammelt werden und eine Datenbank, mit den Daten vergangener Wasser-Desaster sowie der getroffenen administrativen Reaktionen, eingerichtet werden. Als zweites Ziel galt die Ausbildung von Fachkräften in den politischen Ebenen, dazu sollte die Disaster Management Unit ein Trainingsprogramm einrichten. Als dritter Schwerpunkt galten die Öffentlichkeitsarbeit und die Transparenz der

\footnotetext{
${ }^{880}$ DR 004 1994, 99.

${ }^{881}$ DR 040 1995, 1.

${ }^{882}$ Ebd. 2.

${ }^{883} \mathrm{Ebd}$

${ }^{884} \mathrm{Ebd}$
} 
Vorgänge, denn für interessierte Einzelpersonen wie auch für Organisationen sollte die Disaster Management Unit ein öffentliches Informationscenter darstellen. Die vietnamesische Regierung sprach dem Strategieplan noch immer nicht die Priorität zu, die sich die UNMitarbeiter wünschten, denn noch im April 1995 sollte der Plan genutzt werden, „to alert the Government to the importance of water-disaster mitigation” ${ }^{\text {} 885}$. Dazu gehörte ebenfalls die beiden Institutionen, welche im Rahmen der Desasterdekade der UN gegründet wurden, Zentralkomitee für Flut und Sturm Kontrolle (CCFSC) und das Viet Nam National Committee for the International Decade for Natural Disaster Reduction mit dem Mandat auszustatten, mit allen Ministerien zusammen zu arbeiten ${ }^{886}$. Das Department für Deich Management, Flut Kontrolle und Sturm Bereitschaft, stellte aufgrund früherer Operationen im Auftrag des Ministeriums für Landwirtschaft und Rurale Entwicklung bereits eine anerkannte Expertise dar $^{887}$. Die Kapazitäten der vietnamesischen Regierung sollten im Hinblick auf eine ökonomische Bewertung der Projekte gestärkt werden, denn bis dato wurden soziale und umweltbezogene Effekte nicht in die Kosten-Nutzen-Abwägung der Projekte aufgenommen $^{888}$.

Am 28. November 1994 wurde das „Agreement on Cooperation for the Sustainable Development of the Mekong River Basin“ zwischen dem Königreich Kambodscha, der Demokratischen Volksrepublik Laos, der Sozialistischen Republik Vietnam und dem Königreich Thailand unterzeichnet. Das Abkommen basierte auf der Anerkennung der Landesgrenzen, der gleichberechtigten Souveränität und der Beachtung des Umweltschutzes bei der Nutzung natürlicher Ressourcen ${ }^{889}$. Die Staaten erhielten im Gegenzug freie Navigation auf dem Mekongfluss, um die wirtschaftliche Entwicklung der Region zu beschleunigen $^{890}$. Die Unterzeichnung des Abkommens werteten die UNDP-Mitarbeiter als einen förderlichen Schritt zu einer nachhaltigen Entwicklung im Bereich des Desaster Managements ${ }^{891}$. Die UNDP-Mitglieder sahen das Abkommen als Basis an, die zukünftige Hochwasserbekämpfung im gesamten Einzugsgebiet des Mekongflusses voranzutreiben ${ }^{892}$. „Following the record floods of 1994, the DDMFCSP [the already existing department] is to prepare a floodplain management plan for the Vietnamese portion of the Mekong River. This plan will be developed in conjunction with the Mekong River Commission [... $]^{\natural 893}$.

\footnotetext{
${ }^{885}$ DR 040 1995, 2.

${ }^{886}$ DR 0401995.

${ }^{887}$ Ebd.

${ }^{888}$ Ebd.; DR 0041994.

${ }^{889}$ Ebd.

${ }^{890}$ Ebd.

${ }^{891}$ Ebd.

${ }^{892}$ Ebd

${ }^{893}$ DR 040 1995, 8.
} 
Im Februar 1997 versuchte das UNDP auf die Entwicklungen im Desasterdiskurs in Vietnam ein weiteres Mal Einfluss $\mathrm{zu}$ nehmen. Mit einer Machbarkeitsstudie begannen die Verhandlungen zur Gründung eines Desaster Rehabilitationsfonds. Dieser sollte ausschließlich für den Wiederaufbau von Infrastruktur aufgewendet werden, wie beispielsweise Deichbau, dem Bau von Bewässerungs- und Kanalsystemen, Straßen, Brücken, Schulen und Krankenhäusern ${ }^{894}$. Beispiele der UNDP verdeutlichten die geringen finanziellen Ressourcen, die der vietnamesischen Regierung für Wideraufbauprojekte zur Verfügung standen und untermauerten die Wichtigkeit des Desaster Rehabilitationsfonds:

\begin{abstract}
„In Dong Thap Province [north of An Giang Province], two stretches of provincial and district roads, 20 and 15 kilometres, remained unrepaired and unpassable three month after the Mekong flood waters had retreated. 110 kilometres of embankments protecting agricultural land had also not been repaired because there were no funds. The limited capacity of the Central Government for disaster response was demonstrated in 1996, when only \$23.6 million was allocated by the Central Government in response to provincial government requests for all form of disaster relief, against a total estimate of property damage and agricultural losses of $\$ 657$ million. Funds for relief aid were allocated from the central budget reserve [which is determined at $5 \%$ of the total budget], while the funds for infrastructure rehabilitation were extracted from the 1996 ministerial/sectoral budgets." 895
\end{abstract}

Um den Desaster Rehabilitationsfonds zu gründen, wurde von den UNDP-Mitarbeitern vorgeschlagen, dass die Gruppe der Geberorganisationen ein „small percentage of their development assistance" ${ }^{, 896}$ zurück halten und damit den vorgeschlagenen Fonds direkt speisen. Dieser würde sodann von dem Zentralkomitee für Flut und Sturm Kontrolle und dem Department für Deich Management, Flut Kontrolle und Sturm Bereitschaft selbständig verwaltet und in Form von Darlehen an die Provinzen für spezifische Projekte ausgeben ${ }^{897}$. Das Volkskomitee auf der Provinzebene erhält somit eine Schlüsselrolle, denn es übernimmt die Aufgaben der ersten Angebotsprüfung- und Bewertung sowie der Priorisierung der Projektvorschläge der Distrikte und Kommunen ${ }^{898}$. Dabei soll die Definition von Rehabilitation nach Meinung der UNDP-Mitarbeiter flexibel bleiben.

,[...] to allow for the construction of infrastructure at new sites, where disaster victims have been resettled permanently away from devastated areas with a view to mitigating the effects of future disasters. E. g.: Dong Thap province to relocate on a voluntary basis families, who currently live along flood prone canals, to nearby concentrated settlements on newly raised ground. Schools, clinics, and roads will need to be built for these resettled communities. One

\footnotetext{
${ }^{894}$ DR 0411997.

${ }^{895}$ Ebd., 5.

${ }^{896}$ Ebd., 16.

${ }^{897}$ Ebd.

${ }^{898}$ Ebd.
} 
such resettlement site is being implemented by the Giong Gang State Farm in Dong Thap and already 200 families have volunteered to move there." 899

Die Meinungen der bilateralen Geber zu dem Vorschlag gingen auseinander. Zwei der 17 Mitglieder sprachen sich gegen die Einführung des Fonds aus, da sie äußerten, dass die vietnamesische Regierung einen solchen Fonds selbstständig einrichten kann, wenn sie das für richtig hält ${ }^{900}$. Dabei sprach sich ein Berater gegen die strukturelle Art aus, dem Hochwasser zu begegnen, denn er betonte, „donor support could be more effectively be given to disaster preventative and mitigation measures ${ }^{\text {“901 }}$. Drei weitere Sprecher bewerteten das Instrument des Fonds negativ, sprachen sich aber für die Umsetzung von Infrastrukturprojekten aus, wenn diese ,through NGOs and/or their bilateral project aid [...] or in a project to project approach through the bilateral aid programme ${ }^{\text {*902 }}$ finanziert werden. Die vietnamesischen Regierungsmitglieder äußerten ebenfalls unterschiedliche Meinungen. Der Landwirtschaftsminister Nguyen Cong Tan begrüßte am 13. Januar 1997 den Vorschlag einen Infrastrukturfonds einzurichten. Er befürwortete dessen Finanzierung aus Geldern der Entwicklungszusammenarbeit sowie die Übernahme der Koordination durch das Department für Deich Management, Flut Kontrolle und Sturm Bereitschaft ${ }^{903}$. Der Stellvertretende Finanzminister und zugleich derzeitiger Stellvertreter des Zentralkomitees für Flut und Sturm Kontrolle Tao Huu Phung wies am 24. Januar 1997 darauf hin, dass die Mittel der Entwicklungsorganisationen laut Budgetgesetz Teil des staatlichen Budgets sind und somit die Regularien und die Kontrolle des Staats über dem Fonds liegen ${ }^{904}$. Tao Huu Phung bejahte die Koordination durch das Department für Deich Management, Flut Kontrolle und Sturm Bereitschaft und schlug vor, den Ausschuss des Zentralkomitees für Flut und Sturm Kontrolle in der Aufgabe des Fondsmanagers einzusetzen ${ }^{905}$. Er riet dazu, den Fonds von der unabhängigen VACO Joint Venture Accounting and Auditing Company auditieren zu lassen $^{906}$. Für das Ministry of Planning and Investment sprachen am 10. Januar 1997 der stellvertretende Direktor des Department of External Economic Relations Nguyen Thu Phuong, sowie am 29. Januar desselben Jahres der Direktor des Departments Duong Duc Ung $^{907}$, vor. Bezüglich der Finanzierung sowie der Koordination des Fonds forderten sie, die Finanzierung soll nicht auf bilaterale Geber begrenzt bleiben, sondern ebenfalls humanitäre,

\footnotetext{
${ }^{899}$ DR 041 1997, 8.

${ }^{900}$ Aus der Quelle DR 041 ist die genaue Identifikation der Akteure nicht möglich.

${ }^{901}$ Ebd.

${ }^{902}$ Ebd.

${ }^{903}$ Ebd.

${ }^{904} \mathrm{Ebd}$

${ }^{905}$ Ebd.

${ }^{906}$ Ebd.

${ }^{907}$ Ebd.
} 
multilaterale NGOs und den privaten Sektor einbeziehen ${ }^{908}$. Die bilateralen Geber sollen nicht nur Prozente ihres Investitionsvolumens in den Fonds einbezahlen, sondern ihr Volumen insgesamt vergrößern ${ }^{909}$. Sie forderten außerdem, dem Fonds sollen keine öffentlichen Ziele auferlegt werden, er solle aber unter der Kontrolle der vietnamesischen Regierung stehen, damit das Geld direkt an die Provinzen ausgegeben werden kann ${ }^{910}$. Zusätzlich beanspruchten sie, „UNDP sponsored monthly donor meetings““911, um die Ausrichtung durch Beratung zu optimieren. Die Standpunkte der Akteure werden im nächsten Abschnitt unter Berücksichtigung der Rationalitäten interpretiert.

\subsubsection{Interpretation des Desasterdiskurses}

Aus der vorangegangenen Beschreibung ergehen folgende Erkenntnisse. Der Desasterdiskurs in Vietnam wurde auf politischer Ebene durch den Einfluss der Vereinten Nationen 1987/1988 institutionalisiert, obwohl es bereits zuständige Abteilungen für den Deichbau $\mathrm{gab}^{912}$. Zu Beginn der Verhandlungen über den Wasser-Desaster-Strategie- und Aktionsplan 1992 standen die Schäden im Vordergrund der Konferenz. Die Repräsentanten der vietnamesischen Regierung sowie der UNDP konzentrierten sich darauf, die Schäden zu betonen, die die vietnamesische Staatskasse aber auch die Bevölkerung beeinträchtigten. Den UNDP-Mitarbeitern gelang es, eine Kosten-Nutzen-Analyse vorzustellen, die allen Anwesenden verdeutlichen sollte, dass sich Maßnahmen gegen die natürlichen WasserEreignisse als effektiv erweisen. Die Präsentation durch Dr. Lustig von der UN untermauerte die Argumentation von Nguyen Chan Dinh, Roy Morey und K. A. Edwards, denn sie stellte die Schäden in den Vordergrund der Ausführung, während die Kosten für die Maßnahmen nicht betrachtet wurden. Die verhinderten Schäden wurden nicht berechnet, denn ein Mangel an Daten ließ nur die Bewertung durch die Ingenieure der vietnamesischen Behörde zu. Die aufgrund von Umfragen und Statistiken ermittelten Schäden wurden von den Mitarbeitern des Department für Deich Management, Flut Kontrolle und Sturm Bereitschaft in der Regel als zu niedrig bewertet, somit vergrößerten sie den potentiellen Nutzen der Maßnahmen ${ }^{913}$. Die Argumentation mit ökonomischen Kriterien fügte sich in den Rahmen der Gespräche zwischen den an der wirtschaftlichen Entwicklung interessierten vietnamesischen Verantwortungsträgern und den Organisationen, deren Kerngeschäft die wirtschaftliche Entwicklung darstellt. In Vietnam hatte man offensichtlich im ganzen Land mit der

\footnotetext{
${ }^{908}$ DR 0411997.

${ }^{909}$ Ebd.

${ }^{910}$ Ebd.

${ }^{911}$ Ebd., 21.

${ }^{912}$ DR 0021992.

${ }^{913}$ DR 0411997.
} 
unwägbaren Natur zu kämpfen, denn es waren von überall her Vertreter der einzelnen Provinzen nach Hanoi gereist. Die Teilnahme von 66 offiziellen Regierungsmitgliedern sowie 52 Provinzpolitikern zeigte bereits 1992 das Interesse aller politischen Ebenen in Vietnam, die mit dem Strategie- und Aktionsplan verbundenen Entwicklungsgelder in ihrer Region zu investieren. Jeder Teilnehmer war bestrebt, Mittel für seine Projekte zu akquirieren. Aus dem Mekong-Delta war jedoch niemand gekommen, um an dem Desasterdiskurs teilzuhaben. Dies weist darauf hin, dass die Menschen im Mekong-Delta das Hochwasser nicht als Wasserdesaster wahrnahmen.

Aus dem 1994 ausgearbeiteten Strategieplan geht hervor, dass die Provinzen die Möglichkeit zur Partizipation genossen. 15 von insgesamt 18 Maßnahmen wurden auf Vorschlag der Provinzen in den Strategieplan aufgenommen. Der Aktionsplan sollte ein Investitionsvolumen von 83,7 Mio. USD durch die internationalen Geberorganisationen erhalten, davon entfielen jedoch gerade einmal 2,21\% auf die Region im Mekong-Delta. Von den drei im Delta vorgesehenen Projekten wurde nur eines im Kontext zum jährlich auftretenden Hochwasser des Mekongflusses entworfen. Augenscheinlich spielte das Hochwasser in dem südlichen Delta in dem Aktionsplan nur eine geringe Rolle. In Relation zu anderen wasserbasierten Naturgefahren wurde das saisonale Hochwasser nicht als Unglück, sondern als jahreszeitlich bedingte Erscheinung wahrgenommen. Aufgrund der Natürlichkeit des Hochwassers nahmen die Entscheidungsträger die Maßnahmen an anderen Orten als effizienter wahr. Mit der Veröffentlichung des Strategieplans erinnerten die UNDP-Mitarbeiter noch einmal an die Priorität, den neu geschaffenen Institutionen auch politisch Kompetenz zu verleihen, und sie mit Handlungsfähigkeit auszustatten. Das nationale Komitee für Katastrophenvorbeugung wurde bereits nach der Veröffentlichung der UN-Resolution 1987 gegründet, konnte 1994 jedoch immer noch nicht frei mit Ministerien verhandeln. Das weist darauf hin, dass die vietnamesische Regierung nicht wünschte, dass der Desasterdiskurs auf institutioneller Ebene ausgetragen wird, bzw. dass politische Meinungen von außerhalb Vietnams in den bereits vorhandenen vietnamesischen Desasterdiskurs Einfluss nehmen. Deswegen drängten die Mitarbeiter des UNDP 1994 zu mehr Eigenständigkeit der neu geschaffenen Institutionen. Dem Department für Deich Management, Flut Kontrolle und Sturm Bereitschaft wurde die Kontrolle über die internationalen Budgets zugesprochen und die Datenverwaltung übergeben. Die Rolle des Deichdepartments gewann an politischem Gewicht und positionierte die Gruppe der Verantwortlichen 1994 als Kopf des Desasterdiskurses. Mit dieser Verteilung der beiden Arbeitsbereiche wurde eine traditionelle Institution der vietnamesischen Behörden im Gegensatz zu den neu geschaffenen Gremien gestärkt. Nur wenige Monate später justierte 
das UNDP noch einmal die Ausrichtung der Projekte und versuchte den Fokus auf nichtbauliche Maßnahmen zu lenken. Dabei blieb die Organisation ihrer bereits 1992 gefolgten Argumentation treu und versuchte die Vorteilhaftigkeit von nicht-baulichen Maßnahmen anhand der höheren Effizienz gegenüber baulichen Schutzprojekten zu verdeutlichen. Des Weiteren beeinflusste der globale Diskurs über die Folgen des Klimawandels die Fortschreibung des Strategieplans, als beispielsweise der Anstieg des Meeresspiegels in die Projekte aufgenommen wurde. Behördliche Unklarheiten konnten 1995 noch nicht ausgemerzt werden, das Zentralkomitee für Flut und Sturm Kontrolle, sowie das Viet Nam National Committee for the International Decade for Natural Disaster Reduction arbeiteten noch immer ohne Mandat, während das Department für Deich Management, Flut Kontrolle und Sturm Bereitschaft, eine traditionelle Institution darstellte. Mit dem Ziel, die technischen Argumente der Deichbauexperten zu entkräften, wurde ihnen ein Trainingskurs angeboten. Die Ingenieure dieser Behörde sollten lernen, soziale und umweltbezogene Nutzen als auch Kosten in die Effizienzanalysen einzubeziehen. Der Vorschlag, nicht-bauliche Maßnahmen gegenüber baulichen Maßnahmen zu bevorzugen, muss für die Ingenieure der als erfolgreich geltenden und einflussreichen Behörde DDMFCSP absurd geklungen haben. Die Ingenieure sollten für intangible Kosten und Nutzen sensibilisiert werden, damit sie diese künftig offener betrachteten und zumindest deren Existenz eingestehen würden, obzwar sie nicht unmittelbar monetär zu beziffern sind.

Die Fokussierung auf nicht-bauliche Maßnahmen wurde kritisiert. Bereits im Februar 1997 versuchten die Mitarbeiter des UNDP, Graham Alliband und Do Hong Phan, eine neue Diskussion über die Verteilung der Prioritäten zu eröffnen und schlugen die Einführung eines Infrastrukturfonds vor. Das UNDP war bereits seit Beginn des Desasterdiskurses als eindeutiger Fürsprecher von baulichen Projekten auszumachen. Schon in dem ersten Aktionsplan war der Anteil der baulichen Maßnahmen am Gesamtplan übermächtig. Die Verschiebung des Fokus auf nicht-bauliche Aktivitäten wirkt aus der Retrospektive wie eine Strategie, um den bereits etablierten vietnamesischen Behörden größeres Gewicht zukommen zu lassen und deren Position zu stärken. Alliband und Phan zielten darauf ab, den Anteil der Bauprojekte aus den bereits abgestimmten 18 Arbeitspaketen des 1994 verabschiedeten Strategy and Action Plan for Mitigating Water Disasters zu erhöhen. Das Duo arrangierte dazu zwischen dem 08. Januar 1997 und 01. Februar 199716 Treffen mit Entscheidungsträgern des Landwirtschaft-, Finanz- und Planungs- und Investitionsministeriums, sowie mit Verantwortlichen aus den Botschaften von Australien, Norwegen, Großbritannien, Italien, Dänemark, Finnland, Frankreich, der Europäischen 
Union, Schweiz, Kanada, Schweden, Niederlande, Belgien, Japan, Deutschland, Korea und den USA. Die hohe Aktivität der beiden UNDP-Mitarbeiter verdeutlicht das Bestreben, die Investitionsanteile zwischen den beiden Polen der Infrastruktur- und der Präventionsprojekte neu zu verteilen. Um eine Neugewichtung zu erreichen, brauchten sie eine Lobby unter den Mitwirkenden des Strategy and Action Plan for Mitigating Water Disasters. Alliband und Phan trafen sich mit den einzelnen Beteiligten persönlich zum Gespräch, um einen Kreis von Fürsprechern aufzubauen. Vier der 17 ausländischen Berater hegten Zweifel an der Qualität des „Rehabilitationsfonds Wasser Desaster“, ihre Bedenken galten jedoch unterschiedlichen Aspekten. Von den 17 Beteiligten argumentierte nur ein Sprecher dafür, die bereits 1995 abgestimmte Priorität der Präventionsprojekte aufrecht zu erhalten. Er erinnerte noch einmal daran, dass die Investition in präventive Projekte als effizienter eingeschätzt wurde, als die Investition in den Aufbau von Infrastruktur. Diesem Sprecher schlossen sich keine weiteren Akteure an. Er blieb alleine mit seiner Ansicht, Wirtschaftlichkeitskriterien für die Auswahl der Projekte anzulegen. Die anderen Mitglieder der Gebergemeinschaft bezogen in ihre Kosten-Nutzen-Abwägungen das Interesse an einer dauerhaften Zusammenarbeit mit der vietnamesischen Regierung, aufgrund von zukünftigen Kooperationspotentialen, ein. Drei andere Sprecher diskutierten, welche Akteure die Kontrolle über den Infrastrukturfonds haben sollten. Zwei Meinungsführer favorisierten die vermehrten Aktivitäten im Bausektor und plädierten dafür, die Investitionsmittel an den Bau von Infrastruktur zu knüpfen. In ihren Augen sollte die Gebergemeinschaft die Kontrolle über die Verwendung der Gelder behalten, damit sie bei der Selektion der spezifischen Projekte Einfluss ausüben kann. Diese Akteure waren sich über den gesteigerten Einfluss auf noch verschlossene Ressourcen bewusst, den die Geber durch die Einführung eines Infrastrukturfonds in Vietnam ausüben könnten. Besonders die Kommentierung der UNDP-Mitglieder des 1994 neu getroffenen Mekongabkommens weist darauf hin, dass die Akteure das gesamte Flusseinzugsgebiet als potentiellen Handlungsraum zur Umsetzung von Hochwasserschutzprojekten wahrnahmen. Außerdem erschien es ihnen vorteilhaft, dass kein zusätzlicher finanzieller Einsatz durch die Gebergemeinschaft geleistet werden musste, da der Fonds durch Anteile des ohnehin auf der Basis des Aktionsplans Wasserdesaster investierten Kapitals gespeist würde. Ein anderer Sprecher zog es vor, die Kontrolle des Budgets der vietnamesischen Regierung zu übergeben, damit diese eigene Präferenzen für den Einsatz der Gelder zwischen Bau- oder Präventionsprojekten geltend machen könnte. Damit untergrub er die Wirkung des vorgeschlagenen Infrastrukturfonds. 
Die befragten Verantwortlichen des Ministeriums für Landwirtschaft und Rurale Entwicklung, des Finanzministeriums und des Ministeriums für Planung und Investment waren sich einig, dass die Kontrolle des Fonds der vietnamesischen Regierung unterliegen soll. Auffällig wurde die Befürwortung von Infrastrukturmaßnahmen durch den Landwirtschaftsminister Nguyen Cong Tan am 13. Januar 1997, da er seine Abteilung zur Steuerung des Fonds vorschlug. Das Landwirtschaftsministerium übte vermehrt Druck auf die Landwirte im Mekong-Delta aus, die durch neue Hochwasserdeiche geschützten Äcker drei Mal im Jahr zu bewirtschaften ${ }^{914}$. Die Steigerung der Ernte beeinflusste die vietnamesische Volkswirtschaft vor allem aufgrund von Exporten durch das Einbringen von Devisen. Aus der Sicht der Verantwortlichen im Landwirtschaftsministerium war der Infrastrukturfonds zu begrüßen, besonders, da der Fonds künftig durch die Mitglieder des Ministeriums gesteuert werden könnte. Aber auch die anderen Ministerien unternahmen den Versuch, möglichst große Vorteile für ihr Ministerium herauszuschlagen. Finanzminister Tao Huu Phung versuchte seine Vorreiterstellung am 24. Januar 1997 auszubauen, indem er vorschlug, seine Abteilung soll den Fonds betreuen. Er unternahm den Versuch, die Kontrolle parteiisch zu arrangieren und den Mitgliedern des CCFSC und sich selbst Autorität zu verschaffen, als er andiente, die Betreuung des Fonds durch eine ihm persönlich bekannte Firma auditieren zu lassen. Er hob die Machtdebatte damit auf eine neue Ebene. Die Sprecher des Ministeriums für Planung und Investment, Nguyen Thu Phuong und Duong Duc Ung, verstanden es als selbstverständlich, dass die Regierung Vietnams die Kontrolle über den Fund for Disaster Rehabilitation übernehmen wird. Sie versuchten, so viele Investitionsmittel wie möglich in das Land zu holen, denn sie sahen den Nutzen für Vietnam parallel mit der Größe der ausländischen Investitionen steigen. Ihre negativen Äußerungen über Kontrollen der Effizienz der Arbeitsweise des Fonds geben Hinweise, dass die beiden vietnamesischen Politiker nicht daran interessiert waren, ob das Geld für infrastrukturelle oder präventive Maßnahmen eingesetzt wird. An den Äußerungen wird erkennbar, dass 1997 immer noch alle Ministerien der vietnamesischen Regierung unter einem sehr begrenzten Zugang zu Ressourcen litten. Die Ministerien erreichten nur einen geringen Aufgabenerfüllungsgrad, denn $\mathrm{zu}$ wenig Kapital schien vorhanden $\mathrm{zu} \operatorname{sein}^{915}$. Beispielsweise beliefen sich die Schäden im Jahr 1996, ausgelöst durch Naturgefahren, auf 657 Mio. USD ${ }^{916}$. Jedoch setzte die Regierung den Verlusten nur 9,1 Mio. USD zur Nothilfe und weitere 14,4 Mio. USD für die Wiederherstellung der Infrastruktur entgegen ${ }^{917}$. Dies sieht zunächst unverhältnismäßig aus, jedoch entsprachen die Hilfen bereits fast $13 \%$ des

\footnotetext{
${ }^{914}$ Howie 2006.

${ }^{915}$ AG 46 1993, 45.

${ }^{916}$ DR 0411997.

${ }^{917}$ DR 0411997.
} 
nationalen Haushalts, welcher sich 1996 auf 182 Mio. USD belief ${ }^{918}$. Bei der Verteilung der Gelder der Entwicklungszusammenarbeit standen die Ministerien folglich in Konkurrenz zueinander. Alle Verantwortlichen versuchten, für ihr jeweiliges Ressort den größten Anteil einzuwerben und somit die anderen leer ausgehen zu lassen. Der Zugang zu zusätzlichen Mitteln könnte es den Ministerien erleichtern, ihre Pläne umzusetzen, so die Hoffnung der Verantwortlichen. Die Auseinandersetzungen verdeutlichten den Verlauf des Desasterdiskurses, sowie die finanziell eingeschränkte Situation der zentralen Politikebene in Vietnam zwischen 1992 und 1997. Bevor die Dekade 1990 - 2000 von der UNDP zur International Decade of Disaster Reduction ausgerufen wurde, fehlten in Vietnam institutionalisierte Katastrophenschutzmaßnamen zumindest für den Bereich der wasserbasierten Gefahren, die trotz dieser neuen Unterstützung kaum im südlichen Delta eingesetzt wurden. Daraus lässt sich ableiten, dass die Überschwemmungen auch weiterhin nicht als Katastrophe wahrgenommen wurden. Denn auch durch die Einführung des internationalen Desasterdiskurses erhielten sie keinen Katastrophencharakter.

Zum zentralen Thema des Aushandlungsprozesses wurde die Frage, welche Akteure den Desasterdiskurs steuern sollten. Aus der Betrachtung der Debatte erschließt sich der Konflikt zwischen dem nationalen Anliegen, Mittel zu akquirieren und dem internationalen Interesse wirtschaftlichen Einfluss in entlegenen Regionen auszuüben. Die stärkere Gewichtung von Infrastrukturprojekten gegenüber Präventionsprojekten bedeutet, den Zugriff auf Menschen und Ressourcen auszuweiten. Das konkrete Ziel, aus den Überschwemmungsgebieten landwirtschaftliche nutzbare Flächen zu gewinnen, stand für die Mitglieder der Planungskommission nicht im Vordergrund. Ob die Intensivierung der Landwirtschaft der Auslöser für den verstärkten Hochwasserschutz darstellte, wird im folgenden Abschnitt untersucht.

\subsubsection{Diskurs zur Intensivierung der Landwirtschaft und Flächennutzung}

Die vietnamesische Wirtschaft war 1993 durch die Landwirtschaft dominiert ${ }^{919}$. Das Bruttosozialprodukt lag bei 200 USD pro Einwohner ${ }^{920}$, die Sparquote war niedrig und besonders geringe Kapitalallokationen waren in den Bereichen der Bildung und Infrastruktur vorhanden $^{921}$. Die geringe Nachfrage auf dem Binnenmarkt galt als Wachstumshindernis ${ }^{922}$.

\footnotetext{
${ }^{918}$ Ebd.

${ }^{919}$ AG 461993.

${ }_{920}$ Im Juni 1992 entsprachen 200 USD ca. 330 DM. Laut Bundesfinanzministerium festgelegtem Umrechnungsfaktor von 1,95583 DM/Euro entsprechen 330 DM 168,72 Euro.

${ }^{921}$ AG 461993.

${ }^{922}$ Ebd.
} 
Die vietnamesischen Exportgüter bestanden hauptsächlich aus landwirtschaftlichen Erzeugnissen und basierten deswegen auf der Veräußerung natürlicher Ressourcen. Die zentrale Planwirtschaft sollte Schritt für Schritt zu einer dezentralen Wirtschaft umgestaltet werden, jedoch unterstützten die vorhandenen politischen Strukturen noch immer großangelegte und zentral geplante Prozesse ${ }^{923}$. In ruralen und urbanen Gebieten gleichermaßen war die Qualität der vorhandenen Straßen sowie des Telekommunikationsund Elektrizitätsnetz in Relation zum Bruttoinlandsprodukt niedrig ${ }^{924}$. Die Bevölkerung Vietnams konzentrierte sich in den zwei Ballungsgebieten um Hanoi und Ho Chi Minh City, während das Verhältnis von Einwohnerzahlen und Produktivität dort kein höheres Niveau als auf dem Land erreichte ${ }^{925}$. Eine lebenswerte Entwicklung außerhalb der beiden Städte sollte der ländlichen Bevölkerung Perspektiven abseits der Ballungsgebiete bieten und somit die Städte vor weiterer Überlastung bewahren ${ }^{926}$. Die Lebensbedingungen hatten sich seit der Wiedervereinigung verbessert, denn es standen statistisch 2.233 Kalorien pro Tag und Person zu Verfügung. Die Menschen erwartete eine Lebensdauer von ca. 67 Jahren und sie waren mit einer der höchsten Schulbildungen, im Vergleich zu anderen einkommensschwachen Ländern wie Malaysia oder Thailand, ausgestattet ${ }^{927}$. Aufgrund der vorhandenen Bildung schätzten die UN-Analysten Mellor und Qureshi, dass innovative Techniken in der Landwirtschaft sowie die Einführung neuer wirtschaftlicher Strukturen von den Vietnamesen adaptiert werden würden $^{928}$. Die vorhandene Gleichverteilung von Kapital galt als günstige Voraussetzung für die Implementierung neuer Organisationsstrukturen, beispielsweise im Bereich der Bewässerung und somit für wirtschaftliches Wachstum ${ }^{929}$. Der landwirtschaftliche Sektor verfügte aufgrund der hohen Einwohnerdichte über ausreichend Arbeitskraft. Im MekongDelta lebten 359 Personen pro Quadratkilometer ${ }^{930}$.

„The combination of lower than average population growth rates for its income class and high rural population densities is a particularly favourable set for rapid rural growth and strong links to the growth of other sectors. Of course, massive investment in physical infrastructure is required to benefit from these advantages. The income and asset inequalities are still low. This is an important feature facilitating the organization of farmers and hence favourable to rural development. A major barrier to organizing farmers for water control and irrigation maintenance, livestock marketing

\footnotetext{
${ }_{923}$ AG 461993.

${ }^{924} \mathrm{Ebd}$.

${ }^{925}$ Ebd.

${ }^{926}$ Ebd.

${ }^{927}$ Ebd.

${ }^{928}$ Ebd.

${ }^{929} \mathrm{Ebd}$

${ }^{930}$ Ebd., 7.
} 
cooperatives, and credit institutions arise from large inequalities in land holdings, wealth, and income.",931

Das State Planning Committe (SPC) der Sozialistischen Republic Vietnam wurde in seinen Planungsaufgaben im Mai 1993 von dem United Nation Development Programme (UNDP) unterstützt $^{932}$. Sie sondierten gemeinsam in einem zweitägigen Workshop Projekte des Public Investment Programms für die Phase 1993 - 2000. Durch das Public Investment Programm (PIP) sollten die folgenden neun Bereiche modernisiert werden: (1) ländliche Infrastruktur, (2) Bewässerungssysteme, (3) Forschung und Entwicklung, (4) Kreditsysteme, (5) Viehzucht, (6) Fischzucht, (7) industrialisierte Feldfrüchte, (8) Forstwirtschaft und (9) Nutzung der Karsthügel (Bergbau). Für die Projekte des Programms war ein Budget von 17,9 Milliarden USD vorgesehen, $2 / 3$ der Investitionssumme sollte von internationalen Entwicklungsorganisationen und 1/3 vom vietnamesischen Staat in die Projekte investiert werden $^{933}$. Die Verteilung des Budgets wurde durch das SPC und den Mitarbeitern des UNDP abgestimmt und wie folgt verteilt: $45 \%$ des Geldes sollten in Bewässerungsprojekte investiert werden, $20 \%$ für forstwirtschaftliche und $15 \%$ für allgemeine Aufgaben unbestimmter Art bereit gehalten werden, während den Bereichen des Kreditsystems, der Vieh- und Fischzucht und des Bergbaus jeweils zwischen 4 - $6 \%$ des Budgets zugeteilt wurden. Für die Modernisierung des ländlichen Straßennetzes stand nach der Verhandlung keine Allokation finanzieller Mittel bereit, obwohl Mellor und Qureshi abschließend folgende Empfehlung gaben:

„In sum, the characteristics of Vietnam are unusually favourable for agriculture to play a lead role in the development. Thus, the pace of the economic transformation in Vietnam is capable of rivaling [sic] that of its successful neighbours at least after a period of initial structural investment.", 934

Zu Beginn der Verhandlungen im Mai 1993 stellte das vietnamesische SPC zunächst die nationalen Ziele und ihre Budgetpläne für die verschiedenen Projektbereiche vor. Aus der vietnamesischen Perspektive sollte die Bewässerung priorisiert werden, denn 772 Mio. USD (29\% der veranschlagten Summe) wurden für die technokratische Umstrukturierung der Felder zur Intensivierung der Landwirtschaft vorgesehen ${ }^{935}$. Während der Verhandlungen gewichteten die Planungsakteure des SPC und UNDP die Rolle der Bewässerung jedoch noch

\footnotetext{
${ }^{931}$ Ebd., 11.

${ }^{932}$ Ebd.

${ }^{933}$ AG 46 1993, 67.

${ }^{934}$ Ebd., 12.

${ }^{935}$ Ebd., 68.
} 
stärker als die vietnamesische Regierung vorgeschlagen hatte. Im abgestimmten Public Investment Programm wurde die Investitionssumme für die Bewässerungsprojekte vergrößert, obwohl die Gesamtsumme des PIP um $31 \%$ geschmälert wurde. Die Verantwortlichen beider Organe vergrößerten die veranschlagte Investitionssumme für Bewässerungsprojekte von 772 auf 822 Mio. USD. Die Priorisierung der landwirtschaftlichen Projekte ging auf die Zusammenarbeit des UNDP mit Mitgliedern der Food and Agriculture Organization (FAO) zurück. Bei der Beratungsleistung für das vietnamesische Planungskomitee wurde das UNDP von der UN-Sonderorganisation FAO unterstützt, deren Mitarbeiter darauf hinwiesen, dass durch die Entwicklung des Agrarsektors die Herausforderungen Vietnams, wie beispielsweise die fortschreitende Umweltzerstörung und die Armutsbekämpfung, überkommen werden können ${ }^{936}$.

„It was the view of the Food and Agricultural Organization of the United Nations (FAO), that the broadly conceived UNDP effort would benefit from a supplemental, strategic view of the agricultural sector and its role in development. UNDP concurred in this view, as did the SPC., 937

Die Analysten empfahlen den Ausbau der Landwirtschaft und, zu seiner Unterstützung, die Infrastruktur in den entlegenen Gebieten zu modernisieren. Für die Priorisierung der Ausweitung der landwirtschaftlichen Produktion gaben die Autoren des UNDP-Berichtes zwei Gründe an. Sie nannten die unter den Entwicklungsorganisationen vorhandene Expertise, Bewässerungsprojekte umzusetzen, sowie die Konzentration auf schnellen wirtschaftlichen Erfolg, als maßgeblich für die Verteilung der Investitionsgelder, ,[...] the substantial experience of large donors in preparing irrigation projects and a correct emphasis on projects promise direct increases in agricultural production“938. Die Autoren der UNDPStudie betonten die Konsequenzen, würde die vietnamesische Regierung auf die Erschließung der ruralen Gebiete verzichten, durch eine Aufzählung von $\mathrm{zu}$ erwartenden Schreckensszenarien:

„Isolated villages have uncompetitive markets, lack of information, monetary incentives are week, school teachers do not show up, nor do health workers ${ }^{939}$. The result will be slowing of agricultural growth, little progress on poverty reduction, and massing of the bulk of urban growth in the two current centers of population." ${ }^{\text {(940 }}$

\footnotetext{
${ }^{936}$ AG 461993.

${ }^{937}$ Ebd., vi.

${ }^{938}$ Ebd., 70.

${ }^{939}$ Ebd., 45.

${ }^{940}$ Ebd., 69.
} 
Das vietnamesische SPC sah keine Mittel zur Modernisierung des ländlichen Verkehrswesens vor. In beiden Diskussionsentwürfen blieb der Posten der „Rural Physical Infrastructure“ als einziger von zehn ohne Budget ausgestattet ${ }^{941}$. Hohe Investitionsanteile wurden nach dem Abstimmungsprozess neben dem Bewässerungssektor nur im Forstbereich (20\%) gebündelt. Vor der Aushandlung sah das vietnamesischen SPC eine andere Allokation der Gelder vor: Zunächst sollten $18 \%$ im Forstbereich, $15 \%$ für den Bergbau, $13 \%$ für industrialisierte Feldfrüchte und $11 \%$ für Querschnittsaufgaben „for fairly broad purpose“ ausgegeben werden $^{942}$. Mellor und Qureshi bewerteten den vietnamesischen Diskussionsvorschlag mit dem Kommentar: „what is thought foreign assistance donors will support“"943. Die UNDPAutoren schrieben die Abneigung gegen Infrastrukturprojekte den massiven Ausgaben zu, welche für eine befriedigende Infrastruktur erforderlich waren. Diese würden gleichzeitig hohe Opportunitätskosten mit sich ziehen, da diese gleichzeitig die möglichen Investitionen in die Bewässerung schmälern würden ${ }^{944}$. Die Autoren der UNDP-Studie kommentierten, diese Konkurrenzsituation führt wie in anderen Entwicklungsländern dazu, den Verkehrssektor unterentwickelt zu belassen ${ }^{945}$, obwohl ,official pronouncements of the government and the

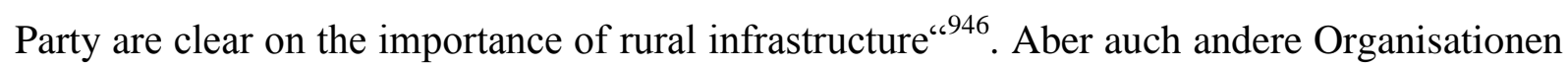
wie die Weltbank kritisierten die Vernachlässigung der Infrastruktur ${ }^{947}$, ,as they [donors] swing into lending action, they will no doubt give attention to this area; however, because the issue is important it needs to be given special attention." 948 Die UNDP-Autoren sahen die Verlegung von „All-Weather Rural Roads“9949 als Schlüssel für die Entwicklung Vietnams zu einer „modern agriculture, rich beautiful and civilized rural areas“, wie es sich die Regierung Vietnams wünschte ${ }^{950}$. Diese Wünsche werden im nachfolgenden Abschnitt in die geschichtlichen Abläufe eingeordnet und interpretiert.

\subsubsection{Interpretation des Landwirtschaftsdiskurses}

Aus den rekonstruierten Entscheidungsprozessen ergehen nachfolgende Ableitungen. Bereits seit der Wiedervereinigung des Landes 1976 instrumentalisierte die Zentralregierung die Bewässerung der Landwirtschaft, um durch eine kontinuierliche Steigerung des landwirtschaftlichen Outputs ihre Macht über Nord- und Südvietnam zu legitimieren. Judith

\footnotetext{
941 Ebd., 68.

942 Ebd., 76.

${ }^{943}$ Ebd., 67.

${ }^{944}$ AG 461993.

${ }^{945}$ Ebd.

${ }^{946}$ Ebd., 45.

${ }_{947}^{9 b d}$.

948 Ebd., 69.

${ }^{949}$ Ebd., 45.

${ }^{950}$ Ebd., 45.
} 
Ehlert beschreibt die Kampagne für die Ausweitung des Bewässerungssystems nach der Wiedervereinigung 1976 im Rahmen des „Rice Everywhere“-Programms:

„This campaign finally marked the drastic upheaval involved towards implementing total hydraulic management in the Mekong-Delta. Large scale irrigation finally became the modern symbol of national pride. The typical rhetoric of recent war propaganda was drawn on to communicate the newly prioritised project of national integration, namely irrigation and development." ${ }^{, 951}$

Dazu gibt Ehlert Beispiele aus der Hau Giang Zeitung (Mekong-Delta) von 1976, denn die Journalisten schrieben zur Mobilmachung von Human Forces, der Irrigation Front sowie über bekanntgewordene Erfolgsgeschichten, „our province mobilised 250.000 work days and excavated 1.420.000 cubic metres of soil“952. Der Erfolg in der landwirtschaftlichen Produktion führte dazu, dass Vietnam 1992 der zweitgrößte Reisexporteur der Welt wurde ${ }^{953}$. Die neue Rolle auf dem Weltmarkt verlieh der Bevölkerung Vietnams eine neue Identität, eine neue Wahrnehmung der nationalen Persönlichkeit. Ehlert schreibt: „During the early 1990s, rice intensification served the formation of national identity after the devastating decades of war and promised a better future" ${ }^{954}$. Das Antlitz ganzer Landstriche war nicht wiederzuerkennen ${ }^{955}$. Die Autoritäten der Provinz priesen die Veränderungen: if we don't restructure plants, there will be no way to improve people's living standard, [...] to bring high-yield plants into Chau Phu [in An Giang] has prioritized internal irrigation system." Geograph Charles Howie konstatiert nach seinen Untersuchungen der sozioökonomischen Auswirkungen von Deichen in der An Giang Provinz, „,whoever controls irrigation, controls agriculture" 957 und beschrieb damit die bis 2010 andauernde Wirkung der Instrumentalisierung der Bewässerungssysteme zum Machterhalt der zentralen Einheitspartei. Die absolute Steigerung der Investitionsmittel für Bewässerungssysteme weist darauf hin, dass auch die Entscheidungsträger des UNDP von der Wichtigkeit der Aktivierung ländlicher Ressourcen überzeugt waren und diese vor allen anderen Maßnahmen priorisierten. Die Argumentation der Berater baute nicht auf einer nachhaltigen Langzeitwirkung für Vietnam, sondern auf bereits erprobten Methoden in dem Geschäft der Entwicklungszusammenarbeit auf $^{958}$. Für die beiden UNDP-Mitarbeiter stand ein Ausbau der ländlichen Produktion

\footnotetext{
${ }^{951}$ Ehlert 2012, 29.

${ }^{952} \mathrm{Ebd}$

${ }^{953}$ Tanh/Singh 2006.

${ }^{954}$ Ehlert 2012, 32.

955 Artikel: Chuyen dich co cau cay trong don bay de phat trien kinh te, Zeitung An Giang, 27.05.1995.

${ }^{956}$ Ebd.

${ }^{957}$ Howie 2011, 6.

${ }^{958}$ In dem Mellor-Bericht wurde die Situation Vietnams mit anderen Nationen verglichen und ein Beispiel der Agrarreformen Pakistans präsentiert. Die Befürwortung des Ausbaus der Bewässerungssysteme wurde argumentativ
} 
synonym mit dem Ausbau der Infrastruktur. Sie sahen einen Bedarf von 5.589 Mio. USD zum befriedigenden Ausbau des befestigten Straßennetzes. Mellor und Qureshi verdeutlichten ihre Präferenz für die Allokation der Entwicklungsgelder, denn sie planten das Straßenwesen wie ein Werkzeug zur Erschließung von entlegenen Gebieten zu nutzen. Damit ermöglichten sie für ihre Organisation den Zugriff auf noch verschlossene Ressourcen wie die Eröffnung von Märkten, Arbeitskraft aber auch natürliche Ressourcen wie Wasser und Boden. Aber auch „easing the world rice market ${ }^{\text {“959 }}$ wurde als Ziel der UNDP genannt. Die FAO war dafür bekannt, durch ihre Beratung Regierungen größere Kontrolle über entlegene Gebiete zu ermöglichen ${ }^{960}$. Jedoch beflügelte die Landwirtschaftsstrategie auch die Ziele der vietnamesischen Regierung, denn auch diese wollte den Zugriff auf entlegene Gebiete ausweiten, um somit ihre Kontrolle über die ländliche Bevölkerung auszubauen:

\begin{abstract}
„FAO funds have thus been highly useful for states keen to enhance their power over grassroots actors, especially in those states where control over rural populations has been relatively weak. In this manner, technical assistance has been relatively weak tool to assert essentially political goals. At a more posaic level, FAO funds have been a boon to cashstarved state agencies. [...] In the process, those grassroots actors for whom these FAO projects are formally intended often see few, if any, of the benefits that FAO claim to be providing through their incentives."
\end{abstract}

Die Mitglieder der FAO betonten ausdrücklich die Unumgänglichkeit des Ausbaus der Infrastruktur $^{962}$. Mit Hilfe dieser Empfehlung versuchten sie, auch zukünftig mit der Regierung zusammenarbeiten zu können.

Die Verantwortlichen der vietnamesischen Delegation sahen die Gelder im Ausbau der Bewässerung effektiver investiert als im Bau entlegener Straßen. Die Meinung, der Ausbau des Straßennetzes sei nicht so effektiv an der wirtschaftlichen Entwicklung Vietnams beteiligt wie andere Investitionsbereiche, kann aus der Erfahrung rühren, dass die vorhandene Infrastruktur bereits ausreichte, um zweitgrößter Reisexporteur der Welt zu sein. Dieser Fakt

\footnotetext{
durch die bereits vorhandene Expertise der Entwicklungsorganisation bestärkt. Die Autoren nannten Pakistan in den Statistikberichten über die Entwicklungen in Südostasien, obwohl das Land nicht in dieser Region liegt. Der Mitautor Sarfraz Qureshi trägt einen pakistanischen Namen, was darauf hinweist, dass der Autor ihm bereits bekannte Beispiele wasserbaulicher Projekte nannte, ohne dass diese mit Vietnam in Relation standen. Die Studie wurde ein Sammelsurium von Daten willkürlicher Agrarnationen, wie z. B. Kolumbien, Costa Rica und Kenia (AG 46 1993, 17). Dabei spielte der Vergleich mit direkten Nachbarländern wie der Volksrepublik China in der Untersuchung keine Rolle. Das Team nutzte statistische Daten der Jahre 1988 bis 1993, während jedoch eigene Erhebungen in den Provinzen nicht unternommen wurden. Lediglich der Vorsitzende des Volkskomitees aus Ba Vi sowie ein Bauer aus der gleichen Region, 55 km westlich von Hanoi, wurden im Rahmen der Erstellung der Studie getroffen (Ebd., 96). Die Studie war folglich das Ergebnis einer Literaturrecherche mit dem Ziel, den Geberorganisationen zukünftig einen Anteil an der Zusammenarbeit mit der vietnamesischen Regierung zu sichern und die agrarpolitische Richtung des südostasiatischen Landes beeinflussen zu können.

${ }_{959}$ AG 46, 69 .

${ }^{960}$ Raymond/Bryant/Bailey 1997.

${ }^{961}$ Ebd., 86.

${ }^{962}$ Ebd.
} 
impliziert, dass beträchtliche Mengen von Reis aus dem Mekonggebiet befördert wurden immerhin stammten $80 \%$ des Exportreis aus dem südlichen Delta ${ }^{963}$.

Die beiden Agrarexperten Mellor und Qureshi waren darauf bedacht, die Entwicklung des Agrarsektors als einzigen Weg für ein wirtschaftliches Wachstum herauszustellen. Dabei hoben sie neben der Bewässerung die Infrastruktur als wichtigsten Faktor hervor, welcher maßgeblich den Erfolg der landwirtschaftlichen Produktion mitbestimmt ${ }^{964}$. Die zwei Berater empfahlen den Bau von Straßen eingehend, wurden aber im Bezug zum Deichbau nicht deutlich genug, um eine Bauempfehlung auszumachen:

„We put [irrigation and dikes] lower in priority than all-weather roads and research, because they are more central and will have a greater aggregate impact on growth. We do not deal at length with the irrigation and dike system needs since they are subject of continued intense analysis. Given the immense requirements for road investment, however, tight priorities must be set for irrigation investment. The Public Investment Program gives far too much relative weight to irrigation over rural roads. ${ }^{965}$

Die beiden Autoren sahen im Rahmen des Investment Planning weder vor, Agrarland vor Hochwasser oder anderen Naturgefahren zu schützen, noch sahen sie die Notwendigkeit, zusätzliche Flächen zur landwirtschaftlichen Nutzung aus den Überschwemmungsgebieten zu gewinnen. Sie setzten sich nicht mit der Frage auseinander, ob eine Bodenkonkurrenz zwischen dem landwirtschaftlichen Anbau und den Überschwemmungen bestand.

Für die vietnamesische Regierung schienen die Investitionen von außen durch die internationale Entwicklungszusammenarbeit einem Window of Opportunity gleich zu kommen. Das neue Geld in den Staatskassen machte nun Projekte möglich, welche dringend notwendig waren und eröffnete zudem die Möglichkeit, politische Interessen auch in entlegenen Gebieten fernab der Hauptstadt durchzusetzen.

1995 wurden die ersten Erfolge der Förderung der Landwirtschaft erzielt ${ }^{966}$, berichtete die vietnamesische Regierung. Der Autor des Artikels „Creating a solid foundation to rise up“ fasste die wichtigsten Ereignisse zwischen 1993 - 1995 zusammen, welche die wirtschaftliche Lage verbessert hatten und unterstrich die Unterstützung der Regierung in der Region Chau Phu (südlich des Hau Arms in der Mitte der Provinz An Giang) ${ }^{967}$. Er nannte die Einführung von hybridem Saatgut, das $40 \%$ ertragreicher als gewöhnlicher Reis sein sollte, die

\footnotetext{
${ }^{963}$ Vormoor 2010.

${ }^{964}$ Ebd.

${ }^{965}$ Ebd., X.

${ }^{966}$ Artikel: Nhin lai va vuon len (Looking back and Rising up), Zeitung: Bao An Giang, 06.09.1995.

${ }^{967}$ Ebd.
} 
Umsetzung von Wasserbauprojekten, den Zugang zu Krediten und die Umstellung auf eine Produktion für den Export als Treiber des neuen wirtschaftlichen Erfolgs. Nguyen Thi Nga, Sekretär des Bezirks Chau Phu, berichtete:

„The province has been trying to maintain the area of seasonal crops to develop subsidiary food crops. We've also defined that it is better to restructure seasonal rice crops as soon as possible, to cultivate high-yield and high-value in exporting plants to improve people's lives." $" 968$

„To keep farmer's mind on production"969, entsprach dem erklärten Ziel der politischen Institutionen. Alle politisch Verantwortlichen unterstützten den neuen Aufschwung, „the party's leaders along with all levels of government have tried to encourage farmers to focus on cultivating efficient plants“970. Des Weiteren wurden Konferenzen organisiert und ein Punktesystem eingeführt, welches die Beteiligung der Ortschaften an der Aussaat der hybriden Samen verglich und kontrollierte. Die Regierung bewahrte ,a clear orientation of what farmers should plant to enrich themselves““971. Zur Unterstützung der Aussagen wurden Kommentare der Landwirte zitiert, welche bereits den Erfolg der Hybridsaat bezeugten. Huynh Tan Man beschwor: „I will never quit hybrid corn“972 und sein Kollege Le Long Thuan schwärmte von einem Ertrag bis zu 11,1 t/ha ${ }^{973} .1993$ wurden zunächst 5,94 km² der hybriden Aussaat in Chau Phu getestet - diese Fläche wurde bereits im darauffolgenden Jahr auf $63,3 \mathrm{~km}^{2}$ ausgeweitet ${ }^{974}$.

Wie gezeigt werden konnte, war die ländliche Bevölkerung Südvietnams, die sich hauptsächlich im Mekong-Delta konzentrierte, für die Regierung nicht einfach zu regieren. Seit der Wiedervereinigung versuchte die Regierung, den Eigensinn der ruralen Bevölkerung mit den Interessen der Regierung in Einklang zu bringen. Aufgrund der internationalen Investitionen verfügte die Regierung seit 1993 über zusätzliches Kapital und somit über die Kapazität, in potentielle Instrumente $\mathrm{zu}$ investieren, die sie bei den Harmonisierungsversuchen unterstützen konnten. Ein solches Instrument stellte die Ausweitung der Bewässerungssysteme dar. Natürliche Wasserressourcen zu kontrollieren war bereits seit 1976 als Zugriffsmethode im Mekong-Delta genutzt worden und sollte jetzt durch die internationale Hilfe zur Entwicklung der Landwirtschaft intensiviert werden. Besonders die Ablehnung der Investition in Allwetterstraßen unterstützt diesen Interpretationsansatz,

\footnotetext{
${ }^{968} \mathrm{Ebd}$

${ }^{969} \mathrm{Ebd}$.

${ }^{970}$ Ebd.

${ }^{971}$ Ebd.

${ }^{972}$ Ebd. Die Größe Chau Phus beträgt $672 \mathrm{~km}^{2}$.

${ }^{973}$ Ebd. Die Entemenge von 11,1 t/ha entspricht $1.110 \mathrm{t} / \mathrm{km}^{2}$.

${ }^{974}$ Ebd.
} 
denn es weist darauf hin, dass die Regierung die Landbevölkerung nicht avancieren wollte. Die bereits vorhandene Infrastruktur wurde für die „renitenten“ Landwirte als ausreichend empfunden. Straßenverbindungen würden die Menschen unnötig auffordern, in die Städte zu kommen, dabei sollten sie sich auf den Landbau konzentrieren. Die Landwirte sollten Exportprodukte erzeugen, um Devisen in Vietnams Staatskasse einzubringen. Die Regierung Vietnams plante den Ausverkauf der ruralen Ressourcen mit der Absicht, den Menschen in entlegenen Gebieten eine lebenswerte Perspektive bieten zu können, damit die Infrastruktur der Städte und die dortige Lebensqualität nicht zunehmend durch diese beansprucht werden würde ${ }^{975}$.

\subsubsection{Diskurs zum Hochwasserschutz im Mekong-Delta}

„Die Entscheidungsträger der vietnamesischen Regierung verstehen Hochwasserschutz als ein technisches Konzept“" ${ }^{976}$, schrieb der Politikwissenschaftler Bach Tan Sinh 2009. Die Hochwasser des Mekong-Deltas wurden zu Beginn der 1990er Jahre als ausreichend negativ bewertet, um einen Hochwasserschutz zu installieren. Deichbau war bis dahin vor allem im Norden des Landes betrieben worden ${ }^{977}$. Der staatliche Hochwasserschutz kann im Delta in zwei Phasen unterteilt werden. Zwischen 1993 und 2002 war der Ansatz der Regierung auf den Deichbau verlegt, der die Felder für den landwirtschaftlichen Anbau frei halten sollte. Ab 2002 versuchte die Regierung auch die Menschen und deren Besitz vor dem Hochwasser zu schützen. Dies sollte durch die Umsiedlung der vom Hochwasser bedrohten Menschen in höher gelegte Cluster gewährleistet werden. Beide Ansätze werden im Folgenden beleuchtet. Ein Deichbauprojekt in der Can Tho Provinz wird zur Veranschaulichung der Abstimmungsabläufe kurz erläutert.

\section{Deichbau zu Gunsten der Landwirtschaft}

Das vietnamesische Ministerium für Landwirtschaft und Rurale Entwicklung (MARD) war zu Beginn der 1990er Jahre bestrebt, das wirtschaftliche Wachstum der Mekongregion zu steigern $^{978}$. Um die Wirtschaft anzuregen, beabsichtigten das MARD und seine Planungsorganisation, das Southern Institute for Water Resource Planning (SIWRP), die Nutzung von Boden und Wasser im Zusammenhang von Landwirtschaft, Forst und Fischerei in intensivieren ${ }^{979}$. Der Mekong-Delta-Master-Plan entstand 1993 im Zuge dieser

\footnotetext{
975 AG 46 1993, 4.

${ }^{976}$ Sinh 2009.

${ }^{977}$ DR 0021992.

${ }^{978}$ White 2002.

${ }^{979}$ Ebd.
} 
Bestrebungen in Zusammenarbeit mit der Weltbank und dem Mekong River Komitee ${ }^{980}$. Zur Ausführung beauftragte das Konsortium die niederländische Umweltplanungsfirma NEDECO. Seit der Fertigstellung 1993 wird der Masterplan dem Bau hydraulischer Großprojekte zu Grunde gelegt ${ }^{981}$. Nicht nur in der Erstellung des Masterplans, sondern auch in der Umsetzungsphase unterstützte die Weltbank den Bau der einzelnen Maßnahmen; dabei finanzierte die Institution sechs überregionale Wasserbauprojekte ${ }^{982}$.

Vorgesehen war, dass die ländliche Bevölkerung sowie auch die nationale Regierung von den Veränderungen profitiert. Die Zentralregierung erhoffte sich steigende Exportzahlen und eine Verbesserung der Lebensqualität im Mekong-Delta. Die Menschen vor Ort würden durch das Eindämmen der natürlichen Gefahren vor Überschwemmungen, Versalzung der Küstenregionen, Versäuerung der Böden und fehlendem Süßwasser für die Bewässerung, günstigere Verhältnisse für die Landwirtschaft vorfinden. Die Regierung beabsichtigte mit dem baulichen Hochwasserschutz folgende Ziele zu erreichen:

- Nahrungsmittelsicherheit ${ }^{983}$

- Steigerung des Einkommens der ländlichen Bevölkerung durch die Intensivierung der Landwirtschaft

- Schaffung von Arbeitsplätzen während der Überschwemmungszeit

- Verringerung von Migration ${ }^{984}$

- Erleichterung des Zugangs zu Märkten, Schulen und Krankenhäusern ${ }^{985}$.

Die niederländischen Experten der Umweltplanungsfirma NEDECO berechneten, dass durch die Umsetzung des Masterplans die landwirtschaftliche Produktivität um 1/3 gesteigert werden kann. Die Planer gingen 1993 von einem Status Quo mit einer bereits vorhandenen Reisanbaufläche von $24.000 \mathrm{~km}^{2}$ aus ${ }^{986}$. Die Ingenieure empfahlen, die landwirtschaftlich genutzte Fläche durch den Einsatz von Deichen um $2.000 \mathrm{~km}^{2}$ zu vergrößern. Dies entsprach einer Erweiterung von 8 \%. Bis zum Jahr 2015 sollten im Mekong-Delta bis zu 16 Mio. t Reis produzieren werden können ${ }^{987}$. Um diese Ziele zu erreichen, musste Fläche gewonnen werden. Hierfür sahen die Planer jene vor, die üblicherweise in den Hochwassermonaten überschwemmt waren. Die Flächenexpansion sollte mit Hilfe von Deichen, Schleusen und

\footnotetext{
${ }^{980}$ Ebd.; Tuan et al. 2008. Das Mekong River Komitee wurde 1957 gegründet.

${ }^{981}$ Evers/Benedikter 2009.

${ }^{982}$ Evers/Benedikter 2009.

${ }^{983}$ Interview John Douglas Flanders, Australian Agency for International Development, März 2012.

${ }^{984}$ Ebd.

${ }^{985}$ Howie 2011.

${ }^{986}$ Tuan et al. 2008.

${ }^{987}$ Ebd., 28.
} 
Abflusskanälen errungen werden. Die dadurch künstlich ermöglichte Anbauzeit würde eine Produktionssteigerung zulassen. Der Masterplan basiert auf technisierten Lösungsmöglichkeiten mit der Absicht, nachhaltiges Wirtschaftswachstum zu generieren ${ }^{988}$. Bereits 1995 wurde im Bezirk An Phu, ein Deich nach einer dreimonatigen Konstruktionszeit fertig gestellt und gestattete fortan eine dritte Aussaat ${ }^{989}$.

Beispielhaft wird nun die Entscheidungsphase des Deichprojekts in der Can Tho Provinz vorgestellt. Die Can Tho Provinz ist die südliche Nachbarprovinz von An Giang und liegt östlich des Hau-Arms. In Can Tho wurde 2004 das Gebiet zwischen der Nationalstraße 80 und 91 im Bezirk Vinh Thanh durch Deiche vom Hau-Arm abgeschlossen. Die drei Nationalstraßen 80, 91 und 921 wurden erhöht, neu befestigt und dienten fortan als Deich. ${ }^{990}$ Die Fläche wurde in fünf Komponenten unterteilt, um den Reisanbau, die Fruchtbaumplantagen und die Fischzucht zu schützen. Die Deichkonstruktionen erreichen in etwa eine Höhe von $5 \mathrm{~m}$. Die Ingenieure orientierten sich hierbei an den außergewöhnlich hohen Wasserständen von 1961 und 1994. Sie verfolgten das Ziel, die Felder auch vor stärksten Hochwassern zu schützen ${ }^{991}$. Finanziert wurde die Planung und Umsetzung durch lokale $(75 \%)$ und nationale $(25 \%)$ Mittel $^{992}$. Die lokale Bevölkerung wurde abhängig vom Nutzenmaß an der Finanzierung beteiligt. Beim Deichbau im benachbarten Bezirk Cho Moi (Provinz An Giang) identifizierte die Regierung für die Beteiligung an der Finanzierung die folgenden Gruppen, die an der Finanzierung beteiligt wurden: Landwirte, Industrielle, Unternehmer, Gastronomen, Maschinenverleiher, und alle Arten von Wasser- oder Landtransporteuren ${ }^{993}$. Der Stichtag der Zahlung wurde an die Erntezeit gebunden, so musste man seinen Beitrag bis zum 28. August eines jeden Jahres geleistet haben.

Das MARD vergab an das SIWRP die Aufgabe, Handlungsalternativen auszuarbeiten. Die weitere Planung, Kommentierung und abschließende Bewertung von vier ministerialen SubInstituten wurde durch die Mitglieder des SIWRP koordiniert. Die vier Sub-Institute waren das Southern Sub-national Institute of Agriculture Planning and Projection des MARD, das Southern Center for Transportation and Economy Sciences des Transportministeriums, die

\footnotetext{
${ }^{988}$ White 2002, 59.

${ }^{989}$ Artikel: Vua chong lu vua noi lien giao thong, Zeitung An Giang, 19.07.1995.

${ }^{990}$ Die Straße 80 führt in $30 \mathrm{~km}$ Länge von Le Te bis Kien Giang. Die Straße 91 ist $50 \mathrm{~km}$ lang und führt von Can Tho bis An Giang. Die Straße 921 verbindet O Mon und Vinh Thanh auf 25,53 km Strecke. Die Karte auf Seite 154 kann bei der Orientierung behilflich sein.

${ }^{991}$ Huu 2011.

${ }^{992}$ Ebd.

${ }^{993}$ Artikel: Xay dung de bao chong lu o Cho Moi, Zeitung An Giang, 06.09.1995.
} 
General Construction Consultant Company des Ministeriums für Transport und Bau sowie das Southern Economy Center des Ministeriums für Planung und Investment.

"[The four institutes] as well as hundreds of other organizations throughout the country. The planers organized hundreds of meetings, seminars/workshops at ministry and local levels to gather information and reach agreements in flood control strategies among the representative leaders of other organizations throughout the country."

Der Aushandlungsprozess kann in vier Schritte unterteilt werden. Zunächst formulierten die oben benannten Institutionen einen ersten Vorschlag für das Deichprojekt ${ }^{995}$. Dieser enthielt vier Handlungsoptionen. Der erste Vorschlag beinhaltete, das Hochwasser durch Kanäle auf die zur Verfügung stehende Fläche zu verteilen und keinen Deich zu bauen. Den Vorteil sahen die Planer bei dieser Handlungsoption in der verbesserten Verteilung des Wassers während der Trockenzeit. Die zweite Alternative sah vor, die Region durch die Nationalstraßen 80 und 91 zu teilen. Die Nationalstraßen 80 und 91 sollten ausgebaut werden, um das Hochwasser künftig zurück zu halten. Die Straßen würden als Deiche funktionieren und das Hochwasser ins Hinterland des Hau-Arms abweisen. Ein Schleusensystem sollte den Ablauf kontrollierbar machen und die Landwirtschaft im Hinterland vor dem Hochwasser schützen. Handlungsmöglichkeit Drei lehnte sich wiederum an das Konzept der zweiten Ausarbeitung an, verzichtete aber auf die weiteren Unterteilungen im tiefen Überschwemmungsgebiet westlich der Nationalstraße 91. Die Ingenieure stellten eine vierte Alternative vor. Dabei sollte eine zusätzliche Zerschneidung der Region südlich der Nationalstraße 80 erfolgen. In dieser Ausarbeitung wurde auf Fruchtbaumplantagen und Reisanbaugebieten östlich der Nationalstraße 91 - entlang des Hau-Arms - Wert gelegt, denn sie würden durch hohe Deiche geschützt. Das SIWRP wertete die Kommentare von allen entscheidungsrelevanten Akteuren aus.

Die Vorschläge wurde durch zwei Gruppen begutachtet ${ }^{996}$. Die eine Gruppe kann als die politischen und administrativen Entscheidungsträger zusammengefasst werden, denn es handelte sich um die Ministerien wie MARD, MONRE, das Verkehrsministerium u. s. w. in Hanoi. Dem gegenüber stand die Kommentierung der lokalen Akteure. Auf den ,unzähligen Veranstaltungen“ während der Planungsperiode wurden die Vor- und Nachteile der vier unterschiedlichen Bauvorschläge evaluiert ${ }^{997}$.

\footnotetext{
${ }^{994}$ Huu 2011, 74.

995 Ebd.

${ }^{996}$ Ebd.

${ }^{997}$ Ebd.
} 
In einem zweiten Schritt wurde nach der Betrachtung des Vorschlags aus den beiden verschiedenen Perspektiven eine Beratungsvorlage erstellt. Die Beratungsvorlage diente dazu, einen Konsens zwischen der staatlichen und lokalen Perspektive im Rahmen eines Workshops herzustellen ${ }^{998}$. MARD richtete einen Workshop aus, auf dem beide Gruppen ein letztes Mal die Gelegenheit nutzen konnten, für ihre Ansichten $\mathrm{zu}$ werben. Die abgestimmte Entscheidung wurde in einem abschließenden Schritt dem Premierminister vorgelegt ${ }^{999}$. Dieser nahm 1998 das Projekt an, somit konnte die Implementierungsphase begonnen werden.

Huu fasste die diskutierten Vor- und Nachteile der vier vorgestellten Handlungsalternativen wie folgt zusammen: Die Handlungsalternative keinen Deich zu bauen und einen Kanal zu nutzen, um das Wasser abfließen zu lassen, wurde aufgrund der Aussicht auf die Inanspruchnahme natürlicher Vorgänge geschätzt. Ein hohes natürliches Fischvorkommen, der leichte Zugang zu Wasserstraßen sowie der für die Landwirtschaft wichtige Auswaschungseffekt auf den Boden zur Befreiung von Chemikalien, Giften und Versalzungen machten den Vorschlag attraktiv. Die Investitionskosten blieben im Vergleich zu den Alternativen gering. Als nachteilig wurde die Beeinträchtigung der Landwirtschaft durch die immer noch zu akzeptierende Überschwemmung sowie der dadurch erzeugte hydrostatische Druck auf den Boden bewertet. Die „Launen des Hochwassers“ wurden als allgemeine Bedrohung der Landwirtschaft empfunden. Der zweite Vorschlag fokussierte die Nationalstraßen 80 und 91 als Deich zu nutzen und damit die Landwirtschaft sowie die Infrastruktur zu schützen, ferner die noch vorhandenen Einstautiefen um $1 \mathrm{~m} \mathrm{zu}$ reduzieren. Aus der Diskussion ging die Befürchtung hervor, dass der Auswascheffekt der Flut nicht mehr zu nutzen sei und der Boden aufgrund der natürlichen Versalzung und der Nutzung von künstlichem Dünger negativ beeinträchtigt werden könne. Auch der Verzicht auf das natürliche Fischvorkommen wurde als negativ bewertet. Die Höhe der Investitionskosten wurde als durchschnittlich und realisierbar eingeschätzt. Das SIWRP hielt zur dritten Option abschließend fest, dass die Möglichkeiten der Wasserstandshöhe zwischen 1-1,20 m sowie die leicht zugänglichen Wasserwege als positiv aufgefasst wurden, allerdings die hohen Investitionskosten und der Verzicht auf Auswascheffekte und Fischvorkommen die Alternative in ihrer Attraktivität beeinträchtigen. Die vierte Handlungsoption bot folgende Vor- und Nachteile: Wie auch in der zweiten und dritten Handlungsoption würde die Infrastruktur und Landwirtschaft durch Deiche geschützt aber die eingestauten Wasserhöhen um 1,30 m reduziert. Dagegen würden sich die Überflutungsperioden verlängern. Der 
Reinigungseffekt durch das Auswaschen des Bodens würde verloren gehen und der Boden in der Folgezeit evtl. negativ beeinflusst. Es würden Einbußen hinsichtlich des Fischvorkommens hinzunehmen sein. Außerdem stellte die vierte Option die kapitalintensivste Bauphase dar, denn die finanziellen Kosten wären „ziemlich hoch“1000. Aufgrund der Gewichtung der diskutierten Vor- und Nachteile erstellte das SIWRP einen Abschlussbericht und empfahl, die vierte Alternative. Obwohl mit der dritten Alternative geringere Investitionskosten verbunden waren als mit der vierte Alternative, stellte sich für die Entscheidungsträger nach Abwägung aller Vor- und Nachteile die letztere Handlungsoption als die vorteilhafteste Wahl heraus. Die SIWRP Mitglieder argumentierten, dass aufgrund von Budgetengpässen nur eine schrittweise Umsetzung des Baus möglich und diese lediglich bei Option 4 zu realisieren sei ${ }^{1001}$.

Die langjährige Ausarbeitungszeit begründete Huu mit der mangelnden Transparenz der Aufgabenverteilung zwischen MARD und dem Ministry of Natural resources and Environment (MONRE) sowie den sich daraus ergebenden Verzögerungen, die sich beide von oben nach unten auf allen Ebenen der vietnamesischen Verwaltung von Provinz zu Stadt/ Kommune und Hamlet durchzögen ${ }^{1002}$. Er präsentierte den Entscheidungsprozess in einem Top-down Stil ohne den Einbezug der lokalen Expertise oder der betroffenen Bevölkerung. Die Eindeichung wurde durch Mitarbeiter des SIWRP sowie der höheren politischen Instanzen ausgehandelt, ohne den betroffenen „Victims““1003 die Möglichkeit zu bieten, sich einzubringen ${ }^{1004}$. Der Einbezug der lokalen Expertise war abhängig von persönlichen Präferenzen der Entscheidungsträger ${ }^{1005}$. Während die örtlichen Planungsebenen in Rücksprache mit der Bevölkerung Vorschläge ausarbeiteten, trafen die Mitglieder des MARD bereits Entscheidungen, ohne mit den Leitern der unteren Organisationseinheiten zu sprechen oder sie zu informieren ${ }^{1006}$. Termine mit den betroffenen Landwirten waren von dem Agricultural and rural Development Office (ARDO), welches auf der Distriktebene angesiedelt ist, zum Zweck der Besänftigung arrangiert worden, sowie, um die Entschädigungszahlungen für nötige Flächenfreigaben organisiert worden ${ }^{1007}$. Die Treffen wurden nicht genutzt, um die Interessen der Landwirte in den Planungsprozess

\footnotetext{
${ }^{1000}$ Huu 2011.

1001 Ebd.

${ }^{1002}$ Ebd. Die Aufgaben von MARD liegen im Bereich der Hydrologie, z. B. Dämme und Deiche zu bauen und gehören dem technischen Bereich an. Die Aufgaben des MONRE beziehen sich auf die Ressourcen wie Wasser und Land. Bei einem Deichbauprojekt muss die Planung des MARD von MONRE genehmigt werden.

${ }^{1003}$ Ebd., 92.

1004 Ebd., 94.

${ }^{1005}$ Ebd., 72.

${ }^{1006}$ Ebd., 115.

${ }^{1007}$ Ebd., 112.
} 
aufzunehmen $^{1008}$, obzwar die Anwohner in die Finanzierung des Deichs einbezogen wurden. Wissenschaftliche Studien zu Veränderungen der hydraulischen Regime des Flusses, eine Veränderung der Schwemmstoffe und Sedimente oder sozial- ökonomischer Studien zur Lebensgrundlage der vor Ort lebenden Bevölkerung fanden keinen Einzug in die Debatten ${ }^{1009}$. Damit blieb der Diskurs durch ingenieurwissenschaftliche Meinungen geprägt. Des Weiteren wurde deutlich, dass institutionalisierte Interessensgruppen wie die Fisch-Schutz-Agentur, die Pflanzenschutz-Agentur oder die lokalen Forschungsinstitute und Universitäten im Planungsprozess als nicht offizielle Planungspartner von einer Partizipation im Gestaltungsprozess ausgeschlossen wurden.

\section{Ein Leben mit der Flut - Umsiedlungen der vom Hochwasser bedrohten Bevölkerung}

Die Richtlinie Living with Floods manifestiert die Eröffnung des Hochwassersicherheitsdiskurses ${ }^{1010}$. Mit dem 2. nationalen Strategie- und Aktionsplan für die Linderung und das Management Wasser bezogener Desaster 2001 - 2020 wurde die Schlüsselstrategie Living with Floods verabschiedet ${ }^{1011}$. Ziel des Aktionsplans ist der Schutz des privaten Eigentums, der durch die Anpassung des Menschen an das saisonale Hochwasser sichergestellt werden soll ${ }^{1012}$.

Die Strategie des Hochwasserschutzes besteht aus zwei Säulen. Drei bauliche Maßnahmen werden durch drei weitere nicht-strukturelle Aktivitäten ergänzt: „A combination of structural and non-structural measures is considered more efficient and long-term sustainable to flood management ${ }^{\star 1013}$; „Support to poor households to elevate their house foundations in [19]90`s failed to provide adequate protection in the devastating 2000 floods." ${ }^{1014}$ Darum sollen durch die drei Baumaßnahmen der Bevölkerung sichere Rückzugsorte zur Verfügung gestellt werden. Sie beinhalten den Bau neuer Deiche zum Schutz vor Überschwemmung, dem Verbreitern und Vertiefen von Ableitungskanälen sowie der Errichtung von öffentlichen

\footnotetext{
${ }^{1008}$ Ebd.

${ }^{1009}$ Ebd., 71 - 75.

${ }^{1010}$ Als Grundsteine der Living with Floods-Leitlinie gelten die „Prime Minister's No. 99 Decision”, vom 02. September 1996 sowie die „Prime Minister's No. 173 Decision” vom 6. November 2001 mit der Vision, dass das wirtschaftliche Wachstum von den Wasserressourcen des Mekongs profitiert und die Kapazität der Menschen, mit Hochwasser zurechtzukommen, gestärkt wird ${ }^{1010}$. Aber erst die „Prime Minister's No. 105 Decision” vom 02. September 2002 beinhaltete, dass Haushalte aus hochwassergefährdeten Gebieten durch den erleichterten Zugang zu Krediten für den Kauf von Land und Häusern unterstützt werden, in die Cluster umzusiedeln. Der Bau der Häuser wurde durch die Entscheidung „Nr. 78 “ in 2004 konkretisiert. Häuser sollten nicht kleiner als $32 \mathrm{~m}^{2}$ sein, während die Straßen in den Clustern $7 \mathrm{~m}$ breit sein durften (Danh 2011).

1011 Tuan 2007.

1012 Ebd.

${ }^{1013} \mathrm{Sanh} / \mathrm{Can} 2009$.

${ }^{1014}$ Lebel/Sinh 2009.
} 
Gebäuden und Privathäusern auf höheren Ebenen ${ }^{1015}$. Den drei technokratischen Ansätzen stehen drei organisatorische Maßnahmen gegenüber: Zum einen soll der landwirtschaftliche Rhythmus von Aussaat und Ernte den natürlichen Bedingungen abermals angepasst werden, zum anderen sollen Kinder während der Hochwasserzeit explizit beaufsichtigt werden und ferner soll die Kommunikation der Administration mit der Bevölkerung in Gefahrengebieten zum Zweck der Steigerung des vorhandenen Gefahrenbewusstseins ausgeweitet werden ${ }^{1016}$. Aber auch die Verbesserung des Frühwarnsystems, die Verteilung von Schadensersatz und die Lagerung von Lebensmitteln und Medikamenten sowie die Vorbeugung von durch Wasser übertragbare Krankheiten gehören zu den Zielen der Richtlinie ${ }^{1017}$.

Durch die Behörden der Provinzebene und in Zusammenarbeit mit Care International wurden die Residenzen im Clusterformat vor Ort konkret geplant ${ }^{1018}$. Die Autorität der Provinz in entschieden mit dem lokalen Personal der Distriktverwaltung gemeinsam, wer eine Aufforderung erhielt, in die Umsiedlungsgebiete einzuziehen. Die potentielle Zielgruppe lebte im Überschwemmungsgebiet verteilt. „We will make conditions for households to resettlement in new Commercial Centre by selling houses' ground with preferential prices. Besides, the poor households will be sold by deferred payment"1019, erklärte Tran Phat Huy, der Bezirksvorsitzende in Tan Chau.

Die Fläche der Umsiedlungscluster beträgt ca. $3 \mathrm{~km}^{2}$. Sie wurden in den Gebieten eingerichtet, die in der Regel am stärksten von den Überschwemmungen betroffen sind ${ }^{1020}$. Ihre Lage hat man 0,5 - 1,0 $\mathrm{m}$ über den Hochwasserpegel von 2000 positioniert, in unmittelbarer Nähe von Verkehrsknotenpunkten wie Kanälen, hochwasserfreien Straßen oder Flüssen ${ }^{1021}$. Ihre Infrastruktur zeichnet sich unter anderem durch einen Marktplatz, Schulen und einem Krankenhaus aus. Anschlüsse an das Telekommunikationssystem und Straßen-, Wasser- und Elektrizitätsanschlüsse sollen den Menschen die Möglichkeit bieten, am modernen Leben teilzuhaben ${ }^{1022}$. Da die Regierung durch das Hochwasserschutzkonzept einen Schutz vor Flutung impliziert, sind die Cluster in der Regel von Deichen geschützt ${ }^{1023}$. Laut Verordnung müssen in jeder Kommune des Überschwemmungsgebiets im MekongDelta mindestens zwei Umsiedlungscluster eingerichtet werden. Zwischen 2001 und 2005

\footnotetext{
1015 Tuan 2007.

${ }^{1016}$ Ebd.

${ }^{1017}$ Sanh/Can 2009.

${ }^{1018}$ Tuan 2007.

${ }^{1019}$ Khu vuc co nguy co sat lo o Tan Chau: Thuc trang va giai phap, Zeitung An Giang, 01.08.2000.

${ }^{1020}$ Danh 2011.

${ }^{1021}$ Ebd.

1022 Ebd.

1023 Ebd.
} 
wurden 1043 Umsiedlungsgebiete für 1 Mio. Menschen angelegt ${ }^{1024}$. In den tief überschwemmten Regionen des Deltas gab es 2011 ca. 1200 Cluster $^{1025}$. Abb. 23 zeigt die Aufnahmen eines Clusters einer islamischen Minderheit im Bezirk An Phu. Die Gebäude stehen in Reih und Glied, auf den kargen Böden wird kaum angebaut. Das Cluster liegt an einer Straße, die als Deich funktioniert, auf dem Weg zur Moschee muss eine steile Treppe überwunden werden.

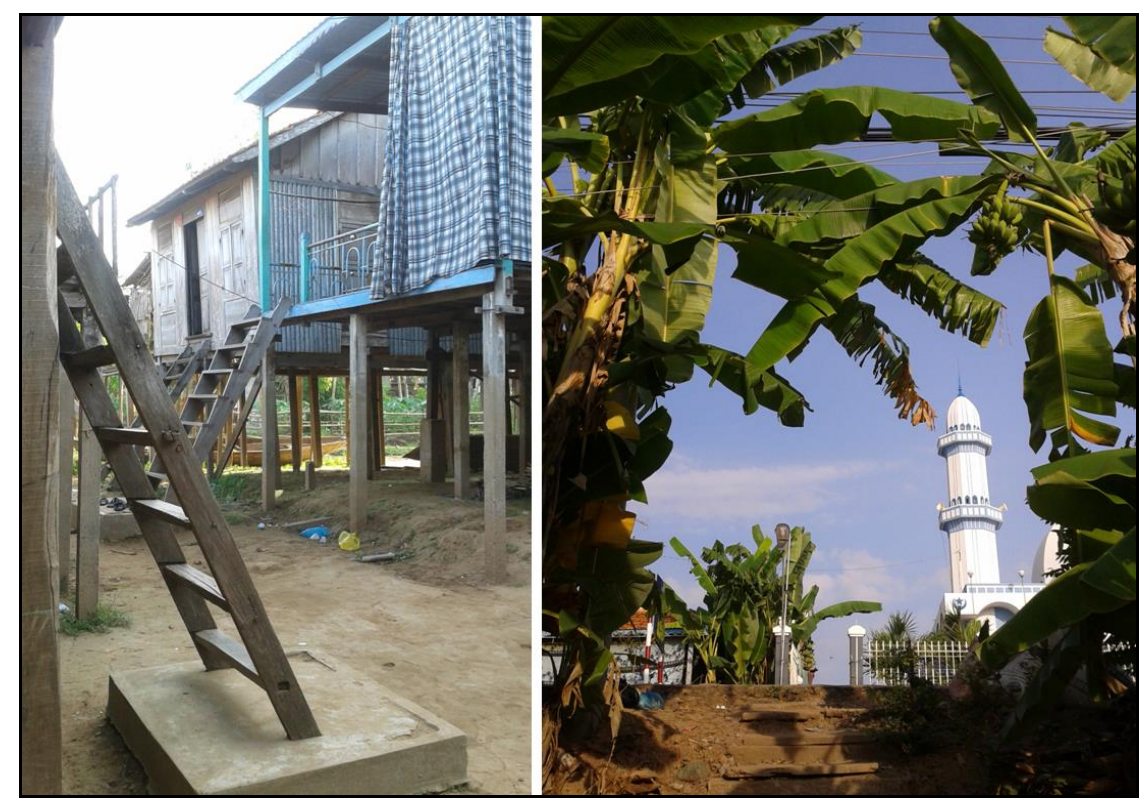

Abb. 23: Hochwassercluster und Umsiedlungsprojekt im An Phu -Distrikt. ${ }^{1026}$

Eine Instandhaltung der Siedlungsstruktur soll für soziale Sicherheit bei Flutzeiten sorgen und das Leben der Bevölkerung normalisieren ${ }^{1027}$. Ein moderner Lebensstil erfordert die Anpassung der Menschen an die natürlichen Gegebenheiten, schloss der Biologe und Ingenieur Le Anh Tuan:

"The concept of „living with floods" is pursued but tempered by recognition of need, for example, $[\ldots]$ that modern agriculture and commercial activities are no longer all floodtolerant. Changes in lifestyles and aspiration as well as migration in of people less familiar with seasonal flood pulse have produced new unanticipated new [sic] risks, for example, for death by drowning for children." 1028

\footnotetext{
${ }^{1024}$ Ebd.

1025 Ebd.

${ }^{1026}$ Eigene Aufnahmen.

1027 Tuan 2007.

${ }^{1028}$ Ebd.; Danh 2011.
} 


\subsubsection{Interpretation des Diskurses zum Hochwasserschutz}

Der Diskurs zum Deichbau im Mekong-Delta konnte in zwei Ansätze unterteilt werden. Zunächst wurden durch die Zentralregierung Deiche gebaut, um die Felder für eine landwirtschaftliche Nutzung hochwasserfrei zu halten. Zu Beginn der 1990iger Jahre manifestierte die Regierung ihre Bauvorhaben im Mekong-Delta-Master-Plan. Ab 2000 betrachtete die Regierung die privaten Verluste der im Delta lebenden Menschen als schützenswert, dazu wurde die Living with Floods-Richtlinie entworfen. Ihre Umsetzung verhindert ein Leben mit dem Hochwasser, da die Menschen in Hochwasserschutzcluster umgesiedelt wurden. Beide baulichen Maßnahmen werden interpretiert.

Aus den Ausführungen zum MDMP ergeben sich die folgenden Erkenntnisse. Die Regionalplanung des Mekong-Delta wurde zwischen der vietnamesischen Regierung und internationalen Akteuren ausgehandelt. Somit steht der Deichdiskurs neben dem Desasterund Landwirtschaftsdiskurs als Produkt internationaler Beratung und Finanzierung. Die staatlichen Reaktionen auf das Hochwasser im Mekong-Delta wurden aufgrund eines homogenen Expertenkreises aus ausschließlich technokratischer Perspektive beeinflusst. Die nationalen und internationalen Experten dominierten die Diskurse mit harten Fakten basierend auf statistischen Extrapolationen, sodass umweltbezogene und sozialökonomische Studien nicht berücksichtigt wurden. Die steigende Anzahl von Publikationen bestätigt, dass erst die verheerende Überschwemmung aus dem Jahr 2000 zum Auslöser wurde, Sozialforschung im Kontext der jährlichen Hochwasser zu betreiben ${ }^{1029}$. Die entscheidenden Faktoren bei der Planung von Deichprojekten bestanden in Vietnam aus dem Ineinandergreifen von Expertenwissen, institutionellen Eigeninteressen und der Gewichtung gesellschaftlicher Prozesse und Ressourcenallokationen einiger weniger Entscheidungsträger ${ }^{1030}$. In Vietnam wurden zentralstaatliche Behörden der Wasserwirtschaft und Wasserbauunternehmen hauptsächlich durch Nordvietnamesen geführt ${ }^{1031}$. Die Führungskräfte stammten ausschließlich von der Universität Hanoi, die Fachkräfte im Bereich des Bewässerung- und Wassermanagements ausbildete ${ }^{1032}$. Das technische Expertenwissen spielte in dem Kanon eine herausragende Rolle, denn es wurden ausschließlich Experten eines bestimmten Fachbereichs (Ingenieure) konsultiert ${ }^{1033}$. Hohes Gewicht wurde dabei ebenfalls internationalen Experten beigemessen ${ }^{1034}$. Anders als in Deutschland wurde der bereits bestehende innervietnamesische Hochwasserschutzdiskurs nicht durch Akteure aus dem

\footnotetext{
${ }^{1029}$ Vgl. Danh, Be, Tuan, Sinh, DRAGON, WISDOM, MRC u. v. a.

${ }^{1030}$ Lebel et al. 2009.

${ }^{1031}$ Evers/Benedikter 2009.

${ }^{1032}$ Ebd.

${ }^{1033}$ Ebd.

${ }^{1034}$ Ebd.
} 
Flusseinzugsgebiet, sondern durch Repräsentanten wirtschaftspolitischer Organisationen amerikanischer Herkunft erweitert.

Die Ziele des MDMP können in zwei Kategorien zusammengefasst werden, sie betreffen einerseits die Intensivierung der Landwirtschaft und andererseits entwicklungspolitische Ziele in Form von ökonomischem Wachstum. Ziele im Bereich des Desasterdiskurses fehlen im MDMP. Die Deichvorrichtungen werden nicht aus dem Grund gebaut, die Anzahl der Todesopfer oder der Verlust des privaten Eigentums oder der öffentlichen Infrastruktur zu senken.

Der Aushandlungsprozess zur Errichtung eines Deiches in der Can Tho Provinz, südlich von An Giang, wurde durch einen Top-Down-Ansatz zentralisiert. Der Deichbau in Can Tho zeigt, dass die politische Ebene vor Ort kein Mitspracherecht hatte und somit „no voice“61035 erheben konnte und sich im Planungsprozess übergangen fühlte. Hier stellt sich heraus, dass die Bezirksvertretung in Form des Planungsbüros ARDO von den höheren politischen Instanzen instrumentalisiert wurde, um vor Ort die Massen zu besänftigen und Einfluss geltend zu machen. Hingegen konnten die Mitglieder des ARDO keinen Einfluss auf die Entscheidungsebene ausüben. Die Betroffenen im Mekong-Delta wurden systematisch von der Planung ausgeschlossen. Um diesen Ausschluss zu gewährleisten, instrumentalisierte die nationale Regierung in Hanoi die politischen Strukturen in der Provinz. Somit wurde die bereits vorhandene Hierarchie durch die Verantwortlichen weiterhin in allen Ebenen der Gesellschaft gefestigt. Die ländliche Bevölkerung wird auch künftig von den oberen Behörden - in Anlehnung an Huu`s Formulierung - geopfert, um mit dem Versprechen, landwirtschaftliche Erträge zu sichern, die Bevölkerung zu lenken. Die Planung der Flächennutzung ist ein wichtiges Instrument, um Kontrolle auszuüben und obliegt deshalb nicht der Provinzverwaltung. Trotzdem bildete das ausschlaggebende Kriterium der begrenzte Zugang zu finanziellen Ressourcen. Die Planer gaben an, eine andere Handlungsoption zu favorisieren, mussten dann aber auf die Gewählte zurückgreifen, da diese sukzessive Bauphasen zuließ. Ist diese Argumentation glaubhaft, oder hatten die Planer sich zu Gunsten der ausgewählten Option entschieden, die aber aufgrund der Abwägung von Vor- und Nachteile zu wenig Zuspruch fand?

In der Argumentation für die abgestimmte Option wird auf den Schutz für Obstbaumplantagen und Reisfelder hingewiesen. Die Hochwasserschäden der letzten Jahre stellten sich wie in Tabelle 8 dar:

\footnotetext{
${ }^{1035}$ Voss 2008.
} 


\begin{tabular}{|c|c|c|c|c|}
\hline & Reisanbaufläche & Wohngebäude & Infrastruktur & Obstbaumplantagen \\
\hline 1991 & 2,59 & 2,33 & 4,83 & 9,83 \\
\hline 1994 & 3,47 & 1,43 & 2,55 & 16,17 \\
\hline 1995 & 0,46 & 0,08 & 1,75 & 1,92 \\
\hline 1996 & 1,81 & 4,38 & 2,69 & 4,00 \\
\hline 2000 & 5,75 & 9,72 & 2,57 & 13,76 \\
\hline 2001 & 1,43 & 3,68 & 2,55 & 3,91 \\
\hline
\end{tabular}

Aus der Tabelle geht hervor, dass in den aufgeführten Jahren vornehmlich die Obstbaumplantagen von den Einbußen aufgrund des Hochwassers betroffen waren. Nur 1996 waren die Verluste aufgrund der Zerstörung von Wohngebäuden höher als an den Obstbaumplantagen. Die geringsten Schäden wurden jeweils in drei andren Bereichen verzeichnet: In vier von sechs betrachteten Jahren wurden diese auf Reisanbauflächen beklagt. Die Schäden an Wohngebäuden blieben verhältnismäßig gering, was daran lag, dass gemauerte Gebäude mit Elektrizität zu Beginn der 1990er Jahre noch nicht weit verbreitet waren. Überraschend sind die geringen Verluste auf Reisfeldern im Vergleich zu Schäden an Unterkünften in den Jahren 1996, 2000 und 2001. Die Probleme am Straßen- und Bewässerungssystemen blieben nahezu konstant, obwohl die Hochwasser von 1994 und 2000 als Jahrhunderthochwasser gelten. Die nachvollziehbarste Reaktion wäre an der Höhe der Schäden orientiert und hätte zum Ziel, diese künftig zu vermeiden. Aufgrund der hier dargestellten Schäden ist der Schutz der Obstbaumplantagen nachvollziehbar.

Die Interpretation der living with floods-Richtlinie wird ebenfalls im Lichte der Kostenentwicklung betrachtet. Das Hochwasser verursachte physische und psychische Kosten, die die Regierung tragen musste, die jedoch im Grunde vom Verhalten der Bürger abhingen. Einerseits gab es jedes Jahr eine hohe Anzahl von Todesopfern zu beklagen, anderseits fielen Kosten an, da die Regierung gelegentlich Bürger aus den überschwemmten Gebieten evakuieren musste, um deren Sicherheit wiederherzustellen. Diese Kosten sollten mit der Einführung der Umsiedlungen zukünftig vermieden werden. Demgegenüber standen die Kosten, die die Umsiedler aufgrund der Veränderungen tragen mussten. Es stellt sich die Frage, ob sich die Umzüge für die vom Hochwasser bedrohten Menschen gelohnt haben.

\footnotetext{
${ }^{1036}$ Daten nach Huu 2011. Der Umrechnungsfaktor von 15000 VND/USD wurde zu Grunde gelegt. Infrastruktur steht in diesem Zusammenhang für Transport und Bewässerungssysteme.
} 
Die Regierung ließ in den letzten Jahren immer wieder Menschen aus den Überschwemmungsgebieten evakuieren; Tabelle 9 beschreibt die Evakuierungen seit 1997. Bei dem zweitgrößten Hochwasser 1994, was unter anderem mit einem Pegel von 4,53 m als Bemessungshochwasser gilt, wurden keine Evakuierungen vorgenommen. Laut Datenmaterial wurden vor 1997 noch keine Anwohner der Überschwemmungsgebiete evakuiert. Bei der Überschwemmung von 2000 stand das Wasser in etwa einen halben Meter höher als bei durchschnittlichen Hochwassern und verursachte aufgrund des früheren Eintreffens höhere Schäden als gewöhnlich ${ }^{1037}$. Es wurden 15.454 Haushalte in der Provinz An Giang evakuiert. Die hohe Evaluierungszahl belegt, dass auch die administrativ Verantwortlichen in Alarmbereitschaft versetzt waren. Nachdem die Umsiedlungscluster seit 2002 eingerichtet wurden, sank die Anzahl der evakuierten Haushalte. Die Maßnahme wurde folglich hinfällig. Neben den Evakuierungen half die Regierung den Opfern auch finanziell. Finanzielle Unterstützung für Privatpersonen war allerdings nur in zwei Jahren nachzuweisen. Da die Hilfen in den Jahren 1994 und 2000 gezahlt wurden, ist anzunehmen, dass der Staat nur bei herausragenden Ereignissen eine direkte Unterstützung an Haushalte leistet.

\begin{tabular}{|l|l|l|l|l|l|l|l|l|}
\hline & 1991 & 1994 & 1995 & 1996 & 1997 & 2000 & 2001 & 2004 \\
\hline Evakuierte Haushalte & 0 & 0 & 0 & 0 & 1.513 & 15.454 & 5464 & 112 \\
\hline Staatliche Unterstützung für Personen & 0 & 10.8131 & 0 & 0 & 0 & 327.909 & 0 & 0 \\
\hline
\end{tabular}

Tabelle 9: Evakuierte Haushalte und staatliche Unterstützung 1991 - 2004. ${ }^{1038}$

Für die Regierung standen Kinder als besonders betroffene Gruppe im Zentrum der Strategie. Dabei argumentierten die Verantwortlichen mit zwei verschiedene Herausforderungen: Zum einen nehmen Kinder mit bis zu $90 \%$ einen großen Anteil an der Summe der ertrunkenen Personen ein und zum anderen gehen die Kinder bei Hochwasser für Monate nicht regelmäßig, die Bildung leidet.

„In 2006 half of the 18 million people living in the Delta were children. Most of drowned children come from the poorer communities although they habituated with the water around. Local people said that almost the death of young children occurred at night when they rowed and fell down the water while sleeping. The vast majority of children drowning are mostly aged under [sic] six years of age."

Eine Betrachtung der Zahlen der Todesopfer verdeutlicht, dass die Anzahl der Todesfälle nicht mit der Hochwasserhöhe korreliert. Während bei dem Hochwasser 2000 mit einem

\footnotetext{
${ }^{1037}$ Artikel: Cong tac phong chong lu duoc tiep tuc dat trung tinh trang bao dong, Zeitung An Giang, 08.08.2000.

${ }^{1038}$ Publikation des CCSFC 1991 - 2004. Internetbasierte Quelle 15.

1039 Tuan 2007, 4.
} 
Pegel von 5,06 m 211 Kinder umkamen, starben 1997 bei einem Hochwasserstand von 4,18 m 524 Kinder. Bei der höchsten Überschwemmung im Betrachtungszeitraum in 2000 waren $75 \%$ der Opfer Kinder. Jedoch war der Anteil der Kinder an den Gesamtopfern bei den drei niedrigsten Fluten in den Jahren 2004, 1995 und 1997 mit 85 - 90 \% am größten. In Tabelle 10 ist die Entwicklung der Opferzahlen zusammenfasst.

\begin{tabular}{|l|l|l|l|l|l|l|l|l|l|}
\hline Jahr & 1994 & 1995 & 1996 & 1997 & 2000 & 2002 & 2004 & 2005 & 2011 \\
\hline Tote (Anzahl) & 407 & 199 & 250 & 607 & 280 & 132 & 35 & 77 & 89 \\
\hline Anteil der Kinder in Prozent & 65 & 90 & 64 & 86 & 75 & 86 & 86 & 84 & 84 \\
\hline Pegel bei Chau Doc in Meter & 4,53 & 4,30 & 4,87 & 4,18 & 5,06 & 4,82 & 4,41 & 4,36 & 4,24 \\
\hline
\end{tabular}

Tabelle 10: Entwicklung der Opferzahlen 1994 - 2005. ${ }^{1040}$

Als in den 1990er Jahren die landwirtschaftliche Produktion ausgeweitet wurde, fanden zwei dieser Hochwasser statt. Wahrscheinlich waren die Kinder in dieser Zeit häufiger unbeaufsichtigt, da die Eltern auf dem Feld arbeiteten oder aber die Kinder ertranken als sie bei der Ernte mithalfen. Zwei Jahre nach der Einführung der Living with Floods-Richtlinie fielen die Opferzahlen rapide ab: In 2004 starben 35 Menschen, darunter 30 Kinder.

„In peak flow periods, all children in the high inundated areas are not attending their schools." ${ }^{1041}$ Neben dem Problem, dass immer wieder Schulen überschwemmt wurden und im Jahr 2000 in An Giang 11.670 Schüler ohne Klassenraum waren ${ }^{1042}$, besteht auch eine Herausforderung bezüglich des Schulwegs. Die Schulwege sind oft viel zu lang, wenn die Kinder aufgrund der Fluten bei Familienmitglieder untergebracht werden müssen ${ }^{1043}$. Nach der Einführung der Wohncluster stieg der Anteil der Kinder, die bei Hochwasser in den Kindergarten und in die Schule gingen auf $96 \%{ }^{1044}$. Die Regierung hat sich zum Ziel gesetzt, durch bessere Bildungsangebote die rurale Bevölkerungsschicht in eine prosperierende Zukunft zu leiten. Deutlich wird hier der Zugriff auf Kinder der Haushalte, die unter traditionellen Umständen in entlegenen Gebieten aufwachsen würden. Die erweiterte Kontrolle über die Ideologien der jungen Generation kann im Licht der seit 1976 geführten Auseinandersetzungen mit den Deltabewohnern betrachtet werden, denn ganz ausdrücklich fordert die Regierung: „Children in flooded areas should be moved to and kept in these

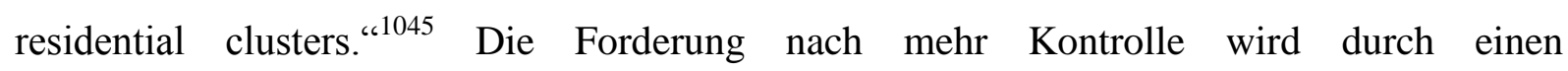
Sicherheitsdiskurs bezüglich des Hochwassers legitimiert. Immerhin gab es im Delta 2006 ca.

\footnotetext{
${ }^{1040}$ Ebd.

1041 Ebd.

1042 Artikel: An Giang truoc nhung yeu cau moi trong phong, chong lu, Zeitung An Giang, 15.09.2000.

${ }^{1043}$ Artikel: Thien tai lu lut o An Giang: Nhung manh doi dang rat can su giup do, Zeitung An Giang, 15.09.2000.

${ }^{1044}$ Danh 2011.

1045 Tuan 2007, 5.
} 
9 Mio. Kinder unter den 18 Mio. Deltabewohnern, die als Indikator zur Messung der Hochwassersicherheit herangezogen werden konnten. Verständlich wird das Bestreben der Regierung, ihre Vormachtsposition zu behalten.

Die Living with Floods-Richtlinie gibt an verschiedenen Stellen sehr genaue Anweisungen, so beispielsweise zur Anpassung des Aussaat- und Ernterhythmus, dieser wirkt wie eine Taktvorgabe durch die Regierung. Wirtschaftliche Entscheidungen werden dem jeweiligen Landwirt abgenommen und auf die Provinzadministration übertragen. Die Häuser und Straßen in den Clustern haben strikte Größenauflagen mit dem Ergebnis, dass alle Cluster gleich aussehen, denn sie folgen einer gleichen Infrastruktur. Die Möglichkeiten des gesellschaftlichen Lebens, die die Bewohner in Anspruch nehmen können, sind uniform. Dies gestaltet die Cluster kontrollierbar, da sie wie Inselsysteme funktionieren. Die gesteigerte Form der Kommunikation zwischen den Behörden und den Bürgern eröffnet den Kontakt zu den ehemals im Überschwemmungsgebiet verteilten Familien. Der verlängerte Arm der Regierung ermöglicht unter Einbezug der Technik, Befehle aus Hanoi vor Ort auszusprechen ${ }^{1046}$. Durch eine Begrenzung der Ressourcen, wie z. B. der begrenzte Zugang zu liquiden Mitteln, die Bindung an die Abtragung von Krediten und die begrenzte Subsistenzwirtschaft beschränkt die freie Kapazität der Landbevölkerung für alternative Interessen. Hinter den Deichen entsteht ein geschlossenes System von einkommensschwachen, landlosen Menschen, die mit der Zeit das traditionelle Wissen über ein Leben mit dem Hochwasser verlieren.

Danh publizierte 2011 eine Evaluierung der Living with Floods-Strategie, in der er die politischen Entscheidungen aufgrund von Befragungen der Umsiedler bewertete. Er kam zu dem Schluss, dass der Erfolg eines Projekts maßgeblich von der erreichten Infrastrukturqualität abhängt. Durch die Auswertung der Äußerungen der Betroffenen fand er heraus, dass die Wasserversorgung sowie die Abwassersysteme, die Versorgung mit Elektrizität und die Anbindung an Straßen unter den Zielvorgaben der Regierung zurückblieben und von den Umsiedlern kritisiert wurden. Die Mehrheit der Umsiedler gehörte vor dem Umzug zur Kategorie der einkommensschwachen Haushalte im Überschwemmungsgebiet $^{1047}$. Es zeigte sich, dass $85 \%$ der Haushalte einen Kredit in Anspruch nahmen, um Land und Unterkunft in dem Cluster erwerben zu können ${ }^{1048}$. Bei der Befragung gaben $45 \%$ an, dass sie nach dem Umzug mit weniger Einkommen, aber höheren

\footnotetext{
${ }^{1046}$ Die Botschaften können aufgrund der Lautsprechersysteme, welche in den Straßen installiert sind, direkt vorgetragen werden. Zwei Mal am Tag, morgens und abends, wird eine Durchsage gemacht. ${ }^{1047}$ Danh 2011. ${ }^{1048}$ Ebd.
} 
Ausgaben konfrontiert waren ${ }^{1049}$. Durch das Verbot der Nutztierhaltung und Fischzucht sowie den begrenzten Zugang zu landwirtschaftlich nutzbaren Flächen verloren die Haushalte die Möglichkeit zur Subsistenzwirtschaft ${ }^{1050}$. Enger wurde der Haushalt auch durch andere Einschnitte. $33 \%$ gaben an, sie müssten geringere Einkünfte aufgrund eines Arbeitsplatzwechsels hinnehmen. Der Anteil an Landbesitz pro Person verkleinerte sich ebenfalls. Von denen, die Land verkauft hatten, gaben $68 \%$ als Begründung an, dass sie in finanzielle Engpässe geraten waren ${ }^{1051}$.

„Many poor households did not want to move into the residential clusters because they were not confident of their capacity to back loans. In one sense it was a shift from a flood to debtrepayment risk. Moreover, clusters have insufficient water and sanitation systems, poor public facilities or employment opportunities." 1052

Wie bereits aufgeführt werden konnte, machte Danh deutlich, dass die nicht-strukturellen Maßnahmen größere Erfolge verzeichneten als die baulichen Maßnahmen. Die Anzahl der Todesopfer ging drastisch zurück, was auf bessere Bildung, ein präziseres Frühwarnsystem, ein höheres Gefahrenbewusstsein und eine intensivere Kommunikation der Behörden mit den Anwohnern zurückgeführt wurde. Die baulichen Maßnahmen waren nicht erfolgreich. Das landwirtschaftlich nutzbare Land verlor hinter den Deichen an Fruchtbarkeit, der schnellere Abfluss des Hochwassers begünstigte Erosion und die Menschen in den Rückzugsgebieten wurden gefährdet, ein Leben an der Armutsgrenze $\mathrm{zu}$ führen. Die Living with FloodsRichtlinie stellte die Menschen in ihrer Lebensqualität schlechter als zuvor und zeigt somit nicht das gewünschte Ergebnis. Fragwürdig wird, welches Ziel der Regierung hinter dem Projekt steht. Welche Intention verfolgte die Regierung mit ihrem Angebot die Lebensstandards der Menschen zu ,modernisieren“?

Louis et al. kommentierten die verstärkten Ambitionen im Hochwasserschutz: „Floods and disaster are political opportunities to produce projects.“ ${ }^{1053}$ Mit der Anzahl der Projekte verdichtet das MARD die flächendeckende Umstrukturierung des Deltas. Doch Lebel wies in der Literatur noch auf einen zweiten Aspekt hin:

„Exaggeration of flood damages in past events or assessments of future threats create opportunities to make promises of bigger infrastructure. Fulfilling protection promises is good business." 1054

\footnotetext{
1049 Ebd.

${ }^{1050}$ Ebd.

${ }^{1051}$ Ebd.

1052 Lebel/Sinh 2009.

${ }^{1053}$ Lebel et al. 2009, 297.

${ }^{1054}$ Ebd.
} 
Seit der Wiedervereinigung unterhielten die staatlichen Politikebenen eigene Baubetriebe, die unter militärischer Führung standen ${ }^{1055}$. Da das nordvietnamesische Verwaltungssystem auf den Süden übertragen wurde, wurden die dortigen Strukturen durch den Einsatz von Experten aus Nordvietnam aufgebaut. Die neu etablierten Baubetriebe standen in keiner Konkurrenz zu privaten Unternehmern und setzten die staatlichen Aufträge um. Seit der Doi Moi Reform 1986 können private Firmen gegründet werden und die Vergabe der staatlichen Projekte unterliegt ab einem Volumen von mehr als 60.000 USD einer Ausschreibungspflicht. Staatliche Baubetriebe wurden daraufhin in private Unternehmen umgeschrieben, wobei $51 \%$ des Firmenkapitals in staatlicher Hand verblieben. Wer einen Auftrag erhält, hängt nicht zuletzt davon ab, wer in der Lage ist informelle Verbindungen zu nutzen. „The system operates in its old manner. [...] always the same companies are contracted to design and carry out the construction works of large-scale schemes [...].“1056

In diesem Sinne kann das Hochwasser als Glücksfall interpretiert werden, welches den staatlichen Organisationen finanzielle Mittel in die leeren Kassen spült. Die zweite Seite der Katastrophe wird hier deutlich. Nicht zuletzt bedeutet die Terminologie Katastrophe eben auch „,öllig umdrehen, auf den Kopf stellen““1057. Eine Umkehr des Machtverhältnisses wird den politisch Verantwortlichen durch den Einsatz von massiven Wasserbauwerken im Delta zuteil $^{1058}$. Aufgrund der Finanzstärke der neuen Verhandlungspartner konnten bereits nach der Wiedervereinigung begonnene Projekte zur Identitätsbildung und Kontrolle in einem neuen Maßstab ausgeführt werden. Die ungehorsame Landbevölkerung lebte bis dahin in Anpassung mit den jahreszeitlich bedingten ,beautiful floods ${ }^{\text {“1059 }}$ als „Katastrophenkultur ${ }^{\text {1060 }}$. Die Menschen hatten ihren Lebensstil an die natürliche Umgebung abgestimmt. Dies lässt sich von der Bauweise der Häuser, dem Saison bedingten Speiseplan, der Nutzung der Wasserstraßen als Transportwege und den verschiedenen Termini für Hochwasser in der vietnamesischen Sprache ableiten ${ }^{1061}$. Tabelle 11 zeigt eine Übersicht über die Entwicklung des Häuserbaus von gemauerten Häusern und Holzhäusern auf Stelzen in den Jahren 1991 1995. Währenddessen änderte sich die Wohnfläche von ca. $7 \mathrm{~m}^{2}$ pro Person nicht. Häuser aus

\footnotetext{
${ }^{1055}$ Evers/Benedikter 2009. Dieser und die nächsten acht Sätze beziehen sich auf diese Literaturangabe.

${ }^{1056}$ Waibel 2010, 19.

1057 Masius 2010.

${ }^{1058}$ Der hier eröffnete Einblick in die Vergabe und Ausführung der staatlichen Aufträge lässt vermuten, weshalb es lediglich einige wenige Veröffentlichungen zum technischen Hochwasserschutz gibt. Diese basieren gewöhnlich auf empirischen Interviews vor Ort und nicht auf archivierten Dokumenten der staatlichen Organisationen.

${ }^{1059}$ Ehlert 2012.

${ }^{1060}$ Bankoff 2009.

${ }^{1061}$ Lebel/Sinh 2009. Das langsam ansteigende Wasser wird mit einem ruhigen Charakter gleichgesetzt. Im MekongDelta wird das Hochwasser mit dem Begriff Nuoc noi betitelt, was etwas Weichem und Fruchtbaren entspricht. Hochwasser in den Gebirgen oder im Delta des Roten Flusses im Norden gleichen zerstörerischen Wassermassen, die plötzlich auftreten und eine Gefahr darstellen. Die reißerischen Hochwasser werden Lu lut genannt.
} 
natürlichen Baustoffen dominierten das Siedlungsbild zu Beginn der 1990er Jahre, wie die Tabelle vorgibt. Nur ca. $5 \%$ der Unterkünfte bestanden in der Phase aus gemauerten Häusern. Der Trend, Häuser fest zu bauen, ist aber bereits erkennbar. Zwischen 1991 und 1995 nahm der Anteil um 16,28 \% zu, während 12,7 \% von den Holzhäusern verschwanden. Die Holzhäuser wurden vermutlich nach einem Hochwasser an einer anderen Stelle aus besten Materialen aufgebaut. Der Ausbau der Deiche und Straßen ermöglichte es den Menschen an vorgeblich sicheren Orten, moderne Häuser zu errichten.

\begin{tabular}{|c|c|c|c|c|c|}
\hline In Prozent $\%$ & 1991 & 1992 & 1993 & 1994 & 1995 \\
\hline Gemauerte Häuser & 4,3 & 4,7 & 5,0 & 5,0 & 5,0 \\
\hline Holzhäuser & 75,6 & 74,3 & 72,5 & 72,4 & 66,0 \\
\hline
\end{tabular}

Die Bewohner des Mekong-Deltas lebten bis in die 1990er Jahre angepasst an die Natur. Sie haben mit Hilfe von Stelzen ihre Häuser an die Hochwasserjahreszeit angepasst. Abb. 24 zeigt ein solch typisches Haus. In An Giang nutzten 1994 nur $31 \%$ der Haushalte Elektrizität, während nur $4 \%$ Zugang zu einer standardisierten Toilette hatten und immer noch $76 \%$ der Haushalte das Flusswasser für den täglichen Gebrauch nutzten ${ }^{1063}$.

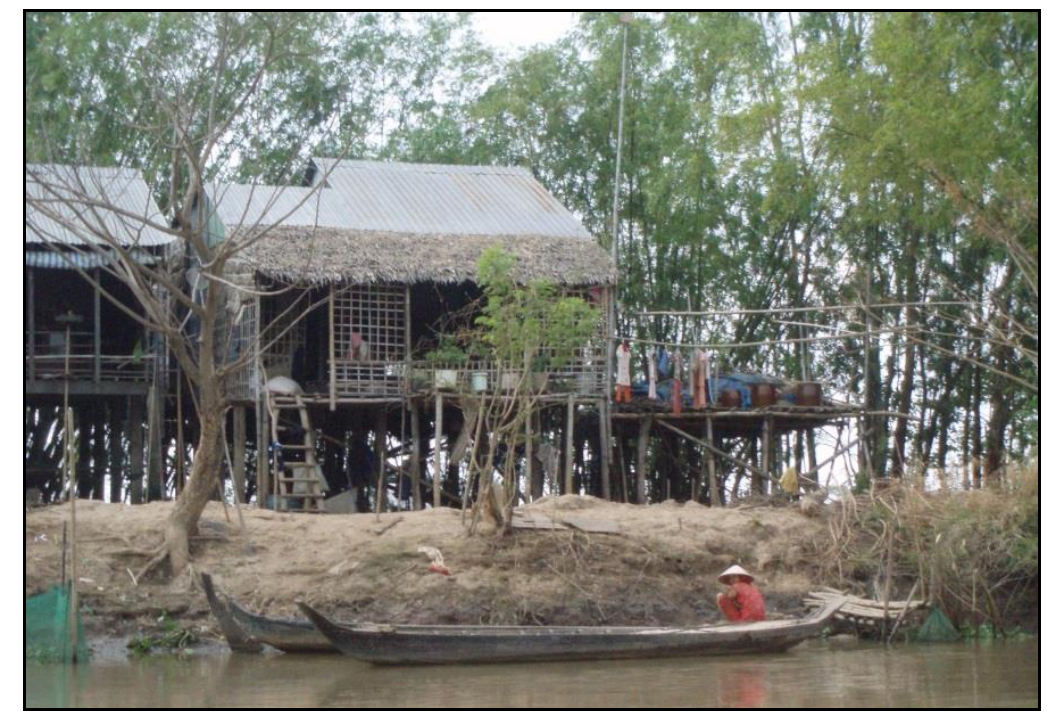

Abb. 24: Typisches Haus auf Stelzen im Tan Chau-Distrikt. ${ }^{106}$

Das Kapital der internationalen Geber verhalf der Regierung in Übereinstimmung mit Entwicklungszielen im Mekong-Delta Tabula Rasa zu machen. Die Regierung nutzt die Medien um den Kampf gegen die Schäden des jährlichen Hochwassers, in dem auch das

\footnotetext{
1062 Daten nach Tong 1997.

${ }^{1063}$ Artikel: An Giang voi giai phap chong o nhiem moi sinh, Zeitung An Giang, 16.03.1995.

${ }^{1064}$ Eigene Aufnahme.
} 
Militär eingesetzt werden muss, zu dramatisieren ${ }^{1065}$. Die saisonalen Überschwemmungen bieten alljährlich eine Vorlage, die bei den Rettungsarbeiten eingesetzten Soldaten zu Ikonen der Wiedervereinigung zu erheben: „The image of Uncle Ho's soldiers of armed forces will be always remembered [sic] forever in the An Giang people`s heart" ${ }^{\text {1066 }}$. Die alljährlichen Hochwasser ersetzten das Feindbild, welches Teile der Gesellschaft während der Kriege jahrzehntelang einte, denn die Regierung verlangt auch im Jahr 2000:

„Firm determination of local people, however, this must ensure unity, obey common guidance, avoid local methods and lack of consistence. With the tradition about winning invaders, the solidarity between An Giang's military and people will help An Giang province overcome disaster, and have peaceful, prosperous lives" ${ }^{, 067}$.

Die künstliche Art des ,nation-building“61068 erschuf vor dem Hintergrund naturaler Extremereignisse den perfekten Landwirt als zivilgesellschaftliches Vorbild in einer nicht zu zivilisierenden Umwelt. Aus der Konfliktsituation gehen jährlich neue Katastrophenszenarien hervor und sorgen dafür, dass der kontinuierliche Kampf fortgesetzt werden kann $^{1069}$.

Die Umstrukturierungen veränderten den traditionellen Lebensstil der Deltabewohner von Grund auf und ermöglichten damit einen Neubeginn. Die Stellung des Landwirts als Entscheidungsträger wurde durch das Ende der Pumping Clubs mit der Verbauung von massiven Deichkonstruktionen markiert ${ }^{1070}$. Seitdem die Hochwasser aus den eingedeichten Gebieten verbannt wurden, bestimmte die kommunale Regierung die Flutungsintervalle, da sie für die Bedienung der Schleusen verantwortlich ist ${ }^{1071}$. Somit wurde die Kontrolle über den Zugang zu Wasser und über den Lebensrhythmus der Landbevölkerung von den Bauern

\footnotetext{
1065 Artikel: Tham sam tinh quan - dan, Zeitung An Giang, 01.08.2000.

1066 Artikel: Sang mai tinh quan dan trong nhung ngay chong lu, Zeitung An Giang, 01.08.2000.

1067 Artikel: Khac phuc kho khan vung vang truoc lu, Zeitung An Giang, 25.07.2000.

${ }^{1068}$ Pfister 2008; Biggs 2010.

1069 „Annual flooding is a recurrent feature of life in the Mekong River Delta. Floods in the Mekong-Delta are not perceived as disasters by many farmers and scientists," schreibt Tuan und weist darauf hin, dass es in der vietnamesischen Bevölkerung Meinungsführer gibt, die die Vorteile der Überschwemmungen größer bewerten als ihre Nachteile (Tuan 2007, 1). Auffällig wird eine immer wiederkehrende Rhetorik in wissenschaftlichen Untersuchungen (Danh 2011; Sanh 2009; Lebel 2009; Tuan 2007), die Nutzen und Kosten des Hochwassers werden in den Erhebungen regelmäßig qualitativ gegenübergestellt. Dabei wird immer wieder betont, dass die Überschwemmung für Mensch, Boden und Wirtschaft, beispielsweise für die landwirtschaftliche Produktivität, von hohem Nutzen ist. Augenscheinlich wird aber sofort, dass die Überschwemmungen nur der mikroökonomischen Ebene Vorteile entgegenbringen. Der Landwirt muss aufgrund der natürlichen Düngereigenschaft der Sedimente, die das Hochwasser über die Felder trägt, geringere Inputkosten aufbringen als ein Landwirt, dessen Felder nicht mehr überschwemmt werden. Einen Vorteil für die Regierung scheint die Inanspruchnahme des natürlichen Wasserzyklus nicht aufzuweisen. Trotzdem gibt es unter den Wissenschaftlern eine Bewegung, die Vorteile des Hochwassers immer wieder mit empirischen Studien nachzuweisen und die massiven Deichbaumaßnahmen zu hinterfragen. Die Parteimitglieder im Bereich der Wissenschaft formieren eine subtile Protesthaltung gegen die vollkommene Abschaffung der Überschwemmungen. Sie diskutieren ihre Ergebnisse auf nationalen und internationalen Konferenzen mit Akteuren der Entwicklungszusammenarbeit sowie der eigenen Regierung und versuchen auf die Aktivitäten im Mekong-Delta Einfluss zu nehmen.

${ }^{1070}$ Howie 2011

${ }^{1071}$ Ebd.
} 
auf die Provinzadministration übertragen. Von dieser Gewalt machen die Behörden Gebrauch, denn: „No one interviewed in any commune reported that gates had been opened once a high dike was completed“1072. Ob das Handeln der Menschen nicht mehr von den natürlichen Gegebenheiten, sondern von der Zentralregierung geprägt sein sollte, bleibt hier offen. Es bestehen philosophische Denkansätze, die Rebound-Effekte der natürlichen Umwelt auf den Menschen suggerieren. Platon benutzte beispielsweise das Narrativ des Wachsdruckes und erklärte damit die Sichtbarkeit eines Umstandes als Voraussetzung für dessen Umsetzung oder Realität ${ }^{1073}$. Die vietnamesische Regierung organisierte ihre Sichtbarkeit im MekongDelta durch massive Wasserbaukunst. Die Denkmäler administrativer Macht sind flächendeckend aufgestellt und visualisieren den verlängerten Arm der Regierung in entlegenen Gebieten. Die Umgestaltung der Heimat entspricht dem Eingriff in das Innerste des Menschen, denn in Anlehnung an Radkaus Formulierung krönt die Intervention die Bemühungen der Regierung, die innere Natur der Menschen durch die äußere Natur zu beeinflussen. Die Kontroverse wird offenbar: „This Government of Vietnam promoted resettlement of people in flood-prone areas in protected residential clusters without giving up rhetoric of living with flood."1074 Durch die Umsiedlungen in hochwassergeschützte Cluster wurden Deltabewohner entwurzelt und mit ihrem persönlichen Neuanfang in einer künstlichen Umwelt konfrontiert. Da die Regierung die Betroffenen an der Finanzierung beteiligte, mussten diese härter arbeiten, um die Kreditraten zurückzahlen zu können ${ }^{1075}$. Mit der zunehmenden finanziellen Abhängigkeit vergrößerte sich ebenfalls der Zugriff auf die Menschen. Der Staat schuf durch den Deichbau einen wirtschaftlichen Druck zur ganzjährigen Arbeit: „To keep farmer's mind on production”1076, ist hier der zentrale Slogan der Regierung. Als messbares Ziel wurde die Produktion von sieben Reiszyklen innerhalb von zwei Jahren ausgerufen ${ }^{1077}$. Die sukzessive Einschränkung des traditionellen Lebensstils bot Raum für eine neue Identität, denn der rurale Bürger entspricht im besten Fall dem perfekten Landwirt, der sich zu jeder Zeit um die Produktion kümmert ${ }^{1078}$. Die Regierung spielt dabei

\footnotetext{
${ }^{1072}$ Howie 2011. Für den Service der Bewässerung der Ackerflächen erhebt die Provinzadministration eine Wassergebühr in Abhängigkeit von der Größe der zu bewässernden Ackerfläche.

1073, ,...] wessen wir uns erinnern wollen von dem Gesehenen oder Gehörten oder auch selbst Gedachten, das drücken wir in diesen [wächsernen] Guß ab, indem wir ihn den Wahrnehmungen und Gedanken unterhalten, wie beim Siegeln mit dem Gepräge eines Ringes. Was sich nun abdrückt, dessen erinnern wir uns und wissen es, solange nämlich sein Abbild vorhanden ist. Hat sich aber dieses verlöscht oder hat es gar nicht abgedruckt werden können, so vergessen wir die Sache und wissen sie nicht,“ (Staudacher et al. 2011, 155).

${ }^{1074}$ Lebel/Sinh 2009.

1075 Howie 2011.

1076 Artikel: Nhin lai va vuon len, Zeitung: Bao An Giang, 06.09.1995.

${ }^{1077}$ Howie 2011.

${ }^{1078}$ Bereits 1995 breitete sich eine Goldrauschmetalität aus: „Obviosly, nowadays, farmers in Chau Phu [...] know how to exploit thoroughly the potentials of their soil: 2 subsidary food crops -1 rice crop or 2 rice crops -1 subsidary
} 
die Rolle des Wohltäters, der für die nötige Infrastruktur sorgt. Aufgrund leerer Staatskassen entspricht die plötzliche Verfügbarkeit von Kapital bei diesem Neubeginn dem zentralen Auslöser für die Baumaßnahmen. Vietnams plötzlicher Zugang zu internationalen Entwicklungsgelder ist somit als ein window of opportunity auszumachen. Die Neuerung im Umgang mit dem Hochwasser wurde nicht durch ein besonders hohes oder verheerendes Hochwasser ausgelöst, sondern durch einen verbreiterten Zugang zu Kapital.

\subsection{Bewertung der Diskurse aus der Kosten-Nutzen-Perspektive}

Die vorliegende Ausarbeitung verdeutlicht, wie die drei Diskursstränge zunächst nebeneinander standen und später willkürlich miteinander verwoben wurden, um den Bau von massiven Hochwasserschutzanlangen und Umsiedlungsclustern zu legitimieren. Der Bau der Schutzanlagen war weder eindeutig auf die Desasterwahrnehmung noch auf die Optimierung der landwirtschaftlichen Entwicklung zurückzuführen, obwohl er unmittelbar von der Regierung durch die Ziele der beiden Diskurse begründet wurde. Um die Bewandtnis der Baumaßnahmen zu entschlüsseln, hilft die Beantwortung folgender Frage: Trieb der bauliche Hochwasserschutz die von der Regierung genannten Ziele an? Mit der Absicht die politischen Ziele zu validieren, werden die eingetroffenen Konsequenzen mit den propagierten Zielen abgeglichen. Damit soll keinesfalls die Effektivität der einzelnen Strategien ausgewertet werden, jedoch lassen sich Tendenzen erkennen, ob die genannten Ziele den tatsächlichen Absichten entsprechen konnten. In den vorgestellten Diskursen wurden die folgenden Ziele benannt:

- Diskurs zum Katastrophenmanagement ${ }^{1079}$

○ Verringerung der Opferzahlen

○ Verringerung der Schadenshöhe

- Diskurs zur Intensivierung der Landwirtschaft ${ }^{1080}$

○ Steigerung des landwirtschaftlichen Outputs

○ Steigerung der Einkommen der lokalen Bevölkerung

4. Diskurs zum Deichbau ${ }^{1081}$

food crop. Soil can genereate gold if farmers know how to use it." Chuyen dich co cau cay trong don bay de phat trien kinh te, Zeitung An Giang, 27.05.1995.

${ }^{1079}$ DR 0021992.

${ }^{1080}$ AG 461993.

${ }^{1081}$ White 2002. 
○ Vergrößerung der landwirtschaftlich nutzbaren Fläche

○ Steigerung des landwirtschaftlichen Outputs

○ Steigerung der Einkommen der lokalen Bevölkerung

- Diskurs zur Hochwassersicherheit Living with Floods ${ }^{1082}$

○ Verringerung der Opferzahlen

○ Verringerung der Schadenshöhen

Die verschiedenen Ziele überschneiden sich. Dies zeigt, dass einzelne Ziele durch verschiedene Methoden verfolgt wurden, was wiederum zunächst die Glaubwürdigkeit der Ziele betont. Für die Validierung können vier Hauptziele zusammengefasst werden:

- Minderung der Opferzahlen und Schadenshöhen

- Vergrößerung der landwirtschaftlich nutzbaren Fläche

- Steigerung des landwirtschaftlichen Outputs

- Steigerung der Einkommen der lokalen Bevölkerung

Die Konsequenzen der Veränderungen lassen sich wie folgt erfassen: Ob es eine Minderung der Opferzahlen und Schadenshöhe zu verzeichnen gab, wird durch einen Vergleich der Schadensbilanzen von vor und nach der Umsetzung der Sicherheitsmaßnahmen festgestellt. Ob die landwirtschaftlich nutzbare Fläche vergrößert wurde, wird durch die Betrachtung von Kartenmaterial erkennbar. Dabei stellt sich die Frage, ob die lokalen Landwirte die gewonnene Zeit tatsächlich für den Anbau nutzen oder wieder zu einer Zweiphasenwirtschaft zurückgekehrt sind. Die Steigerung des landwirtschaftlichen Outputs wird Statistiken entnommen ${ }^{1083}$ und eine Steigerung der lokalen Einkommen wird im Folgenden anhand von Sekundärliteratur ermittelt.

\footnotetext{
1082 Howie 2006.

${ }^{1083}$ Vormoor 2010. Klaus Vormoor gibt in seiner Studie einen Überblick zum Zugang zu statistischen Quellen in ländlichen Gebieten in Vietnam. Statistiken wurden nicht regelmäßig erhoben und weisen deshalb Lücken in dem zu Grunde liegenden Datenmaterial auf. Die Veränderung von administrativen Betrachtungssystemen und die wiederholten Erneuerungen von Definitionen verschoben zudem den Bewertungsmaßstab. Außerdem sind ländliche Statistiken nur in Druckform vor Ort zugänglich, da diese nicht durch Verlage publiziert und vertrieben werden. Vor Ort lassen sich manche Bücher nicht mehr auffinden und somit sind auch die gesammelten Daten verloren. Vormoors Bericht stimmt mit den Erfahrungen der Autorin überein.
} 
Das CCFSC veröffentlicht die eingetretenen Verluste jährlich nach der Hochwasserzeit. Die Entwicklung der Verluste zeigt Abb. 25.

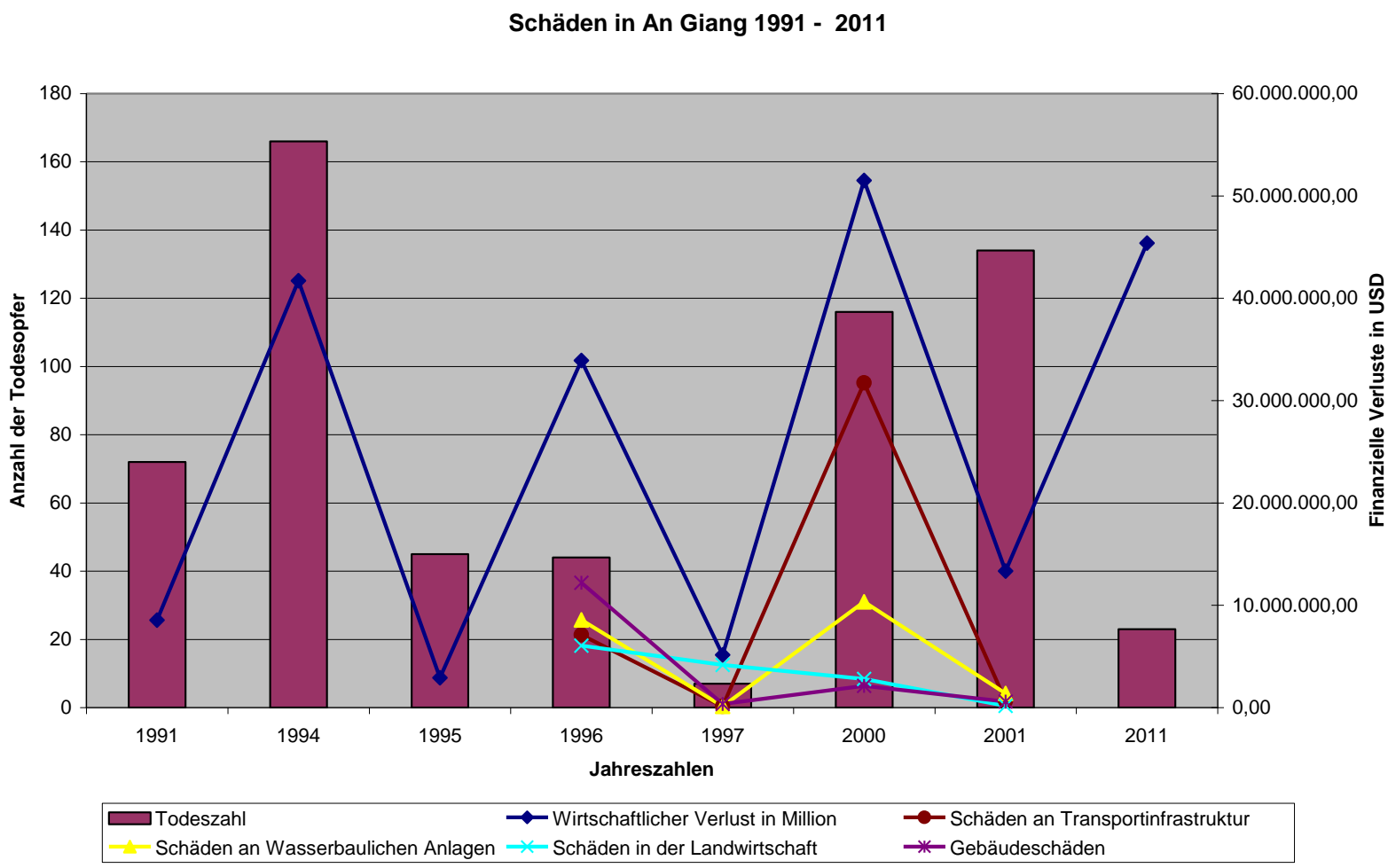

Abb. 25: Hochwasserschäden und Anzahl der Todesopfer 1991 - 2011 in An Giang. ${ }^{1084}$

Die vorliegenden Daten beschreiben acht Überflutungsperioden in einem Zeitraum von 20 Jahren. Für alle acht Beispiele sind die absoluten Schäden sowie die Anzahl der Todesopfer vorhanden. Die bereits zuvor vorgestellten Zahlen sprechen dafür, dass die Opferzahlen seit 2002 verringert werden konnten. Auch die Daten aus dem Jahr 2011 belegen, dass die Opferzahlen erfolgreich reduziert werden konnten. Die Entwicklung der gesamtwirtschaftlichen Schäden hingegen schwankt, da sie zwischen 2,8 - 51 Mio. USD variiert. Dementsprechend waren die Schäden in Jahren mit besonders hohen Pegelmessungen wie 1994 und 2000 hoch. Einen Großteil der Schadenssumme machte im Jahr 2000 die Zerstörung von Infrastruktur und wasserbaulichen Anlagen aus: Während 1991 im Delta $873 \mathrm{~km}$ Straße zerstört wurde, waren es im Jahr 2000 bereits $11.477 \mathrm{~km}^{1085}$. Das Auftreten dieses Verlustes ist relativ neu, da Deiche und Straßen zu Beginn der 1990er Jahre nur geringfügig in der Region installiert waren. Abb. 25 zeigt, dass die Landwirte offenbar zunehmend in der Lage waren, Teile der Ernte zu sichern, denn die landwirtschaftlichen

\footnotetext{
${ }^{1084}$ Internetbasierte Quelle 15. Die USD Angaben entsprechen dem Wert des VND in den jeweiligen Jahren zum jeweils 25. Dezember, da die Schadensaustellungen jeweils zum Jahresende ausgewiesen wurden. Es wurde der Währungsrechner http://fxtop.com/en/ benutzt.

${ }^{1085}$ Huu 2011.
} 
Schäden zeigen trotz großer Überschwemmungen eine sinkende Tendenz. Bereits im Juli 2000 wurden die Landwirte durch das Mitglied des Parteivorstands Vo Van Kiet aufgefordert: „Don’t let famine happen“1086. Für den Verlust der Reisernte gibt es neben der Zerstörung durch Hochwasser eine zweite Begründung. In Anlehnung an die jährlichen Erhebungen des MARD wurden $199470 \%$ des Reises auf der Straße ausgebreitet, um diesen zu trocknen ${ }^{1087}$. Dabei wird der Reis kontinuierlich durch den Verkehr überfahren. Der dabei entstehende Verlust betrug 1994 13,5 \% der Gesamternte. Dies entsprach einer Menge von 108.000 t, die entweder total vernichtet oder für eine weitere Aussaat unbrauchbar wurde. Der Verlust aufgrund des Hochwassers belief sich im gleichen Jahr auf $20-22.000 \mathrm{t}$, was maximal 2,75\% der Ernte entsprach. In An Giang konnten 1994 insgesamt 800.000 t Reis geerntet werden. Obwohl die Hochwasserschutzanlagen bis auf eine Länge von $20.000 \mathrm{~km}$ im Jahr 2000 ausgebaut worden waren ${ }^{1088}$, sind die gesamtwirtschaftlichen Schäden 2011 höher als zu der Zeit vor dem Ausbau der Dämme. Erst seit 1996 weist die Statistik Zahlen für bestimmte Schadensrubriken aus, wie Schäden an wasserbaulichen Anlagen, Schäden an der Transportinfrastruktur, landwirtschaftliche Schäden und Gebäudeschäden. Diese Einteilung verdeutlicht anhand der Schadenshöhe, in welchen Bereichen ein Optimierungsbedarf des Hochwasserschutzes besteht. Die Daten liegen nicht für alle einander folgenden Jahre vor, sodass die entstandenen Datenlücken eine zuverlässige Interpretation nicht möglich machen. Zusammenfassend kann dennoch dargelegt werden, dass die Schäden an landwirtschaftlichen Erzeugnissen sanken, die gesamtwirtschaftlichen Schäden jedoch kaum verringert wurden. Das Ziel der Schadensminimierung wurde nicht erreicht.

Die Fortschreitung der Landgewinnung wird auf Abb. 26 deutlich. Die rote Färbung markiert vor 2010 eingedeichte Flächen, die Farbe Gelb kennzeichnet im Jahr 2011 eingedeichte Gebiete und Grün verdeutlicht für das Hochwasser offenstehende Überschwemmungsgebiete. Die Karte stammt aus dem Jahr 2011 und zeigt alle Deiche, die zwischen 1993 und 2011 gebaut worden sind.

\footnotetext{
${ }^{1086}$ Artikel: Lu dong bang song Cuu Long len cao va co kha nang lan rong, Zeitung An Giang, 25.07.2000.

${ }^{1087}$ Artikel: Doi Moi cach phoi say lua vu he thu: trach nhiem cua nong dan va chinh quyen cac cap, Zeitung An Giang, 26.07.1995,

${ }^{1088}$ Vormoor 2010 .
} 


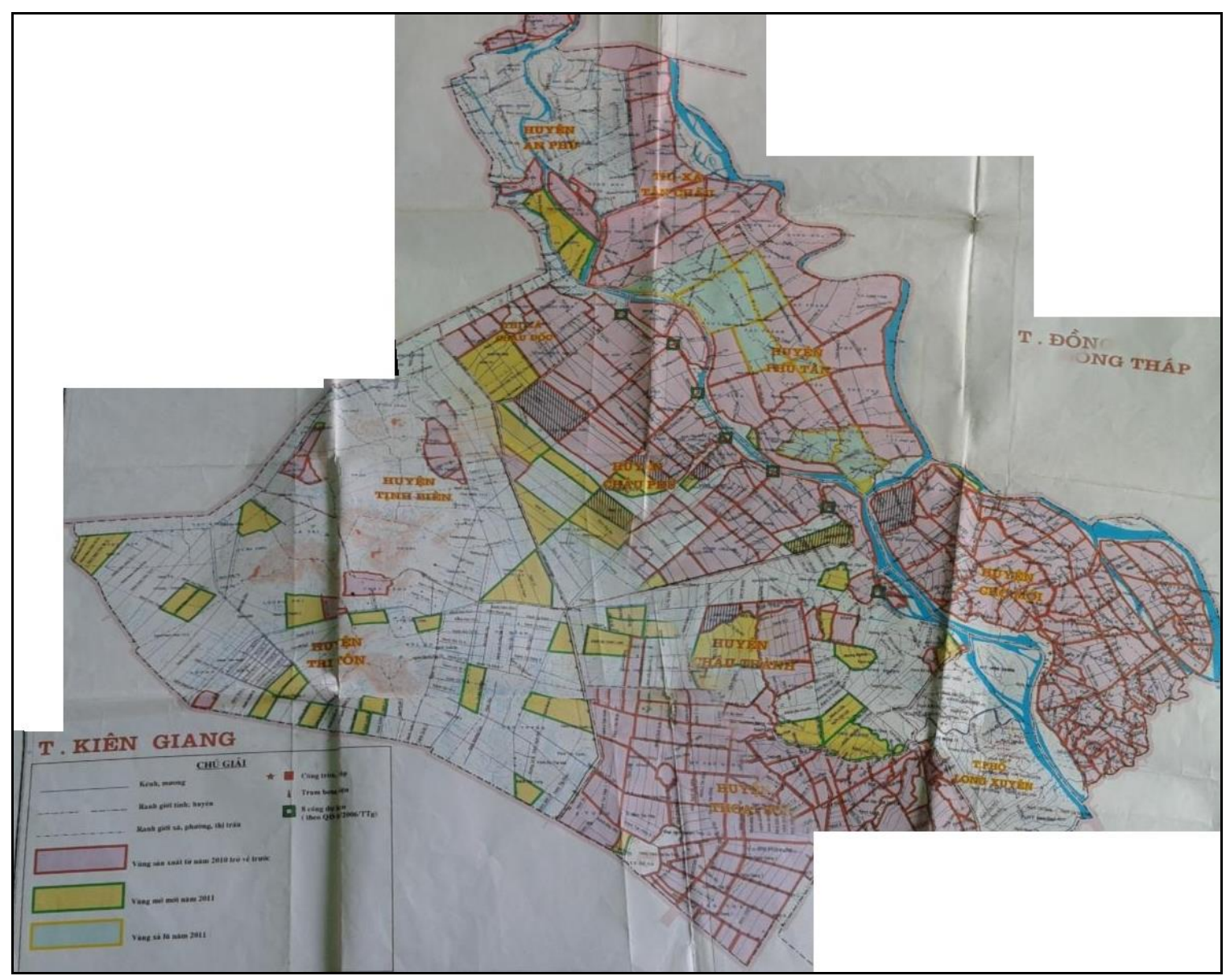

Abb. 26: Deichbau in der Provinz An Giang 2011. ${ }^{1089}$

Besonders deutlich zeigt sich die rote Fläche zwischen den beiden Mekongarmen. Die auf den Inseln gelagerten Bezirke Cho Moi, Phu Tan und Tan Chau sind fast vollständig durch Deiche umgeben. In dem Bezirk Cho Moi ist der Reisanbau mittlerweile durch Obstplantagen ersetzt worden ${ }^{1090}$. Aufgrund der Eindeichung entstand ein Topf, der in der Regenzeit voll Wasser lief und ein neues Schleusen- und Pumpsystem erforderlich machte ${ }^{1091}$. Im Bezirk Tan Chau gibt es westlich der rot markierten Gegenden grün eingefärbte Flächen. Diese Flächen liegen westlich des Deichprojektes von Tan Chau, welches im Rahmen des Desasterdiskurses 1994 entstand $^{1092}$. Die grün markierte Fläche in Tan Chau ist nicht durch einen Deich geschützt und steht dem Abfluss des Hochwassers offen. Im Norden der Provinz liegt An Phu an der Grenze zu Kambodscha. Dort liegen noch uneingedeichte Felder, die lediglich durch den Gebrauch von Augustdeichen geschützt werden. Weitere nicht eingedeichte Flächen sind entlang der

\footnotetext{
${ }^{1089}$ Eigene Aufnahme der Karte Ban Do San Xuat Vu Thu Dong Nam 2011, Tinh An Giang, im Maßstab 1/100.000. Übersetzung der Kartenlegende durch Nuyen Thi Bich Tram: Rot: Production area before 2010, Gelb: Newly-open area in 2011, Grün: Flood discharging area in 2011.

${ }^{1090}$ Interview John Douglas Flanders, Australian Agency for International Development, März 2012.

${ }^{1091}$ Artikel: An Giang truoc nhung yeu cau moi trong phong, chong lu, Zeitung An Giang, 15.09.2000.

${ }^{1092}$ Eine genauere Karte von Tan Chau und eine Luftaufnahme, welche Rückschlüsse auf die Bodennutzung zulässt, sind auf Seite $168 \mathrm{zu}$ finden.
} 
Grenze im Westen der Provinz in den Bezirken Tri Ton und Tinh Bien zu finden. In Tinh Bien besitzt das Gelände zunächst eine hügelige Struktur und erreicht bis zu $720 \mathrm{~m}$ Höhe in der ansonsten mit 0,8 bis $5 \mathrm{~m}$ über dem Meeresspiegel tiefliegenden Provinz ${ }^{1093}$. Aufgrund der Hügel hat sich auf der gezeigten Karte in Abb. 26 eine gelb-weiße Fleckenteppichstruktur entwickelt, welche sich bis zum südlich gelegenen Nachbarbezirk Thoai Son fortsetzt. Dort nutzen die Landwirte noch den natürlichen Überschwemmungszyklus, um die Felder zu düngen. Abb. 27 zeigt einen Augustdeich in Tri Ton sowie die in der Einleitung erwähnten Häuser auf Stelzen entlang eines Kanals. Bis 2005 ist die Reisanbaufläche im Mekong-Delta von zunächst $24.000 \mathrm{~km}^{2}$ auf $38.000 \mathrm{~km}^{2}$ ausgeweitet worden, was einer Vergrößerung der Anbaugebiete um $58 \%$ entspricht $^{1094}$.

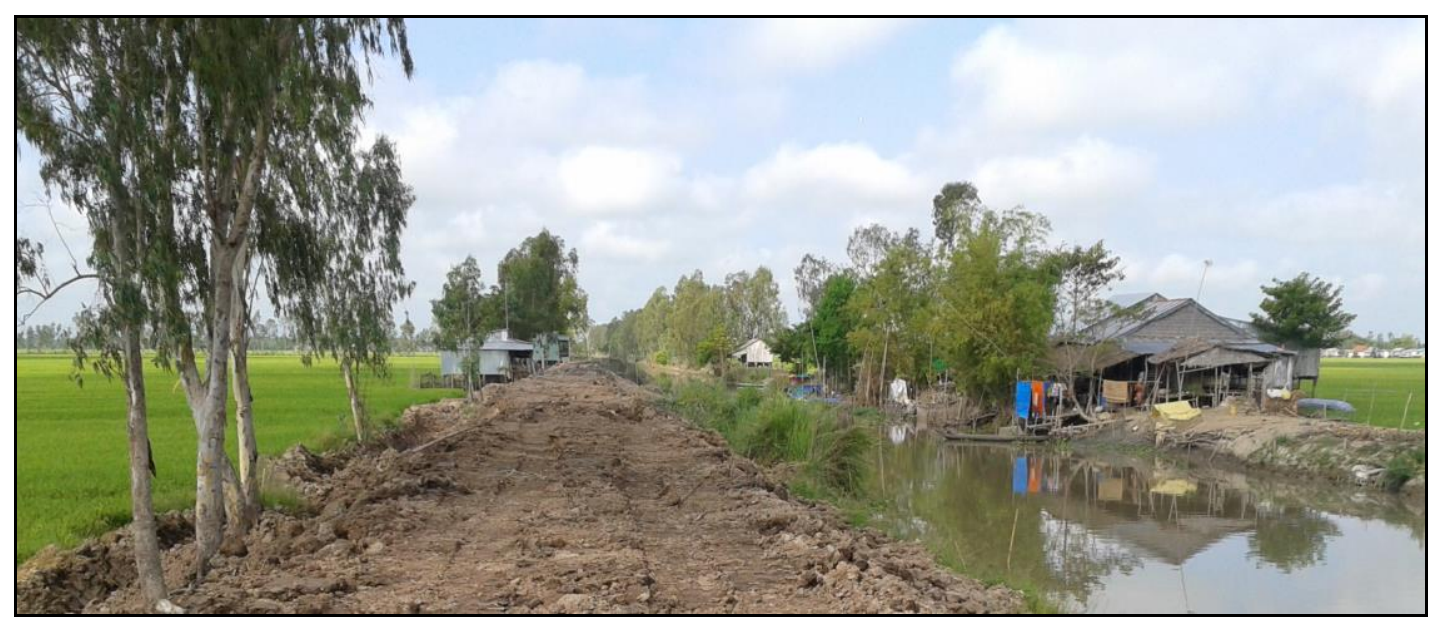

Abb. 27: Ein Augustdeich im Tri Ton-Distrikt. ${ }^{1095}$

Der Bezirk Chau Phu, südlich von Tri Ton, ist in der Karte teilweise weiß, gelb und rot hinterlegt. Neben vereinzelt geschützten Anbauflächen bestehen dort noch uneingedeichte Gebiete. In der Mitte der Provinz, im Bezirk Chau Phu, sind wiederum rot hinterlegte Flächen zu sehen, die bereits vor 2010 eingedeicht wurden. Die Strecke zwischen der Stadt Chau Doc, an der Grenze entlang des Hauarms bis nach Long Xuyen, der Provinzhauptstadt im Bezirk Thoai Son, ist bereits vor 2010 durch Deiche geschützt worden. Die Überschrift der Karte „Deichbau im Jahr 2011“ lässt ableiten, dass die Eindeichungsaktivitäten noch nicht beendet wurden und auch in Zukunft noch weitere Deichkonstruktionen geplant scheinen. Das Ziel, die vorhandenen Flächen aufgrund von Landgewinnungsmaßnahmen in den Überschwemmungsgebieten zu vergrößern, wurde eindeutig erreicht. Von einer tatsächlichen Landgewinnung kann aber nur gesprochen werden, wenn der neu gewonnene Boden auch

\footnotetext{
1093 Tong 1997.

1094 Tuan et al. 2008.

${ }^{1095}$ Eigene Aufnahme.
} 
landwirtschaftlich genutzt wird. Nach der Eindeichung und dem Anbau von drei Aussaaten in einem Jahr berichteten Landwirte jedoch über einen Ernterückgang von $47 \%$ pro eingesetzter Tonne Düngermittel gegenüber einer zweifachen Aussaat bei der Nutzung der natürlichen Überschwemmungen ${ }^{1096}$. Die verringerten Erträge und die gestiegenen Ausgaben für Kunstdünger rechtfertigen den Anbau der zusätzlichen Aussaat nicht mehr. Die gewonnen Flächen eignen sich folglich nicht für einen effizienten Reisanbau. Eine intensive KostenNutzen-Analyse unter Einbezug aller entstandenen Konsequenzen wäre nötig, damit bewertet werden kann, wie erfolgreich dieses Ziel erreicht werden konnte.

Ob das Ziel der niederländischen Ingenieure und der vietnamesischen Regierung, bis 2015 einen Produktionsumfang von 16 Mio. t Reis zu erzielen, erreicht werden kann, zeigt der Abgleich mit Produktionszahlen. Im Jahr 2000 wurden 16,2 Mio. t Reis im Mekong-Delta produziert, dies entsprach $50 \%$ des in Vietnam geernteten Reises und $90 \%$ des Exportreises $^{1097}$. Tabelle 12 zeigt den Anstieg der Reisproduktion in Vietnam in einem gemittelten Fünfjahresintervall. In der Zeit zwischen 1980 und 2004 wurde die Produktion mehr als verdoppelt. Das Ziel der Steigerung wurde somit erreicht. Das historische Verständnis von Natur als Ressource existierte bereits durch die Lehren von Ho Chi Minh in den 1930iger Jahren: Die „Beherrschung der Natur [galt auch Karl Marx] als unaufgebbare Bewährung“ des Menschen ${ }^{1098}$. Marx sah in dem Gebrauchswert der produzierten Sachen die Krönung der Naturbeeinflussung, so solle aus einem „An-sich“ der Natur ein „Für-uns“ entstehen $^{1099}$. Marx prägte den Begriff des Stoffwechsels und damit ein neues Verhältnis zwischen Mensch und Umwelt auf der Basis der Effizienz ${ }^{1100}$.

\begin{tabular}{|l|l|l|l|l|}
\hline $1980-84$ & $1985-89$ & $1990-94$ & $1995-99$ & $2000-04$ \\
\hline $13.740 .200 \mathrm{t}$ & $16.959 .400 \mathrm{t}$ & $21.360 .400 \mathrm{t}$ & $27.885 .000 \mathrm{t}$ & $33.820 .800 \mathrm{t}$ \\
\hline
\end{tabular}

Tabelle 12: Reisproduktion in Vietnam 1980 - 2004. ${ }^{1101}$

Der Wert der landwirtschaftlichen Erzeugung überschritt in An Giang 1991 die 1.000.000 VND pro Kopf Marke und stieg weiterhin steil an. So verdoppelte sich der Wert bis zum Jahresende 1995, und betrug 2005 bereits mehr als 6.000.000 VND pro Person ${ }^{1102}$. Das monatliche Einkommen verdreifachte sich in Vietnam zwischen 1996 und 2006 auf

\footnotetext{
1096 Howie 2011.

1097 Tanh/Singh 2006.

1098 Schmied-Kowarzik 1984, 65.

1099 Schmidt 1978, 74.

1100 Ebd.

1101 Nach Thanh/Singh 2006.

1102 Vormoor 2010.
} 
636.500 VND ${ }^{1103}$. In An Giang lagen die Menschen mit einem Einkommen von 691.000 VND pro Monat über dem landesweiten Durchschnitt, wobei der Gesamtbetrag für das Mekong-Delta etwas unter dem Landesdurchschnitt lag ${ }^{1104}$. Unter Armut litten in Vietnam 1993 noch $58 \%$ der Bevölkerung, der Anteil schrumpfte bis 2006 auf $16 \%{ }^{1105}$. Im MekongDelta zählten 2006 ca. 4 Mio. Menschen als arm, dies entsprach 13,5 \% der Einwohner. In der Provinz An Giang belief sich der Anteil auf 9,7 \% ${ }^{1106}$. Vormoor macht darauf aufmerksam, dass die Menschen, die aus der Armutsstatistik heraus fielen, im Mekong-Delta jedoch nur knapp über der Armutsgrenze leben und ein hohes Potential aufweisen, wieder zu verarmen. Als Gründe für Armut in Vietnam werden in der Literatur vier Faktoren genannt: Zugang zu Boden, Bodenqualität, Zugang zu Märkten und Bildung ${ }^{1107}$. Das Mekong-Delta besaß 2006 die zweitgrößte Gruppe von Landlosen in Vietnam, wobei der Verkauf von Land auf Verbindlichkeiten gegenüber Banken zurückgeführt wurde ${ }^{1108}$. Ein Beispiel für die konstruierte Abhängigkeit wurde mit der Einführung der Living with Floods-Strategie gegeben. Die beiden baulichen Maßnahmen des Hochwasserschutzes hatten negative Effekte auf die Einkommenssituation der Menschen. Jedoch konnte die Entwicklung der landwirtschaftlichen Produktion diese Rückschläge zumindest statistisch kompensieren. Die durchschnittlichen Einkommen stiegen im Mekong-Delta, wie auch in ganz Vietnam, an und die Anzahl der Menschen, die von Armut betroffen waren, konnte verringert werden. Da die statistisch nicht mehr als arm geltenden Bürger allerdings immer noch in prekären Verhältnissen leben, bleibt die statistisch nachgewiesene Armutsreduzierung theoretisch. Zudem weisen die negativen Auswirkungen auf die Einkommenssituation der Bevölkerung, die durch Baumaßnahmen betroffen war, darauf hin, dass der bauliche Hochwasserschutz die ungleiche Verteilung materieller Ressourcen zwischen Arm und Reich beförderte. Das wirtschaftliche Ziel, die Steigerung der Einkommen der ländlichen Bevölkerung, konnte offenbar erreicht werden, jedoch wirkte der Bau der Hochwasserschutzanlagen in die entgegen gesetzte Richtung. Außerdem konnten keine signifikante Korrelation zwischen dem landwirtschaftlichen Output und dem Ausbau der Bewässerungssysteme ermittelt werden ${ }^{1109}$.

\footnotetext{
${ }^{1103}$ Vormoor 2010. 636.500 VND entsprachen 2006 ungefähr 31 Euro laut http://fxtop.com/en/. 1104 Ebd.

${ }^{1105}$ Ebd.. Armut ist hier nach dem vietnamesischen für Amt für Statistik definiert. Es steht in Abhängigkeit zu einem Mindestbetrag, den eine Person zur Verfügung hat. Dieser Betrag wird regelmäßig angepasst. 2006 wurde dieser Betrag mit 213.000 VND/Person festgelegt.

${ }^{1106}$ Ebd.

${ }^{1107}$ Ebd.

${ }^{1108}$ Ebd.

${ }^{1109}$ Ebd., 8.
} 
Abschließend kann festgehalten werden, dass die Minderung der Opferzahlen und die Steigerung des landwirtschaftlichen Outputs positiv zu bewerten sind. Die Höhe der Hochwasserschäden konnten nicht deutlich reduziert werden, denn es fand hauptsächlich eine Verschiebung der Schäden statt: Die einstweilen Schäden in der landwirtschaftlichen Produkten konnten verringert werden, doch die Schäden an der Infrastruktur stiegen steil an. Die Maßnahmen der Landgewinnung in den Überschwemmungsgebieten hatten sich zunächst als erfolgreich erwiesen, jedoch verloren die Felder ihre Furchtbarkeit. Die bereits etablierten landwirtschaftlichen Aktivitäten wurden nach einem Rückgang der Ernten reduziert. Statistisch betrachtet stieg die Lebensqualität in An Giang. Die Gründe für Armut hängen von den vier Faktoren Zugang zu Boden, Bodenqualität, Zugang zu Märkten und Bildung ab. Drei der der vier Faktoren wurden von der Umsiedlungspolitik negativ, und nur der Zugang zu Bildung konnte erheblich verbessert werden. Der Zugang der Bevölkerung zu Boden und insbesondere zu Boden mit hinreichender Qualität wird durch den Bau der Deiche geschmälert. Folglich kann auch die Steigerung der Einkommen aufgrund des baulichen Hochwasserschutzes nicht eindeutig positiv bewertet werden. Die zu Beginn vorgestellten politischen Ziele konnten nur marginal erreicht werden. Der Diskurs zum baulichen Hochwasserschutz erhält somit eine unverständliche Konnotation ${ }^{1110}$, denn es bleibt unklar, weshalb die Baumaßnahmen fortgesetzt wurden, obwohl erhebliche negative Auswirkungen für Boden und Bevölkerung bereits bekannt geworden waren. Es kann gewissermaßen eine Rationalitätslücke festgestellt werden, deren Schließung die tatsächliche Bewandtnis der Baumaßnahmen vervollständigt und nachvollziehbar macht.

\footnotetext{
${ }^{1110}$ Schnädelbach 2000.
} 


\section{Zusammenfassung und Fazit}

Die zentrale Forschungsfrage dieser Arbeit war, ob der Umgang der politischen Entscheidungsträger mit Überschwemmungen rational verlief. Um diese Frage zu beantworten, wurden drei empirische Kapitel aufbereitet. Anhand zweier Beispiele wurde die Einwirkung der Bewertung des Heimatbegriffs, der Vulnerabilität sowie entwicklungspolitischer Ziele in Entscheidungsprozesse dargestellt. Eine Zusammenfassung dieser Beispiele wird im Folgenden vorgestellt.

In der Entwicklung der Argumentationslinien zur Bewahrung des Kölner Stadtbildes sind zwei Phasen erkennbar. Zunächst wurde der Hochwasserschutz als eine lokale Aufgabe betrachtet. Die Kölner Verantwortlichen agierten eigenmächtig und bauten den Hochwasserschutz, den sie für angemessen hielten. Dabei waren die Bautätigkeiten indirekt durch den Wiederaufbau nach dem Zweiten Weltkrieg beeinflusst, das berühmte Stadtbild Kölns verkörperte Heimat, welche unter allen Umständen bewahrt werden musste. Auch wurden die Überflutungsstärken und -intervalle als natürlich wahrgenommen, eine Suche nach Schuldigen fand nicht statt. Besonders nach der Flut von 1955 wurde die Höhe der Überschwemmung in Anbetracht der Zerstörungen durch den Krieg relativiert. Die administrative Ebene Kölns wurde durch das Hochwasser nicht zum Handeln veranlasst. Nach dem Hochwasser von 1970 nahmen die Verantwortlichen in Köln die Bewährung der nach 1960 gebauten Schutzmauer wahr, obwohl diese überspült wurde. Die vorhergegangene Kontroverse über die Einbußen durch die Mauer und deren eigentlichen Zweck verdeutlichte die Unzufriedenheit der Kölner über den Eingriff am Rhein. Obwohl die Mauer nicht primär gebaut wurde, um vor Hochwasser, sondern den Fußgänger vor dem Verkehr zu schützen, waren die Verantwortlichen nur aufgrund eines tatsächlichen Hochwasserereignisses in der Lage, von ihrer Bewährung zu sprechen. Im Jahr 1970 rangen die Verantwortlichen bei den Kölnern noch immer um Akzeptanz für die als Beeinträchtigung des Stadtbildes wahrgenommene Baumaßnahme.

In einer späteren Phase verändert sich die Wahrnehmung des Hochwassers vollkommen. Bereits 1983 wurde an der Natürlichkeit der beiden Frühlings-Hochwasser gezweifelt. In einer Genese von der Dauer einer Dekade ist zu beobachten, wie sich die handelnden Akteure in Köln durch vier konsekutive Hochwasser in ihren Befürchtungen, die Überschwemmungen seien durch die Eingriffe am Oberrhein entstanden, immer mehr bestätigt fühlten. Das Hochwasser von 1993 ließ die Behörden in Köln ein überregionales Konzept erarbeiten, welches nach der Überschwemmung von 1995 inhaltlich auf EU-Ebene institutionalisiert 
wurde. Der Hochwasserschutz wurde seitdem zu einer überregionalen Aufgabe für alle Anlieger von der Quelle bis zur Mündung des Rheins angesehen. Zu Beginn der 1990er Jahre entwickelte die nordrhein-westfälische Landesregierung zudem eigene Vorstellungen von einem landesweiten Hochwasserschutz, welcher von den Kommunen umzusetzen war. Daraufhin verlor die Stadt Köln ihre bisherige Möglichkeit, das Maß des Hochwasserschutzes selbst zu bestimmen, und musste sich fortan mit der administrativen Ebene des Landes auseinandersetzen.

Die zwei Phasen gehen im Laufe der 1970er Jahre ineinander über. Die technischen Veränderungen der hydrologischen Verhältnisse aufgrund der energetischen Wassernutzung des Rheins wurden abgeschlossen. Die letzte Staustufe von insgesamt zwölf Stück am Oberrhein wurde 1977 in Betrieb genommen. Die Eingriffe gaben Grund zur Beunruhigung. Die Rheinstudienkommission studierte in den darauf folgenden Jahren die Auswirkungen der Staustufen und deren Kompensationsmöglichkeiten. Aufgrund dessen wurde im Jahr 1982 der Deutsch-Französische Vertrag über die Einrichtung von Retentionsgebieten zum Ausgleich der Baumaßnahem in Baden-Württemberg, Hessen, Rheinland-Pfalz und Frankreich geschlossen. Das vollständige Einrichten der darin vereinbarten Retentionsräume wurde besonders von den deutschen Bundesländern hinausgezögert. Zwischen 1970 und 1983 fand kein nennenswertes Hochwasser in Köln statt. Deswegen war das Ereignis vom Februar 1970 die letzte Überschwemmung in der ersten Phase, während die Flut vom April 1983 das erste Hochwasser in der zweiten Phase darstellte. Jedoch ist $\mathrm{zu}$ beobachten, dass die Entscheidungsträger der zweiten Phase sehr ähnlich wie in der Nachkriegszeit argumentierten. Die festen Schutzvorrichtungen in der Altstadt wurden nach 1960 nicht mehr in ihrer Höhe verändert $^{1111}$. In der zweiten Phase zeigte sich, dass die Auseinandersetzungen über die Schutzhöhen weiterhin mit Hinweisen auf die Bewahrung der Stadtansicht aufgeladen waren und eine Heimatkonnotation aufwiesen. Die Bewahrung der Stadtansicht wurde im Hinblick auf den Hochwasserschutz weiterhin durch die CDU fortgeführt. Die Argumentation bezog sich immer wieder auf die Stadt am Rhein, bzw. der Tradition des Spaziergangs an Dom und Rhein. Damit können Hinweise auf einen Heimatdiskurs bestätigt werden. Die Präferenzen der an den Debatten beteiligten Kölner Akteure weisen während der gesamten Betrachtungsperiode eine Kontinuität auf. Brüche in der Begründung gegen die Erhöhung der Hochwasserschutzmauer lassen sich nicht auffinden. Monetär nicht bezifferbare Kosten durch die potentielle Zerstörung des Stadtbildes wurden von den Kölnern ausschlaggebend gewichtet.

\footnotetext{
${ }^{1111}$ HWSK 1996, 111.
} 
Im zweiten Kapitel wurden die Rollen der am Hochwasserschutz beteiligten Politiker analysiert. Dazu wurden die Reaktionen der Verantwortlichen nach zwei Hochwasserereignissen einander gegenüber gestellt und mit Krons Ansatz der „Risikopartnerschaft“"1112 kontextualisiert. Die Instrumentalisierung des Vulnerabilitätskonzepts ermöglichte eine tiefere Betrachtung der Gruppe der Hochwasseropfer. Es konnte verdeutlicht werden, dass die partizipative Kapazität unter den Betroffenen unterschiedlich verteilt war und mache Gruppen tatsächlich „,no voice“1113 besaßen. Zunächst waren die Stadtratsmitglieder über die Schäden in dem $0.41 \mathrm{~km}^{2}$ großem Überschwemmungsgebiet nicht erstaunt. Seit sie den Bau der Hochwasserschutzmauer 1960 verabschiedet hatten, wussten sie bereits, dass es nur eine Frage der Zeit sein würde, bis der Rhein wieder über das Ufer treten werde. Die Flussanwohner dagegen hatten offenbar Vertrauen in den Schutz vor Hochwasser gefasst und lagerten nun Wertgegenstände in nicht wasserdichten Räumen. Aufgrund der Schäden gab es im Stadtrat heftige Auseinandersetzungen, denn es galt die Frage $\mathrm{zu}$ beantworten, wer für die Schäden aufzukommen hatte. Die SPD-geführte Verwaltung war der Ansicht, der betroffene Bürger habe selbst Schuld, da zu wenige Besitztümer aus der bedrohten Region heraus gebracht worden wären. Die Verantwortlichen definierten die Anspruchsgruppen und die Schadenshöhen und waren deshalb in der Lage, die Übernahme von Verantwortung abzulehnen. Alternative Handlungsmethoden wurden nicht nur von der Opposition, sondern auch von SPD-Mitgliedern gefordert. Jedoch bot keine andere Strategie als die Gewählte, einen Stillstand zu erzeugen, mehr Vorteile für die Verantwortlichen. Von faktischen Schadenshöhen wollten die Verantwortlichen nichts wissen, und stellten die Schwierigkeiten, Schäden genau zu berechnen, als Hemmnis dar, aktiv zu werden. Mit dem Verweis auf fehlende Anordnungen durch die Landesregierung gab sich die SPD überdies handlungsunfähig, Schäden zu regulieren. Die Verantwortlichen im Landtag diskutierten die Schadensersatzleistungen an die Hochwasseropfer aufgrund der geübten Kritik durch die CDU-Opposition ebenfalls. Der Innenminister (SPD) verdeutlichte jedoch die „Gefährlichkeit“, würden die Richtlinien veröffentlicht. Er glaubte, wie die Mehrheit der Verantwortlichen, dass die Bevölkerung ein nächstes Ereignis ausnutzen würde, um aufgrund von Schäden hohe Ersatzleistungen des Staates zu fordern.

Die Unvorhersehbarkeit menschlichen Handelns stellte für die Entscheidungsträger die größte Gefahr dar, welche mit dem Begriff des unpredictable civil belegt werden konnte. Es zeigte

\footnotetext{
${ }^{1112}$ Kron 2001. Versicherungen gegen Hochwasserschäden wurden im Überschwemmungsgebiet in den rezenten Betrachtungsräumen noch nicht in Anspruch genommen.

${ }^{1113}$ Voss 2008.
} 
sich ein Zwiespalt in dem Streit zwischen Landtag und Stadtrat: Beide Ebenen beschäftigten sich nicht mit der Überschwemmung als Naturgefahr, den Ursachen oder der Bekämpfung. Während der Stadtrat sich mit den Hochwasserschäden auseinandersetzte, wurde im Landtag „Wahlkampf betrieben“ und über Transparenz gestritten. Das Ereignis selbst stand für die Entscheidungsträger 1970 nicht im Vordergrund.

Demgegenüber wurden die Reaktionen nach der Überschwemmung von 1995 betrachtet. Die Kommunalpolitiker fühlten sich nach diesem Hochwasser ausgeliefert und machtlos, die Stadt selbst vor Hochwasser zu schützen. Mit der Aussicht, dass diese großen Hochwasser in Zukunft durch die Kölner Verantwortlichen nicht aufzuhalten sind, war das Potential der Betroffenen, Resilience aufzubauen, erschöpft. Ihre partizipative Kapazität war zu schwach. In ihren Augen wurden die für sie wichtigen Entscheidungen ohne ihre Beteiligung getroffen, konkret kritisierten sie die Landesregierungen von Baden-Württemberg, Hessen und Rheinland-Pfalz, sich der Pflicht des Retentionsbaus zu entziehen. Die Kölner Stadtratsmitglieder verfolgten daraufhin die Ausarbeitung des bereits nach der Flut von 1993 in Auftrag gegebenen Hochwasserschutzkonzeptes. Dieses Konzept beinhaltete hauptsächlich Maßnahmen zur Optimierung des Wasserabflusses in der Fläche, die überregional zur Anwendung kommen sollten. Der Bau von zwei Retentionsräumen wurde favorisiert, während weitere Maßnahmen, wie beispielsweise das Bauverbot in Überschwemmungsgebieten, zunächst an wirtschaftlichen Interessen scheiterten. Unter anderem konnten zwei starke Fürsprecher für die Stadt Köln gewonnen werden. Die nordrhein-westfälische Umweltministerin Höhn (Bündnis 90/DIE GRÜNEN) akzeptierte die Ablehnung der Kommunalpolitiker, höhere Schutzvorrichtungen zu verbauen, nachdem diese die Einrichtung von zwei Retentionsbecken vorgeschlagen hatten. Auf diese Weise wurde die Stadtansicht nicht durch hohen Hochwasserschutz bedroht. Die zweite Fürsprecherin war Merkel (CDU) die als Bundesumweltministerin in der Lage war, einen überregionalen Austausch zu Hochwasserproblemen zu institutionalisieren. Die bereits bestehende Institution der Internationale Kommission zum Schutz des Rheins wurde deshalb mit dem Mandat ausgestattet, Rheinhochwasser zu thematisieren und ist seitdem die Plattform, auf der die Stadt Köln in der Organisation der Hochwassernotgemeinschaft partizipieren darf. Intangible Kosten in Form von Ängsten beeinflussten die Entscheidungen der Kölner Politiker am stärksten, wobei die geringen finanziellen Ressourcen aufgrund der prekären finanziellen Lage der Stadt dazu führten, nach dem Risikoansatz vorzugehen. Der Entscheidungsprozess konnte rational nachvollzogen werden, da die Handlungen gegen Alternativen abgewogen und die Konsequenzen von den involvierten Verantwortlichen kritisiert wurden. Die Rollen der 
Kölner Akteure verschoben sich im Laufe des Betrachtungszeitraums von lokal agierenden Verwaltungsbeauftragten, die den Bürger als Quelle des Schadens ausmachten (unpredictable civil), zu international verhandelnden Hochwasserexperten, die den Hochwasserschutz am Bürger ausrichteten. Sie stellten eine Kommunikation sowohl zu höheren politischen Entscheidungsebenen aufgrund der Partizipation an Entscheidungsprozessen, als auch zu den betroffenen Bürgern im Hochwassergebiet her. Die Untersuchung unter Zuhilfenahme des Vulnerabilitätsansatzes verdeutlichte die Schwierigkeit der Betroffenen, aus der Rolle des Hochwasseropfers herauszukommen, solange kein Mitspracherecht geltend gemacht werden konnte. Voss' „no voice“-Ansatz konnte bestätigt werden, ohne Fürsprecher war für die Betroffenen kein Ausweg zu finden. Die negativen Erwartungen der lokalen Entscheidungsträger über zukünftige Hochwasserschäden bargen hohe intangible Kosten. Die Ängste lassen sich nicht monetär beziffern, jedoch waren sie in den jeweiligen Entscheidungsprozessen ausschlaggebend.

Dieser deutschen Wahrnehmung und Bewältigung der Hochwasserproblematik wurde daraufhin eine südostasiatische gegenübergestellt. Im dritten Kapitel wurden die drei Argumentationslinien der Desaster-, Landwirtschafts- und Hochwassersicherheitsdiskurse in einen Rückblick auf das traditionelle Leben in Vietnams südlicher Region, welches durch zwei jahreszeitliche Zyklen geprägt war, eingebettet. Dies geschah, um die Argumentation weiter zu greifen und den Hochwasserschutz auf eine überregionale Ebene zu heben. Am Beispiel der Provinz An Giang wurden die Beispiele konkretisiert. Die Einwohner der Provinz, hauptsächlich Landwirte, lebten in Anpassung an die Jahreszeiten in Häusern auf Stelzen oder Booten, und nutzten zum Transport vorzugsweise das Kanal- und Flussnetz, denn befestigte Straßen waren bis zu Beginn der 1990er Jahre kaum vorhanden. Die Landwirte und die Regierung Vietnams standen in einem ,dialogischen Verhältnis ${ }^{\text {(1114 }}$ zueinander, denn die Landbevölkerung nahm durch „everyday politics“"1115 Einfluss auf die Entscheidungen politisch Verantwortlicher. Die wehrhafte Haltung der Landbevölkerung hatte der Regierung aufgezeigt, dass ihre Entscheidungs- und Handlungsfähigkeit durch den Widerstand der Gesellschaft begrenzt ist. Nachdem bereits grundlegende Reformen auf den Einfluss der Landbevölkerung zurückzuführen waren, und neben der wirtschaftlichen Öffnung des Landes 1986 auf Druck der Gesellschaft auch die Bodenreform 1988 durchgeführt wurde, kämpfte die Regierung um den Machterhalt. Im Kontext der Situation

\footnotetext{
${ }^{1114}$ Howie 2011. Der Mythos von Son Tin beschreibt den kontinuierlichen Kampf gegen Hochwasser.

${ }^{1115}$ Benedikter/Waibel 2013.
} 
zum Ende der 1980er Jahre wurden drei Diskurse als Reaktion auf Hochwasser am Mekong betrachtet.

Nach einer von Mythen begleiteten Tradition des Deichbaus - im Delta des Roten Flusses (Hanoi) sollen Deiche seit Jahrtausenden gebaut werden - begann mit dem Ausruf der Katastrophendekade der Vereinten Nationen Ende der 1980er Jahre die politische Institutionalisierung des Wasserdesastermanagements in Vietnam ${ }^{1116}$. Die Vereinten Nationen erarbeiteten gemeinsam mit dem Wasserressourcenministerium ein Projektpaket, um Schäden durch wasserbasierte Naturgefahren abzuwenden. Im Laufe des Desasterdiskurses wurde Hochwasserschutz in der Mekong-Region jedoch nur Ziel eines Projekts und lediglich 2,21 \% des Budgets zur Reduzierung von Desasterschäden in die südliche Hochwasserregion investiert. Die anderen wasserbasierten Gefahren besaßen folglich eine größere Priorität, denn für die Entscheidungsträger passte die Bekämpfung des Mekong-Hochwassers nicht in den Kanon der Desasterprojekte.

Im Jahr 1993 setzte der Diskurs zur Hochwassersicherheit im Mekong-Delta ein. Die internationale Entwicklungshilfe finanzierte den Wandel und engagierte niederländische Wasserbauexperten. Bevor die Unterstützung der internationalen Entwicklungsorganisationen für die Umstrukturierungsmaßnahmen gewonnen werden konnte, gab es keine festen Deichkonstruktionen in der flachen Flusslandschaft. Lediglich „Augustdeiche“ provisorische Bauten aus Erde - dehnten die flutfreie Zeit um einige Tage aus, bevor das Hochwasser diese überspülte und die Felder dahinter unter Wasser setzte. Die Planungen der niederländischen Ingenieure zielten darauf $\mathrm{ab}$, die landwirtschaftliche Entwicklung zu fördern. Mit der Implementierung der festen Deichkonstruktionen endete somit der natürliche Überschwemmungszyklus und eine dritte Einsaat der Felder wurde ermöglicht.

Wie gezeigt werden konnte, blieb der Deichdiskurs auch in der Implementierungsphase im Kreise der ingenieurwissenschaftlichen Experten verhaftet, dabei wurden zivilgesellschaftliche und wissenschaftliche Gruppierungen sowie die Anwohner aus dem betroffenen Gebiet von dem elitären Aushandlungsprozess strukturell ausgeschlossen. Es wurde jedoch ersichtlich, dass die Mitglieder des ausgesuchten Kreises in der Entwicklungsphase der spezifischen Projekte verschiedene Handlungsmöglichkeiten gegeneinander abwogen und Konsequenzen der einzelnen Alternativen in die Bewertung einbezogen. Der bauliche Hochwasserschutz ist von dem Desasterdiskurs getrennt zu betrachten, denn erst um die Jahrtausendwende führte die Regierung durch die Living with Floods-Richtlinie einen Schutz für Menschen und dessen Besitz ein. Die Regierung

\footnotetext{
${ }^{1116}$ Lebel et al. 2009.
} 
ermöglichte den Bewohnern des Hochwassergebiets die Umsiedlung in eingedeichte und höher angelegte „Cluster“. Damit blieben die Entscheidungsträger ihren technokratischen Ansätzen treu. Die politisch Verantwortlichen bewarben die Cluster im Überschwemmungsgebiet mit einer Reihe von infrastrukturellen Vorteilen, jedoch stellte der Umzug für die Mehrheit der Menschen einen sozialen Abstieg, statt eine Verbesserung der Lebensqualität dar ${ }^{1117}$. Infolgedessen wurden sie noch abhängiger von der Regierung und somit ebenfalls besser kontrollierbar.

Parallel zu dem Eindeichungsdiskurs begann mit der Beratung der vietnamesischen Regierung durch die UNDP die Intensivierung der Landwirtschaft in ganz Vietnam, insbesondere im Mekong-Delta. Die Regierungsmitglieder Vietnams bewerteten die Chance, internationale Gelder für die wirtschaftliche Entwicklung des Landes nutzen zu können als vorteilhaft, da demgegenüber der Verzicht auf die potentiellen aber unbezifferbaren Entwicklungschancen stand. Da der Bau von Hochwasserbarrieren im Mekong-Delta bereits durch die Landwirte Mitte der 1970er Jahre begonnen worden war, ließe sich vermuten, die Regierung würde durch ein Landwirtschaftsprogramm die bestehenden Barrieren erweitern lassen wollen, um noch weitere Überschwemmungsgebiete vor dem Hochwasser zu schützten. Wie jedoch auch in dem Desasterdiskurs, beabsichtigten die Planer im Laufe des Diskurses zur Intensivierung der Landwirtschaft keine Deichbauaktivitäten. Die Regierungsvertreter planten zunächst fast $30 \%$ der zur Verfügung stehenden Gelder in die Erweiterung der Bewässerungssysteme zu investieren. Die Food and Agriculture Organisation (FAO) riet der UNDP, mit dem Bewässerungssystem die Organisation der Ressourcengewalt, als einflussreiches Kriterium einer erfolgreichen Landwirtschaftsstrategie, dringend auszubauen: Daraufhin wurde der vorgeschlagene Investitionsumfang für Bewässerungsprojekte in Übereinkunft mit den vietnamesischen Verantwortlichen von $30 \%$ auf $45 \%$ des Budgets erhöht. In dem Entscheidungsprozess wurde eine Interessensabwägung zwischen dem Ausbau der Bewässerungssysteme und einer weiteren Investitionsalternative augenscheinlich. Die UNDPMitarbeiter empfahlen den Ausbau von Allwetterstraßen in ländlichen Gebieten als Förderinstrument der landwirtschaftlichen Entwicklung. Der Bau befestigter Allwetterstraßen sollte einen verlässlichen Zugang zu entlegenen Regionen schaffen. Für das Planungskomitee schien diese Handlungsoption mit hohen Opportunitätskosten verbunden gewesen zu sein, da finanzielle Ressourcen dort gebunden und nicht mehr für die favorisierten Bewässerungsprojekte eingesetzt werden konnten. Deswegen entschieden sie sich gegen eine Investition in die Infrastruktur. Diese absolute Priorisierung der Bewässerungsprojekte zeigt,

${ }^{1117}$ Danh 2011. 
dass die Steigerung des landwirtschaftlichen Outputs nicht das favorisierte Ziel der Regierung war, sondern der erweiterte Zugriff auf Ressourcen und Menschen im Vordergrund stand und dieser keinesfalls durch den Infrastrukturausbau geschmälert werden sollte. „Whoever controls water, controls agriculture“, schlussfolgerte der Geograph Howie nach einer Untersuchung zu sozioökonomischen Auswirkungen von Deichbauten in An Giang.

Der Abgleich der propagierten Ziele der jeweiligen Diskurse mit der tatsächlichen Entwicklung im Mekong-Delta deckte zunächst eine Rationalitätslücke auf, denn nicht alle Ziele der Reaktionen auf das jährliche Hochwasser schienen von der Zentralregierung kommuniziert worden zu sein. Die Einordnung der Diskurse in den historischen Kontext erhellte die Betrachtung der Rationalität hinter den Handlungsweisen des Staates. Anscheinend spielten Macht- und Kontrollfragen neben Schadensreduzierungen und entwicklungspolitischen Zielen eine größere Rolle, als vorab durch die Regierung offenbart wurde. Die zentralstaatliche Kontrolle der Wasserressourcen gab seit ihrer Einrichtung den Lebensrhythmus der Deltabewohner vor und prägte deren äußere Welt. Nach Radkaus Vorstellung beeinflusst die äußere Welt die innere Welt der menschlichen Seele. Die Kulisse des perfekten Landwirts wurde durch die Regierung gestaltet und diese sollte die innere Welt der Landbevölkerung formen, um ihr eine neue Identität zu verleihen.

\section{Fazit}

Die vorliegende Untersuchung bestätigte nach zugrunde legen der für diese Forschungsarbeit festgelegten Rationalitätskriterien, dass die politischen Entscheidungsträger jeweils rational mit dem Hochwasser umgingen. Die drei Kriterien erschlossen sich aus dem erarbeiteten Hilfsmodell. Darin wurde der Entwicklungsprozess einer Entscheidung durch die Punkte des Alternativenvergleichs, der kritischen Betrachtung möglicher Konsequenzen sowie der am Entscheidungsprozess beteiligten Akteure widergespiegelt. In dieser Arbeit wurden zwei Organisationsmöglichkeiten des Hochwassermanagements gegenübergestellt. In Deutschland ist die Hochwasserverantwortung föderal geregelt. Diese Regelung wurde nach großen Überschwemmungsereignissen $(1970,1995)$ kritisiert und vorgeschlagen, die föderalistische Organisation auf Organe der Bundesebene zu übertragen ${ }^{1118}$. In Vietnam besteht eine zentralistische Tradition im politischen Umgang mit Hochwasser. Seit Beginn der 1990er Jahre begründen allerdings neue Deichbauprojekte die zunehmende Kritik an der zentral ausgeführten Organisation. Da die kommunalen Gremien vor Ort sowie die betroffene

${ }^{1118}$ Landtag NRW, 74. Sitzung, 22.04.1970; Landtag NRW, Ausschussprotokoll 11/1505, 08.02.1995. 
Bevölkerung in dem Entscheidungsprozess negiert werden ${ }^{1119}$, wird von diesen Akteuren ein lokal ausgeführter Hochwasserschutz herbeigesehnt. Mit den zwei in dieser Arbeit ausgewählten Fallbeispielen wurde es möglich, die Vor- und Nachteile eines Top-DownAnsatzes und eines Bottom-Up-Ansatzes im Bereich der Hochwasserbekämpfung herauszustellen. Beide Organisationstypen sind historisch gewachsen und besitzen somit ihre eigene Rationalität, darüber hinaus untermauert die latent vorhandene Kritik der gewählten Ansätze diese Rationalität. Neben den ausgeübten Beanstandungen waren weitere Gemeinsamkeiten, aber auch Unterschiede festzustellen. Aus den herausgearbeiteten Verhaltensweisen der Akteure werden nun die abzuleitenden Vor- und Nachteile hier in einem Fazit präsentiert.

Wie zu erwarten, konnten in beiden Fällen bei der Auswertung der historischen Quellen keine finanzmathematischen Kosten-Nutzen-Analysen gefunden werden. Die Feststellung konkreter Nutzen und Kosten der Hochwasserschutzmaßnahmen verlief in allen aufgezeigten Fallbeispielen schwierig und stimmt somit mit den Ergebnissen der Studie des Umweltbundesamts überein ${ }^{1120}$. Besonders die Bewertung von Effekten mit zeitlichen und räumlichen Dimensionen bereitete den Entscheidungsträgern Schwierigkeiten. Resümierend ist festzuhalten, dass aus der Kosten-Nutzen-Perspektive betrachtet, in beiden Fällen intangible Kosten weit ausschlaggebender waren als harte ökonomische Fakten. Die Debatte um den Hochwasserschutz war häufig emotional aufgeladen, entweder wie 1960 in Köln, um einen besonderen Stadtteil wieder Heimat werden zu lassen, oder wie 2000 in An Giang, um durch eine Veränderung der Heimat Machtverhältnisse zu konstituieren. Im Kontext des Hochwasserschutzes wurden in beiden Untersuchungsräumen Identitätsfragen aufgeworfen. In Köln wurde zunächst der erstrebte Nutzen als die Verbesserung der Verkehrsprobleme definiert und die diesem Nutzen gegenüber stehenden Kosten als Beeinträchtigung des Stadtbilds dargestellt. Zwei Sprechern gelang die rhetorische Volte, die Beeinträchtigung des mit Heimatbegriffen besetzten Stadtbildes zum Vorteil der Baumaßnahme zu verwandeln. Die Heimat als Welt in den Köpfen ${ }^{1121}$ diente als Folie der Umstrukturierung. Es lässt sich allgemein festhalten, dass intangible Kosten und Nutzen nicht unterschätzt werden dürfen. Entscheidungen wurden in beiden Fallbeispielen durch emotional begründete Überzeugungen beeinflusst, die nicht monetär in die Abwägungsprozesse einbezogen wurden.

Nichtsdestotrotz eigneten sich emotional hervorgerufene Auffassungen nicht allein, um Themen in die Diskussion einzuführen. Denn es war zu beobachten, dass die Diskussionen

\footnotetext{
${ }^{1119}$ Huu 2011.

${ }^{1120}$ Dehnhardt et al. 2008.

${ }^{1121}$ Downs/Stea 1982; Spiegel 1995.
} 
aufgrund eines konkreten Auslösers eröffnet wurden. Beispielsweise wurde als solcher Auslöser die in Vietnam zu Beginn der 1990er Jahre vorherrschende Armut erkennbar, die die Politiker dazu veranlasste, die Landwirtschaft auf Kosten der alljährlichen Überschwemmungen auszubauen. Des Weiteren wurde um die Jahrtausendwende auf die erschreckende Anzahl von ertrunkenen Kindern als Auslöser verwiesen. In Deutschland diente der als verheerend empfundene Straßenverkehr um 1959, die überraschenden Frühlingshochwasser von 1983, die großen Schäden von 1993/95 und die Feststellung, ohnmächtig gegen die Auswirkungen anthropogener Eingriffe am Oberrhein zu sein als Auslöser. Unabhängig vom Ergebnis einer Auseinandersetzung konnte eine solche in beiden Fallbeispielen nur nach einem Schockerlebnis begonnen werden.

Des Weiteren konnte in dieser Untersuchung herausgearbeitet werden, dass die politischen Entscheidungsträger eher zurückhaltend Kritik an Vorschlägen äußerten, sobald die Überschwemmung allgemein als deutliche Beeinträchtigung empfunden wurde oder der Nutzen des Hochwasserschutzes direkt mit den vermiedenen Schäden begründet wurde. Beispielsweise ist hier der Deichbau in Vietnam zu nennen. Durch den Ausbau des Hochwasserschutzes sollte eine Steigerung des landwirtschaftlichen Outputs herbeigeführt werden, die die Lebensqualität der Menschen verbessern sollte. Das Hochwasser zukünftig von den Feldern fernzuhalten erfuhr auf politischer Entscheidungsebene keine spürbare Kritik, obwohl die Überschwemmungen die Grundlage für die Fruchtbarkeit der Felder darstellen. Aber auch in Köln wurde die Erhöhung der Hochwasserschutzmauer 1983 nahezu kritiklos veranlasst, um zukünftig die just angerichteten Schäden zu vermeiden. In beiden Ländern entfiel Kritik an vorgeschlagenen Handlungsoptionen und damit ein Stück Rationalität.

Es wurde deutlich, dass Entscheidungen gefällt wurden, weil mächtige Fürsprecher in der Lage waren, eine Handlungsalternative hervorzuheben, beispielsweise Konrad Adenauer 1959 und Angela Merkel 1995. Adenauer nutzte die Gelegenheit, als er in Köln eine Rede zu einer Brückeneröffnung hielt, in die Stadtratsdebatte einzugreifen. Merkel war ebenso in der Lage eine Gelegenheit für ihre Zwecke zu nutzen, als sie 1995 auf dem Treffen der europäischen Umweltminister die Vertreter der ebenfalls durch die Rheinfluten betroffenen Länder davon überzeugen konnte, Budgets der Raumordnung und Bodennutzung für den Hochwasserschutz $\mathrm{zu}$ akquirieren. Ein weiteres Beispiel für die Notwendigkeit von Fürsprechern ist die städtische Verwaltung zu Köln. Die Stadtratsmitglieder entschlossen sich, politischen Einfluss auf den Umgang der oberrheinischen Verantwortlichen mit dem Rhein auszuüben, jedoch ist dies aufgrund der politischen Struktur in Deutschland nicht unmittelbar möglich. Die 
Stadtratsmitglieder wussten, dass sie mächtige Fürsprecher brauchten, um ihre partizipative Kapazität erhöhen zu können. Sie versuchten diese mit dem Bau von Retentionsräumen im Stadtgebiet Kölns zu beeindrucken. In Vietnam war bereits die Organisation der politischen Struktur darauf eingerichtet, dass der Premierminister die Entscheidungen zum Hochwasserschutz traf. Dort verbargen sich hinter den Entscheidungsprozessen einige wenige Wasserbauexperten nationaler Behörden. Diese erarbeiteten die Entscheidungsgrundlage für den Premierminister. Wie gezeigt werden konnte, wurden wie in Vietnam auch in Deutschland grundlegende Entscheidungen durch Politiker der nationalen Entscheidungsinstanzen beeinflusst. Die sogenannte Kohärenz der Vernünftigen ${ }^{1122}$ führte in Deutschland wie auch in Vietnam dazu, dass Außenstehende kaum Einfluss geltend machen konnten. Teilweise wurden Kritiker strukturell marginalisiert, beispielsweise war das nicht nur in Am Giang der Fall, sondern auch in Köln als die politischen Strukturen eine Mitsprache Kölns zum Umgang mit dem Rheineinzugsgebiet verhinderten. Entscheidungen wurden in Vietnam nur im Kreis von regierungszugehörigen Ingenieuren gefällt, wohingegen Experten anderer Fachgebiete oder die Behörden der betroffenen Regionen durch Regierungsorganisationen trivialisiert wurden. Darüber hinaus wurden hydrologische Gutachten und wissenschaftliche Studien von beiden Gruppen missachtet. Beide Gruppen hielten sich vielmehr selbst für Experten und entschieden selbstständig, welchen Faktoren Gewicht beigemessen werden sollte und welchen nicht. Beide Ansätze gestatten unerwünschte Kritik zu ignorieren, was als Nachteil auszulegen ist.

Abgesehen von einflussreichen Fürsprechern waren ebenfalls starke Allianzen entscheidungswirksam. Neben der Kölner Allianz von Vater Adenauer und Sohn zeigte sich anhand der Darstellung der vier treibenden Kräfte, dass der Oberbürgermeister 1960 trotz seiner Stellung kaum Einfluss ausüben konnte, denn er stand isoliert gegenüber der Allianz aus dem Initiator der Diskussion und den einflussreichen Vorkriegs-Eliten, die den Heimatdiskurs unterstützten. Bei den Aushandlungen nach dem Hochwasser von 1995 entstand eine weitere Allianz. Köln als Stadt konnte ihre partizipative Kapazität durch die Gründung der Hochwassernotgemeinschaft erhöhen. Die Hochwassernotgemeinschaft besteht aus Gemeinden entlang des Flusses und ist seit April 1998 in die Entscheidungsprozesse der IKSR involviert. Außerdem verkürzte der zentralstaatliche Ansatz in einem top-down Verfahren den Prozess aufgrund unklarer Kompetenzverteilung zwischen den politischen Akteuren nicht. In beiden Betrachtungsräumen dauerte die Umsetzungszeit jedoch ungefähr gleich lange. In Köln musste ein Konsens zwischen dem Stadtrat und dem Landtag

\footnotetext{
${ }^{1122}$ Nida-Rümelin 2009.
} 
ausgehandelt werden, dessen Umsetzung jedoch von interpersonellen Beziehungen abhing. Interpersonelle Beziehungen spielen auch beim vietnamesischen Deichbau eine Rolle. Die Vergabe der Aufträge hing beispielsweise davon ab, wie gut jemand vernetzt war. Aus der Untersuchung geht hervor, dass interpersonelle Beziehungen einen entscheidenden Einfluss auf das erfolgreiche Fortschreiten der Aushandlungs- aber auch der Umsetzungsprozesse ausübten und somit wieder die personen-spezifische Abhängigkeit deutlich wurde.

In allen drei empirischen Kapiteln wurde erkennbar, dass sowohl der Hochwasserschutz als auch die Überschwemmung selbst für alternative Interessen der politisch Verantwortlichen instrumentalisiert wurden. In An Giang wurden seit Beginn der 1990er Jahre die Millennium Development Goals der Entwicklungszusammenarbeit instrumentalisiert, um die Identität der Menschen einer entlegenen Region zu beeinflussen. In Köln sollte 1959 der Straßenverkehr neu geregelt werden, um am Rheinufer Heimat entstehen zu lassen. Zur Beeinflussung der Bürger vor einer Wahl sollte auch die Überschwemmung von 1970 durch Mitglieder des Landtags genutzt werden. Nach dem Hochwasser von 1993 versuchte der damalige Bundesumweltminister die Flut zu nutzen, um sich für den bevorstehenden Wahlkampf Profil zu verleihen. Zwar blieben die Politiker in den betrachteten Fällen mit ihren Strategien erfolglos, jedoch ist anzumerken, dass Hochwasserschutz nicht nur in Vietnam sondern auch in Deutschland zur Legitimation der herrschenden politischen Verhältnisse instrumentalisiert wurde. Aus diesen Gemeinsamkeiten leitet sich bereits ab, dass beide Lösungsversuche gleichermaßen von erheblichen Nachteilen begleitet werden, sodass die Differenzierung der beiden Ansätze weitere Auskunft geben muss.

Im Folgenden werden die abgrenzenden Eigenschaften des zentralstaatlichen und des föderalistischen Ansatzes hervorgehoben. Zunächst ist anzuführen, dass anhand der gewählten Beispiele in dieser Arbeit gezeigt werden konnte, dass ein begrenztes Budget dazu anregte, das Verhältnis von ökonomischen Kosten und Nutzen stärker zu betrachten, als in Situationen in denen finanzielle Ressourcen leicht zugänglich waren. Begrenzte finanzielle Mittel sind in diesem Sinne als die eigenen Möglichkeiten zu verstehen, ohne dass sich fremde Investoren engagieren. Eine fehlende Effizienzanalyse führte in Köln 1960 dazu, dass die Ästhetik der Hochwasserschutzmauer und nicht die Schutzaspekte über den Bau entschieden. Ein begrenztes Budget führte dort 1995 jedoch dazu, dass nach dem Risikoansatz verfahren wurde, also die Frage, welcher Schutz zu welchem Preis, im Mittelpunkt der Entscheidung stand. Dementsprechend ist festzuhalten, dass ein begrenzter Zugang $\mathrm{zu}$ finanziellen Ressourcen die Durchführung von Effizienzanalysen befördert. So ist beispielsweise der 
Verhaltenswandel der Kölner Behörden in den 1990iger Jahren zu erklären. Statt nach dem Sicherheitsansatz verfuhr man nun nach dem Risikoansatz und prüfte erstmalig, an welcher Stelle ein höherer Hochwasserschutz die effizientere Wahl ist. Die Verwendung eigener finanzieller Mittel kann daher als ein Vorteil im Rahmen der zugrundeliegenden Rationalitätskriterien interpretiert werden.

Des Weiteren konnte herausgestellt werden, dass normative Zielvorgaben die Kriterien der Rationalität beeinträchtigten. Je klarer ein Schutzziel formuliert wurde, beispielsweise einen 100jährigen Schutz zu verbauen, desto weniger Alternativen wurden in die Diskussionen eingeführt. Da die Erreichung des Ziels kaum mit alternativen Mitteln bewerkstelligt werden konnte, wurde in den angeführten Beispielen der Abwägungsprozess vernachlässigt, und die Rationalität einer Hochwasserschutzhandlung begrenzt. Das vietnamesische System arbeitete während des gesamten Untersuchungszeitraums nach dem Sicherheitsansatz, während die Akteure in Köln ihre Haltung im letzten Betrachtungsbeispiel revidierten und nach dem Risikoansatz verfuhren. Im Rahmen der Kriterien zur Rationalität, die für diese Arbeit aufgestellt wurden, ist ein Verfahren nach dem Sicherheitsansatz als Nachteil abzuleiten, da es den Diskurs behindert.

Dem gegenüber führte ein Verzicht auf eine präzise Formulierung des Schutzziels ebenfalls zu einer Beeinträchtigung der Rationalität, da so ebenfalls erschwert wurde, dem Ziel mit alternativen Maßnahmen beizukommen. Ein unkonkretisiertes Ziel ließ den Vergleich mit potentiellen Handlungsalternativen nur unzureichend $\mathrm{zu}$, da nicht klar verständlich definiert wurde, welche Auswirkungen erstrebenswert und welche zu vermeiden waren. Dagegen bot ein unkonkretes Ziel viel Angriffsfläche für Kritiker, denn die möglichen Konsequenzen stellten das einzige Bewertungskriterium der vorgeschlagenen Handlungsoption dar. Folglich steigerte die ausgeprägte Kritikausübung an der ausgewählten Reaktion paradoxerweise die Qualität der Lösung. Beispielsweise geschah dem so, als 1995 in Köln konstatiert wurde, dass kein baulicher Hochwasserschutz in der Lage wäre, völlige Sicherheit zu garantieren. Daraufhin wurde die Kommunikation mit den betroffenen Bürgern intensiviert und diese in den Hochwasserschutz eingebunden, um zukünftige Schäden zu reduzieren. In Vietnam standen jeweils konkrete Schutzziele im Vordergrund, während Kritik unerwünscht war. Nur in den Untersuchungsbeispielen in Köln wurde sowohl die fehlende Zielvorstellung als auch die ausführliche Debatte deutlich, was dem föderalistischen System einen Vorteil gewährt.

Die Frage, welche Konsequenzen gewünscht und welche unerwünscht sind, war ebenfalls ein Nährboden für Auseinandersetzungen. Die Wahrnehmung potentieller Konsequenzen ist abhängig von den an dem Diskurs teilnehmenden Persönlichkeiten. Deren Bildungsstand, 
Erfahrungsschatz und Lebensgeschichte spannte den Hintergrund ihrer persönlichen Perspektive auf. Deshalb ist es wichtig, zu bekennen, dass eine umfangreiche Kritik aus einem entscheidungsbefugten Personenkreis, den eine große Vielfalt auszeichnet, entspringt. Dies wurde im Hinblick auf die letzten beiden Hochwasser in Köln besonders deutlich. Da die Auseinandersetzungen öffentlich ausgetragen wurden, kommentierten Experten unterschiedlicher Fachgebiete die Geschehnisse und empfahlen Handlungsweisen. Dies steigerte die Qualität des Hochwasserschutzes am Rhein. Negative Konsequenzen wurden aus einer persönlichen Expertise heraus erkannt. In Folge dessen wirkt sich eine Trivialisierung von Meinungsführern negativ auf den Erfolg einer Maßnahme aus, wie am Beispiel von Vietnam gezeigt werden konnte. Da in Vietnam lediglich ingenieurtechnisch geschulte Experten den Diskurs im Hochwasserschutz beeinflussten, muss daraus ein Nachteil für den zentralstaatlichen Ansatz und für die interdisziplinären Auseinandersetzungen im dezentralen System ein Vorteil abgeleitet werden.

Aus den drei vorangegangenen Kapiteln lässt sich schlussfolgern, dass die Beteiligung der lokalen Bevölkerung am Entscheidungsprozess die Planungsgrundlage der politisch Verantwortlichen verbesserte. Dieser Umstand wurde besonders in der Betrachtung der Verteilung der Vulnerabilität in Köln von 1970 deutlich. Außerdem war an der konstatierten Bewährung der Schutzmauer nach dem Hochwasser 1970 zu erkennen, dass die Verantwortlichen sich immer noch nicht gewiss waren, ob der Mauerbau von 1960 den Eingriff in das Stadtbild wert war. In der Diskussion um den zu erhöhenden Hochwasserschutz von 1983 wurde die Bevölkerung in die Planung integriert, was zu einem schnellen und zufriedenstellenden Ergebnis führte. Außerdem wurde erkennbar, dass der Einbezug der betroffenen Bevölkerung in die Entscheidungsprozesse den Erfolg des Kölner Hochwasserschutzkonzeptes von 1996 begründete. In Vietnam wurden die Betroffenen zwar an den Kosten des Hochwasserschutzes beteiligt, aber von den Entscheidungsprozessen ausgeschlossen. Dieses Beispiel zeigte, dass die Marginalisierung der Betroffenen eine negative Entwicklung für die Umsetzung der Bauprojekte mit sich brachte. Das föderalistische System lässt Partizipation eher zu als der zentralstaatliche Ansatz. In Bezug zu den für diese Arbeit aufgestellten Kriterien zur Rationalität ist dies als Vorteil zu erkennen.

Die Gesamtschau der Ergebnisse wurde hier vorangestellt, um die aufgedeckten Vor- und Nachteile der beiden Systeme zu präsentieren. Zur erleichterten Übersicht wurden diese in Gemeinsamkeiten und Unterschiede der beiden Verwaltungssysteme eingruppiert. Dabei ist festzuhalten, dass obgleich die beiden Beispiele aufgrund ihrer grundsätzlichen Unterschiede für diese Untersuchung ausgewählt wurden, im Ergebnis deutlich mehr Gemeinsamkeiten als 
Unterschiede sichtbar wurden. Insbesondere konnten in beiden politischen Systemen ähnliche Nachteile herausgearbeitet werden, die das Ergebnis negativ beeinflussten. Für den föderalistischen Ansatz sind in der späteren Phase vier Vorteile festzuhalten: Die Entscheidungsträger erhoben Effizienzanalysen, äußerten umfangreich Kritik, betrachteten Probleme aus interdisziplinären Perspektiven und berücksichtigten die Bürgermeinungen während des Planungsprozesses.

\subsection{Ausblick}

Die Gesamtschau der Ergebnisse verdeutlicht, dass die Rationalitäten der Entscheidungsträger höchst kontextabhängig waren und von dem jeweiligen Entscheidungsträger abhängig blieben. Für die Zukunft birgt dies einen Unsicherheitsfaktor. Diese Arbeit gibt Hinweise darauf, mit welchem Einfluss personenabhängige Rationalitäten auf die Aushandlungsprozesse einwirkten. Intangible Nutzen und Kosten könnten aufgrund von Zahlungsbereitschaftsanalysen in monetäre Größen transformiert werden, um Anhaltspunkte für eine tatsächliche Bewertung $\mathrm{zu}$ finden. Nichtsdestotrotz werden KNA den Entscheidungsträgern die Entscheidung nicht abnehmen können. Sie sind nicht der Weisheit letzter Schluss, um die vernünftigste Alternative herauszustellen. Vielmehr bleibt die Wertvorstellung der Menschen die subjektive Entscheidungsbasis im Umgang mit Naturgefahren. Wie dargelegt wurde, überfordert die Definition von Vor- und Nachteilen bzw. von Nutzen und Kosten eines Eingriffs in die Umwelt mit Langzeitwirkung bereits die Verantwortlichen. Für den Umgang mit künftigen Naturgefahren, beispielsweise erzeugt durch den Klimawandel, bedeutet dies, dass politisch Verantwortliche faktisch erst Entscheidungen treffen werden, nachdem potentielle Naturgefahren als Schadensereignis in der volkswirtschaftlichen Gesamtrechnung sichtbar wurden.

Es bleibt eine Forschungslücke in der methodischen Annährung an die Frage, was Rationalität eigentlich ausmacht. Dabei ist das hier vorgelegte Modell als Hilfestellung zu verstehen, das in seiner Effizienz sicherlich durch umfangreich vorhandene Rationalitätsdiskurse ergänzt werden kann. Bei dem vorliegenden Versuch wurde auf die praktische Anwendbarkeit Wert gelegt. Es wäre jedoch anmaßend, sich der Illusion hinzugeben, das Hilfsmodell böte die Möglichkeit, die rezenten Rationalitäten von Entscheidungsträgern vollständig zu erfassen es spiegelt vielmehr die Anschauung der Autorin wider, wie historische Rationalität aufgrund von Quellen zu rekonstruieren sein könnte. Überdies sind die Ergebnisse ein Spiegel der untersuchten Fallstudien und keinesfalls universell gültig oder vollständig, denn eine 
Betrachtung weiterer oder alternativer Entscheidungsprozesse könnte zu anderen Aussagen führen.

Weiterer Forschungsbedarf besteht in der Standardisierung von Entscheidungsprozessen für Projekte des Hochwasserschutzes. Aus den untersuchten Fallbeispielen ging hervor, dass Akteure unterschiedliche Arten von Alternativen, wie z. B. Deich und Mauer, oder Deich und Erhöhung des Gefahrenbewusstseins, gegeneinander abwogen. Eine Standardisierung könnte den Entscheidungsträger darauf hinweisen, dass es noch unerschlossene Möglichkeiten gibt, den Hochwasserschutz zu steigern. Das Gleiche gilt für mögliche Konsequenzen, da gezeigt werden konnte, dass die Wahrnehmung spezifischer Grenzen einer Reaktion, insbesondere von zeitlichen und räumlichen Auswirkungen, rein subjektiv verlief. Die Beachtung von Vorund Nachteilen kann aus dem Subjektiven mittels einer Standardisierung des Entscheidungsprozesses auf eine objektive Betrachtungsebene gehoben werden. Ein standardisiertes Verfahren müsste demnach von einer vielfältigen Gruppe erarbeitet werden dies könnte in der Gemeinschaft der Flussanlieger geschehen.

Es erschließt sich für die Zukunft, dass negative Konsequenzen des Hochwasserschutzes unter anderem aus allgemeinem Fortschritt entstehen. In diesem Kontext müssen der Bau der Wasserkraftwerke am Oberrhein sowie die Intensivierung der vietnamesischen Landwirtschaft genannt werden. Die vietnamesischen wie die deutschen Entscheidungsprozesse gestalteten sich repetitiv, denn sie unterlagen einer zeitlichen Spirale. Die Herausforderungen konnten also nur vorläufig bewältigt werden, bevor neue Probleme auftauchten. Deshalb scheinen die Auseinandersetzungen auch künftig kein Ende zu nehmen, wie das Elbhochwasser 2013 zeigte. Dabei besitzt man sowohl im Mekong-Delta - mit jährlichen Hochwassern - als auch am Rhein die Gewissheit, dass das Hochwasser ein wiederkehrendes Ereignis ist. Der Ausspruch „Vor dem Hochwasser ist nach dem Hochwasser“1123 konstituiert diese Anschauung.

\footnotetext{
${ }^{1123}$ Reinhard Vogt, Leiter der Hochwasserschutzzentrale Köln seit 1994 - 2013.
} 


\section{Literatur und Quellen}

\section{Quellen der Stadt Köln}

Stadt Köln, Rat der Stadt Köln, 1. Sitzung, 09.01.1948.

Stadt Köln, Rat der Stadt Köln, 1. Sitzung, 20. 011955.

Stadt Köln, Rat der Stadt Köln, 4. Sitzung, 05.03.1959.

Stadt Köln, Rat der Stadt Köln, 12. Sitzung, 13.10.1959.

Stadt Köln, Rat der Stadt Köln, 10. Sitzung, 20.10.1960.

Stadt Köln, Hochwasserschutzzentrale im Amt für Stadtentwässerung, Vermerk über die Besprechung 23.02.1970.

Tagesbericht des Amtes für Stadtentwässerung, 24.02.1970.

Bericht der Stelle zur Beseitigung von Wohnungsnotständen, 24.02.1970.

Bericht der Berufsfeuerwehr, 25.02.1970.

Bericht der Stelle zur Beseitigung von Wohnungsnotständen, 25.02.1970.

Bericht des Amts für Zivilschutz, 27.02.1970.

Stadt Köln, Rat der Stadt Köln, 2. Sitzung vom 12.03.1970.

Bericht über das Hochwasser 1970, Hochwasserschutzzentrale der Stadt Köln, Dezernat Tiefbau u. Verkehr, Amt für Stadtentwässerung, 03.04.1970.

Stadt Köln, Rat der Stadt Köln, 3. Sitzung, 16.04.1970.

Bericht über das Hochwasser 1983, der Hochwasserschutzzentrale der Stadt Köln, Dezernat Tiefbau u. Verkehr, Amt für Stadtentwässerung 01.06.1983.

Rat der Stadt Köln, Sitzung des Ausschusses für Tiefbau und Verkehr, 09.06.1983.

Rat der Stadt Köln, Sitzung des Ausschusses für Tiefbau und Verkehr, 08.09.1983.

Rat der Stadt Köln, Sitzung 22.09.1983.

Rat der Stadt Köln, Sitzung 27.10.1983.

Rat der Stadt Köln, Sitzung des Ausschusses für Tiefbau und Verkehr, Drucksache 19-214/83, 15.11.1983.

Rat der Stadt Köln, Sitzung des Ausschusses für Tiefbau und Verkehr mit der Bezirksvertretung 1, 01.12.1983.

Brief: Werth, Amt für Brücken- und U-Bahnbau, Ausschuss für Tiefbau und Verkehr an einen durch die Umbauten unmittelbar betroffenen Bürger, 09.05.1984.

Gutachten zur Abflussregelung bei Hochwasser und Steuerung des Kanalnetzes, Erläuterungsbericht, Dr. -Ing. Rolf Pecher, Beratender Ingenieur für Siedlungswasserwirtschaft, Erkrath 1985.

Brief von Egelmann i. A. des Oberstadtdirektors Ruschmeier an Dezernat 1 Tiefbau und Verkehr, 22.12.1993.

Pressekonferenz, Vogt, 07.01.1994, Rat der Stadt Köln, Dezernat für Tiefbau und Verkehr, Amt für Stadtentwässerung. 
Rat der Stadt Köln, 52. Sitzung, 01.02.1994.

Dezernat für Tiefbau und Verkehr, Amt für Stadtentwässerung, Brief von Vogt an Regierungspräsidenten Lampe, 23.02.1994.

Rat der Stadt Köln, 3. Sitzung, 13.12.1994.

Bericht „Hochwasser 1995“ der Hochwasserschutzzentrale der Stadt Köln, Dezernat Tiefbau u. Verkehr, Amt für Stadtentwässerung, 20.03.1995.

Rat der Stadt Köln, 9. Sitzung, 29.06.1995.

Stadt Köln, Drucksachen 1141/095, 29.01.1996, Beschlussvorlage Betreff

Hochwasserschutzkonzept Köln, Anlagen.

Stadt Köln, Drucksachen 1141/095, Anlage 2, 09.10.1995: Änderungsantrag der

Bezirksvertretung Rodenkirchen.

Stadt Köln, Drucksachen 1141/095, Anlage 3, 12.09.1995: Änderungsantrag der

Bezirksvertretung Chorweiler.

Stadt Köln, Drucksachen 1141/095, Anlage 4: Stellungnahme der Verwaltung zu den Änderungsanträgen der Bezirksvertretungen Chorweiler und Rodenkirchen.

Stadt Köln, Drucksachen 1141/095, Anlage 5, 16.11.1995: Ausschuss Umweltschutz und Abfallwirtschaft, Drucksachen Nr. 2012/095, Änderungsantrag.

Stadt Köln, Drucksachen 1141/095, Anlage 6, 30.11.1995: Fraktion der SPD, Drucksachen Nr. 2139/095, Änderungsantrag.

Stadt Köln, Drucksachen 1141/095, Anlage 7, 30.11.1995: Stellungnahme der Verwaltung , Drucksachen - Nr. 1141/095.

Stadt Köln, Drucksachen 1141/095, Anlage 7, 30.11.1995, Anlage I: Stellungnahme des Ausschuss Tiefbau und Verkehr zum Änderungsantrag unter Anlage 5, 30.11.1995.

Stadt Köln, Drucksachen 1141/095, Anlage 7, 30.11.1995, Anlage II: Antwort der Verwaltung an die Bürgervereinigung Rodenkirchen vom 15.11.1995.

Stadt Köln, Drucksachen 1141/095, Anlage 8, 18.01.1996: Stellungnahme des Ausschusses Tiefbau und Verkehr, Drucksachen - Nr. 2139/095.

Stadt Köln, Drucksachen 1141/095, Anlage 9, 18.01.1996: Stellungnahme des Ausschusses Tiefbau und Verkehr, Drucksachen - Nr. 1141/095.

Stadt Köln, Drucksachen 1141/095, Anlage 10, 18.01.1996: Protokoll der Sitzung des Ausschuss Tiefbau und Verkehr, Drucksachen - Nr. 1141/095.

Stadt Köln, Drucksachen 1141/095, Beschlussvorlage Betreff Hochwasserschutzkonzept Köln, 01.02.1996.

Stadt Klön, Besprechungsniederschrift, Zusammenarbeit mit hochwassergefährdeten Gemeinden, Köln, Rathaus, 14.06.1996.

Brief des Oberbürgermeisters Norbert Burger an die IKSR vom 08.04.1998.

Stadt Köln, Besprechungsniederschrift, 16.12.1996.

HWSK 1996: Hochwasserschutzkonzept 1996 der Stadt Köln. Das Konzept entspricht dem Ergebnis der Sitzung des Rats der Stadt Köln vom 01.02.1996, Download über die Homepage der Hochwasserschutzzentrale: http://www.steb-koeln.de/hskkln.html, eingesehen am 01.05.2012. 
Einladung zur Mitgliederversammlung der Hochwassernotgemeinschaft Mittelrhein bzw. konstituierende Sitzung der Hochwassernotgemeinschaft Rhein am 16.11.1996 im Historischen Rathaus zu Köln.

Flugblatt der Hochwasserschutz-Messe 1996.

Dr. Angela Merkel, 21.02.1996, Bundesministerium für Umwelt, Naturschutz und Reaktorsicherheit, Brief an Dr. Heribert Blens, Mitglied des Deutschen Bundestages, Vorsitzender des Vermittlungsausschusses, Anlage des Schreibens von Hubertus Oelmann, Beigeordneter der Stadt Köln, 04.03.1996.

Flugblatt der Hochwasserschutz-Messe 1997.

Reinhard Vogt, 13./14.12.2000, Workshop „Vorbeugender Hochwasserschutz auf kommunaler Ebene“, Umweltbundesamt in Kooperation mit dem Institut für ökologische Raumentwicklung e. V, Dresden.

Stadt Köln, Drucksachen - Nr. 0997/001, 12.06.2001, Beschlussvorlage, Gründung der Stadtentwässerungsbetriebe Köln AöR.

Stadt Köln, Rat der Stadt Köln, 27. Sitzung vom 12.06.2001, Protokoll.

Stadt Köln, Rat, Drucksache 1288/003, 07.10.2003, Anlage 1, Organisation des Hochwasserschutzes, Aufgabenübertragung auf die Stadtentwässerungsbetriebe Köln, AöR Steb.

Stadt Köln, Rat der Stadt Köln, 52. Sitzung vom 07.10.2003, Protokoll.

Präsentation vom 18.07.2011 der Steb, Homepage der Stadtentwässerungsbetriebe: http://www.steb-koeln.de/pool/files/Retentionsraum_Worringen_Endfassung.pdf, eingesehen am 02.06.2012.

Satzung der Stadtentwässerungsbetriebe AöR vom 05.12.2011, http://www.stebkoeln.de/pool/files/AbwGebS_2012-.pdf, eingesehen am 02.08.2012.

Informationen der Bürgerinitiative Ensen - Westhoven e. V.: http://www.bv-ensenwesthoven.de/data/uploads/veranstaltungskalender-bv-2012/bv_kalender2012_web.pdf, eingesehen am 15.07.2012.

\section{Quellen des Landesarchivs Nordrhein-Westfalen, Düsseldorf}

Landesarchiv NRW, NW 112, Nr. 165, 1955 Hochwasserkatastrophengefahr.

\section{Quellen des Landes}

Landtag NRW, 74. Sitzung, 22.4.1970.

Landtag NRW, Drucksache 11/6588, 13.01.1994.

Landtag NRW, Plenarprotokoll 11/118, 20.01.1994.

Landtag NRW, Vorlage, 11/3644, 01.02.1995, Ministerium für Umwelt, Raumordnung und Landwirtschaft des Landes Nordrhein-Westfalen (MURL), Pressetext „ökologischer Hochwasserschutz".

Landtag NRW, Drucksache 11/8422, 06.02.1995, Antrag der Fraktion BÜNDNIS 90/DIE GRÜNEN, Konsequenzen aus der Hochwasserkatastrophe ziehen.

Landtag NRW, Drucksache 11/8420, 08.02.1995, Antrag der Fraktion der SPD, Ökologischer Hochwasserschutz und Hilfsmaßnahmen. 
Landtag NRW, Ausschussprotokoll 11/1505, 08.02.1995, Ausschuss für Umweltschutz und Raumordnung sowie Ausschuss für Landwirtschaft, Forsten und Naturschutz, „Hochwasserschutz in NRW“.

Landtag NRW, Drucksache 11/8490, 16.02.1995, Antrag der Fraktion der CDU, Ökologischer Hochwasserschutz und Hilfsmaßnahmen.

Landtag NRW; Plenarprotokoll 11/157, 17.02.1995, Antwort auf den Antrag der Fraktionen CDU und Bündnis 90/DIE GRÜNEN, Ökologischer Hochwasserschutz und Hilfsmaßnahmen, Konsequenzen aus der Hochwasserkatastrophe ziehen.

Landtag NRW, Drucksache 11/8495, 17.02.1995, Antrag der Fraktion der FDP, Hochwasserschutz in NRW - fundierte Analyse vorlegen.

Landtag NRW, Drucksache 12/248, 11.10.1995, Antwort der Landesregierung auf die Kleine Anfrage 41 der Abgeordneten Arentz und Blömer (CDU), Behindert der Kölner Regierungspräsident den Hochwasserschutz für Köln?

Landtag NRW, Vorlage 12/324, 03.01.1996, MURL an den Ausschuss Umweltschutz und Raumordnung, Einführungsrede zum Haushaltsentwurf 1996.

Landtag NRW, Ausschussprotokoll 12/169, 07.02.1996, Ausschuss für Umweltschutz und Raumordnung, Aktuelle Viertelstunde, Stand der Planung und Umsetzung der Hochwasserschutzmaßnahmen im Verlauf des Rheines.

Landtag NRW, Vorlage 12/428, 01.03.1996, von MURL zur Vorlage an den Haushalts- und Finanzausschuss, Entwurf des Haushaltsgesetzes 1996.

NRW-Umweltministerin Höhn, 08.07.1996, Ministerium für Umwelt, Raumordnung und Landwirtschaft des Landes NRW, Brief an den Oberbürgermeister der Stadt Köln Norbert Burger.

Landtag NRW, Plenarprotokoll 12/38, 10.10.1996, Hochwasserschutz und NRW Untätigkeit der Landesregierung fordert nächste Katastrophe am Rhein heraus.

Landtag NRW, Drucksache 12/1577, 02.12.1996, Antwort auf die Kleine Anfrage 521 der Abgeordneten Hieronymi und Ley (CDU), Hochwasserschutz.

Landtag NRW, Ausschussprotokoll 12/651, 17.09.1997, Ausschuss für Umwelt und Raumordnung, Die Hochwasserkatastrophe in Brandenburg - Konsequenzen für NordrheinWestfalen.

Landtag NRW, Drucksache 12/2452, 07.10.1997, Antwort auf die Kleine Anfrage 815 der Abgeordneten Hieronymi und Ley (CDU), Hochwasserschutz für Köln und Bonn durch Rückhaltebecken am Oberrhein?

Landtag NRW, Vorlage 12/1725, 26.11.1997, von MURL zur Vorlage an den Haushalts- und Finanzausschuss, „Haushaltsgesetz 1998“.

Landtag NRW, Vorlage 12/1900, 29.01.1998, Vorlage an den Ausschuss für Umweltschutz und Raumordnung, Sprechzettel „Aktionsplan Hochwasser-Rhein“.

Landtag NRW, Drucksache 12/2870, 04.03.1998, Antwort der Landesregierung auf die große Anfrage 4 der Fraktion der CDU.

Landtag NRW, Plenarprotokoll 12/91, 18.06.1998, Antwort der Landesregierung auf die Große Anfrage 4 der Fraktion der CDU.

Landtag NRW, Plenarprotokoll 12/98, 04.11.1998, Antwort auf die Dringliche Anfrage 202 des Abgeordneten Stump (CDU), Konsequenzen aus der aktuellen Hochwasserkatastrophe. 


\section{Quellen des Archies NGO-Research-Center, Hanoi/Vietnam}

DR 002, 1992, UNDP Workshop, 22 - 25.06.1992, Hanoi. Proceedings of the International Workshop on Flood Mitigation, Emergency Preparedness and Flood Disaster Management, Vietnam, Ministry of Water Resources, Socialist Republic of Vietnam.

DR 004, 1994, United Nations, Strategy and Action Plan for Mitigating Water Disasters in Viet Nam, Department of Humanitarian Affairs (DHA), United Nations Development Programme (UNDP), Ministry of Water Resources of the Socialist Republic of Viet Nam, New York and Geneva.

DR 037, United Nations, Proceedings of the International Consultation of the Strategy and Action Plan for Mitigating Water Disaster in Viet Nam, 29 - 30.11.1994, Hanoi, Viet Nam.

DR 040, 1995, United Nations, First Update of the Strategy and Action Plan for Mitigating Water Disasters in Viet Nam, United Nations Development Programme (UNDP), Hanoi, Ministry of Water Resources of the Socialist Republic of Viet Nam, United Nations Department of Humanitarian Affairs, Geneva.

DR 041, 1997, February, Feasibility Study for A Disaster Rehabilitation Fund for Vietnam, United Nations Development Programme, Graham Alliband/Do Hong Phan.

AG 46, 1993, Assistance by the Food and Agriculture Organization of the United Nations to the State Planning Committee Socialist Republic of Vietnam, in preparation for the Round Table Meeting of Donors, November 1993, An Agricultural-Led Strategy for the Economic Transformation of Vietnam, Policy and Project Priorities, John Mellor.

\section{Quellen der Internationalen Kommission zum Schutz des Rheins}

Erklärung von Arles, 04.02.1995. Ergebnis des EU-Umweltministertreffens in Arles.

IKSR, PLEN 16/95, Bericht des Vorsitzenden der Projektgruppe Aktionsplan Hochwasser, 03/1995.

IKSR, PLEN 3/95 = K2/95 rev. 06.07.95, Arbeitsplan 1995 - 2000. Luxemburg, 06./07.07.1995.

IKSR, Presseerklärung, 06./07.07.1995, Hochwasserschutz auf dem Prüfstand.

IKSR, Tätigkeitsbericht 1995/1996.

IKSR, Beschlussprotokoll der 63. Vollversammlung der IKSR am 03./04.07.1997 in Schaffhausen.

IKSR, Luxemburg, PLEN 43/97. Beschlussprotokoll der außerordentlichen Plenarsitzung am 10.12.1997 in Echternach.

IKSR, Pressemitteilung, PLEN 42/97, Entwurf 09.01.1998, Aktionsplan Hochwasser für den Rhein fertiggestellt, Neue Rheinpolitik - jetzt auch im Dialog mit

Nichtregierungsorganisationen.

Aktionsplan Hochwasser, IKSR, Koblenz, 1998.

Rheinministertreffen, Rotterdam, 22.01.1998, Download: http://www.wwar.bayern.de/_zentral/doc/folgeseiten/hochwasser/aktionsplan_hw_rhein.pdf, eingesehen am 16.07.2012. 


\section{Deutsche Zeitungsberichte}

Hochwasser - Alarm am Rhein, Kölnische Rundschau, 17.01.1955.

Vater Rhein war schon oft wilder, Kölnische Rundschau, 18.01.1955.

Alarm beim Hochwasser-Schutz, Kölnische Rundschau, 18.01.1955.

Wird in Köln die 9-Meter-Grenze erreicht? Kölnische Rundschau, 18.01.1955.

Nä, nä - wo kütt bloß all dat vile Wasser her! Kölnische Rundschau, 19.01.1955.

Heute wird Höchststand erwartet, Kölnische Rundschau, 19.01.1955.

Das Hochwasser fällt, Kölnische Rundschau, 20.01.1955.

8.80 m über Null um 14 Uhr, Kölnische Rundschau, 20.01.1955.

Hochwasser ruft Verkehrswirrwarr hervor, Kölnische Rundschau, 20.01.1955.

Vater Rhein auf der Mülheimer Promenade, Kölnische Rundschau, 21.01.1955.

Deutz diesmal ohne Laufstege, Kölnische Rundschau, 21.01.1955.

Rheinuferstrasse wieder hochwasserfrei, Kölnische Rundschau, 22.01.1955.

Sie können sich nicht vorstellen, wie das hier aussah, Kölnische Rundschau, 24.01.1955.

Hundert auf einer Insel, Kölner Stadtanzeiger, 25.02.1970.

Sie verließen ihre Häuser doch, Kölner Stadtanzeiger, 25.02.1970.

Ungern umgezogen - Kaum jemand wollte in die Notunterkunft, Kölner Stadtanzeiger, 25.02.1970.

In der guten Stube schwimmen die Möbel, Kölner Stadtanzeiger, 28.02.1970.

Geschädigten muß geholfen werden, Kölner Stadtanzeiger, 28.02.1970.

Mehr als 100000 DM gespendet, Kölner Stadtanzeiger, 11.03.1970.

So wurde den Opfern geholfen, Kölner Stadtanzeiger, 29.04.1970.

Die Schifffahrt ab Köln ist gesperrt, Kölnische Rundschau, 12.04.1983.

Aufgemauert, Kölnische Rundschau, 13.04.1983.

Halten Wälle die Wellen? Kölnische Rundschau, 12. April 1983.

Die Kölner Altstadt überflutet, Kölnische Rundschau, 13.04.1983.

Am Dienstagabend: Statt „Dallas“ gab's nasse Füße, Kölnische Rundschau, 13.04.1983.

1784 war der höchste Rheinwasserstand, Kölnische Rundschau, 13.04.1983.

Ruhe und Gelassenheit, Kölnische Rundschau, 13.04.1983.

Rhein bald hinter höherer Mauer? Kölnische Rundschau, 14.04.1983.

Hochwasser, Kölnische Rundschau, 14.04.1983.

Katastrophe weitet sich noch aus, Kölnische Rundschau, 14.04.1983.

Alle Befürchtungen übertroffen, Kölnische Rundschau, 14.04.1983.

Nun läuft die braune Brühe ab, Kölnische Rundschau, 15.04.1983.

Hochwasser, Kölnische Rundschau, 15.04.1983. 
Nach dem Wasser fließt jetzt wieder das Kölsch, Kölnische Rundschau, 16.04.1983.

Meiner Meinung nach von Stefan Volberg, Kölnische Rundschau, 16.4.1983.

Hochwasser bedroht wieder Kölner Altstadt, Kölnische Rundschau, 28.05.1983.

Die Altstadt wird schon wieder naß, Kölnische Rundschau, 28.05.1983.

Schlimmstes Hochwasser seit 36 Jahren, Kölnische Rundschau, 30.05.1983.

Zukunft der Wirte droht zu ertrinken, Kölnische Rundschau, 30.05.1983.

Rückkehr zur Normalität, Kölnische Rundschau, 31.05.1983.

Aufatmen am Rhein, Kölnische Rundschau, 31.05.1983.

Katastrophe am Rhein, Kölnische Rundschau, 01.06.1983.

Bei Hochwasser Probleme mit Heizung und Campern, Kölnische Rundschau, 07.05.1983.

Landeshilfen für Opfer des Hochwassers, Kölnische Rundschau, 07.05.1983.

Rhein steht bedrohlich vor Häusern der Altstadt, Kölnische Rundschau, 28.03.1988.

Metallwand trennt die Altstadt von der Flut, Kölnische Rundschau, 28.03.1988.

Hochwasser ließ die Altstadt bis in die Nacht bangen, Kölnische Rundschau, 29.03.1988.

Auf- und Abbau der Schutzmauer ist am teuersten, Kölnische Rundschau, 30.03.1988.

Vater Rhein zieht sich weiter in sein Bett zurück, Kölnische Rundschau, 30.03.1988.

Zentimeter trennte die Altstadt von Überflutung, Kölnische Rundschau, 30.03.1988.

Polizeischutz für die Schutzwand, Kölnische Rundschau, 30.03.1988.

Ufermauer hat schon Höchstmaß, Kölnische Rundschau, 30.03.1988.

Köln macht Kanäle hochwassersicher, Kölnische Rundschau, 30.03.1988.

Meiner Meinung nach von M. Burkhard, Kölnische Rundschau, 30.03.1988.

Stadt war zu langsam, Kölnische Rundschau, 02.04.1988.

Hochwasserschutzwand gestern wieder abgebaut, Kölnische Rundschau, 07.04.1988.

Mit dem Wasserspiegel stieg die Angst, Kölnische Rundschau, 23.12.1993.

Der Rhein schaffte die Mauer, Kölnische Rundschau, 23.12.1993.

Jahrhundert - Hochwasser erreicht das Rheinland, Kölnische Rundschau, 23.12.1993.

Der Rhein schaffte die Mauer, Kölnische Rundschau, 23.12.1993.

Keine Entschädigung bei Überschwemmung, Kölnische Rundschau, 23.12.1993.

Mit dem Wasserspiegel stieg die Angst, Kölnische Rundschau, 23.12.1993.

Das Hochwasser treibt Tausende in die Flucht, Kölnische Rundschau, 24.12.1993.

Katastrophe hausgemacht, Kölnische Rundschau, 24.12.1993.

Mehr als tausend Helfer bekämpften Wassermassen, Kölnische Rundschau, 24.12.1993.

Das Hochwasser treibt Tausende in die Flucht, 24.12.1993.

Hochwassergeschädigte können auf Steuererleichterungen hoffen, Kölnische Rundschau 24.12.1993.

Flut kostet die Stadt Millionen, Kölnische Rundschau, 24.12.1993. 
Großeinsatz gegen Schlamm und eine Flut von Sperrgut, Kölnische Rundschau, 27.12.1993. Nach der Flut noch keine Pause, Kölnische Rundschau, 27.12.1993.

Nach Hochwasser beginnt jetzt das große Aufräumen, Kölnische Rundschau, 27.12.1993. Nach der Flut noch keine Pause, Kölnische Rundschau, 27.12.1993.

Rhein streckenweise für Schiffahrt wieder frei, Kölnische Rundschau, 28.12.1993.

Nadelöhr erweitern, Kölnische Rundschau, 29.12.1993.

Noch weiß keiner, wo die Millionen herzuholen sind, Kölnische Rundschau, 29.12.1993.

Komitee hilft Opfern der Katastrophe, Kölnische Rundschau, 30.12.1993.

Leben ohne Strom und ohne Heizung, Kölnische Rundschau, 30.12.1993.

Bitte der Stadt: Öltanks gegen Auftrieb sichern, Kölnische Rundschau, 08.01.1994.

Flut kostet die Kölner über 100 Millionen Mark, Kölnische Rundschau, 08.01.1994.

Grüne fordern: Weißer Bogen als Stauraum, Kölnische Rundschau, 08.01.1994.

Meiner Meinung nach von Volker Kutscher: Weißer Bogen, wer bezahlt den Schutz? Kölnische Rundschau, 10.01.1994.

Natur ist keine Maschine, sie paßt sich der Technik nicht an, Kölnische Rundschau, 10.01.1994.

Umdenken nach dem Hochwasser, Kölnische Rundschau, 11.01.1994.

Wohnungsbau bleibt Thema, Kölnische Rundschau, 15.01.1994.

Naturflächen am Rhein nicht weiter bebauen, Kölnische Rundschau, 23.02.1995.

Zum Wochenende steigt das Hochwasser dramatisch, Kölnische Rundschau, 24.01.1995.

Angst vor bedrohlicher Rheinflut, Kölnische Rundschau, 26.01.1995.

Rodenkirchen: Einsatz der neuen Hochwasserboote, Kölnische Rundschau, 26.01.1995.

Köln Hilfe verweigert: Flut in Altstadt, Kölnische Rundschau, 27.01.1995.

Kölns Altstadt erneut unter Wasser, Kölnische Rundschau, 28.01.1995.

Rheinufertunnel wurde am Abend geschlossen, Kölnische Rundschau, 28.01.1995.

Altstadttiefgaragen sind schon gesperrt, Kölnische Rundschau, 28.01.1995.

Panzerpioniere fahren Insulaner zum Einkaufen und zur Arbeit, Kölnische Rundschau, 30.01.1995.

Wasser im Keller wird Dauerzustand, Kölnische Rundschau, 30.01.1995.

Es kommt noch schlimmer, Kölnische Rundschau, 30.01.1995.

Bei 10,70 Meter läuft die Schäl Sick voll, Kölnische Rundschau, 30.01.1995.

Ehrenamtliche Helfer von Arbeit freistellen, Kölnische Rundschau, 30.01.1995.

Neue Gefahr: „Flut von unten“ bedroht mehr als 300.000 Kölner, Kölnische Rundschau, 31.01.1995.

Jahrhundertflut: Höher als 1926, Kölnische Rundschau, 31.01.1995.

Wir müssen an unsere eigenen Städte denken, Kölnische Rundschau, 31.01.1995.

Solidarabgabe an die Flutopfer? Kölnische Rundschau, 31.01.1995. 
Nach der Flut stehen viele vor dem Nichts, Kölnische Rundschau, 31.01.1995.

Rekord-Flut geht langsam zurück, Kölnische Rundschau, 01.02.1995.

Gemeinsam die Not der Opfer lindern, Kölnische Rundschau, 01.02.1995.

Arbeitslose zum Flut-Einsatz, Kölnische Rundschau, 01.02.1995.

Am ersten Tag Spenden über 100.000 Mark, Kölnische Rundschau, 01.02.1995.

Hilfswelle nach Aufruf zu Spenden in der Rundschau, Kölnische Rundschau, 01.02.1995.

Leserbrief von Walter Heilmann, Bergisch-Gladbach, Kölnische Rundschau 01.02.1995.

Hochwasserkredite werden gewährt, Kölnische Rundschau, 02.02.1995.

Aktuelle Stunde zum Thema Überflutung, Kölnische Rundschau, 02.02.1995.

Versiegelung von großen Flächen, Kölnische Rundschau, 02.02.1995.

Schutz vor Hochwasser: Rasche Lösung Utopie, Kölnische Rundschau, 07.02.1995.

Hochwasser, Köln entging nur knapp der Katastrophe, Kölnische Rundschau, 09.02.1995.

Die ersten Keller sind schon abgedichtet, Kölner Stadtanzeiger, 24.01.1995.

Flutwelle überschwemmt heute die Kölner Altstadt, Kölner Stadtanzeiger, 27.01.1995.

Das Bange Warten auf die Flut in Köln, Kölner Stadtanzeiger, 28./29.01.1995.

Polder am Oberrhein bleiben dicht, Kölner Stadtanzeiger, 28./29.01.1995.

Polder für Köln, Kölner Stadtanzeiger, 28./29.01.1995.

Wir brauchen ein länderübergreifendes Management, auch mit Frankreich und Holland, Kölner Stadtanzeiger, 31.01.1995.

Hilfspakete werden geschnürt, Kölner Stadtanzeiger, 01.02.1995.

240000 Holländer fliehen vor der Flut, Kölner Stadtanzeiger, 01.02.1995.

Im Sommer neues Konzept, Kölner Stadtanzeiger, 01.02.1995.

Viele Polder sind bis heute nicht gebaut, Kölner Stadtanzeiger, 02.02.1995.

Für den Rhein wird ein Konzept gesucht, Kölner Stadtanzeiger, 03.02.1995.

Keine niedrigen Zinsen für Privatleute, Kölner Stadtanzeiger, 03.02.1995.

Merkel will Ökosysteme sichern, Kölner Stadtanzeiger, 03.02.1995.

Beratung für Flutopfer auch am Wochenende, Kölner Stadtanzeiger, 03.02.1995.

Flut-Schäden diesmal „nur“ 65 Millionen DM, Kölner Stadtanzeiger, 09.02.1995.

Hochwasser-Opfer können jetzt Mittel beantragen, Kölner Stadtanzeiger, 18./19.02.1995.

Kohl erklärt die Fluten zur Chefsache, Rhein-Lahn-Zeitung, 31.01.1995.

Aktion gegen Hochwasser geplant, Rhein-Lahn-Zeitung, 06.02.1995.

Warten auf die Schlammschlacht, Süddeutsche Zeitung, 05.06.2013.

Rösler fordert Hilfsfonds für Flutopfer, Süddeutsche Zeitung, 11.06.2013.

Deutschlands teuerste Naturkatastrophe, Süddeutsche Zeitung, 09.07.2013.

Mehr Umweltpolitik wagen, Süddeutsche Zeitung, 16.07.2013. 
Vietnamesische Zeitungsberichte (Die Zeitungsberichte wurden durch Nuyen Thi Bich Tram übersetzt.)

Toan tinh xuong duoc 116.194 ha lua dong xuan, dat $65 \%$ dien tich ke hoach (116.194 ha of Winter-Spring Crop has been seeded, reaching 65\% of the Planned Area), Zeitung An Giang, 29.12.1994.

An Giang voi giai phap chong o nhiem moi sinh (An Giang with Solutions to prevent Pollution of Human Society), Zeitung An Giang, 16.03.1995.

Chuyen dich co cau cay trong don bay de phat trien kinh te (Restructuring Plants - Lever of Economic Development), Zeitung An Giang, 27.05.1995.

Vua chong lu vua noi lien giao thong (Road on Dyke System at Canal Moi in An Phu to confront Foods and to connect Roads), Zeitung An Giang, 19.07.1995.

Doi Moi cach phoi say lua vu he thu: trach nhiem cua nong dan va chinh quyen cac cap (The Innovation of Summer-Autum Rice Drying: Responsebilities of different Levels of Government), Zeitung An Giang, 26.07.1995.

Nhin lai va vuon len (Looking back and Rising up), Zeitung An Giang, 06.09.1995.

Xay dung de bao chong lu o Cho Moi, Zeitung An Giang (Building a Dyke System in Cho Moi), Zeitung An Giang, 06.09.1995.

Lu dong bang song Cuu Long len cao va co kha nang lan rong (Flood in Mekong Delta rise high and have Ability to spread out), Zeitung An Giang, 25.07.2000.

Lu dong bang song Cuu Long len cao va co kha nang lan rong (Overcoming Difficulties, copping with Floods), Zeitung An Giang, 25.07.2000.

Tham sam tinh quan - dan (The good Relationship between Military and People), Zeitung An Giang, 01.08.2000.

Khu vuc co nguy co sat lo o Tan Chau: Thuc trang va giai phap (The Areas are at Risk of Landslides in Tan Chau: Situation and Solution), Zeitung An Giang, 01.08.2000.

Sang mai tinh quan dan trong nhung ngay chong lu (Beautiful Images about the Relationship between Soldiers and People in preventing Flood's Days), Zeitung An Giang, 01.08.2000.

Cong tac phong chong lu duoc tiep tuc dat trung tinh trang bao dong (Floods Prevention in continued to place in the Alert), Zeitung An Giang, 08.08.2000.

An Giang truoc nhung yeu cau moi trong phong, chong lu (An Giang before new Requirements in Flood Prevention and Control), Zeitung An Giang, 15.09.2000.

Thien tai lu lut o An Giang: Nhung manh doi dang rat can su giup do (Flood in An Giang: Lives in Need of Help), Zeitung An Giang, 15.09.2000.

\section{Can Tho Stadt Bibliothek}

Tong 1997 - N. V. Tong, Phan Tich Tinh Hinh Kinh Te Xa Hoi Tinh An Giang 1991 - 1995 (Analysis of economical-social Status of An Giang Province 1991 - 1995), Bo Giao Duc Va Dao Tao Trung Dai Hoc Kinh Te Thanh Pho Ho Chi Minh (Ministry of Education and Taining Ho Chi Minh City University of Economics), Bibliothek der Stadt Can Tho, Archiv der Weltbank, 333.9597, PH 121T, übersetzt durch Nuyen Thi Bich Tram, Juli 2013. 


\section{$\underline{\text { Interviews }}$}

- Reinhard Vogt, Leiter der Hochwasserschutzzentrale Köln, am 17.12.2011 und 09.08.2012 in Köln.

- Anne Schulte-Wülwer-Leidig, Stellvertretende Geschäftsführerin der Internationalen Kommission zum Schutz des Rheines (IKSR), am 06.08.2012 in Koblenz.

- Prof. Dr. Nguyen Van Be, Collage of Environment and Natural Resources, Head of Dragon Institute, Can Tho University, im März 2012 in Can Tho, Vietnam.

- Dr. Van Pham Dang Tri, Integrated Water Resources Management, Vulnerabilities Management and Risk Modelling, Can Tho University, Collage of Environment and Natural Resources, im März 2011 in Can Tho, Vietnam.

- John Douglas Flanders, von 2000 bis 2007 bei der Australian Agency for International Development und Programmleiter des Ringdeich-Projekts „North Vam Nao Water Control Project" in An Giang, im März 2012 in Long Xuyen.

\section{$\underline{\text { Literatur }}$}

Abels $2004-$ H. Abels, Einführung in die Soziologie, Band 2: Der Blick auf die Gesellschaft, 2 Auflage, (Wiesbaden 2004).

Apel 1996 - K.-O. Apel, Die Vernunftfunktion der kommunikativen Rationalität. Zum Verhältnis von konsensual-kommunikativer Rationalität, strategischer Rationalität und Systemrationalität. In: Ders./M. Kettner (Hrsg.), Die eine Vernunft und die vielen Rationalitäten (Frankfurt/Main 1996) 17-41.

Backer 2007 - E. B. Backer, The Mekong River Commission: Does it Work, and How Does the Mekong Basin's Geography Influence Its Effectiveness? Südostasien aktuell, 2007/4, 31 55 .

Bankoff 2003 - G. Bankoff, Cultures of Disaster, Cultures of Coping: Hazard as a Frequent Life Experience in the Philippines. In: Ch. Mauch/Ch. Pfister (Hrsg.), Natural Disasters, Cultural Responses. Case studies toward a Global Environmental History. International Environmental History. Publication of the German Historical Institute (Lanham - Boulder New York - Toronto - Plymouth 2009) 265-284.

Becker 1998 - F. Becker (Hrsg.), Konrad Adenauer. Die Demokratie ist für uns eine Weltanschauung. Reden und Gespräche (1946-1967). Konrad-Adenauer Stiftung (Köln Weimar - Wien 1998).

Benedikter/Waibel 2013 - S. Benedikter, G. Waibel, The formation of water user groups in a nexus of central directives and local administration in the Mekong Delta, Vietnam. Working Paper 112. Zentrum für Entwicklungsforschung, Universität Bonn (Bonn 2013).

Biggs 2004 - D. Biggs, Between the Rivers and Tides: A Hydraulic History of the Mekong Delta, 1820-1975 (Washington 2004).

Biggs 2010 - D. Biggs, Quagmire. Nation-Building and Nature in the Mekong Delta (Seattle 2010).

Blaikie et al. 1994 - P. Blaikie, T. Cannon, I. Davis, B. Wisner, At Risk: Natural hazards, People's vulnerability, and disasters (London 1994). 
Borst 1981 - A. Borst, Das Erdbeben von 1348. Ein historischer Beitrag zur Katastrophenforschung. Hist. Zeitschr. 233/3, 1981, 529-569.

Brixius 2004 - V. Brixius, Der Rheinpark in Köln. Geschichte einer Gartenanlage von 1914 bis heute, Bd. 1. Unveröff. Dissertation Universität Köln (Köln 2004).

Browder/Ortolando 2000 - G. Browder, L. Ortolando, The Evolution of an International Water Resource Management Regime in the Mekong River Basin. Natural Resources Journal 40, 2000, 499-531.

Bryant/Bailey 1997 - R. L. Bryant, S. Bailey, Third World Political Ecology (London - New York 1997).

Cutter et al. 2000 - S. L. Cutter, J. T. Mitchell, M. S. Scott, Revealing the Vulnerability of People and Places: A Case Study of Georgetown County, South Carolina. In: Annals of American Geographers 90(4), 2000, 713-737.

Danh/Mushtag 2011 - V. T. Danh, S. Mushtag, Living with floods: An Evaluation of the Resettlement Program of the Mekong Delta of Vietnam. In: M. A. Stewart/P. A. Coclanis (Hrsg.), Environmental Change and Agricultural Sustainability in the Mekong Delta. Advances in Global Change Research 45 (Heidelberg - London - New York 2011) 181-204.

Dehnhardt et al. 2008 - A. Dehnhardt, D. Drünkler, H. Engel, M. Hammer, J. Hirschfeld, U. Petschow, Kosten-Nutzen-Analysen von Hochwasserschutzmaßnahmen. Bundesumweltamt, Text 31/08 (Dessau-Roßlau 2008).

Deutsch 2007 - M. Deutsch, Untersuchungen zu Hochwasserschutzmaßnahmen an der Unstrut (1500-1900). Göttinger Geographische Abhandlungen 117 (Göttingen 2007).

Downs/Stea 1982 - R. M. Downs, D. Stea, Kognitive Karten. Die Welt in unseren Köpfen (New York 1982).

Döring 1987 - E. Döring, Karl R. Popper, Einführung in Leben und Werk. (Hamburg 1987).

Ehlert 2012 - J. Ehlert, Beautiful Floods. Environmental Knowledge and Agrarian Change in the Mekong Delta, Vietnam. ZEF Development Studies 19 (Zürich - Berlin 2012).

Engel 1999 - H. Engel, Eine Hochwasserperiode im Rheingebiet. Extremereignisse zwischen Dez. 1993 und Febr. 1995. Internationale Kommission für die Hydrologie des Rheingebietes. Bericht 1/17 (Koblenz 1999).

Esser 1990 - H. Esser, „Habits“, „Frames“ und „Rational Choice“. Die Reichweite von Theorien der rationalen Wahl. In: Zeitschrift für Soziologie, Jg. 19, 1990/4, 231-247.

Evers/Benedikter 2009 - H.-D. Evers, S. Benedikter, Strategic Group Formation in the Mekong Delta - The Development of a Modern Hydraulic Society. Working Paper 35. Zentrum für Entwicklungsforschung, Universität Bonn (Bonn 2009).

Fekete 2009 - A. Fekete, Assessment of Social Vulnerability to River Floods in Germany. Graduate Research Series 4 (Bonn 2009).

FitzGerald 1978 - E. V. K. FitzGerald, Public Sector Investment Planning for Developing Countries (London 1978).

Frielingsdorf 2001 - V. Frielingsdorf, Auf den Spuren Konrad Adenauers durch Köln. Konrad Adenauers Wirken als Oberbürgermeister von Köln (1917 - 1933 und 1945). Dokumentiert in zehn Stationen und ausgewählten Zeugnissen seiner Zeit. Gedenkschrift der Stadt Köln zum 125. Geburtstag ihres Ehrenbürgers am 5. Januar 2001 (Köln 2001).

Gert 1996 - B. Gert, Substantielle Rationalität. In: K.-O. Apel/M. Kettner (Hrsg.), Die eine Vernunft und die vielen Rationalitäten (Frankfurt/Main 1996) 318-348. 
Hanley/Spash 2003 - N. Hanley, C. L. Spash, Cost-Benefit Analysis and the Environment (Cheltenham - Northampton 2003).

Heyder 2007 - M. Heyder, KulturSchock Vietnam (Bielefeld 2007).

Howie 2006 - C. Howie, High dykes in An Giang Province, Vietnam, bring environmental pains but social gains. Tropical Agriculture Association Newsletter 26/1, 2006, 29-32.

Howie 2011 - C. Howie, Dike Building and Agricultural Transformation in the Mekong Delta, Vietnam: Dilemmas in Water Management. Online-Publikation: http://www.vietecology.org/Article.aspx/Article/85 (eingesehen am 25.06.2013).

Huu 2011 - P. C. Huu, Planning and Implementation of the Dyke Systems in the Mekong Delta, Vietnam. Unveröff. Dissertation Rheinische Friedrich-Wilhelms-Universität Bonn (Bonn 2011).

Koester/Tangermann 1976 - U. Koester, S. Tangermann, Alternativen der Agrarpolitik. Eine Kosten-Nutzen-Analyse im Auftrag des Bundesministeriums für Ernährung, Landwirtschaft und Forsten. Landwirtschaft-Angewandte Wissenschaft, Heft 182 (Münster-Hiltrup 1976).

Kron 2001 - W. Kron, Versicherung von Hochwasserschäden. In: H. Patt (Hrsg.), Hochwasser-Handbuch. Auswirkungen und Schutz (Berlin u. a. 2001) 461-504.

Lebel/Sinh 2009 - L. Lebel, B. T. Sinh, Risk reduction or redistribution? Flood management in the Mekong region. Asian Journal of Environment Disaster Management 1, 2009, 23-39.

Lebel et al. 2009 - L. Lebel, B. T. Sinh, P. Garden, S. Seng, L. A. Tuan, D. V. Truc, The Promise of Flood Protection: Dikes and Dams, Drains and Diversions. In: F. Molle/T. Foran/M. Käkönen (Hrsg.), Contested Waterscapes in the Mekong Region. Hydropower, Livelihoods and Governance (London - Washington 2009) 283-306.

Masius/Sprenger/Mackowiak 2010 - P. Masius, J. Sprenger, E. Mackowiak, (Hg.) Katastrophen machen Geschichte (Göttingen 2010).

Masius 2010 - P. Masius Naturkatastrophen in der Geschichte: Begegnungen zwischen Machtlosigkeit und Machbarkeit. In P. Masius, J. Sprenger, E. Mackowiak, (Hg.) Katastrophen machen Geschichte (Göttingen 2010) 153 - 171.

Marggraf/Streb 1997 - R. Marggraf, S. Streb, Ökonomische Bewertung der natürlichen Umwelt. Theorie, politische Bedeutung, ethische Diskussion (Heidelberg - Berlin 1997).

Marggraf et al. 2005 - R. Marggraf, Ökonomische Grundlagen der Umweltbewertung. In: Ders./I. Bräuer/A. Fischer/S. Menzel/U. Stratmann/A. Suhr (Hrsg.), Ökonomische Bewertungen bei umweltrelevanten Entscheidungen. Einsatzmöglichkeiten von Zahlungsbereitschaftsanalysen in Politik und Verwaltung (Marburg 2005) 61-83.

Mauch 2009 - Ch. Mauch, Introduction. In: Ders./Ch. Pfister (Hrsg.), Natural Disasters, Cultural Responses. Case studies toward a Global Environmental History. International Environmental History. Publication of the German Historical Institute (Lanham - Boulder New York - Toronto - Plymouth 2009) 1-16.

Miller 2003 - F. Miller, Society-Water Relations in the Mekong Delta: A Political Ecology of Risk. Unveröff. Dissertation, Universität Sydney (Sydney 2003).

Nguyen 2007 - H. N. Nguyen, Flooding in Mekong River Delta, Viet Nam. Human Development Report 53 (Hanoi 2007).

Nida-Rümelin 1996 - J. Nida-Rümelin, Zur Einheitlichkeit praktischer Rationalität. In: K.-O. Apel/M. Kettner (Hrsg.), Die eine Vernunft und die vielen Rationalitäten (Frankfurt/Main 1996) 73-90. 
Nida-Rümelin 2009 - J. Nida-Rümelin, Politische Philosophie der Gegenwart. Rationalität und politische Ordnung (Köln - Weimar - Wien 2009).

Niedermaier 2004 - P. Niedermaier, Gepräge im Wandel: Die mittelalterliche Stadt im Südosten Europas. In: F. Opll (Hrsg.), Bild und Wahrnehmung der Stadt. Beiträge zur Geschichte der Stadt Mitteleuropas 19 (Linz 2004) 119-142.

Oelmann 2008 - H. Oelmann, Die Rheinflut. Der Kölner Hochwasserschutz (Köln 2008).

Pfister 2004 - Ch. Pfister, Von Goldau nach Gondo - Naturkatastrophen als identitätsstiftende Ereignisse in der Schweiz des 19. Jahrhunderts. In: Ch. Pfister/S. Summermatter (Hrsg.), Katastrophen und ihre Bewältigung. Perspektiven und Positionen. Berner Universitätsschriften 49 (Bern 2004) 53-78.

Pfister 2008 - Ch. Pfister, Disasters, interregional solidarity and nation-building: Reflections on the case of Switzerland, 1806-1914. In: R. Favier/Ch. Pfister (Hrsg.), Solidarité et assurance. Les sociétés européennes face aux catastrophes (17e-21e siècle) (Grenoble 2008) $117-141$.

Pfister 2009 - Ch. Pfister, Learning from Nature-Induced Disasters: Theoretical Considerations and Case Studies from Western Europe. In: Ch. Mauch/Ch. Pfister (Hrsg.), Natural Disasters, Cultural Responses. Case studies toward a Global Environmental History. International Environmental History. Publication of the German Historical Institute (Lanham - Boulder - New York - Toronto - Plymouth 2009) 17-40.

Pfister et al. 2010 - Ch. Pfister, E. Garnier, M.-J. Alcoforado, D. Wheeler, J. Luterbacher, M. Nunes, J. Taborda, The meteorological framework and the cultural memory of three severe winter-storms in early eighteenth-century Europe. Climatic Change 101/1-2, 2010, 281-310.

Poliwoda 2007 - G. Poliwoda, Aus Katastrophen Lernen. Sachsen im Kampf gegen die Fluten der Elbe 1784 bis 1845 (Köln - Weimar - Wien 2007).

Radkau 2011 - J. Radkau, Die Ära der Ökologie. Eine Weltgeschichte (München 2011).

Ravallion/van de Walle 2001 - M. Ravallion, D. van de Walle, Breaking up the Collective Farm: Welfare Outcomes of Vietnam's Massive Land Privatization. Policy Research Working Paper 2710 (Washington 2001).

Rheinpark 2007 - Der Rheinpark. Ein Rundgang durch die schönste Gartenanlage Deutschlands. Amt für Landschaftspflege und Grünflächen, Amt für Presse- und Öffentlichkeitsarbeit der Stadt Köln (Köln 2007).

Rohr 2004 - Ch. Rohr, Überschwemmungen an der Traun zwischen Alltag und Katastrophe: die Welser Traunbrücke im Spiegel der Bruckamtsrechnungen des 15. und 16. Jahrhunderts. Jahrbuch des Musealvereines Wels 33, 2004 (2001-2003), 281-327.

Sanh/Can 2009 - N. V. Sanh, N. D. Can, Study on local community institutions to cope with the flood situation of the Mekong region. The Sustainable Mekong Research Network. Mekong Delta Development Research Institute (Can Tho 2009).

A. Schmidt 1978 - A. Schmidt, Der Begriff der Natur in der Lehre von Marx (Frankfurt/Main 1978).

M. Schmidt 2000 - M. Schmidt, Hochwasser und Hochwasserschutz in Deutschland vor 1850. Eine Auswertung alter Quellen und Karten (München 2000).

Schmied-Kowarzik 1984 - W. Schmied-Kowarzik, Das dialektische Verhältnis des Menschen zur Natur. Philosophiegeschichtliche Studien zur Naturproblematik bei Karl Marx (Freiburg München 1984). 
Schnädelbach 2000 - H. Schnädelbach, Rationalitätstypen. Philosophie in der modernen Kultur (Frankfurt/Main 2000).

Schofield 1989 - J. A. Schofield, Cost-Benefit Analysis in Urban \& Regional Planning (London 1989).

Schweizer 1992 - Th. Schweizer, Interkulturelle Vergleichsverfahren. In: H. Fischer (Hrsg.), Ethnologie. Einführung und Überblick (Berlin - Hamburg 1992) 421-439.

Siebenhühner 2002 - B. Siebenhühner, Gesellschaftliches Lernen und kollektive Entscheidungsfindung im Prozess der Nachhaltigkeit. Contribution to the conference "Governance and Sustainability - new Challenges for the State, Business and Civil Society". Institute for ecological economy research, 30.09.-01.10.2002, Berlin.

Spiegel 1995 - E. Spiegel, Heimat. In: Akademie für Raumforschung und Landesplanung (Hrsg.), Handwörterbuch der Raumordnung (Hannover 1995) 467.

Staudacher et al. 2011 - P. Staudacher, A. Diès, F. Schleiermacher, Friedrich F. Eigler (Hrsg.), 6. Aufl., Theaitetos. Der Sophist. Der Staatsmann (Darmstadt 2011).

Stolber 1968 - W. Stolber, Nutzen-Kosten Analysen in der Staatswirtschaft.

Wasserwirtschaftliche Projekte (Göttingen 1968).

Tanh/Singh 2006 - N. C. Tanh, B. Singh, Trend in Rice Production and Export in Vietnam. Omonrice 14, 2006, 111-123.

Tuan 2007 - L. A. Tuan, Children Security as part of the „Living with Floods“ Strategy in the Mekong River Delta, Vietnam. International Development Studies Conference „Mainstreaming Human Security: the Asian Contribution“, Bangkok, 4./5. Oktober 2007.

Tuan et al. 2007 - L. A. Tuan, C. T. Hoanh, F. Miller, B. T. Sinh, Chapter 1: Floods and Salinity Management in the Mekong Delta, Vietnam. Major Reports and Working Papers. The Sustainable Mekong Research Network (2007).

Turner et al 2003 - B. L. Turner, R.E. Kasperson, P.A. Matson, J.J. McCarthy, R.W. Corell, L. Christensen, N. Eckley, J.X. Kasperson, A. Luers, M.L. Martello, C. Polsky, A. Pulsipher and A. Schiller, A framework for Vulnerability Analysis in Sustainability Science. In: Proceedings of the National Academy of Sciences 100(14), 2003, 8074-8079.

TRANS Magazin, 1991, Wenn der Rhein kommt, Stadt Köln, Presse und Informationsamt (Köln 1991).

Vormoor 2010 - K. Vormoor, Water Engineering, Agriculture Development and Socio-

Economic Trends in the Mekong Delta, Vietnam. Working Paper 57. Zentrum für

Entwicklungsforschung, Universität Bonn (Bonn 2009).

Wagner 2008 - K. Wagner, Der Risikoansatz in der europäischen

Hochwassermanagementrichtlinie. Natur und Recht 30/11, 2008, 774-779.

Waibel 2010 - G. Waibel, State Management in Transition: Understanding the Water

Resources Management in Vietnam. Working Paper 55. Zentrum für Entwicklungsforschung, Universität Bonn (Bonn 2010).

Weichselgartner 2000 - J. Weichselgartner, Hochwasser als soziales Ereignis, Gesellschaftliche Faktoren einer Naturgefahr. In: Hydrologie und Wasserwirtschaft, 2000, 44, H.3. 122 - 131.

Weichselgartner 2001 - J. Weichselgartner, Disaster mitigation: the concept of vulnerability revisited. In: Disaster Prevention and Management 2001, 10(2), 85-94. 
Umweltbundesamt 2003, Sichern und Wiederherstellen von Hochwasserrückhalteflächen, http://www.umweltdaten.de/rup/fallstudie-koeln.pdf (eingesehen am 16.07.12).

White 2002 - I. White, Water Management in the Mekong Delta: Changes, Conflicts and Opportunities. Technical Documents in Hydrology 61 (Paris 2002).

Wüstenhube 1999 - A. Wüstenhube, Rationalität und Hermeneutik, Zur Rationalität der Hermeneutik für einen Begriff pragmatischer Rationalität. In: Rationalitätstypen, K. Gloy (Hrsg.), 1999.

\section{$\underline{\text { Internetbasierte Daten }}$}

Internetbasierte Quelle 1: Eingesehen am 13.08.2012, Homepage der Bundesstiftung Baukultur: http://www.bundesstiftungbaukultur.de/service/presse/pressemeldungen/pressemitteilung-detail/article/pressemitteilungunternehmen-oeffnen-sich-dem-thema-baukultur-bsbk-und-partner-zeichnen-in-koe.html.

Internetbasierte Quelle 2: Eingesehen am 13.08.2012, Homepage des Hochwasserkompetenzzentrums: http://www.hkc-koeln.de/de/projekte/projekt1/fallbeispiele/koeln/mobiler-hochwasserschutz/index.html.

Internetbasierte Quelle 3: Eingesehen am 13.08.2012, Homepage der Stadtentwässerungsbetriebe: http://www.steb-koeln.de/presse_archiv.html.

Internetbasierte Quelle 4: Eingesehen am 16.07.2012, Homepage Landesamt für Natur, Umwelt und Verbraucherschutz NRW, Naturschutzgebiet Worringer Bruch, Schutzwürdige Biotope, http://www.naturschutzinformationennrw.de/nsg/de/fachinfo/gebiete/gesamt/K_009.

Internetbasierte Quelle 5: Eingesehen am 13.08.2012, Homepage der Baukunst NRW: http://www.baukunst-nrw.de/objekte/hw-rod-Lagerstaette-fuer-HochwasserschutzelementeKoeln-Rodenkirchen--2335.htm.

Internetbasierte Quelle 6: Eingesehen am 05.07.2012, Homepage der Stadtentwässerungsbetriebe Köln AöR.:http://www.steb-koeln.de/hochwasser.html.

Internetbasierte Quelle 7: Eingesehen am 04.06.2013, Homepage der Hochwasserschutzzentrale Köln: http://www.hw-karten.de/koeln/.

Internetbasierte Quelle 8: Eingesehen am 28.01.2013, Homepage des Dragon Institute, http://www.ctu.edu.vn/institutes/dragon/dragon_eng/index.php.

Internetbasierte Quelle 9: Eingesehen am 03.09.2013, Bilderbuch Köln: Cwww.bilderbuchkoeln.de.

Internetbasierte Quelle 10: Eingesehen am 22.03.2013, OpenSourceMap: http://open.mapquest.de/?q1=50.93319,6.96240\&vs=directions.

Internetbasierte Quelle 11: Eingesehen am 20.09.2013, Homepage der Mekong River Commission: http://portal.mrcmekong.org/gallery/lower-mekong-basin-1968-atlas-series$1.0 \# 2$.

Internetbasierte Quelle 12: Eingesehen am 20.09.2013, Homepage der Internationalen Kommission zum Schutz des Rheins: http://www.iksr.org/index.php?id=13.

Internetbasierte Quelle 13, Eingesehen am 08.04.2013, Homepage der Bundesgartenschau: http://www.bundesgartenschau.de/buga-iga/bisherige-ausstellungen/buga-koeln-1957/. 
Internetbasierte Quelle 14: Eingesehen am 14.08.2013, Homepage der Australian Agency for International Development:

http://www.ausaid.gov.au/HotTopics/Pages/Display.aspx?QID=236.

Internetbasierte Quelle 15: Eingesehen am 05.09.2013, Homepage des Central Committee for Flood and Storm Control: http://www.ccfsc.gov.vn/KW367A21/Trang-chu.aspx. 


\section{Lebenslauf Nicole Kronenberg}

Schulische Laufbahn, Berufsausbildung und Freiwilligendienst

Studium

Johannisstraße 3-4

37073 Göttingen

nkronen@gwdg.de

Staatsangehörigkeit: deutsch

Familienstand: ledig

Führerschein: Klasse B

Geburtsort und -datum: 02.03.1982 in Düren

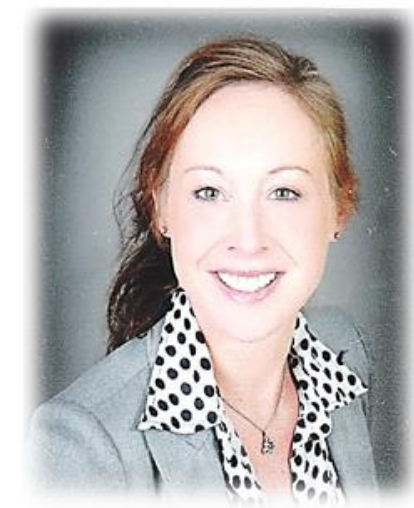

$08 / 92-06 / 98$

Realschule, St. Angela Schule der Ursulinen in Düren

$08 / 98-06 / 00$

Berufskolleg Kaufmännische Schulen des Kreises

Düren, zweijährige Höhere Handelsschule für

Wirtschaft und Verwaltung

09/00 - 06/02 Abgeschlossene Berufsausbildung zur Bankkauffrau, Aachener Bausparkasse AG, Aachen

09/02 - 08/03 European Voluntary Service (EVS) in der Jüdischen

Gemeinde zu Breslau/Polen

09/03 - 08/08

Diplom Wirtschaftsingenieurin (FH) der Umweltplanung, Fachhochschule Trier, UmweltCampus Birkenfeld. Titel der Diplomarbeit: „Innovation and Obstacles in the Circular Economy System of Automobile Recycling - Investigating Political, Economical and Ecological Aspects" am Wuppertal Institut für Klima, Umwelt und Energie $\mathrm{GmbH}$, Forschungsgruppe Stoffstrom- und Ressourcenmanagement, Wuppertal, 03/08-08/08

09/06 - 09/08 Master of Sciences in International Cooperation Policy in Asia Pacific Studies, Ritsumeikan Asia Pacific University, Oita/Japan. Titel der Masterarbeit: „Automobile Recycling under a Material Flow Perspective in Germany and Japan" am Wuppertal Institut für Klima, Umwelt und Energie $\mathrm{GmbH}$, Forschungsgruppe Stoffstrom- und Ressourcenmanagement, Wuppertal, 03/08 - 08/08

09/06 - 03/09 Master of Engineering in International Material Flow Management, Fachhochschule Trier, Umwelt-Campus Birkenfeld. Titel der Masterarbeit: „Resource Efficiency and Decoupling Indicators contributing to Green House Gas Mitigation Strategies" im Sektorvorhaben Ressourceneffizienz, Abteilung Umwelt und Klima, GIZ, Eschborn, 10/08 - 12/08

Stipendiatin

$06 / 09-04 / 10$

Heinz-Nixdorf Stipendiatin, davon 8 Monate in Hanoi/Vietnam im GIZ-Projekt zur Verbesserung der energiepolitischen Rahmenbedingungen für erneuerbare Energien beschäftigt 
Promotion

Beruf

Berufspraktische Erfahrung im Bereich:

- Klimaschutz

- $\quad$ Beratung

- Politik

Soziales Engagement und studentische Initiativen

Sprachkenntnisse
$07 / 10-11 / 13$

$02 / 14$ - heute

09/12 - heute

Mitglied des Foresight Prozesses, „die Junge Perspektive", Fraunhofer-Institut für System- und Innovationsforschung ISI, Karlsruhe

$10 / 04-12 / 04$

09/06 - 09/07

04/07 - 09/07

08/07 - 09/07

09/03 - heute

10/03 - 02/04

04/04 - 07/04

07/05 - 01/06

$07 / 10-07 / 11$

09/13 - heute

Georg-August-Universität Göttingen,

Agrarwissenschaften, Department für Agrarökonomie und Rurale Entwicklung, Abteilung Umwelt- und Ressourcenökonomik. Stipendiatin im DFG-

Graduiertenkolleg 1024 „Interdisziplinäre Umweltgeschichte“, Titel der Promotion: „Nach dem Hochwasser ist vor dem Hochwasser: Zur Rationalität politischer Entscheidungsprozesse in Deutschland und Vietnam im späten 20. Jahrhundert"

Projektmanagerin Klimaschutz/Energie in der NRWLandesgesellschaft Expo Fortschrittsmotor Klimaschutz GmbH, Gelsenkirchen

Wissenschaftliche Hilfskraft im Bereich Eco City Development, Institut für angewandtes Stoffstrommanagement (IfaS), Birkenfeld

Forschungsarbeit „Null-Emissions-Siedlung UmweltCampus-Birkenfeld", nachhaltig Energie- und Abfallsysteme zu kombinieren und zu finanzieren

Analyse der klimarelevanten Material- und EnergieStoffstromflüsse des Campus der Ritsumeikan Asia Pacific University in Oita/Japan

Praktikum im Projekt „Perspektiven dezentraler Infrastrukturen im Spannungsfeld von Wettbewerb, Klimaschutz und Qualität" zur Beratung kommunaler Servicedienstleister im Bereich Abfall, Forschungsgruppe Stoffstrom- und Ressourcenmanagement, Wuppertal Institut für Klima und Umwelt, Energie $\mathrm{GmbH}$, Wuppertal

Diakonie Hannover, Abteilung Diakonisches Jahr im Ausland in Form von Infotagen und Seminaren

Tutorin im Fach Anorganische Chemie Tutorin im Fach Rechnungswesen

EU-Erasmus-Programm an der Akdeniz University, Fakultät für Maschinenbau, Antalya/Türkei

Sprecherin des DFG-Graduiertenkollegs 1024 Interdisziplinäre Umweltgeschichte

Engagement bei der Plattform Arbeiterkind.de

Englisch (verhandlungssicher)

Vietnamesisch

Türkisch

Polnisch

Nicole Kronenberg, Gelsenkirchen den 11.10.2014 